Historic, archived document

Do not assume content reflects current scientific knowledge, policies, or practices. 



\section{ASFGOI}

USDA

Alll National Poultry

United States

Department of Improvement Plan

Agriculture

Animal and

Plant Health

Inspection

Service

APHIS 91-55-069

2003 Directory of Participants

March 2003 Handling Waterfowl, Exhibition Poultry, Game Birds, and Ratites
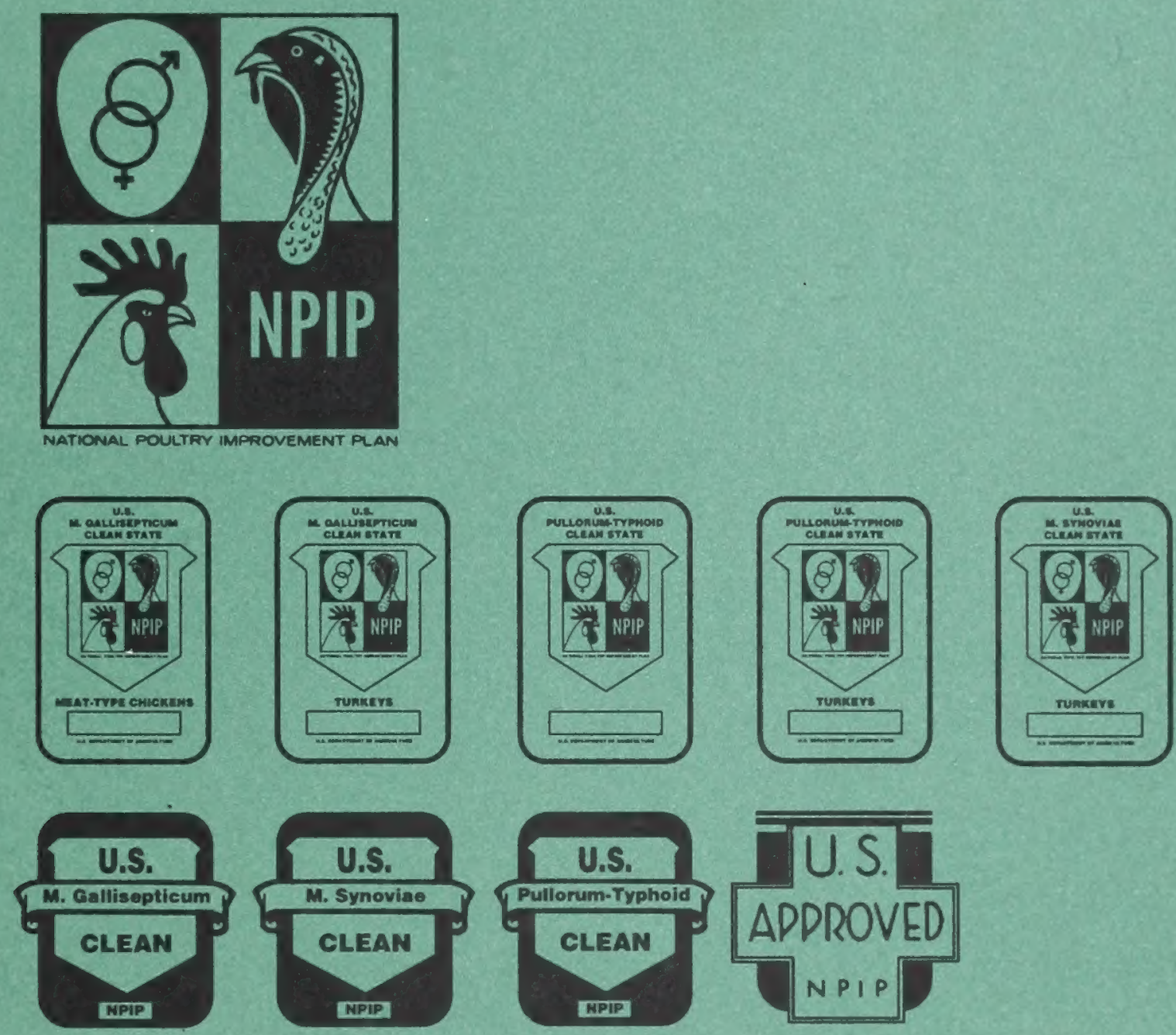
The U.S. Department of Agriculture (USDA) prohibits discrimination in all its programs and activities on the basis of race, color, national origin, sex, religion, age, disability, political beliefs, sexual orientation, or marital or family status. (Not all prohibited bases apply to all programs.) Persons with disabilities who require alternative means for communication of program information (Braille, large print, audiotape, etc.) should contact USDA's TARGET Center at (202) 720-2600 (voice and TDD).

To file a complaint of discrimination, write USDA, Director, Office of Civil Rights, Room 326-W, Whitten Building, 1400 Independence Avenue, SW, Washington, DC 202509410 or call (202) 720-5964 (voice and TDD). USDA is an equal opportunity provider and employer. 
The National Poultry Improvement Plan became operational on July 1, 1935, and the National Turkey Improvement Plan was activated on September 25, 1943, with the approval of the Secretary of Agriculture. On December 3, 1971, the two Plans were combined under one title, "The National Poultry Improvement Plan," with separate provisions applicable to the different types of poultry.

The Plan is implemented by State authorities in cooperation with the U.S. Department of Agriculture (USDA) in administering regulations for the improvement of poultry, poultry products, and hatcheries. Authority for the administration of the Plan is now contained in the Department of Agriculture Organic Act of 1944, as amended (7 U.S.C. 429).

Provisions of the Plan are changed from time to time to conform with the development of the industry and with new information as it becomes available. These changes are based on recommendations made at the biennial national Plan conferences by official delegates representing participating breeders, hatcheries, and flock owners from all cooperating States. Current provisions of the Plan are contained in a publication entitled "The National Poultry Improvement Plan and Auxiliary Provisions." This publication (APHIS 91-55-063) is available from Official State Agencies or on request to the National Poultry Improvement Plan, USDA, Animal and Plant Health Inspection Service (APHIS), Veterinary Services, 1498 Klondike Road, Suite 200, Conyers, GA 30094.

\section{National Poultry Improvement Plan U.S. Pullorum-Typhoid Clean States}

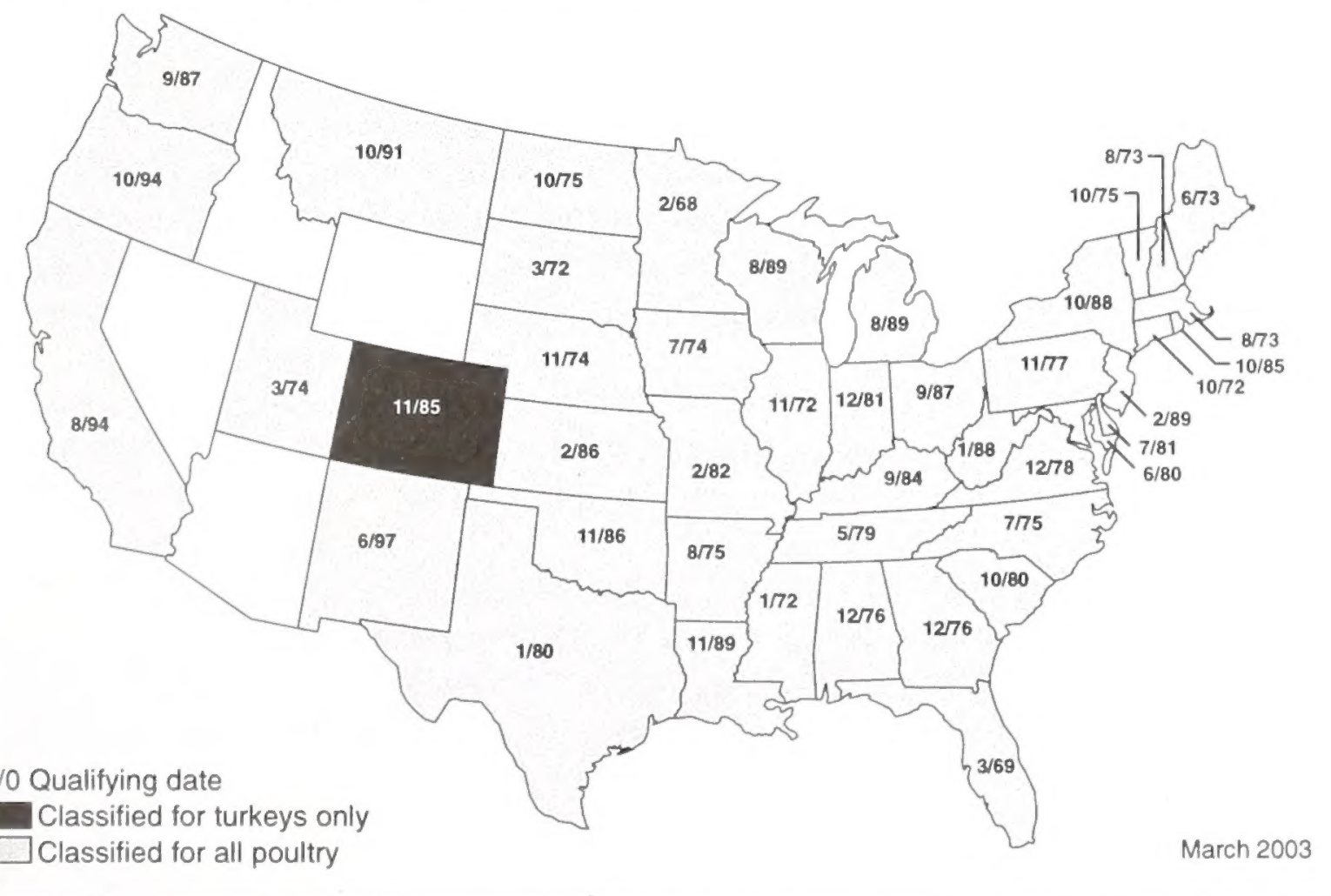


The following States have received recognition for their efforts in controlling certain hatchery-disseminated diseases under the National Poultry Improvement Plan.

\section{U.S. Pullorum-Typhoid} Clean State

\section{U.S. Pullorum-Typhoid Clean State, Turkeys}

\section{U.S. Mycoplasma gallisepticum Clean State, Turkeys}

1975
Arkansas
Connecticut
Florida
Illinois
Maine
Massachusetts
Minnesota
Mississippi
Nebraska
New Hampshire
North Carolina
North Dakota
Ohio
South Dakota
Utah
Vermont
1976
Alabama
Georgia
lowa
1977
Pennsylvania

1978

Virginia

1979

Tennessee

1980

Maryland

South Carolina

Texas

1981

Delaware

Indiana

1982

Missouri

1984

Kentucky

1985

Rhode Island
1986

Kansas

Oklahoma

1987

Washington

1988

New Jersey

New York

West Virginia

1989

Louisiana

Michigan

Wisconsin

1991

Montana

1994

California

Oregon

1997

New Mexico
1985

Colorado

1980

Illinois

Minnesota

1981

Indiana

lowa

Nebraska

1982

North Carolina
1985

Wisconsin

1986

Virginia

1987

West Virginia
1988

New Jersey

1990

Ohio

1993

Arkansas

2001

California 


\section{Using This Publication}

The hatcheries, dealers, and independent flocks listed in this publication are participating in the National Poultry Improvement Plan, according to reports submitted by the Official State Agencies. This list of participants is published for the purpose of developing greater efficiency in the operation of the Plan. Additional information regarding participants may be obtained by communicating with the Contact Representative of the Official State Agency of the respective State. The Contact Representative's name, address, and telephone number are given at the beginning of each State's listings in table 1.

This publication is revised each year because of frequent changes in participation and the need for inspectors, hatchery managers, dealers, and others to have up-to-date information. In addition to this listing, all Official State Agencies are notified of changes in participation that take place during the year.

The National Poultry Improvement Plan provisions, as contained in Title 9, Chapter I, Parts 145 and 147 of the Code of Federal Regulations, provide for recognition of different classes or types of fowl as follows:

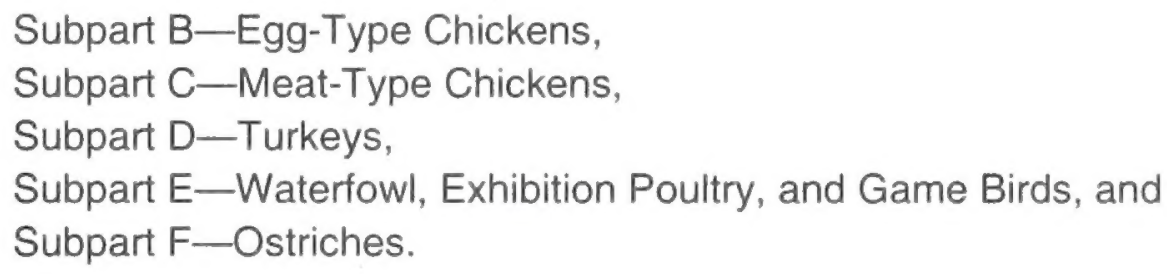

Approval Numbers

\section{Participant's Name and Address}

Hatching-Egg Capacity
Participants handling egg-type and meat-type chickens and turkeys are listed in a separate publication (APHIS 91-55-068) in this series.

In table 1 of this publication, the numerals preceding the name of the State, together with those preceding the name of each participant, constitute the participant's approval number. For example, the approval number for the first hatchery listed under Alabama is 64-800. The number 64 represents the State of Alabama; 800 represents Anderson Creek Hatchery, 6780 Highway 207, Anderson 35610. These assigned numbers may be used by participants on shipping labels, certificates, invoices, and other documents for identification. The numbers may also be used as reference numbers for shipments to member States of the European Economic Community.

Three types of participants are listed in this publication: hatcheries, dealers, and independent flocks. For each State, the type of participant is given immediately above the first name in that category.

Figures in this column refer to the hatching-egg capacity of hatcheries, according to manufacturer's rating, including setting and hatching trays. Letter symbols in this column refer to products that dealers handle, as follows: $E$, hatching eggs; $C$, chicks; $P$, poults; and SP, started pullets. When an independent flock is listed, this column is left blank. 
Products and Classifications
All products handled by participants listed in this publication meet the specific requirements for a "U.S. Pullorum-Typhoid Clean" classification. The combined letter and numeral symbols shown in the fourth column identify the particular breed, strain, or trade name of the participant's products (see tables 3 and 4 in the appendix). Additional classifications for which these products qualify are identified in column five by MG, "U.S. M. gallisepticum Clean," and MS, "U.S. M. synoviae Clean." 


\section{Laboratories Approved To Test for the Following Diseases}

\begin{tabular}{|c|c|}
\hline Code & Disease Name \\
\hline AI & Avian Influenza \\
\hline D & Diagnostic work \\
\hline MG & Mycoplasma gallisepticum \\
\hline MGM & Mycoplasma gallisepticum Monitored \\
\hline MM & Mycoplasma meleagridis \\
\hline MS & Mycoplasma synoviae \\
\hline MSM & Mycoplasma synoviae Monitored \\
\hline $\mathrm{P}$ & Pullorum-Typhoid \\
\hline SALM & Salmonella Monitored \\
\hline SAN MON & Sanitation Monitored \\
\hline SE & Salmonella enteritidis \\
\hline
\end{tabular}

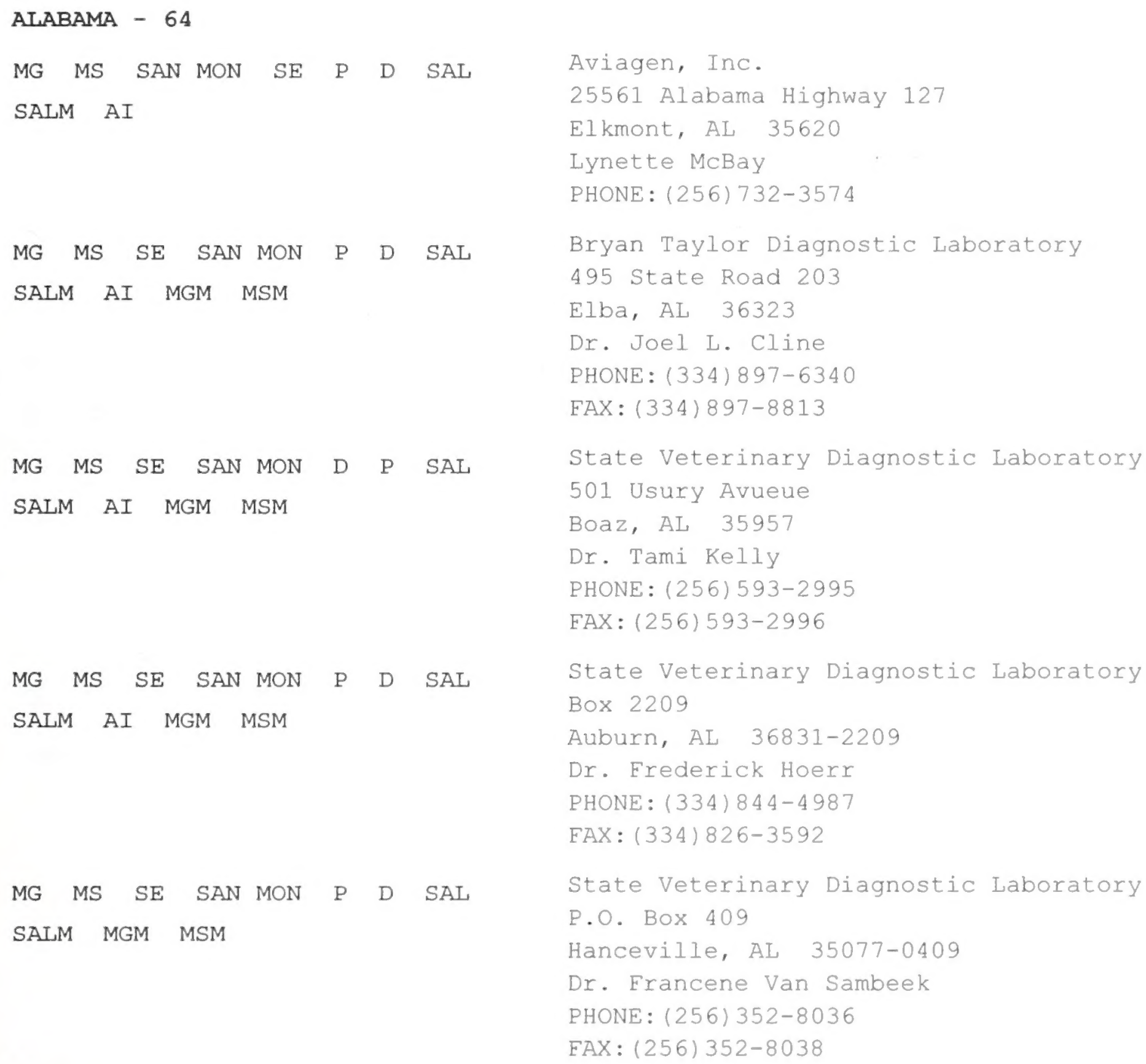




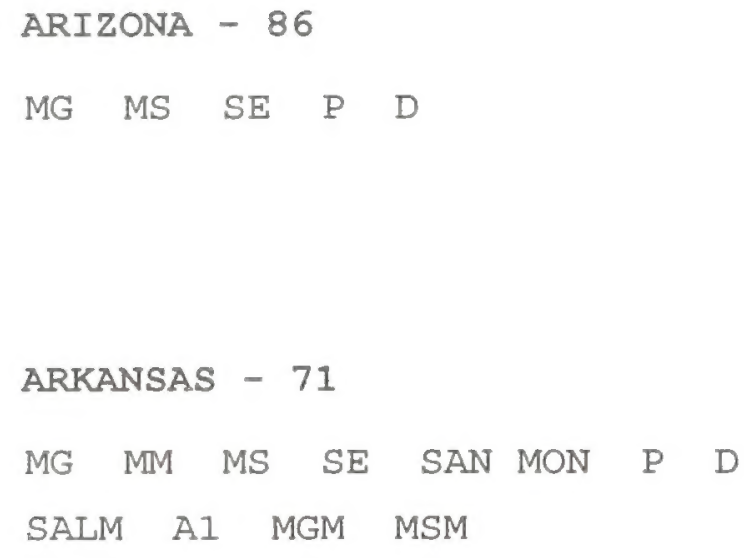

Arizona Veterinary Diagnotic Laboratory 2831 North Ereeway

Tucson, AZ 85721

Dr. John C. Mare at Univ. of Arizona

PHONE: (520)621-4486

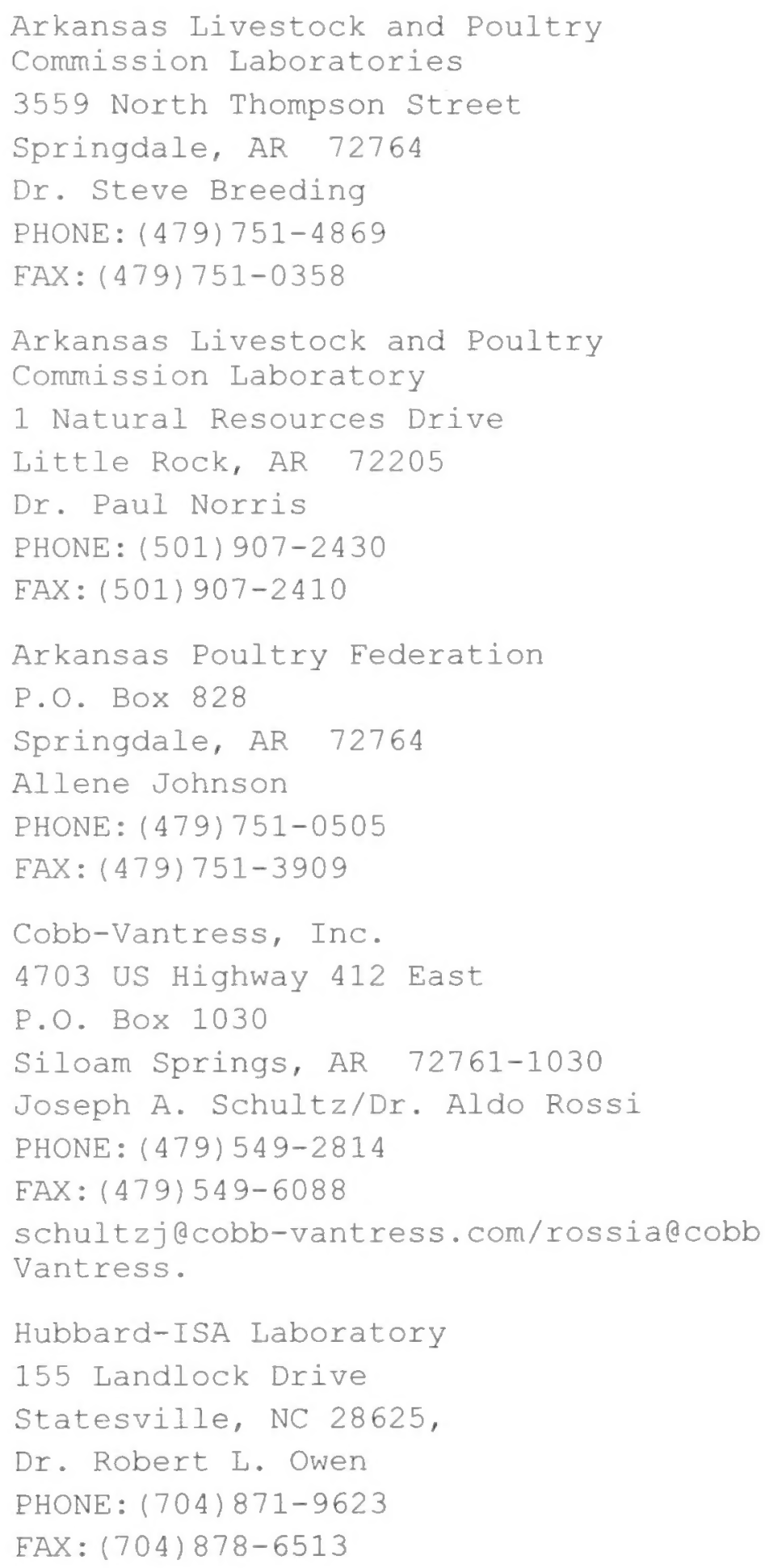


MGM MSM AI P

P MGM MSM

P AI MGM MSM

CALIFORNIA - 93

MG MM MS SE P D

MG MM MS SE $P$ D

MG MM MS SE $P$ D

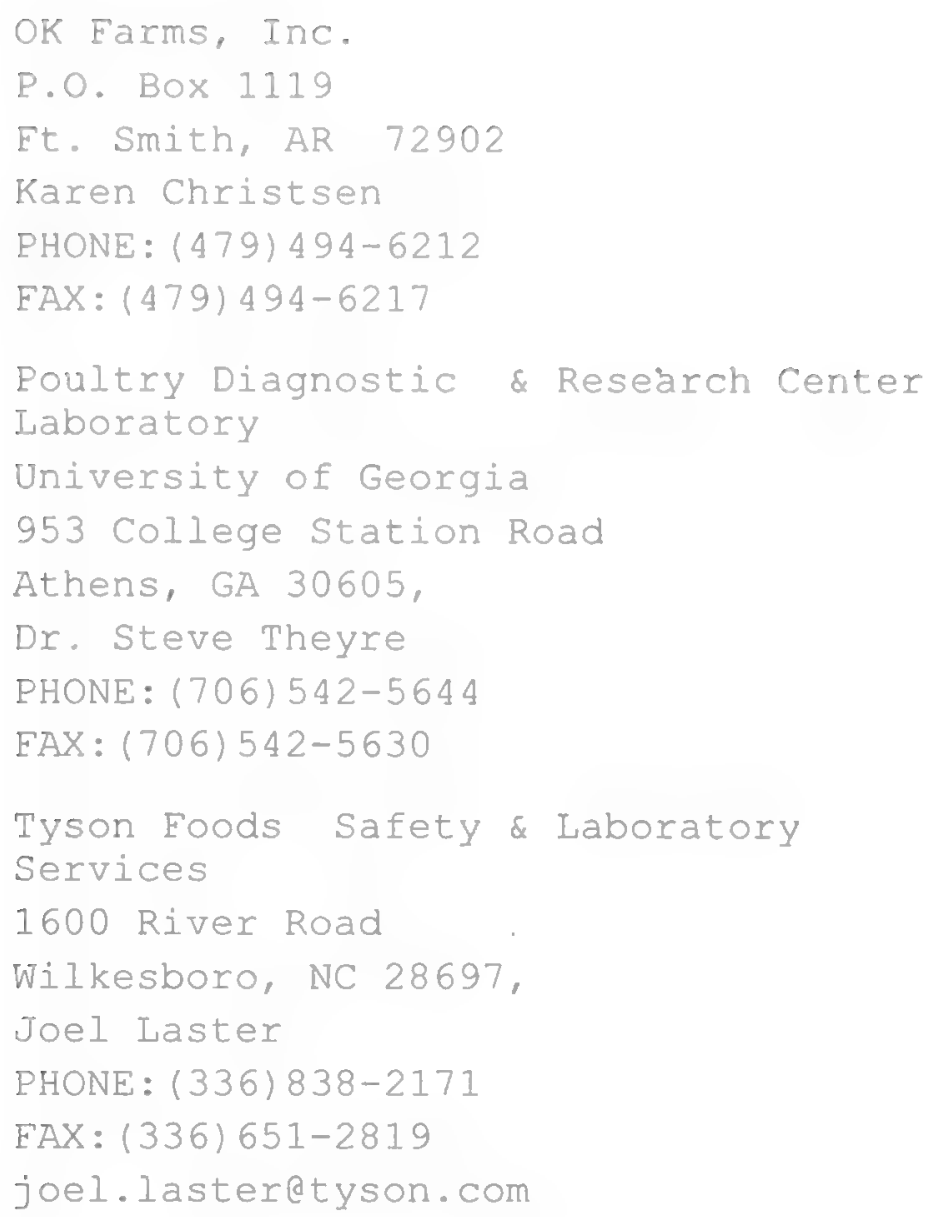

Califormia Animal Health \& Food Safety Davis Branch Laboratory

P.O. Box 1770

Davis, CA 95617-1770

Drs. Sharon K. Hietala \& Richard L.

Walker

PHONE : (530) 752-8700

EAX: (530) $752-6253$

California Animal Health \& Food Safety San Bernardino Branch Laboratory

105 West Central Avenue, P.O. Box 5579

San Bernardino, CA 92412-5579

Dr. Deryck Read

PHONE: (909) 383-4287

EAX: (909) $884-5980$

California Animal Health \& Food Safety Turlock Branch Laboratory

P.O. Box 1522

Turlock, CA 95381

Dr. Bruce Charlton

PHONE: (209) 634-5837

EAX: (209) 667-4261 
MG MM MS SE $P$ D

SE $\quad P \quad D \quad M G \quad M S$

MG MM $\quad$ MS $\quad$ SE $\quad P \quad D$

SE $\quad P \quad D$

SE $\quad P \quad D$

MG MS SE P D
California Animal Health \& Food Safety Laboratory System

Fresno Branch Laboratory

2789 South Orange Avenue

Fresno, CA 93725

Dr. Richard Chin

PHONE: (559) $498-7740$

FAX: (559) 485-8097

Foster Farms Microbiology Laboratory P.O. BOX 457

Livingston, CA 95334

Dr. Duane Olsen \& Judy Lee

PHONE: (209) 394-6914, ext. 4216

FAX: (209) 394-6881

Nicholas Turkey Breeding Farms

Veterinary Laboratory

P.O. BOX Y

Sonoma, CA 95476-1209

Drs. G. Yan Ghazikhanian

PHONE: (707) 938-1111

FAX: (707) 935-0144

San Diego County Veterinarian office Building 4, County Operations Center 5555 Overland Avenue

San Diego, CA 92123-1219

Dr. Kerry Mahoney

PHONE: (619) 694-2838

FAX:(619) $571-4268$

Veterinary Service, Inc. Laboratory

4100 Bangs Avenue

Modesto, CA 95356

Dr. W. D. Woodward

PHONE : (209) 545-5100

FAX: (209) 545-8244

Zacky Farms Microbiology Laboratory

P.O. Box 12556

Fresno, CA 93778

Lyn McDermott

PHONE: (559)486-2310, EXT. 227

FAX: (559) $443-2788$ 
COLORADO - 84

P D MG MS SE

MG MM MS SE P $\quad$ D

MG MS SE P D

CONNECTICUT - 16

MG MS SE SAN MON P D AI

MG MS $P$ D SAN MON SE AI

MG MG SAN MON SE $\mathrm{P}$ D
Colorado State University, Branch

Diagnostic Laboratory

27847 Road 21

Rocky Ford, co 81067

Dr. Charles Dickie

PHONE: (719) 254-6382

EAX:(719)254-6055

Pxairie Poultry Services

1374 East 57 th Street

Loveland, CO 80538-1302

Dr. Douglas Anderson

PHONE : (970) 669-8329

Veterinary School Diagnostic Laboratory

Colorado state University

Eort Collins, co 80523

Dr. Barbara Powers

PHONE : (970) 491-1281

EAX: (970) 491-0320

Arbor Acres, Inc.

Marlborough Road

Glastonbury, CT 06033

Dr. Nick Dorko

PHONE: (860) 633-4681

EAX: (860) 657-9193

Charles River Labs SPAFAS

67 Baxter Road

Storrs, CT 06268

Dr. Ted Girshick

PHONE : (860) 429-7261

EAX: (860) $429-2572$

Diagnostic Testing Services Laboratory

University of Connecticut

Storrs, CT 06268

Dr. Louis van der Heide

PHONE : (860) 486-4000

EAX: (860) 486-2794 


\section{DELAWARE - 50}

SE P D

SE P D

MG MS SE P D

FLORIDA - 58

SE P D AI

MG $M S \quad S E$ P $\quad D \quad A I$

GEORGIA - 57

MG MS SE P D AI
Department of Animal \& Food Sciences

University of Delaware

531 South College Avenue

Newark, DE 19717

Dr. John Rosenberger

PHONE: (302) 831-2524

EAX: (302) 831-8177

Poultry \& Animal Health

Delaware Department of Agriculture

2320 South Dupont Highway

Dover, DE 19901

Dr. H. W. Towers, Jr.

PHONE : (302)739-4811

FAX:(302)697-4451

Poultry Diagnostic Laboratory

Department of Animal and Food Science

University of Delaware, Rt. 6, Box 48

Georgetown, DE 19947

Drs. M. Salem and Ed Odor

PHONE: (302) 856-7303

FAX: (302) 856-1845

Animal Disease Diagnostic Laboratory Florida Department of Agriculture \& Consumer Serv.

P.O. Box 420460

Kissimmee, EL 32742-0460

Dr. John Crews

PHONE: (407) 846-5200

FAX: $(407) 846-5204$

Live Oak Disease Diagnostic Laboratory

Florida Department of Agriculture \&

Consumer Serv.

P.O. Drawer O

Live Oak, FL 32060

Dr. Carolyn Parkins

PHONE : (386) 362-1216

FAX: (386) 362-4213

Georgia Poultry Laboratory

P.O. Box 20

Oakwood, GA 30566

James Scroggs

PHONE: (770) 535-5996

FAX: (770) 535-5941 
MG MS SE P D

MG MS SE P D

MG MS SE P D

MG MS SE P D

MG MS SE P D

MG MS SE P D

MG MS SE P D
Georgia Poultry Laboratory

400 Burson Road

Camilla, GA 31730

Dr. Josh Hatkin

PHONE: (229) 336-0001

FAX: (229) 336-0018

Georgia Poultry Laboratory P.O. Box 6025

Forsyth, GA 31029

Dr. Donna Kelly

PHONE : (478) 994-1219

FAX: (478) 994-1220

Georgia Poultry Laboratory 345 Barr Avenue Bowdon, GA 30108

Dr. Richard D. Whitten

PHONE: (770) 258-0300

Georgia Poultry Laboratory 150 Airport Drive Montezuma, GA 31063

Dr. Donna J. Kelly

PHONE: (478) 472-9904

EAX: (478)472-9905

Georgia Poultry Laboratory 410 North Park Drive

Dalton, GA 30720

PHONE: (706) 278-7306

EAX: (706) 227-7408

Georgia Poultry Laboratory 150 Thomas Frier Drive Douglas, GA 31533

Dr. Yoga Sundram

PHONE: (912) 384-3719

Georgia Poultry Laboratory 1221 Downing Nusgrove Road Glennville, GA 30427

Dr. William J. Martin III

PHONE : (912) 654-0504

EAX:(912) 654-0526 
MG MS SE P D

MG $\quad$ MS SE $\quad \mathrm{P} \quad \mathrm{D}$

IDAHO - 82

MG MS SE $P$ D

ILIINOIS - 33

MS MM SE P $D$ MG

SE $\quad P \quad D$

MG MS SE $\quad P \quad D$
Georgia Poultry Laboratory

159 A.T. Mauldin Drive, P.O. Box 672 Carnesville, GA 30521

Dr. Frank M. Bunnell

PHONE : (706) 384-2387

FAX: (706) 384-2388

Georgia Poultry Laboratory

P.O. Box 349

Canton, GA 30114

Dr. John P. Bohanan

PHONE : (770) 479-2901

EAX: (770) 345-7832

Bureau of Animal Health Laboratories Room 204

2230 Old Penitentiary Road

Boise, ID 83712

Dr. Kendall Eyre

PHONE: (208) 332-8570

FAX:(208) 334-4619

IIlinois Department of Agriculture

Animal Disease Laboratory

2100 South Lake storey Road, P.O. Box $2100 x$

Galesburg, IL 61402

Dr. Dale Webb

PHONE: (309) 344-2451

EAX: (309) 344-7358

Illinois Department of Agriculture Animal Disease Laboratory

9732 Shattuc Road

Centralia, IL 62801-5858

Dr. Gene Niles

PHONE: (618) 532-6701

EAX: (618) 532-1195

University of Illinois

College of Veterinary Medicine

1225 VMBSB, 2001 South Lincoln

Urbana, IL 61801

Dr. John Andrews

PHONE : (217) 333-1620

FAX: (217) 244-2439 


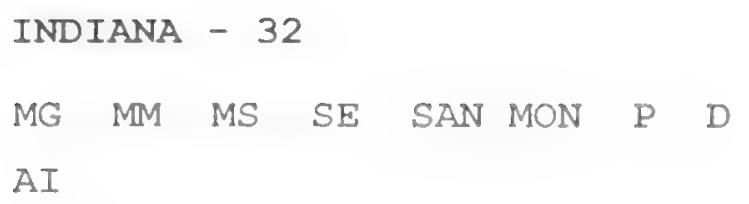

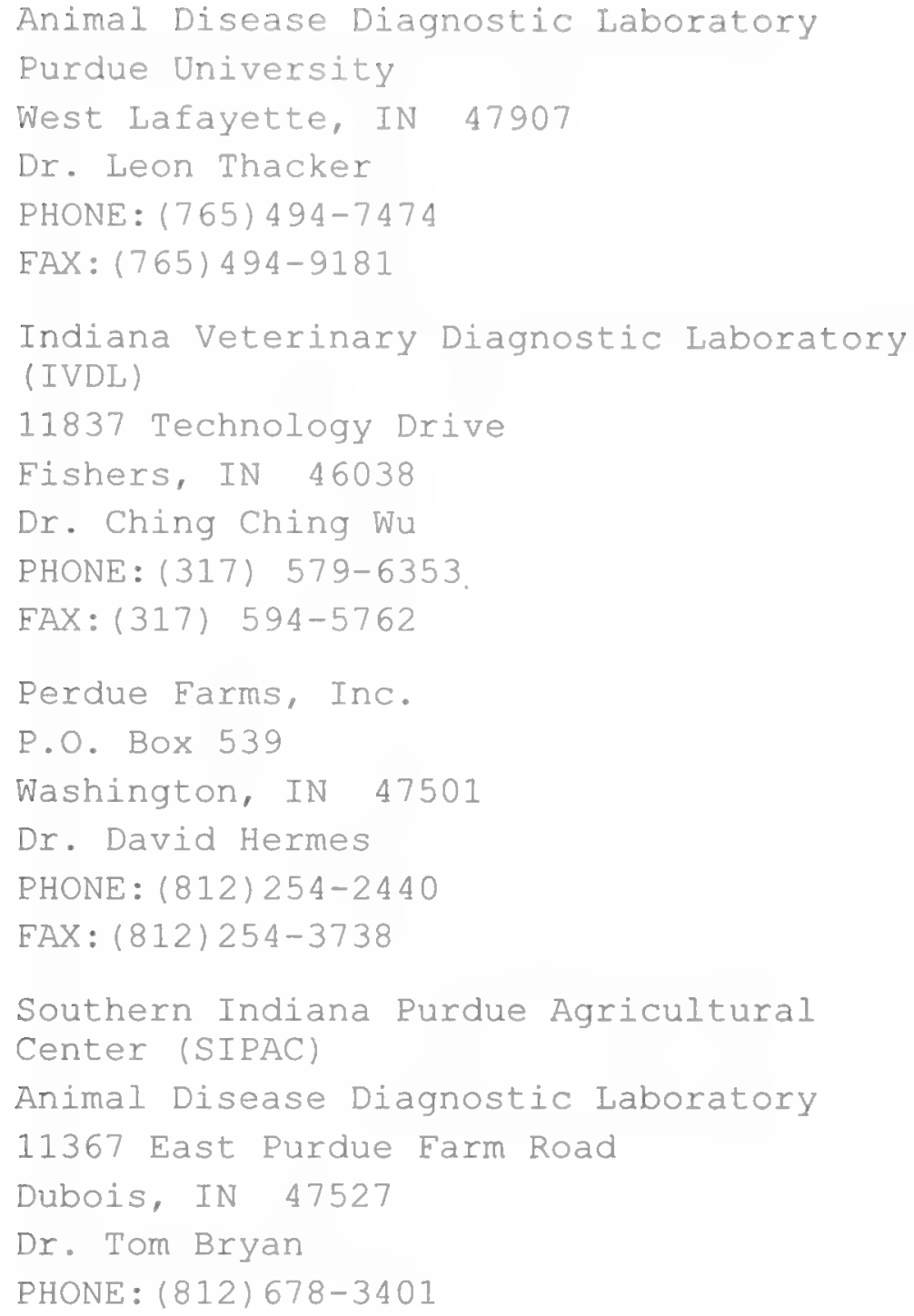


MG

MS SE P D

MG MS SE $P$ D $\quad M$

SALM SE SAN MON P D

\section{KANSAS - 48}

MG MS SE P D

KENTUCKY - 61

$D \quad P$ AI MG MS SALM SAN MO

SAN MON SE

MG MS SE P D
Hy-Vac Laboratory Egg Company

Production and Laboratory

2583240 th Street, P.O. Box 310

Dallas Center, IA 50063

Dr. Michael Hall

PHONE: (515) 992-4173

FAX: (515) 992-3203

USDA, National Veterinary Services

Laboratory

P.O. Box 844

Ames, IA 50010

Dr. Randall Levings

PHONE: (515) 663-7266

FAX:(515) 663-7397

Veterinary Diagnostic Laboratory

Iowa State University

Ames, IA 50010

Dr. Gary Osweiler

PHONE : ( 515 ) 294-1950

EAX:(515) $294-3564$

Kansas State University

1800 Denison Avenue

Vet. Clincical Services Bldg.,

Manhattan, KS 66506

Dr. Crystal Pollock

PHONE: (785) 532-4244

FAX: (785) 532-4481

Cobb-Avian Division

430 West Highway 40 By-Pass

Monticello, KY 42633

Carolyce Dungan

PHONE : (606) 348-3305

FAX: (606) 348-4490

dungancecobb-vantress. com

Department of Veterinary Science

University of Kentucky

Lexington, KY 40546-0099

Dr. Peter J. Timoney

PHONE: (606) 257-1531

EAX: (606) 257-8542 
MG MS SE P D

SE $P \quad D \quad M G \quad M S$

\section{LOUISIANA - 72}

AI D MG SE $P$ SE SALM

MAINE - 11

MG MS SE SAN MON P

MG MS SE P D

MG MS SE P $\quad$ D
Livestock Disease Diagnostic Center 1429 Newton Pike

Lexington, KY 40511

Dr. Lenn R. Harrison

PHONE: (606) 253-0571

EAX: (606) 255-1624

MSU Breathitt Veterinary Center

715 North Drive

P.O. Box 2000

Hopkinsville, KY 42241

Dr. Douglas Cox

PHONE : (502) 886-3959

FAX: (502) $886-4295$

Louisiana Veterinary Medical Diagnostic Laboratory

LSU School of Veterinary Medicine

Skip Bertman Drive

Baton Rouge, LA 70803

Dr. Wayne Taylor

PHONE : (225) 578-9777

FAX:(225) 578-9784

Animal Pathology Laboratory

University of Maine

Orono, ME 04469-5735

Dr. Mike Opitz

PHONE : (207) 581-2771

FAX:(207) 581-4430

Division of Animal Health \& Industry

Department of Agriculture

State House Station

Augusta, ME 04333

Dr. Donald E. Hoenig

PHONE : (207) 287-3701

FAX: (207) $287-7548$

Northeast Laboratory Services

P.O. Box 788

Waterville, ME 04903

PHONE: (207) 873-7711

FAX:(207) 873-7022 
MARYIAND - 51

SE P D

SE $\quad P \quad D$

MS MM SE P D MG AI

SE P D

SE P D

MASSACHUSETTS - 14

MICHIGAN - 34

MG MM MS SAN MON SE P $D$
Animal Health Laboratory

Maryland Department of Agriculture

211 Safety Drive

Centerville, MD 21617-9998

Dr. William D. Higgins

PHONE : (410) 758-0846

FAX:(410)758-9962

Animal Health Laboratory

1840 Rosemont Avenue

Frederick, MD 21702-8212

Dr. Ginny Pierce

PHONE: (301) 663-9528

FAX: (301) 663-6467

Animal Health Laboratory

P.O. Box 2599

Salisbury, MD 21802

Dr. Daniel Bautista

PHONE: (410) 543-6610

EAX:(410) 543-6676

Animal Health Laboratory

8077 Greenmead Drive

College Park, MD 20740

Dr. James Davidson

PHONE: (301) 314-1870

EAX:(301) 935-6072

Animal Health Laboratory

P.O. Box 376

Oakland, MD 21550

Dr. James I Fearer

PHONE: (301) 334-2185

FAX: (301) 334-5967

Animal Health Diagnostic Laboratory

Michigan State University

B629 West Fee Hall

East Lansing, MI 48824-1315

Dr. Willie M. Reed

PHONE: (517) 353-0635

FAX:(517) 353-5096 
MINNESOTA - 41

MG MM MS SE SAN MON P D SALM AI

MG MS MM SE $P$ D $A I$

MISSISSIPPI - 65

MG MS SE $P$ D

MG $M S \quad S E \quad P \quad D$

MISSOURI - 43

MG MM MS SE SAN MON P D AI
Board of Animal Health/university of Minnesota

Minnesota Poultry Testing Laboratory

P.O. Box 126, 622 Business Highway $71 \mathrm{NE}$

Willmar, MN 56201-0126

Dr. Dale Lauer

PHONE: (320) $231-5170$

FAX: $(320) 231-6071$

Department of Diagnostic Laboratories

College of Veterinary Medicine

Univ. Of MN, 1333 Gortner Ave.

St. Paul, MN 55108

Dr. Sagar M. Goyal

PHONE: (612) 625-8787

FAX:(612) 624-8707

Mississippi Veterinary Diagnostic Laboratory

P.O. Box 4389

Jackson, MS 39296

Dr. Lanny Pace

PHONE : (601) 354-6089

FAX: (601) 354-6097

South Central Poultry Research

Laboratory

Mail Stop 9821

Spring Street

Mississippi State, MS 39762

Dr. S. L. Branton

PHONE: (662) 320-7608

EAX: (662) $320-7589$

Missouri Department of Agriculture Veterinary Diagnostic Laboratory P.O. Box 2510

Springfield, MO 65801-2510

Dr. Duke Dana

PHONE: (417) 895-6861

FAX: (417) 831-4766 
SE SAN MON P AI MG MM MS
MS

SE

SAN MON P D AI

MG

MM MS SE SAN MON P D

AI

MONTANA - 81

$S E \quad P \quad D \quad A I$

NEBRASKA - 47

MG MM MS SE $\quad \mathrm{P} \quad \mathrm{D}$

NEVADA - 94

SE P D
Missouri Department of Agriculture

Animal Health Laboratory

216 El Mercado Plaza

Jefferson City, MO 65109

Dr. Charles Massengill

PHONE : (573) $751-3460$

FAX:(573) $751-5279$

Northwest Missouri Veterinary Diagnostic Laboratory

P.O. Box 377

Cameron, MO 64429

Dr. Sam Jameson

PHONE : (816) 632-6594

FAX: (816) 632-3421

School of Veterinary Medicine

University of Missouri

P.O. Box 6023

Columbia, MO 65205

Dr. Stanley Casteel

PHONE : (573) 882-6811

FAX: (573) 882-1411

Montana Department of Livestock

Diagnostic Laboratory Division

P.O. Box 997

Bozeman, MT 59771

Dr. A. W. Layton

PHONE : (406) 994-4885

FAX: (406) 994-6344

Veterinary Diagnostic Center

Department of Veterinary Science

University of Nebraska, Room 151

Lincoln, NE 68583-0907

Dr. Dave Steffen

PHONE : (402) 472-1434

FAX: (402) $472-3094$

Animal Diagnostic Laboratory

Nevada State Department of Agriculture 1351 Elm Street

Elko, NV 89801-3364

Dr. Bruce Branscomb

PHONE: (702)738-8076

FAX: (702) 738-2639 
SE $P \quad D$

NEW HAMPSHIRE - 12

$S E \quad P \quad D \quad M G \quad M S$ AI

NEW JERSEY - 22

MG MS SE P D

NEW MEXICO - 85

SE P D

NEW YORK - 21

SE P D
Animal Disease Laboratory

Nevada State Department of Agriculture 350 Capitol Hill Avenue

Reno, NV 89502-2923

Dr. Alan Bosomworth

PHONE: (702) 688-1182 EXT. 232

FAX: (702) 688-1198

New Hampshire Veterinary Diagnostic Laboratory

State/Eederal Lab, Department of Animal Science

University of New Hampshire

Durham, NH 03824

Drs. Joseph J. Moore, III, Director:

Kendall Hall; \& Roger E. Wells

PHONE: (603) $862-2726$

FAX: (603) 862-0179

New Jersey Department of Agriculture

Division of Animal Health Laboratory

CN 330

Trenton, NJ 08625

Dr. Robert Eisner

PHONE : (609) 984-2250

FAX:(609) 633-2550

Veterinary Diagnostic Services

700 Camino de Salud NE

Albuquerque, NM 87106

Dr. Elint Taylor

PHONE : (505) 841-2576

EAX:(505) $841-2518$

Duck Research Laboratory

192 County Road

Eastport, NY 11941

Dr. T. Sandhu

PHONE : (516) 325-0600

EAX: (516) 325-1843 
P MG MS SE

MG MM MS SE P D

NORTH CAROLINA - 55

MG MM MS SE SAN MON $P$ D SALM AI

SE SAL MON

MG MS SE SAN MON P D SAL SAIM AI

SE SAN MON P D SALM
ISA Breeders, Inc.

P.O. Box 280

Ithaca, NY 14851

Dr. Jarra Jagne

PHONE: $(607) 272-5990$

FAX : (670) 272-5998

Poultry Diagnostic \& Extension Services NYS Animal Health Diagnostic Lab-Cornell Universit

c4-121 Veterinary Medical center Box 5 Ithaca, NY 14853

Dr. Benjamin Lucio-Martinez, Director PHONE: (607) 253-3365

FAX: (607) 253-3384

Griffin Animal Disease Diagnostic Laboratory

P.O. Box 2183

Monroe, NC 28111

Dr. Kim Hagans

PHONE : (704) 289-6448

FAX:(704) 283-9660

Hubbard ISA Diagnostic Laboratory

155 Landlock Drive

Statesville, NC 28625

Dr. Bob Owens

PHONE : (704) 871-9623

FAX:(704) 878-6513

Northwestern Animal Disease Diagnostic Laboratory

P.O. BOX 70

Elkin, NC 28621

Dr. Darrell Rector, Jr.

PHONE: (336) 526-2499

EAX: (336) 526-3014

Poultry Disease Diagnostic Laboratory P.O. Box 476

Robbins, NC 27325

Dr. W. R. Wilson, Jr.

PHONE : (910) $948-2241$

FAX:(910) 948-2241 
$M G$ MS SE SAN MON P D SAL SALM AI

MG MM MS SE SAN MON $P$ D SALM AI

SE

SAN MON P D SALM

NORTH DAKOTA - 45

MG MS SE P D

\section{OHIO - 31}

MG MM MS SE P D

\section{OKCAHOMA - 73}

MG MS SE P D
Rollins Animal Disease Laboratory

P.O. Box 12223

Raleigh, NC 27605

Drs. J.A. Quinn, \& K. Post

PHONE: (919) 733-3986

FAX:(919) 733-0454

Rose Hill Animal Disease Diagnostic

Laboratory

P.O. Box 37

Rose Hill, NC 28458

Dr. Carlton Rouse

PHONE: (910) 289-2635

FAX: (910)289-2070

Western Animal Disease Diagnostic Laboratory

P.O. Box 279

Arden, NC 28704

Dr. R. C. Oliver

PHONE: (828) 684-8188

EAX: (828) $687-3574$

Department of Veterinary science

North Dakota State University

State College Station

Fargo, ND 58105

Drs. Ivan Berg, M.H. Smith, \& George Schamber

PHONE : (701) 231-7511

$\mathrm{EAX}:(701) 231-7514$

State Department of Agriculture

Reynoldsburg Diagnostic Laboratory

8995 East Main Street

Reynoldsburg, OH 43068

Dr. David Glauer

PHONE: (614)728-6210

$\mathrm{FAX}:(614) 759-1467$

Oklahoma state University

Animal Disease Diagnostic Laboratory

Farm Road to Ridge Road

Stillwater, OK 74078-0622

Drs. Bill Edwards, and Stanley Vanhooser

PHONE: (405) 744-6623

EAX: (405)744-8612 
SE P D

OREGON - 92

MG MS SE P D AI SAN MON

D

PENNSYLVANIA -23

MG MS SE $P \quad D$

MG MS SE $P$ D

MG MS SE P D
State Department of Health Laboratory P.O. Box 24106

Oklahoma City, OK 73124

Dr. Keeta Gilmore

PHONE: (405) 271-5070

EAX: (405) 271-4850

Oregon State Department of Agriculture Animal Health Laboratory

635 Capitol street NE

Salem, OR 97310

Lee Effinger

PHONE : (503) 986-4686

FAX:(503) $986-4688$

Veterinary Diagnostic Laboratory

Oregon State University

P.O. Box 429

Corvaliis, OR 97339

PHONE : (541) 737-3261

EAX: (541) 737-6817

Diagnostic Laboratory

New Bolton Center

382 West Street

Kennett Square, PA 19348

Dr. Robert J. Eckroade

PHONE: (610) 444-5800

FAX:(610) 444-5387

Diagnostic Laboratory

Pennsylvania State University

University Park, PA 16802

Dr. Tom Drake

PHONE: (814) 863-0837

FAX: $(814) 865-3907$

Diagnostic Laboratory, Pennsylvania

Dept. of Agriculture

State Veterinary Laboratory

2305 North Cameron Street

Harrisburg, PA 17110-9449

Dr. Helen Acland

PHONE: (717) 787-8808

FAX: (717) 772-3895 
RHODE ISLAND - 15

MG MS SE P D

SOUTH CAROLINA - 56

$M G$ MS SE P $D$ AI

SOUTH DAKOTA - 46

SE P D

\section{TENNESSEE - 63}

SE P D

SE D
Rhode Island State Health Department

Animal Disease Laboratory

Health Laboratory Bldg., 50 Orms Street

Providence, RI 02904

Mr. Leonard LaFazia

PHONE : (401) 222-5600

FAX: (401) 222-6064

Clemson University

Livestock Poultry Health Animal

Veterinary Diagnostic Center, P.O. Box 102406

Columbia, SC 29224

Dr. Tony Caver

PHONE : (803) $788-2260$

FAX: (803) 788-8058

Animal Disease Research and Diagnostic Laboratory

Veterinary Services Department

Box 2175, South Dakota State University

Brookings, SD 57007

Dr. David Zeman

PHONE : (605) 688-5171

FAX: (605) 688-6003

C.E. Kord Animal Disease Laboratory

Ellington Agriculture Center

Box 40627, Melrose Station

Nashville, TN 37204

Dr. Ronald B. Wilson

PHONE: (615) $837-5125$

EAX:(615) $837-5250$

College of Veterinary Medicine

University of Tennessee

P.O. Box 1071

Knoxville, TN 37901-1071

Dr. John New

PHONE: (423) 974-8387

EAX: (423) $974-5640$ 


$D \quad M G$ MS SAN MON SE P AI
SALM

TEXAS - 74

MG MM MS SE SAN MON P D
AI

MG MM MS SE SAN MON P D AI

MG MM MS SE SAN MON P $D$ AI

$\mathrm{UTAH}-87$

MG MS SE P D

MG MS SE $P \quad D$
Crossville Veterinary Laboratory

Aviagen North America

256 Genesis Road, Suite 102

Crossville, TN 38555

Dr. Carolyn Miller

PHONE : (931) 484-3203

EAX: (931) $484-6443$

Texas Veterinary Diagnostic Laboratory

Poultry Diagnostic Laboratory

P. O. Box 187

center, TX 75935

Dr. Tom Blount

PHONE: (936) 598-4451

FAX:(936) 598-2741

Texas Veterinary Medical Diagnostic Laboratory

Poultry Diagnostic Laboratory

P.O. Box 84

Gonzales, TX 78629

Dr. Jose Linares

PHONE: (830)672-2834

FAX: $(830) 672-2834$

Texas Veterinary Medical Diagnostic Laboratory

P.O. Drawer 3040

College Station, TX 77841-3040

Drs. Floyd Golan, Thomas Lester, Melissa Libal \& Sid Anderson

PHONE : (979) 845-4186

EAX: (979) 845-1794

Branch Veterinary Laboratory

2031 south state

Provo, UT 84606

Dr. E. Jane Kelly

PHONE: (801) 373-6383

FAX: $(801) 375-6870$

Moroni Feed Company

Diagnostic Laboratory

15 East 19 South Food Mill Road

Moroni, UT 84646

Dr. Terry Olson

PHONE: (801) 436-8227

FAX: (801)436-8101 
MG MS SE P D

VERMONT - 13

P

VIRGINIA - 52

MG MM $\quad$ MS SE $\quad$ P $\quad$ D

MG MM MS SE P D

SE P D

SE P D
Veterinary Diagnostic Laboratory

950 East 1400 North

North Logan, UT 84341

Dr. Tom Baldwin

PHONE:(435) 797-1895

FAX:(435) $797-2805$

Vermont Department of Agriculture Laboratories

103 South Main Street

waterbury, CT 05671-0101

Mr. John Jaworski

PHONE: (802) 244-4510

FAX: (802) 241-3008

Division of Consolidated Laboratories Bureau of Microbiological Sciences

\#1 North 14th Street

Richmond, VA 23219

Ms. Sally Henderson, Supervisor

PHONE: (804)786-5147

FAX: (804)371-0666

Harrisonburg Regional Laboratory

116 Reservoir street

Harrisonburg, VA 22801

Drs. Gabriel Meza \& Ronald King

PHONE: (540) 434-3897

EAX: (540) $434-3880$

Ivor Regional Laboratory

34591 General Mahone BIvd.

Ivor, VA 23866

Dr. Jerry Dawson

PHONE: (757) 859-6221

EAX: (757) 859-6428

Lynchburg Regional Laboratory

4832 Tyreeanna Road

Lynchburg, VA 24504

Dr. Susan Gardner

PHONE: (804) 947-2518

FAX: (804) 947-2577 
SE P D

SE P D

WASHINGTON - 91

MG MS SE P D MM AI

WEST VIRGINIA - 54

SE $\quad P \quad D$

MG MS MM SAN MON SE SALM

AI

MG

MM MS SE P D
Warrenton Regional Laboratory

272 Academy Hill Road

Warrenton, VA 22186

Dr. Joe Garvin

PHONE : (540) $347-6385$

EAX: (540) $347-6404$

Wytheville Regional Laboratory

250 Cassell Road

Wytheville, VA 24382

C.S. Cunningham, Jr.

PHONE : (540) 228-5501

FAX:(540) 223-1961

Avian Health Laboratory

Washington State University

Puyallup, WA 98371

Dr. A. S. Dhillon

PHONE: (253) 445-4537

EAX:(253) 445-4544

Animal Pathology Laboratory

University of West Virginia

Morgantown, WV 26506-6108

Drs. Sam Barringer \& John Kunkel

PHONE : (304) 293-2406

FAX: (304)293-3740

British United Turkeys of America

Veterinary Laboratory

50 Seneca Trail

Lewisburg, WV 24901

Dr. Tahseen Aziz

PHONE : (304) 647-9695

FAX:(304)647-3038

West Virginia Department of Agriculture

Animal Health Division

State-Eederal Laboratory, Capitol Building

Charleston, WV 25305

Brenda Keavey \& Jeff McClure

PHONE: (304) 558-2214

FAX : (304) 558-2203 
WISCONSIN - 35

MG MM MS SE P D

WYOMING - 83

SE P D
Wisconsin Veterinary Diagnostic

Laboratory-Barron

1521 East Guy Avenue

Barron, WI 54812

Dr. Scott Jones

PHONE: (715) 637-3151

EAX:(715) 637-9220

State Veterinary Laboratory

1174 Snowy Range Road

Laramie, WY 82070

Dr. Lynn Woodward

PHONE: (307) 742-6638

EAX:(307)721-2051 

Officials Authorized To Sign VS Form 9-3,

"Report of Sales of Hatching Eggs, Chicks, and Poults"

\begin{tabular}{|c|c|c|}
\hline ALABAMA & GEORGIA & MAINE \\
\hline Ray Hilburn & James Scroggs & C. W. Ridky \\
\hline \multirow[t]{2}{*}{ Gary Parrish } & Radford Murphy & D. E. Hoenig \\
\hline & Gaynelle N. Beach & Debra Morgan \\
\hline \multicolumn{3}{|l|}{ ARIZONA } \\
\hline \multirow[t]{2}{*}{ Edward J. Bicknell } & IDAHO & MARYLAND \\
\hline & Kendal G. Eyre & Fidelis Hegngi \\
\hline ARKANSAS & Bruce L. Donnell & Kimberly Jones \\
\hline H. M. Ghori & Mark Hindman & \\
\hline Melody Parsley & Heather Jensen & MASSACHUSETTS \\
\hline Paul Norris & Dorothy Stroebel & Edward Hageman \\
\hline James Britt & & Frederick Mach \\
\hline Steve Breeding & ILLINOIS & Lorraine O'Connor \\
\hline $\begin{array}{l}\text { Conley Byrd } \\
\text { Jane Elrod }\end{array}$ & James E. Kunkle & Megan Megarth \\
\hline \multirow[t]{2}{*}{ Robert M. Harbison } & INDIANA & MICHIGAN \\
\hline & Paul Brennan & George House \\
\hline CALIFORNIA & & Sherri L. Merryman \\
\hline Dave Willoughby & IOWA & \\
\hline David Kerr & Kevin S. Vinchattle & MINNESOTA \\
\hline George West & Darrell Trampel & Keith Friendshuh \\
\hline Bill Mattos & Cindy Christian & William Hartmann \\
\hline \multirow[t]{2}{*}{ Doris Pokorny } & & Sandra Ives \\
\hline & KANSAS & Sandra Hinrichs \\
\hline COLORADO & R. Scott Beyer & \\
\hline \multirow[t]{2}{*}{ William C. Lower } & & MISSISSIPPI \\
\hline & KENTUCKY & Danny Thornton \\
\hline CONNECTICUT & Anthony Pescatore & Betty Roberts \\
\hline Louis van der Heide & & Marty Bergin \\
\hline Gabriel Moquin & LOUISIANA & \\
\hline Bruce Sherman & Dwight Raiford & MISSOURI \\
\hline Maurice Dion & Eric Lee & Rose Foster \\
\hline \multirow[t]{2}{*}{ James Sullivan } & Donald Tassin & David Hopson \\
\hline & Darryl Hoover & \\
\hline DELAWARE & Nancy Schleissmann & MONTANA \\
\hline H. Wesley Towers, Jr. & $\begin{array}{l}\text { Hoksan Saing } \\
\text { Martha Littlefield }\end{array}$ & Thomas F. T. Linfield \\
\hline FLORIDA & Mike Barrington & NEBRASKA \\
\hline Leroy Coffman & Johnny Hollis & Delwin Wilmot \\
\hline Jennifer Jennings-Glover & Jimmy Thomas & \\
\hline
\end{tabular}




\begin{tabular}{|c|c|c|}
\hline NEVADA & $\mathrm{OHIO}$ & TEXAS \\
\hline Ronald D. Anderson & $\begin{array}{l}\text { Jack L. Heavenridge } \\
\text { Leslie Stewart }\end{array}$ & Floyd A. Golan \\
\hline NEW HAMPSHIRE & & UTAH \\
\hline \multirow[t]{2}{*}{ Clifford W. McGinnis } & OKLAHOMA & Michael R. Marshall \\
\hline & Gene Eskew & L. Earl Rogers \\
\hline NEW JERSEY & Ralph Duncan & Tom Baldwin \\
\hline Michelle Holloway & & Jane Kelly \\
\hline Ernest Zirkle & OREGON & David D. Frame \\
\hline Leslie Bulaga & Andrew A. Clark & Royd Hatt \\
\hline A. C. Welsh & Becky Rada & Alan Larson \\
\hline Linda Detwiler & Brad R. LeaMaster & Larry Mitchell \\
\hline Cheryl Berthaud & Bruce Mueller & Cliss Mitchell \\
\hline Susan Luchese & Richard TenEyck & John Mathis \\
\hline Tim Schuler & Terry Hensley & Grant C. Jensen \\
\hline Phyllis Rayca & & Terry Olson \\
\hline \multirow[t]{2}{*}{ Anne Peirok } & PENNSYLVANIA & \\
\hline & Phillip Debok & VERMONT \\
\hline NEW MEXICO & & Todd E. Johnson \\
\hline Ron Parker & RHODE ISLAND & Darryl Kuehne \\
\hline Jerry Schickendanz & Susan Littlefield & $\begin{array}{l}\text { Jon Magnant } \\
\text { Ed Jackson }\end{array}$ \\
\hline NEW YORK & SOUTH CAROLINA & \\
\hline John Huntley & Tony Caver & VIRGINIA \\
\hline Jeffry Huse & Julie D. Helm & G. G. Meza \\
\hline Patricia Moore & & Ronald King \\
\hline Sandy McKay & SOUTH DAKOTA & \\
\hline Kathy Crowley & Sam Holland & WASHINGTON \\
\hline \multirow[t]{2}{*}{ Susie Berry } & Charles Seagren & Kathleen Connell \\
\hline & Rick Rieland & Bob Mead \\
\hline NORTH CAROLINA & Thomas Cline & \\
\hline Jo Anna Quinn & Craig Hanson & WEST VIRGINIA \\
\hline \multirow[t]{2}{*}{ Jimmy Collie } & Kay Hall & Jewell Plumley \\
\hline & Terry Peterson & \\
\hline NORTH DAKOTA & & WISCONSIN \\
\hline \multirow[t]{8}{*}{ Iris Stenerson } & TENNESSEE & Donald O'Connor \\
\hline & Ron Wilson & Clarence J. Siroky \\
\hline & Tina Rogers & USDA-Area \\
\hline & Nick Todd & Veterinarian-in-Charge \\
\hline & Philip Gordon & \\
\hline & Kerry Wilkerson & WYOMING \\
\hline & Justin Henson & Accredited veterinarians \\
\hline & Bert Mansfield & \\
\hline
\end{tabular}




\section{Explanation for Tables}

Participants listed in table 1 represent hatcheries, dealers, or independent flocks handling waterfowl, exhibition poultry, and game birds. Participants listed in table 2 handle ostriches. The products they handle have a letter symbol as follows:

A-White Leghorn

B - Rhode Island Red

C-Barred Plymouth Rock

D-White Plymouth Rock

$\mathrm{H}$-Cornish

$\mathrm{N}$ - Game

O-Ostrich

P-Emu

Q-Rhea
$\mathrm{R}$-Other breeds and varieties

S-Egg-production stocks

T-Meat-production stocks

U- Cassowary

W-Bantams

$X$-Ducks

$Y$-Geese

Z-Other birds

These letter symbols, together with accompanying numerals representing specific breed, strain, trade names, or other specific type of birds, are fully explained in tables 3,4 , and 5 . 
Table 1. HATCHERIES, DEALERS. AND INDEPENDENT FLOCKS PARTICIPATING IN

THE NATIONAL POULTRY IMPROVEMENT PLAN

WATERFOWL, EXHIBITION POULTRY AND GAME BIROS

APP. SUB- PARTICIPANTS NAME AND ADDRESS

NO. PART

HATCHING
EGG

CAPACITY
PRODUCTS CLASSIFIED

U.S. PULLORUM-TYPHOID

CLEAN
ADDITIONAL

CLASSIFICATIONS FOR WHICH PRODUCT QUALIFIED

64 - ALABAMA

RAY HILBURN

POULTRY PATHOLOGIST

DEPARTMENT OF AGRICULTURE \& INDUSTRIES

376 BRYAN AVENUE

LUVERNE, AL 36049

Phone: (334)240-7255

Fax: $\quad(334) 223-7352$

Hatcheries

800 E ANDERSON CREEK HATCHERY, 6780 HIGHWAY 207. ANDERSON 35610

Phone: (256)247-3607

\begin{abstract}
848 E RAYMOND AUGHTMAN HATCHERY, RT. 4, BOX 435-B, ANDALUSIA 36420

Phone: (334)222-5613

477 E BEN BABAUTA HATCHERY, 330 HUGULEY ROAD, OPELIKA 36804

742 E BAIN QUAIL FARM HATCHERY, 4223 MONTGOMERY HIGHWAY, DOTHAN 36303

Phone: (334)692-3334

542 E BECK'S DUTCH BANTAM FARM \& HATCHERY, 118 BATES DRIVE, JACKSON 36545

604 E BLACK SHEEP GAME FARM, R. MEGGINSON, 1456 MIDWAY ROAD, THOMASVILLE 36784

Phone: (334)636-5890

828 E CHINNABEE GAME FARM HATCHERY, WOLF, 2146 CHINNABEE ROAD, MUNFORD 36268

Phone: (256)315-0339

612 E CIRCLEL QUAIL FARM. INC., GARY D.LOLLEY, 17734 HIGHWAY 134, ENTERPRISE 36330

794 E DEE DAVIDSON HATCHERY, 80 COUNTY ROAD 441, VERBENA 36091

Phone: (205)280-1899
\end{abstract}

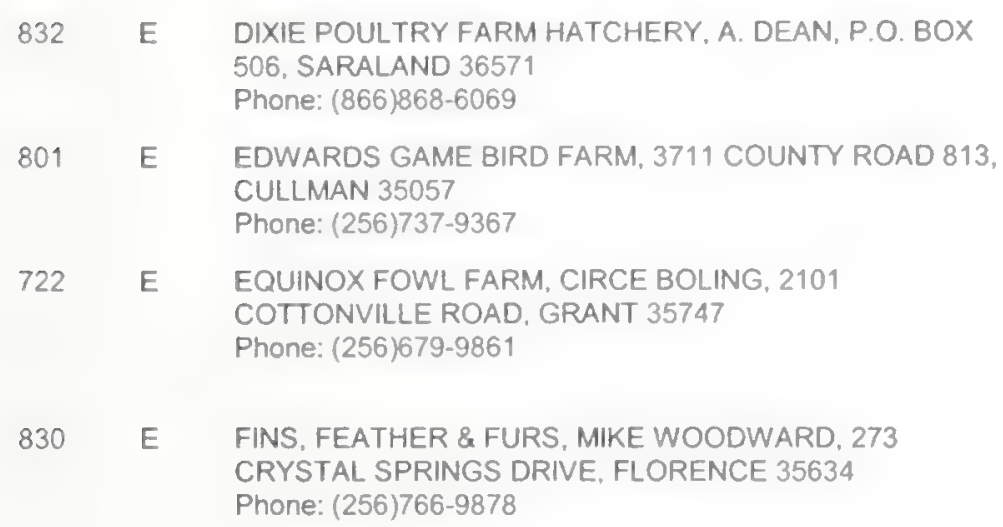

DR.TONY FRAZIER

STATE VETERINARIAN

DEPARTMENT OF AGRICULTURE \& INDUSTRIES

P.O. $80 \times 3336$

MONTGOMERY, AL 36109-0336

Phone: (334)240-7253

Fax: $\quad(334) 240-7198$
R7, W208, W26

N28

$228,000 \quad Z 43$

Al

240 W30, W396, W401, W552, W570, W606, W607

500 N236, N240, N28, N41, N44

N N28, N32, N44, N83, N84

$533,520 \quad Z 43$

Al

4,560 R33, R60, W119, W12, W142. W41, W48, W504, W518, W52. W570, W585, W74, W75, W93. $X 10, X 143, X 17, X 187, X 27, X 28$, X9, Y2, Y9, Z108, Z128, Z202. Z204. Z251, Z9

888 R1, R15, R196, R239, R247, R7. $R 71, W 198, W 25, X 14, X 25, \times 27$, $\times 3, \times 4, \times 6$

$55,000 \quad$ Z247, Z43

1,500 N121, N131, N135, N146, N151, N17, N181, N182, N187, N28, N34, N41, N57, N65, N93, R195, R89

$1,500 \quad \mathrm{Z} 173$. 
Table 1. HATCHERIES, DEALERS, AND INDEPENDENT FLOCKS PARTICIPATING IN

THE NATIONAL POULTRY IMPROVEMENT PLAN

WATERFOWL, EXHIBITION POULTRY AND GAME BIRDS

\begin{tabular}{|c|c|c|c|c|c|}
\hline $\begin{array}{l}\text { APP. } \\
\text { NO. }\end{array}$ & $\begin{array}{l}\text { SUB- } \\
\text { PART }\end{array}$ & PARTICIPANTS NAME AND ADDRESS & $\begin{array}{l}\text { HATCHING } \\
\text { EGG } \\
\text { CAPACITY }\end{array}$ & $\begin{array}{c}\text { PRODUCTS CLASSIFIED } \\
\text { U.S. PULLORUM-TYPHOID } \\
\text { CLEAN }\end{array}$ & $\begin{array}{c}\text { ADDITIONAL } \\
\text { CLASSIFICATIONS FOR } \\
\text { WHICH PRODUCT QUALIFIED }\end{array}$ \\
\hline
\end{tabular}

64 - ALABAMA

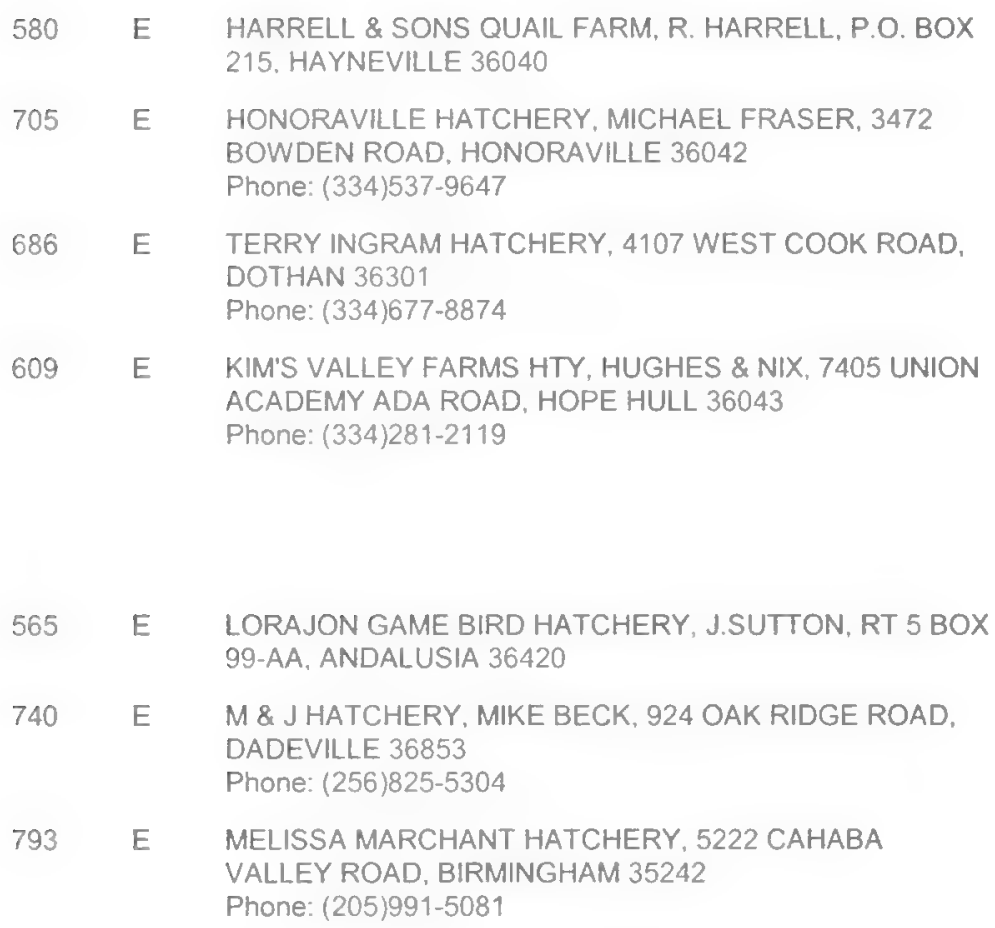

LORAJON GAME BIRD HATCHERY, J.SUTTON, RT 5 BOX 99-AA, ANDALUSIA 36420 740 E M \& J HATCHERY, MIKE BECK, 924 OAK RIDGE ROAD,
DADEVILLE 36853

Phone: (256)825-5304

MELISSA MARCHANT HATCHERY, 5222 CAHABA

VALLEY ROAD, BIRMINGHAM 35242

Phone: (205)991-5081

MARENGO CREEK GAME FARM HTY., S. HARVARD, 151 HUGULEY ROAD, OPELIKA 36804

Phone: (334)448-6050

AL MCGRAW'S HATCHERY, 424 COUNTY ROAD 93, ANDERSON 35610

Phone: (256)247-3769

MCKELVEY HATCHERY, 2506 CHURCH ROAD, JACKSONS GAP 36861

Phone: (256)825-6660

ALLEN MOORE'S QUAIL FARM HATCHERY, 2818 COUNTY ROAD 421, CULLMAN 35057

Phone: (256)734-9494

479 E MOORE'S GAME BIRD FARM HTY, J. MOORE, RR 2, BOX 180. BROWNS 36759

743 E MORTON'S QUALL FARM HATCHERY, 12266 SLAYTON ROAD, DUNCANVILLE 35456

Phone: (205)349-4857 COUNTY ROAD 303. LAWLEY 36793 Phone: (334)366-2752

MUG WUNP MOUNTAIN GAME FARM HTY.R.KELLEY, 15252 SANDLIN MOUNTAIN ROAD, BROOKWOOD 35444 Phone: (205)477-6640

E MUSKOGEE CREEK GAME FARM HTY, J. BOND, 201
$118,000 \quad Z 43$

2,580 R146, R196, R20, R247, R248, R7, W387, X4, X6, Y1, Y2, Y4, Z204, Z205, Z247, Z41, Z9

$370 \mathrm{~W} 121, \mathrm{~W} 18, \mathrm{~W} 23$

1,350 R104, R220, R28, R94, W1 W111, W13, W131, W132, W187, W207, W283, W30, W32, W35, W415, W475, W566. W570, X, Y, Z107, Z11, Z14, Z2 Z202, Z204, Z205, Z219, Z230. $\mathrm{Z} 284, \mathrm{Z3}, \mathrm{Z4}$

$7,200 \quad Z 1, Z 27,243$

800 N. W28, W569, W613

$3,200 \quad R, W, \times 135, \times 143, \times 168, \times 17$, $\times 187, \times 20, X 23, \times 25, X 38, X 4$ $X 6, X 9, X 96, Y 23, Y 3, Y 4, Y 9$, Z108, Z128, Z19, Z202, Z204, $\mathrm{Z2} 30, \mathrm{Z} 4$

3,456 N121, N134, N28, N32, N44, N83.

4,680 R222, R69, W1, W13, W132, W25, W30, W301, W365, W39. W415, W542, W570, W66, W96, Z11, Z231, Z4

2,400 R15, R166, R177, R20, R229, R257, R76, R92

448 W114, W28, W48, W613, W88

16,300 R146, R220, R42, Z27

$228,000 \quad Z 1, Z 43, Z 45, Z 89$

$2,900 \quad Z 173, Z 43, Z 48$

- N105, N28

360 N148, N151, N28, N32, N90

$1,120 \mathrm{~N} 139, \mathrm{~N} 164, \mathrm{~N} 28, \mathrm{~N} 33, \mathrm{~N} 88, \mathrm{~N} 90$, N97
Al THOMLEY ROAD. NEWTON 36352 Phone: (334)692-3328 
Table 1. HATCHERIES. DEALERS, AND INDEPENDENT FLOCKS PARTICIPATING IN

THE NATIONAL POULTRY IMPROVEMENT PLAN

WATERFOWL, EXHIBITION POULTRY AND GAME BIRDS

\begin{tabular}{|c|c|c|c|c|c|}
\hline $\begin{array}{l}\text { APP. } \\
\text { NO. }\end{array}$ & $\begin{array}{l}\text { SUB- } \\
\text { PART }\end{array}$ & PARTICIPANTS NAME AND ADDRESS & $\begin{array}{l}\text { HATCHING } \\
\text { EGG } \\
\text { CAPACITY }\end{array}$ & $\begin{array}{c}\text { PRODUCTS CLASSIFIED } \\
\text { U.S. PULLORUM-TYPHOID } \\
\text { CLEAN }\end{array}$ & $\begin{array}{c}\text { ADDITIONAL } \\
\text { CLASSIFICATIONS FOR } \\
\text { WHICH PRODUCT QUALIFIED }\end{array}$ \\
\hline
\end{tabular}

862 E OTIS ENGLISH HATCHERY, 148 JEFFERSON AVENUE, ATMORE 36502

Phone: (251) 577-6152

284 E AUBURN (SONNY) POE HATCHERY, P.O. BOX 324. HEFLIN 36264

733 E QUAIL START FARM HATCHERY, CLECKLER, 2606 COUNTY ROAD 28, CLANTON 35046

Phone: (205)755-1240

400 E SAND MOUNTAIN QUAIL FARM HTY., D. KIDD, P.O. BOX 246. CROSSVILLE 35962

Phone: (256)561-3471

453 E SCHELL'S HATCHERY, R. SCHELL, HC 65, BOX 196 WAGARVILLE 36585

868 E SHOALS CREEK GAME BIRD HATCHERY, HADDOCK, 5791 CR 47, FLORENCE 35634

Phone: (256)766-4070

870 E WARRIOR RIVER QUAIL FARM, CASEY PATE, 2429 DOVERTOWN ROAD, CORDOVA 35550 Phone: (205)483-7093

687 E DERRELL WILSON HATCHERY, 1175 WALKER FERRY ROAD, ALEXANDER CITY 35010

476 E WILSON'S HATCHERY, 2338 BELL FORK ROAD, ATMORE 36502

Phone: (251)368-9398

803 E WINDHAM'S BIRD HATCHERY, 438 NORTH MAIN STREET, SLOCOMB 36375 Phone: (334)671-9641

359 E WINDY HILL FARM HATCHERY, R.D. HERRING, 4120 UPPER RIVER ROAD, P.O. BOX 780250, TALLASSEE 36078

704 E WIREGRASS QUAIL FARM, J.B. HUGHES, 3404 CO. ROAD 617, ENTERPRISE 36330

Phone: (334)347-2847

810 E JERRY WOLFE, P.O. BOX 918, ASHLAND 36251

Phone: (256)354-0015

Independent Flocks

$863 E$ JERRY ADKINS, 142 WALKER CIRCLE, NAUVOO 35578 Phone: (205)697-5148

769 E JOSEFINO C. AGUILAR, 1123 SCOTT ROAD.

HAZELGREEN 35750

Phone: (256)828-9855

700 E EDDIE AKINS. 3579 HULL ROAD, EMPIRE 35063

Phone: (205)648-0702

268 E RAY ALEXANDER, 44721 US HIGHWAY 78, LINCOLN

35096

Phone: (205)763-9601

804 E R. C. ALEXANDER, 27673 OAK GROVE ROAD, ELKMONT 35620

Phone: (256)423-3021

701 E LOUISE \& DEWAIN ATCHLEY, 1021 EAST LACON ROAD, FALKVILLE 35622

Phone: (256)784-5036
650 R12, R146, R19, R196, R233,

R247, R72, W, X, Z231, Z4

- N28, N32, N41, N44, N83

59,200

Z43, Z45

142,800

Z43.

Al

792 W394, W396, W401, W552, W577

$1,000 \quad Z 43$

10,400

243

6.920

$R, W, X, Y, Z 231, Z 4$

1,440

$\mathrm{R}, \times 20$

R30, W W1, W119, W570, Z1, Z118, Z130, Z26, Z283, Z53

$1,000 \quad \mathrm{~N} 106, \mathrm{~N} 108, \mathrm{~N} 113, \mathrm{~N} 18, \mathrm{Z5}$

$168,480 \quad Z 43, Z 45$

N134, N136, N32

N121, N140, N152, N161, N183, N32, N41, N44

N104, N109, N111, N116, N121, N127, N131, N134, N152, N161. N163, N164, N188, N206, N21, N32, N33, N43, N44, N63

N130, N134, N152, N190, N219, N230, N62

N25, N28, N33, N41, N44, N80, N84

N121, N131, N41, N44, N61.

N134, N32, N43, N61, N83 
Table 1. HATCHERIES, DEALERS, AND INDEPENDENT FLOCKS PARTICIPATING IN

THE NATIONAL POULTRY IMPROVEMENT PLAN

WATERFOWL, EXHIBITION POULTRY AND GAME BIRDS

\begin{tabular}{lllll}
\hline & & HATCHING & PRODUCTS CLASSIFIED \\
APP. SUB- & PARTICIPANTS NAME AND ADDRESS & EGG & U.S. PULLORUM-TYPHOID & CLEAN \\
NO. PART & CAPACITY & & CLASSIFICATIONS FOR \\
\hline
\end{tabular}

64 - ALABAMA

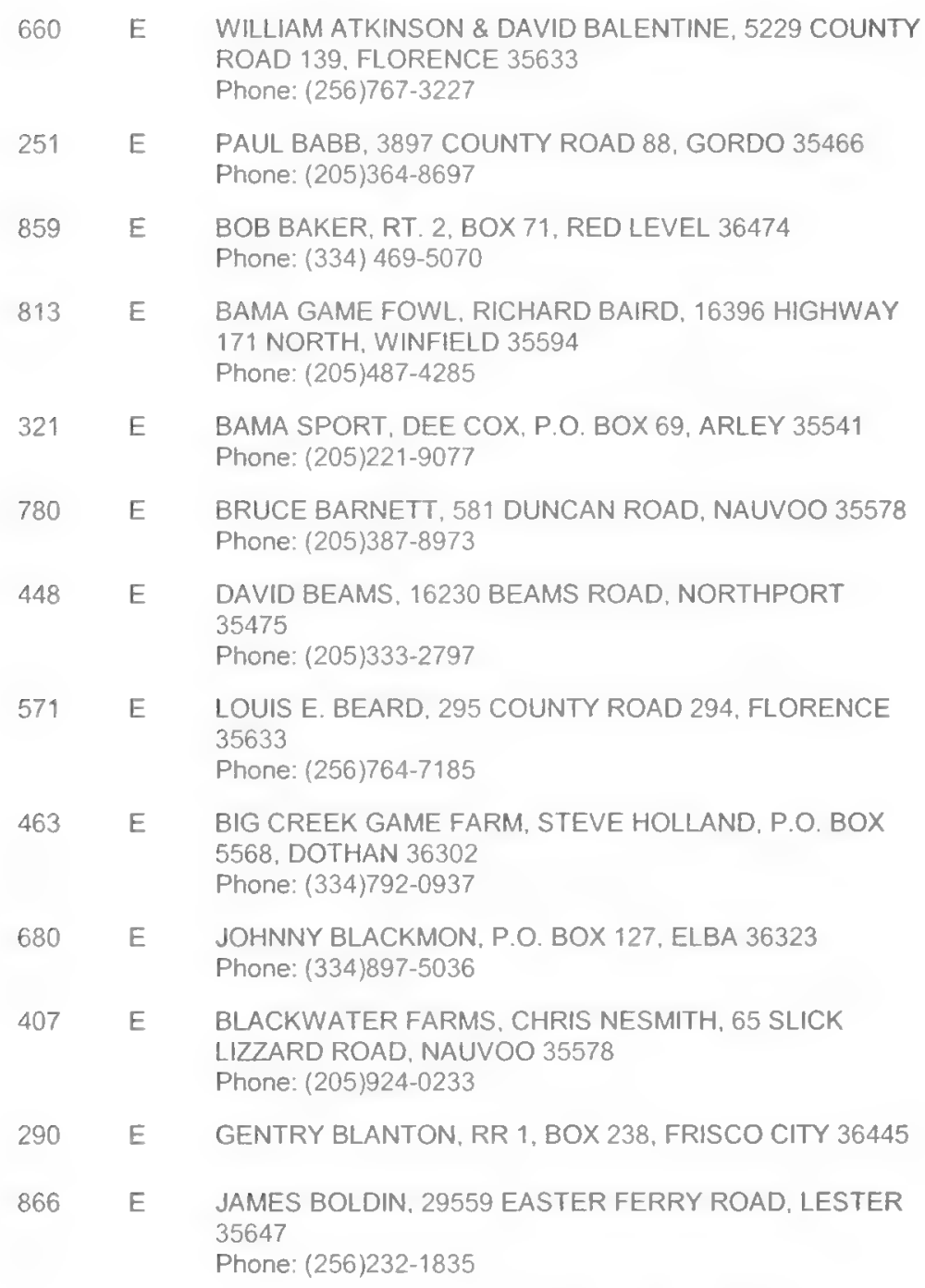

E TERRY CAGLE, 5695 COUNTY ROAD 113, CENTRE 35960

Phone: (256) 475-9271

787 E JAMES W. CALAHAN, 7205 COUNTY ROAD 80,

GAYLESVILLE 35973

Phone: (256)422-6452
N28, N41, N44

W6

N

N28, N44

N28, N32, N33, N41

N134, N152, N167, N44

W187, W518, W615, W74, W75, W88

N152, N28, N32, N44, N90, N93.-

N105, N25, N28, N31, N32, N43, N44, N56, N62, N65, N83

N161, N174, N28, N42, N44, N65. N85

N121, N152, N243, N32, N44_...

N28, N32, N33

N163, N174, N61

N121, N32, N41

w25, w26.

W28, W578, W91

W115, W123, W128, W141 W151, W174, W175, W187. W221, W245, W293, W294, W295. W50, W51

N134, N137, N28, N44, N63, N83.

N121, N131, N150, N152, N168, N83

N121, N134, N142, N155, N161, N168, N21, N28, N33, N34, N43, N44, N57, N65, N83, N98 
Table 1. HATCHERIES, DEALERS, AND INDEPENDENT FLOCKS PARTICIPATING IN

THE NATIONAL POULTRY IMPROVEMENT PLAN

WATERFOWL, EXHIBITION POULTRY AND GAME BIRDS

\begin{tabular}{|c|c|c|c|c|c|}
\hline $\begin{array}{l}\text { APP. } \\
\text { NO. }\end{array}$ & $\begin{array}{l}\text { SUB- } \\
\text { PART }\end{array}$ & PARTICIPANTS NAME AND ADDRESS & $\begin{array}{l}\text { HATCHING } \\
\text { EGG } \\
\text { CAPACITY }\end{array}$ & $\begin{array}{l}\text { PRODUCTS CLASSIFIED } \\
\text { U.S. PULLORUM-TYPHOID } \\
\text { CLEAN }\end{array}$ & $\begin{array}{c}\text { ADDITIONAL } \\
\text { CLASSIFICATIONS FOR } \\
\text { WHICH PRODUCT QUALIFIED }\end{array}$ \\
\hline
\end{tabular}

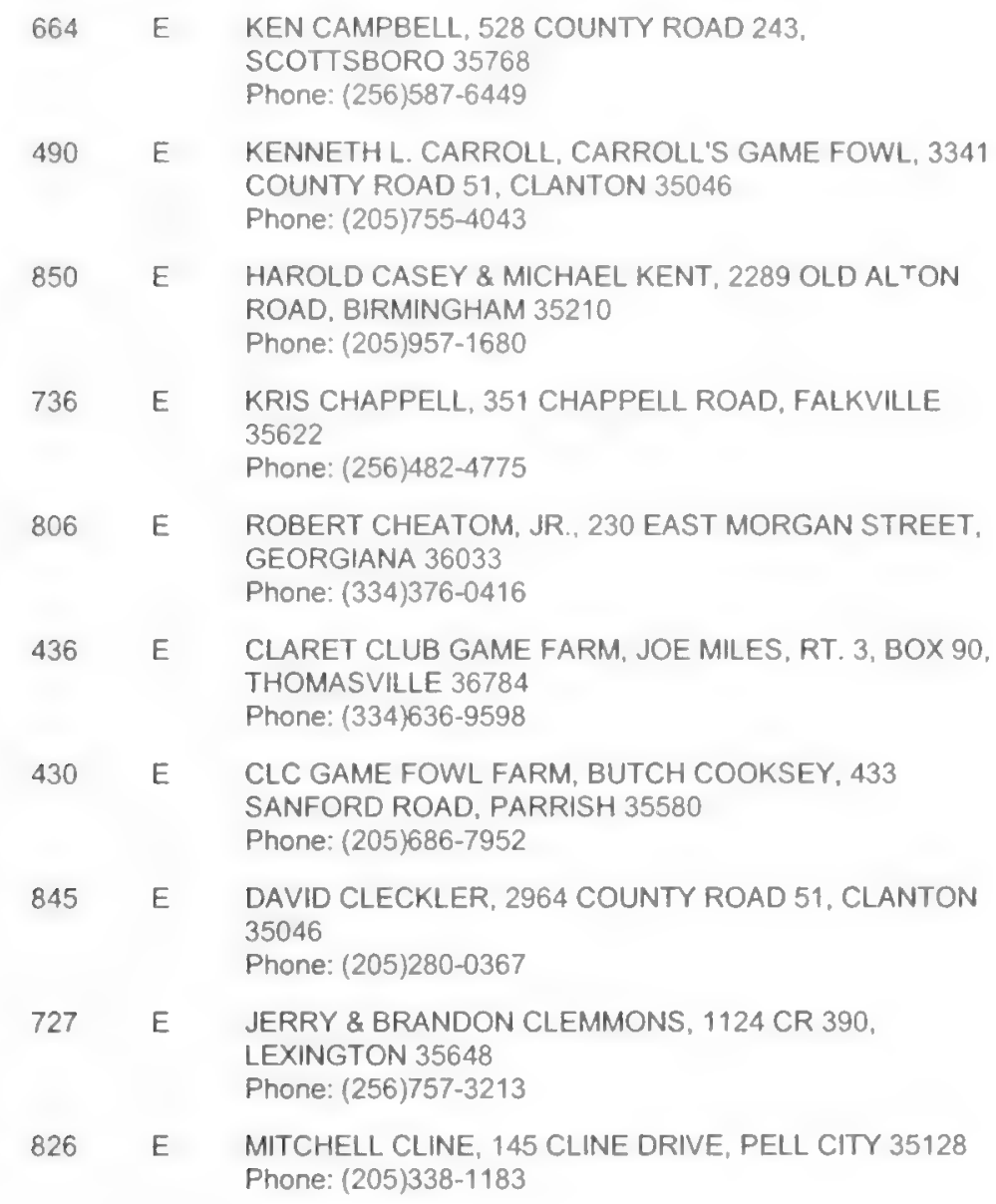

E ROBERT CHEATOM, JR., 230 EAST MORGAN STREET, GEORGIANA 36033 Phone: (334)376-0416

E ClaRET CLUB GAME FARM, JOE MILES, RT. 3, BOX 90 , THOMASVILLE 36784 Phone: (334)636-9598

E CLC GAME FOWL FARM, BUTCH COOKSEY, 433 SANFORD ROAD, PARRISH 35580 Phone: (205)686-7952

E DAVID CLECKLER, 2964 COUNTY ROAD 51, CLANTON 35046 Phone: (205)280-0367

E JERRY \& BRANDON CLEMMONS, 1124 CR 390. LEXINGTON 35648 Phone: (256)757-3213

E MITCHELL CLINE, 145 CLINE DRIVE, PELL CITY 35128 Phone: (205)338-1183

E COAST GAME FARM, RUSSELL HOLLEY, P.O. BOX 157, IRVINGTON 36545

E ELIZABETH COCHRAN, 1099 OLD HARTFORD ROAD, HARTFORD 36344

Phone: (334)588-0630

E JIMMY \& EARNEST CODY, 2901 COUNTY ROAD 73, KILLEN 35648

Phone: (256) 757-8274

E TIM COLE, 831 SPRINGDALE ROAD, HARTSELLE 35640 Phone: (256)773-0265

E JOHN COLE, CHEROKEE COMBINE \#1, 495 COUNTY ROAD 65, CENTRE 35960

Phone: (256)927-6870

E ELLIOTT CONWAY, JR., 612 HIGHWAY 49,

RUSSELLVILLE 35653

Phone: (256)331-9715

E JAMES D. COOK, 361 WATER TOWER LOOP, LACEY SPRINGS 35754

Phone: (256)498-3292
W188, W28

N121, N140, N152, N32, N33

N126, N152, N44, N83, N87

N133, N136, N33, N44, N83

N136, N167, N236, N28

N61.

N130, N152, N44, N83

N152, N236, N243

W14

N104, N106, N109, N114, N121, N131, N133, N134, N139, N161, N162, N167, N183, N194, N198, N236, N24, N243, N247, N28, N32, N33, N34, N41, N77, N84. N87, N98

N162, N34, N44

N121, N152, N32, N44, N93

W128, W142, W151, W187, W28, W50, W88

N138, N150, N155, N161, N164, N25, N33, N34, N41, N43, N46, N56, N83, N85

N28, N32, N90

W34, W48, W89, X28, X4, X6, $X 8, Y 20, Y 8, Y 9$

W74 
Table 1. HATCHERIES, DEALERS, AND INDEPENDENT FLOCKS PARTICIPATING IN

THE NATIONAL POULTRY IMPROVEMENT PLAN

WATERFOWL, EXHIBITION POULTRY AND GAME BIRDS

\begin{tabular}{lllcc}
\hline & & HATCHING & PRODUCTS CLASSIFIED \\
APP. & SUB- & PARTICIPANTS NAME AND ADDRESS & EGG & U.S. PULLORUM-TYPHOID \\
NO. & PART & CAPACITY & CLEAN & CLASSIFICATIONS FOR \\
\hline
\end{tabular}

64 - ALABAMA

\begin{tabular}{|c|c|c|}
\hline 557 & $\mathrm{E}$ & $\begin{array}{l}\text { JIMMY CORNELIUS, } 1455 \text { SLIGO ROAD, BOAZ } 35956 \\
\text { Phone: (256)538-7050 }\end{array}$ \\
\hline 585 & $E$ & $\begin{array}{l}\text { CORNISH CORNER, K. HERRING, } 2344 \text { LOVE LADY } \\
\text { ROAD, TALLASSEE } 36078 \\
\text { Phone: (334)283-4837 }\end{array}$ \\
\hline 696 & $E$ & $\begin{array}{l}\text { PECKERWOOD GAME FARM, MIKE \& JAKE CREW, } 214 \\
\text { WILLOW STREET, JACKSON GAP } 36861 \\
\text { Phone: (205)825-7287 }\end{array}$ \\
\hline 170 & $E$ & $\begin{array}{l}\text { CHARLES GLEN CRYAR, } 18 \text { HAYNES DRIVE, MOODY } \\
35004 \\
\text { Phone: (205)640-5776 }\end{array}$ \\
\hline & $E$ & $\begin{array}{l}\text { W. T. CURBOW, } 107 \text { SIMMONS ROAD, LACEY SPRING } \\
35754 \\
\text { Phone: }(256) 778-7615\end{array}$ \\
\hline
\end{tabular}
618 E DANIEL'S GAME FARM, E. \& N. DANIELS, 184 BURGETT
DRIVE, JASPER 35541

Phone: (205)221-0226
702 E DARK HOLLOW, RONALD \& DONALD HENRY, 295 MATKINS COVE ROAD, LACEY SPRINGS 35754 Phone: (256)778-8905

\begin{tabular}{|c|c|c|}
\hline 676 & $E$ & $\begin{array}{l}\text { DERICK DAUGHTRY, } 900 \text { BRYANT STREET, MOBILE } \\
36608\end{array}$ \\
\hline 772 & $E$ & $\begin{array}{l}\text { MICHAEL DAUGHTRY, 4550-A PINE MEADOW DRIVE, } \\
\text { EIGHT MILE } 36613\end{array}$ \\
\hline 403 & $E$ & $\begin{array}{l}\text { BRENT DAVENPORT, } 5085 \text { COUNTY ROAD } 51 \text {, } \\
\text { CLANTON } 35046 \\
\text { Phone: (205) } 755-2467\end{array}$ \\
\hline 08 & $E$ & $\begin{array}{l}\text { DEEP SOUTH GAME FARM \#2, DAVEY SIPPER, } 101 \\
\text { SIPPER LANE, LUVERNE } 36049 \\
\text { Phone: (334)335-4665 }\end{array}$ \\
\hline 209 & $E$ & $\begin{array}{l}\text { J. V. DEVANEY, } 3094 \text { COUNTY LINE ROAD, MADISON } \\
35756\end{array}$ \\
\hline 61 & $E$ & $\begin{array}{l}\text { JAMES DEVANEY, } 260 \text { LAGRANGE ROAD, TUSCUMBIA } \\
35674 \\
\text { Phone: }(256) 383-3489\end{array}$ \\
\hline 381 & $E$ & $\begin{array}{l}\text { JOHNNIE DIGBY, P.O. BOX 3101, OXFORD } 36203 \\
\text { Phone: (256)831- } 1410\end{array}$ \\
\hline 814 & $E$ & $\begin{array}{l}\text { DIXIELAND GAME FARM, TONY NELSON, } 290 \text { COUNTY } \\
\text { ROAD 495, VERBENA } 36091 \\
\text { Phone: (205)755-3396 }\end{array}$ \\
\hline 132 & $E$ & $\begin{array}{l}\text { HARRY DOUGLAS, } 66 \text { WARRENTON ROAD, } \\
\text { GUNTERSVILLE } 35976 \\
\text { Phone: (256)931-4855 }\end{array}$ \\
\hline 374 & $E$ & $\begin{array}{l}\text { JIMMY EAST GAME CLUB, } 607 \text { WINCHESTER ROAD, } \\
\text { HUNTSVILLE } 35811\end{array}$ \\
\hline 857 & $E$ & $\begin{array}{l}\text { KEITH EDWARDS, CHEROKEE COMBINE \#2, } 1190 \\
\text { COUNTY ROAD 136, CEDAR BLUFF } 35959 \\
\text { Phone: (256) 526-8568 }\end{array}$ \\
\hline & $\mathrm{E}$ & W.T. EPPERSON, 299 CENTER ROAD, ATTALLA 35954 \\
\hline
\end{tabular}

N140, N46.

H10, H5, R116, R86, W96

N104, N127, N131, N236, N32, $\mathrm{N} 41, \mathrm{~N} 44, \mathrm{~N} 63, \mathrm{~N} 83$

W114, W28, W30, W35, W45, W48, W65, W69, W93

W141, W142, W149, W151, W274, W276, W28, W29, W50, W51, W569, W579, W69, W88, W89, X10, X112, X114, X115, $\mathrm{X} 117, \mathrm{X} 122, \mathrm{X} 123, \mathrm{X} 13, \mathrm{X} 28, \mathrm{X} 4$, $X 40, X 49, \times 50, X 52, X 53, X 54$, $X 55, X 70, X 78, X 8, Y 27, Y 31$, Y37, Y8, Y9

N152, N28, N44

N140, N236, N28

N171, N28, N33, N65

N132, N32, N65, N94

W128, W166, W29

N132, N152, N160, N164, N32, N34

N28, N32, N33, N44

N28, N44

N107, N116, N143, N25, N28, N32, N33, N61, N83, N94

N148, N243, N28, N32, N41, N44.

W151, W25, W26

$\mathrm{N} 28, \mathrm{~N} 32, \mathrm{~N} 34, \mathrm{~N} 41, \mathrm{~N} 44$

N138, N150, N155, N161, N164, N25, N33, N34, N41, N43, N46, N56, N83, N85

N28, N41, N44 
Table 4. HATCHERIES, DEALERS, AND INDEPENDENT FLOCKS PARTICIPATING IN

THE NATIONAL POULTRY IMPROVEMENT PLAN

WATERFOWL, EXHIBITION POULTRY AND GAME BIRDS

\begin{tabular}{|c|c|c|c|c|c|}
\hline $\begin{array}{l}\text { APP. } \\
\text { NO. }\end{array}$ & $\begin{array}{l}\text { SUB- } \\
\text { PART }\end{array}$ & PARTICIPANTS NAME AND ADDRESS & $\begin{array}{l}\text { HATCHING } \\
\text { EGG } \\
\text { CAPACITY }\end{array}$ & $\begin{array}{c}\text { PRODUCTS CLASSIFIED } \\
\text { U.S. PULLORUM-TYPHOID } \\
\text { CLEAN }\end{array}$ & $\begin{array}{c}\text { ADDITIONAL } \\
\text { CLASSIFICATIONS FOR } \\
\text { WHICHPRODUCT QUALIFIED }\end{array}$ \\
\hline
\end{tabular}

847 E WILLIAM EVANS, 12605 BARGER ROAD, TUSCALOOSA 35406

860 E RANDALL FARMER, 5121 NESBITH LAKE ROAD, JACKSONVILLE, 36265

Phone: (256)435-9751

632 E FOGGY HOLLOW FARM, HOWARD MAYBEN, 672 WEBSTER CHAPEL ROAD, WELLINGTON 36279 Phone: (256)492-8646

798 E FOWLER FANCY FOWLS, NANCY FOWLER, 358 NEW LIBERTY ROAD, BANKSTON 35542

Phone: (205)689-4337

577 E JOHN FULLER, 209 GEARY DRIVE, MONTGOMERY 36108

Phone: (334)262-3702

815 E RICKY GARGIS, 56 HIGHWAY 227, SPRUCE PINE 35585 Phone: (256)332-9562

697 E DAVID GIBSON, 60001 AARAT ROAD, TOXEY 36921

362 E C. C. GIVENS, 2498 GOSSETT ROAD, HOKES BLUFF 35903 276 E
LARRY GLENN, 1315 COUNTY ROAD 57, PRATTVILLE
36067

747 E JOHNNY GODWIN, 345 JUDGE ALFORD ROAD,

BREWTON 36426

Phone: (251)867-6046

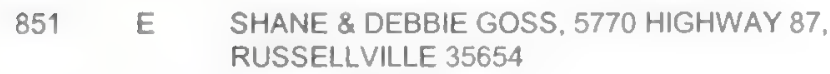

Phone: (256)332-5978

382 E MIKE GREEN, 280 MEEKS ROAD, WELLINGTON 36279 Phone: (256)492-0315

779 E GREEN MOUNTAIN GAME FARM, KELLY GREEN, P.O. BOX 48, BRILLIANT 35548

Phone: (205)465-2214

679 E JOHNNY GRIDER, 11225 LOTT ROAD, CHUNCHULA 36521

Phone: (251)649-5531

134 E S.G. \& GENEVA HAMILTON, 27 PINE VIEW LANE, CORDOVA 35550

Phone: (205)483-1156

816 E RANDY HAMILTON, 207 HIGHWAY 624, RUSSELLVILLE

35653

Phone: (256)332-6943

493 E BILLY HARRIS, 420 HARRIS DRIVE, ARAB 35016

836 E LADONNA A. HART, 1200 CONWAY ROAD, ATMORE

$36502-4840$

Phone: (251)368-4578

717 E MIKE HAYNES, 1315 BOILING SPRINGS ROAD,

OHATCHEE 36271

Phone: (256)892-0283
N162, N239, N28.

W28

N142, N152, N28, N32, N65, N83, N87

W102, W168, W169, W25, W26, W546

$N, R, W, X, Y, Z 2, Z 46, Z 5$

N152, N28, N32, N41, N44

N28, N44

N28, N32, N44

W120, W131, W30, W588

N28, N32, N63

N101, N104, N110, N130, N134, N23, N230, N24, N28, N33, N44

W114, W150, W151, W276, W28, W29, W48, W88, Z153, Z240, Z46, Z47

N152, N32, N41, N44, N63, N83, N90

N32, N63.

W128, W175, W187, W294

N152, N190, N219, N32, N41, N94

W142, W149, W150, W151 W28, W29, W48, W50, W51, W52, W89

W518, W615, W74, W75

N134, N168, N32, N43, N44, N7, R27

N139, N151, N28, N32, N33, N41. 
Table 1. HATCHERIES, DEALERS, AND INDEPENDENT FLOCKS PARTICIPATING IN

THE NATIONAL POULTRY IMPROVEMENT PLAN

WATERFOWL, EXHIBITION POULTRY AND GAME BIRDS

APP. SUB- PARTICIPANTS NAME AND ADDRESS

NO. PART
HATCHING
EGG

CAPACITY
PRODUCTS CLASSIFIED

U.S. PULLORUM-TYPHOID

CLEAN
ADDITIONAL

CLASSIFICATIONS FOR WHICH PRODUCT QUALIFIED

64 - ALABAMA

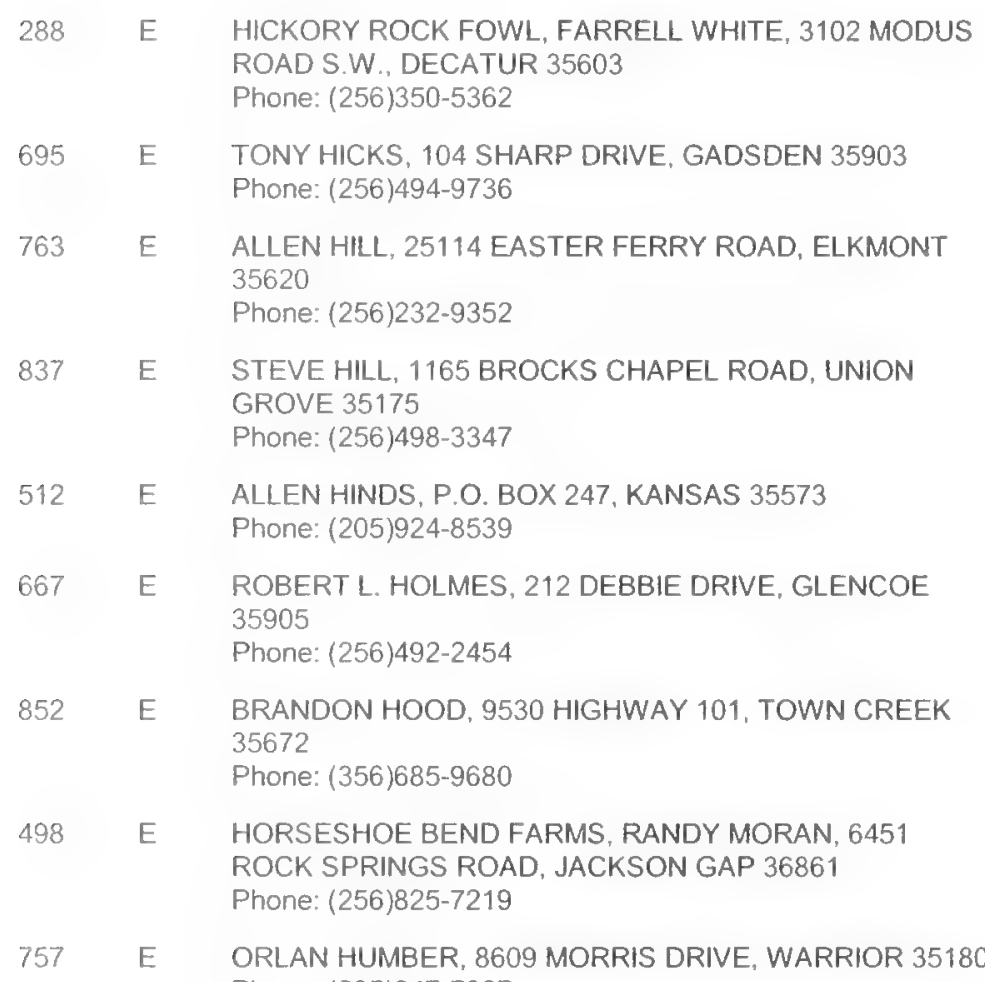

Phone: (205)647-7827
559 E HURTSBORO COMBINE, JOE WORRELL, 5228 SAND
FORT ROAD, SEALE 36875
Phone: (334)855-9274

781 E TOBY HUTCHESON, 693 MCNATT ROAD, SPRUCE PINE
35585

Phone: (256)332-7125

621 E RICHARD HYCHE, 241 HONEYSUCKLE LANE, EMPIRE

Phone: (205)648-6415

656 E J \& J FARMS, JOHN WILLIAMS, 3114-Y WALLACE
AVENUE EAST, FORT PAYNE 35967

Phone: (256)845-0933

838 E JBIRD, JASON ROBERTSON, 95 VICE LANE,

BLOUNTSVILLE 35031

Phone: (256)586-5844

619 E C.D. JARRET, 4410 COUNTY ROAD 818, LOGAN 35098 Phone: (256)747-3891

629 E JIM JOHNSON, 11604 COUNTY ROAD 27, SYLVANIA

35988

Phone: (256)638-1196

681 E CHARLIE JOHNSON, RT. 2, BOX 275-J, ELBA 36323

Phone: (334)897-3223

829 E ANGELA JOHNSON, 235 HIGHWAY 92, ENTERPRISE

36330

Phone: (334)393-5172

734 E BRIAN JOHNSON, 4031-A FOWL RIVER ROAD.

THEODORE 36582

Phone: (251)973-0295
N28, N33, N41, N44, N84

R27

N243, N28, N32, N44, N83

N134, N172, N44, N65

N152, N28, N41, N44, N83

N121, N126, N137, N161, N162, .. N168, N34, N44, N83

N101, N130, N161

N127, N152, N236, N28, N41.

N121, N127, N129, N131, N134, N140, N148, N152, N164, N167. N174, N181, N182, N211, N24, N33, N41, N43, N44, N77, N87, N93

N121, N134, N152, N161, N83_-_

W26, W28, Z2

N113, N121, N134, N146, N161, N219, N222, N90

N28, N41, N83, N97

N113, N140, N161, N189, N28, N44

N134, N150, N227

N121, N28, N34, N44, N83

N183, N27, N44, N84.

R196, X24, X4, Z231

N28, N43, N83 
Table 1. HATCHERIES, DEALERS, AND INDEPENDENT FLOCKS PARTICIPATING IN

THE NATIONAL POULTRY IMPROVEMENT PLAN

WATERFOWL, EXHIBITION POULTRY AND GAME BIRDS

\begin{tabular}{|c|c|c|c|c|c|}
\hline $\begin{array}{l}\text { APP. } \\
\text { NO. }\end{array}$ & $\begin{array}{l}\text { SUB- } \\
\text { PART }\end{array}$ & PARTICIPANTS NAME AND ADDRESS & $\begin{array}{l}\text { HATCHING } \\
\text { EGG } \\
\text { CAPACITY }\end{array}$ & $\begin{array}{l}\text { PRODUCTS CLASSIFIED } \\
\text { U.S. PULLORUM-TYPHOID } \\
\text { CLEAN }\end{array}$ & $\begin{array}{c}\text { ADDITIONAL } \\
\text { CLASSIFICATIONS FOR } \\
\text { WHICH PRODUCT QUALIFIED }\end{array}$ \\
\hline
\end{tabular}

534 E
35058 NETH JONES, 4125 COUNTY ROAD 1682, CULLMAN Phone: (256)796-2193

738 E ROBERT JONES, 11319 LUCAS FERRY ROAD, ATHENS 35611

Phone: (256)232-2127 755 E JONES AND SON BANTAM, 11316 SHORT CUT ROAD,
LESTER 35647

Phone: (256) 232-6531

672 E WALTER JONES, 22913 COUNTY ROAD 62 NORTH, ROBERTSDALE 36567

Phone: (251)978-8360

750 E ARCHIE KEETON, 8356 COUNTY ROAD 10, FLORENCE 35633

Phone: (256)718-3922

839 E KEG MOUNTAIN GAME FARM, BARNS \& ASHLEY, 3775 HIGHWAY 56, RUSSELLVILLE 35654

Phone: (256)332-3483

E LYLE KING, 1800 MOBBS SCHOOL ROAD, ARAB 35016 Phone: (256)586-5530

575 E STEVE KNOX, 5785 EASTERN VALLEY ROAD, MCCALLA 35111

Phone: (205)477-4739

731 E JACKIE LANE, 6310 SHERRY DRIVE, MOBILE 36613 Phone: (251)649-7941

BRAD LANE, 2279 COUNTY ROAD 434, MOULTON 35650 Phone: (256)350-0023

E THOMAS L. LAWSHE, 4675 SCHILLINGER ROAD, SEMMES 36575

CAMP HILL 36850

Phone: (256)896-2739

CLAUDE LIPSCOMB, 10020 VERNANT PARK ROAD, FOLEY 36535

Phone: (251)965-3600

THE LONG GREY LINE FARM, 2752 HOBBS ISLAND ROAD, HUNTSVILLE 35803

Phone: (256)883-9814

E DAVID LOUALLEN, 363 PAYTON PLACE, LINCOLN 35096 Phone: (205)763-0181

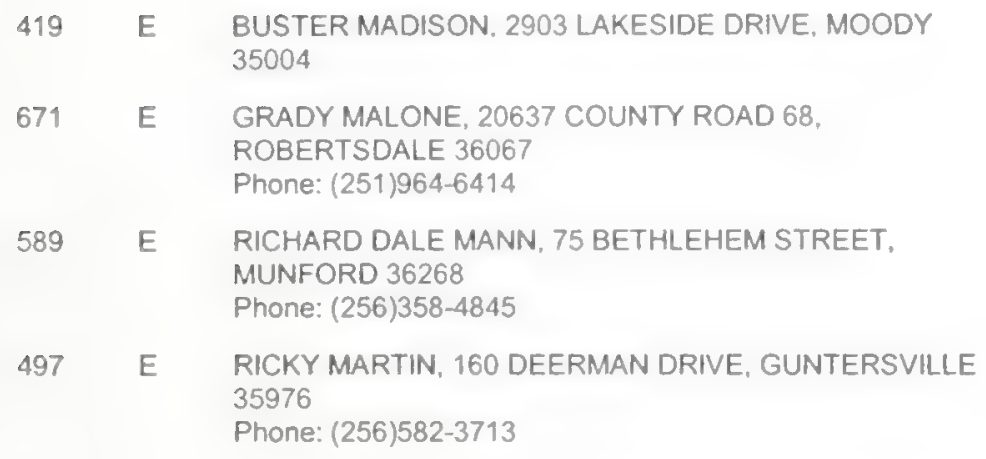

W150, W275, W542, W568 W579

N134, N136, N174, N43, N44, N65

W200, W275, W28, W69, W88, W91

N104, N22, N28, N33.

N161, N32, N33, N44

N139, N140, N152, N28

W25, W26, W88

N152, N25, N28

R222, R237, R94

N140, N28, N32

N132, N28, N41, N84, N85

N236, N268, N28, N41, N44

W

R172, Y22, Z106, Z15, Z205, Z22.

$X 114, X 121, X 141, X 51, X 58, X 59$, Y142, Y145, Y51, Y9, Z102, Z107, Z108, Z11, Z130, Z136, Z137, Z138, Z142, Z147, Z15, Z19, Z242, Z34, Z48, Z49, Z57, Z68

N134, N138, N150, N44, N87.

N28, N32, N41, N44, N83

N25, N41, N43, R27

N28, N33, N34, N41, N44 
Table 1. HATCHERIES, DEALERS, AND INDEPENDENT FLOCKS PARTICIPATING IN

THE NATIONAL POULTRY IMPROVEMENT PLAN

WATERFOWL, EXHIBITION POULTRY AND GAME BIRDS

\begin{tabular}{|c|c|c|c|c|c|}
\hline $\begin{array}{l}\text { APP. } \\
\text { NO. }\end{array}$ & $\begin{array}{l}\text { SUB- } \\
\text { PART }\end{array}$ & PARTICIPANTS NAME AND ADDRESS & $\begin{array}{l}\text { HATCHING } \\
\text { EGG } \\
\text { CAPACITY }\end{array}$ & $\begin{array}{c}\text { PRODUCTS CLASSIFIED } \\
\text { U.S. PULLORUM-TYPHOID } \\
\text { CLEAN }\end{array}$ & $\begin{array}{c}\text { ADDITIONAL } \\
\text { CLASSIFICATIONS FOR } \\
\text { WHICH PRODUCT QUALIFIED }\end{array}$ \\
\hline
\end{tabular}

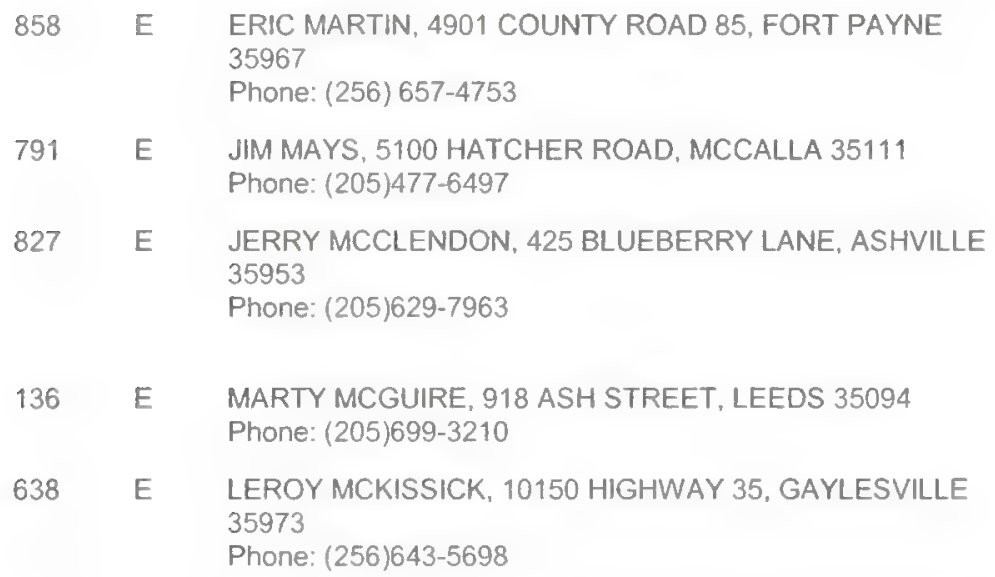

MITCHELL'S GAME FARM, JAMES MITCHELL, P.O. BOX 301. NEW BROCKTON 36351

Phone: (334)897-8283
A.D. MORALIS, 24420 PINE WOOD LANE, ROBERTSDALE 36567 Phone: (251)947-4383

E MATT MORALIS, 24690 PILGRIM ROAD, ELBERTA 36530 Phone: (251)986-8389

\section{$E$} 35959

E TWIN WILLOW, T. L. NALL, 928 IVANWOOD ROAD, BIRMINGHAM 35210

Phone: (205)956-2770

E JOHNNY AARON NICHOLS, P.O. BOX 2, MORRIS 35116 Phone: (205)647-3376

E NO NAME GAME FARM, C. BAKER, 384 HITCHIE ROAO, SEALE 36875

E JOHNNY NUNLEY, 321 COUNTY ROAD 9006, VALLEY HEAD 35989 Phone: (256)635-1059

E DENNIS OAKLEY, 275 DEER TRAIL LANE, FLORENCE 35633

Phone: (256)764-4026

E ROY ODOM, 1275 SCOTT CEMETARY ROAD, CARBON HILL 35549

Phone: (205)924-8600

E OHATCHEE GAME FARM, JERRY ELLARD, P.O. BOX 173, 1045 LOWIMOORE ROAD, ALEXANDRIA 36250 Phone: (256)892-0801
$\mathrm{R} 146, \mathrm{R} 247, \mathrm{X} 2, \times 4, \mathrm{Z}$

N121, N152, N174, N41, R247. $\mathrm{X} 4$

N104, N105, N114, N121, N131, N134, N135, N139, N142, N155, N163, N165, N167, N181, N182. N197, N32, N34, N44, N87

W102, W164, W17, W18, W38, W48, W54

N106, N121, N137, N138, N150, N155, N33, N34, N43, N44, N65, R27

R20, W12, W17, W23, W518, W52, W6, W74, W75, X10, X11, $\mathrm{X} 110, \mathrm{X} 112, \mathrm{X} 113, \mathrm{X} 114, \mathrm{X} 117$ $X 13, X 17, X 22, X 23, X 27, X 28$, $X 38, X 40, X 42, X 44, X 49, \times 5$, $\times 50, \times 52, \times 53, \times 54, \times 55, \times 58$ $X 61, X 62, X 7, X 70, X 73, X 74$, $X 76, X 78, X 8, X 9, Y 10, Y 26, Y 27$, $Y 31, Y 35, Y 37, Y 40, Y 41, Y 49$ $Y 51, Y 53, Y 8, Y 9, Z 118, Z 15$. Z176, Z19, Z27, Z28, Z53, Z57

N20, N24, N28, N33

N28, N41

N112, N183, N28, N41

W145, W19, W57

N136, N140, N152, N161, N224, N227, N28, N33, N41, N44, N83

N134, N152, N161, N28, N32, N44

N236, N28, N41

N138, N140, N24, N46

N140, N143, N152, N28, N44.

N28, N32, N44

N28, N32, N44 
Table 1. HATCHERIES, DEALERS, AND INDEPENDENT FLOCKS PARTICIPATING IN THE NATIONAL POULTRY IMPROVEMENT PLAN

WATERFOWL, EXHIBITION POULTRY AND GAME BIRDS

\begin{tabular}{|c|c|c|c|c|c|}
\hline $\begin{array}{l}\text { APP. } \\
\text { NO. }\end{array}$ & $\begin{array}{l}\text { SUB- } \\
\text { PART }\end{array}$ & PARTICIPANTS NAME AND ADDRESS & $\begin{array}{l}\text { HATCHING } \\
\text { EGG } \\
\text { CAPACITY }\end{array}$ & $\begin{array}{c}\text { PRODUCTS CLASSIFIED } \\
\text { U.S. PULLORUM-TYPHOID } \\
\text { CLEAN }\end{array}$ & $\begin{array}{c}\text { ADDITIONAL } \\
\text { CLASSIFICATIONS FOR } \\
\text { WHICHPRODUCT QUALIFIED }\end{array}$ \\
\hline
\end{tabular}

669 E DENNIS PARKER, 590 NUNNALLYLAKE ROAD,

OHATCHEE 36271

Phone: (256)892-0922

283 E GEORGE R. \& BOBBY PAYTON, 5116 COUNTY ROAD 37. THORSBY 35171

556 E SCOTT PHILLIPS, 4907 COUNTY ROAD 45, HAYDEN 35079

Phone: (205)559-7183

566 E POWERS GAME FOWL, R. POWERS, 12955 PLEASANT POINT ROAD, ATHENS 35611

E TIMOTHYH. PUCKETT, 6773 DANVILLE ROAD, HARTSELLE 35640

269 E SONNY PURSER, 4831 CR 73, KILLEN 35645

799 E GRADY QUATTLEBAUM. 1432 COUNTY ROAD 331, ELBA 36323

Phone: (334)897-6620
RED FOX FOWL, ROCHELLE BROWN, 5858 CLAY

PALMERDALE ROAD, PINSON 35126

Phone: (205)681-6554

E FRANK W. REID III, 1190 DOGWOOD LANE, PIEDMONT 36272

Phone: (256)435-2114

E DALE RIKARD, 121615 TH AVENUE SE, DECATUR 35601 Phone: (256)355-7054

E JACKIE ROGERS, P.O. BOX 303, GERALDINE 35974 Phone: (256)659-6283

E S \& K FARMS, KEN DENNY, RR 1, BOX 527, ROCKFORD 35136

E S \& S GAME FOWL, 18805 HIGHWAY 99, ATHENS 35611 Phone: (256)230-9723

E CARL J. SAIA, 1792 WOODBINE CIRCLE, BIRMINGHAM 35216

Phone: (205)942-5176

E CECIL SAMPLES, 256 COUNTY ROAD 1311, CULLMAN 35058

Phone: (256)739-3676

E KENNETH SAUCER, RR 2, BOX 219, FRISCO CITY 36445 Phone: (251)862-2253

SEBURN GALE BURNS, RISKY BUSINESS, 629 LEE ROAD 169. OPELIKA 36804

Phone: (334)749-5773

E RICKY SHAW, 185 SHAW DRIVE, REFORM 35481 Phone: (205)375-2878

E FLOYD SHIRLEY, 1415 COUNTY ROAD 46, BRILLIANT 35548

Phone: (205)465-9314

E RAIFORD SHIRLEY, 3222 SAND SPRINGS ROAD,

Phone: (205)364-7305

E SHOW TIME GAME FARM, ROBERT A. WARD, 189

HIGHWAY 206. MONTEVALLO 35115-7337

Phone: (205)665-1933 GOROO 35466
N28, N32, N34

N157, N163, N181, N34.

W150, W28, W48.

N121, N137, N138, N140, N151, N155, N161, N32, N33, N34, N44, N65, N84, N90

N32, N43, N44

$\mathrm{R} 52, \mathrm{R} 87$

N134, N224, N230, N27, N44, N65

N216, N219, N28, N32, N33, N41, R27

W28, W29, W50, W69, W88

W28, W578, W91

N28, N32, N33, N41, R27

N183, N41

N181. N33

N104, N110, N114, N121, N28, N41, N89

W149, W200, W275, W328,

W473, W52

N108, N28, N32, N41

N152, N231, N269, N28, N33, N44. N65

R114, R22, W103, W516, W56

N152, N198, N32, N43, N93.

W38, W394, W401, W54

N155: N270, N271, N28, N33.

N41, N44, N83 
Table 1. HATCHERIES, DEALERS, AND INDEPENDENT FLOCKS PARTICIPATING IN

THE NATIONAL POULTRY IMPROVEMENT PLAN

WATERFOWL, EXHIBITION POULTRY AND GAME BIRDS

APP. SUB- PARTICIPANTS NAME AND ADDRESS

NO. PART
PARTICIPANTS NAME AND ADDRESS

HATCHING
EGG
CAPACITY

PRODUCTS CLASSIFIED

U.S. PULLORUM-TYPHOID CLEAN
ADDITIONAL

CLASSIFICATIONS FOR WHICH PRODUCT QUALIFIED

\section{4 - ALABAMA}

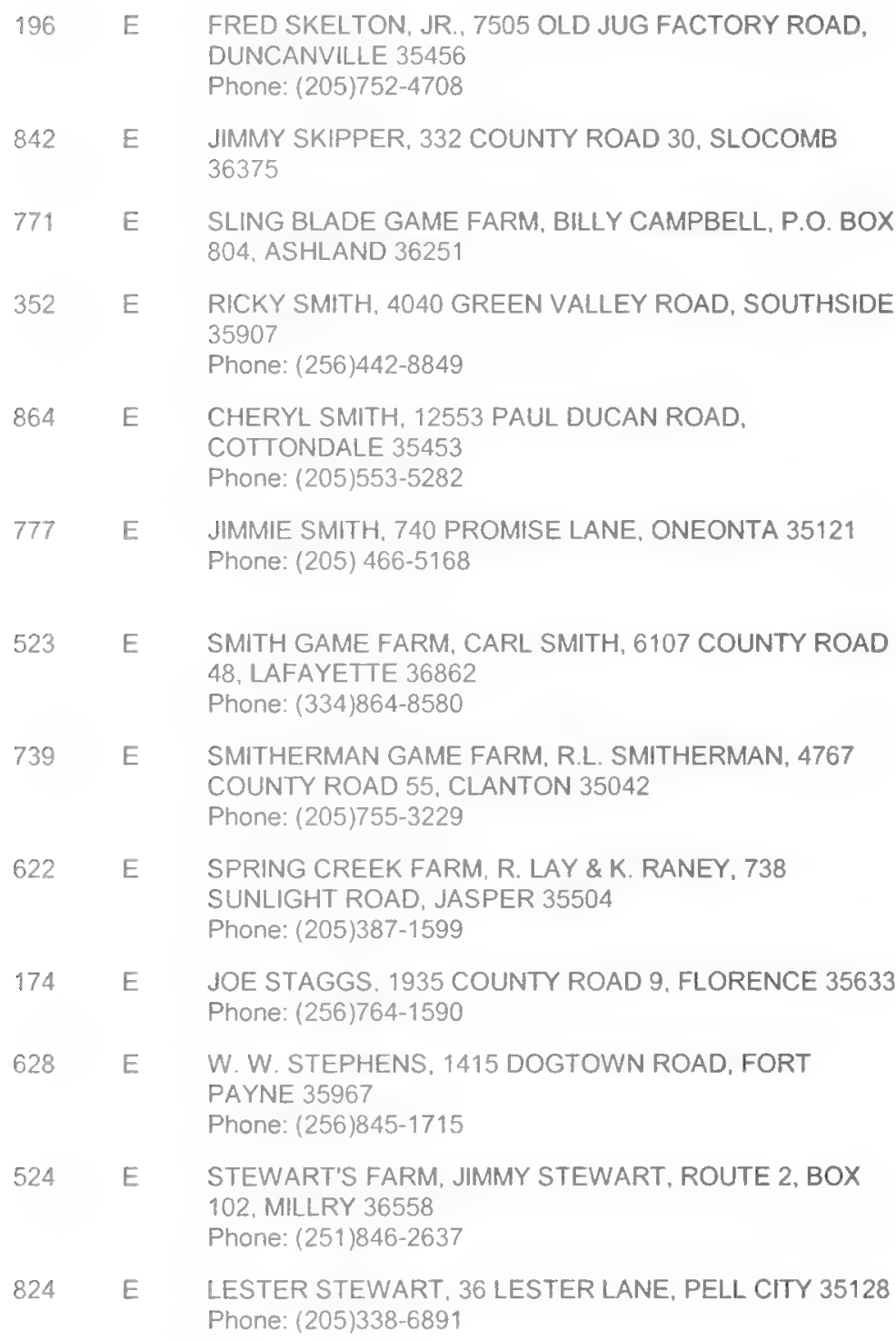

W88.

N51, W2

N108, N131, N98

N28, N32, N41, N44, N90

B7, C6, N, N236, R20, X12

N104, N121, N134, N136, N138, N150, N171, N182, N189, N211, N44, N63, N87

N104, N127, N134, N148, N156, N157, N159, N236, N43, N44

N152, N236, N243

N152, N28, N32, N41

N106, N137, N152, N172, N230, N33, N44, N61, N90, N93, N94

N10, N104, N148, N32, N41, N44, N83

N28, N32, N33, N41, N83, N90 ....

N101, N105, N114, N121, N134, N139, N155, N160, N163, N165 N167, N168, N181, N182, N2, N24, N33, N63, N65, N77, N84

N211, N231, N28, N83

N147, N168, N228, N25, N34, N44, N87

N225, N236, N33, N41, N63, N83

N155, N28, N44

N131, N236, N34, N44

N131, N134, N32, N43, N44 
Table 1. HATCHERIES, DEALERS, AND INDEPENDENT FLOCKS PARTICIPATING IN THE NATIONAL POULTRY IMPROVEMENT PLAN

WATERFOWL, EXHIBITION POULTRY AND GAME BIRDS

\begin{tabular}{|c|c|c|c|c|c|}
\hline $\begin{array}{l}\text { APP. } \\
\text { NO. }\end{array}$ & $\begin{array}{l}\text { SUB- } \\
\text { PART }\end{array}$ & PARTICIPANTS NAME AND ADORESS & $\begin{array}{l}\text { HATCHING } \\
\text { EGG } \\
\text { CAPACITY }\end{array}$ & $\begin{array}{l}\text { PRODUCTS CLASSIFIED } \\
\text { U.S. PULLORUM-TYPHOID } \\
\text { CLEAN }\end{array}$ & $\begin{array}{c}\text { ADDITIONAL } \\
\text { CLASSIFICATIONS FOR } \\
\text { WHICH PRODUCT QUALIFIED }\end{array}$ \\
\hline
\end{tabular}
865 E TAB BANTAMS, TERRY BECK, P.O. BOX 362, JACKSON

Phone: (251)246-4458

760 E JERRY TANNER \& RONNIE JUSTICE, 8797 BAGLEY ROAD, DORA 35062

Phone: (205)647-1278

843 E ROBERT TATE, 10150 KALIOKA ROAD, EIGHT MILE 36613

819 E CARL TEMPLETON, 95 COUNTY ROAD 504, CENTRE 35960

Phone: (256)475-5721

TNT GAME FARM, MICHAEL THOMPSON, 150 BUNNY CREEK ROAD, THOMASVILLE 36784

Phone: (334)736-4250

E CARY TRAVIS, ROUTE 1, BOX 135, MCKENZIE 36456 Phone: (334)374-8197

802 E JAMES R. TRUSSELL, 81 TRUSSELL PVT. DRIVE, DECATUR 35603

Phone: (256)355-7061

JOHN C. TUBBS, 2559 COUNTY ROAD 18, BREMEN 35033

Phone: (256)287-0386

698 E JOHN D. \& WENDY TURNEY, P.O. BOX 385, VINEMONT 35179

VAUGHAN FARMS, BILLY J. VAUGHAN, 53195 STATE HIGHWAY 79, BLOUNTSVILLE 35031

606 E YOU CALL IT GAME FARM, B. VAUGHN, 167 WHITETAIL ROAD, SEALE 36875

Phone: (334)855-2486

746 E CHRIS VOSS, 2235 COUNTY ROAD 12, HEFLIN 36264

Phone: (256)463-4906

699 E NEWTON WADE, P.O. BOX 364, OAKMAN 35579

Phone: (205)622-3850

197 E KEITHWAGONER, 561 COUNTY ROAD 52, JEMISON 35085

Phone: (205)646-2369

510 E MARCELL WAGONER, 715 COUNTY ROAD 52, JEMISON

35085

Phone: (205)646-3651

789 E WALKER COUNTY GAME FARM, KENNITH MOORE, 112

LIBERTY ROAD, JASPER 35501

Phone: (205)384-4086

693 E KENNY WALKER, 627 DEAD LAKE ROAD, CREOLA 36525

Phone: (251)679-1175

590 E JERRY WALLACE, 300 WALLACE ROAD, EASTABOGA 36260

715 E WENDELL WALLACE, 2804 OLD MOULTON ROAD,

DECATUR 35603

Phone: (256)355-0648
W88.

N121, N142, N152, N243, N43, N44, R27

$\times$

N121, N138, N224, N32, N43, N44, N46, N83

N121, N132, N143, N151, N43, N44

N28. N41

N121, N142, N147, N28, N63

N164, N24, N65, N87

N105, N130, N264, N28, N32, N41, N83

N134, N138, N146, N148, N155, N161, N162, N168, N2, N25, N28. N33, N34, N41, N43, N44, N62, N63, N83, N85, N87, R27

N236, N28, N33, N41

N152, N226, N32, N83, N90, R27.

N140, N152, N28, N33, N41, N42, N83

W115, W123, W128, W175. W198, W69, W86

W17. W24

N107, N141, N28, N32, N44

N132, N28, N41, N84, N85

N121, N126, N134, N140, N161, . N167, N168, N22, N28, N32, N58, N65, N83, N87, R27

W141, W149, W363, W365 W596, W7, W74, W91 
Table 1. HATCHERIES, DEALERS, AND INDEPENDENT FLOCKS PARTICIPATING IN

THE NATIONAL POULTRY IMPROVEMENT PLAN

WATERFOWL, EXHIBITION POULTRY AND GAME BIRDS

\begin{tabular}{|c|c|c|c|c|c|}
\hline $\begin{array}{l}\text { APP. } \\
\text { NO. }\end{array}$ & $\begin{array}{l}\text { SUB- } \\
\text { PART }\end{array}$ & PARTICIPANTS NAME AND ADDRESS & $\begin{array}{l}\text { HATCHING } \\
\text { EGG } \\
\text { CAPACITY }\end{array}$ & $\begin{array}{l}\text { PRODUCTS CLASSIFIED } \\
\text { U.S. PULLORUM-TYPHOID } \\
\text { CLEAN }\end{array}$ & $\begin{array}{c}\text { ADDITIONAL } \\
\text { CLASSIFICATIONS FOR } \\
\text { WHICH PRODUCT QUALIFIED }\end{array}$ \\
\hline
\end{tabular}

$\begin{aligned} 260 \text { E } & \text { WAR EAGLE GAME FARM, WILLIAM KENNEY, } 2905 \\ & \text { COUNTY ROAD 63, KILLEN } 35645 \\ & \text { Phone: (256)757-9210 }\end{aligned}$

568 E JIMMY WASHINGTON. 1224 EAST LAWRENCE STREET. RUSSELLVILLE 35654

261 E DARRELL WATSON. 1780 HATTON SCHOOL ROAD, LEIGHTON 35646

Phone: (256)446-6318

786 E WATSON \& GORE, P.O. BOX 292, GARDEN CITY 35070

Phone: (256)352-2402

262 E NOAH WEBSTER, 400 HALLOWAY STREET, HARTSELLE 35640

Phone: (256)773-9059

208 E PAUL B. WEBSTER, 2520 LOCHOPOKA HIGHWAY, AUBURN 36830

820 E EDWARD WELLS, 9936 HIGHWAY 43, SPRUCE PINE 35585

Phone: (256)332-7455

853 E JERRY WESSON, 800 COUNTY ROAD 257, FLORENCE 35633

Phone: (256)766-9892

825 E WHITE CROW GAME FOWL FARM, CRAWFORD, 873

YERBY ACRES LANE, BERRY 35546

Phone: (205)689-1978

657 E LEDON WILBANKS. 1801 ADAMSBURG ROAD EAST, FORT PAYNE 35967

728 E TERRY WILLIAMSON, 1101 VAUGHN ROAD.

BLOUNTSVILLE 35031

Phone: (205)466-5859

854 E JACKIE WOODALL, 818 COUNTY ROAD 1079.

VINEMONT 35179

Phone: (256)339-7691

729 E WOODLAND GAME FOWL FARM, C.R. PATTILLO, 6074 ALABAMA HIGHWAY 199, NOTASULGA 36866

Phone: (334)257-3871

844 E FRANK WORD, 5314 COUNTY ROAD 91, ROGERSVILLE 35652

Phone: (256)247-3094

249 E JACK WRIGHT, 4912 OUTLAW ROAD, EIGHT MILE 36613 Phone: (251)452-1912

198 E ROONEY WRIGHT, 401 PEAR STREET, LEEDS 35094
N216, N222, N28, N41

N137, N231, N28, N32, N41

N28, N32, N44

N130, N207, N222, N28, N87

W128. W142, W151, W187. W28, W50, W88

N22, N33, N43, N44, N83, N84, N98

N28, N44, W115, W48, Z22, Z285, Z4, Z9

N140, N187, N32, N44

N143, N28, N32, N94

N129. N32.

N102, N121, N127, N139, N151, N44, N83, N84

N106, N28, N87

N121, N137, N148, N236, N263, N44, N90

W198, W48, W54, W88

N28, N34, N85

W17, W18

DR. E. J. BICKNELL

EXTENSION VETERINARY SPECIALIST

MARICOPA AGRICULTURAL CENTER

37860 WEST SMITH ENKE ROAD

MARICOPA, AZ 85239

Phone: (520)568-2273

Fax: (520)568-2556 
Table 1. HATCHERIES, DEALERS, AND INDEPENDENT FLOCKS PARTICIPATING IN

THE NATIONAL POULTRY IMPROVEMENT PLAN

WATERFOWL, EXHIBITION POULTRY AND GAME BIRDS

\begin{tabular}{llccc}
\hline & & PATCHING & PRODUCTS CLASSIFIED \\
APP. SUB- & PARTICIPANTS NAME AND ADDRESS & EGG & U.S. PULLORUM-TYPHOID \\
NO. PART & CLEAN & CAPACITY & \\
\hline
\end{tabular}

86 - ARIZONA

Independent Flocks

13 E JEAN G. BARNITZ, 1420 WEST CYPRESS ROAD. TUCSON 85704

16 E CANAAN FARMS, INC., 6016 SOUTH 121ST. AVENUE, TOLLESON 85353

10 E DAVID D. CAVENY, RT. 1, BOX 214-M,1314 WEST ROOSTER DRIVE DOUGLAS 85607-9747

17 E KEITHE. CURRENS, 7434 SOUTH ROOKS ROAD, BUCKEYE 85326

12 E LEON \& MURIEL EWER, 6262 SOUTH SORREL LANE, TUCSON 85748

14 E RICHARD EWER, 5250 S. CAMPBELL, TUCSON 85706

9 E CHRIS HAESE, P.O. BOX 1190, PINEDALE 85934

15 E ROSALINDA WOODS, P.O. BOX 1764, CHINO VALLEY 86323
W3

Z3, Z43, Z93

A. N, R147, R210, R230, R231, R234, R236, R24, R74, R98,

$\mathrm{X} 160$

243

R23, R25, R97, W442, W54

A1, W6

R117, R44, R49, Z205

W. W111, W12, W13, W393

71 - ARKANSAS

DR. H.M. GHORI

ARKANSAS LIVESTOCK \& POULTRY COMMISSION

1 NATURAL RESOURCES DRIVE

LITTLE ROCK, AR 72205

Phone: (501)907-2434

Fax: (501)907-2259

Hatcheries

375 E CHAMBERS QUAIL FARM, RONNIE CHAMBERS, 1900 WEST MISSISSIPPI STREET, BEEBE 72012

233 E DUCKS \& DUCKS GAME FARM, INC., DUTCH NOE, 2823 HIGHWAY 69, LAKE CITY 72437

196 E J\&B BIRD FARM, 1094 STATE LINE ROAD, CORNING 72422

281 E J\&P QUAIL FARM, 503 CLIFFORDS LOOP, SCRANTON 72863

373 E LUPER \& SMITH, 11265 HIGHWAY 74, HUNTSVILLE 72740 NESTLE WAY, JONESBORO 72401
MELODY PARSLEY

ARKANSAS LIVESTOCK \& POULTRY COMMISSION

1 NATURAL RESOURCES DRIVE

LITTLE ROCK, AR 72205

Phone: (501)907-2447

Fax: (501)907-2259 
Table 1. HATCHERIES, DEALERS, AND INDEPENDENT FLOCKS PARTICIPATING IN

THE NATIONAL POULTRY IMPROVEMENT PLAN

WATERFOWL, EXHIBITION POULTRY AND GAME BIRDS

\begin{tabular}{|c|c|c|c|c|c|}
\hline $\begin{array}{l}\text { APP. } \\
\text { NO. }\end{array}$ & $\begin{array}{l}\text { SUB- } \\
\text { PART }\end{array}$ & PARTICIPANTS NAME AND ADDRESS & $\begin{array}{l}\text { HATCHING } \\
\text { EGG } \\
\text { CAPACITY }\end{array}$ & $\begin{array}{l}\text { PRODUCTS CLASSIFIED } \\
\text { U.S. PULLORUM-TYPHOID } \\
\text { CLEAN }\end{array}$ & $\begin{array}{c}\text { ADDITIONAL } \\
\text { CLASSIFICATIONS FOR } \\
\text { WHICH PRODUCT QUALIFIED }\end{array}$ \\
\hline
\end{tabular}

\begin{abstract}
136 E OAK RIDGE GAME FARM, FRANKTIFFANEY RUSH, 13890 GAME FARM ROAD, GRAVETTE 72736

344 E PRETTY GIRL HATCHERY, GUSS \& TAMI JONES. 2758 CR 945, JONESBORO 72401
\end{abstract}

\begin{abstract}
348 E QUAIL MOUNTAIN ENTERPRISES, INC., 811 HYNES, VAN BUREN 72956

303 E UNICORN WOODS HATCHERY, CARLA QUATAEYT, 481
\end{abstract} UNICORN ROAD, SALEM 72576

UNIVERSITY OF ARKANSAS, 0114 POULTRY CENTER, FAYETTEVILLE 72701

328 E V \& C QUAIL FARM, FRANK CARDWELL, HC 68, BOX 1062. PLAINVIEW 72857

379 E WINGS \& RINGS GAMEBIRDS, DEANNA GLOVER, 165 HAWTHICKET, MT. VERNON 72111

Independent Flocks

JOE ALEXANDER, 144 OUACHITA 489, CHIDESTER 71726

ATM GAME FARM, 230 FERREN ROAD, JUDSONIA 72081
E BILLY \& DEBBIE ATWOOD, 1557 GRANT 73, SHERIDAN 72150

E B \& C GAME FARM, BARRY CONDER, 197 JAY BIRD LANE, SEARCY 72143

E BALLARD CREEK FARM, 11115 LITTLE ROAD, SUMMERS 72769

E BEAKS \& BILLS POULTRY FARM, P.O. BOX 4182, LITTLE ROCK 72214

E BIG TIME, JIM OVERTON, 22201 VANCE ROAD, LONSDALE 72087

$E$ BLACK HATCH FARM, 4640 PALESTINE ROAD, HUNTINGTON 72940

E BLAND HICKORY HILL FARM, 1562 CEDAR GLADES ROAD, HOT SPRINGS 71913

E TED BORCHERS, 7590 ORCHARD POINT, HARRISON 72601

E BULL RIDER GAME FARM, 4120 SPRINGHILL ROAD, BENTON 72015

E DAVID CROTTS, 8828 SOUTH GRAPHIC DRIVE, ALMA 72921

E DEAN'S BANTAMS, DEAN LAWSON, 4064 HIGHWAY 242, LEXA 72355

\begin{tabular}{|c|c|}
\hline 30,840 & $\mathrm{X} 4, \mathrm{Z} 1, \mathrm{Z} 247, \mathrm{Z} 41, \mathrm{Z} 43$ \\
\hline 7,500 & $\begin{array}{l}\text { R12, R139, R146, R222, R245, } \\
\text { R30, R53, R66, W119, W120, } \\
\text { W121, W140, W166, W17, } \\
\text { W187, W198, W206, W223, } \\
\text { W23, W3, W301, W33, W338, } \\
\text { W416, W45, W518, W569, } \\
\text { W570, W60, W613, W74, W75, } \\
\text { W81, W94, X131, X2, X25, X4, } \\
\text { Y1, Y2, Y4, Y8 }\end{array}$ \\
\hline 13,000 & $\mathrm{Z1}, \mathrm{Z} 247, \mathrm{Z} 88$ \\
\hline 8,400 & $\begin{array}{l}\text { R20, W115, W12, W160, W174, } \\
\text { W175, W187, W198, W231, } \\
\text { W278, W28, W293, W357, W45, } \\
\text { W48, W600, W628, W629, W86, } \\
\text { W93, Z93 }\end{array}$ \\
\hline 14,500 & A, R55, Z127, Z212 \\
\hline 60,000 & 288 \\
\hline 4,000 & $Z 27, Z 40, Z 41, Z 43$ \\
\hline
\end{tabular}

R229, Z138, Z20, Z202, Z22

N110, N121, N137, N152, N175, N195, N216, N41, N44, N65, N83, N87, N98

R11, R22, W11, W4, W54

N105, N113, N131, N138, N139, N87

N106, N107, N109, N216, N230, -. N24, N28, N32, N41

R247, W7, W8

N161, N44, N63

N134, N249, N250, N251, N252._.

R237, R7, R92

W48.

N121.

R196, W16, W262, W51, W89, W91

W141, W142, W151, W28, W88 
Table 1. HATCHERIES, DEALERS. AND INDEPENDENT FLOCKS PARTICIPATING IN

THE NATIONAL POULTRY IMPROVEMENT PLAN

WATERFOWL, EXHIBITION POULTRY AND GAME BIRDS

APP. SUB- PARTICIPANTS NAME AND ADORESS NO. PART

HATCHING
EGG
CAPACITY

CAPACITY
PRODUCTS CLASSIFIED

U.S. PULLORUM-TYPHOID

CLEAN
ADDITIONAL

CLASSIFICATIONS FOR WHICH PRODUCT QUALIFIED

71 - ARKANSAS

371 E OUR DOUBLE D RANCH, MARLENE DEISS, 265 POLK 141. COVE 71937

311 E BILL DUBOIS \& SON, 11424 AMANDA LANE, FT. SMITH 72916

360 E DUKE'S GAME FARM, 2031 GREENE 424 ROAD. MARMADUKE 72443

395 E ALBERT ENGLISH, 141 HIGHWAY 124 WEST, DAMASCUS 72039

346 E JOSHUA EVATT, 367 BASS ROAD, VILONIA 72173

244 E J.C. GIBSON, 1064 GREENE 843 ROAD, PARAGOULD 72450

376 E GILLIAM BROTHERS BIRD FARM, 25006 LAWSON ROAD, LITTLE ROCK 72210

396 E H\&HPOULTRY, PHILLIP HOWELL, 126 WHITETAIL LANE, HOT SPRINGS 71901

397 E H\&HPOULTRY, MICHAEL HOWELL, 200 REPOP, PEARCY 71964

402 E HALLELUJAH CHICKEN FARM, PAUL BUSH, P.O. BOX 241520, LITTLE ROCK 72223

230 E HARVEST FARMS, JEFF BENDS, 3293 HIGHWAY 775 , LEPANTO 72354

406 E HIDDEN FIELDS, ROY WALLACE, 340 HARGETT ROAD, EX DORADO 71730

388 E ROY HUMMEL, 683 RHEAS MILL ROAD, FARMINGTON 72730

280 E J\& JPOULTRY FARM, 31 LESTER LANE, VILONIA 72173

392 E JERRY JOHNSTON, 715 NORTH 2ND STREET, PARAGOULD 72450

240 E ALFRED JONES, P.O. BOX 256, PRAIRIE GROVE 72753

$257 \quad E$

$301 \quad E$

$258 \quad E$

$367 \quad E$
W. O. JONES, P.O. BOX 120, HATTIEVILLE 72063

KING EDWARD GAMEFOWL FARM, 699 STANLEY ROAD, BALD KNOB 72010

CLARENCE \& RYAN LAFLEUR, 2801 MOUNT HARMONY ROAD, GREENWOOD 72936

E CODY LOUKS, 228 FERREN ROAD, JUDSONIA 72801

$E$

DALE LOVELACE, P.O. BOX 223, RATCLIFF 72951

E MAC, BARBARA, \& DOUG MATHENY, 558 HIGHWAY 63 , RAVENDEN 72459
R20, R69, R7, R71, R83, R89, W160, W211, W248, W313. W361, W518, W74, W96

W120, W208, W215, W23, W28, W290, W314, W7, X10, X143, $\mathrm{X} 17, \times 4, \mathrm{X} 46$

N106, N24, N28, N32, N41, N44, N83

W262, W28, W48, W52, W568, W600, W613, W69, W88

R20, R225, W69, W88

N155, N157, N32

Z1, Z247, Z43

R196, R64, W198, W211, W25, W26, W28, W569, W570

R222, W142, W149, W52, W69, W88, W89, Z205

R247.

N121, N32, N43

Z4, 293 .

W117, W13, W198, W3, W4, W57

R247, W120,W7, W88

N28

N28. N33

R227, W120, W121, W2, X4, Y9, Z182, Z204, Z205, Z22, Z43

N105, N106, N121, N134, N136. N140, N157, N164, N167, N32, N44, N63, N87

W262, W28, W3

N121, N137, N152, N175, N195, . N216, N41, N44, N65, N85, N87, N98

W28, W88.

W119, W121, W138, W140 W142, W145, W149, W169 W17, W176, W198, W208 W211, W25, W26, W270, W276, W3, W301, W33, W42, W427. W45, W568, W6, W60, W69, W7, W74, W81, W84, W86, W88, W91 
Table 1. HATCHERIES, DEALERS, AND INDEPENDENT FLOCKS PARTICIPATING IN

THE NATIONAL POULTRY IMPROVEMENT PLAN

WATERFOWL, EXHIBITION POULTRY AND GAME BIRDS

\begin{tabular}{lllll}
\hline & & & HATCHING & PRODUCTS CLASSIFIED \\
APP. & SUB- & PARTICIPANTS NAME AND ADDRESS & EGG & U.S. PULLORUM-TYPHOID \\
NO. PART & CLEAN & CLASSIFICATIONALS FOR & WHICH PRODUCT QUALIFIED \\
\hline
\end{tabular}

\section{1 - ARKANSAS}

\begin{tabular}{|c|c|c|}
\hline 398 & $\mathrm{E}$ & $\begin{array}{l}\text { CHARLIE MCCLENDON, } 6129 \text { HIGHWAY 261, MARIANNA } \\
72360\end{array}$ \\
\hline 327 & $\mathrm{E}$ & $\begin{array}{l}\text { MCHALFFEY'S GAME FARM, LARRY MCHALFFEY, } 468 \\
\text { CR } 320 \text {, JONESBORO } 72401\end{array}$ \\
\hline 253 & E & $\begin{array}{l}\text { WANDA MEHARG, } 403 \text { EAST MAIN, BOX } 98 \text {, LETONA } \\
72085\end{array}$ \\
\hline 345 & $E$ & $\begin{array}{l}\text { CARL MEYER, } 9815 \text { MEYER VALLEY ROAD, } \\
\text { MOUNTAINBURG } 72946\end{array}$ \\
\hline 394 & $\mathrm{E}$ & $\begin{array}{l}\text { RAY MILLSAP, } 745 \text { CROSSROADS WEST, QUITMAN } \\
72131\end{array}$ \\
\hline 296 & $E$ & $\begin{array}{l}\text { HAROLD MITCHELL, } 3601 \text { PRIVATEWOOD DRIVE } \\
\text { NORTH, PINE BLUFF } 71603\end{array}$ \\
\hline 362 & $E$ & $\begin{array}{l}\text { LEROY MONTGOMERY, P.O. BOX } 397, \text { COLLEGE } \\
\text { STATION } 72053\end{array}$ \\
\hline 298 & $E$ & MOONBEAM ACRES, P.O. BOX 222, GUY 72061 \\
\hline 370 & $E$ & $\begin{array}{l}\text { MOORE FARMS, DAVID \& ZACHARY MOORE, P.O. BOX } \\
\text { 1841, WALDRON } 72958\end{array}$ \\
\hline
\end{tabular}

THOMAS R. NICHOLS, P.O. BOX 151, RUDY 72952
OLD MCROYS FARM, ROY VANCE, 244 BLACKJACK MOUNTAIN ROAD, ROMANCE 72136

PARADISE FARMS, 196 HOSPITALITY TRAIL, JUNCTION CITY 71749
E PASSMORE FARM, JERRY PASSMORE, 171 JACKSON 199, BRADFORD 72020

$\mathrm{E}$ NICK PERRY, 50 LAFAYETTE 5, LEWISVILLE 71845

E

$E$ JAMES PFLASTEREN, 114 JEWETT, CABOT 72023

LUCKY PICKENS FARMS, G \& R PICKENS, HC 66, BOX 89-C. DANVILLE 72833

E PINEY CREEK FARM, JOHNNY PARISH, HC 89, BOX 708 , MT. PLEASANT 72561

E ROBERT H. RHODES, 201 EAST WALKER, OZARK 72949

E ROBINCROFT, P.O. BOX 9, HUMNOKE 72072

E ROBINSON BROTHERS, PAUL ROBINSON, P.O. BOX 582, JUDSONIA 71082

E ROJO'S ROOST, JENKINS, \& SHEPPARD, 21256 OAK PARK LANE. SILOAM SPRINGS 72761
R, R196, W25, W26, Z4

N106, N168, N94

R239, R256, W215, W573, Z22

R139, R225, R229, R254, W102, W119, W121, W140, W17, W18 W2, W206, W25, W361, W86

R229, R237, Z202, Z4.

W28, W88, Z108, Z15, Z19

R247, Z41, Z43

R51, W19, W60

R10, R11, R146, R15, R188, R19, R21, R247, R248, R3, R34, R42, R5, R51, R53, R6, R7, R85, R93 $\mathrm{X} 21, \mathrm{X} 3, \mathrm{Y} 21, \mathrm{Z} 108, \mathrm{Z} 22$

W114, W119, W12, W121. W142, W149, W160, W17, W18, W198, W20, W200, W211, W22 W231, W262, W278, W28, W29, W3, W301, W357, W4, W48. W51, W518, W54, W568, W570 W573, W595, W60, W69, W75. W88, X6, Z15, Z202, Z212, Z230

R146, R227, R229, R237, W2, $W 74, W 75, X 136, Y, Z 202$

R12, R146, R222, R227, R239, R256, R262, R40, R42, R81, W164, W169, W198, W208, W211, W25, W26, W290, W48, W570, W69, Z15, Z20, Z202, Z204, Z205, Z206, Z22, Z230, Z251, Z27, Z295. Z88

N174, N28, N8.

R247, W7

N163, N171

R222, R247, W3, W573, W7

N161, N44

W102, W17

R183, R196, R247, R248.

N104, N136, N225, N28, N32, N85

R62, R89 
Table 1. HATCHERIES, DEALERS, AND INDEPENDENT FLOCKS PARTICIPATING IN

THE NATIONAL POULTRY IMPROVEMENT PLAN

WATERFOWL, EXHIBITION POULTRY AND GAME BIRDS

\begin{tabular}{|c|c|c|c|c|c|}
\hline $\begin{array}{l}\text { APP. } \\
\text { NO. }\end{array}$ & $\begin{array}{l}\text { SUB- } \\
\text { PART }\end{array}$ & PARTICIPANTS NAME AND ADDRESS & $\begin{array}{l}\text { HATCHING } \\
\text { EGG } \\
\text { CAPACITY }\end{array}$ & $\begin{array}{l}\text { PRODUCTS CLASSIFIED } \\
\text { U.S. PULLORUM-TYPHOID } \\
\text { CLEAN }\end{array}$ & $\begin{array}{c}\text { ADDITIONAL } \\
\text { CLASSIFICATIONS FOR } \\
\text { WHICH PRODUCT QUALIFIED }\end{array}$ \\
\hline
\end{tabular}

\begin{abstract}
404 E CLAUDE RUIZ BARN YARD, 4308 LAKE DRIVE, CLINTON 72031

385 E RUSTY ROOSTER POULTRY FARM, MILTON DYER. 181 RUBIN LANE, MALVERN 72104
\end{abstract}

\begin{abstract}
248 E MACK SEALS, $880 \mathrm{KISSINGER} \mathrm{AVENUE,} \mathrm{SPRINGDALE}$ 72762

403 E SHOEMAKER BIRD FARM, HAWKINS \& REID, 1407 SHOEMAKER ROAD, SHERIDAN 72150

350 E JAMES SIMPSON, 6925 SIMPSON DRIVE, ALEXANDER 72002
\end{abstract}

SMITH'S POULTRY, 6789 HIGHWAY 309 SOUTH, PARIS 72855

SMOKE RIDGE POULTRY, DAMON/AMANDA DYER, 102 RUBIN LANE, MAGNET COVE 72104

JOHN SPAIN, 177 HIGHWAY 365, CONWAY 72032

STAR-GAP FARM, 445 STAR GAP ROAD, MOUNTAIN VIEW 72560

E

BILLY STINNETT, 383 OUACHITA 75, CHIDESTER 71726
TRIPLE MW, TAMMY WRIGHT, P.O. BOX 208, PERRYILLE 72126 DAVID TUCKER, 1196 MAIN STREET, VILONIA 72173

STONEYPOINT GAME FARM, 767 SWINGING BRIDGE ROAD, BEEBE 72012

$E$ 72703

E SWEETGUM FARM, DOUG ANDERSON, 420 LITTLE MAZARN, HOT SPRINGS 71913

E T.M.O.FARM, 532 TIMBERLAKE DRIVE, ROYAL 71968

E THOMPSON'S CUTE BRATS, FRANCINE THOMPSON, 579 CR 9570, GREEN FOREST 72638

E JOHN TUNSTILL, 3170 HUNT LANE, FAYETTEVILLE 72701

E ROBERT TUNSTILL, 11668 SOUTH WHITEHOUSE ROAD, FAYETTEVILLE 72701
W119, W124, W26, W29, W570, W613. W88

R237, R247, R257, R46, R7, R84 W115, W128, W129, W142 W16, W187, W188, W198, W25. W26, W276, W28, W295, W357, W45, W48, W50, W51, W54. W56, W569, W6, W613, W69, W7, W74, W88, W89, W91

W11, W142, W149, W250, W28, W5

Z45, Z89

R146, R229, W25, W26, W28, W3, W615, W7, W74, W75, X21, X3, X4, X6, Z20, Z202, Z25, Z4,

Z62, Z89

R7, W141, W142, W149, W151, W200, W25, W26. W262, W28, W29, W48, W50, W52, W568, W570, W579, W613, W616, W69, W88, W89, W91

R225, R248, R44, R7, W119, W13, W160, W176, W22, W220. W286, W484, W82

$\mathrm{X} 3, \mathrm{X} 4, \mathrm{X} 8, \mathrm{Y} 9$

R123, R179, R19, R233, W102, _W151, W74, W75, Z20, Z22

R123, R139, R146, R21, R229, R247, R70, R71, R87, W25, W26. W84, Z118, Z128, Z22, Z230, $\mathrm{Z27}, \mathrm{Z28}$

N28, N32, N94

R247, W142, W200, W28, W303,W45, W480, W84, W88

R109, R111, R21, R76, R92

W102, W17, W18, W60

R103, R15, R227, R233, W1, W100, W106, W11, W121, W125, W129, W13, W179, W18, W26, W566, W70, W86, W90, W91. W98, X17, X21, X46, Y2, Z137, Z138, Z14, Z183, Z283, Z4

R233, R237

R104, R247, R3, R84, W7

R30, W160, W18, W220, W262, W3, W57, W7

R247, W142, W151, W262, W28,W3, W57, W69 
Table 1. HATCHERIES, DEALERS, AND INDEPENDENT FLOCKS PARTICIPATING IN

THE NATIONAL POULTRY IMPROVEMENT PLAN

WATERFOWL, EXHIBITION POULTRY AND GAME BIRDS

\begin{tabular}{|c|c|c|c|c|c|}
\hline $\begin{array}{l}\text { APP. } \\
\text { NO. }\end{array}$ & $\begin{array}{l}\text { SUB- } \\
\text { PART }\end{array}$ & PARTICIPANTS NAME AND ADDRESS & $\begin{array}{l}\text { HATCHING } \\
\text { EGG } \\
\text { CAPACITY }\end{array}$ & $\begin{array}{l}\text { PRODUCTS CLASSIFIED } \\
\text { U.S. PULLORUM-TYPHOID } \\
\text { CLEAN }\end{array}$ & $\begin{array}{c}\text { ADDITIONAL } \\
\text { CLASSIFICATIONS FOR } \\
\text { WHICH PRODUCT QUALIFIED }\end{array}$ \\
\hline & & $71-$ & - ARKANSAS & & \\
\hline 393 & $E$ & $\begin{array}{l}\text { TURKEY'S R US, KEITH SMITH, } 1102 \text { DOGWOOD ROAD, } \\
\text { EL DORADO } 71730\end{array}$ & & $\begin{array}{l}\text { W17, W198, W2, W25, W26, } \\
\text { W570, W74, W88, Z204, Z205, } \\
\text { Z209, Z230, Z24 }\end{array}$ & \\
\hline 269 & $E$ & $\begin{array}{l}\text { BOBBY VANDIVER, } 7214 \text { HIGHWAY } 161 \text { NORTH, NORTH } \\
\text { LITTLE ROCK } 72117\end{array}$ & & $\begin{array}{l}\text { W208, W25, W26, W28, W74, - } \\
Z 22, Z 88\end{array}$ & \\
\hline 355 & $E$ & $\begin{array}{l}\text { JOHN R. \& BARBARA WAGNER, } 700 \text { LONGLEAF LANE, } \\
\text { PINE BLUFF } 71602\end{array}$ & & $\begin{array}{l}\text { R222, W141, W142, W149, } \\
\text { W151, W164, W200, W23, } \\
\text { W231, W25, W26, W262, W270, } \\
\text { W276, W28, W29, W301, W357, } \\
\text { W361, W363, W364, W38, W48, } \\
\text { W50, W52, W53, W54, W542, } \\
\text { W568, W569, W579, W596, } \\
\text { W613, W69, W74, W75, W79, } \\
\text { W86, W88, W89, W91, W94, } \\
\text { W96, Z4 }\end{array}$ & \\
\hline 259 & $\mathrm{E}$ & $\begin{array}{l}\text { WARE'S BANTAMS, GERALD WARE, } 2801 \\
\text { WITCHERVILLE ROAD. GREENWOOD } 72936\end{array}$ & & R254, W276, W28, W48, W88 & \\
\hline 399 & $E$ & $\begin{array}{l}\text { EDDIE WATSON, } 126 \text { ALBERT HILL ROAD, BALD KNOB } \\
72010\end{array}$ & & N28, W48, Y21, Y53, Z15, Z21 & \\
\hline 405 & $E$ & $\begin{array}{l}\text { CHARLES WILLIAMS \& DENIS COWART, P.O. BOX } 456 \text {, } \\
\text { NORMAN } 71960\end{array}$ & & $\mathrm{Z} 27, \mathrm{Z} 89$ & \\
\hline 284 & $E$ & WOOD ENTERPRISES, 192 POLK 289, COVE 71937 & & N28, N32, N83 & \\
\hline 351 & $E$ & $\begin{array}{l}\text { LONNIE YOUNG, } 340 \text { ROOSTER ROAD, BATESVILLE } \\
72501\end{array}$ & & $\mathrm{~N} 121, \mathrm{~N} 161, \mathrm{~N} 32, \mathrm{~N} 44$ & \\
\hline
\end{tabular}

\section{BILL MATTOS}

CALIFORNIA POULTRY HEALTH BOARD

3117-A MCHENRY AVENUE

MODESTO. CA 95350

Phone: (209)576-6355

Fax: $\quad(209) 576-6119$

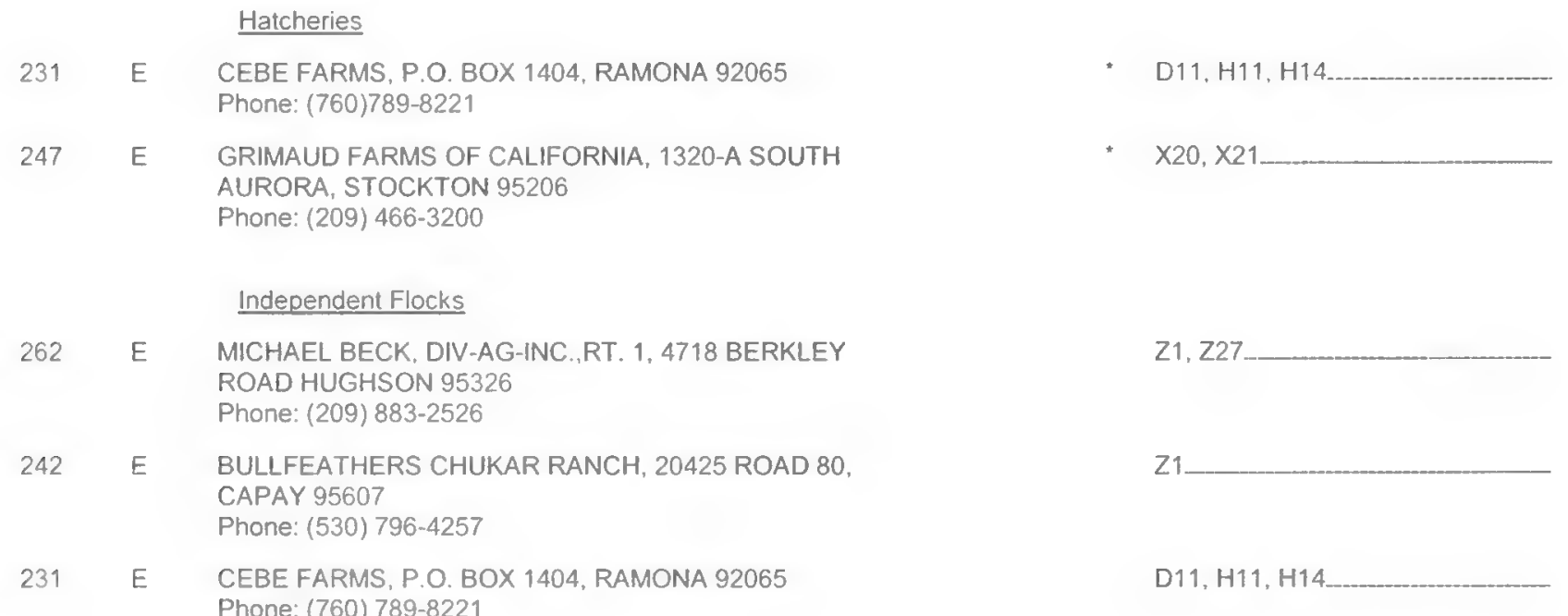

" Does not report Hatching egg capacity 
Table 1. HATCHERIES, DEALERS, AND INDEPENDENT FLOCKS PARTICIPATING IN

THE NATIONAL POULTRY IMPROVEMENT PLAN

WATERFOWL, EXHIBITION POULTRY AND GAME BIRDS

\begin{tabular}{llccc}
\hline & & HATCHING & PRODUCTS CLASSIFIED \\
APP. SUB- & PARTICIPANTS NAME AND ADDRESS & EGG & U.S. PULLORUM-TYPHOID & CLDITIONAL \\
NO. PART & CASIFICATIONS FOR & CLAN & WHICH PRODUCT QUALIFIED
\end{tabular}

93 - CALIFORNIA

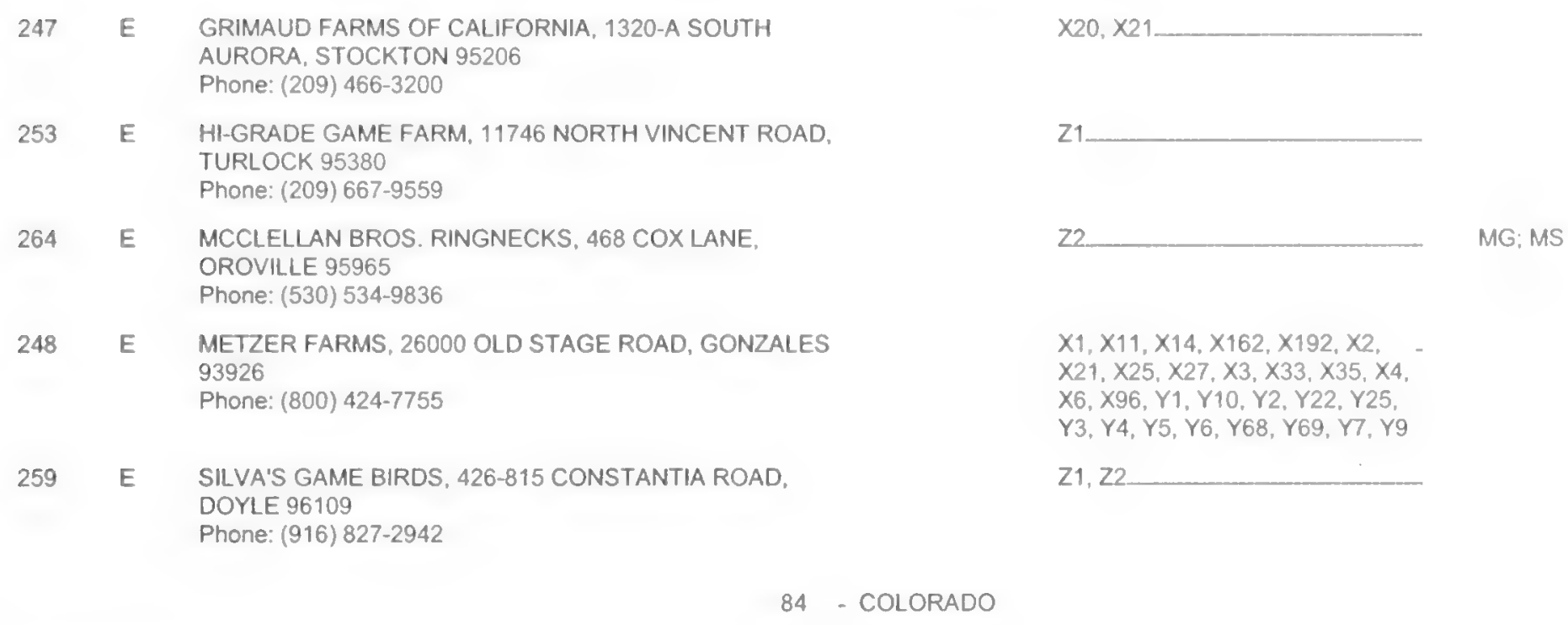

WILLIAM C. LOWER

COLORADO POULTRY IMPROVEMENT BOARD, INC.

4816 EAST COUNTY ROAD \#30

FORT COLLINS, CO 80528-9324

Phone: (970)226-3680

Hatcheries

\begin{tabular}{|c|c|c|c|c|}
\hline 32 & $E$ & $\begin{array}{l}\text { NORMAN L. BRELSFORD, } 2414 \text { GARLAND STREET, } \\
\text { LAKEWOOD } 80215\end{array}$ & 48 & W270, W271, W301, W92 \\
\hline 23 & $E$ & NARD'S OLD ENGLISH, 777 Q STREET, PENROSE 81240 & 109 & W88 \\
\hline 35 & $E$ & $\begin{array}{l}\text { AL PRADO, JR., } 4621 \text { QUIVAS STREET, DENVER } 80211 \\
\text { Phone: }(303) 455-6623\end{array}$ & 150 & W17, W18, W4, W6 \\
\hline 24 & $E$ & $\begin{array}{l}\text { SMITH GAME FARMS, GEORGE SMITH, } 7401 \text { HIGHWAY } \\
\text { 196. LAMAR } 81052 \\
\text { Phone: (719)336-4135 }\end{array}$ & 45,000 & Z1, Z2, Z43 \\
\hline 34 & $E$ & $\begin{array}{l}\text { BRENDA L. STRONG, } 5490 \text { WEST 3RD AVENUE. } \\
\text { LAKEWOOD } 80226\end{array}$ & 300 & $\begin{array}{l}\text { W15, W20, W372, W57, W9, } \\
\times 143\end{array}$ \\
\hline & & Independent Flocks & & \\
\hline 31 & $E$ & $\begin{array}{l}\text { MICHAEL JOHNSON, } 5310 \text { EAST } 88 \text { TH., STRASBURG } \\
80136\end{array}$ & & $R 52, W 128, W 6, X 17, X 9, Y 3$ \\
\hline 37 & E & DONNA LACHEY, 10720 REA ROAD, FDUNTAIN 80817 & & $W 361, X 10, X 18, Y 2 \ldots$ \\
\hline 38 & E & BRET NELSON, 10720 REA ROAD, FOUNTAIN 80817 & & $\begin{array}{l}\text { A19, R118, R49, R52, R8, W12, - } \\
\text { W123, W6, Z204 }\end{array}$ \\
\hline
\end{tabular}


Table 1. HATCHERIES, DEALERS, AND INDEPENDENT FLOCKS PARTICIPATING IN

THE NATIONAL POULTRY IMPROVEMENT PLAN

WATERFOWL, EXHIBITION POULTRY AND GAME BIRDS

$\begin{array}{lll}\text { APP. } & \text { SUB- } \\ \text { NO. } & \text { PART }\end{array}$

$\begin{array}{cc}\text { HATCHING } & \text { PRODUCTS CLASSIFIED } \\ \text { EGG } & \text { U.S. PULLORUM-TYPHOID } \\ \text { CAPACITY } & \text { CLEAN }\end{array}$

ADDITIONAL

CLASSIFICATIONS FOR WHICH PRODUCT QUALIFIED

16 - CONNECTICUT

DR. BRUCE SHERMAN

and

DR. LOUIS VAN DER HEIDE

STATE VETERINARIAN

DEPARTMENT OF PATHOLOGY

DEPARTMENT OF AGRICULTURE

UNIVERSITY OF CONNECTICUT

765 ASYLUM AVENUE

HARTFORD, CT 06105

STORRS. CT 06268

Phone: (860)486-4000

Phone: (860)713-2504

Fax: $\quad(860) 486-2794$

Fax: $\quad(860) 713-2515$

\section{Dealers}

1058 E YANKEE CHICKS, HALL BROTHER'S HTY., P.O. BOX 1026. NORWICH 06360

Independent Flocks

1057 E RAY CROSSEN, 156 WATERFALL ROAD, ASHFORD 06278

1059 E JIM \& MICHELLE CUMMINGS, 305 BOSTON HILL ROAD, ANDOVER 06232

Phone: (860)647-1753

1060 E JOB \& KATE DAY, 1968 BOSTON POST ROAD,

GUILFORD $06437-4339$

48 E EDWARD DYLEWSKI, 605 PALISADO AVENUE, WINDSOR 06095

1056 E G \& M GAME FARM, AKKOURIS, 981 OVERHILL DRIVE, SUFFIELD 06078

77 E JOSEPH JIOVO, 732 THOMPSON STREET, GLASTONBURY 06033

99 E MARKOVER GAME FARM, N. OLSON, COOK HILL ROAD, DANIELSON 06239

750 E SHARON PHEASANT FARM, R. WILBUR, GAY STREET, SHARON 06069

799 E WHISTLING PINE QUAIL FARM, S. MCGRANAHAN, 189 PILFERSHIRE ROAD, EASTFORD 06242
C $\quad \mathrm{X}, \mathrm{Z}, \mathrm{Z} 231$

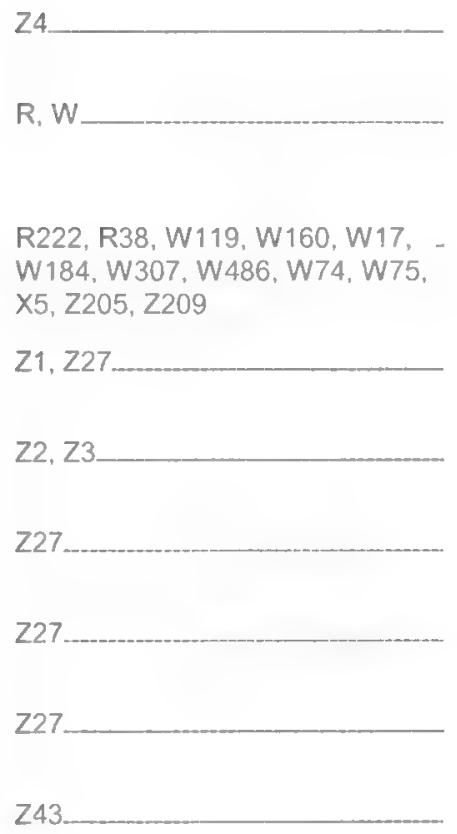

50 - DELAWARE

DR. H. WESLEY TOWERS, JR.

STATE VETERINARIAN

STATE DEPARTMENT OF AGRICULTURE

2320 SOUTH DUPONT HIGHWAY

DOVER, DE 19901

Phone: (302)739-4811

Fax: $\quad$ (302)697-6287 
Table 1. HATCHERIES, DEALERS, AND INDEPENDENT FLOCKS PARTICIPATING IN

THE NATIONAL POULTRY IMPROVEMENT PLAN

WATERFOWL, EXHIBITION POULTRY AND GAME BIRDS

\begin{tabular}{|c|c|c|c|c|c|}
\hline $\begin{array}{l}\text { APP. } \\
\text { NO. }\end{array}$ & $\begin{array}{l}\text { SUB- } \\
\text { PART }\end{array}$ & PARTICIPANTS NAME AND ADDRESS & $\begin{array}{l}\text { HATCHING } \\
\text { EGG } \\
\text { CAPACITY }\end{array}$ & $\begin{array}{l}\text { PRODUCTS CLASSIFIED } \\
\text { U.S. PULLORUM-TYPHOID } \\
\text { CLEAN }\end{array}$ & $\begin{array}{c}\text { ADDITIONAL } \\
\text { CLASSIFICATIONS FOR } \\
\text { WHICH PRODUCT QUALIFIED }\end{array}$ \\
\hline
\end{tabular}

\begin{tabular}{|c|c|c|}
\hline 60 & $\mathrm{E}$ & $\begin{array}{l}\text { PHYLISS DOWNES, RD. 1, BOX 104, GREENWOOD } \\
19950\end{array}$ \\
\hline 39 & $E$ & ROBERT D. ENGLISH, RR 3, BOX 227, SEAFORD 19973 \\
\hline 33 & E & WILLIAM EVANS, 141 MORRIS ROAD, TOWNSEND 19734 \\
\hline 57 & E & $\begin{array}{l}\text { SHELBY HARRINGTON, } 2114 \text { GREEN \& ROD CLUB } \\
\text { ROAD, HOUSTON } 19954\end{array}$ \\
\hline 46 & E & $\begin{array}{l}\text { CHARLES HILLENBRAND, RD 1, BOX 159, WYOMING } \\
19934\end{array}$ \\
\hline 56 & $E$ & $\begin{array}{l}\text { JEFF KANE, } 5709 \text { LIVESTONE ROAD, WILMINGTON } \\
19808\end{array}$ \\
\hline 40 & $E$ & $\begin{array}{l}\text { KENNETH STRAUGHN, } 2076 \text { HARVEY STRAUGHN } \\
\text { ROAD, TOWNSEND } 19734\end{array}$ \\
\hline 35 & E & RICHARDSON TRITT, RR 2, BOX 141-1, DOVER 19901 \\
\hline 55 & E & $\begin{array}{l}\text { DENNIS, JAMES \& VICTORIA TWISSELMANN, RD 2, BOX } \\
\text { 25, FELTON } 19943\end{array}$ \\
\hline 58 & $E$ & CLAUS TWISSELMANN, RD 2, BOX 25, FELTON 19943 \\
\hline 37 & $E$ & $\begin{array}{l}\text { MICHAEL WASKI, } 915 \text { DOVER KENTON ROAD, DOVER } \\
19904\end{array}$ \\
\hline
\end{tabular}

N11, N12, W151, W25, W28, ... W585

W12, W134, W24, W3, W36, W38, W4

$W, W 128, W 20, W 28$

W105, W11, W134, W3, W301, W4, W54

W11, W23, W33

$\times 10, \times 17, \times 28$

W30, w301, w570

W81

W, W166, W301

R86, W115, W166, W24, W603

N7, N97, R85, W3, W4, W54, W57

58 - FLORIDA

JENNIFER JENNINGS-GLOVER

FLORIDA DEPARTMENT OF AGRICULTURE AND

\section{CONSUMER SERVICES}

2145 LAMBERT LANE

TALLAHASSEE, FL 32317

Phone: (850)251-1226

Fax: $\quad(850) 921-3647$

Hatcheries

\begin{tabular}{|c|c|c|}
\hline 470 & $E$ & $\begin{array}{l}\text { GLEN R. BROWN, } 3835 \text { WIGGINTON ROAD, } \\
\text { TALLAHASSEE } 32303\end{array}$ \\
\hline 447 & $E$ & $\begin{array}{l}\text { CAREY BANTAMS, } 3020 \text { EAST VERNON CT., FLORAL } \\
\text { CITY } 34436\end{array}$ \\
\hline 212 & E & MARVIN CUMMINGS, 18001 GERACI ROAD, LUTZ 33549 \\
\hline 500 & E & $\begin{array}{l}\text { H\&B QUAIL FARM, INC., } 6895 \text { SW 32ND LOOP, JASPER } \\
32052\end{array}$ \\
\hline 348 & $E$ & $\begin{array}{l}\text { CHERRIE S. HAGAN, } 1608 \text { SOUTH } 45 \text { TH STREET, } \\
\text { TAMPA } 33619 \\
\text { Phone: }(813) 248-5330\end{array}$ \\
\hline 408 & $E$ & $\begin{array}{l}\text { M \& L GAME BIRD FARM, } 1465 \text { NW C. R. 274, FOUNTAIN } \\
32438\end{array}$ \\
\hline 303 & E & $\begin{array}{l}\text { PETER MERLIN \& TIM BROWN, } 910 \text { NORTH PARSONS } \\
\text { AVENUE, BRANDON } 33510\end{array}$ \\
\hline
\end{tabular}

\author{
DR. CAROLYN PARKINS \\ FLORIDA DEPARTMENT OF AGRICULTURE AND \\ CONSUMER SERVICES \\ P.O. DRAWER "O" \\ LIVE OAK, FL 32064 \\ Phone: (386)362-1216 \\ Fax: (386)362-4213
}


Table 1. HATCHERIES, DEALERS, AND INDEPENDENT FLOCKS PARTICIPATING IN

THE NATIONAL POULTRY IMPROVEMENT PLAN

WATERFOWL, EXHIBITION POULTRY AND GAME BIRDS

\begin{tabular}{|c|c|c|c|c|c|}
\hline $\begin{array}{l}\text { APP. } \\
\text { NO. }\end{array}$ & $\begin{array}{l}\text { SUB- } \\
\text { PART }\end{array}$ & PARTICIPANTS NAME AND ADDRESS & $\begin{array}{l}\text { HATCHING } \\
\text { EGG } \\
\text { CAPACITY }\end{array}$ & $\begin{array}{l}\text { PRODUCTS CLASSIFIED } \\
\text { U.S. PULLORUM-TYPHOID } \\
\text { CLEAN }\end{array}$ & $\begin{array}{c}\text { ADDITIONAL } \\
\text { CLASSIFICATIONS FOR } \\
\text { WHICH PRODUCT QUALIFIED }\end{array}$ \\
\hline
\end{tabular}

\begin{tabular}{|c|c|c|}
\hline 127 & $E$ & $\begin{array}{l}\text { MORRIS TURKEY \& QUAIL, } 18370 \text { SW. 232ND STREET, } \\
\text { GOULDS } 33170\end{array}$ \\
\hline 139 & $E$ & $\begin{array}{l}\text { DANNY PADGETT, RT. 4, BOX 3850, LAKE BUTLER } \\
32054\end{array}$ \\
\hline 291 & $E$ & $\begin{array}{l}\text { PANHANDLE GAME BIRDS, } 7601 \text { LAKESIDE DRIVE, } \\
\text { MILTON } 32583\end{array}$ \\
\hline & $E$ & $\begin{array}{l}\text { PASZEK'S HATCHERY, } 12224 \text { SPOTTSWOOD DRIVE, } \\
\text { RIVERVIEW } 33569\end{array}$ \\
\hline 57 & $E$ & $\begin{array}{l}\text { PREDATOR GAME FOWL, P.O. BOX 1181, GEVENA } \\
32732 \\
\text { Phone: (407) 349-2573 }\end{array}$ \\
\hline & $E$ & $\begin{array}{l}\text { ROCKING W-BAR RANCH, P.O. BOX 106, LA CROSSE } \\
32658\end{array}$ \\
\hline 28 & $E$ & $\begin{array}{l}\text { WANDA SIMMONS, } 3859 \text { WOODLAND HEIGHTS, } \\
\text { CALLAHAN } 32011\end{array}$ \\
\hline 357 & $E$ & $\begin{array}{l}\text { JOHN SIRVENT, } 101 \text { THORNTON LANE, FLORAHOME } \\
32140\end{array}$ \\
\hline 75 & $E$ & $\begin{array}{l}\text { SNAKEYE GUNS AND QUAIL, } 659 \text { O'HARA ROAD, } \\
\text { MIDDLEBURG } 32068\end{array}$ \\
\hline & $E$ & $\begin{array}{l}\text { STRUT YOUR STUFF POULTRY, } 9387 \text { PRESTON ROAD, } \\
\text { BROOKSVILLE } 32601\end{array}$ \\
\hline
\end{tabular}

103 $\begin{array}{ll}507 \text { E } & \text { RAYMOND VALENTINE, HC 3, BOX 579, OLD TOWN } \\ 32680\end{array}$

338 E SCOTT \& MARY WAGNER, $7210 \mathrm{KNIGHTS} \mathrm{GRIFFIN}$ ROAD, PLANT CITY 33565

300 E BOB WARD, 26591 OSAGE STREET, BROOKSVILLE 34601

\section{Dealers}

359 E AVIAN TECHNOLOGY EXPORT PARTNERSHIP, P.O. BOX 522968, MIAMI 33152

472 E DOUBLE R DISCOUNT SUPPLY, 4036 HIELD ROAD NW, PALM BAY 32907

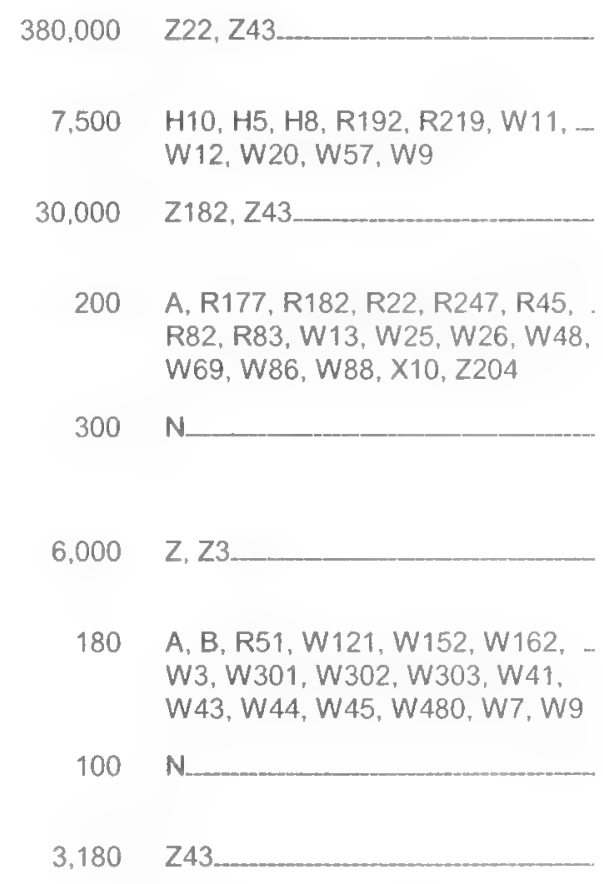

2,600 R105, R12, R139, R19, R20, R27, R45, R62, R70, R83, R89, W247, W248, W33, W559, W74, W86. $\times 1, X 11, X 135, X 17, \times 24, \times 27$, $X 28, X 30, X 64, X 97, X 98, Y 13$ $Y 14, Y 2, Y 21, Y 22, Y 32, Y 4, Y 6$, Y8, Z107, Z108, Z114, Z118, Z121, Z129, Z140, Z15, Z176, Z178, Z188, Z19, Z204, Z205, Z207, Z24, Z26, Z28, Z34, Z4. Z41, Z46, Z5, Z54, Z57, Z61

32,000

Z3.

360 Z111, Z20, Z22, Z239, Z249, ... Z252, Z253

200 R15, R16, R165, R208, R211, R27, R71, W213, W248, W32, W518, W57, W74, W75, X1, X12, $X 15, X 18, X 2, X 3, Y 1, Y 10, Y 12$, $Y 22, Y 7$

R15, R30, W276, W29, W32, W48, W88, X17, X2, Y1

E\&C

C R1, R114, R12, R146, R15, R17, R19, R196, R2, R20, R227, R237, R238, R239, R240, R241, R242, R247, R248, R26, R5, R6, R7, R81, W1, W13, W132, W207. W30, W370, W570, W588, W96 $X 1, X 135, X 154, X 3, X 4, X 6, Y 1$, $Y 13, Y 2, Y 4, Z 11, Z 130, Z 202$, Z204, Z205, Z212, Z247, Z4, Z41, Z43, 29 
Table 1. HATCHERIES, DEALERS. AND INDEPENDENT FLOCKS PARTICIPATING IN

THE NATIONAL POULTRY IMPROVEMENT PLAN

WATERFOWL, EXHIBITION POULTRY AND GAME BIRDS

APP. SUB- PARTICIPANTS NAME AND ADDRESS

NO. PART

HATCHING
EGG
CAPACITY

CAPACITY
PRODUCTS CLASSIFIED

U.S. PULLORUM-TYPHOID CLEAN
ADOITIONAL

CLASSIFICATIONS FOR WHICH PRODUCT QUALIFIED

58 - FLORIDA

428 E NASHVILLE USA, 4785 NW 72 AVENUE, MIAMI 33166

547 E OMNI EXPORT SERVICES, INC., 11350 NW. 38TH TERRACE, MIAMI 33178

Independent Flocks

429 E DAVID ADAMS, 3275 SEARS ROAD, CHIPLEY 32428

392 E BILL ADKINS, 14-C RT. 1, BOX 15, MAYO 32066

441 E TOM ADKINS, 25928 N.W. 130TH AVENUE, HIGH SPRINGS 32643

437 E COREY ALONZO, 13020 OLD KINGS ROAD, JACKSONVILLE 32219

E ROLAND ANDERSON, P.O. BOX 608, LOUGHMAN 33858 Phone: (941)424-6241

E MELODY ANDERSON, 2155 RICH ROAD, MYAKKA CITY 34251

E DALE ANDERSON, P.O. BOX 1669, DEFUNIAK SPRINGS 32435

383 E ASHLEYS FARM, 3394 FRONTIER AVENUE, LAKE WORTH 33467

Phone: (561)568-0940

324 E LYNDA ATKINS, 19973 68TH STREET, LIVE OAK 32060

544 E B \& B POULTRY, 1210 EUCELPTUS AVENUE, ARCADIA 34266

BILLY BALDWIN, RT. 7, BOX 435, LAKE CITY 32055

E LAURELLE BALOG, 19864 KINGFISHER LANE, LOXAHATCHEE 33470

E CAITLIN BANKS, 4598 HIGHWAY 66 EAST, ZOLFO SPRINGS 33890

E CASEY BEATY, 1313 EAST TRAPNELL ROAD, PLANT CITY 33567

E KIM R. BELVIN, 17976 WEST SYCAMORE ROAD, LOXAHATCHEE 33479

E KEN \& FRAN BERDICK, 16121 NOTH RIVER ROAD, ALVA 33920

E JOHN \& JOHNNY BEVERLY, 595 WILLOW AVENUE, BALDWIN 32234

Phone: $(904) 266-4381$

E CHARLES BLACK, 6165 55TH AVENUE NORTH, ST. PETERSBURG 33709

E RICKY BLACKBURN. 5655 BACKWOODS LANE, BASCOM 32423

E CLIFFORD S. BLAKE, 7704 BLAIR DRIVE, ZEPHYRHILLS 33544

E DICKY BLAND, RT. 2, BOX 895, MADISON 32340

E EVIE BLOUNT, 1010 BUCK BLOUNT ROAD, QUINCY 32351

E AL \& JAMES BLUM, 2770 MONICA LANE, CANTONMENT 32533

E\&C R5

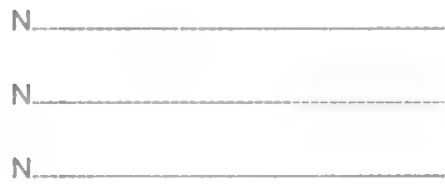

N18, N19, W30

R27

R15, W132, W30, W415, W6, W96

N.

R183, R34, W28, W7

N

R27, R7, Z18, Z19

N

N.

R146, R209, W1, W132, W30

R247

R27

R229, R239, W13, W2, W30, Z140, Z203, Z236, Z250, Z256

N

W28, W91

N.

N

R27

R27

A. D. N18, R116, W11, W160, W19, W20, W23, W26, W54. 
Table 1. HATCHERIES, DEALERS, AND INDEPENDENT FLOCKS PARTICIPATING IN

THE NATIONAL POULTRY IMPROVEMENT PLAN

WATERFOWL, EXHIBITION POULTRY AND GAME BIRDS

APP. SUB- PARTICIPANTS NAME AND ADDRESS

NO. PART
HATCHING
EGG

CAPACITY
PRODUCTS CLASSIFIED

U.S. PULLORUM-TYPHOID CLEAN
ADDITIONAL

CLASSIFICATIONS FOR WHICH PRODUCT QUALIFIED

58 - FLORIDA

\begin{tabular}{|c|c|c|}
\hline 489 & $E$ & $\begin{array}{l}\text { BOBWHITE QUAIL FARM OF HATCHBEND, P.O. BOX } \\
1362 \text {, NEWBERRY } 32669\end{array}$ \\
\hline & $E$ & $\begin{array}{l}\text { BONANZA QUAIL CORP.. } 15625 \text { SW 168TH AVENUE, } \\
\text { MIAMI } 33187\end{array}$ \\
\hline & $E$ & $\begin{array}{l}\text { WILLIAM BRACKEN, } 4535 \text { CROWS BLUFF, LAKE WALES } \\
33859\end{array}$ \\
\hline & $E$ & $\begin{array}{l}\text { RONALD BROOKER, } 10221 \text { NE HIGHWAY 314, SILVER } \\
\text { SPRINGS } 34488\end{array}$ \\
\hline & $E$ & $\begin{array}{l}\text { MILDRED BROOKER, } 3908 \text { WHISPER TRAIL, GREEN } \\
\text { COVE SPRINGS } 32043\end{array}$ \\
\hline & $E$ & $\begin{array}{l}\text { RICH \& SHERRY BROOKER, } 3908 \text { WHISPER TRAIL, } \\
\text { GREEN COVE SPRINGS } 32043\end{array}$ \\
\hline & $E$ & $\begin{array}{l}\text { THEODORE R. BROWN. } 351 \text { NE } 117 \text { TH STREET, MIAMI } \\
33161\end{array}$ \\
\hline
\end{tabular}

474 E BULL FEATHER'S RANCH, DEAN EVANS, HC 04, BOX 990, OLD TOWN 32680

451
JOHN BYRD, 2307 HANDY ROAD, CANTONMENT 32533

E RAY BYRD, 2496 HANDY ROAD, CANTONMENT 32533

E MIGUEL S. CABEZA, 3200 SW 103 RD COURT, MIAMI 33165

E LEON CANADAY, 6810 KEYES ROAD, JACKSONVILLE 32219

E GREG CANNON, 9161 SELLERSVILLE ROAD, JAY 32565

E JOHN \& MARIE CASEY, 223 WILDER ROAD, LAKELAND 38809

E DAVID CLAUSING, P.O. BOX 459, NOCATEE 33864

E BRUCE CLOUGH, 16543 VILLAGE GREEN DRIVE, JACKSONVILLE 32234

E JAMES COLLIER, P.O. BOX 95, WILDWOOD 34785

E CONFEDERATE GAME FOWL FARM, 4828 NORTH ROCK SPRINGS ROAD, APOPKA 32712

E MARVIN E. COX, 11491 SE 40TH STREET, MORRISTON 32668

Phone: (352)486-3922

E CRIPPLE CREEK GAME FARM, 2757 FREEMAN ROAD, ALFORD 32420

E RANDALL \& TINA CROBBY, 4453 193RD DRIVE, LIVE OAK 32060

E CARL CROW. 2120 COX ROAD, COCOA 32926

E MIKE CRUZ, 5501 120TH AVENUE NORTH, LOXAHATCHEE 33411

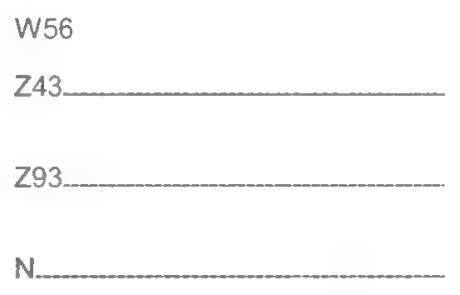

R28, R74, R77, W12, W136, W193, W230, W232, W270. W271, W3, W30, W357, W364, W365, W368, W47, W86, W98

R168, W119, W3, W48

R168, R20, R21, R248, R86

R177, R222, R54, R62, W100, W102, W119, W121, W142, W17, W198, W25, W26, W263, W274, W276, W28, W35, W48, W52, W544, W570, W573, W595, W69, W84, W94

R27

N.

N.

R27.

N.

N, W12, W30, W393.

N15, N9, W570, W69.

$\mathrm{N}$

N

R27

N

R27

N.

N.

R27

N. 
Table 1. HATCHERIES, DEALERS, AND INDEPENDENT FLOCKS PARTICIPATINGIN

THE NATIONAL POULTRY IMPROVEMENT PLAN

WATERFOWL, EXHIBITION POULTRY AND GAME BIRDS

APP. SUB- PARTICIPANTS NAME AND ADDRESS

NO. PART
HATCHING

EGG

CAPACITY
PRODUCTS CLASSIFIED

U.S. PULLORUM-TYPHOID CLEAN
ADDITIONAL

CLASSIFICATIONS FOR WHICH PRODUCT QUALIFIED

\section{8 - FLORIDA}

527 E REGINA CUNINGHAM, 3764 EAST MAIN STREET, WAUCHULA 33873

539 E RICHARD A. EARY, 4329 NW 47TH PLACE, BELL 32619

471 E KENNETHL. EDWARDS, P.O. BOX641, BRISTOL 32321

225 E JESSE ELLIS, P.O. BOX 1066, UMATILLA 32784 Phone: (352)669-4603

449 E CHRISTOPHER ELLIS, 445 HAYES COURT, APOPKA 32712

533 E LAURA EPPENBAUGH, 16060 EAST EPSON DRIVE, LOXAHATCHEE 33470

423 E WILLIAM C. ERNEY, 6970 N.E. 192 PLACE, CITRA 32113

548 E SERAFIN FALCON, 18425 SW 177 TH AVENUE, MIAMI 33187

564 E STEPHEN FARKAS, 12536 TYLER RUN, ODESSA 33556

508 E TAMMY FAYNE, 504 PATCH ROAD, DEFUNIAK SPRINGS 32433

THE FEATHER FARM, 2054 ACOCADO BLVD., BUNNELL 32110

FLATLANDER GAME FARM, RT. 3, BOX 1558-D, LAKE BUTLER 32054

FLORIDA PINE GROVE GAME FARM, 640 BIGBONE LANE, MARIANNA 32446
BILLY GAMBLE, 14-C RT. 1, BOX 120, MAYO 32066

E JIMMY GANDER/JERRY WILLIAMS, P.O. BOX 86 , APALACHICOLA 32320

E DAVID \& JAIMIE GATCHELL, 15199 83RD LANE, NORTH LOXAHATCHEE 33470

E GATOR CREEK GAME FARM, 22406 BELGARD LANE, ASTATULA 34705

E EMMETT GAYLAND, P.O. BOX 2608, HIGH SPRINGS 32655

E KATHY GLISSON, 2545 TIGER CREEK TRAIL, LAKE WALES 33898

E VERBON GOBLE, 12180 HIGHWAY 98 NORTH, LAKELAND 33809

E WILFRED GRANT, 1605 MUSCOGEE ROAD, CANTONMENT 32533

E REXD. GRANTHAM, RT. 2, BOX 141, CARYILLE 32427

E PATTY GREENE, 501 LAKE INEZ TRAIL, PIERSON 32180

E DOYLE GRIFFIS, P.O. BOX 103, GLEN ST. MARY 32040

E RICHARD GRIGGS, 8643 EL PASO DRIVE, LAKE WORTH 33467

E HALE FAMILY FARM, 2038 SW THIGPEN AVENUE, ARCADIA 34266

E MICHAEL HALL, 6570 OLD BAGDAD HIGHWAY, MILTON 32583

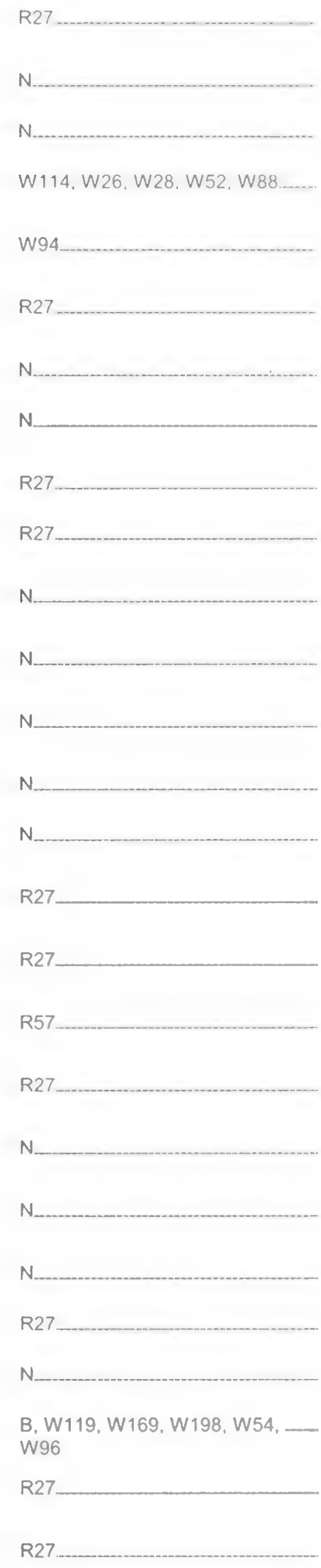


Table 1. HATCHERIES, DEALERS, AND INDEPENDENT FLOCKS PARTICIPATING IN

THE NATIONAL POULTRY IMPROVEMENT PLAN

WATERFOWL, EXHIBITION POULTRY AND GAME BIRDS

\begin{tabular}{lllll}
\hline & & HATCHING & PRODUCTS CLASSIFIED \\
APP. SUB- & PARTICIPANTS NAME AND ADDRESS & EGG & U.S. PULLORUM-TYPHOID \\
NO. PART & CLEAN & CLASSIFICATIONS FOR & WHICH PRODUCT QUALIFIED \\
\hline
\end{tabular}

58 - FLORIDA

\begin{tabular}{|c|c|c|}
\hline 84 & $E$ & BO HAMMOCK, RT. 3, BOX 216, LAKE BUTLER 32054 \\
\hline 323 & $\mathrm{E}$ & $\begin{array}{l}\text { BART HARRELL, } 2833 \text { BRANDY BEACH DRIVE, GRAND } \\
\text { RIDGE } 32442\end{array}$ \\
\hline 20 & E & $\begin{array}{l}\text { JAY \& JOHN HARVEY, } 32443 \text { MEMORY LANE, } \\
\text { LEESBURG } 34788 \\
\text { Phone: (352) } 343-5473\end{array}$ \\
\hline & $E$ & SUSAN HATCH, P.O. BOX 163, ARCADIA 34265 \\
\hline & $E$ & $\begin{array}{l}\text { HEAVENLY ACRES POULTRY \& GAME BIRD FARM, P.O } \\
\text { BOX } 151 \text {, LOXAHATCHEE } 33470\end{array}$ \\
\hline
\end{tabular}

438 E JOHN D. HIGGINBOTHAN, 13278 RANCH ROAD, JACKSONVILLE 32218

372 E ARTHUR HILGERSON, RT. 1, BOX 159-F10, LAKE CITY 32055

431 E BRIM HILL, H.C.R. ONE, BOX 54, OLD TOWN 32680

304 E VALERIE HIRVELA。6230 VIRGINIA LANE, SEFFNER 33584

\begin{tabular}{|c|c|c|}
\hline & $E$ & MARIE HOFFMAN, 2737 HARTLEY LANE, LITHIA 33547 \\
\hline 91 & $E$ & $\begin{array}{l}\text { MURRAY J. HOLT, } 1609 \text { TONI STREET, PENSACOLA } \\
32504\end{array}$ \\
\hline & $E$ & $\begin{array}{l}\text { ED \& SHIRLEY HOWELL, } 11630 \text { SIMMONS ROAD, } \\
\text { JACKSONVILLE } 32218\end{array}$ \\
\hline & E & $\begin{array}{l}\text { DERRICK, BRANDON \& BRITTANY HUFFMAN, } 18085 \\
\text { NORTH COMMONWEALTH AVENUE, POLK CITY } 33868\end{array}$ \\
\hline & E & $\begin{array}{l}\text { EARL HUNTER, } 16922 \text { WATERLINE ROAD, BRADENTON } \\
34202\end{array}$ \\
\hline & $E$ & $\begin{array}{l}\text { LARRY HUTCHINS GAME BIRD FARM, RR 1, BOX } 3890, \\
\text { GLEN ST, MARY } 32040\end{array}$ \\
\hline & $E$ & $\begin{array}{l}\text { GRACE JAYE, } 92 \text { BLUEBERRY LANE. CRAWFORDVILLE } \\
32327\end{array}$ \\
\hline & $\mathrm{E}$ & $\begin{array}{l}\text { JOSE LUIS JIMANEZ, } 23727 \text { NW } 227 \text { DRIVE, HIGH } \\
\text { SPRINGS } 32643\end{array}$ \\
\hline & E & $\begin{array}{l}\text { JIMARY GAME FARM, JIM \& MARY CARDEN, } 9780 \\
\text { EVANS ROAD, POLK CITY } 33868\end{array}$ \\
\hline & $E$ & $\begin{array}{l}\text { MAURICE JOHNSON, } 7751 \text { NW 70TH STREET, } \\
\text { CHIEFLLAND } 32626\end{array}$ \\
\hline 7 & $E$ & $\begin{array}{l}\text { JOHNNY JOHNSON, } 3153 \text { FRANK ARD ROAD, } \\
\text { CANTONMENT } 32533\end{array}$ \\
\hline 50 & $\mathrm{E}$ & $\begin{array}{l}\text { ROY JONES, SR. \& TONY GREEN, } 1715 \text { WEST DETROIT } \\
\text { BLVD., PENSACOLA } 32514\end{array}$ \\
\hline 454 & $\mathrm{E}$ & $\begin{array}{l}\text { ROY JONES, JR., } 2410 \text { FARMS AVENUE, PENSACOLA } \\
32526\end{array}$ \\
\hline 519 & $E$ & $\begin{array}{l}\text { LEANING STAR RANCH, STARLENE KALINSKI, } 2350 \\
\text { SANDY LANE, NAPLES } 34112\end{array}$ \\
\hline 41 & $E$ & $\begin{array}{l}\text { BETTY KEARNS, } 650528 \text { TH AVENUE EAST, PALMETTO } \\
34221\end{array}$ \\
\hline
\end{tabular}

N

Z108, Z128, Z131, Z137, Z15, Z179, Z19, Z235

R27.

R247, W132, W30, W526, Z4

R119, R121, R14, R169, R17, R172, R179, R18, R183, R222, R27, R30, R45, R52, R53, R69, R87, R93, R96, W13, W265, W7

N.

N.

N.

R33, W184, W215, W247, W248, W290, W309, W313, W373, W504, W518, W519, W74, W75

W30

N

R114, R247, R42

$\mathrm{R} 27$

N.

N.

R27

$x 146, \times 147, \times 28, \times 65$

N

R27

N.

N.

N.

R20, R222, R247, R248, R7, R8, W1, W132, W30, W415

W132, Z137, Z204 
Table 1. HATCHERIES. DEALERS, ANO INDEPENOENT FLOCKS PARTICIPATING IN

THE NATIONAL POULTRY IMPROVEMENT PLAN

WATERFOWL. EXHIBITION POULTRY AND GAME BIRDS

APP. SUB- PARTICIPANTS NAME AND ADDRESS
NO. PART

$\begin{array}{cc}\text { HATCHING } & \text { PROOUCTS CLASSIFIED } \\ \text { EGG } & \text { U.S.PULLORUM-TYPHOID } \\ \text { CAPACITY } & \text { CLEAN }\end{array}$

ADDITIONAL

CLASSIFICATIONS FOR WHICH PRODUCT QUALIFIED

58 - FLORIDA

510 E RYAN KEENE, 1402 WEST SANDLEWOOD DRIVE, PLANT CITY 33566

543 E TIFFANY KIMBALL, 3139 CROSS BRANCH ROAD, DELAND 32724

486 E KINARD CHICKEN FARM, 5034 SE TAYLOR ROAD, ARCADIA 34266

552 E RUSSELL KIRKLAND, 1199 PINE RIDGE ROAD, BONIFAY 32425

476 E WAYNE KOLMETZ, 5156 DOUGLAS FERRY ROAD, CARYVILLE 32427

485 E MARY LACKEY \& ANN SWOFFORD, 350 LAKE MARKHAM ROAD, SANFORD 32771

459 E JOHNNY LAWSON, 1127 HIGHWAY 2, WESTVILLE 32464

389 E JERRY LEACH, RT. 1, BOX 227, OLD TOWN 32680

488 E MANUEL LEAL, 19454 NW 61ST AVENUE, MIAMI 33015

462 E MERYL LECHNER, 2573 CHYNN AVENUE, NORTHPORT 34286

351 E STEPHEN \& LINDA LEE, P.O. BOX 914, LAKE CITY 320560914

499 E RICARDO LLORENTE, 11865 SW 46TH STREET, MIAMI 33175

439 E ROY D. LOFTON, 5033 SIESTA DEL RIO DRIVE, JACKSONVILLE 32258

557 E JEFF LOLLIE, 9115 RAVENA ROAD, TALLAHASSEE 32309

339 E LONG SHADOWS, D. EVERMAN, P.O. BOX 527 (CR 417), LAKE PANASOFFKEE 33538

575 E LINDA MACDONALD, 5710 206TH TERRACE, LOXAHATCHEE 33470

553 E WALTER \& FAYE MANN, 12250 MOBLEY POND LANE, MYAKKA CITY 34251

511 E JOE MARTIN, P.O. BOX 1391, LOXAHATCHEE 33470

504 E MARCELO MARTINEZ, 19675 NW 49TH COURT, MIAMI 33055
CHRIS MCGEORGE, 6532 LAMESA CIRCLE, TAMPA 33634

E TERESA MCKINNIE, P.O. BOX 2397, HAVANA 32333

E WILLIAM \& ALMA MCLEAN, 4701 THORPE AVENUE, ORLANDO 32804

E SANDY MEADOWS, 4316 ELLENVILLE PLACE, VALRICO 33594

E JONES H. MERRITT, 19307 N.W. 202 STREET, HIGH SPRINGS 32643

E MESSER POULTRY, P.O. BOX 331, NOCATEE 34268
R27

R27

A, D, R233, R96, W13, W132, W30, W578

R27

R7, Z118, Z89, Z93

A220, R42, R65, Z203, Z230

N

N.

N.

W13

N

N

R248, W248, W30, W4, W48, W52, W610, W69, W88

R27, Z2, Z41, Z43

R87

R27

R247.

R27

W115, W123, W138, W175 W176, W187, W198, W211 W224, W241, W245, W293, W294, W81

D, H10, H5, N180, N4, N8, R244, W128, W19, W306, W74

N.

R27.

R237, R3, R56, R73

N.

R20, R244, W110 
Table 1. HATCHERIES, DEALERS, AND INDEPENDENT FLOCKS PARTICIPATING IN

THE NATIONAL POULTRY IMPROVEMENT PLAN

WATERFOWL, EXHIBITION POULTRY AND GAME BIRDS

APP. SUB- PARTICIPANTS NAME AND ADDRESS

NO. PART
PARTICIPANTS NAME AND ADDRESS

\section{HATCHING \\ EGG}

CAPACITY
PRODUCTS CLASSIFIED

U.S. PULLORUM-TYPHOID CLEAN
ADDITIONAL

CLASSIFICATIONS FOR WHICH PRODUCT QUALIFIED

58 - FLORIDA

\begin{tabular}{|c|c|c|}
\hline 443 & $\mathrm{E}$ & $\begin{array}{l}\text { LARRY METZKER, } 1601 \text { PINE TOP DRIVE EAST, } \\
\text { LAKELAND } 33809\end{array}$ \\
\hline 293 & $E$ & RODNEY MICHAEL, P.O. BOX 244, RIVERVIEW 33569 \\
\hline 356 & $E$ & $\begin{array}{l}\text { CHARLES W. MILLER, } 16386 \text { VILLAGE GREEN DRIVE, } \\
\text { JACKSONVILLE } 32234\end{array}$ \\
\hline 530 & $E$ & $\begin{array}{l}\text { DEBRA A. MILLER, } 16079 \text { WHIDDEN ROAD, SARASOTA } \\
34240\end{array}$ \\
\hline 529 & $E$ & $\begin{array}{l}\text { T. C. MONTGOMERY, } 1446 \text { HIGHWAY } 179 \text {, BONIFAY } \\
32425\end{array}$ \\
\hline 505 & E & $\begin{array}{l}\text { KELVIN MORENO, } 15550 \text { NW 122ND AVENUE, MIAMI } \\
33018\end{array}$ \\
\hline
\end{tabular}

PAULA MOSELEY, RT. 3, BOX 5603, FT. WHITE 32038 LARRY MOSLEY, ROUTE 5, BOX 7036, STARKE 32091

DANIEL E. MULLIN, P.O. BOX 153, SUMMERFIELD 34492 Phone: (352)347-5709 LAURESA MUSGROVE, $5480208 T H$ DRIVE, NORTH LOXAHATCHEE 33470

541

JOHN NEFF, 1351 HOLDER LANE, GENEVA 32732 Phone: (407) 349-0450

E WILLIAM NETTLES, 1763 SUNDALE ROAD, JACKSONVILLE 32218

E ORLANDO RURA-GOMEZ, 9921 SW 157 TERRACE, MIAMI 33157

E ALVIN PAET \& ROBERT W. BIRGE, JR, 2341 HIGHWAY 177-A, BONIFAY 32425

E LINDA PARMETER, P.O. BOX 608, LOUGHMAN 33858

E

TRACY PARROW, 3370 5TH AVENUE NW, NAPLES 34120

E LARRY PEREZ, 10165 CLARONA OCOEE ROAD, APOPKA 32703

E MICHAEL PETERS, 17216 4TTH DRIVE, WELLBORN 32094

E LOUIS PIERCE, 2117 SAGASTA STREET, TAMPA 33619

E LEWIS POWELL, 1751 ROOSTER LANE, WESTVILLE 32464

E RICHARD PRUE, 8087 3RD STREET, LAUREL HILL 32567

E RAINBOW RANCH, 358 SOUTH ARBUCKLE BLVD., AVON PARK 33825
R151, R244, R48, R86, R94, W28.

R57, Z128, Z18

N.

R146, R239, R247, R248, R91, W52, W573, Z207

N

H5, R104, R105, R11, R139, R19. R227, R247, R30, R33, R51, R52, R53, R70, R86, R93, W102, W11, W12, W121, W126, W127, W141, W142, W16, W164 W169, W17, W170, W18, W223, W23, W25, W26, W27, W274, W33, W36, W38, W45, W54 W544, W554, W570, W578, W595, W60, W69, W9, w93. Z102, Z15, Z179, Z22, Z93, Z99

$\mathrm{R} 27$

N

R27

R27

R27

N.

N. N110

N

R27

R27

N.

$\times 187$

R27.

N

N

R27, Z11, Z2

N 
Table 1. HATCHERIES, DEALERS, AND INDEPENDENT FLOCKS PARTICIPATING IN

THE NATIONAL POULTRY IMPROVEMENT PLAN

WATERFOWL, EXHIBITION POULTRY AND GAME BIRDS

APP. SUB- PARTICIPANTS NAME AND ADORESS

NO. PART
HATCHING

EGG

CAPACITY
PRODUCTS CLASSIFIED

U.S. PULLORUM-TYPHOID

CLEAN
ADDITIONAL

CLASSIFICATIONS FOR WHICH PRODUCT QUALIFIED

58 - FLORIDA

346 E LARRY REED, ROUTE 3, BOX 191, LAKE BUTLER 32054

398 E ROBERT \& JOHNNY REYNOLDS, 7930 KIMBRO LANE ORLANDO 32818

DAVID RICHARDS, 5052 JEANETTE DRIVE, MARIANNA 32446

$257 \quad E$

$537 \quad E$

$254 \quad E$

CYNTHIA RIDDINGS, 16985 SW 207 AVENUE, MIAMI 33187

ERIC \& MARIE RIENDEAN, 2658 LOTT ROAD, HILLIARD 32046 MCCOY 32134

E JOHN ROBERTO, 2591 MIKLER ROAD, ORLANDO 32765

E JOHNL. ROBERTS, 1911 DAWN HEIGHTS DRIVE, LAKELAND 33801

E MICHALEROBINSON, 3394 FRONTIER AVENUE, LAKEWORTH 33467

MARIO RODRIGUEZ, P.O. BOX 1083, SAN ANTONIO 33576

Phone: (352)523-1954

E ONIS RODRIGUEZ \& SALVADOR MAYORGA, 14707 HIDDEN TRAIL ROAD, ORLANDO 32824

E ROYAL NEST RANCH,LLC, 1579 NW 87TH PLACE, BELL 32619

LEE SALMON, 7303 WILSON ROAD, WEST PALM BEACH 33413

Phone: (561)686-4774

E ALBERT SANCHEZ, 6705 RAVENWOOD STREET, WESLEY CHAPEL 33544

E AL SARROCA, 4127 O'RILEY ROAD, JACKSONVILLE 32210

DALTON SCONYERS. 1342 NORTH HOLLANDTOWN ROAD, WAUCHULA 33873

AMANDA M. \& BRIAN M. SEMRINEL, 14305 MOSSY HAMMOCK LANE. MYAKKA CITY 34251-2026

E ELLEN SHERMEN, 220 SAUNDERS TRAIL, GENEVA 32732

LISA SMITH, P.O. BOX 1271, OLD TOWN 32680

E SON MIGUELITO GAME FARM, 24 RIDGE ROAD, FROSTPROOF 33843

E SOUTH FORK RANCH, 7383 SOUTH FORK RANCH ROAD, CLERMONT 34711

E GEORGE H. SOWDERS, 3326 SE HASTY DRIVE, ARCADIA 34266

E ROB SPITZE, 10360 SW. CR 18, HAMPTON 32044

E WILLIAM STANFIELD, 8745 36TH AVENUE UE, PALMETTO 34231

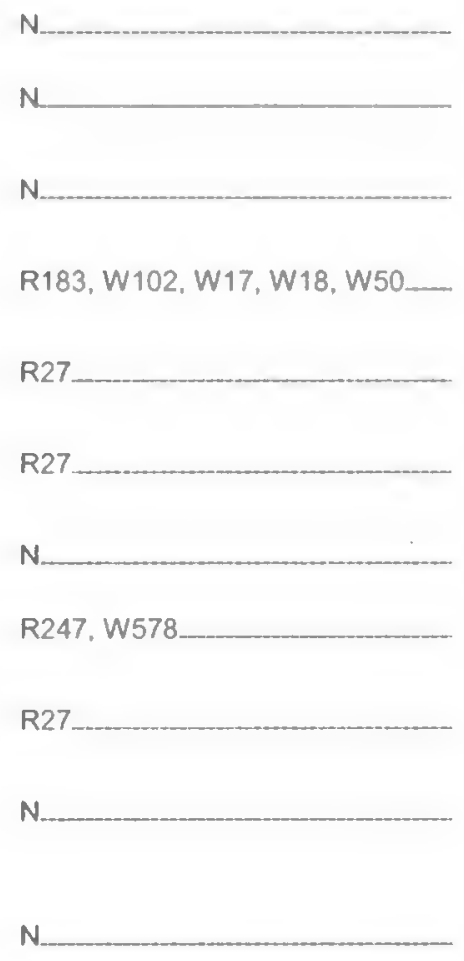

R100, R124, R177, R182, R45, R67, R83, R84, R99, W152, W169, W570, W97, Z22, Z247

C. R1, R2, R27, R51, W12, W121, W126, W16, W17, W19, W33, W5, W54, W570, W66. W74, W75, W8

W52, W570

N.

W1, W132, W30

R12, R15, R19, R22, R7, R71, W106, W12, W23

R183, R94, W13, W415, W46, W588, Z11, Z142, Z2, Z231, Z41. $\mathrm{Z} 60, \mathrm{Z72}$

N.

N

R27

W30, W588

N

W11, W114, W12, W164, W25, W26, W578, W70, W88, W9. W91 
Table 1. HATCHERIES, DEALERS, AND INDEPENDENT FLOCKS PARTICIPATING IN

THE NATIONAL POULTRY IMPROVEMENT PLAN

WATERFOWL, EXHIBITION POULTRY AND GAME BIRDS

\begin{tabular}{llccc}
\hline & & PATCHING & PRODUCTS CLASSIFIED \\
APP. & SUB- & PARTICIPANTS NAME AND ADDRESS & EGG & U.S. PULLORUM-TYPHOID \\
NO. PART & CLEAN & CAPACITY & WHICH PRODATIONS FOR \\
\hline
\end{tabular}

$$
58 \text { - FLORIDA }
$$

\begin{tabular}{|c|c|c|}
\hline 566 & $E$ & $\begin{array}{l}\text { VICTORIA STEVENS, } 13834 \text { 52ND. CT. NORTH, ROYAL } \\
\text { PALM BEACH } 33411\end{array}$ \\
\hline 370 & $\mathrm{E}$ & $\begin{array}{l}\text { CONNIE MACK \& DINO SULLIVAN, } 2829 \text { DENNIS } \\
\text { HOWELL ROAD, PERRY } 32347\end{array}$ \\
\hline 490 & $E$ & $\begin{array}{l}\text { JOE SULLIVAN, } 2781 \text { GLOVER CARLTON ROAD, PERRY } \\
32348\end{array}$ \\
\hline 60 & $E$ & ROBERT SUMNER, HC 5, BOX 45, OLD TOWN 32680 \\
\hline 33 & $E$ & $\begin{array}{l}\text { SUWANNEE RIVER GAME FOWL FARM, RT. 3, BOX } 203 \text {, } \\
\text { JASPER } 32052\end{array}$ \\
\hline 53 & $E$ & $\begin{array}{l}\text { JAMES D. SWANSON, } 600712 \text { TH STREET EAST. } \\
\text { BRADENTON } 34203\end{array}$ \\
\hline 448 & $E$ & $\begin{array}{l}\text { ALAN TAPIA, } 16000 \text { SW } 272 \text { STREET, HOMESTEAD } \\
33031\end{array}$ \\
\hline 494 & $E$ & $\begin{array}{l}\text { ABY CARLIN \& LINDSEY TEATES, } 16032 \text { WINBURN } \\
\text { DRIVE, SARASOTA } 34240\end{array}$ \\
\hline & $E$ & W. G. THOMAS, P.O. BOX 1212, BRADENTON 34206 \\
\hline
\end{tabular}

404 E ROBERT THOMAS, 6624 QUAIL ROAD, CALLAHAN 32011

567 E JIM \& THELMA THOMPSON, 3703 FDC GROVE ROAD, DAVENPORT 33837

568 E KRYSTINA THOMPSON, 3703 FDC GROVE ROAD, DAVENPORT 33837
$E$ JOHN R. THYSSEN, 13877 CR 737, WEBSTER 33597

\section{$E$} MARK TOLAR, 2111 WEST JOHNSON ROAD, PLANT CITY 33567

E LULY TOLBERT, 3127 APPALACIAN TRAIL, POLK CITY 33868

E CAROL DIXIE TURNER, 3233 APPALACHIAN TRAIL, POLK CITY 38868

Phone: (941)984-1676

E JERRY TURNER, 687 BONNIE LANE, WEST PALM BEACH 33415

E HOWARD GLEN VANBUSKIRK, 19895 MANECKE ROAD, BROOKSVILLE 34601

E MARYANNE VANHEUSEN, P.O.BOX 1599 LOXAHATCHEE 33470

E JESSIE S. VERGEL, 180 DEBARRY AVENUE, ORANGE PARK 32073

E CECILA VERNER, 2550 TIGERS CREEK TRAIL, LAKE WALES 33898

E ZELL VONPOHLMAN, 17901 LAMBTURNER ROAD. JACKSONVILLE 32234

E PAMELA JOANNE WAGNER, 8518 GIBSONTON DRIVE \#44. GOBSONTON 33534

E M.K.WATERS, 920 NOWAK ROAD. CANTONMENT 32533

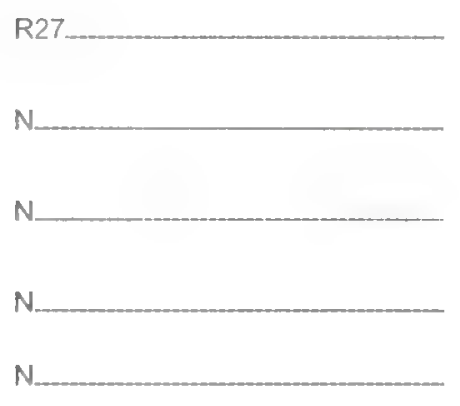

W12, W18, W262, W274, W308, W48, W6, W77, W91

N. N110

R104, R146, R18, R222, R94, R95, W13, W132, W28

R105, R114, R15, R183, R19, R2. R200, R22, R3, R30, R44, R51,

R57, R7, R97, W12, W17, W18,

W20, W25, W26, W33, W372,

W393, W57, W6, W96

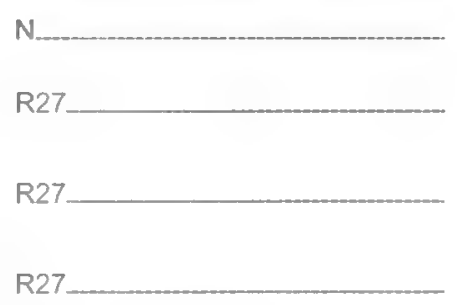

W1, W132, W578

$\mathrm{R} 27$

$\mathrm{R} 27, \mathrm{Z204}, \mathrm{Z205}, \mathrm{Z9}$

W26

N.

$\mathrm{R} 27$

N.

R27

$\mathrm{R} 27$.

W1, W13, w30

W119, W25, W26, W28, W6 
Table 1. HATCHERIES, DEALERS. AND INDEPENDENT FLOCKS PARTICIPATING IN

THE NATIONAL POULTRY IMPROVEMENT PLAN

WATERFOWL. EXHIBITION POULTRY AND GAME BIRDS

\section{APP. SUB- PARTICIPANTS NAME AND ADDRESS}

HATCHING
EGG
CAPACITY

EGG

PRODUCTS CLASSIFIED

U.S. PULLORUM-TYPHOID

CLEAN
ADDITIONAL

CLASSIFICATIONS FOR WHICH PRODUCT QUALIFIED

58 - FLORIDA

\begin{tabular}{|c|c|c|}
\hline 561 & $E$ & WATSON FARM, 1064 LOVE ROAD, BONIFAY 32425 \\
\hline 415 & $E$ & $\begin{array}{l}\text { WEST SIDE QUAIL FARM, } 802 \text { N.W. } 138 \text { STREET. } \\
\text { NEWBERRY } 32669\end{array}$ \\
\hline 434 & $\mathrm{E}$ & BEN WHITE, 1905 LEE RADE, ORLANDO 32810 \\
\hline 532 & $E$ & $\begin{array}{l}\text { JAMES WILLIAMS, } 1158 \text { TAYLOR ROAD, JACKSONVILLE } \\
32234\end{array}$ \\
\hline 475 & $E$ & $\begin{array}{l}\text { TAMARA LUPO \& WILLIS WILSON, } 13400103 R D \\
\text { STREET, SELLSMERE } 32948\end{array}$ \\
\hline 468 & $E$ & $\begin{array}{l}\text { WILLIAM WING-WILLIES WEE WINGS, } 24472 \\
\text { MAELDIGHT ROAD, BROOKSVILLE } 34601\end{array}$ \\
\hline 350 & $E$ & $\begin{array}{l}\text { KELLY O'NEIL WISE \& BRENDA WISE, ROUTE 8, BOX } \\
\text { 349, LAKE CITY } 32055\end{array}$ \\
\hline 384 & $E$ & WOODED WETLANDS, RT. 1, BOX 26, LAMONT 32336 \\
\hline 381 & $E$ & BILL WORTH, RT. 1, BOX 132, BLOUNTSTOWN 32434 \\
\hline 467 & $E$ & RALPH WRIGHT, RT. 14, BOX 878, LAKE CITY 32024 \\
\hline 525 & $E$ & $\begin{array}{l}\text { RICHARD WRIGHT, P.O. BOX 151, LOXAHATCHEE } \\
33470\end{array}$ \\
\hline 469 & $E$ & $\begin{array}{l}\text { ELIZABETH \& TROY YATES, } 2020 \text { KEYSVILLE ROAD, } \\
\text { LITHIA } 33547\end{array}$ \\
\hline 416 & $E$ & $\begin{array}{l}\text { LEWIS ZIMMERMAN, } 31851 \text { LAKESIDE DRIVE, DELAND } \\
32720 \\
\text { Phone: (352)669-1459 }\end{array}$ \\
\hline
\end{tabular}

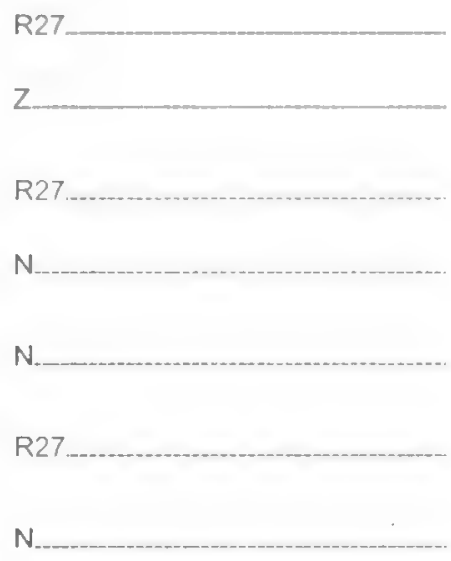

W14, W142 W17, W208, W48, W518, W570, W96, Z118, Z215

N, R94, W132, W30.

R185, R42, R60, R62, Z108, Z138, Z220

$\mathrm{R} 27$.

R16, R247, R7, R94.

N.
JAMES SCROGGS

GEORGIA POULTRY IMPROVEMENT ASSOCIATION, INC.

P.O. BOX 20

OAKWOOD, GA 30566

Phone: (770)535-5996

Fax: $\quad(770) 535-5941$ and DR. DOUG WALTMAN

GEORGIA POULTRY LABORATORY

P.O. BOX 20

OAKWOOD, GA 30566

Phone: (770)535-5996

Fax: $\quad(770) 535-5941$
Hatcheries

551 E QUAIL INTERNATIONAL, 1940 HIGHWAY 15 SOUTH. $_{4}$ GREENSBORO 30642

538 E STRICKLAND GAME BIRD FARM, INC., P.O. BOX 9. POOLER 31322

\section{$\underline{\text { Dealers }}$}

770 E SAMMY BALL, 853 CLYDE BIRD ROAD, LAFAYETTE 30728

548 E JERRY BONDS, P.O. BOX 136, ILA 30647

553 E BORDERS' QUAIL FARM. 10298 US HIGHWAY 441 SOUTH, HOMER 30547
852,480

30.000

N

E\&C

E\&C N

$E \& C \quad Z$ 
Table 1. HATCHERIES, DEALERS, AND INDEPENDENT FLOCKS PARTICIPATING IN

THE NATIONAL POULTRY IMPROVEMENT PLAN

WATERFOWL, EXHIBITION POULTRY AND GAME BIRDS

$\begin{array}{lll}\text { APP. } & \text { SUB- } & \text { PARTICIPANTS NAME AND ADDRESS } \\ \text { NO. } & \text { PART }\end{array}$

$\begin{array}{cc}\text { HATCHING } & \text { PRODUCTS CLASSIFIED } \\ \text { EGG } & \text { U.S. PULLORUM-TYPHOID } \\ \text { CAPACITY } & \text { CLEAN }\end{array}$

ADDITIONAL

CLASSIFICATIONS FOR WHICH PRODUCT QUALIFIED

57 - GEORGIA

\begin{tabular}{|c|c|c|c|c|}
\hline 793 & $E$ & $\begin{array}{l}\text { BRIDGES LAB LUV BANTAMS, P.O. BOX 1131, CALHOUN } \\
30703\end{array}$ & $\mathrm{E \& C}$ & \\
\hline 750 & $E$ & TOMMY BRITT, RT. 1, BOX 134, COLEMAN 31736 & E\&C & \\
\hline 669 & $E$ & $\begin{array}{l}\text { RONNIE BROOKS, } 8101 \text { HOG MOUNTAIN ROAD. } \\
\text { STATHAM } 30660\end{array}$ & $\mathrm{E \& C}$ & W207, W588________ \\
\hline 739 & $E$ & $\begin{array}{l}\text { BROWN BANTAMS, } 2995 \text { HOGANVILLE ROAD, } \\
\text { LAGRANGE } 30240\end{array}$ & $\mathrm{E} \& \mathrm{C}$ & $\begin{array}{l}\text { W26, W276, W28, W29, W52, } \\
\text { W54, W77 }\end{array}$ \\
\hline 765 & $E$ & $\begin{array}{l}\text { ALAN BUTLER, } 1210 \text { BROOKS ROAD, SUMMERVILLE } \\
30747\end{array}$ & $\mathrm{E \& C}$ & \\
\hline 818 & $E$ & $\begin{array}{l}\text { COBB'S BANTAMS, } 159 \text { CENTURY LANE, LAVONIA } \\
30553\end{array}$ & $\mathrm{E} \& \mathrm{C}$ & W149, W81___ \\
\hline 511 & $E$ & $\begin{array}{l}\text { DEER CREEK BANTAMS, } 2790 \text { THOMPSON MILL ROAD, } \\
\text { BUFORD } 30518\end{array}$ & $\mathrm{E} \& \mathrm{C}$ & W _ _ _ \\
\hline 808 & $E$ & $\begin{array}{l}\text { DIXIELAND HATCHERY. RT. 1, BOX 244, LUMBER CITY } \\
31549\end{array}$ & $\mathrm{E} \& \mathrm{C}$ & \\
\hline 809 & $E$ & DEIDRE EDDER, 683 MULBERRY ROAD. WINDER 30680 & $\mathrm{E} \& \mathrm{C}$ & R222. W13. W25, W573 \\
\hline 686 & $E$ & $\begin{array}{l}\text { GQF MANUFACTURING COMPANY, INC., P.O. BOX } 1552, \\
\text { SAVANNAH } 31498\end{array}$ & $\mathrm{E} \& \mathrm{C}$ & Z. \\
\hline 796 & $E$ & $\begin{array}{l}\text { GREEN OAKS GAME FARM. } 9114 \text { DAVIS MOUNTAIN } \\
\text { ROAD, TOCCOA } 30577\end{array}$ & $\mathrm{E} \& \mathrm{C}$ & \\
\hline 784 & $E$ & D.C. GRIZZLE, 3981 WILEY ROAD, GAINESVILLE 30506 & E\&C & W \\
\hline 673 & $E$ & $\begin{array}{l}\text { PAUL HARDY, } 7870 \text { CREEKWOOD ROAD, FAIRBURN } \\
30218\end{array}$ & E\&C & \\
\hline 772 & $E$ & $\begin{array}{l}\text { HATCHET FARMS. ROGER G. ROBERTS, } 963 \text { DEE } \\
\text { KENNEDY ROAD. AUBURN } 30011\end{array}$ & $\mathrm{E \& C}$ & \\
\hline 40 & $E$ & $\begin{array}{l}\text { HULSEY'S QUAIL FARM, } 6552 \text { CAMP CREEK ROAD, MT. } \\
\text { AIRY } 30563\end{array}$ & $\mathrm{E} \& \mathrm{C}$ & $243+\ldots$ \\
\hline 831 & $E$ & $\begin{array}{l}\text { JUNIOR JENKINS, } 349 \text { BUSHY HEAD ROAD, CHERRY } \\
\text { LOG } 30522\end{array}$ & $\mathrm{E \& C}$ & - \\
\hline 833 & $E$ & $\begin{array}{l}\text { JIMARY GAME FARM, } 870 \text { COLVIN ROAD, FORSYTH } \\
31029\end{array}$ & E\&C & $\mathbf{N}$ \\
\hline 828 & $\mathrm{E}$ & RAY JOINER, P.O. BOX 782, EDISON 31746 & $\mathrm{E} \& \mathrm{C}$ & W28, W569, W69, W88, W889 \\
\hline 798 & $E$ & JONES FARM, 2979 MT. GILEAD ROAD. TENNILLE 31089 & E\&C & (2) \\
\hline 827 & $E$ & HAROLD KLECKLEY.P.O. BOX 366. CUTHBERT 31740 & E\&C & W142, W28, W29, W88 \\
\hline 825 & $E$ & $\begin{array}{l}\text { LAZENBY GAME FOWL FARM, } 2540 \text { WRIGHTSBORO } \\
\text { ROAD, THOMASON } 30824\end{array}$ & E\&C & N \\
\hline 742 & $\mathrm{E}$ & $\begin{array}{l}\text { RONNY LEDFORD, } 3803 \text { WHITE SULPHUR ROAD, } \\
\text { GAINESVILLE } 30507\end{array}$ & $\mathrm{E} \& \mathrm{C}$ & W276, W29, W30, W69, w88 \\
\hline 783 & $\mathrm{E}$ & $\begin{array}{l}\text { STEVE LEDFORD, } 4422 \text { AUTRY ROAD, GAINESVILLE } \\
30506\end{array}$ & $\mathrm{E} \& \mathrm{C}$ & W \\
\hline 783 & $E$ & $\begin{array}{l}\text { STEVE LEDFORD, } 4422 \text { AUTRY ROAD. GAINESVILLE } \\
30506\end{array}$ & E\&C & W \\
\hline 826 & $E$ & $\begin{array}{l}\text { JERRY LOW, } 552 \text { WORLEY CROSS ROAD. JASPER } \\
30143\end{array}$ & $\mathrm{E} \& \mathrm{C}$ & N... \\
\hline & $E$ & MAGNET GAME FOWL FARM, BUD ROSS, 4321 TROUP & $\mathrm{E} \& \mathrm{C}$ & $N_{2}$ \\
\hline
\end{tabular}


Table 1. HATCHERIES, DEALERS, AND INDEPENDENT FLOCKS PARTICIPATING IN

THE NATIONAL POULTRY IMPROVEMENT PLAN

WATERFOWL, EXHIBITION POULTRY AND GAME BIRDS

\begin{tabular}{|c|c|c|c|c|c|}
\hline $\begin{array}{l}\text { APP. } \\
\text { NO. }\end{array}$ & $\begin{array}{l}\text { SUB- } \\
\text { PART }\end{array}$ & PARTICIPANTS NAME AND ADDRESS & $\begin{array}{l}\text { HATCHING } \\
\text { EGG } \\
\text { CAPACITY }\end{array}$ & $\begin{array}{l}\text { PROOUCTS CLASSIFIED } \\
\text { U.S. PULLORUM-TYPHOID } \\
\text { CLEAN }\end{array}$ & $\begin{array}{c}\text { ADDITIONAL } \\
\text { CLASSIFICATIONS FOR } \\
\text { WHICH PRODUCT QUALIFIED }\end{array}$ \\
\hline
\end{tabular}

687 E RONNIE MARTIN, 905 NORTH LAKE

DRIVE,THUNDERING SPRINGS LAKE DUBLIN 31027

Phone: (478)275-4556

744 E TOMMY MCCREA, 795 TOMBERLIN ROAD, WAYCROSS 31503

807 E BRUCE MCCULLERS, 3555 MCCULLERS ROAD, LOGANVILLE 30052

829 E JEFF MCKENZIE, 3881 CATO ROAD, GAINESVILLE 30507

815 E PHIL OZBURN, 1250 BROUGHTON LANE, NEWBORN 30056

812 E POLO \& ISABEL PADILLA, 1150 VICKERS ROAD, NORMAN PARK 31771

641 E CHARLES PENNINGTON, 6090 HIGHWAY 219, FORTSON 31808

381 E PINE CREST POULTRY FARM, 1746 TOBACCO ROAD, AUGUSTA 30906

791 E R \& W QUAIL FARM, PERRITT RABUN, 297-A MIDDLEGROUND ROAD, WAYNESBORO 30830

834 E MALINDA RAESE, 332 ULY WHITE ROAD, HARTWELL 30643

830 E ROCKING R BANTAMS, 260 MYRTLE STREET, LAVONIA

399 E ROOSTER VILLAGE, P.O. BOX 104, LITHONIA 30058 Phone: (770)482-8174

634 E S\&C FARMS, 204 PRITCHETT ROAD, LULA 30554

560

$E$

S\&W FOWL, 392 PONDEROSA DRIVE, THOMASTON 30286

710 E MARIE SHOPE, RT. 4, BOX 1425, NASHVILLE 31639

823 E SILVER HILL POULTRY, 3159 FOSTER MANNING ROAD, SUMMERVILLE 30747

635 E RONALD D. SIMPSON, 4210 OLD LEXINGTON ROAD ATHENS 30605

787 E SOUTHERN PRIDE GAME FARM, 2904 WAYMANVILLE ROAD, THOMASTON 30286

824 E SOUTHLAND MALLARDS. 216 GANDER LANE, DAWSON 39842

721 E MATTHEW STEPHENSON, 3681 OLD MILL ROAD, RUTHLEDGE 30663

822 E SWEET BASIL FARM, 2109 SHEFFIELD ROAD, CONYERS 30012

819 E SWEET WILLIAM'S GAME FARM, 994 HICKORY FLAT ROAD, GILLSVILLE 30543

757 E SWEETWATER FARM, 7675 BARNES ROAD, PALMETTO 30268

623 E SWEETWATER GAME FARM, 1135 CENTRAL ROAD, SW., THOMSON 30824

788 E RONNIE TALLENT BANTAMS, 76 ENON GROVE ROAD, FRANKLIN 30217
$E \& C$

E\&C

DEC W114, W30, W50, W568, W596_

E\&C W119, W149, W151, W28, W48, W545, W556, W578, W88, W91

E\&C W142, W151,W26, W28, W29, W50, W51, W556, W569, W596. W613, W88, W91

E\&C N28

E\&C N

E\&C $W$

C 243

E\&C $\quad X_{4}$

E\&C W30

E\&C

E\&C W134,W146, W30

E\&C

E\&C W16, W207, W353, W365

E\&C $W$

E\&C W

E\&C

$E \& C$

E\&C N

E\&C R117, R237, W126, W127. W159, W16, W25, W26, W54

E\&C N

$E \& C$

$E \& C$

E\&C W 
Table 1. HATCHERIES, DEALERS, AND INDEPENDENT FLOCKS PARTICIPATING IN

THE NATIONAL POULTRY IMPROVEMENT PLAN

WATERFOWL, EXHIBITION POULTRY AND GAME BIRDS

\begin{tabular}{|c|c|c|c|c|}
\hline $\begin{array}{l}\text { APP. } \\
\text { NO. }\end{array}$ & $\begin{array}{l}\text { SUB- } \\
\text { PART }\end{array}$ & PARTICIPANTS NAME AND ADDRESS & $\begin{array}{l}\text { HATCHING } \\
\text { EGG } \\
\text { CAPACITY }\end{array}$ & $\begin{array}{c}\text { PRODUCTS CLASSIFIED } \\
\text { U.S. PULLORUM-TYPHOID } \\
\text { CLEAN }\end{array}$ \\
\hline & & 57 & - GEORGIA & \\
\hline 832 & $\mathrm{E}$ & $\begin{array}{l}\text { CORY THOMAS, } 855 \text { ENTERPRISE ROAD, COLQUITT } \\
39837\end{array}$ & $\mathrm{E} \& \mathrm{C}$ & W207, W588________ \\
\hline 835 & $E$ & $\begin{array}{l}\text { THREE ACRE FARM, } 3036 \text { ASH BRANCH ROAD, } \\
\text { PEMBROKE } 31321\end{array}$ & $E \& C$ & N. \\
\hline 679 & $E$ & $\begin{array}{l}\text { DAVID VARNADORE, } 158 \text { UNITY CHURCH ROAD, ALMA } \\
31510\end{array}$ & $E \& C$ & 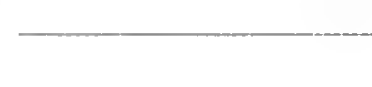 \\
\hline 803 & $\mathrm{E}$ & $\begin{array}{l}\text { WHITE GAME COCK FARM, } 543 \text { ASBURY ROAD, } \\
\text { TEMPLE } 30179\end{array}$ & $\mathrm{E \& C}$ & $N_{-}$ \\
\hline 652 & $E$ & JEFF WILLIAMS, 245 TODD WEAVER ROAD, ALTO 30510 & $E \& C$ & z \\
\hline 813 & $\mathrm{E}$ & LARRY WORSLEY, P.O. BOX 733, EDISON 31713 & $\mathrm{E \& C}$ & W48, W568, W596, W613 \\
\hline 820 & $\mathrm{E}$ & DAN YELTON, P.O. BOX 391, APPLING 30802 & $\mathrm{c}$ & \\
\hline 799 & $E$ & JAMES E. YOUNG, JR., RT. 1, BOX 95-X, METTER 30439 & $\mathrm{E} \& \mathrm{C}$ & \\
\hline
\end{tabular}

$$
82 \text { - IDAHO }
$$

DR. KENDAL G. EYRE

BUREAU CHIEF

BUREAU OF ANIMAL HEALTH LABORATORIES

2230 OLD PENITENTIARY ROAD

BOISE, ID 83712

Phone: (208)332-8570

Fax: $\quad(208) 334-4619$

Hatcheries

49 E B \& C CHUCKERS, BOB WASHBURN, 1849 SOUTH 400

1,000

$\mathrm{Z1}$

MS

WEST, OAKELY 83346

Phone: (208)862-3307

58 E BOHLING GAME BIRDS, ROBERT BOHLING, $4179 \mathrm{TH}$

1,000

MS AVENUE NORTH, BUHL 83316

Phone: (208)543-4556

38 E CURTIS HACKIE COMPANY, DAN CURTIS, 20080 SILVER STREET, RATHDRUM 83858

2,000

C6, N112, N52

Phone: (208)687-5201

57 E DJINVESTMENT COMPANY, DAN JOHNSON, 852 EAST 600 NORTH, FIRTH 83236

Phone: (208)346-6114

20 E DORRIS GAME BIRD FARM, DAVE DORRIS, HC 79, BOX 761, MELBA 83641 Phone: (208)896-4966

6 E DUNLAP HATCHERY, P.O. BOX 507, CALDWELL 83605

24 E FEATHERVILLE GAME BIRD FARM, R. DEBOARD, 25725 HARVEY ROAD, CALDWELL 83605

Phone: (208)459-1408

6,000

Z1, Z27

68,000

158,000 A, B, C, HD, R196, R20, R210A_

$5,000 \quad Z 1, Z 118, Z 132, Z 27, Z 43, Z 96 \ldots$

$60,000 \quad Z 1, Z 27, Z 48$
ADDITIONAL

CLASSIFICATIONS FOR WHICH PRODUCT QUALIFIED 
Table 1. HATCHERIES, DEALERS, AND INDEPENDENT FLOCKS PARTICIPATING IN

THE NATIONAL POULTRY IMPROVEMENT PLAN

WATERFOWL, EXHIBITION POULTRY AND GAME BIRDS

APP. SUB- PARTICIPANTS NAME AND ADDRESS

NO. PART

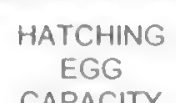

EGG

CAPACITY
PRODUCTS CLASSIFIED

U.S. PULLORUM-TYPHOID CLEAN
ADDITIONAL

CLASSIFICATIONS FOR WHICH PRODUCT QUALIFIED

\section{2 - IDAHO}

46 E SONNY HAIRSTON, LITTLE CANYON SHOOTING PRESERVE \& SPORTING CLAYS.2170 LITTLE CANYON ROAD, P.O. BOX 28 PECK 83545

Phone: (208)486-6235

42 E HAWKS LABRADOR \& GAME BIRD FARM, TOM \& STACEY HAWKS,6410 DALLAS ROAD NEW PLYMOUTH 83655

Phone: (208)278-4826

53 E HENRYS FORK GAME BIRDS, CHARLES HANSON, 844 NORTH 2900 EAST, CHESTER 83421

Phone: (208)624-4811

22 E IDAHO GAME BIRDS, JIM \& CAROL DAVENPORT, RR 1 , BOX 1212, HOMEDALE 83628

Phone: (208)337-4826

60 E KASOTA GAME BIRDS, BROOKS LITTLE, P.O. BOX 562 , BURLEY 83318

Phone: (208) 431-6431

DEE MESSICK, P.O. BOX 104, GRANDVIEW 83624-0401 Phone: (208)834-2461

59 E SHANE MEYERS, 5521 SOUTH FLORIDA, CALDWELL 83607

47 E MINER GAME BIRDS, DARIN MINER, 7400 HIDDEN VALLEY DRIVE, BOISE 83709

Phone: (208)362-2002

25 E MORTENSEN'S PURPLE SAGE GAME BIRD FARM, HAROLD \& KEN MORTENSEN, 13236 GREENWELL LANE CALDWELL 83605

Phone: (208)459-4909

26 E NORTHWEST GAME BIRDS, JERRY COLLINGHAM, 17448 OASIS ROAD, CALDWELL 83605

Phone: (208)454-8282

27 E OAKLEY GAME FARM, DOUGLAS NEWMAN, RR 1, BOX 11-A, OAKLEY 83346

Phone: (208)862-3371

54 E PAYETTE VALLEY GAME BIRDS, R. AUSTIN, 5047 SE 1 1/2 AVENUE, NEW PLYMOUTH 83655

Phone: (208)278-5236

44 E PERDIX, BRETT \& VELVET KILLIAN, 7954 EAST 145 NORTH, IDAHO FALLS 83401

Phone: (208)529-4533

36 E PINE ACRES GAME BIRDS, INC., L.HUBER, ROUTE 1, BOX 431, RUPERT 83350 Phone: (208)436-4241

39 E PORTER ENTERPRISES, CARMA PORTER, 150 NORTH 3RD EAST. FRANKLIN 83237

Phone: $(208) 646-2629$

52 E RYE PATCH ROOSTER RANCH, ROBERT NEIDER, P.O. BOX 12. MURPHY 83650

19 E SKYLINE GAMEBIRD FARM,GEORGE/ DOLLY HYER, RR 2. BOX 14-A, HOMEDALE 83628 Phone: $(208) 337-4443$

56 E REED TAYLOR, 4080 LAPWAI ROAD, LEWISTON 83501

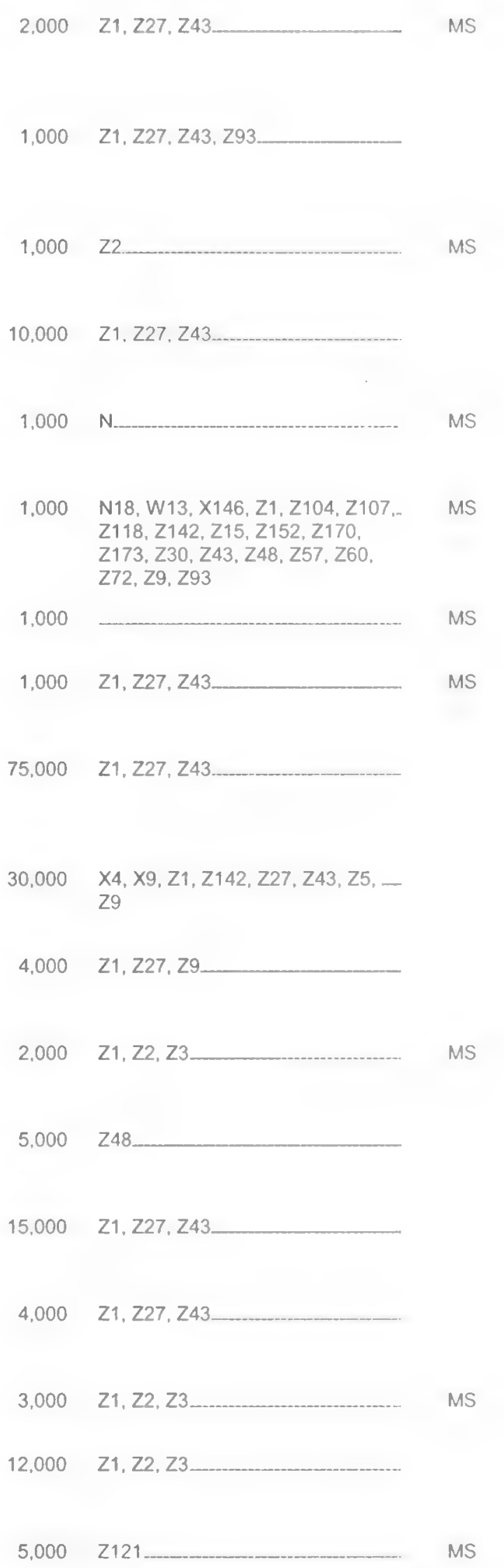


Table 1. HATCHERIES, DEALERS, AND INDEPENDENT FLOCKS PARTICIPATING IN

THE NATIONAL POULTRY IMPROVEMENT PLAN

WATERFOWL, EXHIBITION POULTRY AND GAME BIRDS

APP. SUB- PARTICIPANTS NAME AND ADDRESS

NO. PART

HATCHING
EGG

CAPACITY
PRODUCTS CLASSIFIED

U.S. PULLORUM-TYPHOID

CLEAN
ADDITIONAL

CLASSIFICATIONS FOR

WHICH PRODUCT QUALIFIED

$82-$ IDAHO

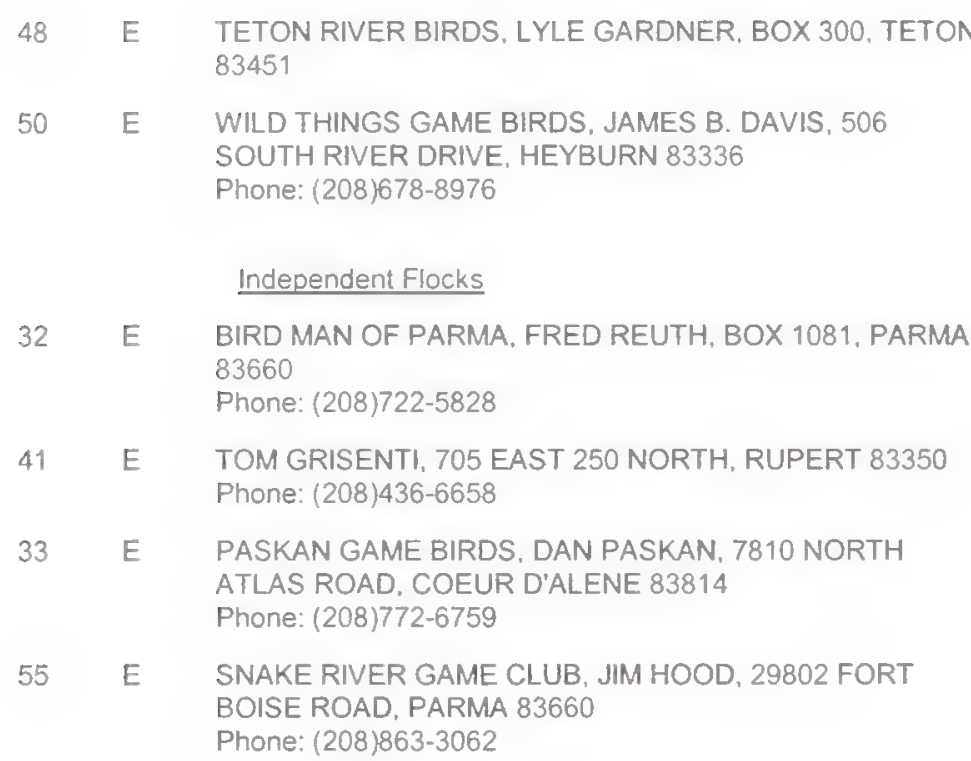

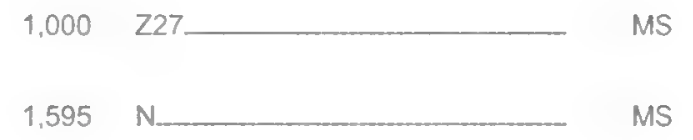

Z1, Z2, Z27, Z43, Z9

Z1, Z27, Z43.

Z1, Z2, Z23, Z28, Z4, Z5, Z94

N9

JAMES E. KUNKLE

ILLINOIS DEPARTMENT OF AGRICULTURE

DIVISION OF ANIMAL INDUSTRIES

P.O. BOX 19281

STATE FAIRGROUNDS

SPRINGFIELD, IL 62794

Phone: (217)782-4944

Fax: (217)524-7702

33 - ILLINOIS

Hatcheries
841 E ALBERS FARMS, 569 NORTH 46TH ROAD, MENDOTA 61342
1011 E TIM ALTHOFF \& MIKE FLETCHER, 17615 EAST 2000TH AVENUE, EFFINGHAM 62401

\begin{tabular}{|c|c|c|}
\hline 326 & $E$ & $\begin{array}{l}\text { AUTUMN FLIGHT GAME FIELDS, GILBERT HOLZ, P.O. } \\
\text { BOX 135, TOLUCA } 61369\end{array}$ \\
\hline & $E$ & $\begin{array}{l}\text { B.F.S. AVIARY, MIKE \& LISA FISHER, } 271816 \text { TH } \\
\text { STREET, MOLINE } 61265\end{array}$ \\
\hline & $E$ & $\begin{array}{l}\text { SAM BAKER, } 1935 \text { VALLEY VIEW DRIVE, BELVIDERE } \\
61008\end{array}$ \\
\hline & $E$ & $\begin{array}{l}\text { BAMBERG POULTRY, ALLISON BAMBERG, } 2506 \text { NORTH } \\
\text { ROCK CITY ROAD, RIDOTT } 61067\end{array}$ \\
\hline & $E$ & $\begin{array}{l}\text { AMANDA BARRIX, } 119 \text { WEST LAMOTTE, PALESTINE } \\
62451\end{array}$ \\
\hline
\end{tabular}

, R22, R247, R78, W11, W113, W13 W142, W2, W25, W357, W475, W48, W570, W6, W88, Z107. Z111, Z139, Z20, Z204, Z205, $\mathrm{Z} 21, \mathrm{Z212}, \mathrm{Z287}, \mathrm{Z45}, \mathrm{Z} 62$

2,000

1,000

60

50

50

$\mathrm{Z27}$

R20, W. Z11, Z165, Z2, Z93

10

R85, W

R183, W134, W3, W69, Z61.

R1, R10, R109, R11, R115, R125. 
Table 1. HATCHERIES. DEALERS. AND INDEPENDENT FLOCKS PARTICIPATING IN

THE NATIONAL POULTRY IMPROVEMENT PLAN

WATERFOWL, EXHIBITION POULTRY AND GAME BIRDS

\begin{tabular}{llccc}
\hline & & HATCHING & PRODUCTS CLASSIFIED \\
APP. SUB- & PARTICIPANTS NAME AND ADDRESS & EGG & U.S. PULLORUM-TYPHOID \\
NO. PART & CAPACITY & CLEAN & CLASSIFICATIONS FOR \\
& & & WHICH PRODUCT QUALIFIED
\end{tabular}

33 - ILLINOIS

\begin{abstract}
941 E BATES GAME BIRD FARM, 1221 BATES ROAD, AVA 62907

877 E KATHY \& JODI BAUMGARTNER, 3494 NORTH 5 CORNERS ROAD, LENA 61048

BEARS, BIRDS \& HATCHERY, C. DAVIDSON, 49 W 049 JERICHO ROAD, BIG ROCK 60511

Phone: (708) 556-3350

551 E BIRDS, LTD., 10235 RIDGE ROAD, ROCK FALLS 61071

717 E ROBERT BOWIE, 1 NORTH 740, RT. 50, WEST CHICAGO 60185

MARION BURETTA. 25535 YOUTH CENTER ROAD, DUBOIS $62831-1426$

431 E GEORGE CAIRNS, 708 HUGHES ROAD, WOODSTOCK 60098

PAT CAIRY'S FUNNY FARM, 11412 WADSWORTH ROAD, BEACH PARK 60099

E MATT CARTON, 15454 EAST 1900 STREET, ATKINSON 61235

E MIKE CHAMNESS, 6680 CARNATION ROAD, PINCKNEYILLE 62274

COUNTRY GARDEN, JACKIE BINKLEY, 10704 SEAMAN ROAD, HERBON 60034

CROME FAMILY POULTRY, JULIE CROME, 3006 EAST WOODBINE ROAD, PAW PAW 61353

BLAKE DALE, 1745 CHESHIRE ROAD, DONGOLA 62926

JOE \& SHIRLEY DIETZ, 11200 LOWER MARINE ROAD, HIGHLAND 62249
\end{abstract}

DOUBLE 8 POULTRY, RON BOESTER, 20652 LOCUST ROAD. HOYLETON 62803

E DEREL D. DOWLAND, 2883 ROCKBRIDGE ROAD, CHESTERFIELD 62630

E DONALD DUTY, 1220 NEW SARATOGA ROAD, ANNA 62901

E BETH ESHELMAN, 1015 THOME ROAD, ALBANY 61230

MYRON EWALT, RR 1, BOX 73, MALDEN 61337

E DWAINE FARRIS, 4871 EDGEWATER DRIVE, GROVELAND 61535
R13, R139, R183, R188, R195. R199, R23, R237, R247, R51, R52, R56, R6, R64, R67, R71, R77, R78, R83, R88, R91, R97, R99, W123, W166, W305, W54. W77

$12,000 \quad Z 1,243$

30 R10, R146, R222

$40,000 \quad 22$

$1,000 \quad R, 211$

84 R146, W16

$1,200 \quad Z 43$

500 W18, W19, W20, W28, W30, W301, W74, W86

100 R20, R62, R89

90 R103, R104, R146

$500 \mathrm{H} 10, \mathrm{~N} 18, \mathrm{R} 20, \mathrm{R} 256, \mathrm{R} 81$. W107, W165, X21, X23, X38, X4, Y $10, Y 5, Z 4$

$42 \mathrm{R} 100, \mathrm{R} 102, \mathrm{R} 19, \mathrm{R} 51, \mathrm{R} 82, \mathrm{R} 96$, W102, W129, W132, W136, W208, W257, W86

90 R196, R260

50 R146, R168, R196, R227, R7, W198, W211, Z4, Z61, 29

40 R146, R175, R178, R19, R21, R33, R34, R53, R71, R9, W136, W138, W18, W19, W198, W256, W262, W45, W585, W590, W74, W75, W83

250 R247, R7, R81, W120, W121, W140

130 W132, W248, W415, W96, Z118, $\mathrm{Z} 4, \mathrm{Z} 53$

1,200 R146, R20, W96, Z61. R11, R146, R17, R196, R222, R6, R77, R85

48 R22, R7.

$216 \quad$ W30

$300 \mathrm{R} 104, \mathrm{R} 2, \mathrm{R} 248, \mathrm{R} 71, \mathrm{R} 88, \mathrm{R} 94$, R95, W28 


\begin{tabular}{|c|c|c|c|c|}
\hline $\begin{array}{l}\text { APP. } \\
\text { NO. }\end{array}$ & $\begin{array}{l}\text { SUB- } \\
\text { PART }\end{array}$ & PARTICIPANTS NAME AND ADDRESS & $\begin{array}{l}\text { HATCHING } \\
\text { EGG } \\
\text { CAPACITY }\end{array}$ & $\begin{array}{l}\text { PRODUCTS CLASSIFIED } \\
\text { U.S. PULLORUM-TYPHOID } \\
\text { CLEAN }\end{array}$ \\
\hline & & 33 & - ILLINOIS & \\
\hline 646 & $E$ & LEO FINN, RT. 1, CHANDLERVILLE 62627 & 150 & R16, R21, R243, R97, W25 ___ \\
\hline 823 & $E$ & $\begin{array}{l}\text { FLO-CHER FARMS, F. STEES, } 112 \text { WEST MASON } \\
\text { STREET, LENA } 61048\end{array}$ & 80 & $\begin{array}{l}\text { R103, R146, R16, R225, R237, } \\
\text { R247, R3, R86, W }\end{array}$ \\
\hline 470 & $E$ & $\begin{array}{l}\text { LESLIE FRAMPTON, } 9710 \text { TELEGRAPH ROAD, } \\
\text { WINNEBAGO } 61088\end{array}$ & 48 & $\mathrm{R}, \mathrm{W}, \mathrm{Z11} \ldots$ \\
\hline 955 & $E$ & $\begin{array}{l}\text { JACKIE FRANCESCONI, } 9014 \text { SEEMAAN ROAD, UNION } \\
60180\end{array}$ & 500 & $\begin{array}{l}\text { R12, R126, R140, R15, R160, } \\
\text { R44, R46, R54, R82, R98, W131, } \\
\text { W179, W2, W208, W290, W75 }\end{array}$ \\
\hline 389 & $\mathrm{E}$ & $\begin{array}{l}\text { MICHAEL FRENCH, } 1606 \text { NORTH MARYLAND, POLO } \\
61064\end{array}$ & 500 & W \\
\hline 957 & $E$ & $\begin{array}{l}\text { LAUREN MARY FRIDAY, } 5678 \text { WHITING ROAD, POPLAR } \\
\text { GROVE } 61065\end{array}$ & 20 & R \\
\hline 981 & $E$ & $\begin{array}{l}\text { ROBERT GALLAGHER, } 15040 \text { MARENGO ROAD, } \\
\text { MARENGO } 60180\end{array}$ & 50 & $\begin{array}{l}\text { R, W, W121, W198, W2, W249, .. } \\
\text { W25, W28, W5, W600 }\end{array}$ \\
\hline 946 & $E$ & $\begin{array}{l}\text { GARFIELD FARM MUSEUM, 3N016 GARFIELD ROAD, } \\
\text { BOX 403, LAFOX } 60147\end{array}$ & 25 & $\mathrm{R}, \mathrm{R} 78,2231 \ldots$ \\
\hline 712 & $E$ & $\begin{array}{l}\text { BILL GARRISON, } 1934 \text { WOODLAWN ROAD, STEWARD } \\
60553\end{array}$ & 200 & $\mathrm{R} 146, \mathrm{R} 15, \mathrm{R} 247, \mathrm{~W}$ \\
\hline 647 & $E$ & DAVE GEMMER, 10650 MIDDLE ROAD, MORRIS 60450 & 100 & $R, W$ \\
\hline 667 & $E$ & AMY GENSLER, RR 1, BOX 83, TOLUCA 61369 & 48 & $\begin{array}{l}\text { R173, R81, W25, W26, W36, } \\
\text { Z202 }\end{array}$ \\
\hline 1020 & $E$ & $\begin{array}{l}\text { DAVID. DAVID, JR \& TRISTAN GILL. } 1581 \text { OAKDALE } \\
\text { BLACKTOP ROAD, NASHVILLE } 62263\end{array}$ & 1,600 & $\begin{array}{l}\mathrm{R} 196, \mathrm{R} 247, \mathrm{R} 248, \mathrm{R} 7, \mathrm{R} 81, \\
\text { W12, Z4, Z62 }\end{array}$ \\
\hline 582 & $E$ & CARL W. GRAY. P.O. BOX 111, VIENNA 62995 & 100 & $\begin{array}{l}\text { H9, R168, R181, W130, W25, } \\
\text { W26, Z118, Z177, Z53 }\end{array}$ \\
\hline 590 & $E$ & $\begin{array}{l}\text { GREEN RIVER GAME FARM, } 7792 \text { NORTH } 2300 \\
\text { AVENUE, GENESEO } 61254\end{array}$ & 1.300 & 22 \\
\hline 927 & $E$ & $\begin{array}{l}\text { ROY GROENNERT. } 6767 \text { COUNTY HIGHWAY } 6 \text {, } \\
\text { OKAWVILLE } 6227 \uparrow\end{array}$ & 200 & $\begin{array}{l}\text { W198, W20, W26, Z15, Z19, } \\
\text { Z202, Z205, Z231, Z4 }\end{array}$ \\
\hline 288 & $E$ & PAUL GUTHRIE, SR., RR 1, BOX 51, GODFREY 62035 & 50 & N18______ \\
\hline 1003 & $E$ & SELAH HADI, 812 EAST 400 NORTH, LODA 60948 & 120 & $R, W_{-}$ \\
\hline 1034 & $\mathrm{E}$ & STACEY HARE, 8517 SOUTH RT. 23, MARENGO 60152 & 36 & $\begin{array}{l}\text { H10, R13, R196, R222, R38, - - } \\
\text { W74, Z202, Z207 }\end{array}$ \\
\hline 944 & $E$ & $\begin{array}{l}\text { LAWRENCE HARPER, } 2819 \text { IMBS STATION ROAD, } \\
\text { MILLSTADT } 62260\end{array}$ & 300 & $\begin{array}{l}\text { R146, R15, R222, R247, R248, } \\
\text { R30, W18, W198, W211, W25, } \\
\text { W26, W28, W368, W48, W52, } \\
\text { Z118, Z148, Z202, Z22, Z24, Z26, } \\
\text { Z49, Z51, Z53, Z57, Z68 }\end{array}$ \\
\hline 1045 & $E$ & $\begin{array}{l}\text { DAN \& ANNA HARTLEY, } 4485 \text { CHINQUIPIN ROAD, } \\
\text { SPRINGFIELD } 62707\end{array}$ & 300 & $\begin{array}{l}\text { H5, H9, N55, N9, R12, R15, R19, } \\
\text { R51, R7, R93, R96, W12, W128, } \\
\text { W130, W165, W207, W213, } \\
\text { W306, W94 }\end{array}$ \\
\hline 919 & $E$ & ROBERT HEEB, 15370 SHARP ROAD, ROCKTON 61072 & 80 & Z14 \\
\hline 325 & $E$ & $\begin{array}{l}\text { JAMES C. HELFRICH WILDLIFE PROP. CENTER, } \\
\text { ILLINOIS DEPTARTMENT OF CONSERVATION, } 101913 \\
\text { 10TH STREET LINCOLN } 61048\end{array}$ & 140,000 & $\mathrm{Z} 2$ \\
\hline 417 & $E$ & HILLTOP HAVEN FARM, 21756 EAST 2450 NORTH & 42 & $\mathrm{R} 53, \mathrm{~W} 387, \mathrm{Z205}$ \\
\hline
\end{tabular}


Table 1. HATCHERIES. DEALERS. AND INDEPENDENT FLOCKS PARTICIPATING IN

THE NATIONAL POULTRY IMPROVEMENT PLAN

WATERFOWL, EXHIBITION POULTRY AND GAME BIRDS

APP. SUB- PARTICIPANTS NAME AND ADDRESS NO. PART

HATCHING
EGG

CAPACITY
PROOUCTS CLASSIFIED

U.S. PULLORUM-TYPHOID

CLEAN
ADDITIONAL

CLASSIFICATIONS FOR WHICH PRODUCT QUALIFIED

33 - ILLINOIS

\begin{tabular}{|c|c|c|c|c|}
\hline 1022 & $E$ & $\begin{array}{l}\text { MARGARET HILTON, } 1071 \text { NORTH } 2401 \text { ROAD, OTTAWA } \\
61350\end{array}$ & 50 & R12, R138, R196, R20, R7 \\
\hline 810 & E & CINOY HOLLOWAY, 318 HELEN, CHILLICOTHE 61523 & 50 & R169, R20, R227, W \\
\hline 678 & E & $\begin{array}{l}\text { HOOKER'S FOWL PLAY FARM, } 3667 \text { STATE ROAD, } \\
\text { POPLAR GROVE } 61065\end{array}$ & 50 & W. \\
\hline 612 & $E$ & $\begin{array}{l}\text { HOPEWELL VIEWS GAME FARM, RR 2, ROCKPOR'- } \\
62370\end{array}$ & 30,000 & Z43. \\
\hline 558 & $E$ & LOU HORTON, IN735 INGALTON, WEST CHICAGO 60185 & 250 & $W, X, Y \ldots$ \\
\hline 228 & E & JOSEPH R. HOY, 14877 EAGLE ROAD, NASHVILLE 62263 & 600 & H9, R262, W74 \\
\hline 687 & $E$ & RICHARD HOYLE, 551 PENROSE RD, DIXON 61021 & 50 & W. \\
\hline 238 & $E$ & $\begin{array}{l}\text { HUDSON POULTRY FARM, RR 2, BOX 12, SAYBROOK } \\
61770\end{array}$ & 2,900 & $\mathrm{R}$ \\
\hline 419 & E & $\begin{array}{l}\text { RAY \& HOLLY HUTCHISON, } 30540 \text { ELKHORN ROAD, } \\
\text { MILLEDGEVILLE } 61051\end{array}$ & 500 & R18, R183, R254, W, Z11, Z2, Z3. \\
\hline 461 & $E$ & $\begin{array}{l}\text { INVERGO QUAIL FARM, } 7151 \text { WEST BLACKJACK ROAD, } \\
\text { HANOVER } 61041\end{array}$ & 3,600 & 293. \\
\hline 472 & $E$ & $\begin{array}{l}\text { PEDRO IRIZARRY, } 11361 \text { WEST WAOSWORTH ROAD, } \\
\text { ZION } 60099\end{array}$ & 100 & N18 \\
\hline 876 & E & $\begin{array}{l}\text { JACKSON POULTRY, } 2082 \text { BASELINE ROAD, SUBLETTE } \\
61369\end{array}$ & 100 & R. W \\
\hline 767 & $E$ & $\begin{array}{l}\text { HARRY JACOBSON, } 7155 \text { SEBERSON ROAD, } \\
\text { ROCKFORD } 61102\end{array}$ & 1,400 & $\mathrm{R}$ \\
\hline 613 & $E$ & JOHNSON FARM, RR 1, P.O. BOX 48, MAZON 60444 & 150 & $\begin{array}{l}\text { R, R146, R7, W4, W54, Z201, } \\
\text { Z202, Z4 }\end{array}$ \\
\hline 462 & $E$ & JONES BACKYARD BANTAMS, BOX 21, MEDORA 62063 & 96 & W30_ \\
\hline 243 & $\mathrm{E}$ & $\begin{array}{l}\text { JONES BANTAMS, G. JONES, RR 4, BOX } 209 \text {, } \\
\text { CARLINVILLE } 62626\end{array}$ & 50 & W19, W20, W48, W57 \\
\hline 319 & $E$ & $\begin{array}{l}\text { BRAD JONES, } 14396 \text { CHRISTMAS TREE ROAD, } \\
\text { JOHNSTON CITY } 62951\end{array}$ & 100 & $\begin{array}{l}\text { R248, W104, W128, W134, W17, } \\
\text { W18, W20, W207, W23, W38, } \\
\text { W4, W478, W54, W578 }\end{array}$ \\
\hline 529 & E & $\begin{array}{l}\text { LES \& CAROL KANTLEHNER, } 9514 \text { WINN ROAD, } \\
\text { RICHMOND } 60071\end{array}$ & 400 & $R, W$ \\
\hline 312 & $\mathrm{E}$ & $\begin{array}{l}\text { KEENE'S FEATHER FARM, } 240 \text { BODY BARN ROAD. } \\
\text { ANNA } 62906\end{array}$ & 30 & $\mathrm{R} 248, \mathrm{~W} 24, \mathrm{~W} 28, \mathrm{~W} 3, \mathrm{~W} 4 \ldots$ \\
\hline 1005 & $E$ & $\begin{array}{l}\text { KINSEY'S GAME BIRD FARM, ANTHONY KINSEY, } 15737 \\
\text { COCHRAN ROAD, MARION } 62959\end{array}$ & 300 & $\begin{array}{l}\text { R229, R245, R247, R57, Z108, } \\
\text { Z11, Z110, Z137, Z18, Z182, Z22 }\end{array}$ \\
\hline 157 & $\mathrm{E}$ & $\begin{array}{l}\text { KNIEPKAMP BANTAM YARD, } 2020 \text { CENTERVILLE } \\
\text { AVENUE, BELLEVILLE } 62221\end{array}$ & 300 & $\begin{array}{l}\text { R237, W17, W18, W26, W481, - } \\
\text { W74 }\end{array}$ \\
\hline 949 & $E$ & $\begin{array}{l}\text { KOCH POULTRY, LINDSE KOCH, } 4453 \text { NORTH DAKOTA } \\
\text { ROAD, DAKOTA } 61018\end{array}$ & 70 & $\begin{array}{l}\text { R179, R248, R71, R94, W, W129. } \\
\text { W131, W132, W370, W4 }\end{array}$ \\
\hline 1007 & E & DANIEL KOCH, 4975 MAEYS ROAD, WATERLOO 62236 & 250 & $\begin{array}{l}\text { R15, R16, R196, R33, R53, } \\
\text { W100, W107, W28 }\end{array}$ \\
\hline 967 & E & $\begin{array}{l}\text { JAMES KONECNY, } 10219 \text { HAEGERS BEND ROAD, } \\
\text { BARRINGTON HILIS } 60102\end{array}$ & 300 & $R, W$ \\
\hline 293 & E & GORDON KRUGER, RR 15, BOX 5, NORMAL 61761 & 1,250 & N18, R, W \\
\hline 499 & E & $\begin{array}{l}\text { GENE W. LEFFELMAN, } 1785 \text { LEE CENTER ROAD, } \\
\text { AMBOY } 61310\end{array}$ & 1,400 & $\begin{array}{l}\text { W. Z107, Z108, Z118, Z126, } \\
\text { Z127, Z131, Z149, Z175, Z177, }\end{array}$ \\
\hline
\end{tabular}


Table 1. HATCHERIES, DEALERS, AND INDEPENDENT FLOCKS PARTICIPATING IN

THE NATIONAL POULTRY IMPROVEMENT PLAN

WATERFOWL, EXHIBITION POULTRY AND GAME BIRDS

\begin{tabular}{|c|c|c|c|c|c|}
\hline $\begin{array}{l}\text { APP. } \\
\text { NO. }\end{array}$ & $\begin{array}{l}\text { SUB- } \\
\text { PART }\end{array}$ & PARTICIPANTS NAME AND ADDRESS & $\begin{array}{l}\text { HATCHING } \\
\text { EGG } \\
\text { CAPACITY }\end{array}$ & $\begin{array}{c}\text { PRODUCTS CLASSIFIED } \\
\text { U.S. PULLORUM-TYPHOID } \\
\text { CLEAN }\end{array}$ & $\begin{array}{c}\text { ADDITIONAL } \\
\text { CLASSIFICATIONS FOR } \\
\text { WHICH PRODUCT QUALIFIED }\end{array}$ \\
\hline
\end{tabular}

\begin{tabular}{|c|c|c|c|c|}
\hline & & & & $\begin{array}{l}\text { Z18, Z260, Z268, Z34, Z48, Z49, } \\
\text { Z50, Z52, Z57, Z68, Z69, Z71, } \\
\text { Z77 }\end{array}$ \\
\hline 890 & E & $\begin{array}{l}\text { LINDON OAKS FARM, L. SCHARNHORST, } 4715 \text { WEST } \\
\text { NORTH, PEOTONE } 60468\end{array}$ & 30 & $\begin{array}{l}\text { R146, R148, R19, R247, R248, - } \\
\text { R256, R40, R52, R55, R7, R87, } \\
\text { R94, R96, W11, W4 }\end{array}$ \\
\hline 501 & E & $\begin{array}{l}\text { JERRY LOTT, } 1008 \text { GROVER STREET, BELVIDERE } \\
61008\end{array}$ & 50 & W2, Z11, Z204, Z26, Z27, Z28__ \\
\hline 321 & $E$ & $\begin{array}{l}\text { MAX MCGRAW WILDLIFE FOUNDATION. P.O. BOX } 9 . \\
\text { DUNDEE } 60118\end{array}$ & 81,500 & $\mathrm{Z2}$ \\
\hline 601 & E & SANDY MCWORTHY, 11675 LOUISA ROAD, LENA 61048 & 50 & $\begin{array}{l}\text { R146, R222, R237, R95, W, } \\
\text { Z202, Z288, Z4 }\end{array}$ \\
\hline 1042 & E & KEN MEAD, 55902500 NORTH AVENUE, TAMPICO 61283 & 50 & $\begin{array}{l}\text { R1, R118, R237, R247, W54, } \\
\text { W570, W610 }\end{array}$ \\
\hline 974 & $E$ & JOHN MESSAMORE, 1840 BALCOMB ROAD, ANNA 62906 & 50 & R18, W4, Z202, Z4 \\
\hline 873 & $\mathrm{E}$ & STEVE MICHELNI, 2950 EAST 103 RD, PERU 61354 & 40 & $W_{-}$ \\
\hline 789 & $\mathrm{E}$ & $\begin{array}{l}\text { MIDWEST PHEASANTRIES, INC., } 20420 \text { - } 104 \text { AVENUE, } \\
\text { CENTER WEST, ILLINOIS CITY } 61259\end{array}$ & 4,000 & $\mathrm{Z2}$ \\
\hline 420 & $E$ & $\begin{array}{l}\text { DARREL H. MILLER, } 632 \text { YOUNGS LANE, FREEPORT } \\
61032\end{array}$ & 400 & W132 _ _ \\
\hline 943 & $E$ & $\begin{array}{l}\text { LINDA MILLER, } 1462 \text { GUN CLUB ROAD, SMITHBORO } \\
62284\end{array}$ & 15 & W208, W257 \\
\hline 911 & $\mathrm{E}$ & $\begin{array}{l}\text { M \& M POULTRY, KEITH MILLER, } 894 \text { HOYLE ROAD, } \\
\text { HARMON } 61042\end{array}$ & 70 & R103, R15, R16, W17_ \\
\hline 1015 & $E$ & DAVID MOHR, 11525 COMLY RD., WINNEBAGO 61088 & 200 & W11.2205_.. \\
\hline 1041 & $\mathrm{E}$ & $\begin{array}{l}\text { MORNING STAR FARM, C \& J GILL, } 7801 \text { NORTH } \\
\text { ROCKTON AVENUE, ROCKFORD } 61103\end{array}$ & 50 & W12, W5, W54, W88 \\
\hline 991 & $E$ & $\begin{array}{l}\text { ANNETTE MRUGACZ, } 7308 \text { SOUTH GRANT HIGHWAY, } \\
\text { MARENGO } 60152\end{array}$ & 20 & R, W, Z11 \\
\hline 359 & E & $\begin{array}{l}\text { NOLLAU'S CUSTOM HATCHERY, } 7319 \text { WOODLAND } \\
\text { SCHOOL ROAD, MASCOUTAH } 62258\end{array}$ & 7,500 & R1, R222, R237, Z176, Z27, Z4_ \\
\hline 715 & E & $\begin{array}{l}\text { DAVID \& KAREN NUNEZ, RR 1, BOX } 7 \text {, LAFAYETTE } \\
61449\end{array}$ & 60 & $R, W$ \\
\hline 1036 & $E$ & $\begin{array}{l}\text { TAMMY OLSON, } 21015 \text { WEST NORTH AVENUE, } \\
\text { ANTIOCH } 60002\end{array}$ & 40 & $\begin{array}{l}\text { R114, R146, R20, R43, R52, R7, - } \\
\text { R85, W12, W13, W164, W33, } \\
\text { W570, W573, W86 }\end{array}$ \\
\hline 882 & E & $\begin{array}{l}\text { DOLORES PASSWATER, } 1245 \text { NORTH 2150, OTTAWA } \\
61350\end{array}$ & 500 & $A, Z 11,2204$ \\
\hline 838 & $\mathrm{E}$ & $\begin{array}{l}\text { JACOB PEARSON. RT. 1. BOX 227-A. SPRING VALLEY } \\
61362\end{array}$ & 40 & R230, R247, R8, W \\
\hline 1016 & $\mathrm{E}$ & $\begin{array}{l}\text { PERSIMMON RIDGE FARM. SCHNEIDER, } 6521 \text { OLD } \\
\text { ORCHARD LANE, WATERLOO } 62298\end{array}$ & 42 & R1, W145, W207, W211, W4 \\
\hline 563 & $\mathrm{E}$ & $\begin{array}{l}\text { A. J. SMITH \& TOM PIERCEALL, } 209 \text { WEST 4TH STREET, } \\
\text { NEW BERLIN } 62670\end{array}$ & 500 & $R, W$ \\
\hline 399 & $\mathrm{E}$ & $\begin{array}{l}\text { PIN OAK FARMS. HAROLD HAMMANN, } 14895 \text { WHEELER } \\
\text { ROAD, CARLINVILLE } 62626\end{array}$ & 6,000 & $\begin{array}{l}\text { W102, W290, W33, W74, W75, } \\
\text { X41, Z101, Z110, Z111, Z128, } \\
\text { Z18, Z19, Z204, Z205, Z22, Z32. } \\
\text { Z4, Z62, Z9 }\end{array}$ \\
\hline 814 & $E$ & DOROTHY PLUMMER, 1316 COUNTY ROAD 900 NORTH, & 50 & R12, R123, R176, R18, R20, W_- \\
\hline
\end{tabular}


Table 1. HATCHERIES, DEALERS, AND INDEPENDENT FLOCKS PARTICIPATING IN

THE NATIONAL POULTRY IMPROVEMENT PLAN

WATERFOWL, EXHIBITION POULTRY AND GAME BIRDS

\begin{tabular}{|c|c|c|c|c|c|}
\hline $\begin{array}{l}\text { APP. } \\
\text { NO. }\end{array}$ & $\begin{array}{l}\text { SUB- } \\
\text { PART }\end{array}$ & PARTICIPANTS NAME AND ADDRESS & $\begin{array}{l}\text { HATCHING } \\
\text { EGG } \\
\text { CAPACITY }\end{array}$ & $\begin{array}{l}\text { PRODUCTS CLASSIFIED } \\
\text { U.S. PULLORUM-TYPHOID } \\
\text { CLEAN }\end{array}$ & $\begin{array}{c}\text { ADDITIONAL } \\
\text { CLASSIFICATIONS FOR } \\
\text { WHICH PRODUCT QUALIFIED }\end{array}$ \\
\hline
\end{tabular}

1023 E SCOTT POHAR, 1087 NORTH 2401 ROAD, OTTAWA 61350

827 E BART PRICE, 19820 183RD AVENUE WEST, REYNOLDS 61279

1018 E DE LA PRYME FARM, NICHOLAS PRIMM, RR 1, BOX 19, WILLIAMSVILLE 62693

1046 E KEVIN \& LAURIE PROBST, P.O. FARM, 9018 EAST 1250TH AVENUE, EFFINGHAM 62401

742 E JOHN PUENTES, 17059 NORTH 2250 AVENUE, GENESEO 61254

938 E ARTHUR PULFORD, 215265 NORTH, HILLSDALE 61257

1010 E QUACKENBERRY FARM, DEBRA PENNINGTON, 2060 BOST ORCHARD ROAD, CREAL SPRINGS 62922

507 E TERRY RAHN, 12777 STONEBRIDGE ROAD, LANARK 61046

961 E ELIZABETH REED, 1400 HICKORY GROVE ROAD, LACON 61540

643 E MARGE RIDER, RR 1, BOX 84-A, 125 SOUTH JACKSON STREET, WINCHESTER 62694

805 E RIGHTWING GAME FARM, DALE DREYER, 11560 EAST STOCKTON ROAD, STOCKTON 61085

1037

$E$

KATHRYN ROSS, DINSMORE ACRES FARM, 27098 NORTH OWENS ROAD, MUNDELEIN 60060 R13, R145, R15, R192, R195, R20, R257, R33, R45, R6, R60, R70, R77, R78, R8, R82, R85. R88, R96, W100, W11, W 117 , W129, W13, W17, W179, W4. W43, W62, X11, X14, X15, X2 $X 25, X 3, Y 1, Y 14, Y 2, Y 21, Y 3$, $Y 4, Y 5, Y 7, Z 201, Z 202, Z 204$. Z205, Z206, Z207, Z209, Z211. Z230, Z4
R1, R14, R200, R202, R247, R28, R33, R4, W

R237, W208, Z107, Z15, Z188, Z4, Z51, Z57, Z93

R146, R196, R247, R248, R7, W504

W573, W593, Z26, Z4

w

A

R42, W13, W2, W60, Z61.

R, W

$\mathrm{Z27}$

R247, W5

W102, W119, W168, W17. W198, W2, W232, W263, W270, 
Table 1. HATCHERIES, DEALERS, AND INDEPENDENT FLOCKS PARTICIPATING IN

THE NATIONAL POULTRY IMPROVEMENT PLAN

WATERFOWL, EXHIBITION POULTRY AND GAME BIRDS

\begin{tabular}{|c|c|c|c|c|c|}
\hline $\begin{array}{l}\text { APP. } \\
\text { NO. }\end{array}$ & $\begin{array}{l}\text { SUB- } \\
\text { PART }\end{array}$ & PARTICIPANTS NAME AND ADDRESS & $\begin{array}{l}\text { HATCHING } \\
\text { EGG } \\
\text { CAPACITY }\end{array}$ & $\begin{array}{l}\text { PRODUCTS CLASSIFIED } \\
\text { U.S. PULLORUM-TYPHOID } \\
\text { CLEAN }\end{array}$ & $\begin{array}{c}\text { ADDITIONAL } \\
\text { CLASSIFICATIONS FOR } \\
\text { WHICH PRODUCT QUALIFIED }\end{array}$ \\
\hline
\end{tabular}

\begin{tabular}{|c|c|c|}
\hline 629 & $E$ & $\begin{array}{l}\text { THOMAS FAMILY POULTRY, } 8712 \text { EAST ULEST ROAD, } \\
\text { ELLISVILLE } 61431\end{array}$ \\
\hline 845 & $E$ & HOLLY TURMAN, P.O. BOX 241, DAVIS 61019 \\
\hline 515 & $\mathrm{E}$ & $\begin{array}{l}\text { TUTTLE'S WATERFOWL AND POULTRY, } 10506 \text { COUNTY } \\
\text { LINE ROAD. MARENGO } 60152\end{array}$ \\
\hline 361 & $E$ & $\begin{array}{l}\text { GARY UNDERWOOD, } 2404 \text { SOUTH SIMMONS, } \\
\text { STOCKTON } 61085\end{array}$ \\
\hline 305 & $E$ & $\begin{array}{l}\text { UNIVERSITY OF ILLINOIS, } 132 \text { ASL, } 1207 \text { WEST } \\
\text { GREGORY, URBANA } 61801\end{array}$ \\
\hline 1009 & $E$ & $\begin{array}{l}\text { SPRING VALLEY FARM, CATHERINE GRAVERT, } 21919 \\
\text { HILLSIDE ROAD, FULTON } 61252\end{array}$ \\
\hline 608 & $E$ & $\begin{array}{l}\text { VANGEENEN'S LITTLE PHEASANT FARM, } 13801 \\
\text { PLEASANT VALLEY ROAD, WOODSTOCK } 60098\end{array}$ \\
\hline 930 & $\mathrm{E}$ & MARIO VELAZQUEZ, 850 ROUTE E 113, MORRIS 60450 \\
\hline 846 & E & $\begin{array}{l}\text { CHARLIE WACHOWSKI, } 3511 \text { HOWLETT ROAD, PAW } \\
\text { PAW } 61353\end{array}$ \\
\hline 970 & $E$ & $\begin{array}{l}\text { BRUCE WACHOWSKI, } 1481 \text { COLLINS ROAD, OSWEGO } \\
60543\end{array}$ \\
\hline 147 & $E$ & $\begin{array}{l}\text { WHISTLING WINGS HATCHERY. } 113 \text { WASHINGTON } \\
\text { STREET, HANOVER } 61041\end{array}$ \\
\hline 533 & $E$ & $\begin{array}{l}\text { LARRY WHITE \& FAMILY, } 7665 \text { DAVIS SCHOOL ROAD, } \\
\text { KINGSTON } 60145\end{array}$ \\
\hline 1019 & E & $\begin{array}{l}\text { WHITE HOUSE POULTRY, RICK WHITE, } 4169 \text { SOUTH } \\
4000 \text { WEST ROAD, KANKAKEE } 60901\end{array}$ \\
\hline 519 & E & ROBERT WHITWOOD, RR 1, BOX 415, HUDSON 61748 \\
\hline 673 & $E$ & $\begin{array}{l}\text { FRANK \& JUNE YOHN, } 155981650 \text { NORTH AVENUE, } \\
\text { PRINCETON } 61356\end{array}$ \\
\hline 940 & E & HEATH ZIMMER, 731 5TH STREET, BECKEMEYER 62219 \\
\hline 914 & $E$ & RALPH ZOLLER, 566 ZOLLER ROAD, VERGENNES 62994 \\
\hline
\end{tabular}

Dealers

660 E BOOI FARM STORE, 117 EAST RIVER STREET, MOMENCE 60954

67 E DICKMAN FARM SERVICE, 500 ROUTE 75, NORTH FREEPORT 61032

$\begin{array}{lll}154 & \text { E } & \text { DILLIER HATCHERY, P.O. BOX 399, GREENUP } 62428 \\ 775 & \text { E } & \begin{array}{l}\text { GARDEN PRAIRIE FEED \& MILLING, 7000 GARDEN } \\ \text { PRAIRIE ROAD, GARDEN PRAIRIE 61038 }\end{array} \\ 226 \text { E } & \begin{array}{l}\text { IL-MO HATCHERY, 7731 HERTEL ROAD, MILLSTADT } \\ \text { 62260 }\end{array}\end{array}$

W271, W28, W30, W52, W573. W74, W75, W86, W92, X143. $\mathrm{X} 163, \mathrm{X} 187$

50
W.

R.

$R, W$

R10, R247, W24, W8

R

R114, R146, R17, R96

$\mathrm{Z2}$

N18

R, W

R

X4

R.

R196, Z205

W12, W30, Z15

W

W262, W28, W316, W319, W88, W96, Z231

W42, W48, W50, W52, W88, Z102, Z182, Z204, Z205, Z24, Z4. Z52, Z57, Z68

C\&P $X 2, X 3, X 4, Y 1, Y 2, Y 3$.

C C2, E3, H10, R146, R72, R95, W304, W578, W96, X14, X2, X21, $X_{22}, X_{2} 5, X 3, X 4, Y 2, Y 3, Y 4$, Z202, Z204, Z205, Z27, Z4, Z43

C A, B, C, D, E, H10, R, W $X 2, X 3, X 4, Y 1, Z 231$

C $B, C, H, R, X, Y$ 
Table 1. HATCHERIES. DEALERS. AND INDEPENDENT FLOCKS PARTICIPATING IN

THE NATIONAL POULTRY IMPROVEMENT PLAN

WATERFOWL. EXHIBITION POULTRY AND GAME BIRDS

$\begin{array}{lll} & \\ \text { APP. } & \text { SUB- PARTICIPANTS NAME AND ADDRESS } \\ \text { NO. PART } & \text { PATS }\end{array}$

\section{HATCHING \\ EGG}

CAPACITY
PRODUCTS CLASSIFIED

U.S. PULLORUM-TYPHOID CLEAN
ADDITIONAL

CLASSIFICATIONS FOR WHICH PRODUCT QUALIFIED

33 - ILLINOIS

\begin{abstract}
92 E LOSTANT HATCHERY \& MILLING COMPANY, 220 MAIN STREET, BOX 208, LOSTANT 61334

700 E MARENGO FEED \& SEED, 20015 EAST GRANT HIGHWAY. MARENGO 60152

870 E NED NICE, 7071 EAST 600TH STREET, LYNN CENTER 61262

644 E POPLAR GROVE FEED COMPANY, 104 MAIN STREET. POPLAR GROVE 61065

129 E TRI COUNTY FEED \& SEED, S. MCELWEE, 7 EAST 3RD STREET, PANA 62557

\section{Independent Flocks}

992 E CARLSON ACRES, DBA KYLER \& CAMERON CARLSON,4415 NORTH MERIDAN ROAD ROCKFORD 61101
\end{abstract}

\begin{tabular}{|c|c|c|}
\hline 478 & $E$ & $\begin{array}{l}\text { ALWERDT'S PHEASANT FARM AND GARDENS, } 3771 \\
\text { EAST } 700 \text { TH AVENUE, ALTAMONT } 62411\end{array}$ \\
\hline 1032 & $E$ & RON BARGER, 22835 HUGO ROAD, CENTRALIA 62801 \\
\hline 986 & E & $\begin{array}{l}\text { CHEYENNE BERGMARK, } 2838 \text { POOLE ROAD, } \\
\text { BELVIDERE } 61008\end{array}$ \\
\hline 525 & $E$ & $\begin{array}{l}\text { BOHLER'S BANTAMS, } 330 \text { WEST 10TH STREET, APT. } \\
\text { 205, MT. CARMEL } 62863\end{array}$ \\
\hline 935 & $E$ & $\begin{array}{l}\text { HARRY BRADDOCK, } 1178 \text { BOATDOCK ROAD, PATOKA } \\
62875\end{array}$ \\
\hline 973 & E & $\begin{array}{l}\text { BRINEY'S BIRD FARM, MILT BRINEY, RR 1, BOX } 237 \text {, } \\
\text { BROWNING } 62624\end{array}$ \\
\hline 1029 & $E$ & $\begin{array}{l}\text { BRONKEMA POULTRY, } 15527 \text { WEST CLAY ROAD, } \\
\text { PEARL CITY } 61062\end{array}$ \\
\hline 972 & $E$ & $\begin{array}{l}\text { JIM BROWN, } 6173 \text { EAST } 1500 \text { N ROAD, FLANAGAN } \\
61740\end{array}$ \\
\hline 987 & $\mathrm{E}$ & $\begin{array}{l}\text { PHILOMENA BUCKMAN, } 8747 \text { MIDDLE ROAD, SOUTH } \\
\text { BELOIT } 61080\end{array}$ \\
\hline 280 & $E$ & BUTLER'S POULTRY, BOX 145, LONDON MILLS 61544 \\
\hline 996 & $E$ & BRIAN CARAKER, 815 WOODS LANE, ANNA 62906 \\
\hline 360 & $E$ & $\begin{array}{l}\text { CAROTHER'S COCHINS, } 1652 \text { NORTH 42ND ROAD, } \\
\text { EARLVILLE } 60518\end{array}$ \\
\hline 988 & $\mathrm{E}$ & $\begin{array}{l}\text { KEITH CARROLL, } 21820 \text { WEST RAILROAD, MARENGO } \\
60152\end{array}$ \\
\hline 932 & $\mathrm{E}$ & $\begin{array}{l}\text { GREG CHAMNESS, } 11439 \text { PAULTON ROAD, PITTSBURG } \\
62974\end{array}$ \\
\hline 483 & $\mathrm{E}$ & $\begin{array}{l}\text { CLIF'S FEATHERED FORTUNE, } 3002134 \text { TH AVENUE } \\
\text { NORTH. HILLSDALE } 61257\end{array}$ \\
\hline 654 & $E$ & $\begin{array}{l}\text { BEN \& BRAD CRAIG, } 1515 \text { SOUTH MAIN, SANDWICH } \\
60548\end{array}$ \\
\hline
\end{tabular}

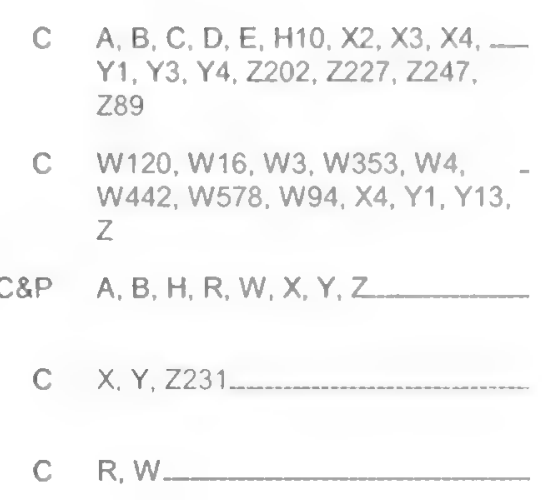

N114, N97, R120, R124, R14, R145, R168, R171, R175, R20, R21, R22, R33, R35, R40, R45, R46, R52, R7, R83, R97, W13, W208, W211, W25, W26, W28, W290, W390, W45, W60, W75

227

R146, W2

W

W276, W613

Z1, Z43, Z93

$\mathrm{Z2}$

R146, R183, R222.

R81, W12, W594

R

R. W

R22, R248, W17, W18, W198, W20, W24, W25, W54, W6

R201, W18

R239, R248, R7

R247, W12, W48, W88

Z14, Z2, Z231, Z4.

$R, W$ 
Table 1. HATCHERIES, DEALERS, AND INDEPENDENT FLOCKS PARTICIPATING IN THE NATIONAL POULTRY IMPROVEMENT PLAN

WATERFOWL, EXHIBITION POULTRY AND GAME BIRDS

\begin{tabular}{|c|c|c|c|c|c|}
\hline $\begin{array}{l}\text { APP. } \\
\text { NO. }\end{array}$ & $\begin{array}{l}\text { SUB- } \\
\text { PART }\end{array}$ & PARTICIPANTS NAME AND ADDRESS & $\begin{array}{l}\text { HATCHING } \\
\text { EGG } \\
\text { CAPACITY }\end{array}$ & $\begin{array}{l}\text { PRODUCTS CLASSIFIED } \\
\text { U.S. PULLORUM-TYPHOID } \\
\text { CLEAN }\end{array}$ & $\begin{array}{c}\text { ADDITIONAL } \\
\text { CLASSIFICATIONS FOR } \\
\text { WHICH PRODUCT QUALIFIED }\end{array}$ \\
\hline
\end{tabular}

725 E DALE'S BIRD FARM, DALE SMITH, 2732 PARK ROAD,
BELLEVILLE 62220

$E$

FEATHERED FRIENDS FARM, JEFF CRUMP, 26973 EAST 2800 ROAD, CHENOA 61726

484

$E$

MERLE DANIELS, 17681 MILLARD ROAD, MORRISON 61270

928 E EDWARD DEMBOWSKI, 43 WEST 922 MAIN STREET. ELBURN 60119

874 E DAVID DIETZ, 17205 MCGUIRE ROAD, HARVARD 60033

661 E CHARLES DOYLE, 3249 SOUTH BEND ROAD, ROCKFORD 61109

872 E $\begin{aligned} & \text { ROBERT DREWES, } 10518 \text { EAST ANGLINBG ROAD. } \\ & \text { MARTINSVILLE } 62442\end{aligned}$

965 E EBERSOLE POULTRY, 26609 AVERS ROAD, STERLING 61081

915 E LEE EILTS, RR 1, BOX 2, WASHBURN 61570

978 E ROBERT EISELE, 4778 WHITING ROAD, POPLAR GROVE 61065

1024 E JERRY ELLIS, 21164 HAILS LANE, TEXICO 62889

975 E CHARLENE FAIRLESS, 19189 WEST FRONTAGE ROAD, RAYMOND 62560
E THE FUNNY FARM, JERRY RUSSELL, 28279 NORTH 2550 EAST ROAD, CHENOA 61726 60449

WILLIAM GECAS, 3530 WILLIAMS STREET, STEGER 60475

ELIZABETHR. GENTZ, 5141 SHATTUCK ROAD, BELVIDERE 61008

E LAUREN GOVEDNIK, 2259-D NORTH BELL ROAD, MINOOKA 60447

E GREEN DIAMOND GAMEFOWL FARM, 29227 NORTH 2300 EAST ROAD, ODELL 60460

E JACOB HABERSTICH, 8227 FRUIT FARM ROAD, BELVIDERE 61008

E DIANA HIGGINS, 885 SOUTH LOCUST, RICHVIEW 62877

E VIRGIL HORSTMAN, 3399 PELL ROAD, METROPOLIS 62960

\section{TIM FIDDER, 8611 HUBER ROAD, BELVIDERE 61008}

W, Z2, Z3

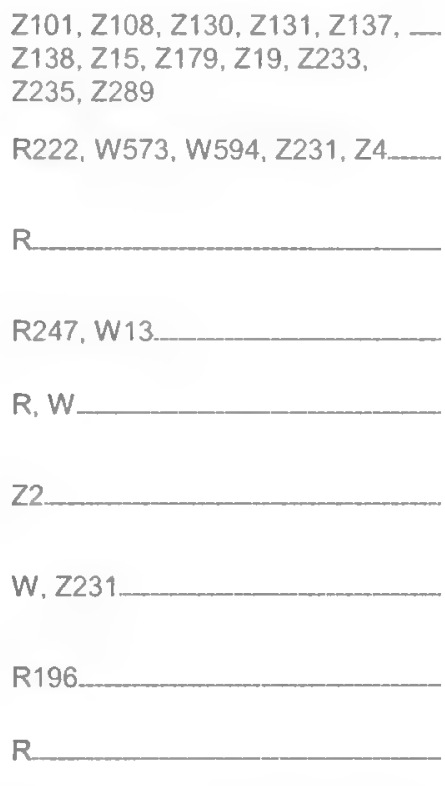

R229, R233, R237, R42, W132, W316, W519, Z20

W142, W23, W26, W28, W29, W48, W53, W538

R. W. Z11

$Z 11, Z 2, Z 205, Z 3, Z 4$

243

w

R. W

R146, R20, R51, R7

N18.

R, W

R139, R30, R52, W120, W586, -. W7, W88

R57, Z102, Z107, Z118, Z22, Z24, Z26, Z27, Z32, Z50, Z52

W. W255, W578, W6

N18, R247, R248, W132, W134, W19, W4, W5, W57, W92, X17, $\times 180, \times 3$

R1, R7, R95, W18, W30 
Table 1. HATCHERIES, DEALERS, AND INDEPENDENT FLOCKS PARTICIPATING IN

THE NATIONAL POULTRY IMPROVEMENT PLAN

WATERFOWL. EXHIBITION POULTRY AND GAME BIRDS

\begin{tabular}{|c|c|c|c|c|c|}
\hline $\begin{array}{l}\text { APP. } \\
\text { NO. }\end{array}$ & $\begin{array}{l}\text { SUB- } \\
\text { PART }\end{array}$ & PARTICIPANTS NAME AND ADDRESS & $\begin{array}{l}\text { HATCHING } \\
\text { EGG } \\
\text { CAPACITY }\end{array}$ & $\begin{array}{l}\text { PRODUCTS CLASSIFIED } \\
\text { U.S. PULLORUM-TYPHOID } \\
\text { CLEAN }\end{array}$ & $\begin{array}{c}\text { ADOITIONAL } \\
\text { CLASSIFICATIONS FOR } \\
\text { WHICH PRODUCT QUALIFIED }\end{array}$ \\
\hline
\end{tabular}

1030 E NEAL KERCHNER, 1340 ATKINSON ROAD, DIXON 61021

1025 E ANTHONY KLEIBOEKER, P.O. BOX 121, BREESE 62230

1006 E GREGG KONITSKI, 6211 MONTAGUE ROAD, ROCKFORD 61102

E EARL KRUG, BOX 85, DWIGHT 60420

E SHIRA LAMBERT, 9724-A SCHLAPP ROAD, YORKVILLE 60560

E DONNA AND JOE LEAHY, 3000 ROTH ROAD, OSWEGO 60543

E JOHN LEFFELMAN, 1297 US RT. 52, AMBOY 61310

ROBERT LIPPERT, 307 IOWA STREET, SHATTUC 62283

RANDY LOTT, 26557 LOCUST ROAD, LANARK 61046

GARY LYTLE, 1377 EAST US HIGHWAY 36, TUSCOLA 61953

E BRIAN MALONE, 4777 WEST 5000 SOUTH ROAD, KANKAKEE 60901

E AMANDA \& HENRY MARQUARDT, 7974 BLUFF ROAD, WATERLOO 62298

E KEN \& SHARON MAUSEY, 5183 MAUSEVILLE ROAD, CREAL SPRINGS 62922

E BRUCE MCCAIN, 56 WEST UNION, MASCOUTAH 62258

MARK MCCOY, 10923 EAST 300TH AVENUE, FLAT ROCK 62427

CHARLES \& LINDA MCKEE, 8116 MAPLE STREET, MARENGO 60152

E KATHRYN MILLER, 4614 SOUTH RIDGEWAY, RINGWOOD 60072

PAULA MORRIS, 21992 CHANDLERVILLE ROAD, VIRGINIA 62691

E CHRISTINE NELSON, 8709 ELEVATOR ROAD, ROSCOE 61073

E BETHANY \& KATHRYN NEWHOUSE, 13407 CAPRON ROAD, CAPRON 61012

E MARK \& PENNY OLDFATHER, 23030 EAST 1500 NORTH, FAIRBURY 61739 JOHN OROZCO, 920 WEST MAIN STREET, ELBURN 60119

QUINTEN E. PAUL, 2554 WYMAN SCHOOL ROAD CALEODNIA 61014 NOKOMIS 62075

E TRAVIS PEDEN, 1415 CINDY LANE, TAYLORVILLE 62568

E PLEASANT VIEW WINGS-N-THINGS, R. \#18, 9112 OLD RIVER ROAD, ROCKFORD 61103
R114

W131, W17, W18, W88

N18

W566

R114.

R

R52, R53, W17, W198, W81, Z26, Z57

R42, W211, W50, W81

R146, Z11

$R, W$

$R, W, Z 231$

$R 12, R 146, R 34, R 7, W 28$

W126, W127, W159, W16

N14, N15, N18, N2, N50, N55, N74, R249, R250, R255

R, W, Z142, Z43, Z45, Z60

$R, W$

B7, R1, R237

R12, R19, R196, R20, R22, R233,. R86, W46, W570, Z202, Z204

W38, W54

R146, R21, R70

W

N18.

$R, W, Z 231$

$R, W$

R20, W

R, W, Z2 
Table 1. HATCHERIES, DEALERS, AND INDEPENDENT FLOCKS PARTICIPATING IN

THE NATIONAL POULTRY IMPROVEMENT PLAN

WATERFOWL, EXHIBITION POULTRY AND GAME BIRDS

APP. SUB- PARTICIPANTS NAME AND ADDRESS

NO. PART
HATCHING

EGG

CAPACITY
PRODUCTS CLASSIFIED

U.S. PULLORUM-TYPHOID

CLEAN
ADDITIONAL

CLASSIFICATIONS FOR WHICH PRODUCT QUALIFIED

\begin{tabular}{|c|c|c|}
\hline 897 & $E$ & DONNIE POLLARD, RT. 2, BOX 13, FAIRFIELD 62837 \\
\hline 63 & $E$ & $\begin{array}{l}\text { GAYLE \& NORMAN PONTIOUS, SR., RT. 1, BOX } 258, \\
\text { EDGEWOOD } 62426\end{array}$ \\
\hline 14 & $E$ & JERRY POOL, 3709 EAST IL 250, ONLEY 62450 \\
\hline 342 & $E$ & $\begin{array}{l}\text { NOEL PORTER, } 11432 \text { WEST RICHARD PLACE, ZION } \\
60099\end{array}$ \\
\hline 619 & $E$ & $\begin{array}{l}\text { RANDALL GAME BIRD FARM, } 2164 \text { EAST STREET } \\
\text { HIGHWAY } 33, \text { BEECHER CITY } 62414\end{array}$ \\
\hline 81 & $E$ & $\begin{array}{l}\text { WALTER RAU, } 880 \text { GILMORE LAKE ROAD, COLUMBIA } \\
62236\end{array}$ \\
\hline 953 & $E$ & $\begin{array}{l}\text { GLADYS RENNER, } 10813 \text { MCCONNELL ROAD, } \\
\text { WOODSTOCK } 60098\end{array}$ \\
\hline 043 & $E$ & MIKE RICHARD, 1245 PINE HILL ROAD, AMBOY 61310 \\
\hline 329 & $E$ & $\begin{array}{l}\text { HAROLD RIGGS, BOX 148, HOFFER LANE, EAST } \\
\text { PEORIA } 61611\end{array}$ \\
\hline 933 & $E$ & TONI ROBERTS, RR 2, BOX 19, GREENFIELD 62044 \\
\hline 921 & $E$ & $\begin{array}{l}\text { SCOTT \& SHERI SAGER, DBA HERITAGE FARM, } 17620 \\
\text { CAPRON ROAD, CAPRON } 61012\end{array}$ \\
\hline 752 & $E$ & $\begin{array}{l}\text { DWAYNE SARGENT GAME BIRD FARM, RR. \#3, BOX } \\
220 . \text { AUBURN } 62615\end{array}$ \\
\hline 622 & $E$ & $\begin{array}{l}\text { DONALD SCHMITT, } 30367 \text { FULFS ROAD, STERLING } \\
61081\end{array}$ \\
\hline & $E$ & $\begin{array}{l}\text { RENEE, HEATHER, \& MICKEY SHOOK, } 800 \text { SOUTHWIND } \\
\text { BLVD., LOT } 25 \text {, ROBINSON } 62454\end{array}$ \\
\hline
\end{tabular}

\begin{tabular}{|c|c|c|}
\hline 918 & $E$ & $\begin{array}{l}\text { SONLIGHT RANCH GAME BIRDS, ZIGTEMA, } 5901 \text { WEST } \\
\text { OFFNER ROAD, MONEE } 60449\end{array}$ \\
\hline 802 & $E$ & $\begin{array}{l}\text { NICK \& JANICE STARR, } 3742 \text { GENOA ROAD, BELVIDERE } \\
61008\end{array}$ \\
\hline 925 & $E$ & $\begin{array}{l}\text { NICOLE STOUT, } 6231 \text { HUNTER ROAD, POPLAR GROVE } \\
61065\end{array}$ \\
\hline 424 & $E$ & JIM STULTS, 1301 LAMSON ROAD, WINNEBAGO 60098 \\
\hline 985 & $E$ & $\begin{array}{l}\text { JEAN SWENSON, } 2905 \text { NORTH } 4650 T H, \text { SANDWICH } \\
60548\end{array}$ \\
\hline 885 & $E$ & $\begin{array}{l}\text { RICHARD \& WENDY TOBIASZ, } 10715 \text { BREEZY LAWN } \\
\text { ROAD, SPRING GROVE } 60081\end{array}$ \\
\hline 49 & $E$ & MIKE TOLKA, 1731 BOONE STATE ROAD, ODIN 62870 \\
\hline
\end{tabular}

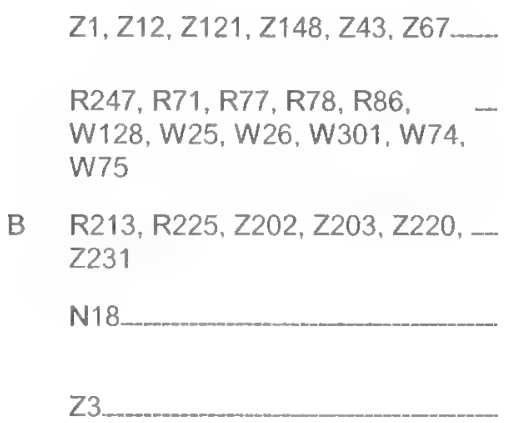

B R213, R225, Z202, Z203, Z220, -Z231

N18.

Z3.

R196, R21, R222, R256, R30. W145, W198, W211, W26, W28, W3, W52, W74, Z108, Z138, Z139, Z204, Z205, Z22, Z9

$\mathrm{R}, \mathrm{W}$

R19, W142, W52, W570.

$\mathrm{R}$

$\mathrm{R} 19, \mathrm{R} 229, \mathrm{~W} 13, \mathrm{~W} 2$

R, W

Z12, Z172, Z2, Z259, Z27

W.

R146, R16, R19, R196, R20, R22, R23, R247, R44, R49, R53, R7, R8, R93, R94, W1, W12, W121, W13, W131, W132, W138, W18, W198, W2, W23, W248, W25 W265, W28, W290, W3, W370, W38, W43, W48, W5, W52, W60. W75, W79, W86, W88, Z128, Z204, Z205, Z22, Z231, Z62

W. X, Y, Z2, Z231

R, W, Z11

R227, R247.

B, W442

R

R104, R11, R115, R12, R139, R140, R146, R168, R175, R18. R19, R192, R196, R232, R247, R248, R260, R3, R30, R35, R42, R51, R53, R56, R60, R7, R71, R73, R77, R87, R88, R90, R97. 
Table 1. HATCHERIES, DEALERS, AND INDEPENDENT FLOCKS PARTICIPATING IN

THE NATIONAL POULTRY IMPROVEMENT PLAN

WATERFOWL, EXHIBITION POULTRY AND GAME BIRDS

\begin{tabular}{|c|c|c|c|c|c|}
\hline $\begin{array}{l}\text { APP. } \\
\text { NO. }\end{array}$ & $\begin{array}{l}\text { SUB- } \\
\text { PART }\end{array}$ & PARTICIPANTS NAME AND ADDRESS & $\begin{array}{l}\text { HATCHING } \\
\text { EGG } \\
\text { CAPACITY }\end{array}$ & $\begin{array}{l}\text { PRODUCTS CLASSIFIED } \\
\text { U.S. PULLORUM-TYPHOID } \\
\text { CLEAN }\end{array}$ & $\begin{array}{c}\text { ADDITIONAL } \\
\text { CLASSIFICATIONS FOR } \\
\text { WHICH PRODUCT QUALIFIED }\end{array}$ \\
\hline
\end{tabular}

33 - ILLINOIS

\begin{abstract}
821 E VIRGINIA TROUT, RR 2, BOX 104, ASHKUM 60911
1038 E DEAN \& SUSAN TROUT, 2767 NORTH 500 EAST ROAD, ASHKUM 60911

407 E JIM VANFLEET, 502 1/2 CHURCH STREET, BELVIDERE 61008-9585

1001 E RAUL VASQUEZ, 45369 HARTER ROAD, SUGAR GROVE 60554

1002 E VICTOR VELAZQUEZ, 539 TERRY AVENUE, AURORA 60506

E JUAN VILLARREAL, 620 2ND STREET, WAUKEGAN 60085

630 E JOE VIVIAN, 1796 LEE CENTER ROAD, AMBOY 61310

858 E HALEY WADE, 9902 POLAND ROAD, WARRENSBURG 62573

375 E ALBERT WALTERS, RR 1, BOX 103, WELDON 61882

993 E LYDIA WANDA, 9712 WRIGHT ROAD, HARVARD 60033

840 E JOHN WELCH, 1527 EAST GERMAN VALLEY ROAD, BAILEYVILLE 61007

487 E ESTELLE WERNLE, 8749 LEPERE SCHOOL ROAD, MILLSTADT 62260
\end{abstract}

578 E WHISPERING WILLOWS, CLYDE GROVER, 8038 WEST STATE, ROCKFORD 61102

543 E JACK WHITWELL, RR 1, BOX 158, SYCAMORE 60178

934 E BRANDY WILCOX, 7677 CENTERVILLE ROAD, CAPRON 61012

998 E JEFF WILSON, 218 SHERMAN STREET, JERSEWILLE 62052

460 E TOM WILTSHIRE, 16376B POLLSGROVE ROAD, MOUNT CARROLL 61053

865 E MERLE D. WITHEFT, 14546 WEST 2000 SOUTH ROAD. BUCKINGHAM 60717

769 E JIM WOLFF, 24811 RIVER ROAD, MARENGO 60152

362 E WARREN ZEHR, 3076 EAST 1700 NORTH, FLANAGAN 61740

980 E RANDAL ZERKEL, 1081 COTTONWOOD AVENUE, GREENVILLE 62246
W100, W108, W11, W115, W12. W121, W128, W132, W138, W142, W166, W187, W198, W20, W207, W23, W24, W245, W25, W27, W28, W3, W33, W38, W39, W41, W44, W5, W54, W56 W57, W570, W576, W587, W64. W69, W7, W74, W75, W9

$R, Z 11, Z 4 \ldots$
$R 229, R 233, R 239, R 248$
$R, W$
$N 18$
$N 18$
$R$

R15, R16, R19, W132.

R, W

H10, R19, R30, R52, R53, R87

R237, R256

Z231

N18, R105, R114, R145, R160,

R179, R183, R19, R20, R22, R42, R62, R68, R70, R89, R94, W13,

W25, W26, W30, W58, W609

R, W, Z11, Z2, Z3

W2, Z, Z11

R

W20

R18, R248

$Z 1, Z 2, Z 3$

w

R169, R237, R248, R90, W56, W6

R20, R96, W166, W215, W570

W, W13, W131, W132, W166, W30, W35, W370, W415, W442, W527, W8, W80 
Table 1. HATCHERIES, DEALERS, AND INDEPENDENT FLOCKS PARTICIPATING IN

THE NATIONAL POULTRY IMPROVEMENT PLAN

WATERFOWL, EXHIBITION POULTRY AND GAME BIRDS

\begin{tabular}{|c|c|c|c|c|c|}
\hline $\begin{array}{l}\text { APP. } \\
\text { NO. }\end{array}$ & $\begin{array}{l}\text { SUB- } \\
\text { PART }\end{array}$ & PARTICIPANTS NAME AND ADDRESS & $\begin{array}{l}\text { HATCHING } \\
\text { EGG } \\
\text { CAPACITY }\end{array}$ & $\begin{array}{l}\text { PRODUCTS CLASSIFIED } \\
\text { U.S. PULLORUM-TYPHOID } \\
\text { CLEAN }\end{array}$ & $\begin{array}{c}\text { ADDITIONAL } \\
\text { CLASSIFICATIONS FOR } \\
\text { WHICH PRODUCT QUALIFIED }\end{array}$ \\
\hline
\end{tabular}

32 - INDIANA

PAUL BRENNAN

INDIANA STATE POULTRY ASSOCIATION, INC.

PURDUE UNIVERSITY

1151 LILLY HALL

WEST LAFAYETTE, IN 47907-1151

Phone: (765)494-8517

Fax: (765)496-1600

Haicheries
279 E CULVER DUCK FARMS, INC., P.O. BOX 910, 12215 COUNTY ROAD 10, MIDDLEBURY 46540
Phone: (219)825-9537
231 E HOOSIER POULTRY BREEDERS ASSOCIATION, 1252 SOUTH 600 WEST, MARION 46953
Phone: (765)384-7017
268 E MAPLE LEAF FARM, INC., R\&D HATCHERY, 1601 WEST CENTER, WARSAW 46580

Phone: (574)658-4121

218 E MAPLE LEAF FARMS, INC., 1307 NORTH 1000 WEST, CROMWELL 46732

Phone: (219)658-4121
12 E PURDUE UNIVERSITY, POULTRY RESEARCH. 5675 WEST 600 NORTH, WEST LAFAYETTE 47906
Phone: (765)583-2950

301 E SCHROCK'S MOTHER GOOSE HATCHERY, 15737 COUNTRY ROAD 28, GOSHEN 46528

Phone: (574)533-2447

\section{$\underline{\text { Dealers }}$}

273 E THE CRITTER CORNER PET SHOP, INC., 1796 EAST VAILE, KOKOMO 46901

Phone: (765) $452-4500$

322 E MORNINGSIDE HATCHERY, SHIREMAN, 7522 WEST STREET ROAD 44, MORGANTOWN 46160 Phone: (317)422-1827

321 E TRACTOR SUPPLY, ANDERSON, 6818 SOUTH SCATTERFIELD ROAD, ANDERSON 46013 Phone: (765)779-0044

\begin{tabular}{|c|c|c|}
\hline 320 & $E$ & $\begin{array}{l}\text { TRACTOR SUPPLY, ANGOLA, } 1807 \text { NORTH WAYNE, } \\
\text { ANGOLA } 46703 \\
\text { Phone: }(219) 624-3766\end{array}$ \\
\hline & $E$ & $\begin{array}{l}\text { TRACTOR SUPPLY, DECATUR, } 829 \text { SOUTH 13TH } \\
\text { STREET, DECATUR } 46733 \\
\text { Phone: (219)728-2181 }\end{array}$ \\
\hline 305 & $E$ & $\begin{array}{l}\text { TRACTOR SUPPLY, EVANSVILLE, } 5210 \text { MORGAN } \\
\text { AVENUE, EVANSVILLE } 47715 \\
\text { Phone: }(812) 477-6497\end{array}$ \\
\hline & $E$ & $\begin{array}{l}\text { TRACTOR SUPPLY, FORT WAYNE, BYPASS } 30 \text { WEST, } \\
2112 \text { COLISEUM BLVD., FORT WAYNE } 46808 \\
\text { Phone: }(219) 484-1596\end{array}$ \\
\hline
\end{tabular}

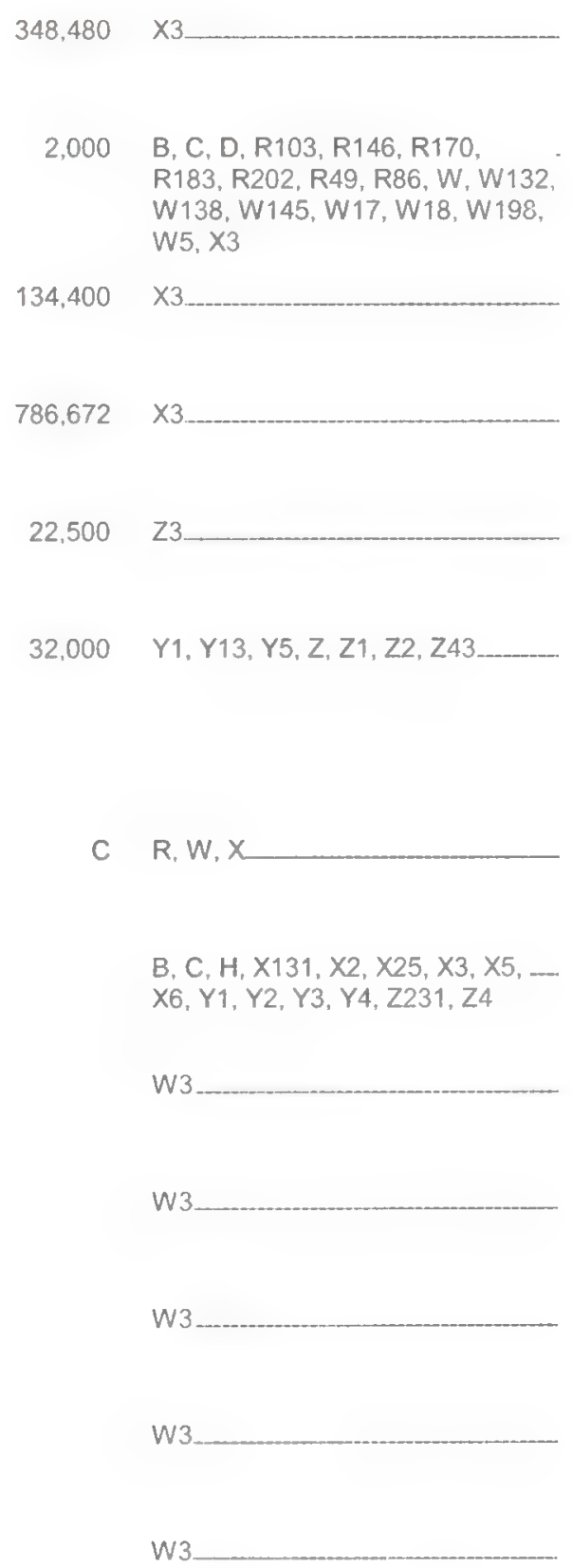
2112 COLISEUM BLVD. FORT WAYNE 46808 
Table 1. HATCHERIES, DEALERS. AND INDEPENDENT FLOCKS PARTICIPATING IN

THE NATIONAL POULTRY IMPROVEMENT PLAN

WATERFOWL, EXHIBITION POULTRY AND GAME BIRDS

\begin{tabular}{lllll}
\hline & & HATCHING & PRODUCTS CLASSIFIED \\
EGG & U.S. PULLORUM-TYPHOID & CLEAN \\
APP. SUPACITY & CAPAICIPANTS NAME AND ADDRESS \\
NO. PART & & & \\
\hline & $32-$ INDIANA
\end{tabular}

314 E TRACTOR SUPPLY, GOSHEN, 2108 LINCOLNWAY EAST, GOSHEN 46526

Phone: (219)533-3703

317 E TRACTOR SUPPLY, GREENCASTLE, 507 NORTH JACKSON, GREENCASTLE 46135

Phone: (765)653-2448

318 E TRACTOR SUPPLY, GREENWOOD, 1460 SOUTHU.3. 31 , GREENWOOD 46142

Phone: (317)882-5800

302 E TRACTOR SUPPLY, INDIANAPOLIS, 7402 EAST

BROOKVILLE ROAD, INDIANAPOLIS 46239

Phone: (317)353-8056

303 E TRACTOR SUPPLY, LAFAYETTE, 3990 STATE ROAD 38 EAST, LAFAYETTE 47905

Phone: (765)447-9331

315 E TRACTOR SUPPLY, NEW CASTLE, 3809 SOUTH TSC

ROAD, NEW CASTLE 47362

Phone: (765)521-2610

316 E TRACTOR SUPPLY, PLAINFIELD, 2640 EAST MAIN

STREET, PLAINFIELD 46168

Phone: (317)839-4792

309 E TRACTOR SUPPLY, PLYMOUTH, 7575 NORTH

MICHIGAN ROAD, PLYMOUTH 46563

Phone: (219)936-2435

313 E TRACTOR SUPPLY, RICHMOND, 4791 NATIONAL ROAD EAST, RICHMOND 47374

Phone: (765)966-2568

304 E TRACTOR SUPPLY, TERRE HAUTE, 4939 SOUTH U.S. HIGHWAY 41. TERRE HAUTE 47802

Phone: (812)299-2161

E TRACTOR SUPPLY, VALPARAISO, 1056 STATE ROAD 2, VALPARAISO 46383

Phone: (219)464-8597

TRACTOR SUPPLY, VINCENNES, 1901 HART STREET, VINCENNES 47591

Phone: (812)882-1955

307 E TRACTOR SUPPLY, WABASH, 1733 SOUTH WABASH STREET, BOX 665, WABASH 46992

Phone: (219)563-2176

312 E TRACTOR SUPPLY, WARSAW, 3925 EAST CARRIDOR DRIVE, WARSAW 46582

Phone: (219)269-2625

319 E TRACTOR SUPPLY, WESTFIELD, 18160 U.S. 31 NORTH, WESTFIELD 46074

Phone: (317)867-3505

Independent Flocks

280 E RON CHEW, 7061 EAST COUNTY ROAD 600 SOUTH, STRAUGHN 47387

Phone: (765) $332-2338$

FEATHERS GAME BIRD FARM, 4701 EAST THOMPSON ROAD, INDIANAPOLIS 46237

Phone: (317)787-9537

275 E HILLSIDE GAMEBIRD FARM, 736 WEST EUREKA ROAD. ROCKPORT 47635

Phone: (812)649-9086
W3.

W3.

w3

w3

W3

W3

W3

W3

w3

w3

w3

W3

w3

W3.

w3

W142, W262, W50

Z43, 293 
Table 1. HATCHERIES, DEALERS, AND INDEPENDENT FLOCKS PARTICIPATING IN

THE NATIONAL POULTRY IMPROVEMENT PLAN

WATERFOWL, EXHIBITION POULTRY AND GAME BIRDS

\begin{tabular}{lllll}
\hline & & HATCHING & PRODUCTS CLASSIFIED \\
APP. SUB- & PARTICIPANTS NAME AND ADDRESS & EGG & U.S. PULLORUM-TYPHOID \\
NO. PART & CAPACITY & CLEAN & CLASSIFICATIONS FOR \\
\hline
\end{tabular}

32 - INDIANA

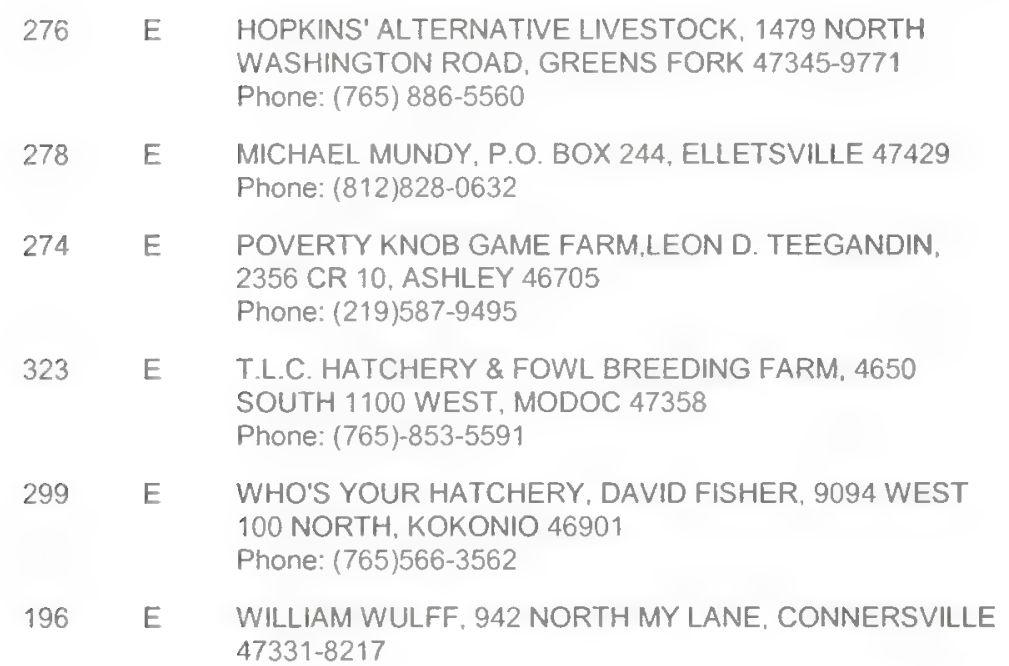

42 - IOWA
Z101, Z108, Z109, Z11, Z130, Z131, Z137, Z138, Z139, Z140. Z15, Z179, Z19, Z235

R225, R31, R7.

Z48.

$A, D, E, H, R, R 104, W, X, Y, Z$, Z11, Z231, Z3

R173, R18, R227, R7, R96

W142, W28, W49, W52, W542, $W 74, W 91, X, Y$

KEVIN S. VINCHATTLE

IOWA POULTRY ASSOCIATION

8515 DOUGLAS AVENUE, SUITE 9

URBANDALE, IA 50322-2924

Phone: (515)727-4701

Fax: $\quad(515) 727-4707$

Hatcheries

$\begin{array}{ll}145 \text { E } & \text { DECORAH HATCHERY, INC., BOX 205, } 406 \text { WEST } \\ & \text { WATER STREET, DECORAH } 52101 \\ 254 \quad \text { E } \quad \text { GUINEA FARM, RALPH WINTER, } 21357 \text { WHITE PINE } \\ \\ \text { LANE, NEW VIENNA 52065 } \\ \text { PhONE: (319)853-4195 }\end{array}$

61 E HOOVER'S HATCHERY, INC., BOX 200, RUDD 50471

233 E KRUSE HATCHERY, 1011 COUNTY ROAD WEST 14, FORT ATKINSON 52144

10 E MURRAY MCMURRAY HATCHERY, 191 CLOSZ, BOX 458, WEBSTER CITY 50595

395 E TIMOTHY MITCHELL, 506 LOCUST STREET, LUCAS 50151

Phone: (641)776-1110

273 E O'CONNELL GAME FARM, 2137 RICHMILL ROAD, FORT DODGE 50501

287 E PINE CREEK GAME FARM, 425 SOUTH 5TH STREET. MONTROSE 52639

251 E PINE TREE LANE, 1043 410TH AVENUE, MILES 52064

229 E SAND PRAIRIE QUAIL FARM, 9690 224TH AVENUE, MAQUOKETA 52060

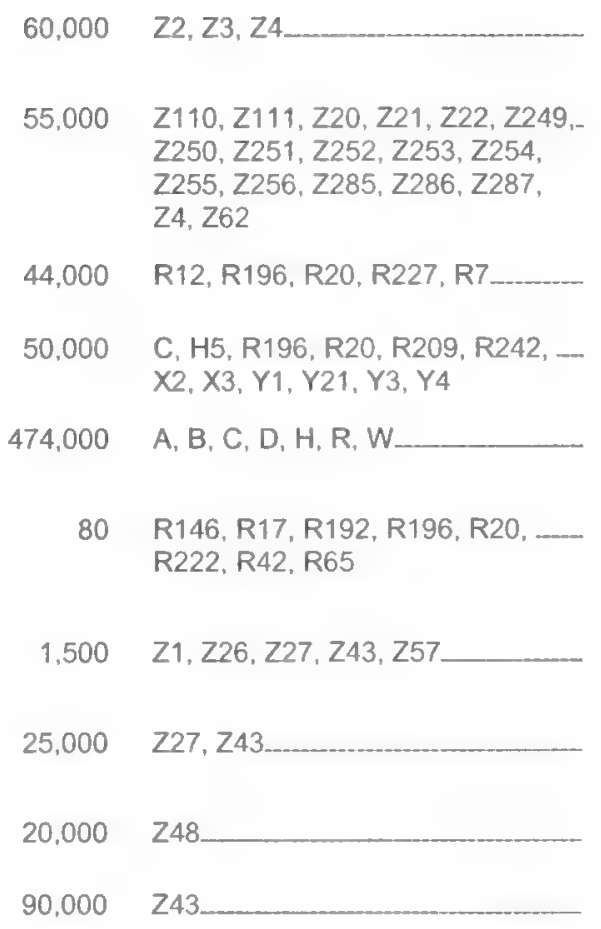


Table 1. HATCHERIES, DEALERS, AND INDEPENDENT FLOCKS PARTICIPATING IN

THE NATIONAL POULTRY IMPROVEMENT PLAN

WATERFOWL, EXHIBITION POULTRY AND GAME BIRDS

APP. SUB- PARTICIPANTS NAME AND ADDRESS

NO. PART
HATCHING
EGG

CAPACITY
PRODUCTS CLASSIFIED

U.S. PULLORUM-TYPHOID CLEAN
ADDITIONAL

CLASSIFICATIONS FOR WHICH PRODUCT QUALIFIED

\section{2 - IOWA}

188 E SCHLECHT FARM \& HATCHERY, 9749 500TH AVENUE, MILES 52064-9704

236 E SUN RAY CHICKS HATCHERY, BOX 300, HAZELTON 50641

\section{Dealers}

E ACORN SUPPLY, ROBERT/JANICE CRISS, 329 16TH AVENUE, COUNCIL BLUFF 50503

Phone: (712)3259282

ALGONA GRAIN \& FEED, 525 SOUTH PHILLIPS STREET. ALGONA 50511

247 E ASPINWALL COOPERATIVE, 204 EAST MAIN STREET. BOX 8, ASPINWALL 51432

220 E BREKKE'S TOWN \& COUNTRY STORE, INC., 23827 580TH AVENUE, AMES 50010

343 E COUNTRY GENERAL, 1405 NORTH LAKE AVENUE, STORM LAKE 50588

145 E DECORAH HATCHERY, INC., BOX 205, 406 WEST WATER STREET, DECORAH 52101

FARMER'S COOPERATIVE, HIGHWAY 63 NORTH, P.O. BOX 151, NEW HAMPTON 50659

HOOVER'S HATCHERY, INC., BOX 200, RUDD 50471

E LAKESIDE COUNTRY STORE, 4040 SOUTH EXPRESS WAY, COUNCIL BLUFFS 51502

Phone: (712)366-8111

LEMARS HATCHERY \& FEED, 4212 ND AVENUE NW, BOX 927, LEMARS 51031

MURRAY MCMURRAY HATCHERY, 191 CLOSZ, BOX 458 WEBSTER 50595

NELSON HATCHERY, INC, P.O. BOX 19, AKRON 51001

E

ORSCHELN FARM \& HOME LLC, ATLANTIC, 1310 EAST 7TH STREET, ATLANTIC 50022

E ORSCHELN FARM \& HOME LLC, BOONE, 1125 SE MARSHALL, BOONE 50036

E ORSCHELN FARM \& HOME LLC, CLAINDA, BOX 25 , CLAINDA 51632

E ORSCHELN FARM \& HOME LLC, FAIRFIELD, 2005 WEST BURLINGTON AVENUE, FAIRFIELD 52556

E ORSCHELN FARM \& HOME LLC, GUTHRIE CENTER, BOX 127, GUTHRIE CENTER 50115

E ORSCHELN FARM \& HOME LLC, IOWA CITY, 655 HOLLYWOOD BLVD., IOWA CITY 52240

E ORSCHELN FARM \& HOME LLC, MARION, $33157 \mathrm{TH}$ AVENUE. MARION 52302

E ORSCHELN FARM \& HOME LLC, MT. PLEASANT, 106 IRIS, BOX 344, MT. PLEASANT 52641

E ORSCHELN FARM \& HOME LLC, MUSKATINE, 1607 GRANDVIEW AVENUE, MUSKATINE 52761
$20,000 \quad Y 1, Y 2, Y 3, Y 4, Y 6$

$64,800 \quad A, B, C, D, E, R$

$A, B, C, D, E, W, Y$

C $\quad B, C, D, R, X, Y, Z$

C $\quad \times 2, Z 217$

C B10, C10, D9, R196, W, X2, X3, $\mathrm{Y} 1, \mathrm{Y} 3, \mathrm{Z} 2$

c

C $\quad X, Z 9$

C $\quad B, C, D, R, X, Y, Z$

C $\quad W, X_{2}, X_{3}, Y_{1}, Y_{3}, Z 2$

C $\quad A, B, C, D, E, N, R, W, X, Y, Z$

C\&SP A, R209, X

C $\quad A, B, C, D, E, H, N, R, W, X, Y, Z$

C B, C, R209, R227, X2, X3, Y1, Y3

C $\quad C, H, R, W, X, Y, Z$

C C, H, R, W, X, Y, Z

C C, $H, R, W, X, Y, Z$

C $\quad C, H, R, W, X, Y, Z$

C $\quad C, H, R, W, X, Y, Z$

C $\quad C, H, R, W, X, Y, Z$

C C, $H, R, W, X, Y, Z$

C $\quad, H, R, W, X, Y, Z$

C $\quad C, H, R, W, X, Y, Z$

C $\quad C, H, R, W, X, Y, Z$ 


\begin{tabular}{|c|c|c|}
\hline 372 & $E$ & $\begin{array}{l}\text { ORSCHELN FARM \& HOME LLC, PERRY, } 2905 \text { EAST } \\
\text { WILLIS, PERRY } 50220\end{array}$ \\
\hline 375 & $E$ & $\begin{array}{l}\text { ORSCHELN FARM \& HOME LLC, RED OAK, } 24004 \text { TH } \\
\text { STREET, RED OAK } 51566\end{array}$ \\
\hline 69 & $\mathrm{E}$ & $\begin{array}{l}\text { ORSCHELN FARM \& HOME LLC, SHENANDOAH, } 208 \\
\text { RYE STREET, SHENANDOAH } 51601\end{array}$ \\
\hline 71 & $E$ & $\begin{array}{l}\text { ORSCHELN FARM \& HOME LLC, CENTERVILLE, } \\
\text { HIGHWAY } 5 \text { SOUTH, BOX } 785, \text { CENTERVILLE } 52544\end{array}$ \\
\hline 46 & $E$ & $\begin{array}{l}\text { ORSCHELN FARM \& HOME LLC, WASHINGTON, } 1613 \\
\text { EAST WASHINGTON, WASHINGTON } 52353\end{array}$ \\
\hline 392 & $E$ & $\begin{array}{l}\text { PINEY WOODS HATCHERY, VICKI BARNCY, RT. 1, BOX } \\
\text { 81, LAMONI } 50140 \\
\text { Phone: }(641) 784-4365\end{array}$ \\
\hline 27 & $E$ & $\begin{array}{l}\text { STATE CENTER GRAIN \& FEED, INC., } 402 \text { 3RD STREET, } \\
\text { NW., STATE CENTER } 50247\end{array}$ \\
\hline 236 & $E$ & $\begin{array}{l}\text { SUN RAY CHICKS HATCHERY, BOX } 300, \text { HAZELTON } \\
50641\end{array}$ \\
\hline 309 & $\mathrm{E}$ & $\begin{array}{l}\text { SUN RAY CHICKS, } 208 \text { EAST FIRST STREET, BOX } 217 \text {, } \\
\text { MECHANICSVILLE } 52306\end{array}$ \\
\hline 206 & $E$ & $\begin{array}{l}\text { WEBB'S FEED, INC., RR 1, BOX 29, ROCKWELL CITY } \\
50579\end{array}$ \\
\hline & $E$ & WELP, INC., P.O. BOX 77, BANCROFT 50517 \\
\hline
\end{tabular}

Independent Flocks

292 E AUSTIN POULTRY FARM, DARREL AUSTIN, 1210236 TH AVENUE, FORT ATKINSON 52144

Phone: (319)534-7749

389 E BLUM FARM, BEN BLUM, 2077 JEFFERSON AVENUE, GEORGE 51237

Phone: (712)475-3219

E BRIGG'S POULTRY FARM, 877 HIGHWAY G-76, KNOXVILLE 501388729

Phone: (641)828-2155

384 E BILL CLINTON, BOX 4, CARROLL 51401

396 E RONALD DINGER, 100 1ST. AVENUE, ARMSTRONG 50514

Phone: (712)868-3254

254 E GUINEA FARM, RALPH WINTER, 21357 WHITE PINE LANE, NEW VIENNA 52065

296 E HAWKEYE GAME BIRDS, 405 2ND AVENUE NE, STATE CENTER 50247 190TH STREET, HONEY CREEK 51542

C $\quad C, H, R, W, X, Y, Z$

C C , H, R, W, X, Y, Z

C $\quad C, H, R, W, X, Y, Z$

C C, H, R, W, X, Y, Z

C $\quad C, H, R, W, X, Y, Z$

E\&C N, Z11, Z2, Z44

C A, B10, C10, D9, R196, W, X2, $\mathrm{X} 3, \mathrm{Y} 1, \mathrm{Y} 3, \mathrm{Z} 2$

E\&C A, B, C, D, E, H, R, W, X, Y, Z

E\&C A, B, D, H, R209

C B, B10, C, C10, D, D9, R196, W. $X_{2}, X_{3}, Y 1, Y 3, Z 2$

C $A, E 3, R, W, X, Y, Z$

$\mathrm{B} 10, \times 2$

$X, Y, Z$

$A, B, D, H, R, W$

$\mathrm{N} 14, \mathrm{~N} 15, \mathrm{~N} 32, \mathrm{~N} 50, \mathrm{~N} 9$

H9, R15, R213, R257, R258, R8, . W119, W16, W160, W26, W435. W501, W567, W573, W6, W621. W7, W74, X2, X99, Y5

Z110, Z111, Z20, Z21, Z22, Z249... Z250, Z251, Z252, Z253, Z254. Z255, Z256, Z285, Z286, Z287, Z4, Z62

243

R178, R53, R72, R85, R89, W121, W142, W17, W25, W26. W4, W427, W48, W518, W52, W568, W6, W60, W74

Z27, Z43 
TabIe 1. HATCHERIES, DEALERS, AND INDEPENDENT FLOCKS PARTICIPATING IN

THE NATIONAL POULTRY IMPROVEMENT PLAN

WATERFOWL, EXHIBITION POULTRY AND GAME BIRDS

\begin{tabular}{|c|c|c|c|c|c|}
\hline $\begin{array}{l}\text { APP. } \\
\text { NO. }\end{array}$ & $\begin{array}{l}\text { SUB- } \\
\text { PART }\end{array}$ & PARTICIPANTS NAME AND ADDRESS & $\begin{array}{l}\text { HATCHING } \\
\text { EGG } \\
\text { CAPACITY }\end{array}$ & $\begin{array}{l}\text { PRODUCTS CLASSIFIED } \\
\text { U.S. PULLORUM-TYPHOID } \\
\text { CLEAN }\end{array}$ & $\begin{array}{c}\text { ADDITIONAL } \\
\text { CLASSIFICATIONS FOR } \\
\text { WHICH PRODUCT QUALIFIED }\end{array}$ \\
\hline
\end{tabular}

288 E JUNGER'S POULTRY FARM, 20545 PARK HOLLOW
ROAD, DURANGO 52039

Phone: (319)552-2021

295 E JULIAN KUHN, 3055 128TH STREET, FORT ATKINSON

381 E MOONLIGHT STABLES, JOE SIMPSON, 1935 UPLAND TRAIL, PROLE 50229

273 E O'CONNELL GAME FARM, 2137 RICHMILL ROAD, FORT DODGE 50501

268 E BART PALS, 1119 EAST STATE STREET, MASON CITY 50401

287 E PINE CREEK GAME FARM, BOX 425 SOUTH $5 T H$ STREET, MONTROSE 52639

251 E PINE TREE LANE, 1043 410TH AVENUE, MILES 52064

336 E BOB PINKERTON, 7855 NE 50TH AVENUE, ALTOONA 50009

RAWSON PEAFOWL FARMS, DUSTIN RAWSON, 2640 VAN AVENUE. WINTHROP 50682

Phone: (319) 934-3196

SAND PRAIRIE QUAIL FARM, 9690-224TH AVENUE, MAQUOKETA 52060

SANDHILL PRESERVATION CENTER, 1878 230TH STREET, CALAMUS 52729

188 E SCHLECHT FARM \& HATCHERY, 9749 500TH AVENUE, MILES 52064-9704

271 E DUSTIN C. SILVER, RR 1. BOX 147, STOCKPORT 52651

269 E ROGER VANDERPLOEG, 510 NORTH JEFFERSON AVENUE, MASON CITY 50401

385 E WILD ROSE FARM, DIANNA MEJSTRIK. 22443 DEVORE AVENUE, GLENWOOD 51534

\section{8 - KANSAS}

Z108, Z128, Z130, Z131, Z137, Z139, Z140, Z179

R15

N28, N32

Z1, Z100, Z25, Z26, Z27, Z28, _ Z43, Z53, Z57, Z89, Z94

W4

$\mathrm{Z} 27, \mathrm{Z} 43$

Z48

R168, R169, R237, R25

214

243

$R, W, X, Y, Z$

$Y 1, Y 2, Y 3, Y 4, Y 6$

$X 142, X 146, X 20, X 28, Y 27, Y 5$, $Y 8, Z 118, Z 15, Z 19, Z 22$

W16, W23, W573, W594

R196, R35, R7, R72, R86.

\section{DR. SCOTT BEYER}

EXTENSION SPECIALIST, POULTRY SCIENCES

CALL HALL, KANSAS STATE UNIVERSITY

MANHATTAN, KS 66506

Phone: (785) 532-1201

Fax: (785) $532-5681$

\section{Hatcheries}

296 E ARK VALLEY PHEASANT FARM, RIETH, 204 SOUTH MAPLE STREET, PIERCEVILLE 67868-9506

293 E DON BADDERS, 715 SOUTH SYCAMORE, IOLA 66749
43,000

Z2.

1,000

10,000

22,23

Z2, Z3 
Table 1. HATCHERIES, DEALERS, AND INDEPENDENT FLOCKS PARTICIPATING IN

THE NATIONAL POULTRY IMPROVEMENT PLAN

WATERFOWL, EXHIBITION POULTRY AND GAME BIRDS

\begin{tabular}{lllll}
\hline & & HATCHING & PRODUCTS CLASSIFIED \\
APP. & SUB- & PARTICIPANTS NAME AND ADDRESS & EGG & U.S. PULLORUM-TYPHOID \\
NO. & PART & CLEAN & CLASSIFICATIONS FOR \\
\end{tabular}

48 - KANSAS

\begin{tabular}{|c|c|c|c|c|}
\hline 230 & $E$ & $\begin{array}{l}\text { BEAVER'S GAME BIRD FARM, HC } 65, \text { BOX } 30 \text {, } \\
\text { PIERCEVILLE } 67868\end{array}$ & 15,700 & Z27_ \\
\hline 299 & $E$ & $\begin{array}{l}\text { BLUE SHOE HATCHERY, } 2033 \text { BECK STREET, } \\
\text { MANHATTAN } 66502\end{array}$ & 2,500 & \\
\hline 141 & $E$ & $\begin{array}{l}\text { COUNTRY HATCHERY \& FEED STORE, RR 3, BOX } 268 \text {, } \\
\text { DODGE CITY } 67801\end{array}$ & 17.000 & $\begin{array}{l}\text { B, C, D, R17, R19, R196, R2, } \\
\text { R38, R7 }\end{array}$ \\
\hline 294 & $E$ & $\begin{array}{l}\text { DYE'S QUAIL FARM, BOBBIE DYE, } 4200 \text { EAST K-4 } \\
\text { HIGHWAY, ASSARIA } 667416\end{array}$ & 1,000 & Z3, Z43_ \\
\hline 261 & $E$ & $\begin{array}{l}\text { FEATHER DUSTER BIRD FARM \& HATCHERY, RT. } 1 \text {, } \\
\text { BOX 14-F, THAYER } 66776\end{array}$ & 1,000 & $\begin{array}{l}X 102, X 135, X 143, X 158, X 38, X 4 \\
Y 10, Y 2, Y 35, Y 5, Y 52\end{array}$ \\
\hline 234 & $E$ & $\begin{array}{l}\text { FRIEND BIRD FARM, ROUTE } 3, \text { BOX 24, FREDONIA } \\
66736\end{array}$ & 21,000 & $\mathrm{X}, \mathrm{Z} 11, \mathrm{Z9}$ \\
\hline 289 & $E$ & $\begin{array}{l}\text { FRANK R. REESE, JR., } 730 \text { SMOKY VALLEY ROAD, } \\
\text { LINDSBORG } 67456\end{array}$ & 5,000 & Z231 \\
\hline 235 & $E$ & ROHRER'S GAME FARM, ROUTE 1, BOX 45, TROY 66087 & 2,000 & $\mathrm{Z} 1, \mathrm{Z27}, \mathrm{Z} 43, \mathrm{Z9}$ \\
\hline 291 & $E$ & $\begin{array}{l}\text { KENNETH SCHORTMANN, } 2501 \text { MEADOWLARK DRIVE, } \\
\text { MILFORD } 66514\end{array}$ & 1,000 & $\mathrm{Z2}$ \\
\hline 288 & $E$ & SPRAGUE HATCHERY, BOX 44, BLUE MOUND 66010 & 1,000 & $\begin{array}{l}\mathrm{Z} 182, \mathrm{Z204}, \mathrm{Z205}, \mathrm{Z209}, \mathrm{Z230}, \ldots \\
\mathrm{Z27}\end{array}$ \\
\hline 165 & $E$ & $\begin{array}{l}\text { THUNDERING WINGS PHEASANT FARM, RR 2, BOX 178, } \\
\text { WESTPHALIA } 66093\end{array}$ & 3,700 & $\mathrm{Z} 129, \mathrm{Z27}, \mathrm{Z60}$ \\
\hline 236 & $E$ & $\begin{array}{l}\text { VERDIGRIS VALLEY GAME FARM, } 817 \text { S.W. BLVD, } \\
\text { MADISON } 66860\end{array}$ & 4,000 & $\mathrm{Z1}, \mathrm{Z} 27$ \\
\hline 298 & $E$ & $\begin{array}{l}\text { KIM VINEYARD, } 2233 \text { BENNETT, WICHITA } 67213 \\
\text { Phone: (316)943-7921 }\end{array}$ & 500 & 22,243 \\
\hline 295 & $E$ & MIKE WEBBER GAME FARM, , OAKLEY 67748 & 1,000 & $\mathrm{Z} 2, \mathrm{Z} 3$ \\
\hline 192 & $E$ & $\begin{array}{l}\text { WHITE'S HATCHERY \& POULTRY FARM, RR 1, BOX 136- } \\
\text { A. GREAT BEND } 67530\end{array}$ & 1,600 & $\begin{array}{l}\mathrm{X} 2, \mathrm{X} 3, \mathrm{Y} 1, \mathrm{Y} 2, \mathrm{Y} 3, \mathrm{Y} 4, \mathrm{Z} 212 \\
\mathrm{Z22}, \mathrm{Z} 62\end{array}$ \\
\hline & $E$ & JACK WILSON, RR 1, BOX 28-A, BRONSON 66716 & 5,000 & Z43 \\
\hline
\end{tabular}

Independent Flocks

237 E MICHAEL ALLIS, 2594 GEORGORIA ROAD, OTTAWA 66067

N. W

R169, R2, R200, W6

\begin{tabular}{|c|c|c|}
\hline 215 & $E$ & $\begin{array}{l}\text { LYLE BAUMGARTEN, } 32285 \text { WEST } 207 \text { STREET, } \\
\text { EDGERTON } 66021\end{array}$ \\
\hline 274 & $E$ & $\begin{array}{l}\text { BUFF CREEK QUAIL FARM, BOX } 923, \text { GREENSBURG } \\
67054\end{array}$ \\
\hline 241 & $E$ & $\begin{array}{l}\text { DAVE CAVENDER, ROUTE 1, BOX 40-A, WELLSVILLE } \\
66092\end{array}$ \\
\hline 216 & E & $\begin{array}{l}\text { GARY COOPER, } 3207 \text { SUMMERSET DRIVE. } \\
\text { HUTCHINSON } 67502\end{array}$ \\
\hline 117 & $E$ & BENNY DEAN, 1139 MAPLE, EUDORA 66025 \\
\hline 278 & $\mathrm{E}$ & JIM EVERITT, RT. 2, BOX 120, FT. SCOTT 66701 \\
\hline 243 & $\mathrm{E}$ & $\begin{array}{l}\text { FREVELE'S FARM, } 25732 \text { EVANS ROAD, TONGANOXIE } \\
66086\end{array}$ \\
\hline & & 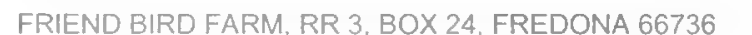 \\
\hline
\end{tabular}


Table 1. HATCHERIES, DEALERS, AND INDEPENDENT FLOCKS PARTICIPATING IN

THE NATIONAL POULTRY IMPROVEMENT PLAN

WATERFOWL, EXHIBITION POULTRY AND GAME BIRDS

APP. SUB- PARTICIPANTS NAME AND ADDRESS

NO. PART

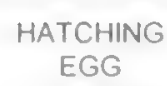

HATCHING

EGG

CAPACITY
PRODUCTS CLASSIFIED

U.S. PULLORUM-TYPHOID CLEAN
ADDITIONAL

CLASSIFICATIONS FOR WHICH PRODUCT QUALIFIED

48 - KANSAS

129 E GADBERRY'S BANTAMS, 944 EAST SHERMAN, HUTCHINSON 67501

200 E ALAN GILLISPIE, 1901 NW. BUCHANAN, TOPEKA 66608

269 E RONALD HINDS, 13067-238TH STREET, LINWOOD 66052

176 E FLOYD HUFFMAN, 21881 LINWOOD ROAD, LINWOOD 66052

245 E ALLEN HUFFMAN, 12375 222ND STREET, LINWOOD 66052

174 E LEVIHUFFMAN, 1830 SOUTH 49TH PLACE, KANSAS CITY 66106

220 E CLAUDE HUFFMAN, 603 SOUTH RACE, SPRINGHILL 66083

179 E CLIFF IKENBERRY, RR 2,1714 HIGH STREET BALDWIN CITY 66006

203 E AL KEEFER, 1045 SOUTH MAIN, OTTAWA 66067

GERALD KLAUS, 1875 KARMAN, MUNJOR 67601

E

JIM KNISLEY, ROUTE 3, BOX 53, AUGUSTA 67010

$E$ BRIAN MOREHEAD, 215 WEST GRANT, GREENSBURG 67054

E CHARLES PASSLER, 25 NORTH 102ND STREET, EDWARDSVILLE 66022

E RALPH PECK, 1943 SOUTH ST. FRANCIS, WICHITA 67211

E JAMES M. RANDALL, ROUTE 3, BOX 201, COLUMBUS 66725

E CHARLES RIDDLE, 3416 NORTH FAIRVIEW, WICHITA 67204

E MARC RIFFEL, 101 EAST 5TH, WOODBINE 67492

E ROBBINS FAMILY, RR 1, BOX 162, OSKALOOSKA 66066

E JOHN SMITH, 441 SOUTH 72ND STREET, KANSAS CITY 66111

E PAUL SODAMANN, RR 1, BOX 244, ST. GEORGE 66535

E JAYSPENCER, 915 EAST BLANCHARD, HUTCHINSON 67501

E JOSEPH TANNER, RT. 3, TONGANOXIE 66086

E PAT VARGAS, 105 SOUTH 145TH STREET, LEAVENWORTH 66048

E CLINT WAITLEY, RT. 3, BOX 294-B, LEAVENWORTH 66048
W23, W4, W54

W150, W151, W16, W568, W94

N.

H...

W25, W26, W568, W88

W19, W20, W7, w8

W94

W149, W17, W29, W573, W58, W88

W13, W3, W30, W576, W585, W83

R87, W305, W570, Z207

N

W126, W13, W14, W80, W83, W84, Z203

N109, N34.

R15. R16, R208

$\mathrm{Z72}$

N...

W25, W26, w81

N.

N

R27

W28, W48, W69, W88

N

R57, Z126, Z182, Z54, Z55, Z71, Z9

N

N

N

N. 
Table 1. HATCHERIES, DEALERS, AND INDEPENDENT FLOCKS PARTICIPATING IN

THE NATIONAL POULTRY IMPROVEMENT PLAN

WATERFOWL, EXHIBITION POULTRY AND GAME BIRDS

\begin{tabular}{|c|c|c|c|c|c|}
\hline $\begin{array}{l}\text { APP. } \\
\text { NO. }\end{array}$ & $\begin{array}{l}\text { SUB- } \\
\text { PART }\end{array}$ & PARTICIPANTS NAME AND ADDRESS & $\begin{array}{l}\text { HATCHING } \\
\text { EGG } \\
\text { CAPACITY }\end{array}$ & $\begin{array}{l}\text { PRODUCTS CLASSIFIED } \\
\text { U.S. PULLORUM-TYPHOID } \\
\text { CLEAN }\end{array}$ & $\begin{array}{c}\text { ADDITIONAL } \\
\text { CLASSIFICATIONS FOR } \\
\text { WHICH PRODUCT QUALIFIED }\end{array}$ \\
\hline
\end{tabular}

48 - KANSAS

$\begin{array}{lll}267 & \text { E } & \begin{array}{l}\text { CHARLES WEIBLE, 3 NORTH CASSIDY, PARTRIDGE } \\ 67566\end{array} \\ 150 & \text { E } & \text { AL WESTLING, 3228 NW. ALICE DRIVE, TOPEKA 66618 } \\ 258 & \text { E } & \begin{array}{l}\text { EDWARD WINELAND, 620 EAST 69TH, HUTCHINSON } \\ 67502\end{array} \\ 285 & \text { E } & \begin{array}{l}\text { WAYNE WOODY, 399 EAST 1700 ROAD, BALDWIN CITY } \\ 66006\end{array}\end{array}$

$61-$ KENTUCKY

DR. ANTHONY PESCATORE

UNIVERSITY OF KENTUCKY

DEPARTMENT OF ANIMAL SCIENCES

604 GARRIGUS BUILDING

LEXINGTON, KY 40546

Phone: (859)257-7529

Fax: (859)323-1027

\begin{tabular}{|c|c|c|c|c|}
\hline \multicolumn{5}{|c|}{ Hatcheries } \\
\hline 36 & E & WADE BAILEY, , LIBERTY 42539 & 15,500 & $\mathrm{Z1}, \mathrm{Z} 43, \mathrm{Z} 60, \mathrm{Z72}$ \\
\hline 54 & E & $\begin{array}{l}\text { DOUBLE R QUAIL FARM, } 115 \text { RUSSELL ROAD, } \\
\text { GREENSBURG } 42743\end{array}$ & 3,600 & Z43_ \\
\hline 47 & $E$ & $\begin{array}{l}\text { FORREST HILL GAME FARM, RR } 8 \text {, BOX 204-X, LIBERTY } \\
42539\end{array}$ & 15,000 & $\mathrm{Z1}, \mathrm{Z43}, \mathrm{Z60}, \mathrm{Z72}$ \\
\hline 55 & E & $\begin{array}{l}\text { DANNY GOODMAN, } 368 \text { JOHNNY GOODMAN LANE, } \\
\text { CECILIA } 42724\end{array}$ & 2,700 & $\begin{array}{l}\mathrm{Z108}, \mathrm{Z118}, \mathrm{Z129}, \mathrm{Z137}, \mathrm{Z138}, \\
\mathrm{Z176}, \mathrm{Z180}, \mathrm{Z237}, \mathrm{Z24}, \mathrm{Z26}, \mathrm{Z27}, \\
\mathrm{Z} 34, \mathrm{Z} 41, \mathrm{Z} 51, \mathrm{Z} 53, \mathrm{Z} 57, \mathrm{Z} 89\end{array}$ \\
\hline 40 & $E$ & $\begin{array}{l}\text { KNOTTY PINE QUAIL FARM, } 2511 \text { COLDWATER ROAD, } \\
\text { MURRAY } 42071\end{array}$ & 30,000 & Z1, Z27, Z43, Z45 \\
\hline \multicolumn{5}{|c|}{ Independent Flocks } \\
\hline 52 & $E$ & $\begin{array}{l}\text { DOUBLE R. QUAIIL FARM, } 115 \text { RUSSELL ROAD, } \\
\text { GREENSBURG } 42743\end{array}$ & & 243 \\
\hline 22 & $E$ & HAROIN COUNTY HATCHERY, RR 1, GLENDALE 42740 & & N N \\
\hline
\end{tabular}

DR. MARTHA LITTLEFIELD

ASSISTANT STATE VETERINARIAN

OFFICE OF ANIMAL HEALTH SERVICES

P.O. BOX 1951

BATON ROUGE, LA 70821

Phone: (225)925-3980

Fax: (225)925-4103 
Table 1. HATCHERIES, DEALERS, AND INDEPENDENT FLOCKS PARTICIPATING IN

THE NATIONAL POULTRY IMPROVEMENT PLAN

WATERFOWL, EXHIBITION POULTRY AND GAME BIRDS

\begin{tabular}{|c|c|c|c|c|c|}
\hline $\begin{array}{l}\text { APP. } \\
\text { NO. }\end{array}$ & $\begin{array}{l}\text { SUB- } \\
\text { PART }\end{array}$ & PARTICIPANTS NAME AND ADDRESS & $\begin{array}{l}\text { HATCHING } \\
\text { EGG } \\
\text { CAPACITY }\end{array}$ & $\begin{array}{l}\text { PRODUCTS CLASSIFIED } \\
\text { U.S. PULLORUM-TYPHOID } \\
\text { CLEAN }\end{array}$ & $\begin{array}{c}\text { ADDITIONAL } \\
\text { CLASSIFICATIONS FOR } \\
\text { WHICH PRODUCT QUALIFIED }\end{array}$ \\
\hline
\end{tabular}

E R \& B FARMS, RITA WARE, 9019 HIGHWAY 182 WEST, FRANKLIN 70538

E\&C W80

Independent Flocks

E B \& D PHENSANTRY, BUD GUIDRY, 122 EAST 132ND STREET, GALLIANO 70354

N207, W102, W13, W146, W173,

W18, W189, W20, W33, W570

Z1, Z101, Z102, Z107, Z118, Z12.

Z126, Z149, Z173, Z174, Z177

Z18, Z24, Z248, Z26, Z27, Z28

Z30, Z34, Z4, Z40, Z41, Z42, Z44,

$Z 48, Z 49, Z 51, Z 52, Z 53, Z 54$

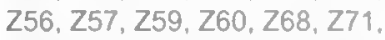

$\mathrm{Z72}, \mathrm{Z74}, \mathrm{Z77}, \mathrm{Z79}, \mathrm{Z} 87, \mathrm{Z} 89$

670 E MAVERICK BOUDREAUX, 135 BOUDREAUX ESTATES HOUMA 70364

R232, R96, W440, W46, W594:

N

W

N30, N33 ROAD, ABBEVILLE 70510

W13

N.

N. 70514

W142, W28, W29, W88, W99

R114, R12, R20, R81, W18, W25, W26

R246, W208, W45

DAVID KELLY, 10426 WOODLAND VIEW DRIVE, GREENWELL SPRINGS 70739

694 E DUSTIN KERBOW. 28357 WOODY BOOTY ROAD, MT. HERMAN 70450

Z43

N28, N32, N41, N63 70560

W110, W304

R11, R183, W119, W5, W54, W60

N149, N187, N216, N236 MICHAEL LEE, 31575 PETE BANKSTON ROAD. HOLDEN 70744

E ROGER LOVE, 452 DALE LOVE ROAD, MARION 71260

W

W25, W28 VENTRESS 70783

577 E JUSTIN OWENS, 913 POPLAR STREET, MORGAN CITY 70380

W11, W30

R183.

W301, W33, Z102, Z107, Z118, Z129, Z141, Z149, Z152, Z170. Z235, Z24, Z26, Z260, Z34, Z44 Z45, Z48, Z52, Z54, Z60, Z68, Z72, Z93 


\begin{tabular}{|c|c|c|c|c|c|}
\hline $\begin{array}{l}\text { APP. } \\
\text { NO. }\end{array}$ & $\begin{array}{l}\text { SUB- } \\
\text { PART }\end{array}$ & PARTICIPANTS NAME AND ADDRESS & $\begin{array}{l}\text { HATCHING } \\
\text { EGG } \\
\text { CAPACITY }\end{array}$ & $\begin{array}{c}\text { PRODUCTS CLASSIFIED } \\
\text { U.S. PULLORUM-TYPHOID } \\
\text { CLEAN }\end{array}$ & $\begin{array}{c}\text { ADDITIONAL } \\
\text { CLASSIFICATIONS FOR } \\
\text { WHICH PRODUCT QUALIFIED }\end{array}$ \\
\hline
\end{tabular}

\section{2 - LOUISIANA}

\begin{tabular}{|c|c|c|}
\hline 556 & $E$ & MANDY PRESENBACK, 101 LOYMAN CT., HOUMA 70364 \\
\hline 704 & $E$ & $\begin{array}{l}\text { MEGAN PURPURA, } 6434 \text { POYDRAS BAYOU, } \\
\text { ERWINVILLE } 70729\end{array}$ \\
\hline 703 & $E$ & JONATHAN PYE, 152 FIELDS ROAD, ARCADIA 71001 \\
\hline 706 & $E$ & $\begin{array}{l}\text { CRAIG RICHARDSON, } 601 \text { LELAND STREET, SULPHUR } \\
70663\end{array}$ \\
\hline 510 & $E$ & $\begin{array}{l}\text { RICHARD RODRIGUEZ, } 188 \text { FRANKS ROAD, HEFLIN } \\
71039\end{array}$ \\
\hline 99 & $E$ & EARL ROLLING, 6639 RIVER ROAD, WESTWEGO 70094 \\
\hline 708 & $E$ & $\begin{array}{l}\text { ANDRE ST. ROMAIN, } 14698 \text { CHENAL ROAD, JARREAU } \\
70749\end{array}$ \\
\hline 538 & $E$ & $\begin{array}{l}\text { WAYNE \& BROOKS ROMERO, } 4305 \text { JEFFERSON ISLAND } \\
\text { ROAD, NEW IBERIA } 70560\end{array}$ \\
\hline 707 & $E$ & $\begin{array}{l}\text { RHONDA ROSE, } 999 \text { CHURCHMAN LANE, DERIDDER } \\
\text { Z70634 }\end{array}$ \\
\hline 707 & $E$ & RHONDA ROSE, \\
\hline 643 & $E$ & GLEN SOPRANO, P.O. BOX 225, CHARENTON 70523 \\
\hline 687 & $E$ & LANE WEST, 232 BERGERON LOOP, CARENCRO 70520 \\
\hline 430 & $E$ & $\begin{array}{l}\text { DENNIS WOLLARD, } 1913 \text { BEAU BASIN ROAD, } \\
\text { CARENCRO } 70520\end{array}$ \\
\hline
\end{tabular}

W578

W

R96, W353

W393, W395, W401

W182, W20, W57

N127, N28, N44

R183, W442

W28, W50, w88

W. Z

W, Z

N139, N142, N160, N32, N33, N34

N, R249

R30, R52, W169, W17, W29, _ W88

DR. DONALDE. HOENIG

STATE VETERINARIAN

MAINE DEPARTMENT OF AGRICULTURE

28 STATE HOUSE STATION

AUGUSTA, ME 04333-0028

Phone: (207)287-3701

Fax: (207)624-5044

Independent Flocks

$\begin{array}{lll}797 & \text { E } & \begin{array}{l}\text { STEPHAINE ANTHANOSIS, 99 ROBINSON HILL ROAD, } \\ \text { SEBAGO 04029 }\end{array} \\ 252 & \text { E } & \text { FELIX ATIENZA, JR., RR 3, BOX 109, GORHAM } 04038 \\ 802 & \text { E } & \begin{array}{l}\text { FELIX ATIENZA, 1334 LONG PLAINS ROAD, BUXTON } \\ 04903\end{array} \\ 322 & \text { E } & \begin{array}{l}\text { JACK BARNES, BOX 73, HIRAM 04041 } \\ 375\end{array} \\ 416 & \text { E } & \text { MARGARET BROWN, RR 2, BOX 1740, MADISON } 04950 \\ & \text { E } & \begin{array}{l}\text { CHARLOTTE BURLEY \& WIN ROBBINS, WILD } \\ \text { ASPARAGUS FARM, P.O. BOX 169, WHITFIELD } 04353\end{array}\end{array}$

R16, R160, R237, R247, W, W34,. W352, Z202

w30.

W30, w69

R115, R41, R54, R56, R64, R7, -$R 88, R 97, Z 205, Z 214$

R222, W12, W129, W136, W223,W33. W570

R42. 
Table 1. HATCHERIES. DEALERS. AND INDEPENOENT FLOCKS PARTICIPATING IN

THE NATIONAL POULTRY IMPROVEMENT PLAN

WATERFOWL, EXHIBITION POULTRY AND GAME BIRDS

APP. SUB- PARTICIPANTS NAME AND ADDRESS

NO. PART

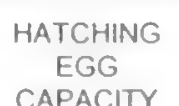

EGG
CAPACITY
PRODUCTS CLASSIFIED

U.S. PULLORUM-TYPHOID CLEAN
ADOITIONAL

CLASSIFICATIONS FOR WHICH PRODUCT QUALIFIED

\section{1 - MAINE}

\begin{abstract}
89 E RAY CANNING, MARSH ROAD, RFD 3, BELFAST 04915
325 E DAN CHARLES, RR 2. BOX 830, NORRIDGEWOCK 04957

803 E WILLIAM CHARLES, RR 2, BOX 1125. NORRIDGEWOCK 04957

160 E CARROLL A. DEAN, RR 1, BOX 608, MADISON 04950

355 E CHARLES \& RORY DEVINE, 2405 ESSEX STREET, ORONO 04473

804 E EDINGTON \& FREEMEN, 683 HOLLIS ROAD, HOLLIS CENTER 04042

805 E LORRAINE FECTEAU, 1975 MIL.TON MILLS ROAD, ACTON 04401

806 E JOHN FITTS, 79 WINNOCKS NECK ROAD, SCARBOROUGH 04074

791 E ALLISON FLAGG, 880 EAST POND ROAD, NOBLEBORO 04555

306 E BRUCE \& MAE GROVER, 182 TEMPLE ROAD, TEMPLE 04984

807 E BILL \& ANN HANSON. 283 GUNEYTOWN ROAD. APPLETON 04862
\end{abstract}

799 E VALERIE D. HATCH, RR 1, BOX 2416, WINDSOR 04363

111 E ROBERT HAWES, 8 SHORT WHARF ROAD, HAMPDEN 04444

260 E FORREST HOOPER, 51 COOPER ROAD, WARREN 04864

384 E ERNIE \& ROSE JOHNSON, P.O. BOX 86, SEARSMONT 04973

800 E WAYNE JONES, 287 BUCK STREET, GORHAM 04038

411 E MYRONLASH, 1155 WASHINGTON ROAD, WALDOBORO 04572

374 E JIM OLMSTED, 213 HIGHLAND ROAD, WARREN 04864

287 E LARRY PEASLEE, 141 PATRICK TOWN ROAD, SOMERVILLE 04348

365 E DENNIS POTTER, 137 ABEN ROAD EXTENTION, EXETER 04435

72 E EBEN PRIME, RR 2, BOX 5807, WINTHROP 04364

27 E NORMAN RAU, 286 BACK RIVER ROAD, BOOTHBAY 04537

272 E DAVID RAYER, 549 HOFFSES DRIVE, FRIENDSHIP 04547

808 E MARK ROSS. JR., 442 WALDOBORO ROAD, FRIENOSHIP 04547

273 E JEFF RYAN, 19 HYDE ROAD, GRAY 04039

247 E SKIP WINSLOW, 466 SMITHFIELD ROAD, NORRIDGEWOCK 04957
R146, R86, W166, W20

R11, R12, R196, R6, Z22

R113, R17, R222, R71, R77, W86

R6, R88, W19, W23, W262

W119. W160, W20, W88

W136, W23, W48, W53, W54, $\times 100, \times 143, \times 145, \times 17$

R229.

W

W142, W556, W69

W187, W262, W28, W29, W50, W69, W88

W102, W12, W132, W18, W20, W208, W28, W352, W365.

W422, W48, W537, W588,

W600. W75, W8, W91

R146, R186, R21.

W104, W121, W24, W470

N129, R186, W23, W484, W606, . W607, W617, W646, W69, W89

R11, W26, W54

W11. W578

W38, W50, W556, W88, W91 _._

$\times 4,227$

R43, W20, W26, W28

W119, W3, W301, W364, W58

W184, W20, W270, W28, W3, W38, W415, W45, W60, W8

W121, W135. W164, W17, W207.

W30.

$\mathrm{R} 42$

D1, W132, W136, W393

R1, R139, R42, R69, R71, R72, R85. W167, W3 


\begin{tabular}{llccc}
\hline & & HATCHING & PRODUCTS CLASSIFIED \\
APP. SUB- & PARTICIPANTS NAME AND ADDRESS & EGG & U.S. PULLORUM-TYPHOID & CLLATIONAL \\
NO. PART & CAPACITY & & CLEAN & WHICH PRODUCTIONS FOR QRALIFIED \\
\hline
\end{tabular}

51 - MARYLAND

DR. FIDELIS HEGNGI

MARYLAND DEPARTMENT OF AGRICULTURE

ANIMAL HEALTH LABORATORY

P.O. BOX 2599

SALISBURY, MD 21802

Phone: (410)543-6610

Fax: $\quad(410) 543-6676$

Hatcheries

\begin{tabular}{|c|c|c|}
\hline 12 & $E$ & $\begin{array}{l}\text { CBT FARMS, } 2640 \text { HARPER ROAD, FEDERALSBURG } \\
21632\end{array}$ \\
\hline 328 & $E$ & $\begin{array}{l}\text { DAVID TRACY, } 17904 \text { GUNPOWDER ROAD, } \\
\text { HAMPSTEAD } 21074\end{array}$ \\
\hline 350 & $E$ & $\begin{array}{l}\text { JOHN L. TUTTLE, } 500 \text { WAVERLY LANE, CENTERVILLE } \\
21617\end{array}$ \\
\hline & & Independent Flocks \\
\hline 63 & $E$ & $\begin{array}{l}\text { JOHN ARBAUGH, } 148 \text { PIPE CREEK ROAD, LINWOOD } \\
21764\end{array}$ \\
\hline 470 & $E$ & $\begin{array}{l}\text { WILSON \& MARY ELLEN ARBAUGH, } 2244 \text { TREVANIAN } \\
\text { ROAD. TANEYTOWN } 21787\end{array}$ \\
\hline 333 & $E$ & $\begin{array}{l}\text { KEITH \& NANCY CARLISLE, } 140 \text { PIPE CREEK ROAD, } \\
\text { LINWOOD } 21764\end{array}$ \\
\hline 12 & $E$ & $\begin{array}{l}\text { CBT FARMS, } 2640 \text { HARPER ROAD, FEDERALSBURG } \\
21632\end{array}$ \\
\hline 136 & $E$ & $\begin{array}{l}\text { NORMAN ENNIS FAMILY, } 4213 \text { WHITESBURG ROAD, } \\
\text { POCOMOKE CITY } 21851\end{array}$ \\
\hline 81 & E & $\begin{array}{l}\text { WILLIAM GLADHILL, } 6051 \text { MT. PHILLIP ROAD, } \\
\text { FREDERICK } 21702-5947\end{array}$ \\
\hline
\end{tabular}

\begin{tabular}{|c|c|c|}
\hline 328 & $E$ & $\begin{array}{l}\text { GUNPOWDER GAME FARM, } 17904 \text { GUNPOWDER ROAD } \\
\text { HAMPSTEAD } 21074\end{array}$ \\
\hline 223 & $E$ & $\begin{array}{l}\text { KENNETH E. KADLEC, JR., } 5626 \text { SHARON DRIVE, GLEN } \\
\text { ARM } 21057\end{array}$ \\
\hline 42 & $E$ & $\begin{array}{l}\text { CHRIS/JASON KEPLINGER, } 655 \text { LONG CORNER ROAD, } \\
\text { MT. AIRY } 21771\end{array}$ \\
\hline 471 & $E$ & $\begin{array}{l}\text { HOMER KINSINGER, } 5790 \text { GARRETT HIGHWAY, } \\
\text { OAKLAND } 21550\end{array}$ \\
\hline 160 & $E$ & $\begin{array}{l}\text { MATTHEWS HATCHERY \& FARM, P.O. BOX } 55 \text {, } \\
\text { OAKLAND } 21550\end{array}$ \\
\hline 326 & $E$ & $\begin{array}{l}\text { THOMAS ROACH, } 5828 \text { DEER PARK ROAD, } \\
\text { REISTERTOWN } 21136\end{array}$ \\
\hline 317 & $E$ & $\begin{array}{l}\text { PRESTON SCOTT, } 7157 \text { ZION CHURCH ROAD, } \\
\text { SALISBURY } 21804\end{array}$ \\
\hline
\end{tabular}

KIM JONES

MARYLAND DEPARTMENT OF AGRICULTURE ANIMAL HEALTH LABORATORY

P.O. BOX 2599

SALISBURY, MD 21802

Phone: (410)543-6610

Fax: $\quad(410) 543-6676$
$R 11, R 25, R 30, R 38, R 39, R 40$, R52, R87, R89, W121, W17. W18, W27, W30, W54, W74, Z109, Z22

R19, R51, R93, W11, W12, W7, W9

R22, R34, W115, W123, W128, W187, W6

A, 293

A1, R227, R34, W103, W104, W120, W134, W15

D1, R14, W114, W115, W128, W142, W262, W29, W393. W394, W396, W401, W51, W52, W552, W56, W566, W577, W6. W69, W77, W88, W89, W91

$\mathrm{Z2}, \mathrm{Z3}$

A1, B1, R30, W17, W250, W270, . W31

W11, W120, W131, W152, W282, W290, W3, W352, W37, W92

$\mathrm{Z11}, \mathrm{Z2}, \mathrm{Z3}$.

C1, E2, R10, R20, R21, R7, R77_

21

W1, W138, W176, W198 
Table 1. HATCHERIES, DEALERS, AND INDEPENDENT FLOCKS PARTICIPATING IN

THE NATIONAL POULTRY IMPROVEMENT PLAN

WATERFOWL. EXHIBITION POULTRY AND GAME BIRDS

\begin{tabular}{|c|c|c|c|c|c|}
\hline $\begin{array}{l}\text { APP. } \\
\text { NO. }\end{array}$ & $\begin{array}{l}\text { SUB- } \\
\text { PART }\end{array}$ & PARTICIPANTS NAME AND ADDRESS & $\begin{array}{l}\text { HATCHING } \\
\text { EGG } \\
\text { CAPACITY }\end{array}$ & $\begin{array}{c}\text { PRODUCTS CLASSIFIED } \\
\text { U.S. PULLORUM-TYPHOID } \\
\text { CLEAN }\end{array}$ & $\begin{array}{c}\text { ADDITIONAL } \\
\text { CLASSIFICATIONS FOR } \\
\text { WHICH PRODUCT QUALIFIED }\end{array}$ \\
\hline
\end{tabular}

347 E LEROY SELLMAN, 11212 LIBERTY ROAD, OWINGS MILLS 21117

348 E MARY ALISA SWANN, P.O. BOX 326. NEWBURG 20646

349 E THOMAS-BIORDI FAMILY, P.O. BOX 459, FULTON 20759

354 E JOE THOMPSON, 301 KENARD AVENUE, EDGEWOOD 21040

351 E BILL VILLAREAL, 295 EDGEMERE DRIVE, ANNAPOLIS 21403

E ROLAND L. VIRTS, 5327 GOLDMINE ROAD, FREDERICK 21701

277 E JAMES H. WEEKS, 512 GARRETTR, OAKLAND 21550 Phone: $301 / 334-4320$
W26, W442.

29

R10, R114, R12, R146, R15, R168, R22, R227, R30, R37, R39, R71, R81, R95, W120, W134, W17, W32, W33, W5, W56, W7

R103, R16, R188, R33, R6, R96, W30

R169, R18, R183, Z61

R110, R172, R196, R203, R6, R72, R73, W112, W327, W37. W422, W50, W58, Z110, Z20, Z204

R100, R15, R19, R20, R21, R30, R53, R54, R83, R87, W2, W74, W75

14 - MASSACHUSETTS

\section{EDWARD HAGEMAN}

MASSACHUSETTS DEPARTMENT OF AGRICULTURE

DIVISION OF POULTRY \& POULTRY PRODUCTS

251 COSWAY STREET, SUITE 500

BOSTON, MA 02114-2151

Phone: $(617) 626-1796$

Fax: (617)626-1850

Hatcheries

1282 E FULL FLIGHT GAME FARM, E. GRAY, 4 BRATTLEBORO ROAD, BERNARDSTON 01337

15 E HARDY HATCHERY, JOHN WISE AVENUE, ESSEX 01929

1284 E ANN MARIE RAPOZA, 91 BAWIEW AVENUE, BERKLEY 02779

Independent Flocks

1268 E HELEN \& RUSSELL BARNES, 114 SOUTH SPENCER ROAD. SPENCER 01562

162 E VITO BUCCO, 55 CONANT STREET, BEVERLY 01915

1274 E COVE CREEK FARM, 489 SPRING STREET, MARSHFIELD 02050

324 E TONY DIPAOLO, 3 BRADFORD ROAD, BEVERLY 01915

1296 E THOMAS DOHERTY, 104 PROVIDENCE ROAD, WESTFORD 01886

809 E RALPH GENERAZZO, 414 NORTH MAIN STREET, SHARON 02067
$20,000 \quad Z 1, Z 12, Z 27, Z 43, Z 48$

$188,000 \quad B 7, C 6, D 5, R 14$

$1,200 \quad Z 27, Z 30, Z 43, Z 48, Z 94$

W131, W17, W35.

W. Z3, Z94

W13, W17, Z11.

W588, Z

$R, Z$

W. Z2 
Table 1. HATCHERIES, DEALERS, AND INDEPENDENT FLOCKS PARTICIPATINC IN

THE NATIONAL POULTRY IMPROVEMENT PLAN

WATERFOWL, EXHIBITION POULTRY AND GAME BIRDS

\begin{tabular}{llccc}
\hline & & HATCHING & PRODUCTS CLASSIFIED \\
APP. & SUB- & PARTICIPANTS NAME AND ADDRESS & EGG & U.S. PULLORUM-TYPHOID \\
NO. PART & CAPACITY & CLEAN & CLASSIFICATIONS FOR \\
\end{tabular}

14 - MASSACHUSETTS

\begin{tabular}{|c|c|c|c|}
\hline 1276 & $E$ & $\begin{array}{l}\text { CONRAD HALBERG. HEATH STAGE, SHELBURN FALLS } \\
01370\end{array}$ & R19 \\
\hline 1297 & $\mathrm{E}$ & IDA HAYNES, 28 BOXFORD ROAD, TOPSFIELD 01983 & W208_ \\
\hline 1292 & $E$ & $\begin{array}{l}\text { H. W. HEUSMANN, } 162 \text { WESTBORO ROAD, UPTON } \\
01568\end{array}$ & W13, W207___ \\
\hline 1287 & $E$ & RANDY KUSY, 12 BRIDGE STREET, AUBURN 01501 & W \\
\hline 213 & $E$ & $\begin{array}{l}\text { BRUCE LAVIOLETTE, } 375 \text { THREE RIVERS ROAD, } \\
\text { WILBRAHAM } 01095\end{array}$ & W, W121, W24, W3, W4 \\
\hline 60 & E & $\begin{array}{l}\text { LUKASIK GAME FARM, HENRY LUKASIK, PEARL } \\
\text { STREET, SOUTH HADLEY } 01075\end{array}$ & $\mathrm{Z} 1, \mathrm{Z2}, \mathrm{Z3}, \mathrm{Z9}$ \\
\hline 1262 & $E$ & KEN MAINVILLE, 229 FLYNT STREET. PALMER 01069 & W____ \\
\hline 1061 & E & $\begin{array}{l}\text { HENRY MCGIBBON, } 262 \text { LAKEWOOD DRIVE, } \\
\text { STOUGHTON } 02072\end{array}$ & W, Z2 \\
\hline 1290 & E & $\begin{array}{l}\text { MONUMENT PHEASANT FARM, DONALD DAUNIAS, } 435 \\
\text { STAFFORD HILL ROAD, CHESHIRE } 01225\end{array}$ & $\mathrm{Z} 247, \mathrm{Z} 43$ \\
\hline 81 & $E$ & $\begin{array}{l}\text { BENJAMIN B. MORRILL. } 407 \text { LOCUST ROAD. DANVERS } \\
01923\end{array}$ & $W$ \\
\hline 373 & $E$ & $\begin{array}{l}\text { ROBERT MURPHY, } 136 \text { FISK STREET, HOLLISTON } \\
01746\end{array}$ & $\begin{array}{l}\text { W104, W134, W220, W276, } \\
\text { W36, W478 }\end{array}$ \\
\hline 1295 & $E$ & $\begin{array}{l}\text { WILLIAM H. PARKINSON, } 211 \text { OLD LITTLETON ROAD, } \\
\text { HARVARD } 01651 \\
\text { Phone: }(978) 456-8404\end{array}$ & $\mathrm{Z126}, \mathrm{Z181}, \mathrm{Z260}, \mathrm{Z} 52, \mathrm{Z70}$ \\
\hline 1298 & $\mathrm{E}$ & $\begin{array}{l}\text { PLAMONDON'S POULTRY, WILLIAM PLAMONDON, } 627 \\
\text { STATE ROAD, WESTPORT } 02790\end{array}$ & $R 222, R 247, W 189$ \\
\hline 61 & $\mathrm{E}$ & ROBERT RHODES. MILLVILLE STREET, MENDON 01756 & $R 27, W$ \\
\hline 1294 & E & KENNETH ROWE, 4 STABLE LANE, ROCKPORT 01966 & R94, W132, W39 \\
\hline 1286 & $E$ & $\begin{array}{l}\text { GUY ROY, } 780 \text { BERNIE AVENUE, WEST SPRINGFIELD } \\
01089\end{array}$ & W- \\
\hline 1288 & E & $\begin{array}{l}\text { RYDER HATCHERY, TERRY RYDER, } 72 \text { MATHEWS } \\
\text { ROAD. CONWAY } 01341\end{array}$ & $w, z$ \\
\hline 1289 & $E$ & $\begin{array}{l}\text { STONEWALL FARM, DAVID ADAMS, } 66 \text { GOLD STREET, } \\
\text { SHEWSBURY } 01545\end{array}$ & $\mathrm{Z247}$ \\
\hline 1272 & E & $\begin{array}{l}\text { WILLY TROMBLEY, } 179 \text { BABCOCK TAVERN ROAD, } \\
\text { WARE } 01082\end{array}$ & W30__ \\
\hline 1277 & $E$ & $\begin{array}{l}\text { EDWIN UPTON, } 74 \text { BIRCH LANE,P.O. BOX } 1227 \\
\text { BREWSTER } 02631\end{array}$ & R146 \\
\hline 1285 & $E$ & $\begin{array}{l}\text { WAREHAM QUAIL FARM, J.TUOMALA, } 150 \text { FEARING } \\
\text { HILL ROAD, WAREHAM } 02571\end{array}$ & 227 \\
\hline 592 & $E$ & $\begin{array}{l}\text { O. FAYNE WHITNEY, } 352 \text { TWITCHELL STREET, ATHOL } \\
01331\end{array}$ & W \\
\hline
\end{tabular}


Table 1. HATCHERIES, DEALERS, AND INOEPENDENT FLOCKS PARTICIPATING IN

THE NATIONAL POULTRY IMPROVEMENT PLAN

WATERFOWL, EXHIBITION POULTRY AND GAME BIRDS

$\begin{array}{ll} & \\ \text { APP. SUB- PARTICIPANTS NAME ANO ADDRESS } \\ \text { NO. }\end{array}$

$\begin{array}{cc}\text { HATCHING } & \text { PRODUCTS CLASSIFIED } \\ \text { EGG } & \text { U.S.PULLORUM-TYPHOID } \\ \text { CAPACITY } & \text { CLEAN }\end{array}$

ADDITIONAL

CLASSIFICATIONS FOR WHICH PRODUCT QUALIFIED

\section{$34-$ MICHIGAN}

GEORGE HOUSE

EXECUTIVE DIRECTOR/NPIP REPRESENTATIVE

5635 FOREST GLEN DRIVE SE

P.O. $80 \times 242$

ADA, Mi 49301

Phone: $(616) 676-5593$

Fax: $\quad(616) 676-1494$

Halcheries

503 E DON GEERILINGS, 7385 96TH AVENUE, ZEELAND 49464

31 E TOWNLINE POULTRY FARM, 4198 96TH AVENUE,

Independent Flocks

513 E JOE AGIUS, 11152 CR 653, GOBLES 49055

495 E ALEX ANCONA, 2907 EAST NORWAY LAKE ROAD, LAPEER 48446

475 E SCOTI ASSENMACHER, 9460 MT. CLEMENTS ROAD, BRIGHTON 48114

292 E JOHN BALL ZOOLOGICAL GARDENS, 1300 WEST FULTON, GRAND RAPIDS 49504

373 E JEANNIE BISSONNETTE, 1685 WEST BLACKMORE ROAD, MAYVILLE 48744

208 E JOHN W. BLEHM, 4599 LANGE, BIRCH RUN 48415

471 E DOUGLAS BOLT, 7942 NORTH RANSOM ROAD WHEELER 48662

474 E ROBERT BOLT, 211 EIGHT STREET, BRECKENRIDGE 48615

416 E JOE BOSCH, 7615 88TH AVENUE, ZEELAND 49464

492 E SPENCER BOUWER, 15 EAST MADISON CT, ZEELAND 49464

470 E TASHA BRECHT, 475 FERRIS ROAD, SUMNER 48889

480 E MEGAN \& MORGAN BURGESS, 322 NORTH PINGREE ROAD, SUMNER 48889

119 E RAY CUTLER, 3805 WASHINGTON ROAD, CARSONVILLE 48419

502 E RANDELL CZACHOWNSKI, 7753 WEST ST. CHARLES ROAD, MUMMER 48889

E DAVIS FARMS, ALLEN MARK DAVIS, 2910 CHAMBERS ROAD, CARO 48723
DR. R. M. (MICK FULTON

AVIAN PATHOBIOLIST

ANIMAL HEALTH DIAGNOSTIC LABORATORY

G-1304 VETERINARY MEDICAL CENTER

MICHIGAN STATE UNIVERSITY

EAST LANSING, MI 48824-1314

Phone: (517)353-3701

Fax: $\quad(517) 355-2152$

11,985 A2, B15, C12, D12, E2, H5, R20, R239, R7, W, Y2, X3, X6, Y1, Y3, $\mathrm{Y} 4, \mathrm{Z} 22$

400,000 B15, C12, D12, H5, R, R196, R20, R239, R7, W

R229, R247, R6, R68

Z101, Z108, Z118, Z131, Z139. Z140, Z179, Z19, Z26, Z28, Z34. $\mathrm{Z} 55$

W142, W28, W415, W588, W69, W88

$X 52, X 58, X 7, X 85, Y 45, Y 8, Y 9$

R12, R146, W96, X2, X3, Y3, $\mathrm{Z} 201, \mathrm{Z202}$

R222, W573

W142, W150, W274, W28 W289, W29, W556, W568, W569, W579, W91

W12, W142, W18, W198, W435, W58

$\mathrm{Z27}$

Z27

B1. R7, R96, W102, W121, W17 B1, R196, R262, W223, W368, W7. W76

Z18, Z24, Z28, Z48

R120, R168, R222, R53, W102, W126, W17, W265, W4

A2, B, R104, R171, R18, R20, R21, R33, R60, R71, R72, R88, R94, W1, W129, W13, W131.

W132, W166, W2, W207, W30, 


\begin{tabular}{llccc}
\hline & & HATCHING & PRODUCTS CLASSIFIED \\
APP. & SUB- & PARTICIPANTS NAME AND ADDRESS & EGG & U.S. PULLORUM-TYPHOID \\
NO. & PART & CLEAN & CLASSIFICATIONS FOR \\
\end{tabular}

\begin{tabular}{|c|c|c|}
\hline 494 & $E$ & $\begin{array}{l}\text { ROBERTA \& TOM DEAN, } 13933 \text { SIMPSON, GREENVILLE } \\
48838\end{array}$ \\
\hline 381 & $E$ & $\begin{array}{l}\text { CHARLES DESANDER, } 2525 \text { WEST PRICE ROAD, ST. } \\
\text { JOHNS } 48879\end{array}$ \\
\hline 491 & E & $\begin{array}{l}\text { JOSEPH DESANDER, } 13170 \text { EAST ROAD, MONTROSE } \\
48457\end{array}$ \\
\hline 425 & E & L.J. DONAHUE, 8305 ALGER ROAD, ITHACA 48847 \\
\hline 477 & $E$ & GLEN FREY, 9660 HUTTONLOCKER, MUNITH 49259 \\
\hline 512 & $E$ & DON GEERLINGS, 7385 96TH AVENUE, ZEELAND 49464 \\
\hline 507 & $E$ & $\begin{array}{l}\text { ROBERTA HIBBARD, } 252 \text { HIGGINS STREET, NILES } \\
49120\end{array}$ \\
\hline 140 & $E$ & $\begin{array}{l}\text { BENARD JAGER, } 11610 \text { 92ND AVENUE, WEST OLIVE } \\
49460\end{array}$ \\
\hline
\end{tabular}

400 E CHERI \& KYLE JESSUP, 4805 EAST ADAMS ROAD, BRECKENRIDGE 48615
224 E JOE JOHNSON, MICHIGAN STATE UNIVERSITY,KELLOGG BIRD SANCTUARY AUGUSTA 49012
414 E CURT JOHNSON, ROLLING HILLS PRESERVE, 17025 MCKENZIE STREET, MARCELLUS 49067
448 E PRECIOUS KLINE, 50781 FULTON ROAD, LEONIDAS 49066
504 E L \& B GAME BIRD FARM, 14825 ALMY ROAD. HOWARD CITY 49329
308 E MICHIGAN DEPT. OF NAT. RESOURCES, MASON WILDLIFE FACILITY, 1219 HAWLEY MASON 48854
465 E MICHIGAN STATE UNIVERSITY. W. PAYNE, DEPARTMENT OF MICROBIOLOGY,458 GILTNER HALL EAST LANSING 48824
469 E MID MICHIGAN ROOSTER RANCH, DAVID STANKE, 5888 DECKERVILLE ROAD, DECKER 48426
198 E EVELYN NUFFER, 7265 SOUTH GRANT STREET, THREE RIVERS 49093

W35. W370, W39, W560, W570 W58, W588, W590, W594, W96 $X 11, X 146, X 2, \times 20, x 28, x 3, x 4$ $X 5, X 9, Y 8, Z 102, Z 114, Z 115$ Z116, Z118, Z125, Z139, Z176 Z181, Z19, Z204, Z22, Z227, Z24, Z25, Z26, Z30, Z35, Z408, Z46. Z5, Z57, Z62, Z90, Z97

$R 229, R 45, \times 25, \times 64, Z 22$

N105, N129, N133, N134, N137, N155, N163, N171, N181, N65, N90, R16, R24, R89, W40, W48

N161, N181, N182, N25, N90

R11, R222, R37, W24, W3, W364, W4, W72, W74, X3

Z129, Z176, 290

$X 2, X 27, X 3, Y 1, Y 3, Y 4, Z 22$

W13, W18, W2, W3, X13, X20, Z14, Z2, Z282, Z3

$\times 109, \times 110, \times 114, \times 115, \times 123$, $\times 150, \times 28, \times 40, X 57, \times 60, \times 61$, $X 70, X 73, X 78, X 8, X 85, Y 26$, Y28, Y75, Z109, Z118, Z123. Z126, Z149, Z248, Z34, Z49, Z52. Z54, Z57, Z68, Z71

A2, B1, R1, R22, W102, W17, W4, W554, W86, Z22

W135, Z109, Z119, Z122, Z123, Z124, Z23

$N, X, Y, Z, Z 11, Z 201$

W504

Z121. Z2

$\mathrm{Z} 27$

R196

Z46.

R30, R89, W102, W11, W119, . W12, W121, W140, W164, W17, W176, W18, W24, W26, W28, W3, w33, w43, w48, w54, W570, W60, W7, W74, W75, W76, W88, W9, W94

Z24, Z282, Z36, Z38 
Table 1. HATCHERIES, DEALERS. AND INDEPENDENT FLOCKS PARTICIPATING IN

THE NATIONAL POULTRY IMPROVEMENT PLAN

WATERFOWL. EXHIBITION POULTRY AND GAME BIRDS

$\begin{array}{lll} & & \\ \text { APP. } & \text { SUB. } & \text { PARTICIPANTS NAME AND ADDRESS } \\ \text { NO. } & \text { PART }\end{array}$

$\begin{array}{cc}\text { HATCHING } & \text { PRODUCTS CLASSIFIED } \\ \text { EGG } & \text { U.S. PULLORUM-TYPHOID } \\ \text { CAPACITY } & \text { CLEAN }\end{array}$

ADDITIONAL

CLASSIFICATIONS FOR

WHICH PRODUCT QUALIFIED

\section{4 - MICHIGAN}

\begin{tabular}{|c|c|c|c|}
\hline 199 & $\mathrm{E}$ & $\begin{array}{l}\text { OLDS PHEASANT FARM, } 7579 \text { WEST } 4 \text { MILE ROAD, } \\
\text { MESICK } 49668\end{array}$ & Z121 \\
\hline 91 & $E$ & $\begin{array}{l}\text { BARBARA L. PIPER, } 2837 \text { KITTER ROAD, OSSINEKE } \\
49766\end{array}$ & $\mathrm{R} 19, \mathrm{R} 51, \mathrm{R} 54$ \\
\hline 510 & $E$ & $\begin{array}{l}\text { CODY \& DANILLE RHYNAND, } 4612 \text { NORTH RANSOM. } \\
\text { BRECKENRIDGE } 48615\end{array}$ & $\begin{array}{l}\text { B1, C1, R1, R146, R248, R7, W7, } \\
\text { W8, X147 }\end{array}$ \\
\hline 484 & $E$ & $\begin{array}{l}\text { DAVID J. RINCKEY, } 605 \text { NORTH THOMPSONVILLE } \\
\text { ROAD, BEULAH } 49617\end{array}$ & Z121. Z25.Z27. Z31 \\
\hline 460 & $E$ & $\begin{array}{l}\text { ANDREA SORENSEN, N149 HIGHWAY M-35, } \\
\text { MENOMINEE } 49858\end{array}$ & $\mathrm{R} 52, \mathrm{~W} 17, \mathrm{~W} 45, \mathrm{Y} 4 \ldots$ \\
\hline 486 & $E$ & TOM STORA, 327 135TH AVENUE, WAYLAND 49348 & $\begin{array}{l}\text { W132, W176, W242, W3, Z101, - } \\
\text { Z118, Z18, Z19, Z23, Z24, Z4, } \\
\text { Z57 }\end{array}$ \\
\hline 164 & E & $\begin{array}{l}\text { FRANCES S. STROUD, } 8550 \text { SOUTH VASSAR ROAD, } \\
\text { MILLINGTON } 48746\end{array}$ & $\begin{array}{l}\text { R20, R247, W28, W30, X2, X46, - } \\
\text { Z138 }\end{array}$ \\
\hline 508 & $E$ & T \& T BIRDS, 14089 AIRPORT ROAD, ROCK 49880 & Z125, Z129, Z265___________._. \\
\hline 315 & E & $\begin{array}{l}\text { TIM TEMPLE, } 3225 \text { WEST MCMILLAN ROAD, MUSKEGON } \\
49445\end{array}$ & W145, W176, W241, W38___ \\
\hline 296 & E & $\begin{array}{l}\text { TOWNLINE POULTRY FARM, } 419896 \text { TH AVENUE, } \\
\text { ZEELAND } 49464\end{array}$ & $\begin{array}{l}\text { A2, B, B1, B3, C, D, D6, H5, R19, } \\
R 20, R 233, R 7, W, X 2, X 3, X 6 \\
Y 1, Y 3, Y 4\end{array}$ \\
\hline 509 & $E$ & RUDY VINCENT, P.O. BOX 122, HERMANSVILLE 49847 & $\begin{array}{l}\text { Z129, Z176, Z257, Z27, Z51, Z53.. } \\
\text { Z67 }\end{array}$ \\
\hline 173 & E & $\begin{array}{l}\text { RUTH WHITNEY, } 3694 \text { WEST MADISON ROAD, ALMA } \\
48801\end{array}$ & $\begin{array}{l}\text { C1, C10, C4, C5, C6, H1, H10, } \\
\text { H12, H2, H3, H5, H6, R146, } \\
\text { R176, R195, R25, W103, W3, } \\
\text { W357, W364, W442, W573, X17, } \\
\text { Z106, Z111, Z22, Z239, Z62 }\end{array}$ \\
\hline 511 & $\mathrm{E}$ & ANGELA WOLVERTON, BOX 366, ONEKAMA 49675 & $W 6, Z 26, Z 22$ \\
\hline
\end{tabular}

41 - MINNESOTA

\section{DR. KEITH FRIENDSHUH}

BOARD OF ANIMAL HEALTH

9O WEST PLATO BLVD., SUITE 119

ST. PAUL, MN 55107

Phone: (651)296-2942

Fax: (651)296-7417

\section{Hatcheries}

574 E AMERICAN HERITAGE HUNTING CLUB, INC., 13604 625TH AVENUE, EAGLE BEND 56446

671 E RICHARD ANDREE, 105 JOHNSON STREET NE., BROWNSDALE 55918

413 E ANTLER'S \& FEATHER'S GAME FARM, 14876 GAME FARM ROAD, LITILE FALLS 56345

349
LLOYD BAUER, 24396 TH STREET SOUTH, SARTELL 56377

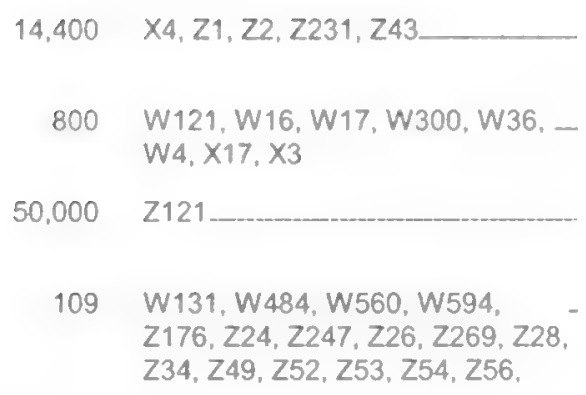
W121, W16, W17, W300, W36, $W 4, \times 17, \times 3$

50,000

Z121

109

W131, W484, W560, W594 Z176, Z24, Z247, Z26, Z269, Z28, Z34, Z49, Z52, Z53, Z54, Z56. 
Table 1. HATCHERIES, DEALERS, AND INDEPENDENT FLOCKS PARTICIPATING IN

THE NATIONAL POULTRY IMPROVEMENT PLAN

WATERFOWL, EXHIBITION POULTRY AND GAME BIRDS

\begin{tabular}{|c|c|c|c|c|c|}
\hline $\begin{array}{l}\text { APP. } \\
\text { NO. }\end{array}$ & $\begin{array}{l}\text { SUB- } \\
\text { PART }\end{array}$ & PARTICIPANTS NAME AND ADDRESS & $\begin{array}{l}\text { HATCHING } \\
\text { EGG } \\
\text { CAPACITY }\end{array}$ & $\begin{array}{l}\text { PRODUCTS CLASSIFIED } \\
\text { U.S. PULLORUM-TYPHOID } \\
\text { CLEAN }\end{array}$ & $\begin{array}{c}\text { ADDITIONAL } \\
\text { CLASSIFICATIONS FOR } \\
\text { WHICH PRODUCT QUALIFIED }\end{array}$ \\
\hline
\end{tabular}

41 - MINNESOTA

796 E LUVERNE BERGS, 28180391 ST AVENUE, HENDERSON 56044

886

$E$

BIRDS NORTH, 4885 FERN LAKE ROAD SW, BEMIDJI

56601

894

635

E

EMIL \& JANET BLOCK, 25553 COUNTY ROAD 34. PAYNESVILLE 56362

E BLUE SHADOW GAME FARM, 68176 205TH STREET, DARWIN 55324
$E$

$E$

$\mathrm{E}$

$E$

$E$

$E$

$E$

$E$

$E$

$\mathrm{E}$

$\mathrm{E}$

$E$
BOWERS' HATCHERY, 10799 STATE 64 SW., MOTLEY 56466

BRAU'S HATCHERY, 20714 COUNTY ROAD 2, BRAINERD 56401

BREHMER POULTRY, 815 50TH AVENUE SE., DEGRAFF 56271

CARRILLO'S PHEASANT FARM, 32560 593RD AVENUE. WARROAD 56763

CEDAR RIDGE MARSH, FLOWERS \& FEATHERS, 38959 TIMBER ROAD, AVON 56310

COUNTRY ACRES FARM, 3170 SW 81 ST STREET, OWATONNA 55060

CRYSTAL LAKE WATERFOWL, 18375 IPAVA AVENUE, LAKEVILLE 55044

DALIMAN'S GAME FARM, 4640 SE 158TH STREET, BLOOMING PRAIRIE 55917

DEER RIDGE GAME FARM, 5050 470TH STREET. HARRIS 55032

DOUBLE DX HATCHERY, 16549 220TH AVENUE NE, THIEF RIVER FALLS 56701

DUSTY CREEK, HAUCK FAMILY, 5900 HODGSON ROAD, SHOREVIEW 55126

FAIRCHILD FUR \& GAME FARM. 2333 120TH STREET, CANBY 56220

\section{Z57, Z71, Z77}

R37, R44, R49, R7, W12, W126, W159, W16, W38, W53, W79

1,500 R, R20, R222, R81, W, W102, W104, W11, W12, W121, W13, W131, W141, W142, W146. W152, W17, W18, W242, W29, W33, W41, W415, W43, W44. W479, W52, W568, W58, W593, W613, W69, W84, W89, X, X146. X174, X20, X6, X99, Z, Z11, Z159 Z188, Z2, Z24, Z247, Z26, Z269, Z28, Z281, Z32, Z34, Z4, Z43, Z52, Z93

600 W. X, Y, Z1, Z14, Z2, Z231, Z3, Z4

$605 \times, \times 109, \times 110, \times 113, \times 114, \times 127$, $\times 144, \times 163, \times 166, \times 167, \times 184$ $\times 188, \times 189, \times 28, \times 4, \times 42, \times 48$. $\times 49, \times 51, \times 52, \times 53, \times 57, \times 60$ $X 62, X 7, X 70, X 73, X 74, X 75$, $X 76, X 77, X 78, X 89, Y, Y 15, Y 16$ $Y 20, Y 26, Y 28, Y 29, Y 31, Y 32$ Y36, Y37, Y40, Y41, Y44, Y50, Y56, Y59, Y60, Y75, Y8, Y9, Z109, Z119, Z122, Z124, Z7

400 R, W573, W6, Z78

$220,000 \quad X_{2}, X_{3}, Y 1, Y 13, Y 6, Z 231, Z 4$

$300 \quad W 166, W 23, W 7, Z 27$

$1,053 \quad \mathrm{Z} 118, \mathrm{Z} 247$

$1,400 \times 109, \times 159, \times 184, \times 187, \times 188, \ldots$ $\times 28, \times 50, \times 53, \times 57, \times 58, \times 60$ $X 61, X 62, X 7, X 72, X 73, X 77$, $X 78, X 79, X 8, X 85, X 89, Y 27$. Z119. Z28

1,200 R11, R12, R7, R81, W117, W38, W53, W538, W54, W56. W587, W619, W620

$850 \times 187, \times 52, \times 53, \times 54, \times 57, \times 61$, $\times 62, \times 7, \times 85$

$3,000 \quad Z 108, Z 18, Z 24, Z 26, Z 28, Z 43, \ldots$ $\mathrm{Z} 48, \mathrm{Z} 57$

$27,000 \quad Z 27$ WHICH PRODUCT QUALIFIED 
Table 1. HATCHERIES, DEALERS, AND INDEPENDENT FLOCKS PARTICIPATINGIN

THE NATIONAL POULTRY IMPROVEMENT PLAN

WATERFOWL, EXHIBITION POULTRY AND GAME BIRDS

\begin{tabular}{|c|c|c|c|c|c|}
\hline $\begin{array}{l}\text { APP. } \\
\text { NO. }\end{array}$ & $\begin{array}{l}\text { SUB- } \\
\text { PART }\end{array}$ & PARTICIPANTS NAME AND ADDRESS & $\begin{array}{l}\text { HATCHING } \\
\text { EGG } \\
\text { CAPACITY }\end{array}$ & $\begin{array}{l}\text { PRODUCTS CLASSIFIED } \\
\text { U.S. PULLORUM-TYPHOID } \\
\text { CLEAN }\end{array}$ & $\begin{array}{c}\text { ADDITIONAL } \\
\text { CLASSIFICATIONS FOR } \\
\text { WHICH PRODUCT QUALIFIED }\end{array}$ \\
\hline
\end{tabular}

229 E FORSGREN'S PHEASANT FARM, INC., 24878 TRILAKES DRIVE. PELICAN RAPIDS 56572

Phone: (218) $863-8803$

895 E GEOBAR GAME \& EXOTIC BIRDS, 19100310 TH STREET, NEW PRAGUE 56071

620 E GIESE WATERFOWL HATCHERY, 2550 50TH STREET SW. APPLETON 56208

861 E RONALD GILBERTSON, 35419 300TH AVENUE, DENT 56528

343 E H\&R SHOOTING PRESERVE, 34934 140TH AVENUE. AVON 56310

304 E FRED HATTEN, 41027 COUNTY ROAD 11, BIRD ISLAND 55310

291 E HEMKER WILDLIFE PARK, P.O. BOX 262, 26715 CO. ROAD 39, FREEPORT 56331

Phone: (320)836-2426

272 E HENSON'S GAME FARM, 263 CALDER AVENUE SE., BUFFALO 55313

734 E HILL COUNTRY GAME FARM, 11146 355TH AVENUE, ASHBY 56309

665 E JEFFREYL. HINES, 4578 36TH AVENUE NW., HACKENSACK 56452

897 E HUS-FEATHER POULTRY, 44676 330TH STREET, GAYLORD 55334

585 E JOHN'S HATCHERY, JOHN PEARSON, 36800 HUNGRY LAKE LANE, FRAZEE 56544

876 E JEFF JOHNSON, 65358 355TH STREET. WATKINS 55389

637 E JOHNSON'S WATERFOWL, 36882 160TH AVENUE NE, MIDDLE RIVER 56737

297

LEROY M. KEHRER, 21280 DODD BLVD., LAKEVILLE 55044

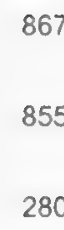

\section{$E$}

ROBERT \& BARBARA KIEWEL, 25305 LAREDO AVENUE, BELLE PLAINE 56011

E LAKEVIEW FARMS, 17626 STATE HIGHWAY 22. GLENCOE 55336

$E$

\begin{tabular}{|c|c|}
\hline 107,000 & Z1. Z2. Z9__. \\
\hline 2,300 & $\begin{array}{l}\text { W, X14, X22, X3, X4, X97, Y1, } \\
Y 13, Y 2, Y 6, Y 9, Z, Z 1, Z 118, \\
Z 131, Z 136, Z 188, Z 2, Z 24, Z 266, \\
Z 27, Z 288, Z 34, Z 43, Z 49, Z 53 \\
Z 56, Z 9\end{array}$ \\
\hline 17,830 & $\begin{array}{l}X_{1}, X_{135}, X_{2}, X_{27}, X_{3}, X_{4}, X_{6},- \\
X_{99}, Y_{1}, Y 2, Y 3, Y 7\end{array}$ \\
\hline 400 & $\begin{array}{l}\text { W121, W13, W166, W17, W23, } \\
\text { W25, W26, W3, W566, W58, W7, } \\
\text { X187, X28, X53, Y9, Z11, Z19, } \\
\text { Z24, Z244, Z26, Z27, Z28, Z283; } \\
\text { Z3, Z43, Z57, Z79 }\end{array}$ \\
\hline 7.400 & Z27 \\
\hline 1.000 & $\begin{array}{l}\text { R19, R237, W11, W12, W38, } \\
\text { W442, W5, W54, W94 }\end{array}$ \\
\hline 1,500 & $\begin{array}{l}X 109, \times 115, X 133, X 149, X 159, \\
\times 172, X 187, X 188, X 28, X 4, X 40, \\
X 52, X 53, X 57, X 58, \times 60, X 62, \\
X 7, X 74, X 77, X 78, X 79, X 85, \\
X 88, Y 28, Y 39\end{array}$ \\
\hline 1.000 & $\begin{array}{l}X 187, X 28, X 40, X 52, X 53, X 57,- \\
X 60, X 61, X 7, X 77, Y 9\end{array}$ \\
\hline 1,900 & Z27, Z94_.......... \\
\hline 3,750 & \\
\hline
\end{tabular}

600 W117, W24, W3, W36, W38, W4, W5, W54, W94, Z202, Z203. Z204, Z205, Z207

$8,500 \quad Z 27, Z 281$

3,500 Z129, Z67

$9,900 \times 10, \times 104, \times 106, \times 107, \times 131$ $\times 135, \times 136, X 143, \times 17, \times 199, \times 2$ $\times 22, \times 23, X 25, X 26, X 3, X 33$ $X 35, X 5, X 9, X 96, X 99, Y 10, Y 2$ $Y 22, Y 53, Y 6, Y 64, Y 65, Y 7, Y 70$, Y72, Y9

$500 \times 110, \times 114, \times 121, \times 149, \times 187$, $\times 28, \times 40, \times 48, \times 49, \times 51, \times 52$ $\times 53, \times 54, \times 57, \times 60, \times 61, \times 62$. $X 7, X 70, X 72, X 73, X 74, X 75$, $X 76, X 77, X 78, X 8, X 85, X 89, Y$, $Y 20, Y 9$

80 R104, R146, R222, R70, W132, W442, W573

$2,000 \quad R, W, X 2, X 3, X 99, Y 4$

800 W102, X114, X117, X118, X127, $\times 146, \times 149, \times 165, \times 171, \times 172$. $X 184, \times 187, \times 189, \times 20, \times 28, X 3$, $X 4, X 40, X 48, X 49, \times 51, X 52$, 
Table 1. HATCHERIES, DEALERS, AND INDEPENDENT FLOCKS PARTICIPATING IN THE NATIONAL POULTRY IMPROVEMENT PLAN

WATERFOWL, EXHIBITION POULTRY AND GAME BIRDS

\begin{tabular}{lllcl}
\hline & & HATCHING & PRODUCTS CLASSIFIED \\
APP. & SUB- & PARTICIPANTS NAME AND ADDRESS & EGG & U.S. PULLORUM-TYPHOID \\
NO. PART & CLEAN & CLASSIFICATIONAL FOR & WHICH PRODUCT QUALIFIED \\
\hline
\end{tabular}

41 - MINNESOTA

$\begin{array}{lll}341 & E & \text { MIKE T. LOSS, } 5869 \text { BARBEAU ROAD, BRAINERD } 56401 \\ 809 & E & \begin{array}{l}\text { MARVIN MARKETON, } 2453 \text { COUNTY ROAD } 8 \text { SW.。 } \\ \text { WAVERLY } 55390\end{array} \\ 675 \quad E & \begin{array}{l}\text { MIDWEST GAMEBIRDS, 2424 1ST STREET SOUTH, ST. } \\ \text { CLOUD 56301 }\end{array} \\ \text { Phone: (320) 259-9774 } & \\ 858 \quad E & \begin{array}{l}\text { MINNESOTA ZOO, 13000 ZOO BLVD., APPLE VALLEY } \\ 55124\end{array}\end{array}$

LEROY MOLENCAMP 13620 BROCKTON AVENUE, ROGERS 55374

E MOUW'S HATCHERY, INC., BOX 337, LEOTA 56153

MYERS TURKEY FARM, 4893 TREE FARM ROAD, PEQUOT LAKES 56472

E TREVOR NAU, RT. 2, BOX 11, ST. JAMES 56081

STEVEN NIETFELD, 34787 COUNTY ROAD 19. BELGRADE 56312

E OAK POINT GAME FARM, 35757 COUNTY HIGHWAY 75. WADENA 56482

Phone: (218) 631-4467

E OAKWOOD GAME FARM, INC., P.O. BOX 274, 30703 HIGHWAY 169. PRINCETON 55371

Phone: (800) 328-6647

E DANIEL L. PASCHE POULTRY, 32736 180TH STREET, STARBUCK 56381-2311

E MARK PETERSON, 53683 108TH STREET, AMBOY 56010

E PHEASANT HEAVEN GAME FARM, RR 1, BOX 90 , DONNELLY 56235

Phone: (320)589-4846

E PHELPS HATCHERY, 18084 280TH AVENUE NE, GOODRIDGE 56725

E R \& M GAME FARM, 21935 COUNTY ROAD 85, OSAKIS 56360

E ADRIAN J.RADEMACHER, 9185 CO. ROAD 10 EAST. WACONIA 55387

E DALE RADEMACHER \& FAMILY, 8250 PAUL AVENUE, WACONIA 55387

E RED OAK WILD BIRD FARM, 15195 BARTON AVENUE NW., MONTICELLO 55362

$\times 53, \times 54, \times 57, \times 58, \times 59, \times 60$ $x 61, x 62, x 7, x 70, x 71, x 73$ $X 74, X 75, X 76, X 77, X 78, X 79$ $X 88, X 89, Y 15, Y 16, Y 26, Y 28$ $Y 31, Y 32, Y 39, Y 40, Y 41, Y 59$ Y7, Y75, Y8, Y9, Z118, Z122,

Z262, Z27, Z57, Z7

600 W, X, Y, Z11, Z3, Z7, Z94

250 W103, W11, W20, W255, W58, W634, W635, W636, W642 W644, W80, X1, X12, X17, X18, $\mathrm{X} 2, \mathrm{Y} 4, \mathrm{Z} 22$

$6,000 \quad Z 108, Z 118, Z 24, Z 48$

2,200 R12, R13, R196, R20, R225. R227, R237, R38, R42, R57, R81, $\mathrm{X}, \mathrm{X} 125, \mathrm{X} 184, \mathrm{X} 187, \mathrm{X} 189, \mathrm{X} 28$, $X 54, X 57, X 58, X 61, X 7, X 76$. $X 77, Y 20, Y 39, Z 122, Z 126, Z 175$. Z2, Z28, Z57, Z9, Z94

500 W. W3, W30, W4, W527, W94

318 Z204, Z205, Z209, Z211, Z230

550 R168, R224, W112, W442, W594.

$480 \quad \mathrm{Z102}, \mathrm{Z118}, \mathrm{Z24}, \mathrm{Z27}, \mathrm{Z} 57$

$16,000 \quad X 4, Z 1, Z 231, Z 27, Z 43$

$607,760 \quad Z 27, Z 94$

400 R, W18, W20, W327, W573, W58, W74, W75, X3, X96, Z28

700 R19, R9, W121, W17

$4,500 \quad X 4, Z 1, Z 2, Z 3$

440 W112, W223, W33, Z118, Z14, Z27. Z43

$22,000 \quad \mathrm{Z} 27, \mathrm{Z30}$

$900 \mathrm{R} 183, \mathrm{R} 247, \mathrm{R} 3, \mathrm{~W} 3, \mathrm{~W} 54$

500 R237, R38, W119, W5, W576, W58, W6, W7

$28.665 \quad \mathrm{Z} 27$ 
Table 1. HATCHERIES, DEALERS, AND INDEPENDENT FLOCKS PARTICIPATING IN

THE NATIONAL POULTRY IMPROVEMENT PLAN

WATERFOWL, EXHIBITION POULTRY AND GAME BIRDS

\begin{tabular}{|c|c|c|c|c|c|}
\hline $\begin{array}{l}\text { APP. } \\
\text { NO. }\end{array}$ & $\begin{array}{l}\text { SUB- } \\
\text { PART }\end{array}$ & PARTICIPANTS NAME AND ADDRESS & $\begin{array}{l}\text { HATCHING } \\
\text { EGG } \\
\text { CAPACITY }\end{array}$ & $\begin{array}{c}\text { PRODUCTS CLASSIFIED } \\
\text { U.S. PULLORUM-TYPHOID } \\
\text { CLEAN }\end{array}$ & $\begin{array}{c}\text { ADDITIONAL } \\
\text { CLASSIFICATIONS FOR } \\
\text { WHICH PRODUCT QUALIFIED }\end{array}$ \\
\hline
\end{tabular}

893 E ROOSTER RANCH, WAYNE AVELSGARD, $727550 T H$ STREET WEST, WEBSTER 55088

426 E RUSHHAVEN PHEASANT CREST, 26475 COE AVENUE, FARIBAULT 55021

\begin{tabular}{|c|c|c|}
\hline 819 & $E$ & $\begin{array}{l}\text { DONALD J. RYAN, } 1555 \text { 245TH STREET EAST, NEW } \\
\text { PRAGUE 56071 } \\
\text { Phone: (952) 758-4007 }\end{array}$ \\
\hline 505 & E & $\begin{array}{l}\text { KENNETH SCHLOTFELDT, } 9216 \text { 14TH STREET SOUTH, } \\
\text { MOORHEAD 56560-7714 } \\
\text { Phone: }(218) 233-5977\end{array}$ \\
\hline 816 & $E$ & $\begin{array}{l}\text { DALE SCHMIDT, } 4716 \text { ATHENS TRAIL, NORTH BRANCH } \\
55056 \\
\text { Phone: (651) } 674-5893\end{array}$ \\
\hline 583 & $E$ & SCHULE'S GAME FARM, 7307 LENT TRAIL, STACY 55079 \\
\hline 310 & $E$ & $\begin{array}{l}\text { SCHULTE HATCHERY, } 2242320 \text { TH AVENUE, MARSHALL } \\
56258 \\
\text { Phone: (507) 532-2893 }\end{array}$ \\
\hline
\end{tabular}

852 E SILKER GAME FARM, 85641 490TH STREET, HECTOR 55342 $\begin{array}{ll}782 \text { E } & \text { SMJ RANCH, } 26831 \text { US HIGHWAY 2, GRAND RAPIDS } \\ 55744\end{array}$

849 E SOME EXOTICS/MARK LAURICH, 3078 120TH STREET, BARNESVILLE 56514

887 E PHILIP SPELTZ, BOX 99, RR 1, ALTURA 55910

347 E STEINHAUER HATCHERY, 16782 290TH STREET NE, THIEF RIVER FALLS 56701

491 E STENDER'S GAME FARM, 5835 VEGA AVENUE, NEW GERMANY 55367-9515

317 E PETER STIER, 3221 COUNTY ROAD 8, SE., ST. CLOUD 56304

672 E JEFF SYMANIETZ, 39443 185TH AVENUE, AVON 56310

739 E TACHENY WILDLIFE, 25195 PILLSBURY AVENUE。 LAKEVILLE 55044

615

842 E TANK POULTRY AND WATERFOWL, 9335 160TH AVENUE NORTH, FELTON 56536

609 E THOMPSON HATCHERY, 2791 8TH STREET SW, BAUDETTE 56623

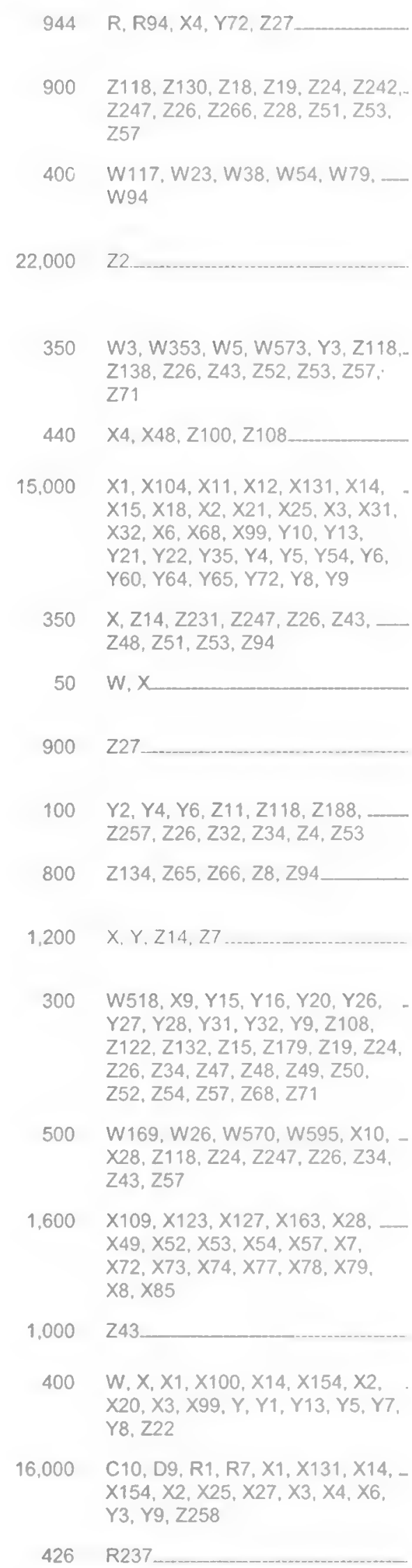

500 W169, W26, W570, W595, X10 X28, Z118, Z24, Z247, Z26, Z34. Z43, Z57

$1,600 \times 109, \times 123, \times 127, \times 163, \times 28$, $\times 49, \times 52, \times 53, \times 54, \times 57, \times 7$ $X 72, \times 73, x 74, x 77, \times 78, \times 79$ $\times 8, \times 85$

$1,000 \quad 243$

$400 \quad W . X, X_{1}, X_{100}, X_{14}, X_{154}, X_{2}$ $X 20, X 3, X 99, Y, Y 1, Y 13, Y 5, Y 7$. YB, Z22

$16,000 \quad$ C10, D9, R1, R7, X1, X131, X14, $X 154, X 2, X 25, X 27, X 3, X 4, X 6$. Y3, Y9, Z258

$426 \quad \mathrm{R} 237$ 
Table 1. HATCHERIES, DEALERS, AND INDEPENDENT FLOCKS PARTICIPATING IN

THE NATIONAL POULTRY IMPROVEMENT PLAN

WATERFOWL, EXHIBITION POULTRY AND GAME BIRDS

\begin{tabular}{llccc}
\hline & & HATCHING & PRODUCTS CLASSIFIED \\
APP. & SUB- & PARTICIPANTS NAME AND ADDRESS & ADDITIONAL \\
NO. PART & & EGG & U.S. PULLORUM-TYPHOID \\
CLEAN & CLASSIFICATIONS FOR \\
\hline
\end{tabular}

41 - MINNESOTA

871 E TWIN PINES HATCHERY, 36879207 TH AVENUE, CLARISSA 56440 815 E TWIN PINES ENT.. 4748 ATHENS TRAIL, NORTH
BRANCH 55056

Phone: (651) 674-8577

253 E UNIVERSITY OF MINNESOTA, POULTRY TEACHING AND RESEARCH, 305 HAECKER HALL, 1364 ECKLES AVE. ST. PAUL 55108-6119

DUANE URCH, 2142 NW. 47TH AVENUE, OWATONNA 55060-1071 Phone: (507) 451-6782

\footnotetext{
740 E TIMOTHY VAN BEUSEKOM, 6844 SE FENNER AVENUE, DELANO 55328

WILD PLUM WATERFOWL, 32765 HIGHWAY 58 BLVD., RED WING 55066

504 E WOODLAND ACRES KENNEL \& GAME FARM, 121 WOODLAND AVENUE. FAIRMONT 56031

Dealers

AG. PARTNERS COOP., 89 MAIN STREET, P.O. BOX 38. WANAMINGO 55983 STREET, P.O. BOX 467, LAKE CITY 55041

690 E AG. PARTNERS CO-OP.., 216 WEST DAKOTA STREET, CANNON FALLS 55009-0308

689 E AG.PARTNERS CO-OP.. 401 GREAT WESTERN AVENUE, BELLECHESTER 55027

641 E AG. SPECIALISTS. INC., P.O. BOX 366, 213 WEST 13TH STREET, ST. CHARLES 55972

AMPI AG. SERVICE, 330 EAST 10TH STREET, BOX 100 , GLENCOE 55336

$E$ ANOKA IND. GRAIN \& FEED, 2315 2ND AVENUE NORTH, ANOKA 55303

E ANOKA-RAMSEY FARM \& GARDEN CENTER, 7435 HIGHWAY 10 WEST. RAMSEY 55303

E BECKER PET \& GARDEN, INC., 204 RUDD STREET, DETROIT LAKES 56501
}

15 W12, W131, W142, W169, W223, W31, W52, W527, W570. W574, W595, X14, X25, X99. Z108, Z4

$1,200 \quad W, X, X 141, X 142, \times 166, X 167,-$ $X 187, X 28, X 58, X 85, Y 16, Y 26$ $Y 9, Z 101, Z 108, Z 109, Z 128$, Z131, Z137, Z138, Z19, Z260, $\mathrm{Z} 270, \mathrm{Z} 274, \mathrm{Z28}, \mathrm{Z41}, \mathrm{Z} 50$

31,055

2,900 R, R105, R109, R11, R115, R13, R139, R14, R15, R16, R181. R186, R192, R195, R196, R199. R213, R22, R222, R225, R23, R25, R257, R258, R30, R31, R35, R37, R38, R40, R41, R42, R49. R51, R52, R53, R64, R72, R76, R78, R81, R86, R87, R88, R92, R97, W, W102, W104, W106, W12, W121, W126, W13, W131, W146, W169, W18, W187. W223, W316, W33, W393. W548, W55, W570, W83, W9, X20, Y, Y2, Y22, Y57, Y6, Y72, $Y 8, Z 61$

$450 \mathrm{R} 136, \mathrm{R} 15, \mathrm{R} 208, \mathrm{~W} 20, \mathrm{X} 10$, $\mathrm{X} 143, \mathrm{X} 17, \mathrm{X99}, \mathrm{Y} 4$

$122,200 \times 2, \times 3, X 4, Z 100, Z 27, Z 43, Z 9$, $\mathrm{Z} 94$

$400 \quad \mathrm{X} 1, \mathrm{X} 10, \mathrm{X} 12, \mathrm{X} 136, \mathrm{X} 137, \mathrm{X} 14$, $X 146, X 17, X 18, X 32, Y 2, Y 4, Y 6$

$41,000 \quad$ Z2, Z43, Z94

C

C\&P $R, X, Y, Z 9$

C R, W, X, Y, Z2

$\times, 2231$

C

C $X, X 4, Y, Z 2, Z 9$

A, C, W96, X2, X3, X4, Y1, Y13, Z2, Z202, Z3, Z4, Z61

A, C, R, W, X, Y, Z

C A, C, R, W, X, Y, Z2, Z4 
Table 1. HATCHERIES, DEALERS, AND INDEPENDENT FLOCKS PARTICIPATING IN

THE NATIONAL POULTRY IMPROVEMENT PLAN

WATERFOWL, EXHIBITION POULTRY AND GAME BIRDS

\begin{tabular}{|c|c|c|c|c|c|}
\hline $\begin{array}{l}\text { APP. } \\
\text { NO. }\end{array}$ & $\begin{array}{l}\text { SUB- } \\
\text { PART }\end{array}$ & PARTICIPANTS NAME AND ADDRESS & $\begin{array}{l}\text { HATCHING } \\
\text { EGG } \\
\text { CAPACITY }\end{array}$ & $\begin{array}{l}\text { PRODUCTS CLASSIFIED } \\
\text { U.S. PULLORUM-TYPHOID } \\
\text { CLEAN }\end{array}$ & $\begin{array}{c}\text { ADDITIONAL } \\
\text { CLASSIFICATIONS FOR } \\
\text { WHICH PRODUCT QUALIFIED }\end{array}$ \\
\hline
\end{tabular}

41 - MINNESOTA

751 E BELLE PLAINE COOPERATIVE, 820 EAST MAIN, BELLE PLAINE 56011

405 E BEMIDJICO-OP ASSOC., 320 3RD STREET NW., P.O. BOX 980. BEMIDJI 56601-0980

384 E BENSON MARKET COMPANY, 1121 ATLANTIC AVENUE, BENSON 56215-1424

458 E BIXBY FEED MILL, INC., 10240 BIXBY LANE, BLOOMING PRAIRIE 55917

750 E BJERGA'S FEED STORES, INC., PINE RIVER, 321 WEST COUNTY ROAD \#1, PINE RIVER 56474

377 E BJERGA'S FEED STORES, INC., LITTLE FALLS, 208 NW 13TH AVENUE, LITTLE FALLS 56345

837 E BJERGA'S FEED STORES, INC., BRAINERD, 915 FRONT STREET, BRAINERD 56401

746 E BJERGA'S FEED STORES, INC., LONG PRAIRIE, P.O. BOX 134, 39 2ND STREET SOUTH, LONG PRAIRIE 56347

836 E BJERGA'S FEED STORES, INC., MOTLEY, P.O. BOX 296 , 81 WEST MAIN, MOTLEY 56466

801 E BLACKDUCK CO-OP AG. SERVICES, INC., 265 CEDAR AVENUE SE, P.O. BOX 159, BLACKDUCK 56630

469 E BOWLUS ELEVATOR, INC., 30 MAIN STREET E, P.O. BOX 68, BOWLUS 56314-0068

644 E BUFFALO QUALITY FEED MILL, 710 1ST AVENUE NE., BUFFALO 55313-1197

550 E BURKEL GRAIN SERVICE, INC., P.O. BOX J, 20463 STATE HIGHWAY 11, GREENBUSH 56726-0306

597 E BURRICHTER FARM CENTER, 207 HIGHWAY 76, BOX 487, EITZEN 55931-0487

880 E CANNON VALLEY COOP., KENYON, 806 2ND STREET, KENYON 55946

881 E CANNON VALLEY COOP., LE CENTER, P.O. BOX 67, LE CENTER 56057

CARL'S FARM STORE, INC., 301 WEST PEARL STREET. P.O. BOX 69, ADRIAN 56110 528 E CARLTON FEED MILL, INC., 340 SOUTH AVENUE, P.O.
BOX 337, CARLTON 55718

626 E CENEX HARVEST STATES, 20 FIRST AVENUE SW, LONG PRAIRIE 56347

907 E CENTRAL FEED SERVICE, INC., PRINCETON, $14067 T H$ STREET NORTH, PRINCETON 55371

579 E CHUCK'S FEED \& GRAIN, INC., 427 MILL STREET, P.O. BOX 27, PETERSON 55962

754 E CLEAR LAKE FARMERS ELEVATOR, BOX 238, 430 STATE STREET, CLEAR LAKE 55319

403 E CONDON FARM SERVICE, 106 5TH AVENUE SW., WASECA 56093

825 E COUNTRY STORE, MUNSON LAKES NUTRITION, D/B/A THE COUNTRY STORE, 510 SOO LANE BUFFALO 55313

869 E COUNTRY GREENHOUSE FEED \& GIFTS, 49274 U.S. 71. BEMIDJI 56601

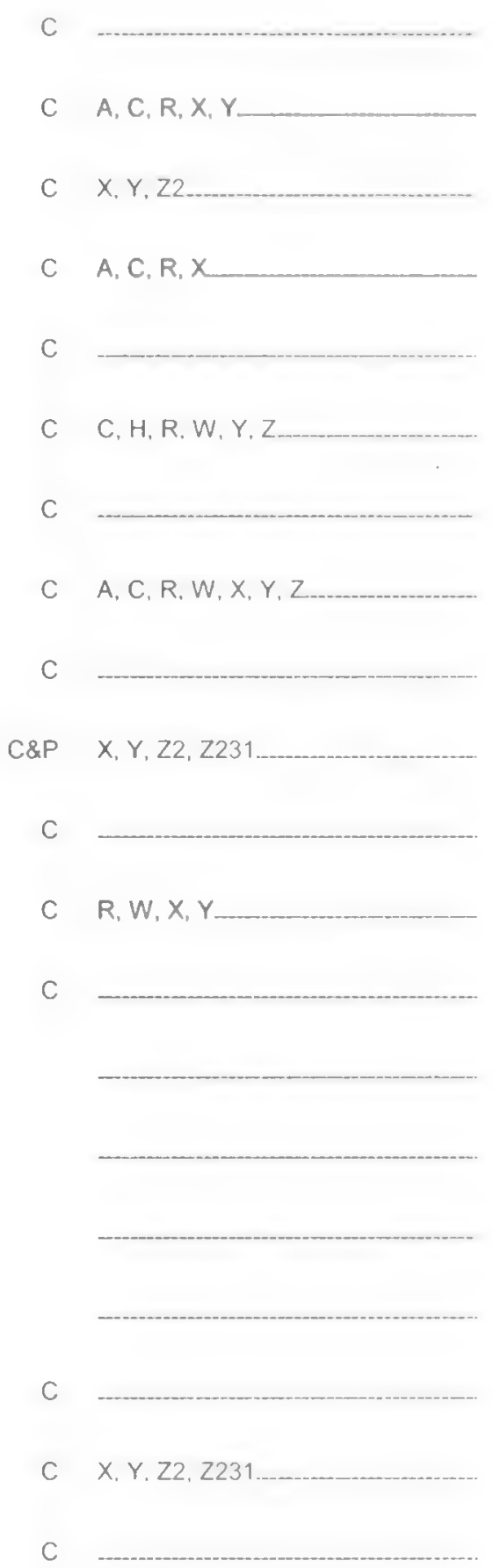

A2, C, D, R209, W, X2, X3, X4, Y1, Y3, Z212, Z27, Z4

C A2, C, D, R209.

C $\quad B, D, H, R, W, X, Y, Z, Z 2, Z 4$

C $X, Y, Z 2, Z 3$

$X, Y, Z 1, Z 14, Z 2, Z 231, Z 4, Z 43$ 
HATCHING

EGG

CAPACITY
PRODUCTS CLASSIFIED

U.S. PULLORUM-TYPHOID CLEAN
ADDITIONAL

CLASSIFICATIONS FOR WHICH PRODUCT QUALIFIED

\section{1 - MINNESOTA}

843 E COUNTRY STORE, MUNSON LAKES NUTRITION, P.O. BOX 549. HOWARD LAKE 55349

726 E COUNTRY STORE FARMERS COOP GRAIN \& SEED, BOX 525, THIEF RIVER FALLS 56701

402 E CRYSTAL CO-OP. ELEVATOR, P.O. BOX 425, 303 RAILROAD STREET, VERNON CENTER 56090

247 E CRYSTAL VALLEY, 701 E 1ST. STREET, P.O. BOX 1, JANESVILLE 56048

633 E CRYSTAL VALLEY COOP.. 51711221 ST AVENUE, ELYSIAN 56028

889 E DEER CREEK AG. (ARNESON AG., INC.), P.O. BOX 296, 300 EAST MAIN AVENUE, DEER CREEK 56527

329 E DEPOT FEEDS, INC., 109 MAIN STREET, P.O. BOX 28 SAUK CENTRE 56378

524 E DILL COMPANY, 423 2ND STREET WEST, WABASHA 55981-1279
DOANE GRAIN SERVICE, INC., 1100 WEST BROADWAY, PLAINVIEW 55964

E DOUBLEK SPECIALTY, INC., 109 4TH STREET SE., AUSTIN 55912

E EQUITY SUPPLY, INC., 2023 3RD AVENUE, MANKATO 56001

E FARM COUNTRY COOP., 417 NORTH MAIN, P. O. BOX 1037, PINE ISLAND 55963-1037

E FARMER'S CO-OP. ELEVATOR, P.O. BOX 676, 300 NORTH GRANT, HOUSTON 55943

E FARMER'S COOP. FEED STORE, 105 RAILROAD AVENUE, P.O. BOX 82, BROWERVILLE 56438

E FARMER'S FEED \& GRAIN, INC., 2020 MAHOGANY STREET, MORA 55051

E FARMER'S FEED \& PET SUPPLY, 616 NORTH CEDAR, OWATONNA 55060

E FARMER'S MILL \& ELEVATOR, INC., P.O. BOX 488, 27920 DANVILLE AVENUE. CASTLE ROCK 55010-0488

E FARMERS CO-OP ASSOCIATION-JACKSON, SHERBURN DIVISION,BOX 743. SECOND STREET WEST SHERBURN 56171

E FARMERS CO-OP. CREAMERY COMPANY, P.O. BOX 38 , 214 MAIN AVENUE, FORESTON 56330

E FARMERS CO-OP. ELEVATOR, 215 EAST FIRST STREET, MINNEOTA 56264

E FARMERS COOPERATIVE ASSOCIATION, 340 WEST MAIN, P.O. BOX 270, TRIMONT 56176

E FARMERS UNION OIL COMPANY, 1500 CENTER STREET WEST, P.O. BOX 250, ROSEAU 56751

E FARMERS UNION OIL COMPANY, P.O. BOX 48, GRYGLA 56727

E FEDERATED COOPS, INC., 303 DAHLIN AVENUE, P. O. BOX 117, ISANTI 55040

$E$
C

$X, Y, Z 2$

C B, D, H, R209, X, Y

A16, B10, D9, R209, X, Z231

$B, C, H, X, X 4, Y, Z 2, Z 3, Z 94$

C $\mathrm{B}, \mathrm{C}, \mathrm{H}$

C

C B, C, H

$B, C, H, X 2, X 3, Y 1, Y 13, Z 201, \ldots$ $\mathrm{Z202}$

C\&P $\quad X, Y, Z 231$

C,P C, D, R209, W352, X, Y, Z2

C

C $D, X, Y, Z$

C R, R196, R209, X2, X3, X4, Y1, Y13, Z2, Z202, Z227

C R, R196, R209

C $\quad R, X, Z, Z 2$

227

C R, R196, R209, X, Z, Z2.

C R, R196, R209, X, Z, Z2

c

C $X, Y, Z$

C A2, C, D, R209, X, Y, Z2 
Table 1. HATCHERIES, DEALERS, AND INDEPENDENT FLOCKS PARTICIPATING IN

THE NATIONAL POULTRY IMPROVEMENT PLAN

WATERFOWL, EXHIBITION POULTRY AND GAME BIRDS

\begin{tabular}{|c|c|c|c|c|c|}
\hline $\begin{array}{l}\text { APP. } \\
\text { NO. }\end{array}$ & $\begin{array}{l}\text { SUB- } \\
\text { PART }\end{array}$ & PARTICIPANTS NAME AND ADDRESS & $\begin{array}{l}\text { HATCHING } \\
\text { EGG } \\
\text { CAPACITY }\end{array}$ & $\begin{array}{l}\text { PRODUCTS CLASSIFIED } \\
\text { U.S. PULLORUM-TYPHOID } \\
\text { CLEAN }\end{array}$ & $\begin{array}{c}\text { ADDITIONAL } \\
\text { CLASSIFICATIONS FOR } \\
\text { WHICH PRODUCT QUALIFIED }\end{array}$ \\
\hline
\end{tabular}

474 E FEDERATED COOP., INC., 10 1ST. STREET, P.O. BOX 8. PEASE 56363

864 E FEDERATED COOP., INC., P.O. BOX 49, 825 SOUTH BREMER STREET, RUSH CITY 55069

791 E FEDERATED COOP. INC., 6467100 TH AVENUE, PRINCETON 55371

588 E FEDERATED COOPS., INC., 206 UNION STREET SOUTH, MORA 55051

860 E THE FEED STORE, 18961 STATE HIGHWAY 23, P.O. BOX 696. RICHMOND 56368

443 E FEED COMPANY, 521 WELLS STREET, BELGRADE 56312

833 E FEED STORE AND MORE, 104 HOOSAC STREET WEST, WATERVILLE 56096

534 E FIRST DISTRICT AG. SERVICE, P.O. BOX 629, 204 EAST ATLANTIC AVENUE, ATWATER 56209
FIRST DISTRICT AG. SERVICE, 25 WEST DEPOT STREET, BOX 842. LITCHFIELD 55355

FLUEGEL'S LAWN, GARDEN \& PET SUPPLY, 1278 NORTH FRONTAGE ROAD, HASTINGS 55033

FRENZEL FERTILIZER, INC., 405 RAILROAD STREET, BEMIDJI 56601

FRENZEL FERTILIZER, INC., P.O. BOX 430, BLACKDUCK 56630

E G.B. FEED AND SUPPLY, 303 GREAT WESTERN AVENUE, BELLECHESTER 55027

GAYLORD FEED MILL, INC., 109 MAIN, P.O. BOX 295 GAYLORD 55334-0295

GENEVA GRAIN SERVICES, INC. 101 3RD STREET NE., P.O. BOX 89, GENEVA 56035

E STREET, ZUMBROTA 55992

E GLACIAL PLAINS COOPERATIVE, 1008 ATLANTIC AVENUE, BOX 378, KERKHOVEN 56252

E HAMBURG FARM SUPPLY, 411 SOPHIA AVENUE HAMBURG 55339

E HAYWARD COOPERATIVE, P.O.BOX 337, HAYWARD 56043

E HEIM MILLING COMPANY, 32311 COUNTY ROAD 1, ST. CLOUD 56303

E HENNEPIN COOP. SEED EXCHANGE, 5135 OAK STREET, MAPLE PLAIN 55359

E HENNEPIN COOP. SEED EXCHANGE, 11810 BROCKTON LANE NORTH, OSSEO 55369

E HIBBING FEED \& SEED, 608 WEST 41ST STREET, HIBBING 55746

E HOLDINGFORD MILL, INC., P.O. BOX 240 , HOLDINGFORD 56340
FLUEGEL ELEVATOR, INC., 14700 SOUTH ROBERT TRAIL, ROSEMOUNT 55068
$X, Y, Z 2$

C

C\&P $\quad X, Z 231$

C

C\&P A, C, R, X, Y, Z231

C

C $X, Y$

C D, X3, Y13, Z202

D. X2, X3, Y3, Z212

C\&P

C $\mathrm{x}$

$X, Y, Z, Z 2$

C $R, X, Y$

C $\quad X, 2231$

$R, X, Z$

C B, C, H, X, Y, Z, Z231

C $\quad W, X, Y, Z 2, Z 231$

A, B, C, D, E, H, N, R, R209, W219, X, Y, Z210

$X, Y, Z 231$.

C A, B, C, D, E, H, N, R, X, Y, Z231.

C $A, B, C, D, E, H, N, R, X 2, X 3, Y 1$, $\mathrm{Y} 3, \mathrm{Z} 4$

C A, B, C, D, E, H, N, R, X, Y, Z, Z231

C

C $A, B, C, D, E, H, N, R, X 2, X 3, Y 1$. Y3, Z4 
Table 1. HATCHERIES, DEALERS, AND INDEPENDENT FLOCKS PARTICIPATING IN

THE NATIONAL POULTRY IMPROVEMENT PLAN

WATERFOWL, EXHIBITION POULTRY AND GAME BIRDS

\begin{tabular}{llccc}
\hline & & HATCHING & PRODUCTS CLASSIFIED \\
APP. SUB- & PARTICIPANTS NAME AND ADDRESS & EGG & U.S. PULLORUM-TYPHOID \\
NO. PART & CAPACITY & CLEAN & CLASSIFICATIONS FOR \\
\end{tabular}

\section{1 - MINNESOTA}

\begin{tabular}{|c|c|c|c|c|}
\hline 845 & $E$ & $\begin{array}{l}\text { HOMESTEAD MILLS, } 221 \text { NORTH RIVER STREET, COOK } \\
55723\end{array}$ & & \\
\hline 374 & $E$ & $\begin{array}{l}\text { E. J. HOULE, INC., } 55 \text { S.W. 2ND STREET, FOREST LAKE } \\
55025\end{array}$ & & $\begin{array}{l}A, B, C, D, E, H, N, R, W, X, Y, Z,- \\
Z 11, Z 4\end{array}$ \\
\hline 607 & $E$ & $\begin{array}{l}\text { HOULE'S FARM, GARDEN AND PET, } 10010 \text { NORTH } 60 T H \\
\text { STREET, STILLWATER } 55082\end{array}$ & $\mathrm{C}$ & $\begin{array}{l}A, B, C, D, E, H, N, R, W, X, Y, Z_{1-} \\
Z 11, Z 4\end{array}$ \\
\hline 389 & $E$ & $\begin{array}{l}\text { HUGO FEED MILL \& ELEVATOR COMPANY, } 5582146 \text { TH } \\
\text { STREET NORTH, BOX } 75 \text {, HUGO 55038-0075 }\end{array}$ & & $X_{2}, X_{3}, X_{4}, Y_{1}, Y 3, Z 1, Z 2, Z 3$ \\
\hline 337 & $E$ & $\begin{array}{l}\text { HUNT'S FEED AND GARDEN CENTER, } 906 \text { HIGHWAY } 10 \text {, } \\
\text { ELK RIVER } 55330\end{array}$ & & B, C, H, R209, X, Y, Z2, Z9 \\
\hline 513 & $E$ & $\begin{array}{l}\text { HUTCHINSON COOP., } 1060 \text { 5TH AVENUE SE., P.O. BOX } \\
\text { 158, HUTCHINSON } 55350\end{array}$ & & R, R196, R209, X, Z \\
\hline 803 & $E$ & $\begin{array}{l}\text { ISLE FEED \& FARM, } 450 \text { WEST MAIN STREET, P.O. BOX } \\
34, \text { ISLE } 56342\end{array}$ & $\mathrm{c}$ & $x_{4}$ \\
\hline 875 & $E$ & $\begin{array}{l}\text { J \& L FEEDS, BOX 206, HIGHWAY } 2 \text { WEST, DEER RIVER } \\
56636\end{array}$ & $\mathrm{C}$ & \\
\hline 399 & $E$ & $\begin{array}{l}\text { J \& S FARMER'S MILL, INC., } 104 \text { 5TH AVENUE SE, } \\
\text { BARNESVILLE } 56514\end{array}$ & C\&P & B, H, R209, X, Y, Z202, Z227_ \\
\hline 744 & $E$ & $\begin{array}{l}\text { KOLLOS FEEDS, } 8065 \text { TH AVENUE NORTH, ST. JAMES } \\
56081\end{array}$ & C & $X, Y, Z 231$ \\
\hline 753 & $E$ & $\begin{array}{l}\text { KRAUSE LIVESTOCK \& FEED COMPANY, } 9352 \text { SW } 37 \text { TH } \\
\text { AVENUE, P.O. BOX } 86 . \text { HOPE } 56046\end{array}$ & C\&P & \\
\hline 286 & $E$ & $\begin{array}{l}\text { KUPIETZ FEED SALES, } 120 \text { EAST 2ND STREET, } \\
\text { WINONA } 55987-3410\end{array}$ & & $x, z$ \\
\hline 872 & $E$ & $\begin{array}{l}\text { L \& M FLEET SUPPLY, PARK RAPIDS, } 1404 \text { 1ST. STREET } \\
\text { E, PARK RAPIDS } 56470\end{array}$ & C\&P & \\
\hline 873 & $E$ & $\begin{array}{l}\text { L \& M FLEET SUPPLY, CLOQUET, } 620 \text { HIGHWAY } 33 \\
\text { SOUTH. CLOQUET } 55720\end{array}$ & C\&P & \\
\hline 874 & $E$ & $\begin{array}{l}\text { L \& M FLEET SUPPLY, VIRGINIA, } 8497 \text { ENTERPRISE } \\
\text { DRIVE NORTH, VIRGINIA } 55792\end{array}$ & C\&P & \\
\hline 834 & $E$ & $\begin{array}{l}\text { L\& M SUPPLY, INC., BEMIDJI, } 2740 \text { PAUL BUNYAN } \\
\text { DRIVE NW., BEMIDJI } 56601\end{array}$ & C\&P & \\
\hline 835 & $E$ & $\begin{array}{l}\text { L \& M FLEET SUPPLY, GRAND RAPIDS, P.O. BOX } 280, \\
1200 \text { EAST HIGHWAY } 169, \text { GRAND RAPIDS } 55744\end{array}$ & C\&P & \\
\hline 896 & $E$ & L \& M SUPPLY, 3923 1ST AVENUE, HIBBING 55746 & & \\
\hline 789 & $\mathrm{E}$ & $\begin{array}{l}\text { LEAF RIVER AG. SERVICES, } 10 \text { WEST VAN AERMAN } \\
\text { STREET, BOX } 280, \text { NEW YORK MILLS } 56567\end{array}$ & C & W. W442, W578, X, Z2, Z231 \\
\hline 802 & $E$ & $\begin{array}{l}\text { LEAF RIVER AG. SERVICE, P.O. BOX } 6 \text {, COUNTY ROAD } \\
19, \text { BLUFFTON } 56518\end{array}$ & $\mathrm{C}$ & \\
\hline 640 & $E$ & $\begin{array}{l}\text { LITTLE MOUNTAIN FEED, INC., } 410 \text { MAPLE STREET, } \\
\text { P.O. BOX } 428, \text { MONTICELLO } 55362\end{array}$ & C & W. Z3 \\
\hline 659 & $E$ & $\begin{array}{l}\text { LONSDALE FEED MILL, INC., P.O. BOX } 283,209 \text { WEST } \\
\text { ASH, LONSDALE } 55046-0283\end{array}$ & $\mathrm{C}$ & $\mathrm{X}, \mathrm{Y}, \mathrm{Z231}$ \\
\hline 9 & $E$ & LOWRY HATCHERY, 28627 HIWAY 55, LOWRY 56349 & & A, X, Y, Z231 \\
\hline 703 & $E$ & $\begin{array}{l}\text { LUXEMBURG FEED SERVICE, INC., } 21967 \text { STATE } \\
\text { HIGHWAY } 15 \text { SOUTH. ST. CLOUD } 56301\end{array}$ & $\mathrm{C}$ & A. Z27 \\
\hline 339 & E & MARTIE'S FARM SERVICE, 100 DUNDAS ROAD, P.O. & C & $A, B, D, H, N, X, X 20, Y$ \\
\hline
\end{tabular}


Table 1. HATCHERIES, DEALERS, AND INDEPENDENT FLOCKS PARTICIPATING IN THE NATIONAL POULTRY IMPROVEMENT PLAN

WATERFOWL, EXHIBITION POULTRY AND GAME BIRDS

APP. SUB- PARTICIPANTS NAME AND ADDRESS

NO. PART

HATCHING
EGG
CAPACITY

PRODUCTS CLASSIFIED

U.S. PULLORUM-TYPHOID CLEAN
ADDITIONAL

CLASSIFICATIONS FOR WHICHPRODUCT QUALIFIED

\section{1 - MINNESOTA}

362 E MARTIE'S FARM SERVICE, 345 JACKSON, ELK RIVER 55330

655 E MARTIE'S FARM SERVICE, INC., 3950 227TH AVENUE NE, ST. FRANCIS 55070

798 E MARTY BROTHERS, INC., 105 GREAT NORTHERN DRIVE SW., P.O. BOX 699, BAGLEY 56621

765 E MATAWAN GRAIN \& FEED, INC., BOX 304, MINNESOTA LAKE 56068

766 E MATAWAN GRAIN \& FEED. INC., 20820 MATAWAN AVENUE, NEW RICHLAND 56072

590 E MEADOWLAND FARMERS COOP., P.O. BOX 393, 120 EAST TIN STREET. REDWOOD FALLS 56283

MEYER FARMS, 17937 290TH STREET, NEW PRAGUE 56071

MIDWEST LIVESTOCK SERVICE, 114 NORTH HIGHWAY 86, BOX 857, LAKEFIELD 56150

E MILOMA AGRI. SERVICE, DENMARK ENTERPRISES, INC.,35319 880TH STREET HERON LAKE 56137

E MINNESOTA VALLEY AG. COOP., P.O. BOX 10, 4455 40TH STREET WEST, WEBSTER 55088

E NERSTRAND AGRI. CENTERIAGP GRAIN, LTD., 415 MAIN STREET, P.O. BOX 38, NERSTRAND 55053

E NEW PRAGUE FARM CENTER, 442 WEST MAIN STREET, NEW PRAGUE 56071

E NICOLLET FARMERS EXCHANGE, 716 2ND STREET, P.O. BOX 47, NICOLLET 56074-0047

E NORTHERN COUNTRY COOP., CONGER, BOX 297 , CONGER 56020

NORTHERN COUNTRY COOP. P.O. BOX 295, ALDEN 56009

E NORTHLAND FEED \& SEED, 209 EAST 3RD STREET, PARK RAPIDS 56470

E NUTRIFLEX, INC., P.O. BOX 154, ZUMBRO FALLS 55991

E NUTRIPRO FEEDS, 501 OXFORD STREET, WORTHINGTON 56187

E OSAKIS AG SERVICE, P.O. BOX 250, 101 CENTRAL AVENUE, OSAKIS 56360

E PARK RAPIDS SEED HOUSE, $811 \mathrm{E}$ 1ST STREET, PARK RAPIDS 56470

E PERHAM CO-OP CREAMERY, 459 3RD AVENUE SE., P.O. BOX 247, PERHAM 56573

E PERHAM COOP. CREAMERY (DETROIT LAKES). 815 ROOSEVELT AVENUE, DETROIT LAKES 56501

E PERHAM GRAIN \& FEED, INC., P.O. BOX 180, $2407 T H$ AVENUE NE, PERHAM 56573

E PETERSEN SERVICE CENTER, INC. 13526 CSAH 26. HUTCHINSON 55350

E PETERSON'S NORTH BRANCH MILL, INC., 638 BRANCH AVENUE, BOX 218, NORTH BRANCH 55056

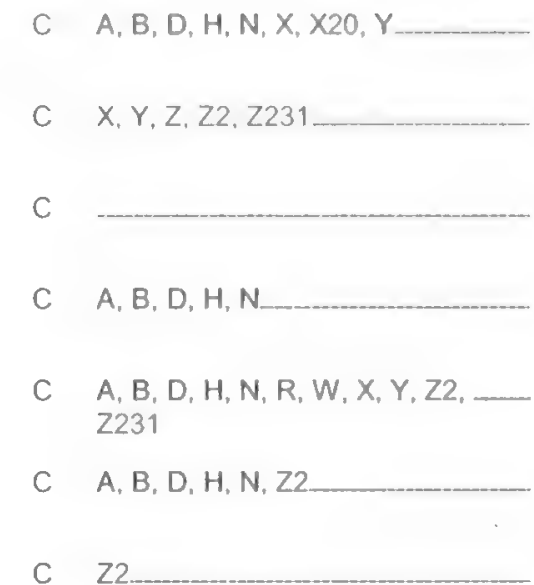

$X, Y, Z 2, Z 231$.

C A, B, D, H, N

C

C $\quad W, X, Y, Z 2$

$X, Y$

C C, D, X, Y

$\times 2, X 3, Y 13$

$X, Y$

C C , D , X, Z

C $\mathrm{R}$

C

C

C H. R

C B, C. H

C H.R

C\&P H, R

C 
Table 1. HATCHERIES, DEALERS, AND INDEPENDENT FLOCKS PARTICIPATING IN

THE NATIONAL POULTRY IMPROVEMENT PLAN

WATERFOWL, EXHIBITION POULTRY AND GAME BIRDS

$\begin{array}{lll}\text { APP. SUB- } & \text { PARTICIPANTS NAME AND ADDRESS } \\ \text { NO. PART } & \end{array}$
HATCHING

EGG

CAPACITY
PRODUCTS CLASSIFIED

U.S. PULLORUM-TYPHOID CLEAN
ADDITIONAL

CLASSIFICATIONS FOR WHICH PRODUCT QUALIFIED

\section{1 - MINNESOTA}

$729 \quad E$

PINE CITY MILL, INC., 320 3RD STREET SOUTH EAST, PINE CITY 55063

545 E PIPESTONE GRAIN COMPANY, 318 NORTH HIAWATHA AVENUE,P.O. BOX 274 PIPESTONE 56164

648 E PRAIRIE FARM SUPPLY, INC., 115 WEST CHURCH STREET, P.O. BOX 63, BELLE PLAINE 56011-0063

453 E PRO-AG FARMERS COOPERATIVE, P.O. BOX 38, 104 EAST FRONT STREET, BRANDON 56315

455 E PRO-AG FARMERS COOPERATIVE, 13 CENTRAL AVENUE SOUTH, PARKERS PRAIRIE 56361

430 E PRO-HEALTH FEEDS, INC., 710 3RD AVENUE WEST, ALEXANDRIA 56308

244 E ROBBINSDALE FARM \& GARDEN (TMC STORES), 4719 42ND AVENUE NORTH, ROBBINSDALE 55422

\footnotetext{
716 E ROCHESTER FEED \& PET SUPPLY, 3434 MARION ROAD SE, ROCHESTER 55904

807 E SCANDIA FEED AND PET, 21150 OZARK AVENUE NORTH, SCANDIA 55073

850 E SCHMITT FEED \& PRODUCE, INC., P.O. BOX 235, 503 EAST WISCONSIN AVENUE, STAPLES 56479

294 E SCHNEIDER'S SALES \& REPAIR, 714 EAST MAIN, ALBERT LEA 56007

667 E LARRY SCHULTZ, 4950 SW. 82ND AVENUE, OWATONNA 55060

E SODERBERG'S WILLOW CREEK NURSERY, 4430 HIGHWAY 63 SOUTH, ROCHESTER 55904 586 E SPRINGFIELD COOP. CREAMERY ASSOCIATION, 120
SOUTH O'CONNELL, P.O. BOX 7, SPRINGFIELD 56087

495 E ST. FRANCIS FEED, P.O. BOX 747, 3645 BRIDGE STREET, ST. FRANCIS 55070

STATE LINE FARM SUPPLY, P.O. BOX 306, 139 SOUTH MAIN, KIESTER 56051

STEVE'S FEED \& FARM SUPPLY, 709 5TH AVENUE NE, SLEEPY EYE 56085

E STILLWATER FARM STORE, 401 SOUTH MAIN STREET, STILLWATER 55082

360 E STROMBERG CHICKS \& GAMEBIRDS, INC., P.O. BOX 400. PINE RIVER 56474

759 E UNITED FARMER'S CO-OP, 2 NORTH MAIN STREET, P.O. BOX 461, WINTHROP 55396

UNITED FARMER'S CO-OP, P.O. BOX 4, 840 PIONEER AVENUE. LAFAYETTE 56054-0004 SUEUR 56058

826 E UNITED FARMERS CO-OP.. 2825 MARKET STREET, BOX UNITED FAR

C $H_{n} R$

C $H, R, X, Y$

C C, D, R

c

$X, Y, Z 2$

B, C, D, R12, R146, R19, R21, R24, R7, W, W219, X14, X2, X22, $X_{2} 5, X_{3}, X_{4}, X_{6}, X_{8}, Y 1, Y 2, Y 3$, Y4, Y9, Z202, Z204, Z205, Z207, Z212, Z22, Z230, Z27, Z43, Z61, Z62, Z9, Z94

C\&P $\quad N, W, X, Y, Z$

C\&P $\quad \mathrm{X}, \mathrm{Z2}, \mathrm{Z231}$

$X, Y, Z 2$

C $X, Y$

$A 16, B 10, D 9, R 209, X, X 2, Y, Y 1$, $\mathrm{Y} 13, \mathrm{Z} 202$

C B, D, H, R209, X

c $\mathrm{x}$

C B, D, H, R209

B. D, H, R209, X

C

$W, X, X 28, X 4, X 8, Y, Z, Z 11, Z 2$

C $H, X_{2}, X_{3}, Y_{1}, Y_{13}$

C

$X, Y$

C\&P $\quad W, X, Y, Z 2, Z 231$

W3, Z11, Z202, Z22, Z227, Z4 
Table 1. HATCHERIES, DEALERS, AND INDEPENDENT FLOCKS PARTICIPATING IN

THE NATIONAL POULTRY IMPROVEMENT PLAN

WATERFOWL, EXHIBITION POULTRY AND GAME BIRDS

APP. SUB- PARTICIPANTS NAME AND ADDRESS

NO. PART
HATCHING

EGG

CAPACITY
PRODUCTS CLASSIFIED

U.S. PULLORUM-TYPHOID CLEAN
ADDITIONAL

CLASSIFICATIONS FOR WHICH PRODUCT QUALIFIED

\section{1 - MINNESOTA}

\begin{abstract}
441
E

JPSALA CO-OP., 303 NE 6TH STREET, LITTLE FALLS

56345
\end{abstract}

832 E UPSALA CO-OP. CREAMERY ASSOCIATION, P.O. BOX 666, 21825 COUNTY ROAD 54, ALBANY 56307

632 E UPSALA COOP. ASSOCIATION, P.O. BOX 160,119 WEST ELM AVENUE, UPSALA 56384

839 E VERGAS COUNTRY STORE, 101 1ST. AVENUE NORTH, VERGAS 57587

629 E VERMILLION ELEVATOR, INC. 203 MAIN STREET,BOX 49 VERMILLION 55085

479 E WACONIA FARM SUPPLY, 801 SOUTH HIGHWAY 284. WACONIA 55387

334 W WADERICH FEED AND GARDEN.17860 PANAMA AVENUE, PRIOR LAKE 55372

747 E WATONWAN FARM SERVICE, 309 SOUTH RICE STREET, P.O. BOX 65, BLUE EARTH 56013

748 E WATONWAN FARM SERVICE, 200 WENBERG STREET, DUNNELL 56127

WATONWAN FARM SERVICE, 404 HIGHWAY 22 SOUTH, MAPLETON 56065 WEISS MILLING, INC., 104 SOUTH DUGAN STREET, BOX 315, WELCOME 56181-0315

E WEST-CON COUNTRY STORE., 975 BORDSON STREET, APPLETON 56208

E WEST-CON,P.O.BOX65, GRACEVILLE 56240-0065

E WESTERN CONSOLIDATED COOP., 121 CENTRAL AVENUE, P.O. BOX 94, SUNBURG 56289

E WINDMILL FEED \& PET SUPPLY, 350 MAIN STREET, P.O. BOX 10, NEW MARKET 55054

E ZUMBRO AG. SERVICE, 314 SOUTH MAIN STREET, BOX 628. PINE ISLAND 55963-0745

Independent Flocks

502 E B \& T GAME FARM, 12755 LAMB AVENUE, DENNISON 55018

CHARLES KAMM, RT. 1, BOX 87, COURTLAND 56021

LUNZ POULTRY, 2364 90TH STREET, FAIRMONT 56031

MEZZENGA DAGO RANCH, 33253 MEZZENGA LANE, CROSSLAKE 56442

Phone: (218) 692-1004 56263

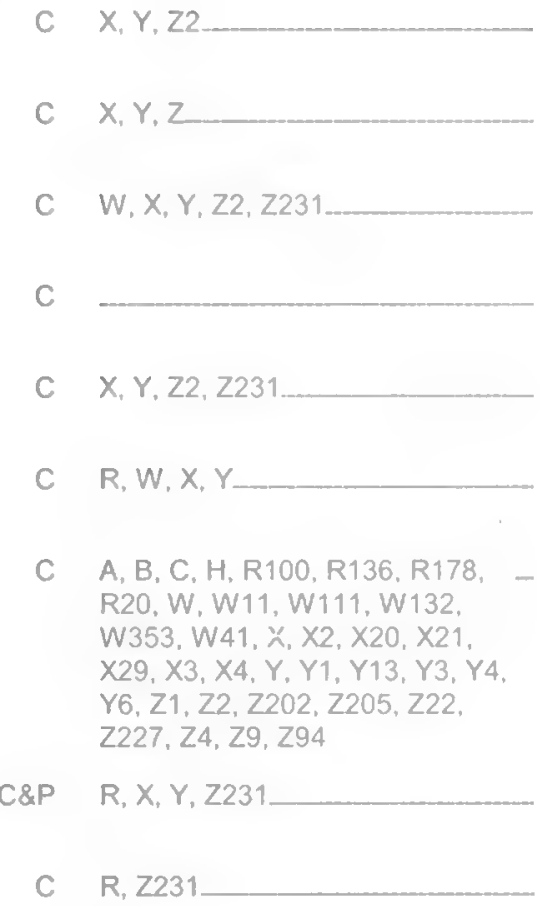

$H, X 2, X 3, Y 1, Y 13$

C A, B, C, D, H, R100, R178, R20, $X, Y, Z$

A, B, C, D, H, R100, R178, R20, $\mathrm{Z} 202$

C A, B, C, D, H, R100, R178, R20, Z202

C A, B, C, D, H, R100, R20, Z202

C

Z, Z108, Z118, Z136, Z24, Z26, Z260, Z288, Z51, Z53, Z57, Z71

$\times 109, \times 111, \times 142, \times 187, \times 28$ $\times 40, \times 52, \times 53, \times 60, \times 61, \times 62$ X79, X85, Y39, Z48

R247, W104, W38, W4, W5 W54, W7

Z118, Z24, Z26, Z34, Z51, Z53, .... Z57

227

W, W18, W33, W573, W578, W610. 24 


\begin{tabular}{|c|c|c|c|c|}
\hline $\begin{array}{l}\text { APP. } \\
\text { NO. }\end{array}$ & $\begin{array}{l}\text { SUB- } \\
\text { PART }\end{array}$ & PARTICIPANTS NAME AND ADDRESS & $\begin{array}{l}\text { HATCHING } \\
\text { EGG } \\
\text { CAPACITY }\end{array}$ & $\begin{array}{l}\text { PRODUCTS CLASSIFIED } \\
\text { U.S. PULLORUM-TYPHOID } \\
\text { CLEAN }\end{array}$ \\
\hline & & 41 & - MINNESOTA & \\
\hline 484 & E & SUNNYSIDE FARM, RR 2, BOX 119, PRESTON 55965 & & $\begin{array}{l}Y, Y 15, Y 16, Y 20, Y 26, Y 31, Y 40, . \\
Y 75, Y 9\end{array}$ \\
\hline 407 & $E$ & $\begin{array}{l}\text { WOODEN POULTRY, } 531 \text { SHORE ACRES ROAD, LA } \\
\text { CRESCENT } 55947\end{array}$ & & R44, W23 \\
\hline
\end{tabular}

$65-$ MISSISSIPPI

DANNY THORNTON

POULTRY EPIDEMIOLOGIST

4239 EBENEZER ROAD

CARTHAGE, MS 39051

Phone: (601)267-3844

Fax: (601)359-1177

Hatcheries

709

566 E AUSSIE GAME FARM, 367 ROAD 1500, BELDEN 38826

662 E RALPH BEDWELL, 232 SHADY GROVE CHURCH ROAD, FOXWORTH 39483

704 E BELL GAMEFOWL FARM, 77 COUNTY ROAD 43, DENNIS 38838

Phone: (662) $438-7698$ $\begin{array}{ll}518 \text { E } & \text { BLUE MOUNTAIN GAME FARM, P.O. BOX 191, BLUE } \\ \text { MOUNTAIN } 38610\end{array}$

642 E ALLEN BOUNDS GAME FARM, 268 COUNTRY ESTATES ROAD, FLORENCE 39073

710 E FRED BOYKIN, 12376 HIGHWAY 37 NORTH, TAYLORSVILLE 39168

689 E DAVID BRADY, 3000 COUNTY ROAD 408, FALKNER 38629

262 E BRADY FOWL FARM, RR 4, BOX 156, RIPLEY 38663

263 E BROOKHAVEN GAME BIRD FARM, 788 MT, ZION ROAD, WESSON 39191

615 E CALCOTE GAME FOWL FARM, RT. 1, BOX 87-E, SARA 38665

519 E CEDAR HILL GAME FARM, 1200 A COUNTY ROAD 805, BLUE MOUNTAIN 38610

514 E CHANDLER'S QUAIL FARM, RT. 1, BOX 11, WOODLAND 39776

507 E CLEAR CREEK QUAIL FARM, 156 OILFIELD ROAD, BAXTERVILLE 39455

446 E COAST QUAIL FARM, 5188 CC ROAD, SAUCIER 39574

678 E COUNTRY FARMS QUAIL, 4254 DICKERSON SAWMILL ROAD, LUCEDALE 39452
DR. JIM WATSON

STATE VETERINARIAN

MISSISSIPPI BOARD OF ANIMAL HEALTH

P.O. BOX 3889

JACKSON. MS 39207

Phone: (601)359-1170

Fax: (601)359-1177
ADDITIONAL

CLASSIFICATIONS FOR WHICH PRODUCT QUALIFIED 
Table 1. HATCHERIES, DEALERS, AND INDEPENDENT FLOCKS PARTICIPATING IN

THE NATIONAL POULTRY IMPROVEMENT PLAN

WATERFOWL, EXHIBITION POULTRY AND GAME BIRDS

APP. SUB- PARTICIPANTS NAME AND ADDRESS

NO. PART

\section{HATCHING \\ EGG}

CAPACITY
PRODUCTS CLASSIFIED

U.S. PULLORUM-TYPHOID CLEAN
ADDITIONAL

CLASSIFICATIONS FOR WHICH PRODUCT QUALIFIED
CRAWFORD FARMS, BOX 1017, WOODVILLE 39669

CYPRESS CREEK GAME BIRD FARM, RR 1, BOX 137. ASHLAND 38603

D AND J GAMEBIRD FARM, 6479 HIGHWAY 570, SMITHDALE 39664

654 E LEROY DERICK, HIGHWAY 4 EAST \#22591, RILEY 38663

655 E DOC MAUNEY, 7911 CR 500, RIPLEY 38663

693 E CARLOS \& JUSTIN DOUGLAS, 963 HIGHWAY 583 S.E., BROOKHAVEN 39601

694 E ART DOWD, 60 MARCUS MITCHELL ROAD, PICAYUNE 39466

405 E DRY CREEK FARM, RR 3, BOX 235, BOONEVILLE 38829

669 E WANDA ELLIS QUAIL FARM, BOX 208, BUCKINGHAM STREET, OKOLONA 38860

673 E FAMILYTRADITIONS, 123 MERRITT ROAD, LUCEDALE 39452
FULL FLIGHT GAMEBIRD FARM, PICKERING, P.O. BOX 453, COLLINS 39428

GRAY EAGLE FOWL FARM, 1415 WEST CHAMBERS DRIVE, BOONEVILLE 38829

E GULF HAVEN GAME FARM, 2544 SOUTH SHORE DRIVE, BILOXI 39532

E CLAUDE HAMMONS, 183 HURRICANE CREEK ROAD, SANDY HOOK 39478

E JOHNNY HOLDENESS QUAIL FARM, 3366 MIDDLETON ROAD, LOUISVILLE 39339

E RAY HOLMES, HOUSE 1020 CR603, RIPLEY 38663

E HOOPER GAME FARM, 16250-A HIGHWAY 2 EAST, RIPLEY 38663

E HWY 14 GAME FARM, RT 1, BOX 118, MCCOOL 39108

E RUDYIBELE, 12167 SPIKES ROAD, SAUCIER 39574

E L. H. PIERCE, RT. 5, BOX 336, LUCEDALE 39452

E

CHARLES D. LEWELLEN GAME FARM، 351-8 CRD 540 RIPLEY 38663

E MATTHEW LOTT, 214 SPRINGHILL DRIVE, HATTIESBURG 39402

E FRED LUSK,P.O.BOX 242, BILOXI 39533

E MISSISSIPPI COMBINE, E. GOBER, P.O. BOX 625 , CRYSTAL SPRINGS 39059

E MOORE \& SON POULTRY FARM, 580 SPARKS ROAD, LAKE CORMORANT $3864^{\circ}$

E MEDFORD NOLES, RT. 2, BOX 204, NETTELTON 38838

E
J.D. O'NEAL, 37 CR 572, RIENZI 38865

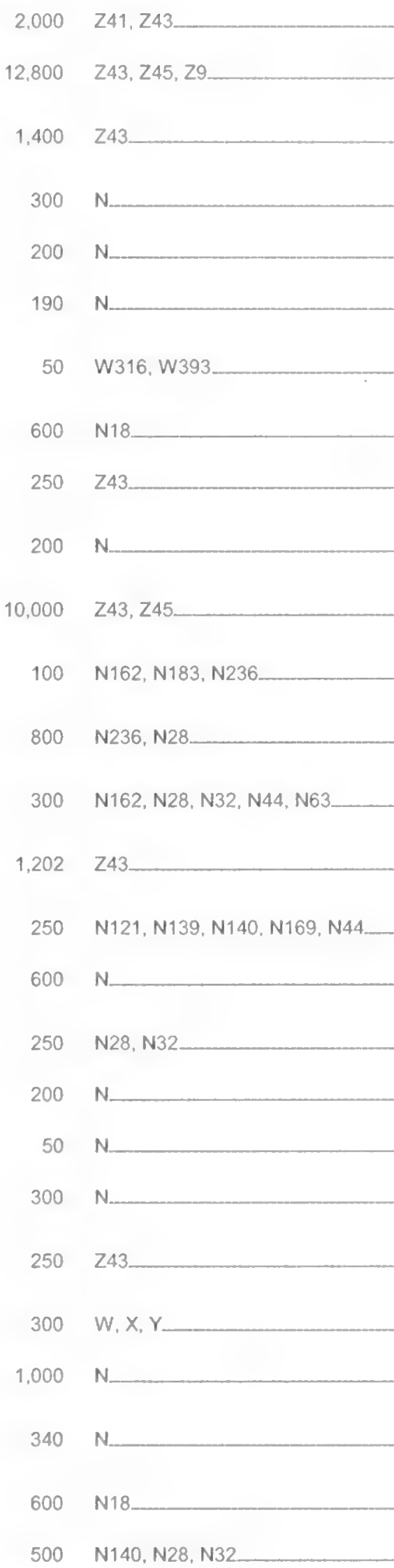




$\begin{array}{lll}\text { APP. } & \text { SUB- } \\ \text { NO. PARTICIPANTS NAME AND ADDRESS } & \text { PART }\end{array}$

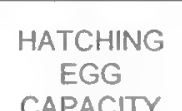

EGG
CAPACITY
PRODUCTS CLASSIFIED

U.S. PULLORUM-TYPHOID CLEAN
ADDITIONAL

CLASSIFICATIONS FOR WHICH PRODUCT QUALIFIED

\section{5 - MISSISSIPPI}

\begin{tabular}{|c|c|c|c|c|}
\hline 568 & E & $\begin{array}{l}\text { OAK GROVE GAME GARM, P.O. BOX } 213 . \\
\text { PHILADELPHIA } 39350\end{array}$ & 1.000 & N121. N161 \\
\hline 340 & E & $\begin{array}{l}\text { OAK HILL QUAIL FARM, } 79 \text { SAM SMITH ROAD, } \\
\text { POPLARVILLE } 39470\end{array}$ & 43,200 & Z43 \\
\hline 506 & E & $\begin{array}{l}\text { OAKRIDGE GAME FOWL FARM, P.O. BOX } 411, \\
\text { BELMONT } 38827 \\
\text { Phone: } 601 / 454-7328\end{array}$ & 100 & $N$ \\
\hline 695 & E & $\begin{array}{l}\text { OLD PEARL GAME FARM, } 210 \text { COUNTRY ESTATES, } \\
\text { FLORENCE } 39073\end{array}$ & 100 & N236, N28, N33 \\
\hline 106 & $E$ & $\begin{array}{l}\text { PEE JAY GAME BIRD FARM, 14081-A HIGHWAY } 4 \text {, } \\
\text { RIPLEY } 38663\end{array}$ & 1,027 & N56, N57 \\
\hline 656 & $E$ & $\begin{array}{l}\text { PIPEDREAM GAME FOWL FARM, } 3767 \text { SPRING RIDGE } \\
\text { ROAD, RAYMOND } 39154\end{array}$ & 360 & N183, N28, N32, N33, N41, N44 \\
\hline 480 & $E$ & $\begin{array}{l}\text { PLEASANT HILL QUAIL FARM, RR 1, BOX } 183 \text {, } \\
\text { LEAKSVILLE } 39451\end{array}$ & 18,000 & $\mathrm{Z1}, \mathrm{Z43}$ \\
\hline 454 & $E$ & $\begin{array}{l}\text { POLLARD QUAIL FARM, RR 1, BOX 99, MATHISTON } \\
39752\end{array}$ & 40,000 & Z43 \\
\hline 659 & $E$ & THE QUAIL FARM. RT. 1, BOX 66, UTICA 39175 & 3,900 & Z43 \\
\hline 618 & $E$ & $\begin{array}{l}\text { R \& R GAME FOWL FARM \#1, CR } 500 \text { HOUSE } \# 7990 \text {, } \\
\text { RIPLEY } 38663\end{array}$ & 1,200 & $\mathrm{~N} 140, \mathrm{~N} 28, \mathrm{~N} 32$ \\
\hline 619 & E & R \& R GAME FOWL FARM \#2, CR 500, RIPLEY 38663 & 1,200 & $\mathrm{~N} 140, \mathrm{~N} 28, \mathrm{~N} 32$ \\
\hline 570 & E & $\begin{array}{l}\text { RAM TUFF GAME FARM, } 1114 \text { HARDEN CHAPEL ROAD, } \\
\text { FULTON } 38843\end{array}$ & 800 & $\mathrm{~N} 121, \mathrm{~N} 152, \mathrm{~N} 167, \mathrm{~N} 28, \mathrm{~N} 43$ \\
\hline 666 & E & $\begin{array}{l}\text { BILLY REED QUAIL FARM, } 30012 \text { HIGHWAY } 41 \text { EAST, } \\
\text { OKOLONA } 38860\end{array}$ & 3,600 & Z88_ \\
\hline 687 & $E$ & $\begin{array}{l}\text { ROCKYTOP FARM, } 39 \text { JOE LUMPKIN ROAD, CARRIERE } \\
39426\end{array}$ & 300 & N28, N44 \\
\hline 657 & $E$ & $\begin{array}{l}\text { RUSHING HILL GAME FARM, } 102 \text { SOUTH COUNTY LINE } \\
\text { ROAD, LUMBERTON } 39455\end{array}$ & 70 & $\mathrm{~N} 28, \mathrm{~N} 44 \ldots$ \\
\hline 697 & E & $\begin{array}{l}\text { SAUCIER'S QUAIL FARM, } 355 \text { GOSS-BUNKER HILL } \\
\text { ROAD, COLUMBIA } 39429\end{array}$ & 2,400 & $\mathrm{Z} 43$ \\
\hline 453 & $E$ & $\begin{array}{l}\text { SOUTHSIDE QUAIL FARM, } 14211 \text { SOUTH SWAN ROAD, } \\
\text { GULFPORT } 39503\end{array}$ & 23,360 & Z43 \\
\hline 515 & $\mathrm{E}$ & $\begin{array}{l}\text { JOE SPARKMAN QUAIL FARM, RT. 1, BOX 39, SCOOBA } \\
39358\end{array}$ & 44,000 & Z43 \\
\hline 527 & E & $\begin{array}{l}\text { JIMMY SPENCE QUAIL FARM, } 2560 \text { RAYMOND } \\
\text { WILLIAMS ROAD, STURGIS } 39769\end{array}$ & 600 & 288 \\
\hline 620 & E & $\begin{array}{l}\text { DARRELL STROUPE GAME FARM, RT. 3, BOX 297-B, } \\
\text { BONNEVILLE } 38829\end{array}$ & 250 & $\mathrm{~N} 28, \mathrm{~N} 32, \mathrm{~N} 44$ \\
\hline 565 & $E$ & $\begin{array}{l}\text { SULLIVAN'S GAME FARM, RT. 3, BOX 99, SALTILLO } \\
38866\end{array}$ & 1,300 & $\mathrm{~N} 28, \mathrm{~N} 32, \mathrm{~N} 44$ \\
\hline 488 & E & $\begin{array}{l}\text { TANNER BANTAM FARM, RT.1, BOX } 6570 \text {, } \\
\text { TAYLORSVILLE } 39168\end{array}$ & 80 & W28 \\
\hline 378 & $\mathrm{E}$ & $\begin{array}{l}\text { THORNHILL QUAIL FARM, } 91 \text { THRONHILL ROAD, } \\
\text { JAYESS } 39641\end{array}$ & 3,900 & Z43 \\
\hline 692 & E & JEREMY THRASHER, RT. 5, BOX 230, CORINTH 38834 & 200 & N._- \\
\hline 561 & $\mathrm{E}$ & TRAVIS BRADY GAME FOWL, 530 CR 415, RIPLY 38663 & 100 & $N$ \\
\hline
\end{tabular}


Table 1. HATCHERIES, DEALERS, AND INDEPENDENT FLOCKS PARTICIPATING IN

THE NATIONAL POULTRY IMPROVEMENT PLAN

WATERFOWL, EXHIBITION POULTRY AND GAME BIRDS

\begin{tabular}{llll}
\hline & & HATCHING & PRODUCTS CLASSIFIED \\
APP. SUB- & PARTICIPANTS NAME AND ADDRESS & A.S. PULLORUM-TYPHOID & CLEAN \\
NO. PART & CAPACITY & CLASSIFICATIONS FOR & WHICHPRODUCT QUALIFIED \\
\hline
\end{tabular}

65 - MISSISSIPPI

\begin{abstract}
286 E WILSON GAME BIRD FARM, RR 1, BOX 186, FULTON 38843

708 E BOBBY WREN FOWL FARM, 41 C.R. 551, RIENZI 38865
\end{abstract}

493 E YAZOO GAME FARM, RR 6, BOX 235, YAZOO CITY 39194

\section{Dealers}

229 E A\&R FARM SUPPLY, 1820 HIGHWAY 11 BYPASS, HATTIESBURG 39401

579 E ALCORN COUNTY COOPERATIVE, 211 HIGHWAY 72 WEST, CORINTH 38834

676 E ASHCRAFT FEED, INC., 100 EAST CHURCH ROAD, BOONEVILLE 38829

E B \& W FEED, P.O. BOX 108, LAWERENCE 39336

203 E BARNES FARM \& GARDEN CENTER, 405 EAST SUNFLOWER, CLEVELAND 38732

214 E BATESVILLE FEED \& SEED SUPPLY, 201 HAYS STREET. BATESVILLE 38606

630 E BI-COUNTY FARM SUPPLY, 903 HIGHWAY 311 NORTH, HOLLY SPRINGS 38635

582 E BJR FEED, P.O. BOX 1434, CORINTH 38834

199 E PAUL BOUNDS, 104 EAST MAGNOLIA STREET, POPLARVILLE 39470

585 E BRAMLETTS FEED AND MILLING, 3540 OLD HIGHWAY 51 SOUTH,P.O. BOX 506 HERNANDO 38632

603 E BRUCE FARM SUPPLY,P.O. BOX 751, BRUCE 38915

586 E CO-LIN FEED AND SEED, 1232 HIGHWAY 51 NE, BROOKHAVEN 39601
CROSS COUNTRY SEEDS, 1815 SOUTH COMMERCE STREET, GRENADA 38901

DELTA FEED COMPANY, P.O. BOX 396, GREENWOOD 38935

E EAST MISSISSIPPI CO-OP, P.O. BOX 84, DEKALB 39328

E FARMER'S FEED AND SUPPLY, 608 NORTH MAIN STREET, LELAND 38756

E FARMER'S SUPPLY STORE, P.O.BOX 2192, COLUMBUS 39704

E FAZZIO HOME AND GARDEN CENTER, 23506 HIGHWAY 53, GULFPORT 39501

E GAMBERI FEED, 2 MORGANTOWN ROAD, NATCHEZ 39120

E GRAYSON'S FEED AND SEED STORE, 103 EAST GEORGETOWN, CRYSTAL SPRINGS 39059

E GREENLEAF AGRICULTURAL PRODUCTS, P.O. BOX 406. SUMMIT 39666

E HOODS FEED AND SEED, 12312 HIGHWAY 57. VANCLEAVE 39565

$\begin{array}{ll}5,400 & \text { N } \\ 100 & \text { N183, N28, N32 } \\ 120 & \text { N28, N32 }\end{array}$

C $B, C, D, E$

C B, C, D, E

C B, C, D, N28, N32

C B, C, D, E

C $\quad B, C, D, E$

C B, C, D, E

C B, C, D, E, N28, N32

C B, C, D, E

C B, C, D, E

C B, C, D, E

C B, C, D, E

C B, C.D.E

C B, C, D, E

C B, C, D, E

C B, C, D, E

C B, C, D, E

C B, C, D, E

C B, C, D, E

C $B, C, D, E$

C B, C, D, E

C B, C, D, E

C $\quad B, C, D, E$ 


\begin{tabular}{llccc}
\hline & & PRODUCTS CLASSIFIED & ADDITIONAL \\
APP. & SUB- & PARTICIPANTS NAME AND ADDRESS & HATCHING & PRA \\
NO. & PART & EGG & U.S. PULLORUM-TYPHOID & CLEAN \\
\end{tabular}

\section{$65-$ MISSISSIPPI}

\begin{tabular}{|c|c|c|c|c|}
\hline 592 & $E$ & $\begin{array}{l}\text { HUBBARDS FEED AND SEED, } 100 \text { ADAMS AVENUE, } \\
\text { EUPORA } 39744\end{array}$ & C & $B, C, D, E$ \\
\hline 593 & $E$ & $\begin{array}{l}\text { HURLEY FARM AND FEED, P.O. BOX 601, HURLEY } \\
39555\end{array}$ & C & B, C, D, E \\
\hline 307 & $E$ & $\begin{array}{l}\text { J \& B FEEDS, INC., } 39 \text { VIRGINA STREET, LUCEDALE } \\
39452\end{array}$ & C & $B, C, D, E$ \\
\hline 594 & $E$ & $\begin{array}{l}\text { JENKINS FEED AND FARM SUPPLY, } 340 \text { SOUTH } \\
\text { PONOTOC ROAD, BRUCE } 38915\end{array}$ & C & $B, C, D, E$ \\
\hline 308 & $E$ & $\begin{array}{l}\text { JENKINS FEED SEED AND PECANS, } 145 \text { DELTA, } \\
\text { CLARKSDALE } 38614\end{array}$ & C & $B, C, D, E$ \\
\hline 462 & $E$ & $\begin{array}{l}\text { KRAMERTOWN FEED AND SEED, P.O. BOX } 310 \text {, } \\
\text { GLOSTER } 39638\end{array}$ & c & $B, C, D, E$ \\
\hline 595 & E & $\begin{array}{l}\text { L \& H FEED AND FARM SUPPLY, } 6919 \text { EAST } \\
\text { HOMOCHITTO ROAD, GLOSTER 39638-6037 }\end{array}$ & C & $B, C, D, E$ \\
\hline 597 & $E$ & $\begin{array}{l}\text { LOWNDES FARM SUPPLY, 3192-2102 MAIN STREET, } \\
\text { COLUMBUS } 39701\end{array}$ & C & $B, C, D, E$ \\
\hline 216 & $E$ & $\begin{array}{l}\text { M \& E FEEDS, } 4413 \text { MCINNIS AVENUE, MOSS POINT } \\
39563\end{array}$ & C & B, C, D, E \\
\hline 682 & $E$ & $\begin{array}{l}\text { MAGNOLIA FARM AND HOME SUPPLY, } 244 \text { EAST } \\
\text { HICKORY, P.O. BOX 6524, LAUREL } 39440\end{array}$ & C & $\mathrm{B}, \mathrm{C}, \mathrm{D}, \mathrm{E}$ \\
\hline 538 & $E$ & $\begin{array}{l}\text { MATTOX FEED MILL, INC., P.O. BOX 146, ABERDEEN } \\
39730\end{array}$ & C & $B, C, D, E$ \\
\hline 116 & $E$ & $\begin{array}{l}\text { MONTICELLO FEED, SEED AND HARDWARE, P.O. BOX } \\
\text { 1764, MONTICELLO } 39654\end{array}$ & $\mathrm{C}$ & $B, C, D, E$ \\
\hline 540 & E & $\begin{array}{l}\text { MOORE'S FEED STORE, } 157 \text { HIGHWAY } 15 \text { BY-PASS, } \\
\text { PONTOTOC } 38863\end{array}$ & C & B, C, D, E \\
\hline 599 & $E$ & $\begin{array}{l}\text { NESHOBA COUNTY CO-OP, } 338 \text { WEST MAIN STREET, } \\
\text { PHILADELPHIA } 39350\end{array}$ & C & $B, C, D, E$ \\
\hline 600 & $E$ & $\begin{array}{l}\text { OAK GROVE FEED AND SEED, } 4985 \text { OLD HIGHWAY } 11, \\
\text { HATTIESBURG } 39402\end{array}$ & $\mathrm{C}$ & B, C, D, E \\
\hline 601 & $E$ & $\begin{array}{l}\text { PEARL RIVER FARM AND GAS SUPPLY, } 829 \text { PITTMAN } \\
\text { STREET, COLUMBIA } 39429\end{array}$ & C & $B, C, D, E$ \\
\hline 464 & $E$ & $\begin{array}{l}\text { PLUNKETT'S FARM SUPPLY, RR 2, BOX } 88 \text {, } \\
\text { MONTICELLO } 39648\end{array}$ & C & $B, C, D, E$ \\
\hline 400 & $E$ & $\begin{array}{l}\text { REEDER FARM SUPPLY, INC., P.O. BOX } 506 \text {, } \\
\text { PONOTOCO } 38863-0506\end{array}$ & C & $B, C, D, E$ \\
\hline 602 & $E$ & $\begin{array}{l}\text { REEDERS FARM STORE, EAST MAIN STREET, } \\
\text { BALDWYN } 38824\end{array}$ & C & $\mathrm{B}, \mathrm{C}, \mathrm{D}, \mathrm{E}$ \\
\hline 605 & $E$ & $\begin{array}{l}\text { ROY'S FEED OF CORINTH, } 511 \text { COX STREET, CORINTH } \\
38834\end{array}$ & C & B, C, D, E \\
\hline 606 & $E$ & $\begin{array}{l}\text { SHOWS BROTHERS FARM SUPPLY, } 201 \text { SHELBY } \\
\text { STREET, ELLISVILLE } 39437\end{array}$ & C & $B, C, D, E$ \\
\hline 539 & $E$ & $\begin{array}{l}\text { SMITH'S FARM AND GARDEN, RT. 1, BOX 1-B, WEST } \\
\text { POINT } 39773\end{array}$ & C & $B, C, D, E$ \\
\hline 175 & $E$ & $\begin{array}{l}\text { SOUTHERN FEED AND SUPPLY, } 119 \text { WILMINGTON, P.O. } \\
\text { BOX } 8574 \text {. JACKSON } 39204\end{array}$ & C & B, C, D, E \\
\hline 699 & $\mathrm{E}$ & $\begin{array}{l}\text { STEWART HOME \& GARDEN, } 3200 \text { HIGHWAY } 11 \\
\text { NORTH, PICAYUNE } 39466\end{array}$ & C & $B, C, D, E$ \\
\hline 357 & E & SUMRALL'S FARM SUPPLY, 1200 HIGHWAY 15 SOUTH, & C & $B, C, D, E$ \\
\hline
\end{tabular}


Table 1. HATCHERIES, DEALERS, AND INDEPENDENT FLOCKS PARTICIPATING IN

THE NATIONAL POULTRY IMPROVEMENT PLAN

WATERFOWL, EXHIBITION POULTRY AND GAME BIRDS

\begin{tabular}{llccc}
\hline & & HATCHING & PRODUCTS CLASSIFIED \\
APP. SUB- & PARTICIPANTS NAME AND ADDRESS & EGG & U.S. PULLORUM-TYPHOID & CLASSIFICATIONS FOR \\
NO. PART & CAPACITY & CLEAN & WHICHPRODUCT QUALIFIED \\
\hline
\end{tabular}

\begin{abstract}
469 E TAYLORSVILLE FEED MILL, P.O. BOX 417, TAYLORSVILLE 39168

424 E TOWN AND COUNTRY FEED STORE, 510 LUMBERTON ROAD, COLUMBIA 39429

535 E TRACE FEED AND FARM SUPPLY, 4855 CLIFF GOOKIN BLVD., TUPELO 38801

532 E TUPELO FARM AND RANCH SUPPLY, 2921 EASON BLVD., TUPELO 38801

607 E UNION COUNTY CO-OP, 200 MOSS HILL DRIVE, P.O. BOX 126, NEW ALBANY 38652

179 E UNION COUNTY FEED AND SEED, 118 WEST MAIN STREET, NEW ALBANY 38652

625 E VAIDEN FARM SUPPLY, ROUTE 2, BOX 59-A, VAIDEN 39176

207 E VENUS FARM SUPPLY, 10263 GORENFLO ROAD, D'IBERVILLE 39532

148 E WAND SEED STORE, 219 SOUTH JACKSON STREET, BROOKHAVEN 39601

365 E WARD FEED COMPANY, 8170 LAMAR, OLIVE BRANCH 38654

471 E WARRENTON FARM AND GARDEN, 5320 HIGHWAY 61 SOUTH, VICKSBURG 39180

472 E WILKINSON FARMERS COOPERATIVE, P.O. BOX 1217, WOODVILLE 39669

608 E YAZOO FARMERS SUPPLY, 534 NORTH WASHINGTON STREET, YAZOO CITY 39194
\end{abstract}

Independent Flocks

703 E WELL KEY \& DONNIE ARNOLD, 254 COUNTY ROAD 500, RIENZI 38865

703 E. WELL KEY \& DONNIE ARNOLD, 256 COUNTY ROAD 611, WALNUT 38683

698 E CIRCLE M PLANTATION, LANIER LONG, QUAIL,

TURKEY, DEER HUNTING PRESERVE, ROUTE 3, BOX 710 MACON 39341

Phone: (662)726-5791

702 E PAUL A. COPELAND FOWL FARM, 477 CR. 550, RIENZI 38865

690 E LOYAL DULANEY, 60111 HIGHWAY 23, SMITHVILLE 38870

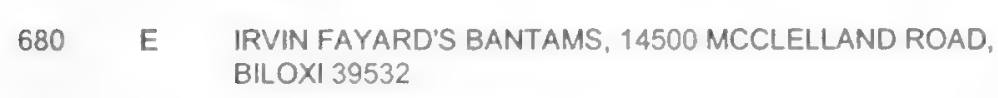

576 E BILLY GARRISON GAME FARM, P.O. BOX 235. ASHLAND 38603

637 E TIM HARE GAME FARM, HOUSE \#239, HIGHWAY 30 EAST, BOONEVILLE 38829

713 E STEVE MORRIS, P.O. BOX 165, PURVIS 39475

523 E PINE BURR GAME FOWL FARM \#2, 12433 BALL DIAMOND ROAD, COLLINSVILLE 39325

511 E PINE BURR GAME FOWL FARM \#1, RR 3, BOX 111. COLLINSVILLE 39325

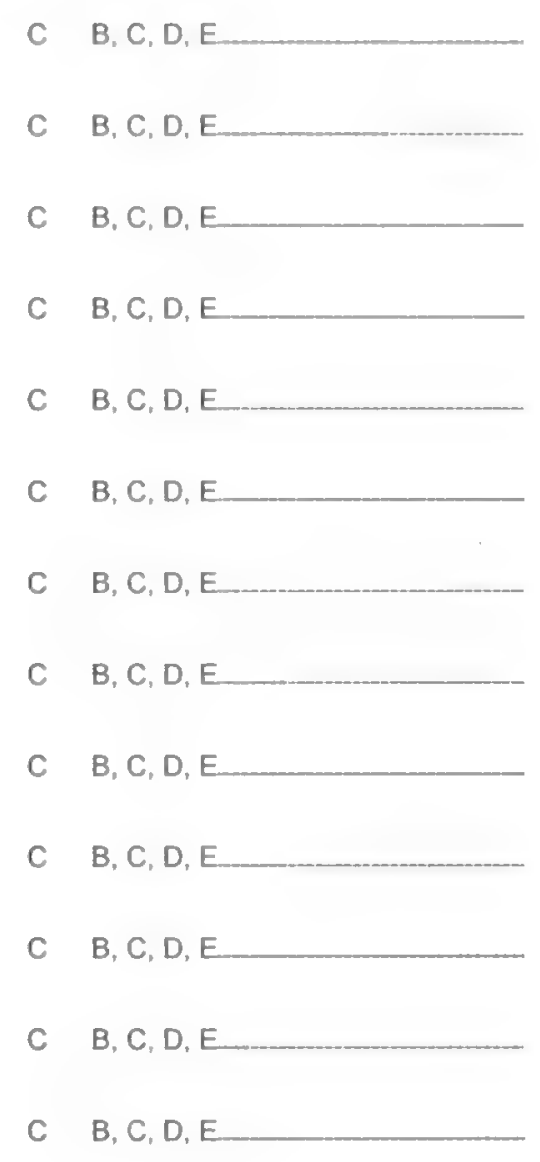

N28.

N28.

$\mathrm{Z} 88$

N28, N33

N28.

W30

N140, N28

N.

w30

N28

N28 
Table 1. HATCHERIES, DEALERS, AND INDEPENDENT FLOCKS PARTICIPATING IN THE NATIONAL POULTRY IMPROVEMENT PLAN

WATERFOWL, EXHIBITION POULTRY AND GAME BIRDS

\begin{tabular}{|c|c|c|c|c|c|}
\hline $\begin{array}{l}\text { APP. } \\
\text { NO. }\end{array}$ & $\begin{array}{l}\text { SUB- } \\
\text { PART }\end{array}$ & PARTICIPANTS NAME AND ADDRESS & $\begin{array}{l}\text { HATCHING } \\
\text { EGG } \\
\text { CAPACITY }\end{array}$ & $\begin{array}{c}\text { PRODUCTS CLASSIFIED } \\
\text { U.S. PULLORUM-TYPHOID } \\
\text { CLEAN }\end{array}$ & $\begin{array}{c}\text { ADDITIONAL } \\
\text { CLASSIFICATIONS FOR } \\
\text { WHICH PRODUCT QUALIFIED }\end{array}$ \\
\hline
\end{tabular}

$65-$ MISSISSIPPI

\begin{tabular}{|c|c|c|c|}
\hline 531 & $E$ & $\begin{array}{l}\text { PRAIRIE MILLS, } 10111 \text { PRAIRIE MILLS ROAD, PRAIRIE } \\
39756\end{array}$ & W578 \\
\hline 703 & $E$ & $\begin{array}{l}\text { MICHAEL STEWART, } 254 \text { COUNTY ROAD 500, RIENZI } \\
38865\end{array}$ & N28 \\
\hline 703 & $E$ & $\begin{array}{l}\text { MICHAEL STEWART, } 256 \text { COUNTY ROAD } 611 \text {, WALNUT } \\
38683\end{array}$ & N28 \\
\hline 516 & $E$ & $\begin{array}{l}\text { THUNDER HEART GAME FARM, RT. 2, BOX } \\
\text { 77,HIGHWAY } 5 \text { SOUTH ASHLAND } 38603\end{array}$ & W262, W50. \\
\hline 638 & E & $\begin{array}{l}\text { MARY WALKER, } 2108 \text { NEW LIGHT ROAD, STARKVILLE } \\
39759\end{array}$ & $\mathrm{R} 114, \mathrm{~W} 7$ \\
\hline
\end{tabular}

ROSE FOSTER

MISSOURI DEPARTMENT OF AGRICULTURE

P.O. BOX 630

JEFFERSON CITY, MO 65102

Phone: (573)522-3377

Fax: $\quad(573) 751-5279$

Hatcheries

$\begin{array}{ccl}218 & E & \begin{array}{l}\text { ALBIN'S QUAIL FARM, 2445 CARTER RD, MOSCOW } \\ \text { MILLS 63362 }\end{array} \\ 234 & \text { E } & \begin{array}{l}\text { BAKER'S HATCHERY, 158 HIGHWAYN, HUMANSVILLE } \\ 65674\end{array} \\ 260 \quad \text { E } & \begin{array}{l}\text { BIROLAND, DAVE \& BECKY BONNER, 239 RED TOP } \\ \text { ROAD, BUFFALO 65622 }\end{array} \\ 188 \text { E } & \begin{array}{l}\text { BISON HILL CHUKAR FARM, 74 FRAKER ROAD, } \\ \text { BUFFALO 65622 }\end{array}\end{array}$

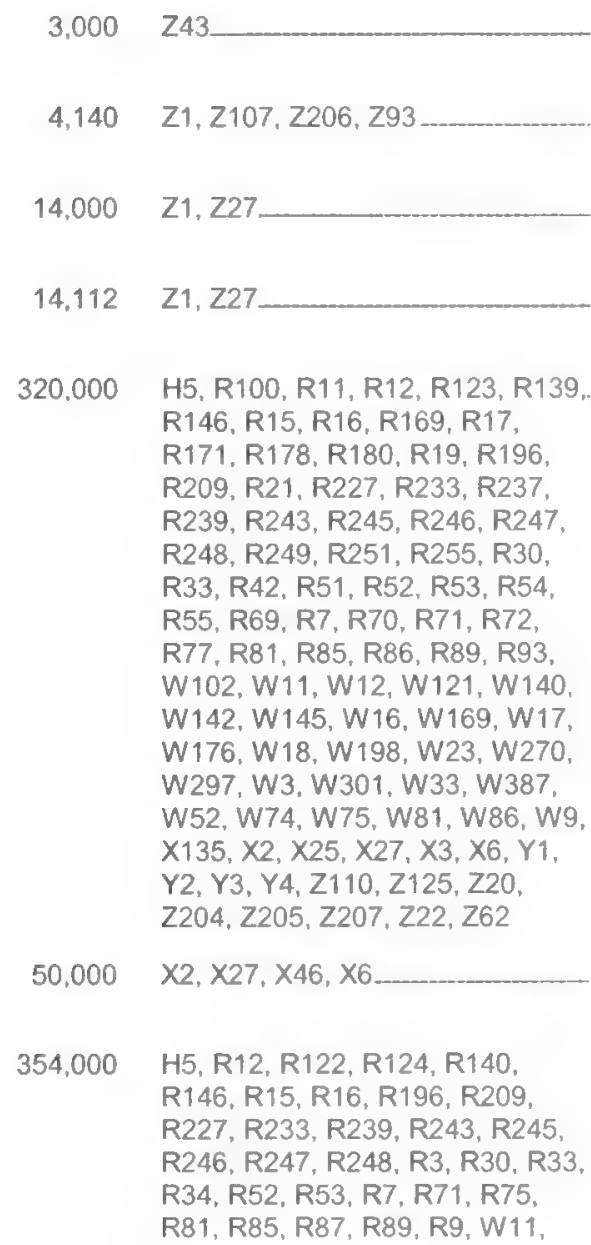

$\times 2, \times 27, \times 46, \times 6$

$354,000 \quad H 5, R 12, R 122, R 124, R 140$, R146, R15, R16, R196, R209, R227, R233, R239, R243, R245 R246, R247, R248, R3, R30, R33, R34, R52, R53, R7, R71, R75, R81, R85, R87, R89, R9, W11,

63 E C. MARK ESTES HATCHERY, INC., 805 NORTH METEOR, 
Table 1. HATCHERIES, DEALERS, ANO INDEPENDENT FLOCKS PARTICIPATING IN

THE NATIONAL POULTRY IMPROVEMENT PLAN

WATERFOWL, EXHIBITION POULTRY AND GAME BIRDS

\begin{tabular}{|c|c|c|c|c|c|}
\hline $\begin{array}{l}\text { APP. } \\
\text { NO. }\end{array}$ & $\begin{array}{l}\text { SUB- } \\
\text { PART }\end{array}$ & PARTICIPANTS NAME AND ADDRESS & $\begin{array}{l}\text { HATCHING } \\
\text { EGG } \\
\text { CAPACITY }\end{array}$ & $\begin{array}{l}\text { PRODUCTS CLASSIFIED } \\
\text { U.S. PULLORUM-TYPHOID } \\
\text { CLEAN }\end{array}$ & $\begin{array}{c}\text { ADDITIONAL } \\
\text { CLASSIFICATIONS FOR } \\
\text { WHICH PRODUCT QUALIFIED }\end{array}$ \\
\hline
\end{tabular}

43 - MISSOURI

$\begin{array}{lll}239 & E & \text { FIRST CHOICE GAME BIRD FARM, 32126 U.S. HIGHWAY } \\ & \text { 136, UNIONVILLE 63565 } \\ 271 & E & \begin{array}{l}\text { GOOD EARTH GAME FARM AND HATCHERY, } 8960 \\ \text { HIGHWAY E, BONNE TERRE 63628 }\end{array} \\ 464 & \text { E } & \begin{array}{l}\text { GRIFFITH POULTRY FARM HATCHERY, 4791 COUNTY } \\ \text { ROAD 105, FULTON 65251 }\end{array} \\ 262 & \text { E } & \text { HEARTLAND HATCHERY, RT. 1, BOX 177-A, } \\ & & \text { AMSTERDAM 64723 }\end{array}$

MARTI POULTRY FARM, P.O. BOX 27, 603 EAST JACKSON, WINDSOR 65360

MCKINNEY \& GOVERO POULTRY, 4717 HIGHWAY B, PARK HILLS 63601-9102

RICE CHUKAR FARM, 320 DURINGTON ROAD, BUFFALO 65622

E ROYAL FLUSH GAME BIRD FARM, 18078 STATE HIGHWAY 149, UNIONVILLE 63565

E SNOW WHITE ENTERPRISES, RR 1, BOX 40 , CARROLLTON 64633

E WINDY OAKS FARMS, 1427 BIG BUFFALO ROAD STOVER 65078
W119, W12, W121, W140, W17. W198, W208, W211, W25, W26 W270, W28, W290, W573, W60 W86, W9, X11, X2, X27, Y1, Y2. $Y 3, Y 4, Y 6, Z 111, Z 125, Z 20$ Z204, Z205, Z206, Z207, Z22 262

$25,000 \quad 227$

$10,000 \quad \mathrm{Z} 1$

$88,000 \quad R 146, R 247, R 248, R 6, \times 2,222$

2,400 H5, R100, R11, R12, R122, R123, R124, R146, R168, R178, R196. R21, R222, R227, R233, R239. R247, R34, R37, R42, R53, R55, R69, R7, R71, R75, R77, R89. R90, W176, W184, W208, W211, W247, W25, W26, W290, W309, W313, W368, W518, W519. W573, W74, W75, X135, X2, X25 $X 27, X 3, X 6, Y 1, Y 3, Y 52, Z 108$, Z11, Z111, Z15, Z20, Z202, Z204, Z206, Z22, Z62

190,000 R100, R11, R12, R123, R139, R146, R15, R16, R168, R17. R171, R178, R19, R192, R196. R21, R227, R237, R243, R245. R247, R248, R30, R34, R49, R52, R53, R55, R6, R7, R70, R71, $R 72, R 85, R 86, R 87, R 89, W 102$, W119, W12, W121, W129 W138, W140, W141, W142, W145, W16, W160, W169, W17 W176, W18, W19, W198, W20, W223, W25, W259, W26, W270, W28, W3, W302, W48, W52. W57, W573, W6, W60, W64, W69, W74, W75, W81, W86, W88

1,602 R10, R100, R104, R11, R119, R12, R120, R123, R13, R139, R140, R146, R15, R16, R171, R178, R180, R183, R19, R201, R21, R22, R222, R223, R23. R245, R246, R247, R248, R260, $\mathrm{R} 261, \mathrm{R} 262, \mathrm{R} 3, \mathrm{R} 30, \mathrm{R} 33, \mathrm{R} 34$, $R 37, R 38, R 40, R 50, R 51, R 52$. R53, R56, R60, R69, R7, R71. R72, R81, R86, R87, R91, R93. R95, R97

$10,500 \quad Z 1$

$25.000 \quad Z 1, Z 12, Z 27$

$20,000 \quad Z 1, Z 43, Z 44, Z 60$.

22,000 Z44, Z60, Z73 
Table 1. HATCHERIES, DEALERS AND INDEPENDENT FLOCKS PARTICIPATING IN

THE NATIONAL POULTRY IMPROVEMENT PLAN

WATERFOWL, EXHIBITION POULTRY AND GAME BIRDS

\begin{tabular}{|c|c|c|c|c|c|}
\hline $\begin{array}{l}\text { APP. } \\
\text { NO. }\end{array}$ & $\begin{array}{l}\text { SUB- } \\
\text { PART }\end{array}$ & PARTICIPANTS NAME AND ADDRESS & $\begin{array}{l}\text { HATCHING } \\
\text { EGG } \\
\text { CAPACITY }\end{array}$ & $\begin{array}{l}\text { PRODUCTS CLASSIFIED } \\
\text { U.S. PULLORUM-TYPHOID } \\
\text { CLEAN }\end{array}$ & $\begin{array}{c}\text { ADDITIONAL } \\
\text { CLASSIFICATIONS FOR } \\
\text { WHICH PRODUCT QUALIFIED }\end{array}$ \\
\hline
\end{tabular}

$$
43 \text { - MISSOURI }
$$

Independent Flocks

\begin{tabular}{|c|c|c|}
\hline 476 & $E$ & $\begin{array}{l}\text { BONITA \& RILEY ANDERSON, P.O. BOX 109, BATES } \\
\text { CITY } 64011\end{array}$ \\
\hline 479 & E & $\begin{array}{l}\text { PAT ARCHDEKIN, } 14248 \text { COUNTY ROAD 305, ST. } \\
\text { JOSEPH } 64505\end{array}$ \\
\hline 325 & E & $\begin{array}{l}\text { SCOTT A. \& AMANDA BAKER, } 1211 \text { WEST JOSEPH } \\
\text { STREET, ST. JOSEPH } 64504\end{array}$ \\
\hline 500 & E & $\begin{array}{l}\text { ALAN BARNETT, } 470 \text { NORTH HIGHWAY } 65 \text {, TRENTON } \\
64683\end{array}$ \\
\hline 363 & E & $\begin{array}{l}\text { HOWARD BEAMER, RT. 1, BOX 15, SOUTHWEST CITY } \\
64863\end{array}$ \\
\hline 508 & $E$ & $\begin{array}{l}\text { PATRICK H. BENNETT, HC 3, BOX 3585, GREENVILLE } \\
63944\end{array}$ \\
\hline 485 & E & $\begin{array}{l}\text { DALE BIESER, } 10487 \text { STATE HIGHWAY } 72 \text {, } \\
\text { MILLERSVILLE } 63766\end{array}$ \\
\hline 471 & $E$ & EARL BROWN, 12947 HIGHWAY E, ELDRIDGE 65463 \\
\hline 481 & $\mathrm{E}$ & $\begin{array}{l}\text { WADE BURKHALTER, } 3113 \text { SOUTH STEWART, SEDALIA } \\
65301\end{array}$ \\
\hline
\end{tabular}
NIANGUA 65713

422 E KIM A. CASH, 7800 HICKORY HILL ROAD, HENLEY 65040

\begin{tabular}{|c|c|c|}
\hline 374 & $E$ & $\begin{array}{l}\text { FRANK CHILES, } 25404 \text { EAST BLUE MILLS ROAD, } \\
\text { INDEPENDENCE } 64058\end{array}$ \\
\hline 510 & $E$ & $\begin{array}{l}\text { CHUBBY CHUKARS, RT. 1, BOX 577, BONNOTS MILL } \\
65016\end{array}$ \\
\hline 276 & $E$ & $\begin{array}{l}\text { PHIL CHURCH, } 3289 \text { STATE HIGHWAY JJ, SPARTA } \\
65753\end{array}$ \\
\hline 254 & $E$ & $\begin{array}{l}\text { TRAVIS CLARK, } 320 \text { COUNTY SHOP ROAD, LAMPE } \\
65681\end{array}$ \\
\hline 438 & $E$ & $\begin{array}{l}\text { HERMAN CLARK, } 3822 \text { STATE HIGHWAY } 86 \text { WEST, } \\
\text { LAMPE } 65681\end{array}$ \\
\hline 493 & $E$ & $\begin{array}{l}\text { TY \& CODI BETH COATS, RT. 1, BOX 85, BRAYMER } \\
64624\end{array}$ \\
\hline & $E$ & KEN COLEMAN, RT. 2, BOX 853, SALEM 65560 \\
\hline
\end{tabular}

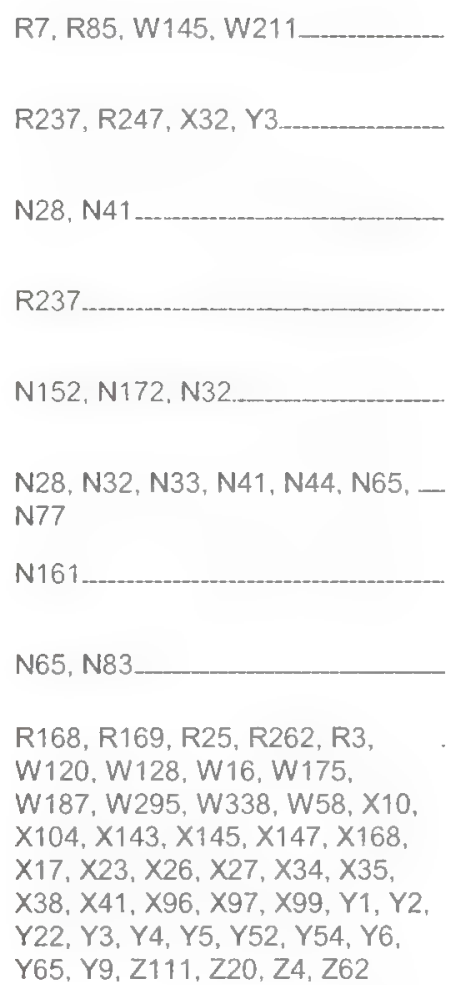

N28, N63

Z1, Z27, Z43

N174, N28

N148, N28, N32, N33, N44

N28, N32, N41, N44, N90

W28, W52

N33, N41, N65 
Table 1. HATCHERIES, DEALERS, AND INDEPENDENT FLOCKS PARTICIPATING IN

THE NATIONAL POULTRY IMPROVEMENT PLAN

WATERFOWL, EXHIBITION POULTRY AND GAME BIRDS

APP. SUB- PARTICIPANTS NAME AND ADDRESS
NO. PART

HATCHING
EGG
CAPACITY

HATCHING

CAPACITY
PRODUCTS CLASSIFIED

U.S. PULLORUM-TYPHOID CLEAN
ADDITIONAL

CLASSIFICATIONS FOR WHICH PRODUCT QUALIFIED

$43-$ MISSOURI

\begin{abstract}
253 E CONAWAY'S BANTAMS, 4376 SOUTH FARM ROAD 107, BROOKLINE 65619

494 E BRAD CORBIN, 304 NORTH RICHEY AVENUE, BRAYMER 64624

495 E DEREK COX, 3275 SE SUNNY ALE ROAD, BRAYMER 64624

483 E RACHEL M. CRAIG, 9620 EAST VEMERS FORD ROAD, COLUMBIA 65201

292 E JOSEPH FRANCISCO CRUZ, P.O.BOX 1097, DIXON 65459

$E$

E

E

E

$E$

E

E

E

$E$

$E$

$E$

LANE, BUFFALO 65622

DARRELL FAUSET, 4384 SPRINGCREEK ROAD, BILLINGS 65610
\end{abstract} NORTH PENNY LANE, SPRINGFIELD 65803

E JAMES FERRILL, RT. 2, BOX 278-A, DONIPHAN 639359624

E RALPHL. FIDLER, 34621 SOUTH O'BANNON, CREIGHTON 64739

E MARK FIELDS, 5000 DRISKEL ROAD, COLUMBIA $65202-$ 7294

E FLIGHTS OF FANCY, 22445 STATE ROUTE BB, KING CITY 64463

E ZACK FORD, 3169 SE PENN ROAD, COWGILL 64637

E HENRY FORSEE, 3150 SOUTH OLIVET ROAD, COLUMBIA 65201

E
W142, W25, W26, W28, W50, W51, W568, W7

W394

W120, W54

R183, R247, W387, W7

N121, N164, N174, N216, N222, N230, N24, N28, N32, N33, N43, N44, N63, N84, N94

R

W119, W142, W160, W52 W552, W568, W607, W88, W91

N152, N33, N83

R146, R19, R196, R222, R42, R7

R34, W23, W302, W4, W54

W142, W28, W568, W88, W89

R12, R146, R15, R19, R196, R227, R239, R247, R7, R81, R85 N134, N174, N28, N32, N44, N83. W119, W160, W22, W307, W39. W484. W486

Z1.

W111, W119, W136, W141, W149, W22, W28, W30, W307. W361, W39, W393, W484 W492, W552, W77

W208, W45

N148, N28, N32, N44, N83

N137, N227

R42, R6, W387

R12, R146, R171, R222, R247, W102, W198, W206, W208, W45, W74, W86, Z107, Z108. Z11, Z137, Z139, Z19, Z20, Z22, Z41

W120, W142

R183, R247, R248, W7, W8

R183, R247, R33 
HATCHING

EGG

CAPACITY
PRODUCTS CLASSIFIED

U.S. PULLORUM-TYPHOID CLEAN
ADDITIONAL

CLASSIFICATIONS FOR WHICH PRODUCT QUALIFIED

\section{3 - MISSOURI}

\begin{tabular}{|c|c|c|}
\hline 290 & $E$ & $\begin{array}{l}\text { GILLISPIE'S OF PAYDOWN, } 11601 \text { MARIES ROAD } 302 \text {, } \\
\text { VIENNA } 65582\end{array}$ \\
\hline 348 & $E$ & $\begin{array}{l}\text { GOLD DIGGER FANCIES, MIKE BURKHART, P.O. BOX } \\
771 \text {, CROCKER } 65452\end{array}$ \\
\hline 497 & $E$ & $\begin{array}{l}\text { CHANCE GUFFEY, } 501 \text { BOBCAT AVENUE, BRAYMER } \\
64624\end{array}$ \\
\hline 312 & $E$ & $\begin{array}{l}\text { DENNIS HALL, } 36701 \text { EAST J.W. CUMMINS ROAD, OAK } \\
\text { GROVE } 64075\end{array}$ \\
\hline 473 & $E$ & $\begin{array}{l}\text { HARDING GAMEBIRD FARM. } 24637 \text { EAST HIGHWAY T, } \\
\text { RIDGEWAY } 64481\end{array}$ \\
\hline 513 & $E$ & $\begin{array}{l}\text { JERRY, CAROLE \& CYDNEY HARTEL, } 19176 \text { MONROE } \\
\text { ROAD 249, PARIS } 65275\end{array}$ \\
\hline
\end{tabular}

$442 \quad E$

$443 \quad E$

$397 \quad E$

$328 \quad E$

$505 \quad E$

$344 \quad E$

$490 \quad$ E

333

206

421

$373 \quad E$

$219 \quad E$

$386 \quad$ E

$470 \quad E$

$480 \quad E$

335

$506 \quad E$

$439 \quad E$
DON HAYES, RT. 4, BOX 789, SALEM 65560

HEGGEMEIER GAME FARM \& KENNELS, 6822 HIGHWAY B, HIGBEE 65257-9710

HENDRICKS GAME FARM, 24265 WEST 130TH PLACE, EAGLEVILLE 64442

JOHN HICKS, 5373 WEST FARM ROAD 6, BRIGHTON 65617

DANNY HILL, RT. 1, BOX 253, CAMDENTON 65020

JOHN HOLDREN, P.O. BOX 115, BARING 63531

CURT HOLMQUIST, 13510 STATE MM S.E., AGENCY 64401

A. J. HOUSTON, 16494 RUDD ROAD, LAWSON 64062

E JOHN HUTCHINS, 9459 RYE CREEK ROAD, LONEDELL 63060

E J.R.'S QUAIL RANCH, JAMES SUITER, RT. 1, BOX 122-B, OSCEOLA 64776

DONALD \& SHEILA JAMES, D \& S GAME FOWL, 4951 STATE ROAD J, ROACH 65787

JAMES RIVER GAME FARM, 187 HICKORY HOLLOW DRIVE, GALENA 65656

JEFF JENSEN, 2088 MARBLE ROAD, CONWAY 65632

JOHN \& PAT JOHNSON, 39 OSAGE ROAD, URBANA 65767-8109

EAN KLEINLEIN, 18805 EVERGREEN TERRACE, ST. JOSEPH 64505

E JOHN KOGER, 7370 NORTHWEST OLD 36 HIGHWAY, CAMERON 64429

GERALD \& ANITA KRACHER, 5853 HIGHWAY F,

E JESSE LEATHERMAN, 9050 HIGHWAY 165, HOLLISTER 65672 HARTVILLE 65667
W114, W23, W28, W29, W290, W38, W4, W5, W518, W69, W88, $\times 10$

$\mathrm{R}$

W7

N134, N32, N83, N84

Z27. 288

R11, R146, R19, R227, R237, R53, R7, R85, R86, W120, W121, W17, W198, W208, W223, W26, W28, W3, W4, W52, W54, W7, W94

N253, N254, N255

$\mathrm{X} 46, \mathrm{Z1}, \mathrm{Z27}, \mathrm{Z} 43$

$\mathrm{X} 46, \mathrm{Z1}, \mathrm{Z27}, \mathrm{Z} 43$

R34, W25, W28, W4, W48, W88, W91, Z205

N28, N41, N83

N105, N172, N198, N199, N25, N41

R237, R6, W164, Y5, Z22

N134, N32, N65, N83

Z118, Z24, Z26, Z3, Z53, Z57.

R146, R247, R248, Z22, Z43

N106, N28, N32, N44, N63.

N105, N28, N32, N44

N109, N148, N28, N32, N82.

R139, R52, R53, R7, R86, W198, W208, W74

Z1, Z177, Z2, Z27, Z93

N131, N183, N44, N46

Z27, Z43

N28, N32, N33 
Table 1. HATCHERIES, DEALERS, AND INDEPENDENT FLOCKS PARTICIPATING IN

THE NATIONAL POULTRY IMPROVEMENT PLAN

WATERFOWL, EXHIBITION POULTRY AND GAME BIRDS

\begin{tabular}{lllll}
\hline & & HATCHING & PRODUCTS CLASSIFIED \\
APP. SUB- & PARTICIPANTS NAME AND ADDRESS & EGG & U.S. PULLORUM-TYPHOID \\
NO. PART & CLEAPACITY & CLASSIFICATIONS FOR & WHICHPRODUCT QUALIFIED \\
\hline
\end{tabular}

43 - MISSOURI

\begin{abstract}
484 E CHRISTOPHER L. LINK, 3683 HOMEYER ROAD, OWENSVILLE 65066

258 E LONESOME DOVE, RR 3, BOX 186, PRINCETON 64673

460 E JESSE LYONS, 8209 DUSTY RHODES LANE, COLUMBIA 65202-6991

MANHAWKA GAMEFOWL FARM, 25279 OMAHA ROAD, FOREST CITY 64451

E DAVID MATTHEWS, 5166 STATE HIGHWAYE, OAK RIDGE 63769

MAYLEE GAME FARM, 11847 HIGHWAY 54 EAST, MEXICO 65265

E SHARON MEHLER, 5756 NEWTON ROAD, ODESSA 64076-9313

E MILLER'S HIDEAWAY POULTRY, 27700 SMASAL ROAD, SEDALIA $65301-0908$

\section{E GERALD MITCHELL, 1559 COUNTY ROAD 60, SARCOXIE} 64862

E JAMES MURPHY, 510 SPRING STREET, MOUND CITY 64470

E MATT MURRAY, RT. 2, BOX 67, BRAYMER 64624

E PHIL MURRAY, RT, 2, BOX 67, BRAYMER 64624

E THOMAS NELSON, 238 STATE ROAD BB, URBANA 65767

E ANDYNORMAN, 307 SOUTH GULF STREET. HUMANSVILLE 65674-8826

E CLINTON J. NOVAK, SR., 14140 SW HIGHWAY JJ, DEKALB 64440

E MIKE O'BRIEN, RT. 1, BOX 404-B, GOODMAN 64843-9730

E DENNIS O'CALLAGHAN, 14031 ROAD 459, AMAZONIA 64421

E LORRIE OLDHAM, 6446 SE 240TH STREET, LATHROP 64465-9620
N28, N32, N83, W150, W51 W569, W579, W91

N148, N28, N32, N97

R168, R169, R227, R237

W114, W142, W149, W28, W29, W30, W50, W542, W568, W569, W579, W644, W88, W89, X28 $Y 6, Y 7, Z 22, Z 249$

N147, N32

R100, R119, R123, R146, R17, = R178, R182, R196, R20, R222, R225, R245, R246, R247, R248, R33, R42, R69, R7, R81, R86, W100, W1C2, W119, W12, W13, W132, W149, W17, W176, W19 W198, W207, W231, W24, W25, W26, W270, W3, W301, W357. W364, W38, W387, W442, W48, W504, W518, W54, W573, W594, W7, W75, W86, W88. Z108, Z111, Z19, Z22, Z24, Z251. Z293, Z3, Z4, Z42, Z86, Z93

N104, N105, N131, N134, N197, N28, N32, N33, N43, N44, N65, N83, N90

R211, R248, W4

W102, W121, W132, W140, W164, W168, W169, W17, W18, W206, W223, W25, W26, W265, W33, W40, W415, W60, Y22, Z220

N198, N225, N23, N28, N32, N33. N41

R

W53

W3.

N183, N28, N32, N44, N83

W17, X10, X2, X46, X97, X98, Y60, Y64, Y9, Z204, Z209, Z93

N183, N28, N41, N44, N90, N97_

N104, N43, N65, N90

N109, N129, N28, N41, N84

R114, R168, R169, R175, R183, R237, R247, R90, W7, W8, X6, 


\begin{tabular}{|c|c|c|c|c|c|}
\hline $\begin{array}{l}\text { APP. } \\
\text { NO. }\end{array}$ & $\begin{array}{l}\text { SUB- } \\
\text { PART }\end{array}$ & PARTICIPANTS NAME AND ADDRESS & $\begin{array}{l}\text { HATCHING } \\
\text { EGG } \\
\text { CAPACITY }\end{array}$ & $\begin{array}{c}\text { PRODUCTS CLASSIFIED } \\
\text { U.S. PULLORUM-TYPHOID } \\
\text { CLEAN }\end{array}$ & $\begin{array}{c}\text { ADDITIONAL } \\
\text { CLASSIFICATIONS FOR } \\
\text { WHICH PRODUCT QUALIFIED }\end{array}$ \\
\hline
\end{tabular}

E DALE OSBORN, RT. 1, BOX 520, FLEMINGTON 65650

P \& H GAME FOWL FARM, RT. 2, BOX 45, BRUNSWICK 65236

E JERRY C. PERRYMAN, 303 NORTH 10TH STREET, UNIT 5, OZARK 65721

E DALE POSTMA, 1881 WEST BLUE SPRINGS ROAD, NIXA 65714

E MAYNARD RANDALL, 14973 IRIS ROAD, NEOSHO 64850

E JEFFREY AND DAWN RECTOR, HCR 2, BOX 2239, VAN BUREN 63965

\section{E DARRYL REED, 22299 CHESTNUT RIDGE ROAD,} FARMINGTON 63640

E BRENDA, JEFFREY \& ETHAN REKER, 212 ARIKARA LANE, CAPE GIRARDEAU 63701

E SANDRA RICE, 43691 HORN HILL ROAD, RICHMOND 64085

E RIDGE TOP RANCH, 3903 WEST RIDGE TRAIL ROAD, CLARK 65243-9525

E TROY ROBERTS, 35 MARKET STREET, DEARBORN $64439-9764$

E JULIE ROBERTSON, P.O. BOX 3, POWERSITE $6573 \uparrow$

E ROCKING R \& R ENTERPRISES, FISCUS, HCR 33, BOX 300, ROLLA 65401

E RICHARD ROSS, 119 MEADOW BROOK LANE, POPLAR BLUFF 63901

E DELMAR \& KAREN SCHEBAUM, 702 LINCOLN, WESTBORO 64498

E ROBERT SCHIPPER, 22 HAFLINGER ROAD, MONTGOMERY CITY 63361

E ROBERT SCHULTZ, P.O. BOX 20, PITTSBURG 65724

E DON SCHUPP, 25177 HIGHWAY 179, BOONVILLE 65233

E JOHN SCHWAN, P.O. BOX 78, MARQUAD 63655

E JERRY SEMSCH, 11925 NORTH SHERMAN ROAD, KANSAS CITY 64156

E CHARLES F. SHAW, 140 FROELICH ROAD, HILLSBORO 63050

E HARRY \& VERONICA SHAW, 3571 SUNSET ROAD GROVESPRING 65662
N32, N44, N83

N104, N110, N134, N161, N24, N32, N33, N44, N82

$X 10, X 17, X 2, X 21, X 23, X 24, X 3,-$ $X 46, X 9, X 96, X 97, Y 1, Y 14, Y 2$, Y22, Y3, Y4, Y8, Z109, Z284

W115, W245, W28, W4, W48, W54, W7

N32, N43

R19, R51, R93, W11, W12, W121, W140, W145, W164. W17, W176, W18, W33, W415. W7, W74, W75, W86, W9

N104, N28, N32

R124, R171, R178, R196, R21, $R 89$, W208, W33

R12, R139, R196, R52, W119, W160, W415, W75

R42.

$R$

W11, W23, W38

R

N121, N134, N28, N32, N33

W20, W57

W200, W569, W58

N32, N33, N41, N90

N109, N134, N43, N44, N65, N83.

Z108, Z111, Z118, Z182, Z24, Z26, Z44, Z53, Z57

W142, W263, W28, W88, W89

N109, N142, N161, N183, N24, N25, N28, N32, N41, N44, N83

R220, R86, W142, W176, W198, W211, W25, W26, W270, W28, W297, W45, W477, W52, W74, W75, W86, X135, X98, Y1, Y2, Y5, Y6, Z108, Z111, Z118, Z139. Z19, Z20, Z202, Z204, Z205. Z206, Z207, Z209, Z22, Z250, Z251, Z252, Z253, Z262, Z285, Z286 
Table 1. HATCHERIES, DEALERS, AND INDEPENDENT FLOCKS PARTICIPATING IN

THE NATIONAL POULTRY IMPROVEMENT PLAN

WATERFOWL, EXHIBITION POULTRY AND GAME BIRDS

\begin{tabular}{|c|c|c|c|c|c|}
\hline $\begin{array}{l}\text { APP. } \\
\text { NO. }\end{array}$ & $\begin{array}{l}\text { SUB- } \\
\text { PART }\end{array}$ & PARTICIPANTS NAME AND ADDRESS & $\begin{array}{l}\text { HATCHING } \\
\text { EGG } \\
\text { CAPACITY }\end{array}$ & $\begin{array}{l}\text { PRODUCTS CLASSIFIED } \\
\text { U.S. PULLORUM-TYPHOID } \\
\text { CLEAN }\end{array}$ & $\begin{array}{c}\text { ADDITIONAL } \\
\text { CLASSIFICATIONS FOR } \\
\text { WHICH PRODUCT QUALIFIED }\end{array}$ \\
\hline
\end{tabular}

43 - MISSOURI

\begin{abstract}
359 E SHOME GAME FARM, W.L. JOHNSON, 13912 COUNTY ROAD 7240, NEWBURG 65550

509 E DEAN \& JULIE SHUCK, 29797 MEXICO ROAD, PERRY $63462-2507$
\end{abstract}

PHYLLIS SINN, 413 COUNTY ROAD 623, CAPE GIRARDEAU 63701

\begin{abstract}
323 E STEVE \& DAVE SMITH, 23591 LAWRENCE 1170, VERONA 65769
361 E SMITH GAME FOWL FARM, P.O. BOX 529, LEBANON 65536

437

E

491

E

ANGELA STANLEY, 777 BLUEGRASS ROAD, CONWAY 65632

AMBER \& KAYLA STAPP, 1082 SWITCHGRASS ROAD, FORDLAND 65652
\end{abstract}

FRED TORREY, 14747 STATE HIGHWAY 129 , UNIONVILLE 63565

E

PETER \& LAURA STEPHENS, RR3, BOX 3205J, LINCOLN 65338

STRING CREEK GAME BIRD FARM 31676 SUNNYSIDE ROAD. CALIFORNIA 65018-3546 CREIGHTON 64739

LARRY TESTERMAN, 42 TRACEY LANE NORTH. BRANSON WEST 65737

CLIFF THORNBURG, RT. 2, BOX 219, HUNTSVILLE 65259

GEORGE THORNBURG, RT. 2, BOX 219, HUNTSVILLE 65259

E

E

LUKE THORNBURG, RT. 1, BOX 32, CLIFTON HILL 65244

RICK THORNBURG, RR 2, BOX 227, HUNTSVILLE 65259

LORI THORPE, 1950 SW 200 ROAD, KINGSVILLE 64061

E

TAMMY TURNER, 414 BLUEGRASS ROAD, CONWAY 65632

E

UCHEE PINES GAME FARM, 320 CURBOW ROAD, GALENA 65656-8212
N109, N141, N152, N43, N44, N63, N83, N84

R268, R269, W130, W149, W303, W40, W439, W637. W638, W639, W640, W641. W642, W643, W644, W645, W74, $\times 23$

R123, R19, R30, R52, R87, W11, W121, W138, W140, W145.

W17, W18, W198, W248, W270, W28, W297, W33, W41, W415,

W43, W45, W60, W74, W75

W86, W91, Z204, Z205, Z22,

$\mathrm{Z} 283$

N28, N41, N44

N.

R117, R146, R196, R233, R237. R239, R247, R260, R261, R44, $\mathrm{R} 69, \mathrm{R} 7, \mathrm{X} 2, \mathrm{X} 98$

R12, R124, R146, R168, R171, R196, R222, R245, R290, R34, R42, R53, R7, R70, R71, R85. R86, R89, W121, W166, W17. W198, W25, W26, W278, W28 W301, W62, X2, X20, X27, Z202, $\mathrm{Z204}$

R114, R237, W17, W6

Z1, Z27, Z43

R249, R250, R253, R255

W119, W142, W160, W22, W220, W274, W30, W307, W39, W484, W569

R19. W119

R247

W115, W128

R15, R7, W11, W12, W16

R81, W100, W102, W117, W18, W20, W26, W475, W86, Z118, Z182, Z204, Z205, Z207

R211, W121, W138, W145, W169, W176, W198, W24, W3, W305, W372, W41, W415, W74, W75, W86

R146, R15, R19, R196, R233, R239, R247, R7, R85, R89, W74. W75

N104, N105, N109, N230, N248, N28, N32, N33, N41, N7, N97 
Table 1. HATCHERIES, DEALERS, AND INDEPENDENT FLOCKS PARTICIPATING IN

THE NATIONAL POULTRY IMPROVEMENT PLAN

WATERFOWL, EXHIBITION POULTRY AND GAME BIRDS

APP. SUB- PARTICIPANTS NAME AND ADDRESS

NO. PART

HATCHING
EGG

CAPACITY

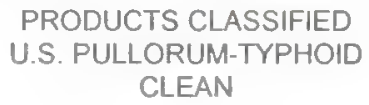

PRODUCTS CLASSIFIED

U.S. PULLORUM-TYPHOID

CLEAN

ADDITIONAL

CLASSIFICATIONS FOR

WHICH PRODUCT QUALIFIED

\section{3 - MISSOURI}

\begin{tabular}{|c|c|c|}
\hline 434 & $E$ & VINE HILL FARM, RT. 2, BOX 713-F, GALENA 65656 \\
\hline 384 & $E$ & $\begin{array}{l}\text { JOHN L. WALKER, } 7316 \text { EAST FARM ROAD } 50 \text {, } \\
\text { STRAFFORD } 65757\end{array}$ \\
\hline 482 & $E$ & AL WILEY, RT. 1, BOX 146, BIRCH TREE 65438 \\
\hline 502 & $E$ & $\begin{array}{l}\text { JOE WOODWARD, } 14088 \text { SE STATE ROUTE CC, } \\
\text { FAUCETT } 64448\end{array}$ \\
\hline 257 & $E$ & $\begin{array}{l}\text { BEVERLY WRIGHT, } 8618 \text { EAST } 30 \text { TH TERRACE, } \\
\text { KANSAS CITY } 64129\end{array}$ \\
\hline 227 & $E$ & $\begin{array}{l}\text { GARRY YOUNG, } 14714 \text { MONROE ROAD 1022, MADISON } \\
65263\end{array}$ \\
\hline & $E$ & FRED ZILLICH, RT. 1, BOX 190, MERCER 64661-9777 \\
\hline
\end{tabular}

81 - MONTANA
$\mathrm{N} 121, \mathrm{~N} 161$

N109, N183, N28.

R115, R12, R120, R139, R16, R213, R225, R42, R56, R7, R71, R72, R81, W248, W518, W75. Z118

R

R85

$\mathrm{N} 28, \mathrm{~N} 41, \mathrm{~N} 83$

R114, R168, R169, R176, R187, R2, R200, R237, R25, R3, R56, R6, R9, R97, W11, W12, X1, X10 $X 104, X 135, X 143, X 145, X 147$. $\mathrm{X} 15, \mathrm{X} 16, \mathrm{X} 168, \mathrm{X} 2, \mathrm{X} 23, \mathrm{X} 25$ $X 26, X 3, X 34, X 5, Y 21, Y 53$

DR. THOMAS F.T. LINFIELD

ASSISTANT STATE VETERINARIAN

MONTANA DEPARTMENT OF LIVESTOCK

ANIMAL HEALTH DIVISION

P.O. BOX 202001

HELENA, MT 59620-2001

Phone: (406)444-2043

Fax: $\quad(406) 444-1929$

Hatcheries

52 E BLUE SKY HATCHERY \& GAME BIRD FARM, ROBERT WADDELL, 407 QUARTER CIRCLE ROAD, WINSTON 59647

20 E BRAND E. CAEKAERT, 8519 KING AVENUE WEST, BILLINGS 59105

32 E OTTER CREEK GAME BIRD (STEVE EIDE), HC 87, BOX 2220, BIG TIMBER 59011

44 E ROCKY MOUNTAIN HATCHERY, EILEEN JACKSON, P.O. BOX 1086, HAMILTON 59840

Phone: (406)642-3253

51 E ZAK'S GAME BIRD FARM \& HATCHERY, LEROY ZAK, 624 COUGAR LANE, STEVENSVILLE 59870

Phone: 406/772-2263

\section{Dealers}

$60 \quad E$

Z1, Z2, Z27, Z9 
Table 1. HATCHERIES, DEALERS. AND INDEPENDENT FLOCKS PARTICIPATING IN

THE NATIONAL POULTRY IMPROVEMENT PLAN

WATERFOWL, EXHIBITION POULTRY AND GAME BIRDS

\begin{tabular}{|c|c|c|c|c|c|}
\hline $\begin{array}{l}\text { APP. } \\
\text { NO. }\end{array}$ & $\begin{array}{l}\text { SUB- } \\
\text { PART }\end{array}$ & PARTICIPANTS NAME AND ADDRESS & $\begin{array}{l}\text { HATCHING } \\
\text { EGG } \\
\text { CAPACITY }\end{array}$ & $\begin{array}{l}\text { PRODUCTS CLASSIFIED } \\
\text { U.S. PULLORUM-TYPHOID } \\
\text { CLEAN }\end{array}$ & $\begin{array}{c}\text { ADDITIONAL } \\
\text { CLASSIFICATIONS FOR } \\
\text { WHICH PRODUCT QUALIFIED }\end{array}$ \\
\hline
\end{tabular}

Independent Flocks

66 E B \& B GAME BIRDS KEVIN BURTON, 6260 CANYON FERRY ROAD. HELENA 59602

57 E ALFRED BASSETT \& BARBARA CLARK, HC 60, BOX 19, BRUSSETT 59318

Phone: (406)577-2318

58 E BERGSTROM LAND \& LIVESTOCK, HCR 75, BOX 497. BRADY 59416

Phone: (406)627-2488

49 E BIG SKY GAME BIRDS, VAN TAMPLIN, 135 WEST VIEW DRIVE, VICTOR 59875

Phone: (406)642-3373

65 E BROKEN WING FARM, DONIELLE A. SLANINA, 2425 FLY CREEK ROAD, POMPEYS PILLAR 59064

61 E RICHARD BURNETT, 3144 OLD DARBY ROAD, DARBY 59829

Phone: (406)821-4710

53 E CACTUS HILL GAME BIRDS, JODIE BUTLER, P.O. BOX 174,329 3RD AVENUE, CARTER 59420

Phone: (406)734-5367

20 E BRAND E. CAEKAERT, 8519 KING AVENUE WEST, BILLINGS 59106

64 E MARIE \& HENRY CANTRELL, 5925 BUNDY ROAD, WORDEN 59088
E DAVID H. COUCH, P.O.BOX 177, ST, XAVIER 59075

E AUGUST D. EGDORF, P.O. BOX 434, HARDIN 59034

EVER FOWL FARM, DIANE ARGANBRIGHT, , CARTER 59420

E LAZY AC GAME BIRDS, AARON \& DEBBIE CLARK, P.O. BOX 1274, 240 CANTON LANE, TOWNSEND 59644 Phone: (406)266-3321

E FRANK MARULLO, 1131 OLD PINE DRIVE, BILLINGS 59101

E MONTANA GAME BIRDS, ROY EREAUX, P.O. BOX 1287. MALTA 59538

Phone: (406)654-2089

E WAYNE A. ROBISON, BOX 213, HYSHAM 59038

E MICHELLE RUARK, 1859 SOUTH CAMAS LANE, HAMILTON 59840

Phone: $(406) 375-9067$

E TRIPLE CREEK OUTFITTERS, BOX 1173, MALTA 59538

E VALLEY BIRD FARM, CHERYLE \& BILL WATSON, HC 45, BOX 1004, LODGEGRASS 59050

E WALKER CREEK FARMS, BOB UPTON, 2275 DILLON ROAD, WHITEFISH 59937 69 COTTONWOOD ROAD, CARDWELL 59721

Phone: (406)285-6715
E WHISPERING WIND RANCH, SHARON L. BUCKALLEW,
R15, R16, R21, Z121, Z31

$\mathrm{Z} 27$

$\mathrm{Z27}$

R176, R239, W12, W13, Z1, Z129, Z173, Z183, Z27, Z28, Z43, Z48

R117, R12, R213, R222, R225. $R 229, R 253, R 254, R 81, \times 2, \times 23$

W13, Z1, Z27, Z48

$\mathrm{Z1}, \mathrm{Z177}$

H5, R103, R117, R12, R16, R179, R213, R222, R229, R240, R248, R253, R254, R51, R7, R8, R83, W13, W573, Z4

Z1, Z142, Z27, Z43, Z48, Z60

Z43, Z60

N18, R104, R26, W247, W309

Z112, Z27, Z31, Z67

Z231, Z27

Z1, Z27, Z48

227

R87, W13, W370, X109, X110, $\times 112, \times 187, \times 188, \times 28, \times 51, \times 52$. $X 75, X 77, Z 2202, Z 260, Z 269, Z 28$, $Z 52, Z 57, Z 9$

227.

227.

Z1, Z27

W3, Z11, Z114, Z204, Z205, Z211, Z221, Z27, Z29, Z30, Z4, Z43, Z48, Z60, Z62 
Table 1. HATCHERIES, DEALERS, AND INDEPENDENT FLOCKS PARTICIPATING IN

THE NATIONAL POULTRY IMPROVEMENT PLAN

WATERFOWL, EXHIBITION POULTRY AND GAME BIRDS

\begin{tabular}{|c|c|c|c|c|c|}
\hline $\begin{array}{l}\text { APP. } \\
\text { NO. }\end{array}$ & $\begin{array}{l}\text { SUB- } \\
\text { PART }\end{array}$ & PARTICIPANTS NAME AND ADDRESS & $\begin{array}{l}\text { HATCHING } \\
\text { EGG } \\
\text { CAPACITY }\end{array}$ & $\begin{array}{c}\text { PRODUCTS CLASSIFIED } \\
\text { U.S. PULLORUM-TYPHOID } \\
\text { CLEAN }\end{array}$ & $\begin{array}{c}\text { ADDITIONAL } \\
\text { CLASSIFICATIONS FOR } \\
\text { WHICH PRODUCT QUALIFIED }\end{array}$ \\
\hline
\end{tabular}

47 - NEBRASKA

DR. DELWIN WILMOT

DEPUTY STATE VETERINARIAN

DEPARTMENT OF AGRICULTURE

BUREAU OF ANIMAL INDUSTRY

P.O. BOX 94787

LINCOLN, NE 68509-4787

Phone: (402)471-2351

Fax: $\quad(402) 471-3252$

Hatcheries
$81 \quad E$
$73 \quad E$ 68748-9401
NORFOLK HATCHERY, P.O. BOX 132, 1000 EAST OMAHA, NORFOLK 68702-0132
370 E SWANSON'S HUNTING ACRES, INC., RR 1, BOX 24, NIOBRARA 68760
Dealers
715
E
AG-WEST FEEDS, P.O. BOX 368, 470 SOUTH HIGHWAY 11, BURWELL 68823-0368 Phone: (308)346-5851
650 E AGLAND COOP. (FREMONT), 234 EAST JACKSON, FREMONT 68025
631 E AURORA COOP, P.O. BOX 125, 605 12TH STREET, BRYON 68325
641 E BAR THREE FEEDS, INC., P.O. BOX 100, ASHBY 69333
262 E BILL'S FARM \& RANCH SERVICES, P.O. BOX 512, WEST HIGHWAY 30, LEXINGTON 68850

249 E BLAIR FEED MILL, INC., 1643 STATE STREET, BLAIR 68008

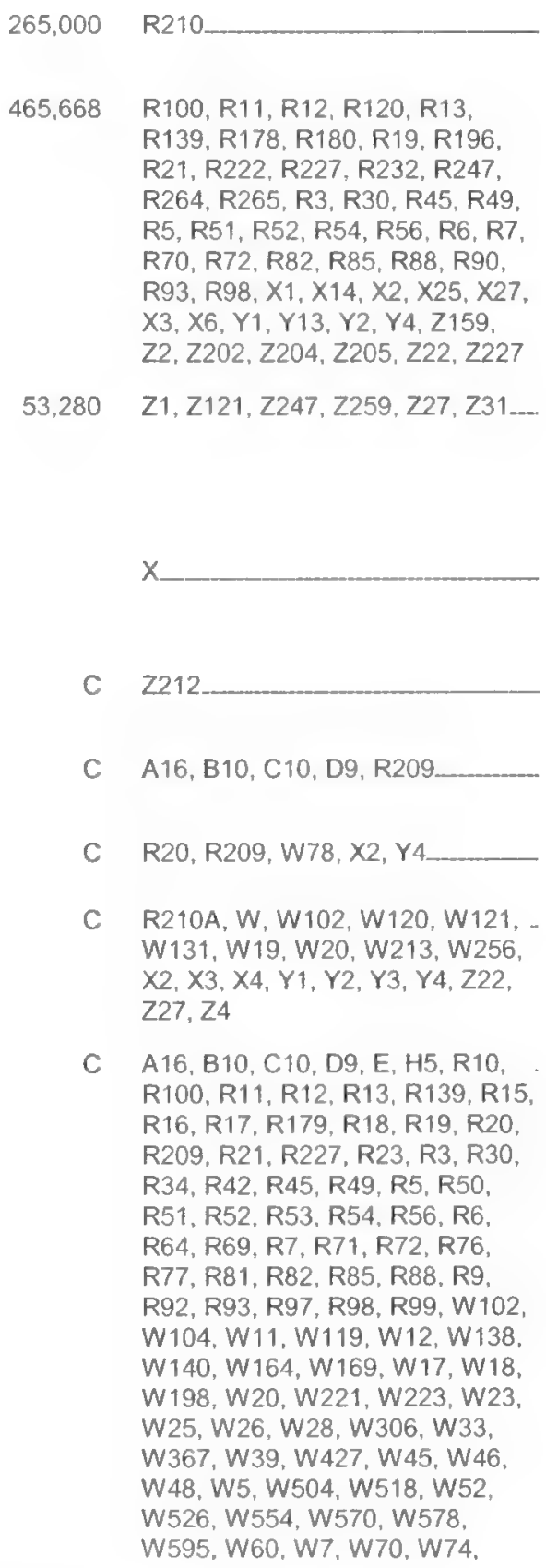

C A16, B10, C10, D9, R209.

C R20, R209, W78, X2, Y4

C R210A, W, W102, W120, W121, . W131, W19, W20, W213, W256, $X 2, X 3, X 4, Y 1, Y 2, Y 3, Y 4, Z 22$, $\mathrm{Z27}, \mathrm{Z4}$

C A16, B10, C10, D9, E, H5, R10, R100, R11, R12, R13, R139, R15, R16, R17, R179, R18, R19, R20, $R 209, R 21, R 227, R 23, R 3, R 30$, R34, R42, R45, R49, R5, R50, R51, R52, R53, R54, R56, R6 $R 64, R 69, R 7, R 71, R 72, R 76$, $R 77, R 81, R 82, R 85, R 88, R 9$, R92, R93, R97, R98, R99, W102, W104, W11, W119, W12, W138, W140, W164, W169, W17, W18, W198, W20, W221, W223, W23, W25, W26, W28, W306, W33, W367, W39, W427, W45, W46 W48, W5, W504, W518, W52, W526, W554, W570, W578, W595. W60, W7, W70, W74. 
Table 1. HATCHERIES, DEALERS, AND INDEPENDENT FLOCKS PARTICIPATING IN

THE NATIONAL POULTRY IMPROVEMENT PLAN

WATERFOWL, EXHIBITION POULTRY AND GAME BIRDS

$\begin{array}{llccc} & & \text { HATCHING } & \text { PRODUCTS CLASSIFIED } \\ \text { APP. } & \text { SUB- } & \text { PARTICIPANTS NAME AND ADDRESS } & \text { EGG } & \text { U.S. PULLORUM-TYPHOID } \\ \text { NO. } & \text { PART } & \text { CAPACITY } & \text { CLEAN }\end{array}$

ADDITIONAL

CLASSIFICATIONS FOR

WHICH PRODUCT QUALIFIED

47 - NEBRASKA

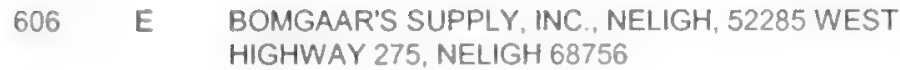

BOMGAAR'S SUPPLY, INC., SCHUYLER, BOX 587, WEST HIGHWAY 30 , SCHUYLER 68661

Phone: (402) 352-7900

BOMGAAR'S SUPPLY, INC. ALBION, BOX 233, NORTH HIGHWAY 14, ALBION 68620
BOMGAAR'S SUPPLY, INC. AINSWORTH, 843 EAST 4TH STREET, AINSWORTH 69210
W75, W81, W9, W91, W96, X1, $X 2, X 3, X 4, X 5, X 6, Y 1, Y 2, Y 3$ $\mathrm{Y} 4, \mathrm{Z} 15, \mathrm{Z} 159, \mathrm{Z} 176, \mathrm{Z2}, \mathrm{Z212}$ $\mathrm{Z} 22, \mathrm{Z} 27, \mathrm{Z} 43, \mathrm{Z} 61, \mathrm{Z} 79, \mathrm{Z} 80$

C E, H5, R10, R100, R11, R12, R13, R139, R15, R16, R17, R179, R18, R19, R20, R21, R227, R23, R3, R30, R34, R42, R45, R49, R5, R50, R51, R52, R53, R54, R56, R6, R64, R69, R7, R71, R72, R76, R77, R81, R82, R85, R88, R9, R92, R93, R97, R98. R99, W102, W104, W11, W119, W12, W138, W140, W164, W169, W17, W18, W198, W20 W221, W223, W23, W25, W26, W28, W306, W33, W367, W39 W427, W45, W46, W48, W5, W504, W518, W52, W526, W554, W570, W578, W595 W60, W7, W70, W74, W81, W9. W91, W96, X1, X2, X3, X4, X5, $X 6, Y 1, Y 2, Y 3, Z 15, Z 159, Z 176$. $\mathrm{Z2}, \mathrm{Z22}, \mathrm{Z27}, \mathrm{Z} 43, \mathrm{Z} 61, \mathrm{Z} 7 \mathrm{Y}, \mathrm{Z} 80$

C A16, B10, C10, D9, R209.

C A16, B10, C10, D9, E, H5, R10 R100, R11, R12, R13, R139, R15 R16, R17, R179, R18, R19, R20, R209, R21, R227, R23, R3, R30, R34, R42, R45, R49, R5, R50, $R 51, R 52, R 53, R 54, R 56, R 6$, R64, R69, R7, R72, R76, R77, $R 81, R 82, R 85, R 88, R 9, R 92$, R93, R97, R98, R99, W102, W104, W11, W119, W12, W138, W140, W164, W169, W17, W18, W198, W20. W221, W223, W23, W25, W26, W28, W306, W33, W367, W39, W427, W45, W46, W48, W5, W504, W518, W52, W526, W554, W570, W578, W595, W60, W7, W70, W74. W75, W81, W9, W91, W96, X1, $X_{2}, X_{3}, X_{4}, X_{5}, X 6, Y 1, Y 2, Y 3$, Z15, Z159, Z176, Z2, Z22, Z27. Z43, Z61, Z79, Z80

C A16, B10, C10, D9, E, H5, R10, R100, R11, R12, R13, R139, R15, R16, R17, R179, R18, R19, R20, R209, R21, R227, R23, R3, R30, R34, R42, R45, R49, R5, R50, R51, R52, R53, R54, R56, R6, R64, R69, R7, R71, R72, R76 $R 77, R 81, R 82, R 85, R 88, R 9$, R92, R93, R97, R98, R99, W102. W104, W11, W119, W12, W138, W140, W164, W169, W17, W18, W198, W20, W221, W223, W23, W25, W26, W28, W306, W33, W367, W39, W427, W45, W46, W48, W5, W504, W518, W52. W526, W554. W570, W578, W595, W60, W7, W70, W74, W75, W81, W9, W91, W96, X1 


\begin{tabular}{|c|c|c|c|c|c|}
\hline $\begin{array}{l}\text { APP. } \\
\text { NO. }\end{array}$ & $\begin{array}{l}\text { SUB- } \\
\text { PART }\end{array}$ & PARTICIPANTS NAME AND ADDRESS & $\begin{array}{l}\text { HATCHING } \\
\text { EGG } \\
\text { CAPACITY }\end{array}$ & $\begin{array}{l}\text { PRODUCTS CLASSIFIED } \\
\text { U.S. PULLORUM-TYPHOID } \\
\text { CLEAN }\end{array}$ & $\begin{array}{c}\text { ADDITIONAL } \\
\text { CLASSIFICATIONS FOR } \\
\text { WHICH PRODUCT QUALIFIED }\end{array}$ \\
\hline
\end{tabular}

BOMGAAR'S SUPPLY, INC., CREIGHTON, 1605 MAIN STREET. CREIGHTON 68729

BOMGAAR'S SUPPLY, INC., TEKAMAH, 660 SOUTH HIGHWAY 75, TEKAMAH 68061

Phone: (402)374-2399

BOMGAAR'S SUPPLY, INC., BLAIR, 1410 SOUTH HIGHWAY 30 , BLAIR 68008

Phone: (402)533-8133
47 - NEBRASKA
$X 2, X 3, X 4, X 5, X 6, Y 1, Y 2, Y 3$, Z15, Z159, Z176, Z2, Z22, Z27, $\mathrm{Z} 43, \mathrm{Z61}, \mathrm{Z79}, \mathrm{Z} 80$

C A16, B10, C10, D9, E, H5, R10, R100, R11, R12, R13, R139, R15, R16, R17, R179, R18, R19, R20, R209, R21, R227, R23, R3, R30, R34, R42, R45, R49, R5, R50. R51, R52, R53, R54, R56, R6, R64, R69, R7, R71, R72, R76, $R 77, R 81, R 82, R 85, R 88, R 9$, R92, R93, R97, R98, R99, W102. W104, W11, W119, W12, W138, W140, W164, W169, W17, W18 W198, W20, W221, W223, W23, W25, W26, W28, W306, W33, W367, W39, W427, W45, W46, W48, W5, W504, W518, W52, W526, W554, W570, W578. W595, W60, W7, W70, W74 W75, W81, W9, W91, W96, X1. $X 2, X 3, X 4, X 5, X 6, Y 1, Y 2, Y 3$, Z15, Z159, Z176, Z2, Z22, Z27. $\mathrm{Z} 43, \mathrm{Z} 61, \mathrm{Z79}, \mathrm{Z} 80$

A16, B10, C10, D9, E, H5, R10, R100, R11, R12, R13, R139, R15, R16, R17, R179, R18, R19, R20, R21, R227, R23, R3, R30, R34, R42, R45, R49, R5, R50, R51. R52, R53, R54, R56, R6, R64, R69, R7, R71, R72, R76, R77, R81, R82, R85, R88, R9, R92, R93, R97, R98, W, W20, W221, W223, W23, W26, W28, W306, W33, W367, W39, W427, W45, W46, W48, W5, W504, W518, W52, W526, W554, W570, W578, W595, W60, W7, W70, W74, W75, W81, W9, W91, X1, $X 2, X 3, X 4, X 5, X 6, Y 1, Y 2, Y 3$, $Y 4, Z 15, Z 159, Z 176, Z 2, Z 22$, Z27, Z43, Z61, Z79, Z80

A16, B10, C10, D9, E, H5, R10, . R100, R11, R12, R13, R139, R15。 R16, R17, R179, R18, R19, R20, R21, R227, R23, R3, R30, R34, R42, R45, R49, R5, R50, R51. $R 52, R 53, R 54, R 56, R 6, R 64$, R69, R7, R71, R72, R76, R77, R81, R82, R85, R88, R9, R92, R93, R97, R98, W, W20, W221, W223, W23, W25, W26, W28, W306, W33, W367, W39, W427, W45, W46, W48, W5, W504, W518, W52, W526, W554, W570, W578, W595, W60, W7. W70, W74, W81, W9, W91, X1, $X_{2}, X_{3}, X_{4}, X_{5}, X_{6}, Y_{1}, Y_{2}, Y_{3}$, $Y 4, Z 15, Z 159, Z 176, Z 2, Z 22$, Z27, Z43, Z61, Z79, Z80

A16, B10, C10, D9, E, H5, R10, R100, R11, R12, R13, R139, R15, R16, R17, R179, R18, R19, R20, R21, R227, R23, R3, R30, R34, R42, R45, R49, R5, R50, R5 . R52, R53, R54, R56, R6, R64. 
Table 1. HATCHERIES, DEALERS, AND INDEPENDENT FLOCKS PARTICIPATINGIN

THE NATIONAL POULTRY IMPROVEMENT PLAN

WATERFOWL, EXHIBITION POULTRY AND GAME BIRDS

APP. SUB- PARTICIPANTS NAME AND ADDRESS

NO. PART
HATCHING

EGG

CAPACITY
PRODUCTS CLASSIFIED

U.S. PULLORUM-TYPHOID

CLEAN
ADDITIONAL

CLASSIFICATIONS FOR

WHICH PRODUCT QUALIFIED

47 - NEBRASKA

692 E BOMGAAR'S SUPPLY, INC. WAHOO, 441 EAST FIRST,
WAHOO 68066

Phone: (402)443-1177
694 E
HIMGAAR'S SUPPLY, INC., HARTINGTON, 56285
HIGHAY 84, HARTINGTON 68739

Phone: (402)254-2134
R69, R7, R71, R72, R76, R77, R81, R82, R85, R88, R9, R92, R93, R97, R98, W, W20, W221, W223, W23, W25, W26, W28, W306, W33, W367, W39, W427, W45, W46, W48, W5, W504, W518, W52, W526, W554. W570, W578, W595, W60, W7. W70, W74, W75, W81, W9, W91. W96, X1, X2, X3, X4, X5, X6, Y1。 Y2, Y3, Z15, Z159, Z176, Z2, Z22, Z27, Z43, Z61, Z79, Z80

A16, B10, C10, D9, E, H5, R10, 。 R100, R11, R12, R13, R139, R15, R16, R17, R179, R18, R19, R20. R21, R227, R23, R3, R30, R34, R42, R45, R49, R5, R50, R54, R52, R53, R54, R56, R6, R64, R69, R7, R71, R72, R76, R77, R81, R82, R85, R88, R9, R92, R93, R97, R98, W, W20, W221. W223, W23, W25, W26, W28, W306, W33, W367, W39, W427, W45, W46, W48, W5, W504 W518, W52, W526, W554, W570, W578, W595, W60, W7 W70, W74, W75, W81, W9, w91, W96, X1, X2, X3, X4, X5, X6, Y1, Y2, Y3, Y4, Z15, Z159, Z176, Z2, $\mathrm{Z} 22, \mathrm{Z27}, \mathrm{Z43}, \mathrm{Z61}, \mathrm{Z79}, \mathrm{Z} 80$

A16, B10, C10, D9, E, H5, R10, R100, R11, R12, R13, R139, R15, $R 16, R 179, R 18, R 19, R 20, R 21$. R227, R23, R3, R30, R34, R42, R45, R49, R5, R50, R51, R52, R53, R54, R56, R6, R64, R69, R7, R71, R72, R76, R77, R81, $R 82, R 85, R 88, R 9, R 92, R 93$, R97, R98, W, W20, W221, W223, W23, W25, W26, W28, W306, W33, W367, W39, W427, W45. W46, W48, W5, W504, W518, W52, W526, W554, W570, W578, W595, W60, W7, W70 W74, W75, W81, W9, W91, W96, $X_{1}, X_{2}, X_{3}, X_{4}, X 5, X 6, Y 1, Y 2$, $Y 3, Y 4, Z 15, Z 159, Z 176, Z 2, Z 22$. $Z 27, Z 43, Z 61, Z 79, Z 80$

C A16, B10, C10, D9, E, H5, R10. R100, R11, R12, R13, R139, R15, R16, R17, R179, R18, R19, R20, R21, R227, R23, R3, R30, R34, R42, R45, R49, R5, R50, R51, R52, R53, R54, R56, R6, R64, R69, R7, R71, R72, R76, R77, $R 81, R 82, R 85, R 88, R 9, R 92$, R93, R97, R98, R99, W, W102, W104, W11, W119, W12, W138, W140, W164, W169, W17, W18, W19, W20, W221, W223, W23, W25, W26, W28, W306, W33. W367, W39, W427, W46, W48, W5, W504, W518, W52, W526, W554, W570, W578, W595, W60, W7, W70, W74, W75, W81, W9, W91, W96, X1, X2, X3, X4 , $X_{5}, X 6, Y 1, Y 2, Y 3, Y 4, Z 15$. 


\begin{tabular}{|c|c|c|c|c|c|}
\hline $\begin{array}{l}\text { APP. } \\
\text { NO. }\end{array}$ & $\begin{array}{l}\text { SUB- } \\
\text { PART }\end{array}$ & PARTICIPANTS NAME AND ADDRESS & $\begin{array}{l}\text { HATCHING } \\
\text { EGG } \\
\text { CAPACITY }\end{array}$ & $\begin{array}{c}\text { PRODUCTS CLASSIFIED } \\
\text { U.S. PULLORUM-TYPHOID } \\
\text { CLEAN }\end{array}$ & $\begin{array}{c}\text { ADDITIONAL } \\
\text { CLASSIFICATIONS FOR } \\
\text { WHICH PRODUCT QUALIFIED }\end{array}$ \\
\hline
\end{tabular}

47 - NEBRASKA

685

\section{E}

BOMGAARS SUPPLY, INC., WEST POINT, 1310 SOUTH LINCOLN STREET, WEST POINT 68788

BOMGAARS SUPPLY, INC., WAYNE, 614 NORTH VALLEY DRIVE, WAYNE 68787

BOMGAARS SUPPLY, INC., NORFOLK, 1201 OMAHA AVENUE, NORFOLK 68701
Z159, Z176, Z2, Z22, Z27, Z43. $\mathrm{Z} 61, \mathrm{Z79}, \mathrm{Z80}$

C A16, B10, C10, D9, E, R10, R100 R11, R12, R13, R139, R15, R16, R17, R179, R18, R19, R20, R21, R227, R23, R3, R30, R34, R42, R45, R49, R5, R50, R51, R52, R53, R54, R56, R6, R64, R69, R7, R71, R72, R76, R77, R81, R82, R85, R88, R9, R92, R93, R97, R98, R99. W. W102, W104, $W 11, W 119, W 12, W 138, W 140$. W164, W169, W17, W18, W19. W20, W221, W223, W23, W25, W26, W28, W306, W33, W367, W39, W427, W45, W46, W48, W5, W504, W518, W52, W526. W554, W570, W578, W595, W60, W7, W70, W74, W75, W81, W9, W91, W96, X1, X2, X3, X4, $X 5, X 6, Y 1, Y 2, Y 3, Y 4, Z 15$, Z159, Z176, Z2, Z22, Z27, Z43, $\mathrm{Z} 61, \mathrm{Z} 79, \mathrm{Z} 80$

C A16, B10, C10, D9, E, H5, R10, R100, R11, R12, R13, R139, R15, R16. R17, R179, R18, R19, R20,

R21, R227, R23, R3, R30, R34, R42, R45, R49. R5, R50, R51, $R 52, R 53, R 54, R 56, R 6, R 64$ R69, R7, R71, R72, R76, R77. R81, R82, R85, R88, R9, R92, R93, R97, R98, R99, W, W102, W104, W11, W119, W12, W138, W140, W164, W169, W17, W18 W19, W20, W221, W223, W23, W25, W26, W28, W306, W33, W367, W39, W427, W45, W46, W48, W5, W504, W518, W52, W526. W554, W570, W578. W595, W60, W7, W70, W74, W75, W81, W9, W91, W96, X1, $X_{2}, X_{3}, X 4, X 5, X 6, Y 1, Y 2, Y 3$, $Y 4, Z 15, Z 159, Z 176, Z 2, Z 22$, $\mathrm{Z} 27, \mathrm{Z} 43, \mathrm{Z} 61, \mathrm{Z} 79, \mathrm{Z} 80$

C A16, B10, C10, D9, E, H5, R10, R100, R11, R12, R13, R139, R15, R16, R17, R179, R18, R19, R20。 R21, R227, R23, R3, R30, R34, R42, R45, R49, R5, R50, R51, R52, R53, R54, R56, R6, R64, R69, R7, R71, R72, R76, R77, R81, R82, R85, R88, R9, R92. R93, R97, R98, R99, W102, W104, W11, W119, W12, W138, W140, W164, W169, W17, W18 W198, W20, W221, W223, W23, W25, W26, W28, W306, W33, W367, W39, W427, W45, W46, W48, W5, W504, W518, W52. W526, W554, W570, W578, W595, W60, W7, W70, W74 W75, W81, W9, W91, W96, X1. $X_{2}, X 3, X 4, X 5, X 6, Y 1, Y 2, Y 3$. $Y 4, Z 15, Z 159, Z 176, Z 2, Z 22$. Z27, Z43, Z61, Z79, Z80 
Table 1. HATCHERIES, DEALERS, AND INDEPENDENT FLOCKS PARTICIPATING IN THE NATIONAL POULTRY IMPROVEMENT PLAN

WATERFOWL, EXHIBITION POULTRY AND GAME BIRDS

APP. SUB- PARTICIPANTS NAME AND ADDRESS
HATCHING
EGG

CAPACITY
PRODUCTS CLASSIFIED

U.S. PULLORUM-TYPHOID CLEAN
ADDITIONAL

CLASSIFICATIONS FOR WHICH PRODUCT QUALIFIED

47 - NEBRASKA

\begin{abstract}
351 E CHICKEN DEPOT, 2523 DAKOTA AVENUE, SOUTH SIOUX CITY 68776

78 E COLUMBUS FEED \& HATCHERY, 221611 STREET, COLUMBUS 68601
\end{abstract}

673 E COOPERATIVE SUPPLY, INC., P.O. BOX 358, HOWELLS 68641

Phone: (402)986-1212

333 E CRAWFORD GRAIN, INC., P.O. BOX 454, 210 SOUTH WASHINGTON, LEXINGTON 68850

352 E CRETE LUMBER \& FARM SUPPLY, P.O. BOX 283, WEST HIGHWAY 33, CRETE 68333

671 E DICKENSON SEED \& FEED, 3354 MCKELVIE ROAD, SEWARD 68434

682 E DICKINSON SEED \& FEED, 3354 MCKELVIE ROAD, SEWARD 68434

237 E DORCHESTER FARMERS COOP, BOX 263, DORCHESTER 68343

90 E ELGIN FEEDERS SUPPLY, INC., BOX 387, 2ND \& CEDAR, ELGIN 68636

567 E EVAN'S FEED COMPANY, 142 SOUTH 10TH, BROKEN BOW 68822

449 E FARMER'S COOP., GORDON, 201 EAST ORIN STREET. GORDON 69349

\begin{abstract}
415 E FARMER'S ELEVATOR COMPANY, 232 SOUTH RAILROAD, P.O. BOX 725, WILBER 68465

710 E THE FEED SHED, INC. 444 WEST 29TH STREET, SOUTH SIOUX CITY 68776

Phone: (402)494-1828
\end{abstract}

502 E HERITAGE SEED COMPANY, P.O. BOX 544, 324 MAIN
STREET, CRAWFORD 69339

675 E MARLAN IDEUS, P.O. BOX 38, 202 WEST JEFFERSON AVENUE, AMHERST 68812

Phone: (308)826-5265

681 E JUSTUS FEEK MILL, HC 64, BOX 66A, 620 B STREET, BENKELMAN 69021-9143

365 E KENWOOD FEED STORE, 4801 NORTH 30TH, OMAHA 68111
C A16, B10, C10, D9, R209, X2, X3. $\mathrm{Y} 1, \mathrm{Y} 13, \mathrm{Y} 3$

C R209, R210, W, X2, X25, X3, X5。Y1, Y2, Y3, Y4, Z2, Z202, Z204. Z205, Z227, Z4

C $\quad \mathrm{X} 3, \mathrm{X} 41, \mathrm{Z212}, \mathrm{Z22}$

C B10, R209

C\&E N

C B10

C B10, R209

C R240, W219

C $\mathrm{B} 10$

C R100, R11, R12, R120, R13, R139, R178, R180, R19, R196, R21, R222, R227, R232, R247, $R 264, R 265, R 3, R 30, R 45, R 49$. R5, R51, R52, R54, R56, R6, R7. R70, R72, R82, R85, R88, R90, $R 93, R 98, X 1, X 14, X 2, X 25, X 27$. $X 3, X 6, Y 1, Y 13, Y 2, Y 4, Z 159$,

Z2, Z202, Z204, Z205, Z22, Z227

P A16, B10, C10, D9, R209, X, Y

R146, R169, R20, R5, R6, R7, R95, W1, W102, W120, Wi21, W131, W19, W20, W213, W256, W257, W259, W26, W424, W86

C A16, B10,D9, X131,X14,X2,X3, $X 4, X 5, X 6, Y 1, Y 2, Y 3, Y 4, Z 227$, $\mathrm{Z27}$

R94, W132

$R, R 20, R 209, \times 2, \times 6, Z 4$

C A16, B10, C10, D9, E, H5, R10, R100, R11, R12, R13, R139, R15. $R 16, R 17, R 179, R 18, R 19, R 20$, $R 209, R 21, R 227, R 23, R 3, R 30$, $R 34, R 42, R 45, R 49, R 5, R 50$, R51, R52, R53, R54, R56, R6, R64, R69, R7, R71, R72, R76, R77, R81, R82, R85, R88, R9, R92, R93, R97, R98, R99, W104, W11, W119, W12, W138, W140, W164, W169, W17, W18, W198, W20, W221, W223, W23, W25, 
Table 1. HATCHERIES, DEALERS, AND INDEPENDENT FLOCKS PARTICIPATING IN

THE NATIONAL POULTRY IMPROVEMENT PLAN

WATERFOWL, EXHIBITION POULTRY AND GAME BIRDS

\section{APP. SUB- PARTICIPANTS NAME AND ADDRESS}

NO. PART
HATCHING

EGG

CAPACITY
PRODUCTS CLASSIFIED

U.S. PULLORUM-TYPHOID

CLEAN
ADDITIONAL

CLASSIFICATIONS FOR

WHICH PRODUCT QUALIFIED

47 - NEBRASKA

$\begin{array}{ccc}311 & \text { E } & \begin{array}{l}\text { KRUGER FEED AND SEED, 131 EAST 7TH STRET, P.O. } \\ \text { BOX 250, NORTH BEND 68649 }\end{array} \\ 273 & \text { E } & \begin{array}{l}\text { LIVESTOCK FEEDER'S SERVICE, INC., P.O. BOX 106,515 } \\ \text { SOUTH 3RD STREET HOLDREGE 68949 }\end{array} \\ 611 & \text { E } & \begin{array}{l}\text { LOOP VALLEY SUPPLY, } 302 \text { HOWARD AVENUE, ST. } \\ \text { PAUL 68873 }\end{array} \\ 337 & \text { E } & \begin{array}{l}\text { MEYER FEED \& SUPPLY, INC., P.O. BOX 390, 108 } \\ \text { HIGHWAY 2, CAIRO 68824 }\end{array} \\ 280 & \text { E } & \begin{array}{l}\text { MILLER FARMER'S FEED \& SEED, INC., 418 EAST } \\ \text { ELLINOIS STREET, SIDNEY 69162 }\end{array} \\ 642 & \text { E } & \begin{array}{l}\text { NORTH PLATTE FEED CENTER, 1415 EAST 8TH, } \\ \text { NORTH PLATTE 69101 }\end{array} \\ 206 \quad \text { E } & \begin{array}{l}\text { NORTHWEST FEED \& GRAIN COMPANY, INC., 8625 } \\ \text { MiLITARY AVENUE, OMAHA 68134 }\end{array}\end{array}$

OGALLALA FEED \& SEED, INC., 2 EAST B, P.O. BOX 81, OGALLALA 69153

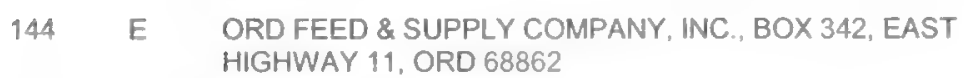

653
ORSCHELN, AUBURN, 1020 CENTRAL AVENUE, AUBUN 68305
W26, W28, W306, W33, W367, W39, W427, W45, W46, W48, W5, W504, W518, W52, W526, W554, W570, W578, W595, W60, W7, W70, W74, W75, W81. W9, W91, W96, X1, X2, X3, X4. $X 5, X 6, Y 1, Y 13, Y 2, Y 3, Y 4, Z 15$ Z159, Z176, Z2, Z22, Z27, Z43, $\mathrm{Z} 61, \mathrm{Z79}, \mathrm{Z} 80$

C A16, B10, C10, D9, R209, X, Y

C $X_{1}, X_{11}, X_{14}, X_{2}, X_{2} 5, X_{3}, X_{5}$, $X 6, Y 1, Y 13, Y 2, Y 3, Y 4, Z 202$. Z205, Z212

C A16, B10, C10, D9, R209

$P \quad X, Y$

C B10, C10, R6, X, Z227

C Z204, Z209, Z212, Z227, Z9

C A, B, D, E, H5, R11, R12, R146, R15, R16, R19, R20, R21, R22, R27, R30, R33, R42, R45, R53, R54, R69, R7, R71, R72, R77, R81, R85, R87, R89, R96, R98. W. W11, W119, W12, W120. W121, W126, W129, W136. W138, W140, W142, W145. W160, W164, W17, W176, W18, W19, W193, W198, W20, W211, W248, W25, W259, W26, W262, W270, W28, W29, W297, W33, W41, W45, W48, W5, W50, W54. W60, W7, W74, W75, W81, W86, W88, W89, W9, W96, X131. $X 135, X 136, X 2, X 21, X 23, X 24$ $X 25, X 27, X 3, X 4, X 6, Y 1, Y 2, Y 3$, Y4, Y5, Y54, Z1, Z110, Z172, Z20, Z202, Z204, Z205, Z206, Z207, Z22, Z227, Z27, Z43, Z45, Z62, Z9, 293

P A, C, X14, X2, X3, Y2, Y4

C A16, B10, C, D9, R12, R20, Y1, Y $2, Y 3, Y 4, Y 7, Z 202, Z 212$

C H5, R100, R11, R12, R123, R146, R15, R16, R169, R17, R171, R178, R19, R196, R20, R209, R21, R22, R227, R237, R245. R247, R248, R33, R34, R42, R51, R52, R53, R54, R55, R69, R7. R70, R71, R72, R77, R81, R85, R89, W11, W119, W121, W129, W138, W140, W142, W145, W16, W169, W17, W176, W18, W19, W198, W20, W248, W25, W26, W270, W28, W297, W3, W301, W346, W363, W368, W41. W427, W45, W48, W552, 
Table 1. HATCHERIES, DEALERS. AND INDEPENDENT FLOCKS PARTICIPATING IN

THE NATIONAL POULTRY IMPROVEMENT PLAN

WATERFOWL, EXHIBITION POULTRY AND GAME BIRDS

\begin{tabular}{|c|c|c|c|c|c|}
\hline $\begin{array}{l}\text { APP. } \\
\text { NO. }\end{array}$ & $\begin{array}{l}\text { SUB- } \\
\text { PART }\end{array}$ & PARTICIPANTS NAME AND ADDRESS & $\begin{array}{l}\text { HATCHING } \\
\text { EGG } \\
\text { CAPACITY }\end{array}$ & $\begin{array}{l}\text { PRODUCTS CLASSIFIED } \\
\text { U.S. PULLORUM-TYPHOID } \\
\text { CLEAN }\end{array}$ & $\begin{array}{c}\text { ADDITIONAL } \\
\text { CLASSIFICATIONS FOR } \\
\text { WHICH PRODUCT QUALIFIED }\end{array}$ \\
\hline
\end{tabular}

47 - NEBRASKA

654 E ORSCHELN, NEBRASKA CITY, 1707 FRONTAGE ROAD,
NEBRASKA CITY 68140

ORSCHELN. FALLS CITY, RR 2, BOX 268, FALLS CITY 68355

ORSCHELN FARM \& HOME LLC, SEWARD, 701 SOUTH 6TH STREET, SEWARD 68434

Phone: (402)643-9797
W57, W60, W74, W75, W81.

W86, W9, W96, X135, X2, X25

$X_{27}, X_{3}, X_{6}, X 96, Y 1, Y 2, Y 3, Y 4$,

Y6, Z1, Z125, Z172, Z20, Z202.

Z204, Z205, Z206, Z207, Z22

Z227, Z27, Z4, Z43, Z45, Z62, Z9,

293

C H5, R100, R11, R12, R123, R146,

R15, R16, R169, R17, R171

R178, R19, R196, R20, R209,

$R 21, R 22, R 227, R 237, R 245$,

R247, R248, R33, R34, R42, R51,

R52, R53, R54, R55, R69, R7.

R70, R71, R72, R77, R81, R85

R89, W11, W119, W121, W129,

W138, W140, W142, W145.

W16, W169, W17, W176, W18,

W19, W198, W20, W248, W25,

W26, W270, W28, W297, W3

W301, W346, W363, W368.

W41, W427, W45, W48, W552,

W57, W60, W74, W75, W81

W86, W9, W96, X135, X2, X25,

$X 27, X 3, X 6, Y 1, Y 2, Y 3, Y 4, Y 6$,

Z1, Z125, Z172, Z20, Z202, Z204, Z205, Z206, Z207, Z22, Z227,

Z27, Z43, Z45, Z62, Z9, Z93

C H5, R100, R11, R12, R123, R146, R15, R16, R169, R17, R171, R178, R19, R196, R20, R209. R21, R22, R227, R237, R245, R247, R248, R33, R34, R42, R51, R52, R53, R54, R55, R69, R7, R70, R71, R72, R77, R81, R85. R89, W11, W119, W121, W129, W138, W140, W142, W145. W16, W169, W17, W176, W18, W19, W198, W20, W248, W25 W26, W270, W28, W297, W3, W301, W346, W363, W368. W41, W427, W45, W48, W552 W57, W60, W74, W75, W81, W86, W9, W96, X135, X2, X25. $X 27, X 3, X 6, X 96, Y 1, Y 2, Y 3, Y 4$, Y6, Z1, Z125, Z172, Z20, Z202, Z204, Z205, Z206, Z207, Z22. Z227, Z27, Z4, Z43, Z45, Z62, Z9, 293

C H5, R100, R11, R12, R123, R146. R15, R16, R169, R17, R171, R178, R19, R196, R20, R209. R21, R22, R227, R237, R245, R247, R248, R33, R34, R42, R51, R52, R53, R54, R55, R69, R7, R71, R72, R77, R81, R85, R89, W11, W119, W121, W129. W138, W140, W145, W16. W169, W17, W176, W18, W19, W198, W20, W248, W25, W26. W270, W28, W297, W3, W301, W346, W363, W368, W41, W427, W45, W48, W552, W57, W60, W74, W75, W81, W86, W9. $W 96, X_{135}, X_{2}, X_{25}, X_{27}, X_{3}, X_{6}$, X96, Y1, Y2, Y3, Y4, Y6, Z1 Z125, Z172, Z20, Z202, Z204. Z205, Z206, Z207, Z22, Z227. 
Table 1. HATCHERIES, DEALERS, AND INDEPENDENT FLOCKS PARTICIPATING IN

THE NATIONAL. POULTRY IMPROVEMENT PLAN

WATERFOWL, EXHIBITION POULTRY AND GAME BIRDS

$\begin{array}{lll}\text { APP. } & \text { SUB- } \\ \text { NO. } & \text { PART }\end{array}$

$\begin{array}{cc}\text { HATCHING } & \text { PRODUCTS CLASSIFIED } \\ \text { EGG } & \text { U.S.PULLORUM-TYPHOID } \\ \text { CAPACITY } & \text { CLEAN }\end{array}$

ADDITIONAL.

CLASSIFICATIONS FOR WHICH PRODUCT QUALIFIED

\footnotetext{
47 - NEBRASKA
}

ORSCHELN FARM \& HOME LLC, BEATRICE, 2415 NORTH 6TH STREET, BEATRICE 68310-1217

Phone: (402)228-3323
ORSCHELN FARM \& HOME LLC, BROKEN BOW, 334 EAST SOUTH E STREET, BROKEN BOW 68822 Phone: (308)872-6459
C H5, R100, R11, R12, R123, R146, R15, R16, R169, R17, R171, R178, R19, R196, R20, R209. R21, R22, R227, R237, R245, R247, R248, R33, R34, R42, R51, R52, R53, R54, R55, R69, R7, R70, R71, R72, R77, R81, R85, R89, W11, W119, W121, W129, W138, W140, W142, W145, W16, W169, W17, W176, W18. W19, W198. W20, W248, W25 W26, W270, W28, W297, W3. W301, W346, W363, W368, W41, W427, W45, W48, W552, W57. W60, W74, W75, W81, W86, W9, W96, X135, X2, X25, $X_{2} 7, X 3, X 6, X 96, Y 1, Y 2, Y 3, Y 4$, Y6, Z1, Z125, Z172, Z20, Z202. Z204, Z205, Z206, Z207, Z22. Z227, Z27, Z4, Z43, Z45, Z62. Z9. $\mathrm{Z93}$

C H5, R100, R11, R12, R123, R146, R15, R16, R169, R17, R171. R178, R19, R196, R20, R209. $R 21, R 22, R 227, R 237, R 245$, R247, R248, R33, R34, R42, R51, R52, R53, R54, R55, R69, R7. R70, R71, R72, R77, R81, R85 R89, W11, W119, W121, W129, W138, W140, W142, W145. W16, W169, W17, W18, W19. W198, W20, W248, W25, W26. W270, W28, W297, W3, W301. W346, W363, W368, W41, W427, W45, W48, W552, W57. W60, W74, W75, W81. W86. W9, $X 135, X 2, X 25, X 27, X 3, X 6, X 96$. $Y 1, Y 2, Y 3, Y 4, Y 6, Z 1, Z 125$, Z172, Z20, Z202, Z204, Z205. Z206, Z207, Z22, Z227, Z27, Z4, Z43, Z45, Z62, Z9, Z93

C H5, R100, R11, R12, R123, R146, R15, R16, R169, R17, R171, R178, R19, R196, R20, R209. R21, R22, R227, R237, R245, R247, R248, R33, R34, R42, R51, R52, R53, R54, R55, R69, R7. R70, R71, R72, R77, R81, R85. R89, W11, W119. W121, W129. W138, W140, W142, W145, W16, W169, W17, W176, W18. W19. W198, W20. W248, W25. W26, W270, W28, W297, W3, W301, W346, W363, W368, W41, W427, W45, W48, W552. W57, W60, W74, W75, W81, W86, W9, W96, X135, X2, X25 $X 27, X 3, X 6, X 96, Y 1, Y 2, Y 3, Y 4$, $Y 6, Z 1, Z 125, Z 172, Z 20, Z 202$. Z204, Z205, Z206, Z207, Z22. Z227, Z27, Z4, Z43, Z45, Z62, Z9, 293

C H5, R100, R11, R12, R123, R146. R15, R16, R169, R17, R171. R178, R19, R196, R20, R209, 
Table 1. HATCHERIES. DEALERS. AND INDEPENDENT FLOCKS PARTICIPATING IN

THE NATIONAL POULTRY IMPROVEMENT PLAN

WATERFOWL, EXHIBITION POULTRY AND GAME BIRDS

\begin{tabular}{|c|c|c|c|c|c|}
\hline $\begin{array}{l}\text { APP. } \\
\text { NO. }\end{array}$ & $\begin{array}{l}\text { SUB- } \\
\text { PART }\end{array}$ & PARTICIPANTS NAME AND ADDRESS & $\begin{array}{l}\text { HATCHING } \\
\text { EGG } \\
\text { CAPACITY }\end{array}$ & $\begin{array}{l}\text { PRODUCTS CLASSIFIED } \\
\text { U.S. PULLORUM-TYPHOID } \\
\text { CLEAN }\end{array}$ & $\begin{array}{c}\text { ADDITIONAL } \\
\text { CLASSIFICATIONS FOR } \\
\text { WHICHPRODUCT QUALIFIED }\end{array}$ \\
\hline
\end{tabular}

47 - NEBRASKA

ORSCHELN FARM \& HOME LLC, HASTINGS, 1315 WEST J STREET, HASTINGS 68901-6708

Phone: (402)463-9494

R21, R22, R227, R237, R245, R247, R248, R33, R34, R42, R51, R52, R53, R54, R55, R69, R7.

R70, R71, R72, R77, R81, R85, R89, W11, W119, W121, W129, W138, W140, W142, W145, W16, W169, W17, W18, W19, W198, W20, W248, W25, W26, W270, W28, W297, W3, W301, W346, W363, W368, W41, W427, W45, W48, W552, W57, W60, W74, W75, W81, W86, W9, W96, X135, X2, X25, X27, X3, X6, $X 96, Y 1, Y 2, Y 3, Y 4, Y 6, Z 1$. Z125, Z172, Z20, Z202, Z204。 Z205, Z206, Z207, Z22, Z227. Z27, Z4, Z45, Z62, Z9, Z93

C H5, R100, R11, R12, R123, R146, R15, R16, R169, R17, R171, R178, R19, R196, R20, R209, $R 21, R 22, R 227, R 237, R 245$, R247, R248, R33, R34, R42, R51, R52, R53, R54, R55, R69, R7. R70, R71, R72, R77, R81, R85, R89, W11, W119, W121, W129, W138, W140, W142, W145. W16, W169, W17, W176, W18, W19, W198, W20, W248, W25, W26, W270, W28, W297, W3, W301, W346, W363, W368, W41, W427, W45, W48, W552. W57, W60, W74, W75, W81 W86, W9, W96, X135, X2, X25, $X 27, X 3, X 6, X 96, Y 1, Y 2, Y 3, Y 4$ Y $6, Z 1, Z 126, Z 172, Z 20, Z 202$, Z204, Z205, Z206, Z207, Z22. Z227, Z27, Z4, Z43, Z45, Z62, Z9. Z93

C H5, R100, R11, R12, R123, R146, R15, R16, R169, R17, R171. R178, R19, R196, R20, R209, R21, R22, R227, R237, R245, R247, R248, R33, R34, R42, R51. R52, R53, R54, R55, R69, R7. R70, R71, R72, R77, R81, R85, R89, W11, W119, W121, W129. W138, W140, W142, W145, W16, W169, W17, W176, W18, W19, W198, W20, W248, W25. W26, W270, W28, W297, W3, W301, W346, W363, W368, W41, W427, W45, W48, W552. W57, W60, W74, W75, W81, W86, W9, W96, X135, X2, X25, $X 27, X 3, X 6, X 96, Y 1, Y 2, Y 3, Y 4$, Y $6, Z 1, Z 125, Z 172, Z 20, Z 202$ Z204, Z205, Z206, Z207, Z22 Z227, Z27, Z4, Z43, Z45, Z62, Z9. $\mathrm{Z} 93$

C H5, R100, R11, R12, R123, R146, R15, R16, R169, R17, R171, R178, R19, R20, R209, R21, R22, R227, R237, R245, R247, R248, R33, R34, R42, R51, R52, R53, R54, R55, R69, R7, R70, R71, R72, R77, R81, R85, R89, W11, W119, W121, W129, W138. 
Table 1. HATCHERIES, DEALERS, AND INDEPENDENT FLOCKS PARTICIPATING IN

THE NATIONAL POULTRY IMPROVEMENT PLAN

WATERFOWL, EXHIBITION POULTRY AND GAME BIRDS

HATCHING

EGG

CAPACITY
PRODUCTS CLASSIFIED

U.S. PULLORUM-TYPHOID CLEAN
ADDITIONAL

CLASSIFICATIONS FOR WHICH PRODUCT QUALIFIED

47 - NEBRASKA

ORSCHELN FARM \& HOME LLC, LINCOLN, 5640 CORNHUSKER HIGHWAY, LINCOLN 68507 Phone: (402)466-1191

ORSCHELN FARM \& HOME LLC, MCCOOK, NORTH HIGHWAY 83. MCCOOK 69001

Phone: (308)345-5390
ORSCHELN FARM \& HOME LLC. NORTH PLATTE, 2501 EAST 4TH STREET, NORTH PLATTE 69101-7452 Phone: (308)532-6138
W140, W142, W145, W16. W169, W17, W176, W18, W19. W198, W20, W248, W25, W26, W270, W28, W297, W3, W301, W346, W3636, W368, W41, W427, W45, W48, W552, W57, W60, W74, W75, W81, W86, W9, W96, X135, X2, X25, X27, X3, X96, Y1, Y2, Y3, Y4, Y6, Z1, Z125, Z172, Z20, Z202, Z204, Z205, Z206, Z207, Z22, Z227. Z27, Z4, Z43, Z45, Z62, Z9, Z93

C H5, R100, R11, R12, R123, R146, R15, R16, R169, R17, R171. R178, R19, R196, R20, R209. R21, R22, R227, R237, R245, R247, R248, R33, R34, R42, R51, R53, R54, R55, R69, R7, R70. R71, R72, R77, R81, R85, R89 W11, W119, W121, W129. W138, W140, W142, W145, W16, W169, W17, W176, W18, W19, W198, W20, W248, W25, W26, W270, W28, W297, W3, W301, W346, W363, W41, W427, W45, W48, W552, W57, W60, W74, W75, W81, W86, W9, $W 96, X 135, X 2, X 25, X 27, X 3, X 6$. $X 96, Y 1, Y 2, Y 3, Y 4, Y 6, Z 1$. Z125, Z172, Z20, Z202, Z204, Z205, Z206, Z207, Z22, Z227, Z27, Z4, Z43, Z45, Z62, Z9, Z93

C H5, R100, R11, R12, R123, R145, R15, R16, R169, R17, R171. R178, R19, R196, R20, R209, R21, R22, R227, R237, R245, R247, R248, R33, R34, R42, R51, R52, R53, R54, R55, R69, R7. $R 70, R 71, R 72, R 77, R 81, R 85$. R89, W11, W119, W121, W129, W138, W140, W142, W145, W16, W169, W17, W176, W18, W19, W198, W20, W248, W25, W26, W270, W28, W297, W3, W301, W346, W363, W368, W41, W427, W45, W48, W552, W57, W60, W74, W75, W81 W86, W9, W96, X135, X2, X25 $X 27, X 3, X 6, X 96, Y 1, Y 2, Y 3, Y 4$ $Y 6, Z 1, Z 125, Z 172, Z 20, Z 202$ Z204, Z205, Z206, Z207, Z22 Z27, Z4, Z43, Z45, Z62, Z9, Z93

C H5, R100, R11, R12, R123, R146, R15, R16, R169, R17, R171, R178, R19, R196, R20, R209, R21, R22, R227, R237, R245, R247, R248, R33, R34, R42, R51, R52, R53, R54, R55, R69, R7. $\mathrm{R} 70, \mathrm{R} 71, \mathrm{R} 77, \mathrm{R} 81, \mathrm{R} 85, \mathrm{R} 89$, W11, W119, W121, W129, W135, W138, W140, W142, W145, W16, W169, W17, W176, W18, W19, W198, W20, W248 W25, W26, W270, W28, W297. W3, W301, W346, W363, W368, W41, W427, W45, W48, W552 W57, W60, W74, W75, W81. 
Table 1. HATCHERIES, DEALERS, AND INDEPENDENT FLOCKS PARTICIPATING IN

THE NATIONAL POULTRY IMPROVEMENT PLAN

WATERFOWL, EXHIBITION POULTRY AND GAME BIRDS

\begin{tabular}{|c|c|c|c|c|c|}
\hline $\begin{array}{l}\text { APP. } \\
\text { NO. }\end{array}$ & $\begin{array}{l}\text { SUB- } \\
\text { PART }\end{array}$ & PARTICIPANTS NAME AND ADDRESS & $\begin{array}{l}\text { HATCHING } \\
\text { EGG } \\
\text { CAPACITY }\end{array}$ & $\begin{array}{l}\text { PRODUCTS CLASSIFIED } \\
\text { U.S. PULLORUM-TYPHOID } \\
\text { CLEAN }\end{array}$ & $\begin{array}{c}\text { ADDITIONAL } \\
\text { CLASSIFICATIONS FOR } \\
\text { WHICH PRODUCT QUALIFIED }\end{array}$ \\
\hline
\end{tabular}

47 - NEBRASKA

699 E ORSCHELN FARM \& HOME LLC, ORD, RT. 3, BOX 152 , NORTH HIGHWAY 11, ORD 68862

Phone: (308)728-9920

ORSCHELN FARM \& HOME LLC, YORK, 110 MARQUIS AVENUE, YORK 68467-9780

Phone: (402)362-3375

ORTMEIER'S SEED, FEED \& PRODUCE, 309 S. MAIN STREET, WEST POINT 68788

E PETE'S FEED \& SEED, 1121 CENTRAL AVENUE, NEBRASKA CITY 68410
W86, W9, W96, X2, X25, X27. $X 3, X 6, X 96, Y 1, Y 2, Y 3, Y 4, Y 6$, Z1, Z125, Z172, Z20, Z202, Z204. Z205, Z206, Z207, Z22, Z227. Z27, Z4, Z43, Z45, Z62, Z9, Z93

C H5, R100, R11, R12, R123, R146, R15, R16, R169, R17, R171, R178, R19, R196, R20, R209, R21, R22, R227, R237, R245, $R 247, R 248, R 33, R 34, R 42, R 51$, R52, R53, R54, R55, R69, R7. $\mathrm{R} 70, \mathrm{R} 71, \mathrm{R} 72, \mathrm{R} 77, \mathrm{R} 81, \mathrm{R} 85$, R89, W11, W119, W121, W129, W138, W140, W142, W145. W16, W169, W17, W176, W18, W19, W198, W20, W248, W25, W26, W270, W28, W297, W3. W301, W346, W363, W368, W41, W427, W45, W48, W552, W57, W60, W74, W75, W81. W86, W9, W96, X135, X2, X25, $X 27, X 3, X 6, X 96, Y 1, Y 2, Y 3, Y 4$, $Y 6, Z 1, Z 125, Z 172, Z 20, Z 202$, Z204, Z205, Z206, Z207, Z22. Z227, Z27, Z4, Z43, Z45, Z62, Z9, 293

C H5, R100, R11, R12, R123, R146, R15, R16, R169, R17, R171, R178, R19, R196, R20, R209, R21, R22, R227, R237, R245, R247, R248, R33, R34, R42, R51, R52, R53, R54, R55, R69, R7. R70, R71, R72, R77, R81, R85, R89, W11, W119, W121, W129. W138, W140, W142, W145, W16, W169, W17, W176, W18, W19, W198, W20, W248, W25, W26, W270, W28, W297, W3. W301, W346, W363, W368, W41, W427, W45, W48, W552. W57, W60, W74, W75, W81, W86, W9, W96, X135, X2, X25, $X 27, X 3, X 6, X 96, Y 1, Y 2, Y 3, Y 4$, $Y 6, Z 1, Z 125, Z 172, Z 20, Z 202$, Z204, Z205, Z206, Z207, Z22. Z227, Z27, Z4, Z43, Z45, Z62, Z9, 293

C B10, R29, X2, X3, X4, Y1, Y3, Z182, Z205, Z227

C A16, B10, C10, D, E, H, H5, R10, R100, R11, R12, R13, R139, R15. R16, R17, R179, R18, R19, R20, $R 209, R 21, R 227, R 23, R 3, R 30$, R34, R42, R45, R49, R5, R50, R51, R52, R53, R54, R56, R6, R64, R69, R7, R71, R72, R76, R77, R81, R82, R85, R88, R9, R92, R93, R97, R98, R99, W 102. W104, W11, W119, W12, W138, $W 140, W 164, W 169, W 17, W 18$, W198, W20, W221, W223, W23, W25, W26, W28, W306, W33, W367, W39, W427, W45, W46, W48, W5, W504, W518, W52. W526, W554, W570, W578, W596, W60, W7, W70, W74, 
Table 1. HATCHERIES, DEALERS, AND INDEPENDENT FLOCKS PARTICIPATING IN

THE NATIONAL POULTRY IMPROVEMENT PLAN

WATERFOWL, EXHIBITION POULTRY AND GAME BIRDS

\begin{tabular}{lllll}
\hline & & HATCHING & PRODUCTS CLASSIFIED \\
APP. SUB- & PARTICIPANTS NAME AND ADDRESS & EART & U.S. PULLORUM-TYPHOID \\
NO. & CLLEAN & CAPACITY & CLASSIFICATIONS FOR \\
\hline
\end{tabular}

47 - NEBRASKA

\begin{abstract}
340 E PLEASANTON LIVESTOCK SERVICE, P.O. BOX 123, 108 NORTH SYCAMORE, PLEASANTON 68866
\end{abstract}

651 E SAMSON FEED SERVICE, RR 1, BOX 136, PLATTE CENTER 68653

229

E F.H.SCHAFER ELEVATOR, INC, P.O. BOX 963,909 SOUTH BROADWAY, SCOTTSBLUFF 69361

ST. HELENA STORE, P.O. BOX 50, 6TH AND HELENA STREET, ST. HELENA 68774
W75, W81, W9, W91, W96, X1. $X 2, X 3, X 4, X 5, X 6, Y 1, Y 2, Y 3$, $Y 4, Z 15, Z 159, Z 176, Z 2, Z 22$. $\mathrm{ZZ7}, \mathrm{Z43}, \mathrm{Z61}, \mathrm{Z79}, \mathrm{Z} 80$

C $\quad 2227$

C R210

C $A, B, C, D, E, H 5, R 11, R 12$, R125, R146, R15, R16, R19, R196, R20, R209, R21, R22, R30, R33. R42, R45. R53, R54, R69. R7, R71, R72, R77, R81, R85, R87, R89, R98, W, W11, W119, W12, W120, W121, W126, W129, W136, W138, W140. W142, W145, W160, W164, W17, W176, W18, W19, W193. W198, W20, W211, W248, W25, W259, W26, W262. W270, W28 W29, W297, W33, W41, W45 W48, W5, W50, W54, W60, W7. W74, W75, W81, W86, W88 W89, W9, W96, X131, X135, $X 136, X 2, X 21, X 23, X 24, X 25$, $X 27, X 3, X 4, X 6, Y 1, Y 2, Y 3, Y 4$, $Y 5, Y 54, Z 1, Z 110, Z 180, Z 2, Z 2.0$, Z202, Z204, Z205, Z206, Z207. Z217, Z22, Z2227, Z239, Z27, Z3 Z4, Z43, Z62, Z9, Z93

C A16, B10, C10, D9, E, H5, R10, R100, R11, R12, R13, R139, R15, R16, R17, R179, R18, R19, R20, R209, R21, R227, R23, R3, R30, R34, R42, R45, R49, R5, R50. R51, R52, R53, R54, R56, R6, R64, R69, R7, R71, R72, R76, R77, R81, R82, R85, R88, R9, R92, R93, R97, R98, R99, W102, W104, W11, W119, W12, W138, W140, W164, W169, W17, W18, W198, W20, W221, W223, W23, W25, W26, W28, W306, W33, W367, W39, W427, W45, W46, W48, W5, W504, W518, W52, W526, W554, W570, W578, W595, W60, W7, W70, W74 W75, W81, W9, W91, W96. X1, $X 2, X 3, X 4, X 5, X 6, Y 1, Y 2, Y 3$, $Y 4, Z 15, Z 159, Z 176, Z 2, Z 22$, Z27, Z43, Z64, Z79, Z80

C A, B, D, E, H5, R11, R12, R125, R146, R15, R16, R19, R196, R20, $R 209, R 21, R 210, R 22, R 30, R 33$, R42, R45, R53, R54, R69, R7. R71, R72, R77, R81, R85, R87, R89, R96, R98, W, W11, W119. W12, W120, W121, W126. W129, W136, W138, W140, W142, W145, W160, W164, W17, W176, W18, W19, W193, W198, W20, W211, W248, W25. W259, W26, W262, W270, W28, W29, W297, W33, W41, W45, W48, W5, W50, W54, W60, W7, 
Table 1. HATCHERIES. DEALERS. AND INDEPENDENT FLOCKS PARTICIPATING IN

THE NATIONAL POULTRY IMPROVEMENT PLAN

WATERFOWL. EXHIBITION POULTRY AND GAME BIRDS

\begin{tabular}{|c|c|c|c|c|c|}
\hline $\begin{array}{l}\text { APP. } \\
\text { NO. }\end{array}$ & $\begin{array}{l}\text { SUB- } \\
\text { PART }\end{array}$ & PARTICIPANTS NAME AND ADDRESS & $\begin{array}{l}\text { HATCHING } \\
\text { EGG } \\
\text { CAPACITY }\end{array}$ & $\begin{array}{l}\text { PRODUCTS CLASSIFIED } \\
\text { U.S. PULLORUM-TYPHOID } \\
\text { CLEAN }\end{array}$ & $\begin{array}{c}\text { ADDITIONAL } \\
\text { CLASSIFICATIONS FOR } \\
\text { WHICH PRODUCT QUALIFIED }\end{array}$ \\
\hline
\end{tabular}

$\begin{array}{ccl}633 \text { E } & \begin{array}{l}\text { SUNDANCE FEED \& SEED COMPANY, 4155 EAST } \\ \text { HIGHWAY 30, GRAND ISLAND 68801 }\end{array} \\ 184 \quad \text { E } & \begin{array}{l}\text { TNT FARM SERVICE, INC., RR 2, BOX 57-A, ARAPAHOE } \\ 68922\end{array} \\ 686 \quad \text { E } \quad \begin{array}{l}\text { TRACTOR SUPPLY COMPANY, COLUMBUS, } 3920 \text { 23RD. } \\ \text { STREET, COLUMBUS 68601 } \\ \text { PhONe: (402)564-8102 }\end{array}\end{array}$

TRACTOR SUPPLY COMPANY, LINCOLN, 1940 CORN HUSKER HIGHWAY, LINCOLN 68521

Phone: (402)476-2292
W74, W75, W81, W86, W88, W89, W9, W96, X, X131, X135. $X 136, X 2, X 21, X 23, X 24, X 25$ $X 27, X 3, X 6, Y 1, Y 2, Y 3, Y 4, Y 5$, Y54, Z1, Z110, Z172, Z20, Z202. Z204, Z205, Z206, Z207, Z217, Z22, Z227, Z27, Z43, Z45, Z62. Z9. Z93

C A, B, C, D, E3, H, N, R33, W, X, $Y, Z$

C A, A2, D, E, H, R209

R1, R114, R12, R14, R146, R15, . R17, R175, R19, R196, R209. R21, R210, R22, R222, R227, R230, R239, R241, R242, R245, R247, R248, R256, R3, R34, R41. R42, R5, R51, R54, R56, R6. R64, R69, R7, R71, R72, R77, R81, R82, R85, R89, R9, R90, R96, R99, W1, W102, W11, W114, W12, W120, W121, W129, W13, W132, W138, W140, W142, W164, W169. W17, W176, W182, W198, W20, W207, W208, W211, W215, W219, W223, W248, W25, W26, W28, W289, W290, W3, W30, W302, W304, W33, W34, W37 W393, W442, W518, W519. W52, W544, W554, W57, W570, W573, W578, W595, W60, W600, W74, W81, W88, W89, W9, X1, X14, X154, X161, X2, $X_{27}, X_{3}, Y_{1}, Y_{2}, Y_{3}, Y 4, Z 129$, Z159, Z165, Z176, Z182, Z183, Z202, Z203, Z204, Z205, Z206, Z209, Z22, Z227, Z247, Z264, Z27, Z29, Z30

R1, R114, R12, R14, R146, R15, R17, R175, R19, R196, R209, R21, R210, R22, R222, R227. R230, R239, R241, R242, R245, R247, R248, R256, R3, R34, R41。 R42, R5, R51, R54, R56, R6, R64, R69, R7, R71, R72, R77. R81, R82, R85, R86, R89, R9, R90, R99, W1, W102, W11, W114, W12, W120, W121, W129, W13, W132, W138, W140, W142, W164, W169, W17, W176, W182, W198, W20, W207, W208, W211, W215, W219, W223, W248, W25, W26, W28, W289, W290, W3, W30, W302, W304, W33, W34, W37, W393, W442, W518, W519, W52, W544, W57, W570, W573. W578, W595, W60, W600, W74, W81, W88, W89, W9, X1, X14, $X 154, X 161, X 2, X 27, X 3, Y 1, Y 2$, Y3, Y4, Z129, Z159, Z165, Z176, Z182, Z183, Z202, Z203, Z204. Z205, Z206, Z209, Z22, Z227. Z247, Z264, Z27, Z29, Z30 
Table 1. HATCHERIES, DEALERS, AND INDEPENDENT FLOCKS PARTICIPATING IN

THE NATIONAL POULTRY IMPROVEMENT PLAN

WATERFOWL, EXHIBITION POULTRY AND GAME BIRDS

APP. SUB- PARTICIPANTS NAME AND ADDRESS
NO. PART

$\begin{array}{cc}\text { HATCHING } & \text { PRODUCTS CLASSIFIED } \\ \text { EGG } & \text { U.S. PULLORUM-TYPHOID } \\ \text { CAPACITY } & \text { CLEAN }\end{array}$

ADDITIONAL

CLASSIFICATIONS FOR WHICH PRODUCT QUALIFIED

47 - NEBRASKA

TRACTOR SUPPLY COMPANY, BEATRICE, 2420 NORTH SIXTH STREET, BEATRICE 68310 Phone: (402)223-4596

TRACTOR SUPPLY COMPANY, OMAHA, 7910 "L" STREET, OMAHA 68127

Phone: (402)339-2800
R1, R114, R12, R14, R146, R148. R15, R17, R175, R19, R196, R209, R21, R210, R22, R222. R227, R230, R239, R241, R242. R245, R247, R256, R3, R34, R41, R42, R5, R51, R54, R56, R6 R64, R69, R7, R71, R72, R77. R81, R82, R85, R86, R89, R9, R90, R99, W1, W102, W11. W114, W12, W120, W121, W129, W13, W132, W138 W140, W142, W164, W169, W17, W176, W182, W198, W20. W207, W208, W211, W215, W219, W223, W248, W25, W26, W28, W289, W290, W3, W30 W302, W304, W33, W34, W37, W393, W442, W518, W519, W52, W544, W554, W57, W570. W573, W578, W595, W60. W600, W74, W81, W88, W89, W9, X1, X14, X154, X161, X2, $X 27, X 3, Y 1, Y 2, Y 3, Y 4, Z 129$, Z159, Z165, Z176, Z182, Z183, Z202, Z203, Z204, Z205, Z206, Z209, Z22, Z2227, Z247, Z264, Z27, Z29, Z30

E37, R1, R114, R12, R14, R146, R15, R17, R175, R19, R196. R209, R21, R210, R22, R222, $R 227, R 230, R 239, R 241, R 242$, R245, R247, R248, R256, R3, R34, R41, R42, R5, R51, R54, R56, R6, R64, R69, R7, R71, $R 72, R 77, R 81, R 82, R 85, R 86$, R89, R9, R90, R99, W1, W102, W11, W114, W12, W120, W129, W13, W132, W138, W140, W142, W164, W169, W17, W176, W182, W198, W20, W207, W208, W211, W215. W219, W223, W248, W25, W26. W28, W289. W290, W3. W30. W302, W303, W304, W33, W34, W442, W518, W519, W52. W544, W554, W57, W570, W573, W578, W595, W60, W600, W74, W81, W88, W89, $W 9, X 1, X 14, X 154, X 161, X 2$, $X 27, X 3, Y 1, Y 2, Y 3, Y 4, Z 129$ Z159, Z165, Z176, Z182, Z183, Z202, Z203, Z204, Z205, Z209, Z22, Z247, Z264, Z27, Z29, Z30

R1, R114, R12, R134, R14, R146,. R15, R17, R175, R19, R196. R209, R21, R210, R22, R222. R227, R230, R239, R241, R242, R245, R247, R248, R256, R3 R41, R42, R5, R51, R54, R56, R6, R64, R69, R7, R71, R72, R77, R81, R82, R85, R86, R89, R9, R90, R99, W1, W102, W11. W114, W12, W121, W129, W13, W132, W138, W140, W142 W164, W169, W17, W176, W182, W198, W20, W207 W208, W211, W215, W219, 
Table 1. HATCHERIES, DEALERS, AND INDEPENDENT FLOCKS PARTICIPATINGIN

THE NATIONAL POULTRY IMPROVEMENT PLAN

WATERFOWL, EXHIBITION POULTRY AND GAME BIRDS

\begin{tabular}{|c|c|c|c|c|c|}
\hline $\begin{array}{l}\text { APP. } \\
\text { NO. }\end{array}$ & $\begin{array}{l}\text { SUB- } \\
\text { PART }\end{array}$ & PARTICIPANTS NAME AND ADDRESS & $\begin{array}{l}\text { HATCHING } \\
\text { EGG } \\
\text { CAPACITY }\end{array}$ & $\begin{array}{l}\text { PRODUCTS CLASSIFIED } \\
\text { U.S. PULLORUM-TYPHOID } \\
\text { CLEAN }\end{array}$ & $\begin{array}{c}\text { ADDITIONAL } \\
\text { CLASSIFICATIONS FOR } \\
\text { WHICH PRODUCT QUALIFIED }\end{array}$ \\
\hline
\end{tabular}

47 - NEBRASKA

\begin{abstract}
587 E TRI-VALLEY FEED AND SUPPLY, P.O. BOX 235, INDIANOLA 69034

666 E UNITED FARMER'S COOP., BOX 128, 120 ELM, BEE 68314

E VALLEY FEEDS, 2945 NORTH 10THSTREET, P.O.BOX 205, GERING 69341

313 E WASHCO FEED AND SUPPLY, 217 NORTH 10TH, BLAIR 68008
\end{abstract}

Independent Flocks

ADAM'S ANGUS RANCH, SCOTT \& JAWAIN ADAMS, RR 1. BOX 102-A, LITCHFIELD 68852

679

BUNKER HILL HUNTING RESORT, OWEN NELSON, RR 1, BOX 22-A, FAIRFIELD 68938

$647 \quad E$

D\&D EXOTIC BIRDS, QUINCYIDEAN DICKAU, HC69, BOX 69, ATKINSON 68713

347 E D. DAVID DELAND, 7218 SARPY AVENUE, OMAHA 68147

RANDY DUNN, P.O. BOX 26, 202 WEST 4TH, SHUBERT 68437

$717 \quad E$
FIELDING BIRDS, RT. 3, BOX 60, MCCOOK 69001 Phone: (308)345-2752

BUTCH HASSLER, 31140 ROAD Y, SUTTON 68979

E JACK F. HOPKINS, 324 WEST IRVING, P.O. BOX 162. BLUE SPRINGS 68318

E

HOTOWY TURKEY FARM, 102 ELM STREET, P.O. BOX 38, DWIGHT 68635

KEVIN HURTIG, RT. 1, P.O. BOX 1641, NORFOLK 68702

LEBBY JENSEN, RR 2, BOX 572, BAYARD 69337 Phone: (308)586-2410

E K-D HUNTING ACRES, 2980 COUNTY ROAD I, TEKAMAH 68061

E MICKEYW. LEMBURG, 1772 2ND AVENUE, CAIRO 68824
W223, W248, W25, W26, w28. W289, W290, W3, W30, W302. W304, W33, W34, W37, W393, W442, W518, W519, W52. W544, W554, W57, W570. W573, W578, W595, W60 W600, W74, W81, W88, W89, $W 9, X 1, X 14, X 154, X 161, X 2$. $X_{2} 7, X 3, Y 1, Y 2, Y 3, Y 4, Z 129$ Z159, Z165, Z176, Z182, Z183, Z202, Z203, Z204, Z205, Z206, Z207, Z209, Z22, Z247, Z264. Z27, Z29, Z30

C $B, Y 2$

E\&C R210

C Z231

C $\quad B, R 209, X 2, Y 12$

R11, R30, R52, R87, W123, W164, W17, W18, W27, W38, W54, W60

$\mathrm{Z1}, \mathrm{Z3}$

$R 20, R 7, R 83, R 94, W 18, X, Y, Z$ W120

w30

D. R13, R16, R9, W18, W93

N12, N9, W119, W160, W17, W18, W197, W220, W494, W70

R53, W102, W11, W12, W121, W140, W17, W33, W442, W573. W60, Z204, Z206, Z229

$X 17, Y 9, Z 247, Z 252$

A, B, R186, R20, W12, W208, W282, W42, W96

R27

$x 117, x 160, x 28, x 4, \times 5, x 53$, $X 57, X 58, X 60, X 7, X 70, X 8, X 85$, $\mathrm{Y} 28$

$\times 109, \times 117, \times 28, \times 29, \times 4, \times 50$ $\times 52, \times 53, \times 54, \times 57, \times 58, \times 60$, $X 61, X 62, X 7, X 8, X 85, X 89, X 99$, $Y 20, Y 28, Y 3, Y 31, Y 51, Y 9$, 


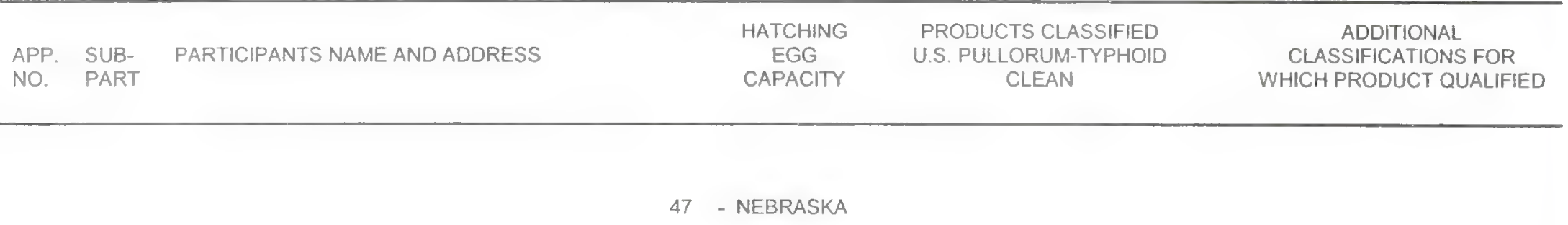

\begin{tabular}{|c|c|c|c|}
\hline & & & $\mathrm{Z} 122, \mathrm{Z123}, \mathrm{Z27}, \mathrm{Z8}$ \\
\hline 429 & $E$ & $\begin{array}{l}\text { LOVE-A-LOP FARM, V. ISOM, P.O. BOX 165, ALLEN } \\
68710\end{array}$ & $W 7, X 161, X 20, Y 8, Y 9$ \\
\hline 298 & $E$ & $\begin{array}{l}\text { GARY \& VERONA A. NEUMAN, } 324 \text { W. BROAD, RR } 1, \\
\text { BOX 9A, BLUE SPRINGS } 68318\end{array}$ & W, W190, W578_____ \\
\hline 664 & $E$ & $\begin{array}{l}\text { DENNIS \& JENEANE OESTREICH, } 55630 \text { \#847 ROAD, } \\
\text { NORFOLK } 68701\end{array}$ & $\begin{array}{l}\text { W. W131, W132, W136, W162, } \\
\text { W20, W262, W278, W42, W594, } \\
\text { W606, W84, W93, X, Z, Z231 }\end{array}$ \\
\hline 718 & $\mathbf{E}$ & $\begin{array}{l}\text { PF GAMEBIRD RANCH, PAUL J. FORCH, HC } 2, \text { BOX } 124, \\
\text { TRENTON } 69044\end{array}$ & - \\
\hline 711 & $E$ & $\begin{array}{l}\text { PRAIRIE THUNDER GAME BIRDS, RR 1. BOX 136-A, } \\
\text { SARGENT } 68874 \\
\text { Phone: (308)527-3679 }\end{array}$ & 227 \\
\hline 536 & $E$ & $\begin{array}{l}\text { PRAIRIE HILL HUNTING CLUB, JOHN MCELROY, } 1267 \\
\text { 2ND AVENUE, DANNEBROG } 68831\end{array}$ & $\mathrm{Z}_{2}$ \\
\hline 561 & $E$ & $\begin{array}{l}\text { RAVEN WING FARM, MCALEXANDER, RR 1, BOX 90-A, } \\
\text { BROWNVILLE } 68321\end{array}$ & $\begin{array}{l}\text { R103, R94, W132, W30, W35, - } \\
\text { W554 }\end{array}$ \\
\hline 218 & $E$ & RICHARD L. RIEKEN, BOX 21, CLARKS 68628 & $\begin{array}{l}\text { R19, W130, W223, W290, W60, - } \\
\text { W7, W75 }\end{array}$ \\
\hline 505 & $E$ & MARVIN ROUBAL, P.O. BOX 21, BRUNO 68014 & 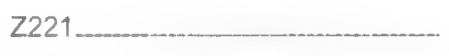 \\
\hline 661 & $E$ & GARY SANDERS, RR 2, BOX 69, SPENCER 68777 & W. Z231, Z4 \\
\hline 659 & E & $\begin{array}{l}\text { JOE SCHUKAR, } 9185 \text { RAWNEE ROAD, P.O. BOX } 507 \text {, } \\
\text { GIBBON } 68840\end{array}$ & $\begin{array}{l}\text { R195, R253, R40, R52, R78, X24,. } \\
\text { Z188, Z257, Z26, Z260, Z34, Z46, } \\
\text { Z49, Z71 }\end{array}$ \\
\hline 402 & E & DIX SCRANTON. 55009 840TH ROAD, NORFOLK 68701 & $\mathrm{R} 16, \mathrm{X} 146, \mathrm{Y}, \mathrm{Z11} \ldots$ \\
\hline 658 & $E$ & $\begin{array}{l}\text { STONE'S THROW FARM, W \& A OTT, } 2527 \text { COUNTY } \\
\text { ROAD V, MORSE BLUFF } 68648\end{array}$ & N194, X135, X2, X3 \\
\hline 637 & $E$ & $\begin{array}{l}\text { MARCIA WEAVERS, } 8498 \text { WEST PLATTE RIVER DRIVE, } \\
\text { DONIPHAN } 68832\end{array}$ & $\begin{array}{l}\text { R166, R20, W12, W25, W570, } \\
\text { W58, W74, Z78 }\end{array}$ \\
\hline 678 & $E$ & $\begin{array}{l}\text { WHISPERING PINES POULTRY, PETRMICHL, } 4105 \\
\text { GILES ROAD, BELLEVUE } 58147 \\
\text { Phone: (402)733-9483 }\end{array}$ & $\begin{array}{l}\text { R146, R15, R168, R247, R3, R52, } \\
\text { R56, R6 }\end{array}$ \\
\hline 170 & $E$ & URBAN WIENKE, 108 IVY, CRETE 68333 & C. W12, W54 \\
\hline 644 & $E$ & $\begin{array}{l}\text { WINDMILL QUAIL FARM, NORMAN MORTENSEN, RR } 1, \\
\text { BOX 183, CRAB ORCHARD } 68332\end{array}$ & 243 \\
\hline 667 & $E$ & $\begin{array}{l}\text { LORENE ZIMMERMAN, } 62514 \text { CORSO, NEBRASKA CITY } \\
68410\end{array}$ & $X 174, X 20, X 25, Y, Z 11, Z 138$ \\
\hline
\end{tabular}

DR. RONALD D. ANDERSON

NEVADA ANIMAL DISEASE LABORATORY

350 CAPITOL HILL AVENUE

RENO, NV 89502

Phone: (702)688-1182 EXT.233

Fax: (702)688-1198 
Table 1. HATCHERIES, DEALERS, AND INDEPENDENT FLOCKS PARTICIPATING IN

THE NATIONAL POULTRY IMPROVEMENT PLAN

WATERFOWL, EXHIBITION POULTRY AND GAME BIRDS

APP. SUB- PARTICIPANTS NAME AND ADDRESS
NO. PART

$\begin{array}{cc}\text { HATCHING } & \text { PRODUCTS CLASSIFIED } \\ \text { EGG } & \text { U.S. PULLORUM-TYPHOID } \\ \text { CAPACITY } & \text { CLEAN }\end{array}$

ADDITIONAL

CLASSIFICATIONS FOR WHICH PRODUCT QUALIFIED

\section{2 - NEW HAMPSHIRE}

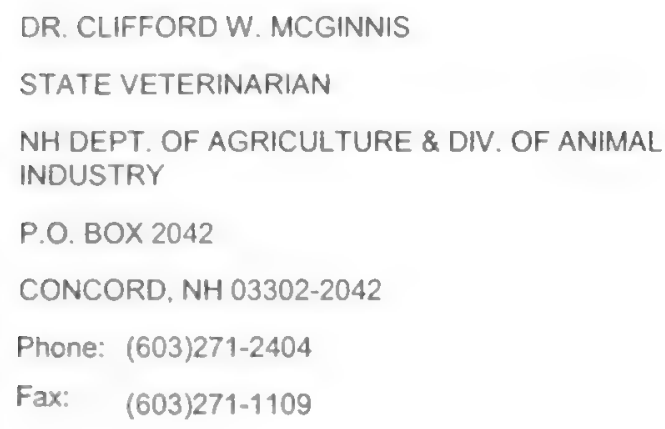

C, D, E3, R11, R115, R117, R14, -

R15, R16, R19, R196, R213,

R225, R227, R233, R239, R247,

R37, R42, R44, R49, R51, R7,

$R 85, R 93, X 1, X 14, \times 2, \times 20, X 3$,

X99, Z202, Z204, Z205, Z209.

$\mathrm{Z} 227$

R237.

W75, Z118, Z15, Z22, Z53, Z57_-

R146, R15, R20, R21, R227, R7 -

W111, W48, Z108, Z136, Z204, $\mathrm{Z24}, \mathrm{Z26}, \mathrm{Z283}, \mathrm{Z} 53, \mathrm{Z78}$

$X 193, X 20, X 4, X 5, Y 2, Y 5, Y 6$, $Y 72, Z 11, Z 2, Z 4$

R12, R146, R237, R247, R7, W26, W393, X17, X2, X22, X3, $\mathrm{X} 4, \mathrm{Z118}, \mathrm{Z53}$

R227, X20, Y4, Y5, Y70, Z108, Z19, Z204, Z227, Z26, Z283, Z4

W578
DR. KAREN M. FROBERG

DIVISION OF ANIMAL HEALTH

DEPARTMENT OF AGRICULTURE

P.O. BOX CN-330

TRENTON, NJ 08625

Phone: (609)292-3965

Fax: (609)633-2550 and DR. ERNEST ZIRKLE

DIVISION OF ANIMAL HEALTH

DEPARTMENT OF AGRICULTURE

P.O. BOX CN-330

TRENTON. NJ 08625

Phone: (609)292-3965

Fax: (609)633-2550 
Table 1. HATCHERIES, DEALERS, AND INDEPENDENT FLOCKS PARTICIPATING IN

THE NATIONAL POULTRY IMPROVEMENT PLAN

WATERFOWL, EXHIBITION POULTRY AND GAME BIRDS

\begin{tabular}{llccc}
\hline & & HATCHING & PRODUCTS CLASSIFIED \\
APP. SUB- & PARTICIPANTS NAME AND ADDRESS & ADG & U.S. PULLORUM-TYPHOID \\
NO. PART & CLEAN & CAPACITY & WHICHSIFICATIONS FOR PRODUCT QUALIFIED \\
\hline
\end{tabular}

22 - NEW JERSEY

\begin{tabular}{|c|c|c|}
\hline 229 & $E$ & $\begin{array}{l}\text { BUTTONWOOD FARM, RR 3, BOX 326, PHILLIPSBURG } \\
08865\end{array}$ \\
\hline 153 & $E$ & FORKED RIVER GAME FARM, , FORKED RIVER 08731 \\
\hline 30 & $E$ & LEROY H. FRITTS, RR 3, BOX 362, PHILLIPSBURG 08865 \\
\hline 37 & $E$ & $\begin{array}{l}\text { MONGOLIAN CROSS PHEASANT FARM, RR 4, BOX } 266 \text {, } \\
\text { SUSSEX } 07461\end{array}$ \\
\hline 36 & E & $\begin{array}{l}\text { FRANCIS L. REINER, RR 2, } 80 \times 155 \text {, FLEMINGTON } \\
08822\end{array}$ \\
\hline 150 & $E$ & ROCKPORT GAME FARM, RR 1, HACKETSTOWN 07840 \\
\hline 33 & $E$ & $\begin{array}{l}\text { STAN'S PHEASANT FARM, RR 2, BOX 533, RINGOES } \\
08551\end{array}$ \\
\hline 319 & $E$ & $\begin{array}{l}\text { TOP FLIGHT QUAIL FARM, P.O. BOX 262, BELVIDERE } \\
07823\end{array}$ \\
\hline & & Independent Flocks \\
\hline 228 & $E$ & $\begin{array}{l}\text { ADRIENNE BLANKENSHIP, WEST VALLE BROOK ROAD } \\
\text { BOX } 43 \text { LONG VALLEY } 07853\end{array}$ \\
\hline 245 & $E$ & $\begin{array}{l}\text { JENNIFER \& BRANDI DEMBESKI, } 43 \text { SCHOOL ROAD, } \\
\text { WHITEHOUSE STATION } 08889\end{array}$ \\
\hline 195 & $E$ & $\begin{array}{l}\text { CHRISTOPHER ELMERS, RR } 3 \text {, BOX 409-l, } \\
\text { WILLIAMSTOWN } 08094\end{array}$ \\
\hline 194 & $E$ & $\begin{array}{l}\text { WILLIAM F. GRINER, RR 1, BOX 39-A, BORDENTOWN } \\
08505\end{array}$ \\
\hline 242 & $E$ & BILL HAASE, P.O. BOX 992, SPARTA 07871 \\
\hline 248 & $E$ & $\begin{array}{l}\text { KIMBERLY \& MICHAEL KAVANAUGH, P.O. BOX } 356 . \\
\text { OLDWICK } 08858\end{array}$ \\
\hline 320 & $E$ & $\begin{array}{l}\text { KENNY KEEN, } 17 \text { PENNSVILLE-AUBURN ROAD, } \\
\text { CARNEYS POINT } 08069\end{array}$ \\
\hline 28 & E & PAUL KUHL, 43 KUHL ROAD, FLEMINGTON 08822 \\
\hline
\end{tabular}

M \& M GAME FARM, 2 WINSLOW ROAD, PENNSVILLE 08070

290 E M \& M HUNTING PRESERVE, WINSLOW \& HOOK ROAD, PENNSVILLE 08070

247 E KATHY MALLET, 367 MILL LANE, NESHANIC 08853

227 E STEVE MORGAN, 35 MOREHOUSE ROAD, NEW EGYPT 08533

243 E LYNN \& DAVID MYERS, 110 INWOOD AVENUE, COLONIA
07067

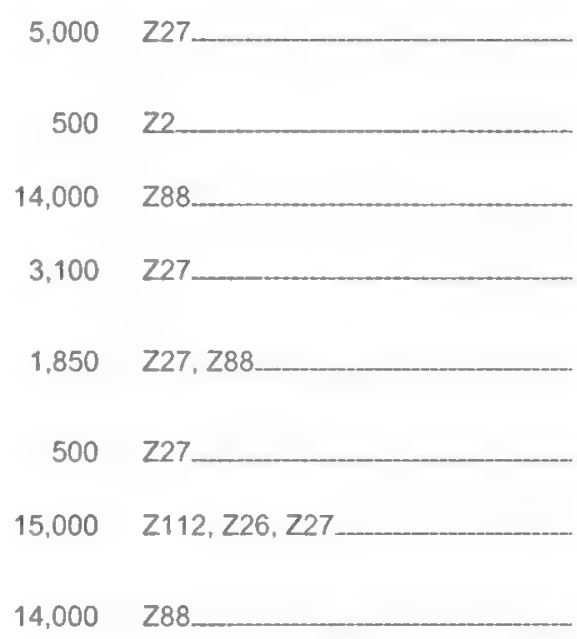

R185, W23, W38, W5, W546

W11, W117, W32, W34, W43, W96

W102, W17, W190, W265. W305, W527, W53, W535, X31, Y13

W102, W132, W17, W20, W38, W4, W54, W57, Y6

$\mathrm{H} 10, \mathrm{H} 9, \mathrm{Z27}$

A, R95, W16

Z129, Z24, Z26, Z27, Z94

R123, R182, W103, W107, W13, W130, W135, W165, W193. W232, W27, W270, W281. W306, W34, W365, W395. W396, W549, W57, W6, W67, W77, W86, W90, W92, W97

$\mathrm{X} 10, \mathrm{X} 17, \mathrm{X} 24, \mathrm{X} 38, \mathrm{X} 47, \mathrm{X} 65$, $Y 15, Y 24, Y 26, Y 4, Y 8, Y 9, Z 105$ Z108, Z109, Z15, Z19, Z204, Z27, 29

$\times 4$

$\times 140, \times 4$

W43, W5, W94, Z14

$\mathrm{X}, \mathrm{Z14}, \mathrm{Z27}, \mathrm{Z3}$

$B, W 131, W 30, W 370$ 
Table 1. HATCHERIES, DEALERS, AND INDEPENDENT FLOCKS PARTICIPATING IN

THE NATIONAL POULTRY IMPROVEMENT PLAN

WATERFOWL, EXHIBITION POULTRY AND GAME BIRDS

\begin{tabular}{llcccc}
\hline & & HATCHING & PRODUCTS CLASSIFIED \\
APP. SUB- & PARTICIPANTS NAME AND ADDRESS & EGG & U.S. PULLORUM-TYPHOID & CLEAN \\
NO. PART & CAPACITY & & CLASSIFICATIONALS FOR \\
\hline
\end{tabular}

22 - NEW JERSEY

241 E JOHN PELACHATY, 53 MORAIS AVENUE, MILLVILLE
08332

85 - NEW MEXICO
Z24, Z26, Z28, Z33, Z34, Z52, $253,255,257,277$

\begin{abstract}
DR. RON PARKER
DEPARTMENT HEAD

BOX $3 A E$

LAS CRUCES, NM 88003

Phone: $(505) 646-1709$

Fax: $\quad(505) 646-3164$
\end{abstract}

AGICULTURAL EXTENSION SERVICE

Hatcheries

36 E JIM \& MARY CLARY, 821 MILLER ROAD, LOS LUNAS
87031
7 E PRIVETT HATCHERY, P.O. BOX 176, PORTALES 88130

QUAILCO GAME BIRD FARM, BOX 368, DORA 88115

Independent Flocks
42 E ALBERT \& MARGARET BARNARD, 5 MEIKLE ROAD, TIJERAS 87059
44 E DESERT POULTRY, JAMES MONK, 6505 SEVEN RIVERS HIGHWAY, ARTESIA 88210

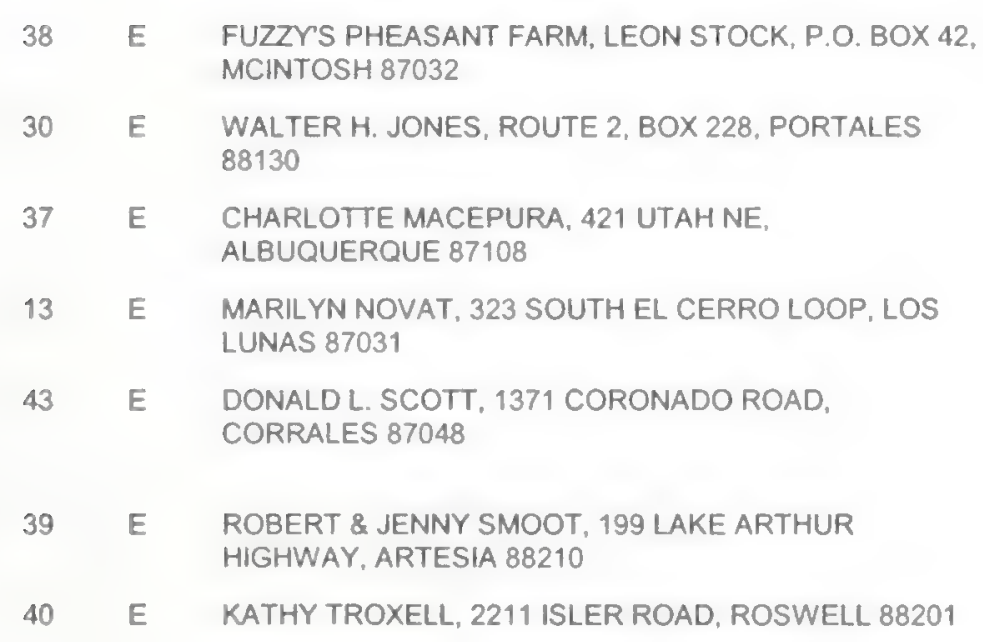

40 E KATHY TROXELL, 2211 ISLER ROAD, ROSWELL 88201

5,000 R55, R57, X28, X8, Z118, Z126, Z24, Z30, Z34, Z43, Z49, Z50, Z52, Z57, Z93

320,000 R196, R7, W102, W119, W12, W121, W13, W146, W164, W17. W18, W19, W21, W26, W28, W33, W368, W48, W56, W57, W60, W7, W86, W89, W94, X2. $X 20, X 25, X 3, X 5, X 6, Y 1, Y 2, Y 3$, Y4, Z11, Z20, Z205, Z211, Z22, Z62

$150,000 \quad Z 1, Z 182, Z 183, Z 27, Z 41, Z 43$

$\mathrm{Z118}, \mathrm{Z138}, \mathrm{Z140}, \mathrm{Z19}, \mathrm{Z2}, \mathrm{Z26}$, Z260, Z283, Z34, Z49, Z50, Z52. Z54, Z57, Z77

R225, W115, W123, W128, W38, W511, W531, W537, W79, W9, $\mathrm{X} 10, \mathrm{X} 147, \mathrm{X} 161, \mathrm{X} 162, \mathrm{X} 17, \mathrm{X} 28$, Y22, Z, Z107, Z220, Z283

$\mathrm{Z} 27$

N104, N139, N164, N32, N33, N44, N83

R229, R30, R57, Z237, Z248, Z34, Z52, Z54, Z68, Z71

W164, W17, W18, X143, X147, $\times 167, \times 38$

$\mathrm{Z260}, \mathrm{Z269}, \mathrm{Z28}, \mathrm{Z34}, \mathrm{Z49}, \mathrm{Z50}$, Z57, Z60, Z70, Z71, Z72, Z77.

Z88

$\mathrm{Z1}, \mathrm{Z27}$

W119, W19, W248, W25, W26, W44, W45, W518, W594, W615, W68, W74, W75, W84, W94 
Table 1. HATCHERIES, DEALERS, AND INDEPENDENT FLOCKS PARTICIPATING IN

THE NATIONAL POULTRY IMPROVEMENT PLAN

WATERFOWL, EXHIBITION POULTRY AND GAME BIRDS

\begin{tabular}{lllll}
\hline & & & HATCHING & PRODUCTS CLASSIFIED \\
APP. & SUB- & PARTICIPANTS NAME AND ADDRESS & EGG & U.S. PULLORUM-TYPHOID \\
NO. PART & CLEAN & CAPACITY & CLASSIFICATIONS FOR \\
\hline
\end{tabular}

85 - NEW MEXICO

\begin{abstract}
33 E MARGE USSERY, 37 CHACO LOOP, SANDIA PARK 87047

34 E VASQUEZ FANCY POULTRY, E.P. VASQUEZ, RT. 12, BOX 503-V. SANTA FE 87505
\end{abstract}

41 E DAVID WEST, P.O. BOX 657, FORT SUMNER 88119
W247, W248, W48, W604, W74, W75, W89

N1, N180, N19, N2, N5, N74, N94, R224, R86, W128, W166, W175, W187, W262, W263, W30, W31, W316, W32, W322, W323, W326, W35, W38, W427. W88, W91

E86, R213, R260, R261, R262, R263, R264, R265, R41, R64, R70, R77, R86, W119, W129, W160, W22, W220, W270, W39, W46, W492, W624, W625 W626, W627

21 - NEW YORK

DR. JOHN HUNTLEY

STATE VETERINARIAN

DIVISION OF ANIMAL INDUSTRY

DEPARTMENT OF AGRICULTURE \& MARKETS

$\uparrow$ WINNERS CIRCLE

ALBANY, NY 12235

Phone: (518)457-3502

Fax: (518)485-7773

Hatcheries

\begin{tabular}{|c|c|c|}
\hline 647 & $E$ & WAYNE BARR, 1775 NYS ROUTE 13, CAYUTA 14824 \\
\hline 259 & $\mathrm{E}$ & $\begin{array}{l}\text { CLARENCE, EMMET, MICHEAL BENNETT, } 63 \text { SPRINGS } \\
\text { ROAD. EAST HAMPTON } 11937\end{array}$ \\
\hline 152 & $E$ & $\begin{array}{l}\text { ROBERT \& WENDY BERKEMEIER, } 10764 \text { EAST ROAD, } \\
\text { PAVILION } 14525\end{array}$ \\
\hline 477 & $\mathrm{E}$ & $\begin{array}{l}\text { MARYAN BILLS, SEMINARY HILL ROAD, RD 13, CARMEL } \\
10512\end{array}$ \\
\hline 897 & $E$ & $\begin{array}{l}\text { STACY L. BROCKETT, } 6384 \text { FISHER HILL ROAD, } \\
\text { CANANDAIQUA } 14424\end{array}$ \\
\hline 367 & $E$ & $\begin{array}{l}\text { RUDY BROVILLETTE, } 1555 \text { JEFFRIS-BIRD ROAD, } \\
\text { MADISON } 13402\end{array}$ \\
\hline 122 & $E$ & $\begin{array}{l}\text { FREDERICK J. CANETTO, RR 1, BOX 401, HILLSDALE } \\
12529\end{array}$ \\
\hline 322 & E & $\begin{array}{l}\text { HENRY L. CLAUSS, } 6170 \text { ROUTES } 5 \text { \& } 20 \text { WEST, } \\
\text { CANANDAIGUA } 14424\end{array}$ \\
\hline 324 & $E$ & $\begin{array}{l}\text { WILLIAM \& PATRICIA EHLERS, RD 2, P.O. BOX } 480 \text {, } \\
\text { TULLY } 13159\end{array}$ \\
\hline 522 & $\mathrm{E}$ & $\begin{array}{l}\text { STANELY A. FOSTER, } 128 \text { TUTTLE HILL ROAD. BOX } 128, \\
\text { CANDOR } 13743\end{array}$ \\
\hline 395 & $E$ & $\begin{array}{l}\text { FAY H. FOWARD, CROW HILL ROAD, BOX } 130 \text {, } \\
\text { BOUCKVILLE } 13310\end{array}$ \\
\hline
\end{tabular}

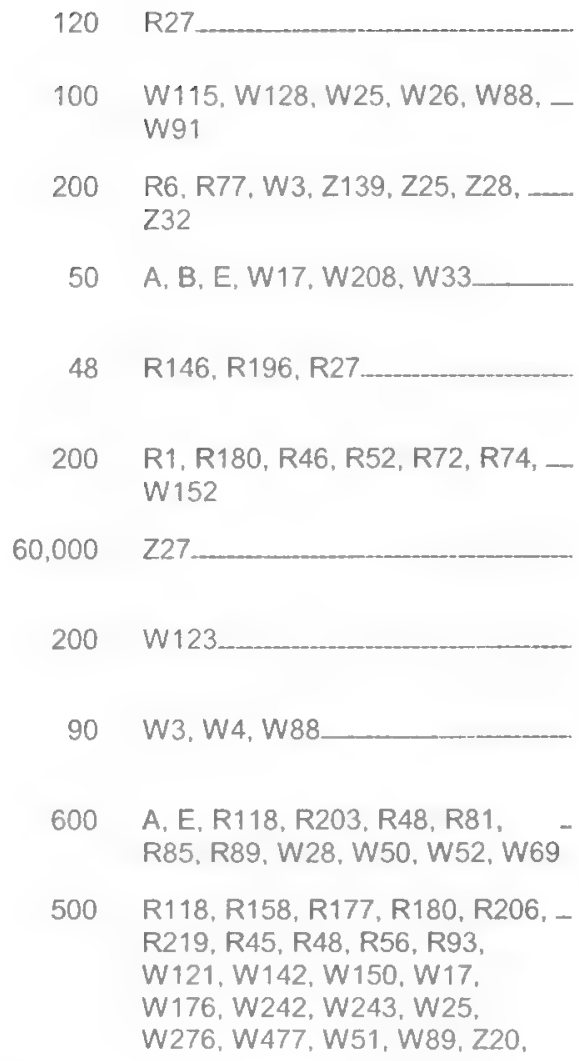

120

R27 W115, W128, W25, W26, W88, W91

$\mathrm{Z32}$

A, B, E, W17, W208, W33

R146, R196, R27

R1, R180, R46, R52, R72, R74, W152

Z27.

W123

W3, W4, W88

A, E, R118, R203, R48, R81, R85, R89, W28, W50, W52, W69

R118, R158, R177, R180, R206, R219, R45, R48, R56, R93, W121, W142, W150, W17. W176, W242, W243, W25, W276, W477, W51, W89, 220 , 
Table 1. HATCHERIES, DEALERS, AND INDEPENDENT FLOCKS PARTICIPATING IN

THE NATIONAL POULTRY IMPROVEMENT PLAN

WATERFOWL. EXHIBITION POULTRY AND GAME BIRDS

\begin{tabular}{llll}
\hline APP. SUB- & PARTICIPANTS NAME AND ADDRESS & $\begin{array}{c}\text { HATCHING } \\
\text { EGG } \\
\text { CO. PART }\end{array}$ & $\begin{array}{c}\text { PRODUCTS CLASSIFIED } \\
\text { C.S. PULLORUM-TYPHOID } \\
\text { CLEAN }\end{array}$ \\
\hline & & \\
& $21-$ NEW YORK
\end{tabular}

21 NEW YORK

\begin{abstract}
245 E RICHARD A. HARE, S-8971 STATE ROAD, COLDEN 14033

876 E EDWARD \& KIM HAYDEN, 4848 MT. RODERICK R2, CINCINNATUS 13040

188 E JOHNHAYES, 1689 ST. HIGHWAY 8, MT. UPTON 13809

846 E MICHAEL HERRICK, 4322 ROUTE 21, MARION 14505

783 E TAYNA HOPPER, 2891 SOUTH LYNDONVILLE ROAD, MEDINA 14103

812 E NICOLE \& ERICKA HORNING, 9320 WILKANSON ROAD, BATAVIA 14020

888 E KEITH, BARBARA, WILLIAM JAROSINSKI, 16024 GLIDDEN ROAD, HOLLEY 14470

291 E SAMUEL A. KUCHAREK, 226 STELLA IRELAND ROAD, BINGHAMTON 13905

508 E MARY M. LACKEY, RR 1, BOX 222, DAVIS ROAD, SALT POINT 12578

587 E ALICE LEE, 131 HOWLAND HILL ROAD, BERKSHIRE 13736
\end{abstract}

E DEVIN,JEFF,COLLEEN,GALEN \& ROBIN LEWIS, 1683 OAK ORCHARD ROAD, ALBION 14411
254 E ARTHUR LUNDGREN, 2168 BUFFALO STREET EXT., JAMESTOWN 14701
847 E C.R.C. MAYNARD, 1384 LAWRENCE ROAD, HILTON 14668

\begin{tabular}{|c|c|c|}
\hline 256 & $E$ & $\begin{array}{l}\text { CATHERINE MCHUGH, } 35 \text { CHURCH LANE, EAST } \\
\text { HAMPTON } 11937\end{array}$ \\
\hline 458 & $E$ & $\begin{array}{l}\text { NY DEPT. OF ENCON., BUREAU OF WILDLIFE, } \\
\text { REYNOLDS GAME FARM, GAME FARM ROAD ITHACA } \\
14850\end{array}$ \\
\hline 668 & $E$ & $\begin{array}{l}\text { TRAVIS PIMM, } 465 \text { MALLOCK ROAD, CHURCHVILLE } \\
14428\end{array}$ \\
\hline 282 & $E$ & $\begin{array}{l}\text { JOHN P. REBHAHN, } 3908 \text { ABBEY ROAD, SYRACUSE } \\
13215\end{array}$ \\
\hline 250 & $E$ & $\begin{array}{l}\text { HERBERT ROGERS, } 2306 \text { RIVER ROAD, CALVERTON } \\
11933\end{array}$ \\
\hline 965 & $E$ & $\begin{array}{l}\text { DAVID ROSS, } 802 \text { DAISY HOLLOW ROAD, DRYDEN } \\
13053\end{array}$ \\
\hline 856 & $E$ & $\begin{array}{l}\text { TOM \& JOHN ROSSITER, } 6570 \text { COE ROAD, LIVONIA } \\
14487\end{array}$ \\
\hline
\end{tabular}

$\mathrm{Z21}, \mathrm{Z22}, \mathrm{Z4}, \mathrm{Z} 62$

500 R89. W119

$50 \quad W 17, W 270, W 7, W 86$

600 R12, R39, R56, R97, W119, W22. W231, W58

92 A, C, R13, R7, W11, W211, W26, Z118, Z15, Z19

90 B, D, R19, R192, R40, R7, R82, R88, W2, W213, W215, W518, $\mathrm{Z} 212$

$48 \quad W 12, W 17, W 18, W 570$

80 W2, W25, W26, Z24, Z26, Z28

42 R19, R52, W121, W17, W198, W23

$50 \mathrm{R} 243, \mathrm{R} 34, \mathrm{~W} 4$

24 R15, R16, R2, R222, R24, R3, R56, R84, R85, R91, R97, R98, W160, W17, W18, W30, W33,

W48, W5, W88, W89, Z204

48 R1, R11, R12, R139, R222, R229, R247, R34, R40, R53, R54, R70. R85, R93, R96, W12, W121, W18, W28, Z202, Z22

1,200 R19, W23, W38, W54

148 R123, R16, R196, R21, R222, R237, R248, R45, R56, R7, R76. W102, W17, W20, W26, W32. W477, W54, W6

$100 \quad W 17, W 54, W 81$

$60,000 \quad 227$

45 R19, W249, W52, W570

130 D. N1, R85, W11, W115, W12, W123, W527, W556, W93

200 R19, W25, W26, W74

400

$\mathrm{Z27}, \mathrm{Z43}$

20 B, E, R123, R196, R21, R222, R229, R40, R7, W11, W211, W27, W33, W38, W56, W570, W573, W60, Z22, Z62

436 W28, W54 
Table 1. HATCHERIES, DEALERS, AND INDEPENDENT FLOCKS PARTICIPATING IN

THE NATIONAL POULTRY IMPROVEMENT PLAN

WATERFOWL, EXHIBITION POULTRY AND GAME BIRDS

APP. SUB- PARTICIPANTS NAME AND ADDRESS

NO. PART

HATCHING
EGG

CAPACITY
PRODUCTS CLASSIFIED

U.S. PULLORUM-TYPHOID CLEAN
ADDITIONAL

CLASSIFICATIONS FOR WHICH PRODUCT QUALIFIED

21 - NEW YORK

\begin{tabular}{|c|c|c|}
\hline 599 & $E$ & $\begin{array}{l}\text { JOSEPH A. SEAVEY \& FAMILY, RR } 3, \text { BOX } 80, \text { CANTON } \\
13126\end{array}$ \\
\hline 233 & $E$ & $\begin{array}{l}\text { ALBERT SNOLIS, } 22 \text { KALMIA STREET, EAST } \\
\text { NORTHPORT } 11731\end{array}$ \\
\hline 826 & $E$ & $\begin{array}{l}\text { SPEEDWELL FARM, TOBY FOOR-PESSIN, HCR 66, BOX } \\
\text { 9, SOUTH OTSELIC } 13155\end{array}$ \\
\hline 192 & $E$ & $\begin{array}{l}\text { VALLEY GAME FARM, } 62 \text { HIGH HILL LOOP, CATSKILL } \\
12414\end{array}$ \\
\hline 750 & $E$ & $\begin{array}{l}\text { LEAH WALTON, } 4742 \text { WOLCOTT ROAD, GAINESVILLE } \\
14066\end{array}$ \\
\hline & $E$ & $\begin{array}{l}\text { GARY J. WILLIAMS, BOX 31, RD 2, LEBANAN ROAD, } \\
\text { EARLVILLE } 13332\end{array}$ \\
\hline
\end{tabular}

WALTER D. WRIGHT, 1093 WILBUR ROAD, PHELPS 14532

\section{Independent Flocks}

E CORNERS 12514

$E$

ANGELA ALBANO \& MARY JANE KANE, 2016 MAXON ROAD, VARYSBURG 14167

E ROBERT M. ALLISON, P.O. BOX 37, ROUTE 30-A, CENTRAL BRIDGE 12035

E HERMAN ALTMANN, 314 SPRING STREET, GROTON 13073

E JOHN R. AMEND, JR., 1449 SWEET ROAD, EAST AURORA 14052

E FRAN ANDREWS, 5907 RAILROAD AVENUE, BOX 72 , KANONA 14856

E

DOUGLAS ARK, 2400 FULLER ROAD, BURT 14028-2905

E ANN ARMBRUSTER, 2096 FULTON FOLSOMDALE ROAD, COWLESVILLE 14037

E CLAUD N. ARNOLD, 7279 CAMPBELL CREEK ROAD, BATH 14810

E EVELYN AVERY, 2576 STATE HIGHWAY 7, BAINBRIDGE 13733

E RICHARD BAKER, 6336 CAMEL HILL ROAD, CONESUS 14435

E RENEE BAKER, 79 WATSON HOLLOW ROAD, WEST SHOKAN 12494

E RICHARD H. BAKER, 3313 WAYNE CENTER ROAD, LYONS 14489

E LAURA BARBANO \& FAMILY, P.O. BOX 303 , WAMPSVILLE 13163

E GEORGIA BARRETT, BOX 226, IVES ROAD, HARPURSVILLE 12787
150 R238, W119, W4, W568, W91, _Z22

100 W25, W26, W30

70 B, R12, R24, R39, W17, W23, .... W67

$130,000 \quad Z 27$

41 A, B, R222, R67, R77, W208, W290, W554, Z108, Z139, Z19, Z22, Z28

$30 \quad \mathrm{~N} 12, \mathrm{~N} 18, \mathrm{~N} 9, \mathrm{R} 52, \mathrm{~W} 103, \mathrm{~W} 11$, W12, W120, W142, W17, W18. W198, W223, W23, W24, W302. W33, W38, W4, W45, W48, W5, W50, W53, W561, W6, W60. W69, W72, W9

72 A, N18, R103, R229, W13, W30, W39, W442, W578, W593, Z11 Z2

W6

R146, R16, R21, R22, R222, R23, R248, R34, R9, W18, W249,

W573, Z22

R229, R27, W2

$\mathrm{R} 229, \mathrm{R} 27, \mathrm{~W} 2$

$\mathrm{R} 229, \mathrm{R} 27, \mathrm{~W} 2$

W54. W6

R19, R20, R48, R53, R87, W17, ..W18, Z204, Z22

B, C, R169, R222, R3, R7, R96, W587, W6

R196. W11

R229, R27, W2.

N18, W30

R229, R27, W2

R229, R27, W2.

R229, R27, W2

R229, R27, W2 
Table 1. HATCHERIES, DEALERS, AND INDEPENDENT FLOCKS PARTICIPATING IN

THE NATIONAL POULTRY IMPROVEMENT PLAN

WATERFOWL, EXHIBITION POULTRY AND GAME BIRDS

APP. SUB- PARTICIPANTS NAME AND ADDRESS

NO. PART
HATCHING
EGG

CAPACITY
PRODUCTS CLASSIFIED

U.S. PULLORUM-TYPHOID

CLEAN
ADDITIONAL

CLASSIFICATIONS FOR WHICH PRODUCT QUALIFIED

21 - NEW YORK

\begin{abstract}
751 E THE BATZ FAMILY, 7519 PARKER HILL ROAD, DANSVILLE 14437

873 E SARAH BEAN, 327 WELLMAN ROAD, ASHVILLE 14710

451 E JANET BERKEMEIER, 10901 EAST ROAD, PAVILION 14525

682 E BERKEMEIER FAMILY, 5481 RAYMOND ROAD, WYOMING 14591

365 E GEORGE BEYER, 1291 WEST BLOOD ROAD, EAST AURORA 14052

904 E ALLEN BITTER, 3251 ROSE HILL ROAD, SKANEATELES 13152
\end{abstract}

915 E BLACKWELL FAMILY, 5176 OLD BALD HILL ROAD, HAMLOCK 14466

916 E LEER. BOWHALL, 550 SMITH ROAD, GOUVERNEUR 13642

806 E JUDY G. BOYER, 1806 COUNTY ROUTE 28, LISBON 13658

917 E ALLAN BRESSETT, 397 CLAY HILL ROAD, FORT ANN 12827

918 E DEBORAH \& DERREK BUJAK, HC 1, BOX 42-A, RT. 23, ACRA 12405

919 E DAINA BULLWINKEL, 24 CLIFTON ROAD, CLIFTON 14428

920 E RONALD BURCH, BOX 330 , SUNSET BLVD., STOTTVILLE 12172

921 E BURKHARDT FAMILY, SARAH ANN BURKHARDT, 5748

\section{BARUM ROAD, AKRON 14001}

E PATRICK BURNS. 6207 STATE HIGHWAY 58, GOUVERNEUR 13642

E AARON CARMICHAEL, 4550 ROUTE 19-A, SILVER SPRINGS 14550

E DAVID CARPENTER, 2749 OLD STATE ROAD, SCHENECTADY 12303

E CHARLES CASPER, 4606 ROUTE 34-B, UNION SPRINGS 13160

E JOHN CHAMBERS, 51 CHURCH ROAD, MONSEY 10952

E DONNA CHILDS, 293 WEST STREET EXT., GLOVERSVILLE 12078

E JOSEPH D. CLARK \& MATT MORRIS, 5242 MERRILL ROAD, HOLLEY 14470

E WARREN \& REBECCA CLARK \& FAMILY, 2417 COUNTY ROUTE 4, FULTON 13069

E BARBARA A. CLARKSON, 8135 IRISH ROAD, COLDEN 14033

E DEBORAHS. CONDON, 7677 GOWANDA STREET ROAD, EDEN 14057
A, D, R222, R3, R77, R83, W2, W26

R229, R27, W2

R22, R229

R229, R6, R77, W149, W208, W552, Z139, 228

W11, W516, W56, W6

B, C, R114, R12, R3, R98, W102, W142, W23, W25, W26, W262,

W28, W29, W3, W4, W50, W52,

W53, W568, W578, W74, W88,

Z118, Z26

R229, R27, W2

R229, R27, W2

W141, W275, W28, W30, W50

R229, R27, W2

R229, R27, W2

R229, R27, W2

$\mathrm{R} 229, \mathrm{R} 27, \mathrm{~W} 2$

R229, R27, W2

R229. R27, W2.

R229, R27, W2

R229, R27, W2

$R 229, R 27, W 2$

R229, R27, W2.

B1, R196, R86, W17, W25, W52, W569, W69, W88

R12, R222, W25, W26, W33, W74, W81, Z107, Z43, Z48, Z53. Z57, 294

R229, R27, W2

R179, R180, R196, W119, W17, W176, W20, W327, W396.

W552, W57

$R 85, W 190, W 330$ 
Table 1. HATCHERIES, DEALERS, AND INDEPENDENT FLOCKS PARTICIPATING IN

THE NATIONAL POULTRY IMPROVEMENT PLAN

WATERFOWL, EXHIBITION POULTRY AND GAME BIRDS

\begin{tabular}{llcccc}
\hline & & HATCHING & PRODUCTS CLASSIFIED \\
APP. & SUB- & PARTICIPANTS NAME AND ADDRESS & EGG & U.S. PULLORUM-TYPHOID & CLEAN \\
NO. & PART & CAPACITY & CLASSIFICATIONS FOR & WHICHPRODUCT QUALIFIED \\
\hline
\end{tabular}

21 - NEW YORK

\begin{abstract}
927 E SAM \& CARLA COX, 2305 ANDERSON ROAD, LINWOOD 14486

871 E AMY CRAFT, RR 1, BOX 107 B1, BLOOMVILLE 13739

548 E JEAN \& LANGDON CURTIS, RT. 23, HILLSDALE 12529

\begin{tabular}{|c|c|c|}
\hline 896 & E & $\begin{array}{l}\text { R. J. DALEY, ROSSWAY ROAD, BOX 75-B, PLEASANT } \\
\text { VALLEY } 12569\end{array}$ \\
\hline 929 & $E$ & $\begin{array}{l}\text { KEITH E. DAVENPORT, P.O. BOX 792, WEEDSPORT } \\
13166\end{array}$ \\
\hline & E & GORDON DAVID, 7177 STONE HILL ROAD, LIMA 14485 \\
\hline & E & ROGER F. DAVIS, 5186 ST. RTE 365, VERONA 13478 \\
\hline & E & $\begin{array}{l}\text { JERRY DAVIS, } 1375 \text { CHURCH ROAD, BALDWINSVILLE } \\
13027\end{array}$ \\
\hline & $\mathrm{E}$ & $\begin{array}{l}\text { BARRY P. DEBYAH, BOX 291, COUNTY ROAD } 53 \text {, } \\
\text { NORTH BANGOR } 12966\end{array}$ \\
\hline & $E$ & $\begin{array}{l}\text { LEE \& SHIRLEY DELONG, } 10015 \text { SOUTH LANDING } \\
\text { ROAD. MANNSVILLE } 13661\end{array}$ \\
\hline
\end{tabular}
\end{abstract}

936 E JOHN DELONG. JR., 321 COUNTY ROUTE 41-A, PULASKI 13142

907 E TAMARA \& LEE DELONG, JR., 1805 NYS ROUTE 3, MANNSVILLE 13661

350 E SUE DI GASPARI, RR 1, OLMSTEAD ROAD,
CHITTENANGO 13037

200 E JOHN DINATALE, 46 CASEMENT AVENUE, CENTRAL ISLIP 11722

775 E JOHATHAN DODSON, JR., 5112 CHUGG ROAD, HOLLEY 14470

931 E STEVE DORROUGH, 5617 CROOKED STREET. BROADALBIN 12025

874 E KAREN DUDLEY, 166 MCCLOUD ROAD, WEST MONROE 13167 214 E ADDISON EARLEY, P.O. BOX 57, EARLEY ROAD,
PRATTSBURG 14873

109 E WILLIAM EGLIN, 7341 HIGHWAY 109 PROKOP ROAD. BROADALBIN 12025
R229, R27, W2

R120, R139, R180, R19, R20, R23, R24, R30, R51, R54, R70, R77, R85, R88, R94, W117, W160, W2, W22, W52, W6

R45, R50, R87, W102, W119, . W121, W129, W138, W164, W17, W18, W198, W208, W25, W26, W33, W45, W570, W58, W6, W69, W70, Z101, Z108 Z118, Z19, Z20, Z204, Z205, Z22, Z26, Z51, Z53, Z57, Z62, Z67, Z9

A, B, R12, R243

R229, R27, W2

W11, W12, W25, W26, W28, W38, W48, W88

R22, W14, W15, W94

R229, R27, W2

W300, W570, W67, W77, 226

H8, N1, N10, N6, N9, R114, R168, R179, R214, R222, R33, R51, R53, R62 R7, R70, R71. R72, R86, R89, R9, W102, W128. W166, W17, W18, W198, W211, W24, W265, W29, W302, W33, W36, W4, W45, W475, W479, W50, W573, W578, W6, W67, W92

R229, R27, W2

R178, R20, R214, R33, R8, W142, W149, W151, W17, W200. W276, W28, W29, W3, W50, W52, W6, W74, W86, Z4

W102, W164, W17, W74

R139, R30, R52, W17, W18, W198, W25, W26. W48

R12, R222, R96, W544

R229, R27, W2

R115, R139, R180, R182, R45, R70, R83, R86, W 129, W424, W585, W68

R229, R27, W2

$R 229, R 27, W 2$ 
Table 1. HATCHERIES. DEALERS. AND INDEPENDENT FLOCKS PARTICIPATING IN

THE NATIONAL POULTRY IMPROVEMENT PLAN

WATERFOWL, EXHIBITION POULTRY AND GAME BIRDS

\begin{tabular}{|c|c|c|c|c|c|}
\hline $\begin{array}{l}\text { APP. } \\
\text { NO. }\end{array}$ & $\begin{array}{l}\text { SUB. } \\
\text { PART }\end{array}$ & PARTICIPANTS NAME AND ADDRESS & $\begin{array}{l}\text { HATCHING } \\
\text { EGG } \\
\text { CAPACITY }\end{array}$ & $\begin{array}{l}\text { PRODUCTS CLASSIFIED } \\
\text { U.S. PULLORUM-TYPHOID } \\
\text { CLEAN }\end{array}$ & $\begin{array}{c}\text { ADDITIONAL } \\
\text { CLASSIFICATIONS FOR } \\
\text { WHICH PRODUCT QUALIFIED }\end{array}$ \\
\hline
\end{tabular}

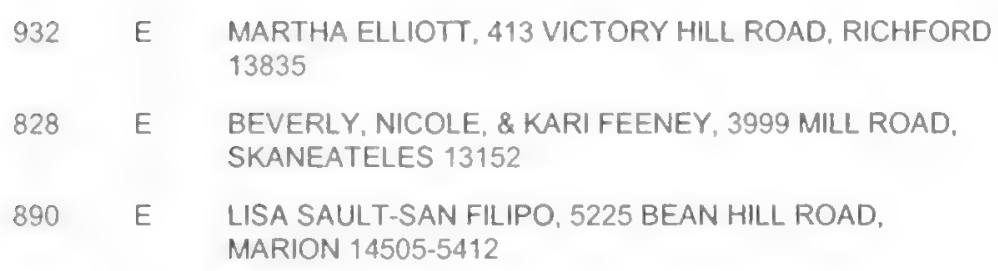

R229, R27, W2

W150, W151, W20, W28

$A, B, C, R 213, R 227, R 42, R 45,-$ R47, R84, R93, W208, W309,

$\mathrm{Z} 22$

W189, W301, W357, W364, W74.

W121, W140, W164, W169,

W17, W18, W208, W215, W25,

W26, W3, W33, W5, W570, W60,

W69, W7, W74, W89, W91

R229, R27, W2

Z108, Z137, Z14, Z201, Z202, Z204, Z205, Z207, Z9

B, C, R222, R56, R98, W118,

W16, W2, W25, W26

E

$\mathbf{Z 1 1}, \mathbf{Z 1 2 6}$

W114, W149, W262, W29, W48, W88, Z24, Z26, Z27, Z34, Z51,

Z52, Z53, Z57

R229, R27, W2

Z18, Z204

R229, R27, W12, W19, W2, W20, W25, W74

R229, R27, W12, W19, W2, W20.. W25, W74

R199, R222, R42, R60, R70, R71,. R89, W1, W13, W132, W25,

W26, W30, W570, W7

R12, R20, R227, R247, R51

Z202, Z227

R179, R180, R182, W45, W68_

W115, W25, W261, W293,

W556, W91

N10, N16, N18, N9, R24, W30, W74

R168, W102, W119, W142, W17,W244, W25, W26, W364, W48

R229, R27, W2.

R229, R27, W2

R229, R27, W2 
Table 1. HATCHERIES, DEALERS, AND INDEPENDENT FLOCKS PARTICIPATING IN

THE NATIONAL POULTRY IMPROVEMENT PLAN

WATERFOWL, EXHIBITION POULTRY AND GAME BIRDS

\begin{tabular}{|c|c|c|c|c|c|}
\hline $\begin{array}{l}\text { APP. } \\
\text { NO. }\end{array}$ & $\begin{array}{l}\text { SUB- } \\
\text { PART }\end{array}$ & PARTICIPANTS NAME AND ADDRESS & $\begin{array}{l}\text { HATCHING } \\
\text { EGG } \\
\text { CAPACITY }\end{array}$ & $\begin{array}{l}\text { PRODUCTS CLASSIFIED } \\
\text { U.S. PULLORUM-TYPHOID } \\
\text { CLEAN }\end{array}$ & $\begin{array}{c}\text { ADDITIONAL } \\
\text { CLASSIFICATIONS FOR } \\
\text { WHICH PRODUCT QUALIFIED }\end{array}$ \\
\hline
\end{tabular}

21 - NEW YORK

\begin{tabular}{|c|c|c|}
\hline 289 & $\mathrm{E}$ & PAUL A. JONES, 380 WEST ROAD, RD 2, ONEIDA 13421 \\
\hline 223 & $E$ & $\begin{array}{l}\text { HAROLD E. KNAPP, } 5299 \text { JERICO ROAD, EAST } \\
\text { BETHANY } 14054\end{array}$ \\
\hline 855 & $E$ & $\begin{array}{l}\text { SUSAN KOWALSKI, } 65 \text { WHITE SCHOOLHOUSE ROAD, } \\
\text { RHINEBECK } 12572\end{array}$ \\
\hline 937 & E & $\begin{array}{l}\text { KRAJEWSKI FAMILY, } 1615 \text { HALLS COURNER ROAD, } \\
\text { WARSAW } 14569\end{array}$ \\
\hline 91 & $E$ & $\begin{array}{l}\text { KREHER'S POULTRY FARM, } 5411 \text { DAVISON ROAD, } \\
\text { CLARENCE } 14031\end{array}$ \\
\hline 411 & E & $\begin{array}{l}\text { PAUL F. KROLL, } 4325 \text { TWO ROD ROAD, EAST AURORA } \\
14052\end{array}$ \\
\hline 79 & $E$ & RICHARD \& KARL KUEHNLE, 6774 RT. 31, CICERO 13039 \\
\hline 885 & E & $\begin{array}{l}\text { THOMAS LAMBERT, 291-A SCHOOL HOUSE ROAD, } \\
\text { HYDE PARK } 12580\end{array}$ \\
\hline 355 & $E$ & $\begin{array}{l}\text { RICHARD LANG, } 4957 \text { HOLLENBECK ROAD, LOCKPORT } \\
14094\end{array}$ \\
\hline 113 & $\mathrm{E}$ & $\begin{array}{l}\text { RICHARD LANGENBACH, BOX } 355, \text { MEADOWDALE, RD } \\
\text { 2, ALTAMONT } 12009\end{array}$ \\
\hline 769 & $\mathrm{E}$ & $\begin{array}{l}\text { MARK J. \& BRADLEY LANGERMAN, } 2231 \text { ROUTE } 98, \\
\text { ATTICA } 14011\end{array}$ \\
\hline 558 & $\mathrm{E}$ & $\begin{array}{l}\text { RONALD \& VERONICA LARSON, } 4613 \text { SOUTH GRAVEL } \\
\text { ROAD, MEDINA } 14103\end{array}$ \\
\hline 962 & $\mathrm{E}$ & $\begin{array}{l}\text { JOHN \& HEATHER LATHROP, } 333 \text { BINGHAM ROAD, } \\
\text { SHERBURNE } 13460\end{array}$ \\
\hline 336 & $\mathrm{E}$ & KENNETH \& MARY LEARN, 7194 RT. 54, BATH 14810 \\
\hline 694 & $\mathrm{E}$ & $\begin{array}{l}\text { RUBY MARIE LEGGETT, } 426 \text { CROTON AVENUE, } \\
\text { PEEKSKILL } 10566\end{array}$ \\
\hline 825 & $\mathrm{E}$ & $\begin{array}{l}\text { LESSWING FAMILY, } 14 \text { MASSACHUSETTS STREET,P.O. } \\
\text { BOX } 637 \text { NUNDA } 14517\end{array}$ \\
\hline 957 & $E$ & $\begin{array}{l}\text { KIMBERLY LYNN \& KELLY WATERS, } 3221 \text { COUNTY LINE } \\
\text { ROAD, MIDDLEPORT } 14105\end{array}$ \\
\hline 939 & $E$ & CHRIS MACARI, 404 LOWER ROAD, CONSTANTIA 13044 \\
\hline 964 & $E$ & $\begin{array}{l}\text { PAMELA MARSHALL, P.O. BOX 351, ROUTE 22-N, } \\
\text { AMENIA } 12501\end{array}$ \\
\hline 457 & $E$ & $\begin{array}{l}\text { EDWIN MARTIN, } 16 \text { MARKET STREET, ROTTERDAM } \\
\text { JUNCTION } 12150\end{array}$ \\
\hline 940 & $E$ & $\begin{array}{l}\text { CLYDE MARTIN, } 6197 \text { STATE HIGHWAY } 58, \\
\text { GOUVERNEUR } 13642\end{array}$ \\
\hline 391 & $E$ & $\begin{array}{l}\text { JAMIE L. \& TERRI MATTS, RD 1, BOX 48-A, } \\
\text { HARPURSVILLE } 13787\end{array}$ \\
\hline 938 & $E$ & $\begin{array}{l}\text { MCCORMICK FAMILY, } 118 \text { O'BRIEN ROAD, } \\
\text { CHURCHVILLE } 14424\end{array}$ \\
\hline 941 & $\mathrm{E}$ & $\begin{array}{l}\text { KIM MEDECKY, RD 2, BOX } 289 \text { NORTH ROAD, } \\
\text { JEFFERSON } 12093\end{array}$ \\
\hline 942 & $E$ & $\begin{array}{l}\text { ADAM MIHALIK, } 116 \text { GIFFORD VALLEY ROAD, } \\
\text { NORTHVILLE } 12134\end{array}$ \\
\hline
\end{tabular}

N10, N14, N15, N50, R12, R8, - W208

B, R15, R186, R229, R6, R78, W144, W2

R196, R47, W208, Z62.

R229, R27, W2

W

W138, W145, W176, W198

W211, W81

R227, R42, W102, W120, W121, W17, W387, W4, Z126, Z2, Z24, Z26, Z28, Z34, Z53, Z55, Z57

W142, W30, W48, W50, W51, W88, W89

$W_{102}, W_{12}, W 17, W_{60}$

W142, W216, W23, W25, W28, W3, W54, W89

R169, R89, W576, W58, W6, Z204, Z227

A, C, E, R112, R12, R15, R35,

R45, R53, W573, W58, W74, Z22

B, R222, W115, W121, W17, W175

R42, W11, W12, W25, W26, Z118, Z138, Z22

A, B, C, R19, R21, R222, R56, R94, W140, W17, W208, W25. W26, W45, W519, W69

R45, R89, W145, W176, W198

R229, R27, W2

R229, R27, W2

R126, R213, R225, W189, W208. W26, Z204, Z216

B, C, R168, R21, R52, R64, W76

R229, R27, W2

R94, W102, W121, W164, W17, W206, W87

R229, R27, W2

R229, R27, W2

R229, R27, W 
Table 1. HATCHERIES, DEALERS, AND INDEPENDENT FLOCKS PARTICIPATING IN

THE NATIONAL POULTRY IMPROVEMENT PLAN

WATERFOWL, EXHIBITION POULTRY AND GAME BIRDS

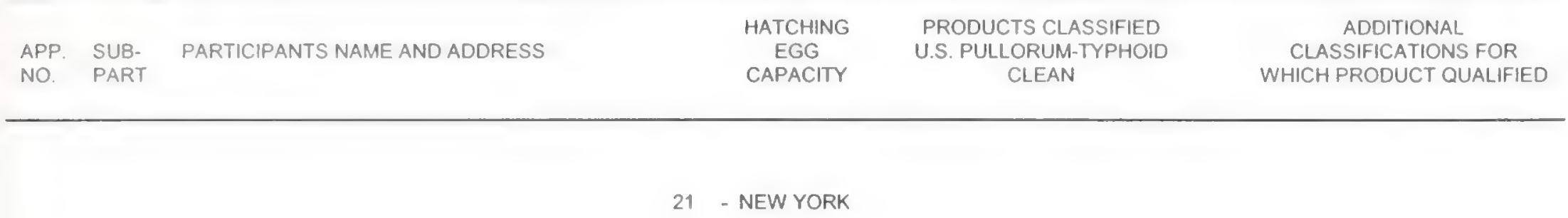

\begin{tabular}{|c|c|c|}
\hline 878 & E & $\begin{array}{l}\text { SARA MILONOVICH, } 740 \text { BULLS HEAD ROAD, } \\
\text { AMSTERDAM } 12010\end{array}$ \\
\hline 943 & E & $\begin{array}{l}\text { PHILIP \& SAM MINCEY, } 148 \text { SOUTH MAIN STREET, } \\
\text { PERRY } 14530\end{array}$ \\
\hline 970 & E & $\begin{array}{l}\text { MOON DISK FARM, NEAL BURGOYNE, } 101 \text { SANTAMORE } \\
\text { ROAD, CHURUBUSCO } 12923-2721\end{array}$ \\
\hline 501 & $E$ & $\begin{array}{l}\text { SUE MYERS, } 2005 \text { NEW BOSTON ROAD, CHITTENANGO } \\
13037\end{array}$ \\
\hline 397 & $E$ & $\begin{array}{l}\text { RICHARD \& JUDY NASON, RR 1, BOX 128, MALONE } \\
12953\end{array}$ \\
\hline 963 & $E$ & $\begin{array}{l}\text { LAUREN NELSON, } 171 \text { MAPLE DRIVE, EAST CHATHAM } \\
12060\end{array}$ \\
\hline 725 & $E$ & $\begin{array}{l}\text { KAREN \& SETH O'DELL, RD 1, BOX 70, CO RT. 51, COX } \\
\text { SACKIE } 12051\end{array}$ \\
\hline 632 & $E$ & $\begin{array}{l}\text { VALERIE ORLOWSKI, } 4608 \text { STATE ROUTE } 30 \\
\text { AMSTERDAM } 12010\end{array}$ \\
\hline 203 & $E$ & MERLE PALMITER, 2675 GENESEO ROAD, AVON 14414 \\
\hline 863 & $E$ & $\begin{array}{l}\text { HAROLD PASSMORE \& TIM FOWLER, } 11461 \text { KELLY HILL } \\
\text { ROAD. PINE CITY } 14871\end{array}$ \\
\hline
\end{tabular}

945

900

900

28 SCIPIP CENTER 13147

E

E LARRY PERRIN, RR 1, BOX 130-A CROOKS ROAD, NORTH BANGOR 12966

E JOHN PIERCE, 3832 MAKYES ROAD, SYRACUSE 13215

E ROBERT PIOSELI, RD 1, BOX 93, CHESTER 10918

WILLIAM POST, RD 3, BOX 90, BERLIN 13411

E CAROL PUESCHEL, 2997 STATE ROUTE 5S, LITTLE FALLS 13365

E QUAIL VALLEY FARM, D.R. PERRIN, 2825 DODGESON ROAD, ALEXANDER 14005

E RADLEY FAMILY, 5485 CLINTON STREET ROAD, BATAVIA 14020

E ROBERT RAMSEY SR., P.O. BOX 95, WING ROAD. GREENFIELD CENTER 12833

Phone: $(518) 587-0116$
B, C. R120, R139, R20, R34, R7, R9, W28, W60

R229, R27, W2

B, C, D, R120, R139, R30, R81, R86, W, W102, W119, W121,

W17, W223, W265, W54, W570 W578

R22, W15

R13, R147, R222, R34, R52, R73. W38, Z204, Z227

A, C, D, R139, R20, R222, R64, R74, R84, W119, W12, W198, W211, W223, W25, W427

B, R42, W216, W516

B1, C1, R19, R229, R85, R9, W102, W119, W36

W136, W301, W58

R107, R168, R72, W11, W119, W128, W17, W176, W198, W2 W211, W25, W26, W33, W36, W466, W475, W53, W548, W560, W570, W576, W590, W6, W60, W9, Z118, Z159, Z202, Z204, Z22, Z26, Z4, Z57

R229, R27, W2

R229, R27, W2

C, D, R222, R9, W102, W119, W126, W129, W138, W151, W17, W176, W18, W193, W198. W223, W230, W257, W271, W4, W561, W573, W6, W75, W81, W88

B, R21, R3, W28, W69, W7

W121, W168, W17, W208, W290, W309, w60

W23, W38, W7

R229, R27, W2

Z111, Z21, Z22

R229, R27, W2

R229, R27, W2 
Table 1. HATCHERIES, DEALERS, AND INDEPENDENT FLOCKS PARTICIPATING IN

THE NATIONAL POULTRY IMPROVEMENT PLAN

WATERFOWL, EXHIBITION POULTRY AND GAME BIRDS

\begin{tabular}{|c|c|c|c|c|c|}
\hline $\begin{array}{l}\text { APP. } \\
\text { NO. }\end{array}$ & $\begin{array}{l}\text { SUB- } \\
\text { PART }\end{array}$ & PARTICIPANTS NAME AND ADDRESS & $\begin{array}{l}\text { HATCHING } \\
\text { EGG } \\
\text { CAPACITY }\end{array}$ & $\begin{array}{l}\text { PRODUCTS CLASSIFIED } \\
\text { U.S. PULLORUM-TYPHOID } \\
\text { CLEAN }\end{array}$ & $\begin{array}{c}\text { ADDITIONAL } \\
\text { CLASSIFICATIONS FOR } \\
\text { WHICH PRODUCT QUALIFIED }\end{array}$ \\
\hline
\end{tabular}

21 - NEW YORK

\begin{tabular}{|c|c|c|c|}
\hline 399 & E & $\begin{array}{l}\text { ARTHUR W. RANKIN, RR 1, BOX 165, PULP MILL ROAD, } \\
\text { CHATEAUGAY } 12920\end{array}$ & $\mathrm{R} 42, \mathrm{~W} 262, \mathrm{~W} 4$ \\
\hline 947 & $E$ & $\begin{array}{l}\text { RAYMO'S ROOSE, DEREK W. RAYMO, } 1618 \text { STATE } \\
\text { HIGHWAY 420, NORFOLK } 13667\end{array}$ & R229, R27, W2 \\
\hline 948 & E & $\begin{array}{l}\text { KAREN REBEHN, } 18 \text { ORIENT AVENUE, AVERILL PARK } \\
12018\end{array}$ & $\mathrm{R} 229, \mathrm{R} 27, \mathrm{~W} 2$ \\
\hline 394 & $E$ & $\begin{array}{l}\text { ROBERT E. RICHIE, } 5069 \text { LEE VALLEY ROAD, ROME } \\
13440\end{array}$ & R34, W23 \\
\hline 661 & E & $\begin{array}{l}\text { CAROL ROBINSON, } 12404 \text { TREVETT ROAD, } \\
\text { SPRINGVILLE } 14141\end{array}$ & R46, R84, W100, W18, W564 \\
\hline 808 & E & $\begin{array}{l}\text { TOM ROEBUCK, SR., } 1796 \text { ALBANY POST ROAD, } \\
\text { ULSTER } 12589\end{array}$ & W13, W17, W38, W53 \\
\hline 779 & $E$ & $\begin{array}{l}\text { FLOYD ROOD, } 11 \text { NORTH WOODS ROAD, HERMAN } \\
13652\end{array}$ & W114, W48, W545 ___ \\
\hline 949 & $E$ & GERALD ROSS, 2320 EMO ROAD, WAYLAND 14572 & R229, R27, W2 \\
\hline 950 & $E$ & $\begin{array}{l}\text { MICHAEL \& CHRISTINA ROSS, } 5319 \text { LAKEVILLE ROAD, } \\
\text { GENESEO } 14454\end{array}$ & $R 229, R 27, W 2$ \\
\hline 857 & $E$ & $\begin{array}{l}\text { JOSEPH D. ROSSI, RD 3, BOX 135, HAPEMAN HILL } \\
\text { ROAD, RED HOOK } 12571\end{array}$ & R10, R21, R222. R34, R49, R72 \\
\hline 866 & $E$ & $\begin{array}{l}\text { WILLIAM C. RYAN \& C. JANIE CARLE, BOX } 212 \text {, } \\
\text { MAYFIELD } 12117\end{array}$ & $\begin{array}{l}\text { R21, R52, R86, W119, W132, } \\
\text { W166 }\end{array}$ \\
\hline 951 & $E$ & $\begin{array}{l}\text { TERRY SANFORD, } 3535 \text { COUNTY ROUTE 23-A, DUNDEE } \\
14837\end{array}$ & $\mathrm{R} 229, \mathrm{R} 27, \mathrm{~W} 2$ \\
\hline 839 & $E$ & $\begin{array}{l}\text { KATIE, TINA, \& JANET SCHIE, } 2836 \text { MAXON ROAD, } \\
\text { VARYSBURG } 14167\end{array}$ & R15, R222, R6, W74 \\
\hline 543 & $E$ & $\begin{array}{l}\text { DANIEL D. \& MARIETTA SCHUTH, } 2279 \text { TRANSIT ROAD, } \\
\text { KENT } 14477\end{array}$ & N11, N6, W115 \\
\hline 206 & $E$ & TED SCHWABROW, RD, BOX 321, HANNACROIX 12087 & B. W164, W18, W6, W8 \\
\hline 877 & $E$ & $\begin{array}{l}\text { TRUDI D. SCOTI, } 196 \text { BART BULL ROAD, MIDDLETOWN } \\
10941\end{array}$ & W54 _ _ _ _ _ \\
\hline 884 & $E$ & WILLIAM SEBAST, STAR ROUTE BOX 13, SALEM 12826 & $\begin{array}{l}\text { A, C, R15, R196, R7, W121, } \\
\text { W32, W33, W45, W6, W7, Z2205 }\end{array}$ \\
\hline 343 & $E$ & $\begin{array}{l}\text { SHELDON FAMILY, } 160 \text { MALDEN BRIDGE ROAD, } \\
\text { NASSAU } 12123\end{array}$ & $\begin{array}{l}\text { C1, R109, R34, R56, R85, W13, } \\
\text { W140, W18, W200, W23, W26, } \\
\text { W28, W3, W5, W52, W54, W7 }\end{array}$ \\
\hline 886 & $E$ & DAN SIDOR, 1483 ROUTE 43. STEPHENTOWN 12168 & $\begin{array}{l}\text { B1, E1, N18, N4, R196, W104, } \\
\text { W11, W13, W132, W25, W26, } \\
\text { W3, W30, W361, W54, W74 }\end{array}$ \\
\hline 966 & $E$ & $\begin{array}{l}\text { SNYDER COOP.. DAVID SNYDER, } 990 \text { COUNTY ROUTE } \\
53, \text { BRASHER FALLS } 13613-4001\end{array}$ & $\begin{array}{l}\text { W142, W149, W247, W262, } \\
\text { W28, W48, W69, W89 }\end{array}$ \\
\hline 952 & $E$ & JOHN SONGER. 548 DRUMM ROAD. WEBSTER 14580 & $\mathrm{R} 229, \mathrm{R} 27, \mathrm{~W} 2$ \\
\hline 969 & $\mathrm{E}$ & $\begin{array}{l}\text { SPRINGDALE FARM, DAN PIETERS, } 696 \text { COLBY } \\
\text { STREET, SPENCERPORT } 14559\end{array}$ & $\begin{array}{l}\text { B, R229, R53, R84, R87, W17, - } \\
\text { W208, Z15, Z202, Z204, Z227 }\end{array}$ \\
\hline 519 & $E$ & $\begin{array}{l}\text { DAVID ST.MARY, RD 2, BOX 307, LIMEKILN ROAD, } \\
\text { MALONE } 12953\end{array}$ & $\begin{array}{l}\text { W104, W119, W142, W24, W28, } \\
\text { W3, W33, W4, W456, W48, W50, } \\
\text { W52, W570, W69, W88, W89, } \\
\text { W91, Z118, Z32, Z67, Z80, Z90 }\end{array}$ \\
\hline 887 & E & RICHARD STAIE, 610 GOUVERNEUR, P.O. BOX 239 , & W365 \\
\hline
\end{tabular}


Table 1. HATCHERIES, DEALERS, AND INDEPENDENT FLOCKS PARTICIPATING IN

THE NATIONAL POULTRY IMPROVEMENT PLAN

WATERFOWL, EXHIBITION POULTRY AND GAME BIROS

\begin{tabular}{|c|c|c|c|c|c|}
\hline $\begin{array}{l}\text { APP. } \\
\text { NO. }\end{array}$ & $\begin{array}{l}\text { SUB- } \\
\text { PART }\end{array}$ & PARTICIPANTS NAME AND ADDRESS & $\begin{array}{l}\text { HATCHING } \\
\text { EGG } \\
\text { CAPACITY }\end{array}$ & $\begin{array}{c}\text { PRODUCTS CLASSIFIED } \\
\text { U.S. PULLORUM-TYPHOID } \\
\text { CLEAN }\end{array}$ & $\begin{array}{c}\text { ADDITIONAL } \\
\text { CLASSIFICATIONS FOR } \\
\text { WHICH PRODUCT QUALIFIED }\end{array}$ \\
\hline
\end{tabular}

832 E MARY \& RAYMOND STARR III, 881 BRIDGE STREET, ROUTE 396, RAVENA 12143

868 E STELLER FARM, SHADY DELL ROAD, RR 2, BOX 54-A, MILLBROOK 12545

740 E STORRER FAMILY, BROOKLYN STREET, EATON 13334

840 E TERRY \& MATT STROBEL, 7238 NORTH BERGEN ROAD, BERGEN 14416

967 E SUNRISE FARMS, INC., 175 CAUTERSKILL ROAD, CATSKILL 12414

953 E THERON SWORTS, 9500 GRAHAM ROAD, MIDDLEPORT 14105

746 E CAMERON TALLON. 151 SAND STREET, HAMMOND 13646

844 E PAUL, LAURA, ASHTON, \& JORDAN THURBER, 7389 ALLEGANY ROAD, BASOM 14013

E LEE A. TRAVER, RD 4, BOX 350B, WYNNATSKILL 12198

E TOM TUCKER, 8357 U.S. ROUTE 11-S, ADAMS 13605

E DAVID URDA, P.O. BOX 209, 7824 NORTH MAIN STREET, KANONA 14856

E PETER M. VAUGHN, 65 CARGIN ROAD, MALONE 12953

E LIZ WAGNER, 9475 RIDGE ROAD, MIDDLEPORT 14105

WALCH FAMILY, 1271 MOSCOW ROAD, HAMLIN 14464

E DICK \& THOLA WALDOU, 433 COUTNY RT. 23, CONSTANTIA 13044

E JOE \& MARGARET WALKER, 375 ORTNER ROAD, DARIAN CENTER 14040

E DUFF WEHLE, ENGLISHMAN'S BAY FARM,3062 OATKA TRAIL ROAD MUMFORD 14511

E MARY WEINHEIMER, 2682 WEST CHURCH STREET, EDEN 14057

E GARYA. WELLS, 2421 STATE HIGHWAY 310 , MADRID 13660

E MATTHEW WELLS, 4614 COUNTY RT. 14, MADRID 13660

E MATTHEW J. WHITEMAN, 2028 PAINE ROAD, DALTON 14836

E WHITNEY BROTHERS, 8000 MT. WASHINGTON ROAD, BATH 14810

E WILLIAM D. WHITNEY, 7411 FAUCETT ROAD, BATH 14810

E DEAN A. \& DESAREE E. WILKIE, 36883 NEW YORK STATE RT. 12, CLAYTON 13724

E RONALD WILLIAMSON, 1309 NORTH GREENWICH ROAD, ARAYLE 12809

R114, R200, R3, W141, W207, W23, W28, W363, W48, Z4

R180, R182, R247, R50, R54, R82, W102, W138, W570

W25, W26, W28, W33, W561, W89

R168, R214, Z107, Z118, Z15, Z206, Z26

R229, R27, W2

R229, R27, W2

W12, W2

N4, W115, W125, W188, W245, W293

A, B, R30, W208, W211, W6, W7.

R229, R27, W2

R17, R227.

R229, R27, W2

A, B, R222, R229, R81, W223, W270, W28, W33, W48, W86

W102, W17, W18, W193, W270, W271, W86

R229, R27, W2

W106, W17, Z108, Z15, Z179, Z22

R11, R146, R19, R35, R44, R7, W12, W17, W28, W54, W74. $\mathrm{Z27}, \mathrm{Z28}$

W30

R27

R229, R27, W2

R22, R97, W142, W24, W50, W52

A, R168, R19, R34, R39, W36, W5, W54

R196, R7, W102, W17, W415

D. R10, R196, R7, R86, W126, W16, W17, W24, W3, W352, W353 
Table 1. HATCHERIES, DEALERS, AND INDEPENDENT FLOCKS PARTICIPATING IN

THE NATIONAL POULTRY IMPROVEMENT PLAN

WATERFOWL, EXHIBITION POULTRY AND GAME BIRDS

\begin{tabular}{|c|c|c|c|c|c|}
\hline $\begin{array}{l}\text { APP. } \\
\text { NO. }\end{array}$ & $\begin{array}{l}\text { SUB- } \\
\text { PART }\end{array}$ & PARTICIPANTS NAME AND ADDRESS & $\begin{array}{l}\text { HATCHING } \\
\text { EGG } \\
\text { CAPACITY }\end{array}$ & $\begin{array}{l}\text { PRODUCTS CLASSIFIED } \\
\text { U.S. PULLORUM-TYPHOID } \\
\text { CLEAN }\end{array}$ & $\begin{array}{c}\text { ADDITIONAL } \\
\text { CLASSIFICATIONS FOR } \\
\text { WHICH PRODUCT QUALIFIED }\end{array}$ \\
\hline & & 21 & - NEW YORK & & \\
\hline 786 & $E$ & $\begin{array}{l}\text { STEVEN E. WOJTKOWIAK, } 2111 \text { TRANSIT ROAD, ELMA } \\
14059\end{array}$ & & $R 19, R 51, W 12, W 4$ & \\
\hline 959 & $E$ & $\begin{array}{l}\text { PEGGY ZAEPFEL, } 8474 \text { CHAPMAN ROAD, GASPORT } \\
14067\end{array}$ & & $R 229, R 27, W 2$ & \\
\hline 902 & $E$ & $\begin{array}{l}\text { DONALD C. ZIEGLER JR., } 3164 \text { THREE ROD ROAD, } \\
\text { EAST AURORA } 14052\end{array}$ & & $R 229, R 27, W 2$ & \\
\hline 300 & $E$ & $\begin{array}{l}\text { WILLIAM ZIEHM, } 7171 \text { SLAYTON SETTLEMENT ROAD, } \\
\text { LOCKPORT } 14094\end{array}$ & & W121, W17, W170, W41, W88 & \\
\hline
\end{tabular}

DR. JO ANNA QUINN

VETERINARY DIVISION

DEPARTMENT OF AGRICULTURE AND CONSUMER

SERVICES

P.O. BOX 12223

RALEIGH, NC 27605

Phone: (919)733-3986

Fax: $\quad(919) 733-0454$

$\underline{\text { Hatcheries }}$

\begin{tabular}{|c|c|c|}
\hline 772 & $E$ & $\begin{array}{l}\text { MICHELLE GRANTER, } 6211 \text { LEMON SPRINGS ROAD, } \\
\text { LEMON SPRINGS } 27332\end{array}$ \\
\hline 356 & $\mathrm{E}$ & $\begin{array}{l}\text { KENNEDY GAME FARM HATCHERY, } 4623 \text { NORTH } \\
\text { CAROLINA HIGHWAY } 705 \text {, ROBBINS } 27325\end{array}$ \\
\hline 348 & $E$ & $\begin{array}{l}\text { QUAIL VALLEY FARMS, } 9219 \text { CONCORD HIGHWAY, } \\
\text { INDIAN TRAIL } 28079\end{array}$ \\
\hline & & Independent Flocks \\
\hline 737 & $E$ & $\begin{array}{l}\text { STEVE ALEXANDER, } 8486 \text { ISLAND POINT ROAD, } \\
\text { SHERRILLS FORD } 28673\end{array}$ \\
\hline 626 & $E$ & TOM ANDERSON. P.O. BOX 263, WILLARD 28478 \\
\hline 649 & $E$ & $\begin{array}{l}\text { MARK ATWOOD, } 3740 \text { CEDAR TRAIL, THOMASVILLE } \\
27360\end{array}$ \\
\hline 59 & $E$ & APRIL BRENDLE, 9 PIGEON LANE, SYLVA 28779 \\
\hline
\end{tabular}

300

200,000

356,000

Z43

R, Z11

W35

R1, R175, R74, R90, W105, W281, W56

E52, N31, R123, R225, R23, - R245, R42, R53, R75, R83,

W102, W13, W142, W17, W18,

W211, W215, W248, W262,

W265, W48, W568, W613.

W615, W69, W98

\section{Z43}

W115, W177, W211, W224, W56, W576, W6, W75

Z1, Z43

E211, W115, W177, W198, W224, W56, W576, W6, W75

$\mathrm{Z1}, \mathrm{Z2}$ 
Table 1. HATCHERIES, DEALERS. AND INDEPENDENT FLOCKS PARTICIPATING IN THE NATIONAL POULTRY IMPROVEMENT PLAN

WATERFOWL, EXHIBITION POULTRY AND GAME BIRDS

\begin{tabular}{|c|c|c|c|c|c|}
\hline $\begin{array}{l}\text { APP. } \\
\text { NO. }\end{array}$ & $\begin{array}{l}\text { SUB- } \\
\text { PART }\end{array}$ & PARTICIPANTS NAME AND ADDRESS & $\begin{array}{l}\text { HATCHING } \\
\text { EGG } \\
\text { CAPACITY }\end{array}$ & $\begin{array}{l}\text { PRODUCTS CLASSIFIED } \\
\text { U.S. PULLORUM-TYPHOID } \\
\text { CLEAN }\end{array}$ & $\begin{array}{c}\text { ADDITIONAL } \\
\text { CLASSIFICATIONS FOR } \\
\text { WHICH PRODUCT QUALIFIED }\end{array}$ \\
\hline
\end{tabular}

\begin{abstract}
429 E WILLIAM CHURCH, 4272 ROCKY ROAD, LENOIR 28645
644 E DAVID CLARK, 2623 LOVICK ROAD, KINSTON 28501
\end{abstract}

\begin{tabular}{|c|c|c|}
\hline 760 & E & $\begin{array}{l}\text { COCK-A-DOODLES, } 5489 \text { STAG PARK ROAD, BURGAV } \\
28425\end{array}$ \\
\hline 653 & $E$ & $\begin{array}{l}\text { SAMMIE COLLINS, } 229 \text { CLEVE STREET, MOUNT AIRY } \\
27030\end{array}$ \\
\hline 229 & $E$ & $\begin{array}{l}\text { ROBERT CRANE, } 1169 \text { DUCKWORTH DRIVE, } \\
\text { MORGANTON } 28655\end{array}$ \\
\hline 738 & $E$ & $\begin{array}{l}\text { KEN DEAL, } 1470 \text { PATTERSON STREET, CHINA GROVE } \\
28023\end{array}$ \\
\hline 551 & E & $\begin{array}{l}\text { DENNIS NED DEVINE, } 104 \text { EAST BALLARD STREET, } \\
\text { CHERRYILLE } 28021\end{array}$ \\
\hline 761 & $E$ & JOHATHAN DOSS, 200 OAK AVENUE, MT. AIRY 27030 \\
\hline 762 & $E$ & $\begin{array}{l}\text { CHARLES DULIN, } 14679 \text { HOPE WILL CHURCH ROAD, } \\
\text { MIDLAND } 28107\end{array}$ \\
\hline 748 & $E$ & DON DYSART, 933 KELLY ROAD, CAROLEEN 28019 \\
\hline
\end{tabular}

725 E JAMES EATON, 410 LINVILLE STREET, KERNERSVILLE 27284

234 E DAVID ELLIS, 132 BEN CARPENTER ROAD, CHERRYIILLE 28021

235 E C. D. ESSEX, 2080 WELFARE ROAD, WINSTON-SALEM 27127

481 E JOHN IVERY FLOWERS, 1055 DOGWOOD DRIVE, GASTONIA 28056

754 E PATTY FORSTER, 4929 PURNELL ROAD, WAKE FOREST 27587

FROG FLAT FARM, DON EDWARDS, 3108 OLD BEULAH ROAD, SELMA 27576

\section{5}


Table 1. HATCHERIES, DEALERS, AND INDEPENDENT FLOCKS PARTICIPATING IN

THE NATIONAL POULTRY IMPROVEMENT PLAN

WATERFOWL, EXHIBITION POULTRY AND GAME BIRDS

\begin{tabular}{lllcc}
\hline & & HATCHING & PRODUCTS CLASSIFIED \\
APP. & SUB- & PARTICIPANTS NAME AND ADDRESS & EGG & U.S. PULLORUM-TYPHOID \\
NO. & PART & CAPACITY & CLEAN & CLASSIFICATIONS FOR \\
\end{tabular}

55 - NORTH CAROLINA

\begin{tabular}{|c|c|c|}
\hline 366 & $E$ & $\begin{array}{l}\text { JAMES GRAVELY, } 714 \text { DEPOT STREET, MOUNT AIRY } \\
27030\end{array}$ \\
\hline & $E$ & $\begin{array}{l}\text { H \& C QUAIL FARM, } 1580 \text { BOONE GAP ROAD, BOOMER } \\
28606\end{array}$ \\
\hline & E & $\begin{array}{l}\text { DAVID HAGER, } 165 \text { CENTENARY CHURCH ROAD, MT. } \\
\text { ULLA } 28125\end{array}$ \\
\hline & $E$ & $\begin{array}{l}\text { CHUCK HAGLER, } 7929 \text { WILL HILL ROAD, MINT HILL } \\
28227\end{array}$ \\
\hline & E & $\begin{array}{l}\text { JAMES T, HAMILTON, JR, } 613 \text { MAUNEY ROAD, } \\
\text { STANLEY } 28164\end{array}$ \\
\hline & E & $\begin{array}{l}\text { HENRY HARBOR, } 223 \text { EDWARD DRIVE, MOUNT AIRY } \\
27030\end{array}$ \\
\hline & $\mathrm{E}$ & $\begin{array}{l}\text { JOE HARRINGTON, } 1076 \text { PINNACLE DRIVE, } \\
\text { WAYNESVILLE } 28786\end{array}$ \\
\hline & $E$ & $\begin{array}{l}\text { BENNY HARTMAN, } 142 \text { HARTMAN LANE, MOCKSVILLE } \\
27028\end{array}$ \\
\hline & E & $\begin{array}{l}\text { DONALD HARTMAN, } 7868 \text { BELEWS CREEK ROAD, } \\
\text { BELEWS CREEK } 27009\end{array}$ \\
\hline & $E$ & $\begin{array}{l}\text { ZACH \& JACOB HARTMAN, } 434 \text { VOGLER ROAD, } \\
\text { ADVANCE } 27006\end{array}$ \\
\hline & E & STEVE HELMS, 5320 DUDLEY ROAD, MONROE 28112 \\
\hline & $E$ & $\begin{array}{l}\text { CHARLIE HILDEBRAN, } 97 \text { LITTLE BRIDGE ROAD, } \\
\text { TAYLORSVILLE } 28681\end{array}$ \\
\hline & $E$ & $\begin{array}{l}\text { GENE HOOVER, } 207 \text { EDDINGER ROAD, THOMASVILLE } \\
27360\end{array}$ \\
\hline & $E$ & $\begin{array}{l}\text { BILLL HURT, } 130 \text { OAK FOREST DRIVE, STATESVILLE } \\
28677\end{array}$ \\
\hline
\end{tabular}

721 E CHARLES HUSBAND, 2615 STUTTS ROAD, ASHEBORO 27203

741 E KENNETH JARRELL, 216 CRITZ STREET, TOAST 27049

592 E J.B. JAYNES, 1330 REMBRANT CIRCLE, CHARLOTTE 28211

735 E MAUREEN JOHNSON, 8377 STONEY POINT ROAD, FAYETTEVILLE 28301

768 E BERNICE JOHNSON, 724 GEORGE CHANDLER ROAD, MT. AIRY 27030

\begin{tabular}{|c|c|c|}
\hline 742 & $E$ & $\begin{array}{l}\text { DALY KENNEDY, } 697 \text { CASWELL STATION ROAD, } \\
\text { KINSTON } 28501\end{array}$ \\
\hline 659 & $E$ & $\begin{array}{l}\text { C. D. KIRKMAN, } 1830 \text { ROSSWOOD ROAD, PLEASANT } \\
\text { GARDEN } 27319\end{array}$ \\
\hline 734 & $E$ & $\begin{array}{l}\text { JOHNNY LEONARD, } 2377 \text { BENJAMIN STREET, } \\
\text { MORGANTON } 28055\end{array}$ \\
\hline 486 & $E$ & $\begin{array}{l}\text { NEIL R. MAHAFFY, } 1381 \text { OLD THOMASVILLE ROAD, } \\
\text { WINSTON-SALEM } 27107\end{array}$ \\
\hline 595 & $E$ & $\begin{array}{l}\text { JOHN MARSHALL, } 8743 \text { HIGHWAY } 150 \text { SOUTH, } \\
\text { LINWOOD } 27299\end{array}$ \\
\hline
\end{tabular}

W138, W198

Z41

W115, W187, W28, W88, W89

W17, W170, W18, W223, W596

W121, W23, W38, W53, W54, W94

W52, W69, W91, Z28

W395, W397, W477, W559

W28, W50, W69, W91

R77, W11, W278, W28, W301, _. W357, W48

W104, W23, W4, W53, W54, W568, W578, W7

N55.

R15, R179, R222, R94, W1, W13,. W132, W28, W291, W38, W415, W599, W65

W56, W7

N28, N33, N44, N6, N63, R45, R82, R83, W13, W132, W141, W274, W276, W387, W578, W69, W88

W121, W57

W25, W28, W364

N106, N131, N32, N43, N44, N46, N65, N87, N90

Z108, Z19, Z233, Z283, Z4

W121, W129, W17, W179, W198, W253, W270, W297. W45, W52, W570, W588, W69, W74. W80, W86, W88

N18

W18

Z1, Z107, Z141, Z172, Z173, Z3, $\mathrm{Z} 30, \mathrm{Z} 48, \mathrm{Z} 60, \mathrm{Z72}, \mathrm{Z74}, \mathrm{Z} 88$

W29, W50, W51, W52, W76

R118, R195, R196, R214, R22, R222, R25, R34, R56, R74, R89. 
Table 1. HATCHERIES, DEALERS. AND INDEPENDENT FLOCKS PARTICIPATING IN

THE NATIONAL POULTRY IMPROVEMENT PLAN

WATERFOWL, EXHIBITION POULTRY AND GAME BIRDS

$\begin{array}{ll}\text { APP. SUB- } & \text { PARTICIPANTS NAME AND ADDRESS } \\ \text { NO. PART } & \end{array}$

$\begin{array}{cc}\text { HATCHING } & \text { PRODUCTS CLASSIFIED } \\ \text { EGG } & \text { U.S. PULLORUM-TYPHOID } \\ \text { CAPACITY } & \text { CLEAN }\end{array}$

ADDITIONAL

CLASSIFICATIONS FOR WHICH PRODUCT QUALIFIED

55 - NORTH CAROLINA $\begin{array}{ll}728 \text { E } & \text { BENJAMIN T. MATTHEWS, JR., } 863 \text { NEW BETHEL } \\ & \text { CHURCH ROAD, HENDERSON } 27536\end{array}$

743

RICHARD SCHOCK, 3036 WOODRUFF ROAD, BOONVILLE 27011

DAVID MCCOY, 128 WILLS HELM ROAD, MONROE 28110

JOHN MCCRAW, 1436 ARMSTRONG FORD ROAD, BELMONT 28012

RONNIE MCCRAW, 1436 ARMSTRONG FORD ROAD, BELMONT 28012

BRYAN MILLER, 1555 WILDCAT ROAD, DEEP GAP 28618

$E$

E

E BURLINGTON 27215

27030 28655

E JOENEY

E

$E$

PARKER QUAIL FARM, GEORGE V. PARKER, 768 MOORES LANE, LUMBERTON 28358

E LARRY PARSONS, 2761 HENDERSON MILL ROAD, MORGANTON 28655

E KEITH \& KATHY PROCTOR, 4840 ANTHONY ROAD, KINGS MOUNTAIN 28086

$E$ 28164

$E$

ROBIN'S NEST, 49 EPPS-MARTIN ROAD, ROXBORO 27573

E SEVEN OAKS GAME FARM, 7823 MASONBORO SOUND ROAD, WILMINGTON 28407-2672
W169, W34, W573

R15, R225, R247, R258, R42, R7. R85, W120, W126, W127, W159 W43, W527, W609, W95, X100. $X 12, X 136, X 137, X 18, X 31, X 6$, $X 99, Y 14, Y 35, Y 4, Y 5, Y 64, Y 65$ $Y 72, Z 4$

$\mathrm{R}$

W142, W151, W3, W4, W5, W88.

W142, W151, W3, W4, W5, W88.

N193, R26, R30, R62, W252, W271, W297, W363, W508, W72, W86

R247, R248, R97, W4, W7

W115, W187. W35

W28, W50, W52, W88

R27, R28

Z101, Z108, Z128, Z130, Z137. Z139, Z15, Z179, Z18, Z19, Z234, $\mathrm{Z} 270, \mathrm{Z} 274, \mathrm{Z} 288$

Z101, Z108, Z128, Z140, Z19, Z28, Z283, Z57

R20, R42, R93, W113, W130, W208, W26, W28, W30, W81

Z43, Z93

W115, W128, W20

W28, W50, W88.

W25. W26.

R145, R146, R19, R196, R203. R214, R222, R30, R53, R7, R71. R89, W100, W129, W176, W190, W20, W224, W25, W26, W363, W469, W74, W81, W86

R19, R208, R22, R237, R25, R3, R60, R88, W166, W21, W440

N20, N34, N65, N94, N98, R1, R100, R105, R109, R119, R120。 R122, R124, R126, R139, R140. R15, R153, R16, R160, R169, R172, R177, R179, R19, R192, R20, R201, R21, R22, R222, R30. R33, R45, R50, R52, R53, R54, R55, R56, R57, R60, R70, R72.

R76, R87, R88, R91, R92, R93. R94, W100, W102, W106, W11. 
Table 1. HATCHERIES, DEALERS, AND INDEPENDENT FLOCKS PARTICIPATING IN

THE NATIONAL POULTRY IMPROVEMENT PLAN

WATERFOWL, EXHIBITION POULTRY AND GAME BIRDS

\begin{tabular}{|c|c|c|c|c|c|}
\hline $\begin{array}{l}\text { APP. } \\
\text { NO. }\end{array}$ & $\begin{array}{l}\text { SUB- } \\
\text { PART }\end{array}$ & PARTICIPANTS NAME AND ADDRESS & $\begin{array}{l}\text { HATCHING } \\
\text { EGG } \\
\text { CAPACITY }\end{array}$ & $\begin{array}{c}\text { PRODUCTS CLASSIFIED } \\
\text { U.S. PULLORUM-TYPHOID } \\
\text { CLEAN }\end{array}$ & $\begin{array}{c}\text { ADDITIONAL } \\
\text { CLASSIFICATIONS FOR } \\
\text { WHICH PRODUCT QUALIFIED }\end{array}$ \\
\hline
\end{tabular}

55 - NORTH CAROLINA

\begin{tabular}{|c|c|c|}
\hline 770 & $E$ & $\begin{array}{l}\text { CNP FARM, PAUL SEWPAUL, } 13135 \text { DUNN ROAD, } \\
\text { GODWIN } 28344\end{array}$ \\
\hline 625 & E & $\begin{array}{l}\text { SHADY KNOLL GAME BIRD FARM, } 3642 \text { SHADY KNOLL } \\
\text { DRIVE, ASHEBORO } 27203\end{array}$ \\
\hline 771 & $E$ & $\begin{array}{l}\text { SHORESH NATURAL POULTRY \& CAPRINE, } 75 \text { BLOOM } \\
\text { COTTAGE PLACE, FRANKLIN } 28734\end{array}$ \\
\hline 495 & $E$ & TOMMY SHORT, 3215 GOBLE DRIVE, MAIDEN 28650 \\
\hline 321 & $E$ & $\begin{array}{l}\text { JERRY H. SHOUSE, } 268 \text { WILDWOOD LANE, WINSTON- } \\
\text { SALEM } 27107\end{array}$ \\
\hline 749 & $E$ & NICK SMITH, P.O. BOX 87, CAROLEEN 28019 \\
\hline 322 & $E$ & $\begin{array}{l}\text { B. L. SPARKS, } 2856 \text { FRATERNITY CHURCH ROAD, } \\
\text { WINSTON-SALEM } 27127\end{array}$ \\
\hline 634 & $E$ & $\begin{array}{l}\text { DAVID SWANGER, } 4105 \text { BRIDGETT COURT, GRAHAM } \\
27253\end{array}$ \\
\hline 690 & $E$ & CONNIE W. TART, JR., P.O. BOX 875, PRINCETON 27569 \\
\hline
\end{tabular}

W113, W115, W12, W121, $W 128, W 129, W 13, W 138, W 14$ W140, W145, W146, W15, W16. W164, W166, W167, W168, $W 169, W 17, W 170, W 173$ W175, W176, W179, W18, W180, W187, W189, W198, W206, W208, W209, W223 W24, W243, W244, W247. W248, W25, W251, W253. W259, W26, W262, W265, W274, W28, W290, W294, W3, W31, W313, W33, W331, W332, W333, W334, W335, W337. W36, W370, W4, W416, W424. W435, W439, W440, W453, W457, W466, W468, W469, W501, W504, W507, W509, W510, W518, W542, W544 W55, W554, W569, W573 W574, W578, W58, W588, W60, W7, W74, W75, W89, W95, W96, W98, X1, X10, X103, X11, X12, $X 131, X 134, X 135, X 137, X 14$, $\times 140 \times 143, \times 145, \times 147, \times 16$ $\times 17, \times 2, \times 20, \times 21, \times 22, \times 23$, $\times 24, \times 25, \times 26, \times 27, \times 28, \times 3$, $\times 31, \times 32, \times 34, \times 38, \times 4, \times 40$ $X 41, X 48, X 49, X 50, X 6, X 65$, $X 68, X 8, X 85, X 9, X 96, X 97, Y 1$ $Y 10, Y 13, Y 14, Y 2, Y 21, Y 22$. $Y 25, Y 4, Y 5, Y 6, Y 7, Y 9, Z 1$, Z101, Z107, Z108, Z12, Z120 Z141, Z142, Z152, Z170, Z172, Z173, Z176, Z182, Z24, Z25, Z26, Z27, Z28, Z30, Z33, Z37, Z38, $Z 39, Z 40, Z 41, Z 42, Z 44, Z 45$, Z46, Z47, Z48, Z5, Z51, Z57, Z59, $\mathrm{Z} 600, \mathrm{Z72}, \mathrm{Z73}, \mathrm{Z78}, \mathrm{Z} 86, \mathrm{Z} 88$, Z89, Z9, Z90, Z93

$\mathrm{R}, \mathrm{X}$

Z43

R14, R146, R213, R225, R247, R42. R94

R175, W103, W105, W23, W281, W299, W300, W38, W56, W67. W76

W216, W23, W301

R12, R229, R239, R247, R7, W102, W121, W13, W140, W17. W18, W313, W518, W7, W74 W75, W86

W39, W4

N18, W30

W100, W129, W141, W142, W149, W166, W176, W200, W248, W25, W26, W262, W275, 
Table 1. HATCHERIES, DEALERS, AND INDEPENDENT FLOCKS PARTICIPATING IN

THE NATIONAL POULTRY IMPROVEMENT PLAN

WATERFOWL. EXHIBITION POULTRY AND GAME BIROS

HATCHING

EGG

CAPACITY
PRODUCTS CLASSIFIED

U.S. PULLORUM-TYPHOID

CLEAN
ADDITIONAL

CLASSIFICATIONS FOR

WHICH PRODUCT QUALIFIED

55 - NORTH CAROLINA

DARRELL THOMAS, 6419 HOWEY BOTTOMS ROAD. INDIAN TRAIL 28079

715 E FARRELL THORNBURG, 3330 LASSITER MILL ROAD, ASHBORO 27205

730 E TW FRIENDLY FOWL AND FEATHERS, 2755 TABERNACLE CHURCH ROAD, PLEASANT GARDEN 27313

632 E KARL F. URSHEL, 1048 NEW LEBANON CHURCH ROAD REIDSVILLE 27320

731 E LENNY WAHL, 1415 AURORA ROAD, ERNUL 28527

397 E SAMMY WESTMORELAND, BOX 585, CORNELIUS 28031

691 E WIGHTMAN BANTAMS, 5409 B OLD STAGE ROAD, RALEIGH 27603

750 E JONATHAN WOOTEN, 5854 MARY LANE, CLEMMONS 27012

732 E RON WORLEY, 50 WORLEY BIRD LANE, SWANNONOA 28778
W28, W29, W313, W368, W44. W48, W50, W52, W569, W570, W573, W579, W58, W590, W600, W69, W81, W88, W89, W98

W25, W26, W48, W568.

W17

W104, W12, W121, W128, W18_

W271, W297, W365, W526, W527. W86

N104, N17, N207, W132, W511, W554

W198, W274, W28, W568, W88_ W114, W142, W151, W25, W26, W276, W28, W29, W363, W48, W52, W58, W69, W88

W26, W3, W6, W60, W81

227

45 - NORTH DAKOTA

WILLIAM J. DAVIS

NORTH DAKOTA DEPARTMENT OF AGRICULTURE

600 EAST BLVD.

6TH FLOOR

BISMARCK, ND 58505

Phone: (701)328-4763

Fax: (701)328-4567

Hatcheries

\begin{tabular}{|c|c|c|}
\hline 110 & $E$ & $\begin{array}{l}\text { DAKOTA RANCHES, } 9525 \text { COUNTY ROAD 22, FAIRMONT } \\
58030\end{array}$ \\
\hline 107 & $E$ & $\begin{array}{l}\text { GREAT PLAINS PHEASANT FARM, RR 1, BOX } 2 \text {. } \\
\text { CARRINGTON } 58421\end{array}$ \\
\hline 113 & $E$ & $\begin{array}{l}\text { LOREN KITTELSON, } 8777 \text { 39TH STREET SE, } \\
\text { JAMESTOWN } 58401\end{array}$ \\
\hline 38 & $E$ & $\begin{array}{l}\text { KLOSES CANADIANS PLUS, } 4071 \text { 97TH AVENUE SE, } \\
\text { ECKELSON } 58481\end{array}$ \\
\hline
\end{tabular}

15 E MAGIC CITY HATCHERY, P.O. BOX 1771, MINOT 58702

111 E SMITH FARMS POULTRY, 14604 34TH STREET SE, WHEATLAND 58079

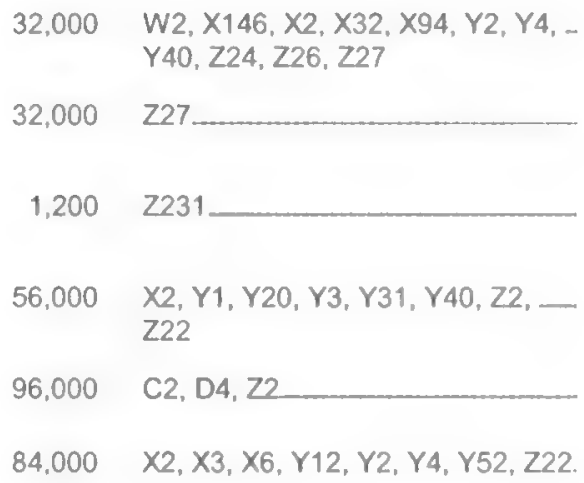


HATCHING

EGG

CAPACITY
PRODUCTS CLASSIFIED

U.S. PULLORUM-TYPHOID CLEAN
ADDITIONAL CLASSIFICATIONS FOR WHICH PRODUCT QUALIFIED

\section{5 - NORTH DAKOTA}

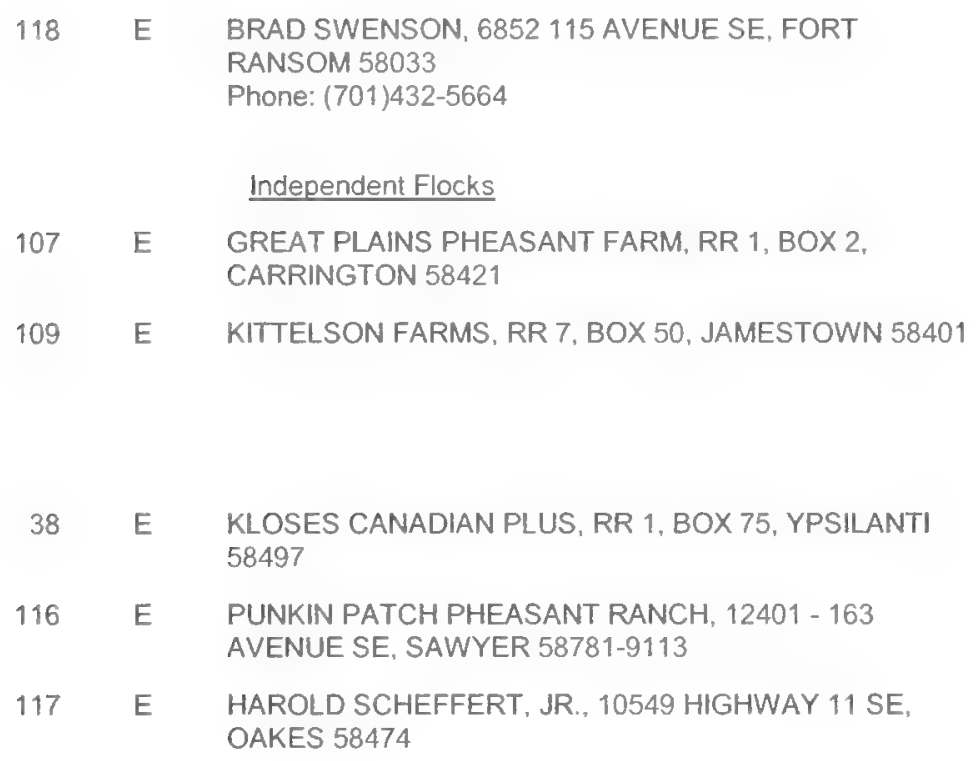

9 E MT. HEALTHY HATCHERY, R. O'HARA, 9839 WINTON ROAD, CINCINNATI 45231

13 E RIDGWAY HATCHERY, INC. LARUE 43332

244 E S \& C BIRD AND ANIMAL FARM, 2758 FOX ROAD, FT. RECOVERY 45846

163 E WINDY RIDGE PHEASANT FARM, 6063 STOUGH ROAD, TIRO 44887

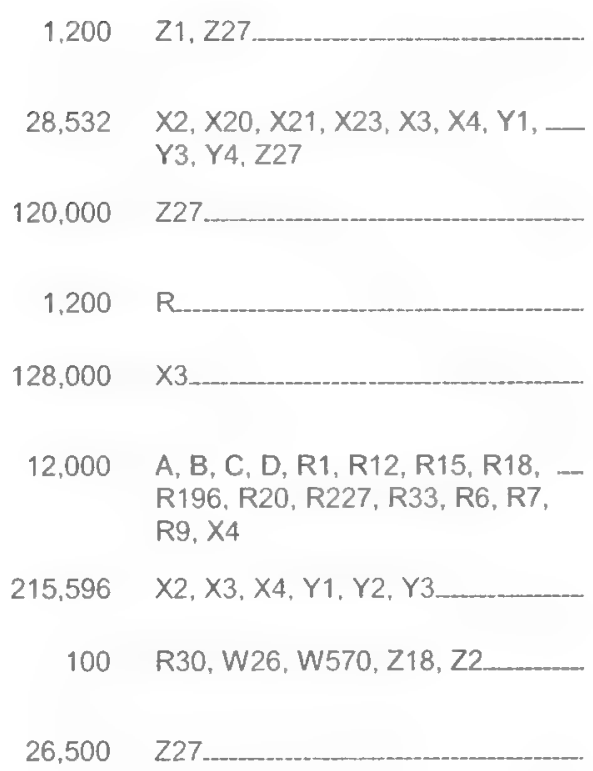

$R 89, X 4, X 48, X 49, X 50, X 62, X 7$ $\mathrm{X} 8, \mathrm{Y} 16, \mathrm{Y} 20, \mathrm{Y} 26, \mathrm{Y} 28, \mathrm{Y} 31$, Y32, Y40, Y41, Y5, Y7, Y9, Z140, Z22, Z24, Z25, Z26, Z28, Z32, Z49, Z53

$X_{2}, Y_{1}, Y_{2} 0, Y_{3}, Y_{31}, Y_{40}, Z 2, \ldots$ $\mathrm{Z22}$

Z

$\mathrm{Z1}, \mathrm{Z48}, \mathrm{Z} 60$

\section{LESLIE STEWART}

OHIO POULTRY ASSOCIATION

5939 SHARON WOODS BLVD.

COLUMBUS, OH 43229

Phone: (614)882-6111

Fax: $\quad$ (614)882-9444

Dealers 
Table 1. HATCHERIES, DEALERS, AND INDEPENDENT FLOCKS PARTICIPATING IN

THE NATIONAL POULTRY IMPROVEMENT PLAN

WATERFOWL, EXHIBITION POULTRY AND GAME BIRDS

\begin{tabular}{llccc}
\hline & & HATCHING & PRODUCTS CLASSIFIED & ADDITIONAL \\
APP. SUB- PARTICIPANTS NAME AND ADDRESS & CAPACITY & C.S. PULLORUM-TYPHOID & CLEAN \\
NO. PART & & & \\
\hline & $31-\mathrm{OHIO}$
\end{tabular}

DARBY CREEK AG. ENTERPRISES, 110 SOUTH MILL STREET, MILFORD CENTER 44887

Independent Flocks

252 E CLELL AGLER, P.O. BOX 682, GROVE CITY 43123

235 E TOM ARNOLD, BOX 433, TWP. RU 67, KILLBUCK 44637

MATT BARMAN, 136 PARK ROAD, FT. RECOVERY 45846

BERAN ACRES GAME BIRDS, MARK BERAN, 1060 POE ROAD, BUCYRUS 44820

Phone: (419)562-2492

E ROLLAND A. BLOCKBERGER, 9299 STATE ROUTE 66 NORTH, DELPHOS 45833
BOWMAN'S POULTRY, WILLIAM BOWMAN, 5626 RUDYARD ROAD, SYLVANIA 43560

E

BROWN \& MAGER, 2241 DEAN ROAD, BETHEL 45106

BOB COLE, 832 BLACKS ROAD, HEBRON 43025

\section{4}

C $A, B, C, D, E, H$

W.

N106, N28, N83, N99, Y16

W123, W142, W175, W187, W245, W276, W50, W545, Z28. $\mathrm{Z72}$

$\mathrm{Z} 48$

W527

B, C, D, R10, R105, R11, R114, R117, R12, R124, R13, R15, R16 R169, R175, R181, R183, R19, $R 195, R 2, R 21, R 22, R 227, R 23$, R25, R3, R30, R34, R35, R38, R39, R4, R40, R42, R44, R45, R46, R47, R49, R51, R52, R53, R6, R71, R75, R81, R85, R87, R9, R90, R91, R92, R93, R96. W11, W114, W115, W119, W12, W120, W121, W128, W134。 W138, W15, W160, W17, W18. W187, W19, W198, W20, W23. W232, W24, W25, W26, W27. W270, W282, W293, W3, W301, W302, W33, W357, W38, W4, W48, W5, W54, W55, W568 W57, W576, W58, W6, W7, W72, W8, W81, W86, W88, W9, W94

W11, W12

W207, W216, W35

W207, W216, W270, W282, W293, W3, W301, W302, W33, W35, W357, W38, W4, W48, W5 W54, W55, W568, W57, W576 W58, W6, W7, W72, W8, W81, W86, W88, W9, W94

R20, W102, W17, W18, W20, W208, W74

$A, B, C, D, E, H$

R18, R42, R60, R94, R96, W13, W131. W132, W16, W30, W370, W440, W477, W570

Z11. Z2

N100, N101, N2, N28, N32, N34, N62, N63, N65, N98, N99

N. N112, N25, N28, N44 
Table 1. HATCHERIES, DEALERS, AND INDEPENDENT FLOCKS PARTICIPATING IN

THE NATIONAL POULTRY IMPROVEMENT PLAN

WATERFOWL, EXHIBITION POULTRY AND GAME BIRDS

\begin{tabular}{|c|c|c|c|c|c|}
\hline $\begin{array}{l}\text { APP. } \\
\text { NO. }\end{array}$ & $\begin{array}{l}\text { SUB- } \\
\text { PART }\end{array}$ & PARTICIPANTS NAME AND ADDRESS & $\begin{array}{l}\text { HATCHING } \\
\text { EGG } \\
\text { CAPACITY }\end{array}$ & $\begin{array}{l}\text { PRODUCTS CLASSIFIED } \\
\text { U.S. PULLORUM-TYPHOID } \\
\text { CLEAN }\end{array}$ & $\begin{array}{c}\text { ADDITIONAL } \\
\text { CLASSIFICATIONS FOR } \\
\text { WHICH PRODUCT QUALIFIED }\end{array}$ \\
\hline
\end{tabular}

$$
31 \text { - OHIO }
$$
255 E $\begin{aligned} & \text { RICHARD B. FRYER \& COMPANY, } 16575 \text { JONES ROAD, } \\ & \text { VENEDOCIA } 45894\end{aligned}$

248 E G \& S GAME BIRDS, T. SANDERS, 07599 STOVER ROAD, DEFIANCE 43512

257 E ALTON \& BETTY GROVES, 1530 TIFFIN ROAD. BUCYRUS 44820 Phone: (419)562-6088

284 E HARLEY HAMRICK, 9630 NORTH MORRISON ROAD, DRESDEN 43821

271 E RON HUERKAMP, 5991 GERMANTOWN-LIBERTY ROAD, GERMANTOWN 45327

Phone: (937)855-3131

277 E ROSE ISGRIGG, 59 LONGVIEW HEIGHTS ROAD, ATHENS 45701

270 E JEFFS QUAIL FARM, J. UHRIG, 7251 BLAINE HIGHWAY, CHILLICOTHE 45601

249 E RAY JOHNSON, RT. 3, BOX 139, ALBANY 45710

240 E LARRY D. KELLY, 10132 ASHVILLE PIKE, LOCKBOURNE 43137

Phone: 6149834811

THE L-BAR RANCH'S TURKEY FARM \& HATCHERY, 34310 SR 260. RINARD MILLS 45774

CRAIG MIDDAUGH, 3075 MAINSVILLE RD., JUNCTION CITY 43748

258
KIM MITCHELL, 2165 ALEX WHITE ROAD, JACKSON 45640

Phone: (614)820-4507

\section{$\mathrm{Z2}, \mathrm{Z27}, \mathrm{Z43}$}

W102, W13, W570, Z118, Z44, $\mathrm{Z} 60$

W112, W131, W304, W370 W442, W548, W594, W609, W96

N. N148, N181, N182, N224, N240, N241, N41, N43, N90

B, R146, R222, R26, R71, R95, R96. Z231

Z180.

N28, N32, N44, N63, W83

N

R20, R40, R95, W1, W100, W105, W111, W126, W13, W132, W26, W30, W330, W352, W527, W7, W96, Z, Z1, Z15, Z204. Z211, Z213, Z220, Z221. Z9

N. N150, N151, N43, N44, N63, N65, N98

N90, R10, R100, R11, R116, R119, R12, R120, R122, R124, R139, R140, R145, R146, R15, R16, R168, R17, R171, R172. R176, R177, R178, R19, R192, R196, R20, R201, R203, R21. R211, R213, R219, R222. R227. R244, R245, R247, R248, R30, R33, R35, R37, R45, R47, R50, R52, R53, R54, R57, R6, R60, R62, R7, R70, R71, R73, R75, R76, R77, R81, R83, R84, R85, R86, R87, R88, R89, R9, R92, R93, R96, W102, W114, W13. W139, W142, W17, W18, W184. W198, W20. W208, W215, W24, W247, W248, W25, W26, W265, W290, W309, W313, W33, W380, W435, W518, W519 W52, W560, W57, W570, W573, W595, W60, W600, W74, W75, $W 98, \times 131, \times 135, \times 18, \times 24, \times 25$, $X 32, X 4, X 46, X 68, X 9, X 96, Y 1$, Y5, Y6, Y9, Z1, Z102, Z110, Z111, Z118, Z129, Z138, Z142, Z15, Z182, Z19, Z20, Z202, Z204, Z205, Z206, Z209, Z21, Z211. Z22, Z227, Z24, Z247, Z26. Z27. Z28, Z3, Z41, Z43, Z44, Z45, Z51, Z53, Z57, Z60, Z62, Z72, Z73, Z88, Z9, Z93 
Table 1. HATCHERIES, DEALERS, AND INDEPENDENT FLOCKS PARTICIPATING IN

THE NATIONAL POULTRY IMPROVEMENT PLAN

WATERFOWL, EXHIBITION POULTRY AND GAME BIRDS

\begin{tabular}{|c|c|c|c|c|c|}
\hline $\begin{array}{l}\text { APP. } \\
\text { NO. }\end{array}$ & $\begin{array}{l}\text { SUB- } \\
\text { PART }\end{array}$ & PARTICIPANTS NAME AND ADDRESS & $\begin{array}{l}\text { HATCHING } \\
\text { EGG } \\
\text { CAPACITY }\end{array}$ & $\begin{array}{l}\text { PRODUCTS CLASSIFIED } \\
\text { U.S. PULLORUM-TYPHOID } \\
\text { CLEAN }\end{array}$ & $\begin{array}{c}\text { ADDITIONAL } \\
\text { CLASSIFICATIONS FOR } \\
\text { WHICH PRODUCT QUALIFIED }\end{array}$ \\
\hline
\end{tabular}

\begin{tabular}{|c|c|c|}
\hline 219 & $E$ & $\begin{array}{l}\text { EDGAR K. MONGOLD, } 48 \text { PRIVATE DRIVE } 830 \text {, IRONTON } \\
45638\end{array}$ \\
\hline & $E$ & $\begin{array}{l}\text { TOM MOORE, } 602 \text { WOOSTER ROAD, MT. VERNON } \\
43050\end{array}$ \\
\hline & $E$ & DENNIS MYERS, 4225 SYLVAN ROAD, WOOSTER 44691 \\
\hline & $E$ & $\begin{array}{l}\text { CHARLES NUGENT, } 72474 \text { MICA ROAD, KIMBOLTON } \\
43749\end{array}$ \\
\hline & $E$ & $\begin{array}{l}\text { RANDALL PERRY, } 428 \text { SECOND STREET, MARIETTA } \\
45750\end{array}$ \\
\hline
\end{tabular}

289 E PATTY PICARD, 27101 TURNPIKE ROAD 47,
COSHOCTON 43812

Phone: (740)622-1440

222 E RAY POWERS, 1073 LONG ROAD, XENIA 45385

256 E LINDA QUINN, 2806 WOODBURY ROAD, BELLVILLE

Phone: (419)886-3872

262 E KENNETH E. RICER, 2014 WRIGHT ROAD, AKRON 44320

Phone: (330)864-4276

\begin{tabular}{|c|c|c|}
\hline 291 & $E$ & $\begin{array}{l}\text { CAROL RINEHART, } 18211 \text { COUNTY ROAD } 297, \\
\text { COSHOCTON } 43812\end{array}$ \\
\hline 253 & $E$ & $\begin{array}{l}\text { RON RISNER, } 6310 \text { SHAWBAKER ROAD, WINCHESTER } \\
45697\end{array}$ \\
\hline 210 & $E$ & $\begin{array}{l}\text { GEORGE SHREFFLER, } 1192 \text { NORTH COUNTY ROAD } \\
42, \text { BOX } 83 \text { GIBSONBURG } 43431\end{array}$ \\
\hline 285 & $E$ & $\begin{array}{l}\text { GORDON SMITH, } 13391 \text { WEST TOUSSAINT NORTH, OAK } \\
\text { HARBOR } 43449\end{array}$ \\
\hline
\end{tabular}

ROY STACY, 31223 RT. 30, HANOVERTON 44423

STAUFFER POULTRY FARM, 8716 EMERSON ROAD, APPLECREEK 44606
W120, W128, W13, W247 W308, W88

Z, Z102, Z108, Z137, Z19, Z28, ... Z 257

$\mathrm{B}$

W. Z65, Z66, Z8

R13, R20, R220, R83, R95, W1, W106, W13, W251, W286, W415, W588, Z107, Z118, Z123, Z138, Z153, Z19, Z242, Z247 Z27, Z3, Z4, Z43, Z47, Z57, Z93

R12, R185, R39, W104, W282, W570. W647

N.

Z108, Z110, Z111, Z118, Z131, ... Z137, Z139, Z19, Z53, Z78

$W 13, W 132, W 415, X 146, X 20$, X28, X58, X60, X61, X8, Y22, Y27, Y4, Y51, Y8, Z123, Z124, Z27, Z28, Z49, Z55, Z57

W132, W23, W588

W. Z180, Z27

D, R14, R9, W28, W556, W7, W9.

H10, H5, R10, R104, R109, R110. R111, R114, R12, R139, R146, R169, R19, R192, R195, R203, R206, R21, R22, R227, R23, R3, R34, R37, R38, R4, R42, R45, R51, R54, R60, R64, R71, R72, R81, R82, R83, R87, R9, R90. R91, R93, R95, R97, W34, W35, W578, W7, W8, Z201, Z204 Z230, Z231, Z94

w30, w7

A, D, E, H10, H8, N52, R10, R100, R109, R11, R114, R12, R123, R124, R13, R14, R146, R15, R16, R168, R176, R178. R180, R183, R188, R19, R195. R196, R2, R21, R22, R23, R25 $R 3, R 30, R 34, R 35, R 37, R 38$. R39, R4, R40, R42, R45, R49. R51, R52, R53, R54, R6, R64. R7, R71, R75, R81, R85, R86, R87, R9, R90, R91, R92, R93, W11, W115, W119, W12, W121, W123, W128, W138, W145. W15, W17, W176, W18, W187, W19, W198, W20, W23, W25. W26, W27, W28, W3, W301, W32, W33, W38, W4, W43, W44. 
Table 1. HATCHERIES, DEALERS, AND INDEPENDENT FLOCKS PARTICIPATING IN

THE NATIONAL POULTRY IMPROVEMENT PLAN

WATERFOWL, EXHIBITION POULTRY AND GAME BIRDS

\begin{tabular}{|c|c|c|c|c|c|}
\hline $\begin{array}{l}\text { APP. } \\
\text { NO. }\end{array}$ & $\begin{array}{l}\text { SUB- } \\
\text { PART }\end{array}$ & PARTICIPANTS NAME AND ADDRESS & $\begin{array}{l}\text { HATCHING } \\
\text { EGG } \\
\text { CAPACITY }\end{array}$ & $\begin{array}{l}\text { PRODUCTS CLASSIFIED } \\
\text { U.S. PULLORUM-TYPHOID } \\
\text { CLEAN }\end{array}$ & $\begin{array}{c}\text { ADDITIONAL } \\
\text { CLASSIFICATIONS FOR } \\
\text { WHICH PRODUCT QUALIFIED }\end{array}$ \\
\hline
\end{tabular}

\section{1 - OHIO}

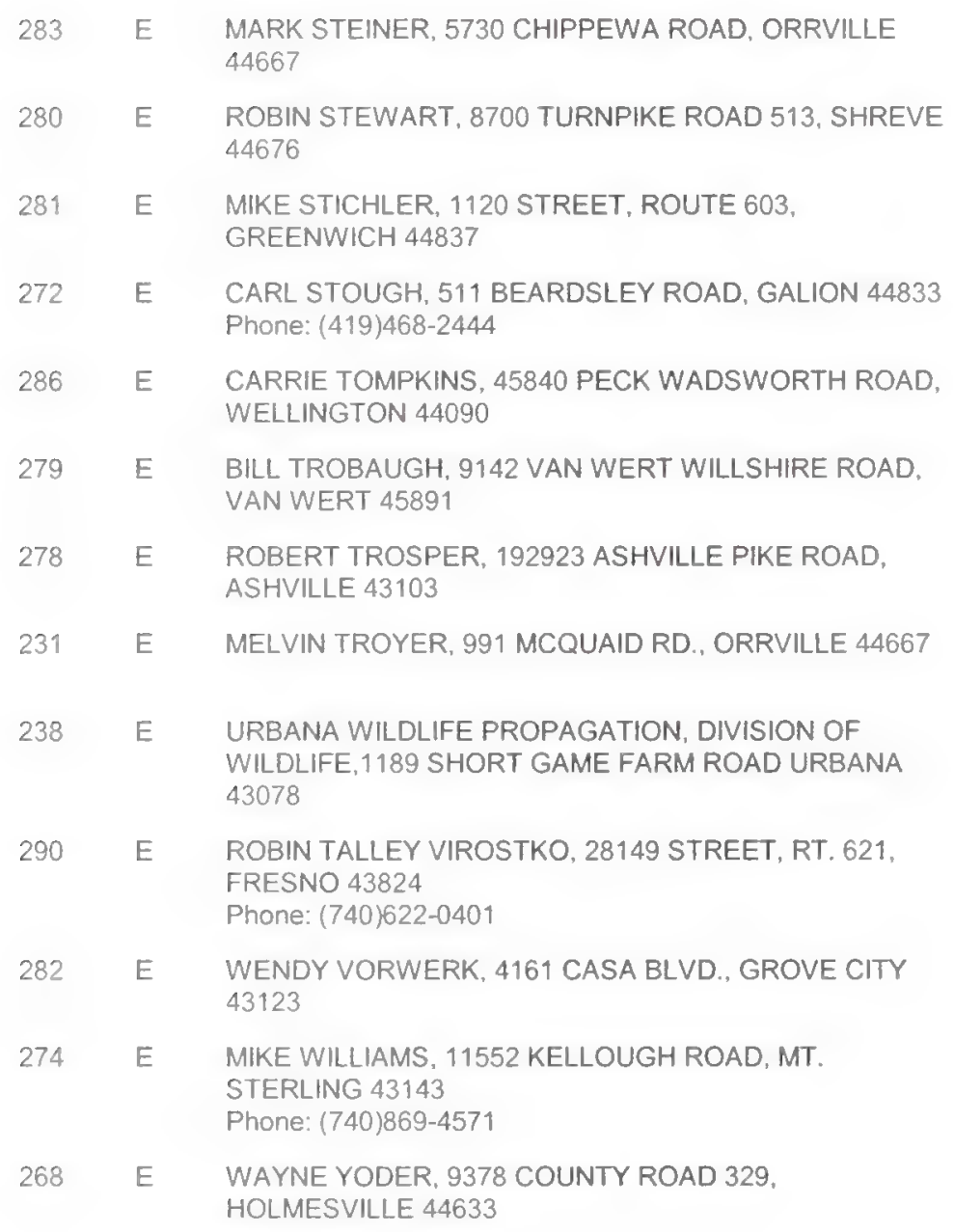

W45, W48, W5, W527, W54 W55, W57, W570, W576, W58, W6, W7, W74, W75, W8, W81. W88, W9, W94

R19, R34, R9, W442

Z110, Z249, Z255, Z285, Z286. Z287. Z62

R42

w

Z205

Z101, Z108, Z131, Z138, Z15, Z19, Z235

N106, N134, N138, N183, N41, N44, N90

N. W198, Z18, Z19, Z26, Z27, Z28, Z49, Z53, Z55, Z9

$\mathrm{Z2}$

W30

Z1, Z107, Z231, Z43

N28, N41

Z. Z183

RALPH DUNCAN

ANIMAL INDUSTRY DIVISION

OKLAHOMA STATE DEPARTMENT OF AGRICULTURE

2800 NORTH LINCOLN BLVD.

OKLAHOMA CITY, OK 73105

Phone: (405)521-3864

Fax: $\quad(405) 522-0756$

\section{Hatcheries}

274 E B \& D GAME FARM, BERT \& DIANE TUMEY, RT 1, BOX 812. HARRAH 73045

2741 E BILL POULTRY FARM, RT. 1, BOX 750, POTEAU 74953
Z120, Z22, Z27, Z283, Z29, Z39, Z41, Z42, Z45, Z88, Z93

360 W231, W301, W357, W359, W360, W361, W363, W364 
Table 1. HATCHERIES, DEALERS, AND INDEPENDENT FLOCKS PARTICIPATINGIN

THE NATIONAL POULTRY IMPROVEMENT PLAN

WATERFOWL, EXHIBITION POULTRY AND GAME BIRDS

\begin{tabular}{llllll}
\hline & & HATCHING & PRODUCTS CLASSIFIED \\
APP. & SUB- & PARTICIPANTS NAME AND ADDRESS & EGG & U.S. PULLORUM-TYPHOID & CLEAN \\
NO. PART & CAPACITY & CLASSIFICATIONS FOR & WHICHPRODUCT QUALIFIED \\
\hline
\end{tabular}

73 - OKLAHOMA $\begin{array}{ll}1222 \text { E } & \text { BRIAR PATCH GAME BIRD FARM, G. HOLT, RT. 2, BOX } \\ & \text { 307-1, CHELSEA 74016 }\end{array}$

2483 E CHAIN FARM, VIRGINIA CHAIN, RT. 1, BOX 64 , GERONIMO 73543

67 E COUNTRY HATCHERY, DENNIS SMITH, RT. 1, BOX 174. WEWOKA 74084

996 E D \& D GAME BIRDS, D. TERRY, 2719 EISENHOUER DRIVE, CHOCTAW 73020

1456 E HAMM'S SPORTMAN OASIS, RT. 4, BOX 82, FREDERICK 73542

1843 E KING'S GAMEBIRDS, INC., 23750 COUNTY ROAD 200, MORRISON 73061

463 E LEWIS GAME BIRD FARM \& HATCHERY, 5013 WOODLAND PARK TERRACE, SPENCER 73084

2126 E MILLER'S GAME BIRD FARM \& HATCHERY, RT. 1, BOX 163, HENNESSEY 73742

1837 E OKLAHOMA FARMS, INC.,(STIGLER, P.O. BOX 119, STIGLER 74462

2637 E RED ROSE HATCHERY/ARLUS \& LINDA WALTERS, RT. 3, BOX 1409, STILWELL 74960

2159 E SPORTSMAN WORLD HATCHERY, RT. 3, BOX 5800, PRYOR 74361

1673 E TENNON QUAIL FARM \& HATCHERY, 4404 WOODLAND PARK TERRACE, SPENCER 73084

2270 E TENNONS QUAIL FARM \& HATCHERY, 4404 WOODLAND PARK TERRACE, SPENCER 73084

Dealers

67 E COUNTRY HATCHERY, DENNIS SMITH, RT, 1, BOX 174, WEWOKA 74884

$1462 \quad E$

FEEDERS SUPPLY, 12 WEST OAK, STILLWELL 74960

Independent Flocks

1698 E 4C GAME FARM, C \& K COULTER, 618 WEST OKLAHOMA, SULPHUR 73086

2612 E MICHAEL ADAIR, 1509 EAST ALLEN ROAD, TAHLEQUAH 74464

2300 E CLIFFORD ADAMS, RT. 1, BOX 134-A, COYLE 73027

2589 E JOE ADAMS, RT. 2, BOX 240-A, MARIETTA 73448

2695 E ADAMS POULTRY, 18120 SE 70TH, NEWALLA 74857

2206 E ROCKIE ADKISSON, 2116 SW 35TH, OKLAHOMA CITY 73119

2512 E RITCHIE \& WILLIAM ADREAN, 11440 SOUTH 26TH WEST AVENUE, TULSA 74066

2512 E RITCHIE \& WILLIAM ADREAN, RT. 3, BOX 245, SAPULPA 74066

2742 E JACKIE ALBERT, 4855 TAMOURA LANE, BEGGS 74421

\begin{tabular}{|c|c|}
\hline 2,000 & $\mathrm{Z} 182, \mathrm{Z183}, \mathrm{Z27}$ \\
\hline 1,500 & $z$ \\
\hline 38,000 & R17, R8, W26, X2, X3, Z22, Z62 \\
\hline 4,400 & $\begin{array}{l}\mathrm{Z} 118, \mathrm{Z} 169, \mathrm{Z22}, \mathrm{Z24}, \mathrm{Z26}, \mathrm{Z27},- \\
\mathrm{Z} 32, \mathrm{Z} 34, \mathrm{Z} 4, \mathrm{Z} 43, \mathrm{Z} \text {, } 57\end{array}$ \\
\hline 18,250 & $z$ \\
\hline 900 & $z$ \\
\hline 39.000 & $\mathrm{Z1}, \mathrm{Z} 43 \ldots$ \\
\hline 88,000 & $Z 1, Z 2, Z 3 \ldots$ \\
\hline $3,849,120$ & C5, R, R508 \\
\hline 6,200 & $\begin{array}{l}\text { Z182, Z201, Z202, Z204, Z205, - } \\
\text { Z206, Z209, Z230 }\end{array}$ \\
\hline 5,200 & Z \\
\hline 8,720 & Z \\
\hline 8,720 & $\mathrm{Z} 1, \mathrm{Z} 43 \ldots$ \\
\hline
\end{tabular}

E\&C R17

$X, Y, Z$

N43, N90

N

N

N34.

R146, R168, R196, R22, R247, R248, R7, W12, W38, W576, W6 N17.

N. N17

N. N17.

N17 


\begin{tabular}{|c|c|c|c|c|c|}
\hline $\begin{array}{l}\text { APP. } \\
\text { NO. }\end{array}$ & $\begin{array}{l}\text { SUB- } \\
\text { PART }\end{array}$ & PARTICIPANTS NAME AND ADDRESS & $\begin{array}{l}\text { HATCHING } \\
\text { EGG } \\
\text { CAPACITY }\end{array}$ & $\begin{array}{c}\text { PRODUCTS CLASSIFIED } \\
\text { U.S. PULLORUM-TYPHOID } \\
\text { CLEAN }\end{array}$ & $\begin{array}{c}\text { ADDITIONAL } \\
\text { CLASSIFICATIONS FOR } \\
\text { WHICH PRODUCT QUALIFIED }\end{array}$ \\
\hline
\end{tabular}

\begin{tabular}{|c|c|c|}
\hline 2494 & $E$ & KEVIN ALEXANDER, RT. 2, BOX 462, COMANCHE 73534 \\
\hline 2207 & $E$ & DALLAS ALLEN, RT. 1, BOX $87 A A$, SALLISAW 74955 \\
\hline 2658 & $E$ & H.R. ALLEN, RT. 1, BOX 92-C. SPIRO 74959 \\
\hline 2037 & $E$ & EDGAR ALLISON, P.O. BOX 128, MANNFORD 74044 \\
\hline 2408 & $E$ & BERNARD ALPERS, P.O. BOX 665, DAVIS 73030 \\
\hline 2638 & $E$ & LARRY ALSUP, 497 SOUTH MUNGLE, ATOKA 74525 \\
\hline 2247 & $E$ & DAVID ANDERSON, RT. 2, BOX 373, MEDFORD 73759 \\
\hline 672 & $E$ & $\begin{array}{l}\text { SHADY ANDERSON, } 6505 \text { SE 59TH, OKLAHOMA CITY } \\
73135\end{array}$ \\
\hline 2185 & $E$ & JASON ANDREWS, 33300 SOUTH 650 ROAD, JAY 74346 \\
\hline 2301 & $E$ & ALVIN ANDREWS, P.O. BOX 708, DRUMRIGHT 74030 \\
\hline 2242 & $E$ & ALLEN ANDREWS, HC 80, BOX 95. ATOKA 74525 \\
\hline 2553 & $E$ & KEITH ANDREWS, RT. 2, BOX 1475, CUSHING 74023 \\
\hline 1967 & $E$ & $\begin{array}{l}\text { HARVEY ANNESLEY, } 12251 \text { CEDAR LANE, NORMAN } \\
73026\end{array}$ \\
\hline 2547 & $\mathrm{E}$ & MARCIA ARANDA, P.O. BOX 22, ARAPAHO 73620 \\
\hline 1642 & $E$ & $\begin{array}{l}\text { MIKE ARDIZZONE/OKIE COMBINE GAME FARM, RT. } 1 \text {, } \\
\text { BOX 226, BLANCHARD } 73010\end{array}$ \\
\hline 2513 & $E$ & TOMMY ARDRY, 202 EAST JONES, HOLLIS 73550 \\
\hline 849 & $E$ & BOB ARMS, RT. 1, BOX 425, SULPHUR 73086 \\
\hline 1939 & $E$ & $\begin{array}{l}\text { CHARLES ARMSTRONG, } 100 \text { KNIGHT DRIVE, DURANT } \\
74701\end{array}$ \\
\hline 2169 & $E$ & JIM ARMSTRONG, RT. 1, BOX 70, LAVERNE 73848 \\
\hline 2707 & $E$ & $\begin{array}{l}\text { JOSHUA ARNOLD, } 495 \text { BOBBY TOMLINSON ROAD, } \\
\text { BENNINGTON } 74723\end{array}$ \\
\hline 1552 & $E$ & PATE ASHFORD, RT, 2, BOX 226, BLANCHARD 73010 \\
\hline 1234 & $E$ & LONNIE ATKERSON, P.O. BOX 641, WARNER 74469 \\
\hline 2004 & $E$ & LAWRENCE AUSTIN, RT. 1, BOX 2725, APACHE 73006 \\
\hline 2157 & $E$ & $\begin{array}{l}\text { JOHN AUTRY, } 7645 \text { SOUTH } 65 T H \text { WEST AVENUE, } \\
\text { TULSA } 74131\end{array}$ \\
\hline 2026 & $E$ & MARVIN BABB, 218 TEXAS, WOODWARD 73801 \\
\hline 2708 & $E$ & DAVID BACKON, 321 WEST FRISCO ROAD, ROFF 74865 \\
\hline 2198 & $E$ & DALE BADDERS, RT. 1, BOX 79, KENEFIC 74748 \\
\hline 2170 & $E$ & $\begin{array}{l}\text { CHARLES BAILEY (RIPLEY COMBINE), P.O. BOX } 121 \text {, } \\
\text { RIPLEY } 74062\end{array}$ \\
\hline 2644 & $E$ & BILLY BAILEY, P.O. BOX 140, SMITHVILLE 74957 \\
\hline
\end{tabular}

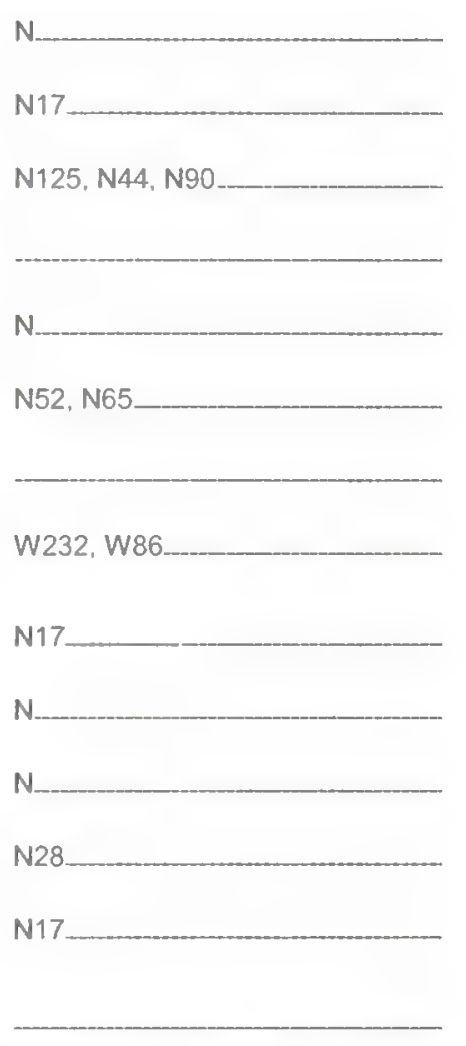

N28, N32, N41, N44, N90.

N.

N.

W108, W23

W119, W17, W18, W94

N157, N162, N34

$\mathrm{N}$

N110, N127, N28

$\mathrm{H} 10, \mathrm{H} 5$

W11, W126, W127, W155, W16, W578

N.

N17

N121, N152, N167, N32, N41, N44, N83

N41 
Table 1. HATCHERIES, DEALERS, AND INDEPENDENT FLOCKS PARTICIPATING IN

THE NATIONAL POULTRY IMPROVEMENT PLAN

WATERFOWL, EXHIBITION POULTRY AND GAME BIRDS

\begin{tabular}{|c|c|c|c|c|c|}
\hline $\begin{array}{l}\text { APP. } \\
\text { NO. }\end{array}$ & $\begin{array}{l}\text { SUB- } \\
\text { PART }\end{array}$ & PARTICIPANTS NAME AND ADDRESS & $\begin{array}{l}\text { HATCHING } \\
\text { EGG } \\
\text { CAPACITY }\end{array}$ & $\begin{array}{l}\text { PRODUCTS CLASSIFIED } \\
\text { U.S. PULLORUM-TYPHOID } \\
\text { CLEAN }\end{array}$ & $\begin{array}{c}\text { ADDITIONAL } \\
\text { CLASSIFICATIONS FOR } \\
\text { WHICH PRODUCT QUALIFIED }\end{array}$ \\
\hline
\end{tabular}

1940 E BRUCE BAILEY, 615 NORTH TAFT, OKMULGEE 74447

2757 E ROBIN \& BRIAN BAIRD, 11414 SOUTH PERKINS ROAD, PERKINS 74059

2367 E DANNY BAKER, 8101 EAST 156TH NORTH, COLLINSVILLE 74021

2613 E DARRELL BALES, P.O. BOX 843, JAY 74346

2456 E JIM BALLARD, RT. 2, BOX 47-1, TAHLEQUAH 74464

2527 E JOHN BANKS, 59000 EAST 130 ROAD, MIAMI 74354

522 E BANTAM'S ACRE, JOHNNY SAMPLE, RT. 2, B0X 180, BENNINGTON 74723

1631 E W. L. BARBER, 1010 WEST CHOCTAW, LINDSAY 73052

1759 E CARL BARDSHER, RT. 1, BOX 209-B, LAWTON 73552

1695 E KELLY BARGER, RT. 2, BOX 368, PAWNEE 74058

256 E BARNES BANTAMS, ANDY JOE BARNES, RT. 2, BOX 450, COMANCHE 73529

BUD BARNES, P.O. BOX 254, CORDELL 73632

E

TERRY BARNES, 40641 NORTH 3950 ROAD, SKIATOOK 74070

JUNIOR L. BARNETI, RT. 1, BOX 1255, ROSE 74364

CHEPARNEY BARNETTE, 3120 EAST DAVIS FIELD ROAD, MUSKOGEE 74403

E BRILEY BARNHART, RT. 2, BOX 91, GEARY 73040

E STEVE BARRETT, RT. 2, BOX 82-12, SEMINOLE 74868

E RONNIE BARRETT, 445 SOUTH 4 TTH WEST AVENUE, TULSA 74127

E FRED BARTON, RT. 1, BOX 63-A, MCALESTER 74501

E TERRY BATCHELOR, HC 80, BOX 95, ATOKA 74525

E RONNIE BAUDERS, P.O. BOX 496, COLLINSVILLE 74021

E JOHN T. BAUMANN, P.O. BOX 451, BLANCHARD 73010

E WALTER LEE BAYLES, P.O. BOX 432, ATOKA 74525

E JIMMY BEAL, 297 LOWER W. RIVER ROAD, COLBERT 74733

E GREG BEAM, 9615 SOUTH FRANKLIN ROAD, NORMAN 73069

E TOM BEASON, P.O. BOX 253, WYNNEWOOD 73098

E FORREST BEAUFORD, 18077 SOUTH HIGHWAY 88 , CLAREMORE 74017

E BOYD BEAUMONT, 1202 COMMERCIAL, MOUNDS 74047

E ROBERT WAYNE BECKHAM, RT. 2, BOX 92-13, SPERRY 74073

N17

R20, R7, R92, W12, W176, W18, W427, W573, Z22, Z62

N17.

N104, N28, N32, N41, N90

N17

R19, R227, R71, W120

W142, W151, W276, W28, W29, W50, W7

W136

N28, N37, N44

N17

W28, W88

W28

N

H5

N.

N17

N17

N28

N28, N33

N28, N32, N33

N

N17

N

N127, N133, N143.

N

N 
Table 1. HATCHERIES, DEALERS, AND INDEPENDENT FLOCKS PARTICIPATING IN

THE NATIONAL POULTRY IMPROVEMENT PLAN

WATERFOWL, EXHIBITION POULTRY AND GAME BIRDS

\begin{tabular}{|c|c|c|c|c|c|}
\hline $\begin{array}{l}\text { APP. } \\
\text { NO. }\end{array}$ & $\begin{array}{l}\text { SUB- } \\
\text { PART }\end{array}$ & PARTICIPANTS NAME AND ADDRESS & $\begin{array}{l}\text { HATCHING } \\
\text { EGG } \\
\text { CAPACITY }\end{array}$ & $\begin{array}{c}\text { PRODUCTS CLASSIFIED } \\
\text { U.S. PULLORUM-TYPHOID } \\
\text { CLEAN }\end{array}$ & $\begin{array}{c}\text { ADDITIONAL } \\
\text { CLASSIFICATIONS FOR } \\
\text { WHICH PRODUCT QUALIFIED }\end{array}$ \\
\hline
\end{tabular}

73 - OKLAHOMA

\begin{tabular}{|c|c|c|}
\hline 2709 & $E$ & $\begin{array}{l}\text { BILLIE BECKNELL, } 1100 \text { SE 108TH STREET, NORMAN } \\
73026\end{array}$ \\
\hline 1899 & E & $\begin{array}{l}\text { M. L. BEDFORD GAME RANCH, P.O. BOX 294, MOYERS } \\
74557\end{array}$ \\
\hline 1547 & E & $\begin{array}{l}\text { LEWIS BEENE, } 16193 \text { STREET HIGHWAY 39, PURCELL } \\
73080\end{array}$ \\
\hline 1488 & E & J.W. BEESON, RT. 1, BOX 158, SAYRE 73662 \\
\hline 2410 & $E$ & MARK BELECKI, RT. 1. BOX 37X. HAWORTH 74740 \\
\hline 2639 & E & JACK BELL, RT. 4, BOX 340, MADILL 73446 \\
\hline 2005 & E & LESTER BELT, JR., RT. 1, BOX 738, COLLINSVILLE 74021 \\
\hline 2586 & E & ABUNDIO BELTRAN, RT. 3, BOX 209-D, OKEMAH 74859 \\
\hline 2659 & $\mathrm{E}$ & JOEL F. BENCH, P.O. BOX 73, COUNTYLINE 73425 \\
\hline 1870 & E & $\begin{array}{l}\text { RUSSELL BENEFIELD, HC } 64, \text { BOX } 3535 \text {, HEAVENER } \\
74937\end{array}$ \\
\hline 2191 & E & ZENIADO BENITEZ, HC 60, BOX 79, SPRINGER 73458 \\
\hline 2457 & $\mathrm{E}$ & $\begin{array}{l}\text { RONNIE BETTERTON, RT. 1, BOX 279-Z, ARDMORE } \\
73401\end{array}$ \\
\hline 2758 & $E$ & JEFF BETTS, RT. 1, BOX 227, WYANDOTTE 74370 \\
\hline 2544 & $E$ & BRIANNE BEWLEY, P.O. BOX 882, OOLOGAH 74053 \\
\hline 2710 & $\mathrm{E}$ & EDDIE BIGBOW, RT. 2, BOX 285, MT. VIEW 73062 \\
\hline 2411 & $\mathrm{E}$ & $\begin{array}{l}\text { LARRY BIGGS, } 6016 \text { SOUTH LUTHER ROAD, MIDWEST } \\
\text { CITY } 74857\end{array}$ \\
\hline 2645 & E & KENNETH BILLINGS, P.O. BOX 41, EARLSBORO 74840 \\
\hline 2381 & $E$ & WENDALL BILLS. P.O. BOX 121, VERDEN 73092 \\
\hline 907 & $\mathrm{E}$ & $\begin{array}{l}\text { OREN BILLS, } 11202 \text { SOUTHERN SHORE DRIVE, } \\
\text { NORMAN } 73071\end{array}$ \\
\hline 1401 & $\mathrm{E}$ & BENNIE BILYEU, RT. 1, BOX 810, BUNCH 74931 \\
\hline 1602 & $E$ & JOE BINGHAM, P.O. BOX 99, JAY 74346 \\
\hline 1942 & E & JACKIE BINGHAM, RT, 3, BOX 111-A, DUNCAN 73533 \\
\hline 2801 & $E$ & BILLY BINGHAM, RT. 1, BOX 339, TUTTLE 73089 \\
\hline 2098 & $\mathrm{E}$ & $\begin{array}{l}\text { ROBERT L. BISHOP, RT. 2, BOX 190-C3. SEMINOLE } \\
74868\end{array}$ \\
\hline 2683 & $E$ & PETER BLACK, RT. 1, BOX 119, ROFF 74865 \\
\hline
\end{tabular}

2683 E PETER BLACK, RT. 1, BOX 119, ROFF 74865 2311 E WARREN BLACKBURN, 2221 NORTH 84 TH WEST.
MUCKOGEE 74401

1968 E WARREN BLACKBURN, 2221 NORTH 84TH WEST, MUSKOGEE 74401

1750 E COY BLAKE, RT. 1, BOX 196, HENRYETTA 74437

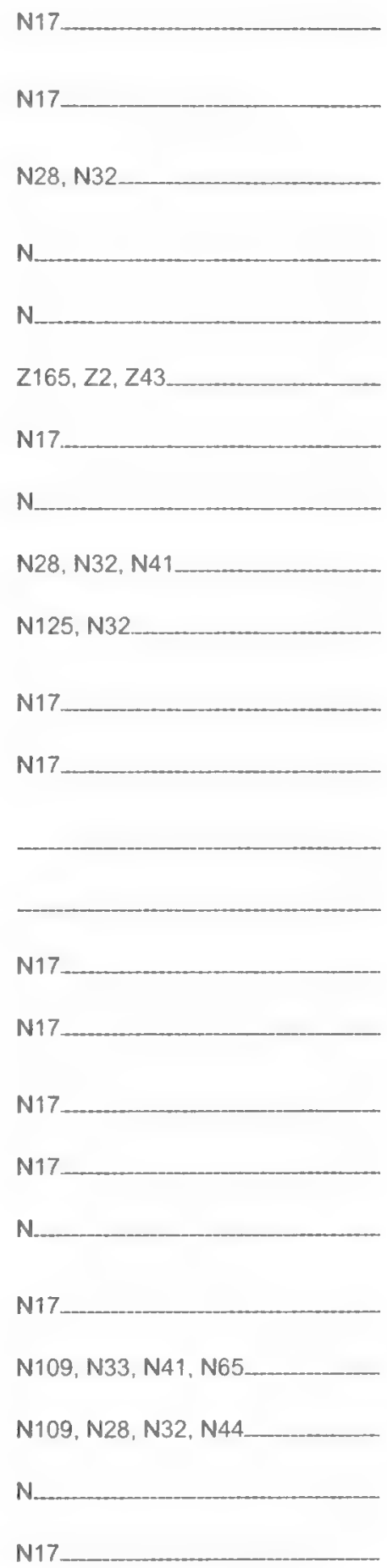

N1, N94, W100, W115, W128, W175, W245, W293, W294. W295. W453, W455, W544, Z207, Z209, Z211

N17

N17

N. 
Table 1. HATCHERIES. DEALERS. AND INDEPENDENT FLOCKS PARTICIPATING IN

THE NATIONAL POULTRY IMPROVEMENT PLAN

WATERFOWL. EXHIBITION POULTRY AND GAME BIRDS

APP. SUB- PARTICIPANTS NAME AND ADDRESS NO. PART

HATCHING
EGG
CAPACITY

PRODUCTS CLASSIFIED

U.S. PULLORUM-TYPHOID CLEAN
ADDITIONAL

CLASSIFICATIONS FOR WHICH PRODUCT QUALIFIED

73 - OKLAHOMA

2711 E BILL BLAS, 15600 VIKING ROAD, NOBLE 73068

2347 E GLEN D. BLEVINS, RT. 1, BOX 5905, ANTLERS 74523

151 E JACK BLOOMER, 6901 EAST COVELL ROAD, EDMOND 73034

1872 E HARVEY BOHALL, P.O.BOX 34, MAYSVILLE, AR 72747

1586 E BONANZA GAME FARM, J.DOUGLASS, P.O. BOX 388 , COLLINSVILLE 74021

2073 E MICHAEL BOND, RT. 3, BOX 205-28, SALLISAW 74955

$2646 \quad E$

$2745 \quad E$

$2348 \quad E$

2587

2700

$2320 \quad$

2780

1696

69

1921

225

2524

$2412 \quad E$

821

1850

2268

1226

122

2107

965

693

2064

BOBBY BOND, 615 NORTH 5TH, CLINTON 73601

BONNIE \& CLYDE, 142 CHOCTAW STREET, BOKOKSHE 74930

JOHN BOTTOMS, RT. 1, BOX 2040, HEAVENER 74937

RONNIE BOWDERS, P.O. BOX 496, COLLINSVILLE 74021

THE BOYS BANTAMS, P.O. BOX 542, HINTON 73047

RONALD BOYLE, 3800 HORSE LANE, MOORE 73160

BILLY BRADEN, 515 WYANDOTTE, LOCUST GROVE 74352

E J.C. BRADY, HC 63, BOX 226-A, EUFAULA 74432

$E$

DONNIE BRANDT, RT. 1, BOX618, BLANCHARD 73010

$E$

E SKIATOOK 74070 DARNELL BRANNON, P.O. BOX 101, EARLSBORO 74840

JERRY BRAZIEL, 29603 HOMER LANE ROAD, MCLOUD 74851

THOMAS R. BREEDLOVE, RT. 1, BOX 159-1, GARVIN 74736-9755

E BRESSLER'S BANTAMS, RT. 1, BOX 6, GUYMON 73942

E MIKE BREWSTER, RT. 2, BOX 165, ELGIN 73538

E BRIAR PATCH GAME FARM, GARY HOLT, RT. 2, BOX 307-1. CHELSEA 74016

E BRIAR PATCH GAME BIRD FARM, G. HOLT, RR 2, BOX 307-1, CHELSEA 74016

$E$

LYNDOL BRIDGERS, RT. 1, HASTINGS 73548

$E$

J. R. BRISTOW, 18608 BRANGUS ROAD, SHAWNEE 74801

E HUSTON BROOKSHER, RT. 1, BOX 107, ELMORE CITY 73035

E

BROOKSHIRE POULTRY FARM, RT. 2, BOX 204, HINTON 73047

N17

N17

R19, R23, R42

N28, N44

N

N17

N.

N41

N28, N41

N17

W28, W48, W6

N127, N28, N41

R13, R22, R223

N17

N.

N17

N17.

W276, W29, W50, W568

N.

W28, W88

R225, R42, X21, Z204.

Z183.

N. Z

N28, N32

N.

N17

W119, W12, W307, W48, W484, W69 
Table 1. HATCHERIES, DEALERS, AND INDEPENDENT FLOCKS PARTICIPATING IN

THE NATIONAL POULTRY IMPROVEMENT PLAN

WATERFOWL, EXHIBITION POULTRY AND GAME BIRDS

APP. SUB- PARTICIPANTS NAME AND ADDRESS

NO. PART

$\begin{array}{cc}\text { HATCHING } & \text { PRODUCTS CLASSIFIED } \\ \text { EGG } & \text { U.S. PULLORUM-TYPHOID } \\ \text { CAPACITY } & \text { CLEAN }\end{array}$

ADDITIONAL CLASSIFICATIONS FOR WHICH PRODUCT QUALIFIED

\section{3 - OKLAHOMA}

\begin{tabular}{|c|c|c|}
\hline 694 & $\mathrm{E}$ & BOB BROWN, RT. 1, BOX 3320, ELK CITY 73644 \\
\hline 1132 & $E$ & FLOYD N. BROWN, 701 EAST 12TH, PAWHUSKA 74056 \\
\hline 1529 & $E$ & $\begin{array}{l}\text { CECIL R. BROWN, RT. 1, BOX } 130 \text { A R, TUSKAHOMA } \\
74574\end{array}$ \\
\hline 1262 & $E$ & DENVER BROWN, RT. 2, BOX 4445, CAMERON 74932 \\
\hline 2349 & $E$ & ORVIL BROWN, RT. 2, BOX 223, MARIETTA 73448 \\
\hline 2413 & E & $\begin{array}{l}\text { THERESA \& CHARLES BROWN, P.O. BOX 1413, JAY } \\
74346\end{array}$ \\
\hline 2554 & $\mathrm{E}$ & JERRY M. BROWN, RT. 5, BOX 950, STILWELL 74960 \\
\hline 2382 & $E$ & CARL BROWN, P.O. BOX 337, ALEX 73002 \\
\hline 2495 & $E$ & $\begin{array}{l}\text { LOUIE BROWN. } 1205 \text { EAST RANDOLPH. OKMULGEE } \\
74447\end{array}$ \\
\hline 2722 & $\mathrm{E}$ & BRUCE BROWN, 15975 VIKING ROAD, NOBLE 73068 \\
\hline 1861 & $E$ & BROWN'S POULTRY, P.O. BOX 188, HENNESSEY 73742 \\
\hline 1447 & E & $\begin{array}{l}\text { CHRISTOPHER BRUMLEY, RT. 1, BOX 104-C, PAOLI } \\
73074\end{array}$ \\
\hline 1943 & $\mathrm{E}$ & $\begin{array}{l}\text { JACKIE BRUMLEY, RT. 2, BOX 146-D6, PAULS VALLEY } \\
73075\end{array}$ \\
\hline 1577 & $\mathrm{E}$ & FRANK BRUMMETT, P.O. BOX 117, SPERRY 74073 \\
\hline 2588 & $E$ & KEEVIN BRYANT, RT. 1, BOX 575, CLEVELAND 74020 \\
\hline 2607 & $E$ & $\begin{array}{l}\text { LARRY BUCK, } 5621 \text { WEST BROADWAY, ARDMORE } \\
73401\end{array}$ \\
\hline 2235 & $E$ & $\begin{array}{l}\text { JIM BUCKNER, } 3636 \text { SOUTH CEMETARY ROAD, YUKON } \\
73099\end{array}$ \\
\hline 2648 & $E$ & DARRELL BULLARD, 18630 NE 178TH, LUTHER 73054 \\
\hline 2525 & E & EDDIE BURNS, RT. 5, BOX 1085, COALGATE 74538 \\
\hline 975 & $\mathrm{E}$ & EMMITT BURNS. P.O. BOX 105. SAND SPRINGS 74063 \\
\hline 2209 & E & GLEN BURR, P.O.BOX 122, TERLTON 74081 \\
\hline 2496 & $E$ & JOE BURRIS, RT. 3, BOX 345, OAKLAND 73446 \\
\hline 1771 & $E$ & $\begin{array}{l}\text { ALLEN BURROUGH, RT. 1, BOX 5210, CARTWRIGHT } \\
74731\end{array}$ \\
\hline 2312 & $E$ & JOHN BUSBY, P.O. BOX 83, KELLVILLE 74039 \\
\hline 2319 & E & $\begin{array}{l}\text { BUSHWACKER, LARRY COWAN, } 2227 \text { SOUTH 116TH } \\
\text { AVENUE, TULSA } 74101\end{array}$ \\
\hline 1760 & $E$ & KURT BUSS, RT. 2, BOX 27, SELMAN 73834 \\
\hline 2548 & $E$ & RICHARD L. BUTLER, RT. 1, BOX 702-6, IDABEL 74745 \\
\hline
\end{tabular}

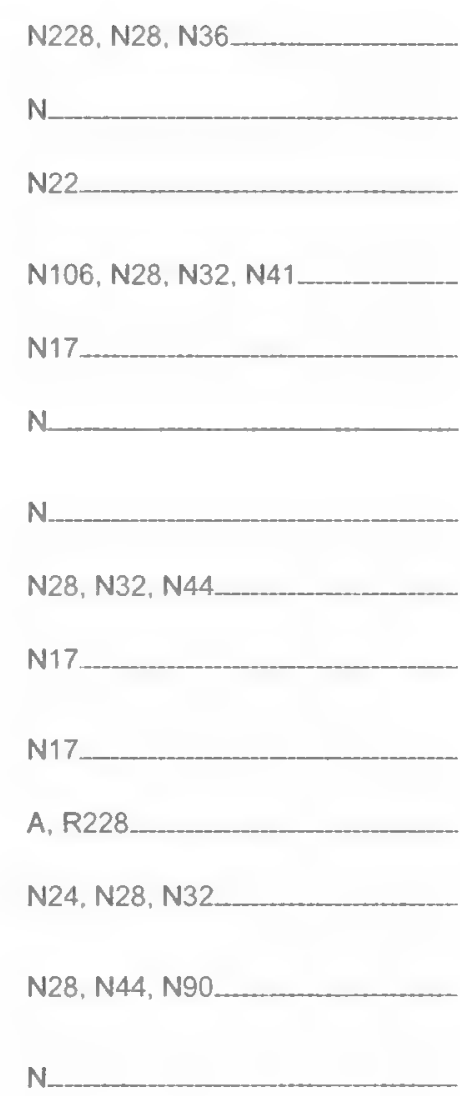

N28, N41, N44

N17

N19

N20, N28, N41

N.

N.

N107, N28, N32, N90.

N17

N32, N41, N44

N

N.

R12, R222, R7, W119, W160, W164, W23, W262, W278, W28, W294, W357, W50, W568, W606, X10, X135, X143, X145 $X 147, X 168, X 17, X 2, X 28, X 3$, $X 4, X 6, X 8, Y 14, Y 2, Y 6, Y 9$ 
Table 1. HATCHERIES, DEALERS, AND INDEPENDENT FLOCKS PARTICIPATING IN

THE NATIONAL POULTRY IMPROVEMENT PLAN

WATERFOWL, EXHIBITION POULTRY AND GAME BIRDS

\begin{tabular}{lllll}
\hline & & HATCHING & PRODUCTS CLASSIFIED \\
APP. SUB- & PARTICIPANTS NAME AND ADDRESS & EGG & U.S. PULLORUM-TYPHOID \\
NO. PART & CLAPACITY & CLASSIFICATIONS FOR & WHICHPRODUCT QUALIFIED \\
\hline
\end{tabular}

73 - OKLAHOMA

\begin{tabular}{|c|c|c|c|}
\hline 2608 & E & PHILLIP BUTLER, P.O. BOX 35, REYDON 73660 & $\begin{array}{l}\text { R21, W142, W276, W28, W48, - } \\
\text { W70, Z20, Z22 }\end{array}$ \\
\hline 2803 & $E$ & CECIL BYRD, HC 60, BOX 2930, LAWTON 73501 & $\mathrm{~N} 17$ _ \\
\hline 1807 & $\mathrm{E}$ & BILLY BYRD, RT. 2, BOX 66, WETUMKA 74883 & N17 \\
\hline 2028 & E & RANDALL BYRD, P.O. BOX 135, LEQUIRE 74943 & $\mathrm{~N} 32, \mathrm{~N} 41, \mathrm{~N} 43, \mathrm{~N} 44, \mathrm{~N} 90$ \\
\hline 2192 & E & $\begin{array}{l}\text { C \& S FARMS, CARRY \& SMITH, RT. 3, BOX } 7000, \\
\text { STIGLER } 74462\end{array}$ & $\mathrm{~N} 134, \mathrm{~N} 28, \mathrm{~N} 32, \mathrm{~N} 83$ \\
\hline 2609 & E & C \& S GAME FARM, RT. 3, BOX 7000 , STIGLER 74462 & H57, N134, N28, N32, N83_ \\
\hline 2414 & E & $\begin{array}{l}\text { KENNETH CAIN, RT. 3, BOX 267-C, PAULS VALLEY } \\
73075\end{array}$ & N24, N28, N32 - \\
\hline 2610 & $E$ & $\begin{array}{l}\text { JAMES CALDWELL, } 29206 \text { SOUTH } 337 \text { WEST AVENUE, } \\
\text { BRISTOW } 74010\end{array}$ & W28, W52, W67, W88 \\
\hline 2415 & $E$ & JERRY CALLICOAT, RT. 2, BOX 445, BOKCHITO 74726 & $\mathrm{~N} 32, \mathrm{~N} 41$ \\
\hline 2416 & $E$ & TONY CALLICOAT, HC 80, BOX 970, ATOKA 74525 & N41, N44, N63, N83 _ _ _ _ _ \\
\hline 1168 & $E$ & MIKE CAMPBELL, RT. 1, BOX 255, HASKELL 74436 & N105, N136, N41, N43, N65, N87_ \\
\hline 2248 & $E$ & JOE CAMPO, P.O. BOX 112, LONE GROVE 73443 & $\mathrm{~N} 32, \mathrm{~N} 41, \mathrm{~N} 44, \mathrm{~N} 63, \mathrm{~N} 77, \mathrm{~N} 83$ \\
\hline 1563 & $E$ & CHARLES CANADY, RT. 1, BOX 279, ARDMORE 73401 & $\mathrm{~N}$ \\
\hline 982 & $E$ & BILLY CANTRELL, RT. 1, BOX 488, DEPEW 74028 & $\mathrm{~N}$ \\
\hline 2171 & $E$ & SUSAN CANTWELL, RT. 1, BOX 27, ELMORE CITY 73433 & $\mathrm{~N} 28, \mathrm{~N} 44, \mathrm{~N} 90$ \\
\hline 2555 & $E$ & PHILLIP CAPSHAW, RT. 4, BOX 2930, ATOKA 74525 & 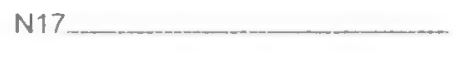 \\
\hline 801 & E & $\begin{array}{l}\text { CAREY \& SMITH GAME FOWL, RT. 3, BOX } 7000 \text {, } \\
\text { STIGLER } 74462\end{array}$ & $\mathrm{H} 57, \mathrm{~N} 134, \mathrm{~N} 28, \mathrm{~N} 32, \mathrm{~N} 83$. \\
\hline 2271 & E & $\begin{array}{l}\text { JESSICA CARMACK, } 13208 \text { NORTH 83RD AVENUE, } \\
\text { COLLINSVILLE } 74021\end{array}$ & \\
\hline 2417 & E & ODIE CARPITCHER, P.O. BOX 372, CANADIAN 74425 & N17. \\
\hline 2188 & $E$ & $\begin{array}{l}\text { DUB CARR, } 13635 \text { SOUTH } 49 \text { TH WEST AVENUE, } \\
\text { SAPULPA } 74066\end{array}$ & \\
\hline 1752 & E & JORGE CARRION, RT. 1, BOX 67, WYANDOTTE 74370 & \\
\hline 335 & E & BUD CARTER, RT. 2, BOX 372, MADILL 73446 & N161___ \\
\hline 1675 & E & TOM CARTER, HC 64, BOX 172, MARLOW 73055 & N125, N28, N32, N41_. \\
\hline 1079 & $E$ & DONALD CARTER, HC 61, BOX 43, ALEX 73002 & $\mathrm{~N} 28, \mathrm{~N} 41, \mathrm{~N} 44$ \\
\hline 2556 & $E$ & FRED CARTER, RT. 2, BOX 13800, PORUM 74455 & $\mathrm{~N}$ \\
\hline 2383 & $E$ & JOSE CASAS. 218 SOUTH TTH. CLINTON 73601 & N17 \\
\hline 1960 & $E$ & CLAYTON CASS, P.O. BOX 1186, TALIHINA 74571 & N28, N41, N44 \\
\hline 2384 & $E$ & $\begin{array}{l}\text { WILLIAM CASTLEBERRY, RT. 1, BOX 1090, GRANDFIELD } \\
73546\end{array}$ & N24 \\
\hline 2549 & E & $\begin{array}{l}\text { TERRY CASTLEBERRY, RT. 3, BOX 759, BLANCHARD } \\
73010\end{array}$ & $\mathrm{~N} 17 \ldots$ \\
\hline
\end{tabular}


HATCHING EGG CAPACITY
PRODUCTS CLASSIFIED U.S. PULLORUM-TYPHOID CLEAN
ADDITIONAL

CLASSIFICATIONS FOR WHICH PRODUCT QUALIFIED

$\begin{array}{lll}281 & \text { E } & \begin{array}{l}\text { WILLIAM CASTO, JR., 76455 65TH WEST AVENUE, } \\ \text { TULSA 74131 }\end{array} \\ 1873 & \text { E } & \text { STEVE CAVINESS, RT. 3, BOX 558, TAHLEQUAH 74465 } \\ 1325 & \text { E } & \begin{array}{l}\text { J.W. (JIM) CHADWICK, RT. 1, BOX 124-A, MILL CREEK } \\ 74856\end{array} \\ 2483 & \text { E } & \begin{array}{l}\text { CHAIN FARM, VIRGINIA CHAIN, RT. 1, BOX 64, } \\ \text { GERONIMO 73543 }\end{array} \\ 2006 & \text { E } & \begin{array}{l}\text { MIKE CHAMBLIN, P.O. BOX 394, SOUTH COFFEEVILLE } \\ 74072\end{array} \\ 1906 & \text { E } & \begin{array}{l}\text { TOMMY CHANEY, RT. 4, BOX 55-B, ANADARKO 73005 } \\ 1103\end{array} \\ \text { E } & \begin{array}{l}\text { DONALD CHARLES, 16800 SOUTH 4170 ROAD, } \\ \text { CLAREMORE 74017 }\end{array} \\ 1722 & \text { E } & \text { DON CHEEK, RT. 1, BOX 455, BOSWELL 74727 } \\ 2775 & \text { E } & \begin{array}{l}\text { CHICKEN CREEK ACRES, 6351 164 STREET, NOBLE } \\ 73068\end{array}\end{array}$

2172 E RYAN CHRISTENSEN, 121 SOUTH LOCUST, APACHE 73006

1774 E GLENN CHRISTIAN, HC 68, BOX 14, KINGSTON 73439

2611 E RUSTY CHRISTMAN, P.O. BOX 12, PITTSBURG 74560

2276 E VICKY CINDLE, RT. 4, BOX 90, BRISTOW 74010

2321 E FRANK CLARK, P.O. BOX 681, DEWAR 74431

2459 E DAVID CLARK, 708 NORTH ROCKWELL, BLANCHARD 73010

2186 E DANNY CLAUNCH, P.O. BOX 69, WAYNE 73095

1874 E SAMMY CLAUNTS, SR., P.O. BOX 164, CAMERON 74932

743 E HERB CLAY, 108 PICKARD DRIVE, MCLOUD 74851

1530 E KEVIN CLAY, RT, 1, ANTLER 74523

2160 E BILLY CLAY, HC 67, BOX 255, ANTLERS 74523

2302 E CLEAR BAY GAME FARM, TIM \& ELAINE BILLS, P.O. BOX 603, TECUMSEH 74873

2322 E CLIFF CLEMMER, P.O. BOX 1025, KELLYYILLE 74039

2640 E THOMAS CLICK, 509 ANDERSON SE, ARDMORE 73401

2787 E CLINE FAMILY POULTRY, 2225 NW 12TH AVENUE, NORMAN 73072

1651 E RANDY CLINTON, RT. 1, BOX 75-C, GERONIMO 73543

1885 E BUDDY CLINTON, P.O. BOX 331, COOKSON 74427
W20. W38

N.

N98

Z, Z41, Z43

N

N10, N27, N28, N32

N125, N28, N32, N41

N17, N43

W1, W117, W119, W12, W129, W132, W152, W162, W208, W248, W26, W270, W282,

W290. W31. W313, W368. W41. W415, W42, W43, W45, W560, W561, W573, W74, W75, W86. W96, W98

W394.

N.

N17

N17.

N28, N32, N41

N17.

N28, N32

N28, N32, N41, N44

N17

N17

N104, N148, N24, N25, N28, N44.

N113, N32, N41

N17.

W48, W89

N17, N65

N 
Table 1. HATCHERIES, DEALERS, AND INDEPENDENT FLOCKS PARTICIPATING IN

HATCHING

EGG

CAPACITY
PRODUCTS CLASSIFIED

U.S. PULLORUM-TYPHOID CLEAN
ADDITIONAL

CLASSIFICATIONS FOR WHICH PRODUCT QUALIFIED
2210 E DAVID COBB, RT. 1, BOX 335, WELLING 74471

2369 E MARK COCHELL, 11221 JEFFOROS AVENUE, NICOMA PARK 73066

2641 E STACYL. COGBURN, RT. 1, BOX 95, CYRIL 73029

953 E RUPERT COGBURN, RT. 4, BOX 66, BROKEN BOW 74728

1276 E WILLIAM COKER, P.O. BOX 176, CALERA 74730

2685 E RAFE COKER, P.O.BOX 1092, TUTTLE 73089

2219 E ROLAND COLE, RT. 4, BOX 308, BRISTOW 74010

2665 E MARVIN COLE, RT. 1, BOX51, ARDMORE 73401

2128 E DANNY COLE, 1879 NORTH 505 ROAD, ROSE 74364

2174 E DOYLE COLEMAN, 3607 NW DELLA, NORMAN 73072

2173 E GARY COLLINS, RT, 1, BOX 153, CHANDLER 74834

1637 E RANDYL COLLINS, 140-25 NORTH JARBIS ROAD, TAHLEQUAH 74464

2129 E HAROLD COLLINS, P.O. BOX 175, KENEFIC 74748

2418 E LARRY COLSTON, P.O. BOX 1987, SALLISAW 74955

2529 E GLEN D. COLVIN, HC 70, BOX 1035, KINGSTON 73439

2460 E JOE COOLEY, RT. 3, BOX 305-K, TECUMSEH 74873

2557 E DAVID COOPER, 26519 WEST 17TH STREET, SAND SPRINGS 74063

1875 E LARRY RAY COOPER, P.O. BOX 387, PRAGUE 74864

2292 E COPPERHEAD GAME FARM, J. BEDDO, 6251120 TH STREET, NOBLE 73068

1922 E HEATH CORMANY, 507 NORTHN STREET, DUNCAN 73533

2065 E LEONARD COTTAM, RT, 5, BOX 1338, MULDROW 74948

1652 E B.L. COZAD, JR., P.O. BOX 136, INDIAHOMA 73552

2220 E BILL CRAGG, RT. 1, BOX 170, STILWELL 74960

2697 E GARY CRAIG, 13504 SOUTH 4190 ROAD, CLAREMORE 74017

1704 E KEITH CRAIG, RT. 1, BOX 655, MANNFORD 74044

1371 E RICHARD CRAMBERG, 17764 SOUTH 273RD WEST AVENUE, BRISTOW 74010

1677 E RICHARD CRENWELGE, 214 WEST GATLIN ROAD, MARLOW 73055

1739 E RAYMOND CRIPPS, P.O. BOX 82, HEAVENER 74937

1080 E CHRIS CRITES, 1011 WEST PINKY ROAD, CADDO 74729

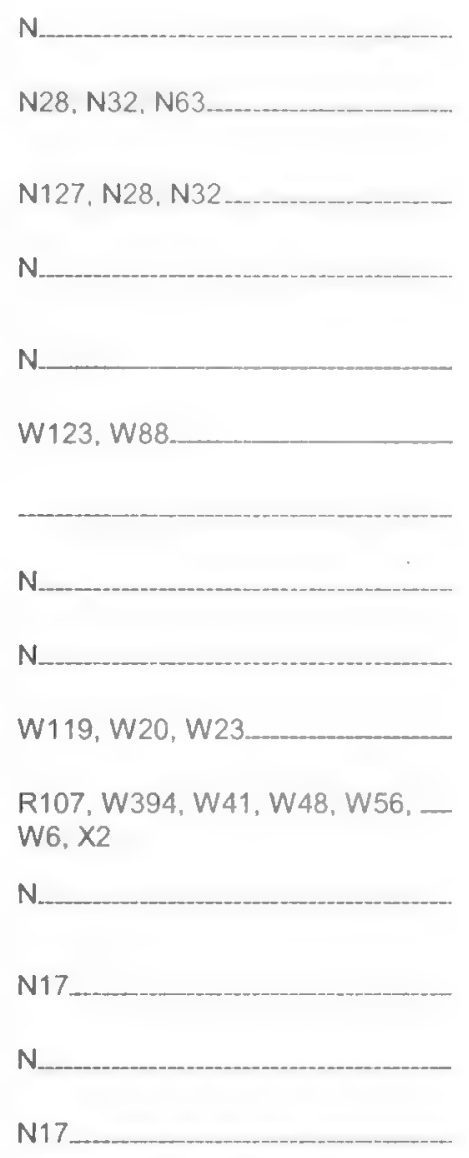

$\mathrm{N} 17, \mathrm{~N} 30, \mathrm{~N} 56, \mathrm{~N} 63, \mathrm{~N} 65$

N28, N32, N41, X2, Z247, Z88

N17

N127, N28, N32

N17.

N148, N28, N32, N41, N44, N63, N90

N17

N28, N32, N83

N17.

W12, W15, W3, W301, W4, W52, W7, X18, Y2, Y25, Y6

N28, N41, N44

N28, N32, N44, N63, N90

N17 
Table 1. HATCHERIES, DEALERS, AND INDEPENDENT FLOCKS PARTICIPATING IN THE NATIONAL POULTRY IMPROVEMENT PLAN

WATERFOWL, EXHIBITION POULTRY AND GAME BIRDS

\begin{tabular}{|c|c|c|c|c|c|}
\hline $\begin{array}{l}\text { APP. } \\
\text { NO. }\end{array}$ & $\begin{array}{l}\text { SUB- } \\
\text { PART }\end{array}$ & PARTICIPANTS NAME AND ADDRESS & $\begin{array}{l}\text { HATCHING } \\
\text { EGG } \\
\text { CAPACITY }\end{array}$ & $\begin{array}{c}\text { PRODUCTS CLASSIFIED } \\
\text { U.S. PULLORUM-TYPHOID } \\
\text { CLEAN }\end{array}$ & $\begin{array}{c}\text { ADDITIONAL } \\
\text { CLASSIFICATIONS FOR } \\
\text { WHICH PRODUCT QUALIFIED }\end{array}$ \\
\hline
\end{tabular}

$\begin{array}{ccl}2558 & \text { E } & \text { BOBBY CRITTENDEN, RT. 2, BOX 2172, STILWELL } 74960 \\ 2647 & \text { E } & \text { LLOYD CROSS, RT. 3, BOX 18-A, HOLDENVILLE } 74846 \\ 2419 & \text { E } & \text { RICKY CRUM, RT. 2, BOX 33, DOVER 73734 } \\ 2257 & \text { E } & \text { MICHAEL CUBBAGE, RT. 1, BOX 158-H, DUNCAN 73533 } \\ 931 & \text { E } & \text { FRANK CULLUM, RT, 1, BOX 159, SEMINOLE 74868 } \\ 2221 & \text { E } & \text { ROBERT CUMMINS, P.O. BOX 483, COLBERT 74733 } \\ 2559 & \text { E } & \begin{array}{l}\text { MITCHELL \& CHRISSY CUSTRED, RT. 2, BOX 56-1, BIG } \\ \text { CABIN 74332 }\end{array} \\ 1223 & \text { E } & \begin{array}{l}\text { D \& D GAME BIRDS, D. TERRY, 2719 EISENHOUR } \\ \text { DRIVE, CHOCTAW 73020 }\end{array} \\ & & \end{array}$

2286 E TODD DALE, RT. 2, BOX 919, MANNFORD 74044

2670 E DICK DALE, 32 REMUDA DRIVE, MANNFORD 74044

2497 E LARRY DANKER, P.O. BOX 35 , WELLSTON 74881

1633 E RONALD DANSBY, RT. 2, BOX 299, ADA 74820

2420 E RODNEY DAUGHERTY, RT. 3, BOX 2545, STILWELL 74960

2421 E HARDING DAVIS, RT. 1, BOX 198, DRUMRIGHT 74030

2514 E MIKE DAVIS, RT. 5, BOX 924, BLANCHARD 73010

2635 E JOHNL. DAVIS, RT. 3, BOX 92, DUNCAN 73533

767 E ANCEL DAVIS, P.O. BOX 270, BOSWELL 74727

646 E JERRY L. DAVIS, RT. 1, BOX 6540, ANTLERS 74523

1876 E BILL DAVIS, 10813 GRANADA LANE, MOORE 73165

868 E JIMMY DAVIS, P.O. BOX 783, VELMA 73491

817 E LONNIEN. DAVIS, RT. 1, BOX 279-R. ARDMORE 73401

1225 E ARCHIE L. DAVIS, RT. 1, BOX 107, DELAWARE 74027

824 E D. H. DAVIS, RT. 1, BOX 37-A. HANNA 74845

1338 E GEORGE R. DAY, RT, 4, BOX 276, MULDROW 74948

2007 E JOHN DAY, RT. 1, BOX 27, MENO 73760

1643 E GERALD DEARMAN, 1310 WEST 7 TH, OKMULGEE 74447

2759 E TERRY DEATHERAGE, RT. 6, BOX 246, MCALESTER 74501

2370 E JASON DEATON, P.O. BOX 12, STONEWALL 74871

1719 E BOYD DEAY, 68735 EAST 70TH ROAD, QUAPAW 74363

N112, N41, N44

N140, N160, N28

N17

N113, N28, N32, N41

N106, N28, N32, N41, N83, N94...

R89, W119, W17, W18, W198, W270, W554, W561, W60, X10. X147, Z118, Z179, Z19, Z202, Z204, Z205, Z27, Z34, Z46, Z47, Z57, Z88

N28, N32, N44, N90

N28, N41, N44, N83

N17

N17

N.

N

N17

N28, N32, N41.

N

N17

N

N28, N32, N41

N17

N28, N32, N41.

N28.

N125, N157, N21, N41

N17

N17

N28, N32, N34, N41, N90.

N17

N23, N28. 
Table 1. HATCHERIES, DEALERS, AND INDEPENDENT FLOCKS PARTICIPATING IN

THE NATIONAL POULTRY IMPROVEMENT PLAN

WATERFOWL, EXHIBITION POULTRY AND GAME BIRDS

\begin{tabular}{lllll}
\hline & & HATCHING & PRODUCTS CLASSIFIED \\
APP. SUB- & PARTICIPANTS NAME AND ADDRESS & EGG & U.S. PULLORUM-TYPHOID \\
NO. PART & CLEAN & CLASITIONALICATIONS FOR & WHICH PRODUCT QUALIFIED \\
\hline
\end{tabular}

73 - OKLAHOMA

\begin{tabular}{|c|c|c|c|}
\hline 2530 & E & L. D. DELAROSA, P.O. BOX 625, CALERA 74730-0625 & N17 _._._..... \\
\hline 2461 & E & $\begin{array}{l}\text { GUILLERMO DELATORRE, } 508 \text { BILL HALSTIED, } \\
\text { MARIETTA } 73448\end{array}$ & N17 \\
\hline 2189 & E & ANTHONY DELBRE, HC 62, BOX 248, DURANT 74701 & N17 _._. \\
\hline 2371 & E & PAUL DELOZIER, RT. 1, BOX 76, KINGFISHER 73750 & $\mathrm{~N} \ldots$ \\
\hline 2560 & $E$ & ARNOLD DELOZIER, 2000 WEST 32ND, ADA 74820 & $\mathrm{~N}$ \\
\hline 1644 & $\mathrm{E}$ & DON DELOZIER, RT. 2, P.O. BOX 155-A, ADA 74820 & N17. \\
\hline 2132 & E & DON DELOZIER, JR., RT. 2, BOX 155-A, ADA 74820 & N17 .... \\
\hline 1725 & $E$ & JACKIE DEMPSEY, RT. 2, BOX 36, SNYDER 73566 & N17 \\
\hline 2690 & $E$ & TYLER ZEKE DENION, P.O. BOX 821, APACHE 73006 & N127, N28 \\
\hline 2498 & E & JOSEPH DEVIN, HC 71, BOX 165, KINGSTON 73439 & $\mathrm{~N}$ \\
\hline 662 & $E$ & $\begin{array}{l}\text { DIAMOND C RANCH, SAM CAUDLE, JR., RT. 3, BOX 648, } \\
\text { BROKEN BOW } 74728\end{array}$ & N197, N28, N44, N63____ \\
\hline 2323 & E & PAT DIGNAN, RT. 1, BOX 545, HOMMINY 74035 & $\begin{array}{l}\text { N104, N129, N131, N17, N32, } \\
\text { N33, N43, N6, N63, N90, N97 }\end{array}$ \\
\hline 1726 & $E$ & JIM DILL, RT. 1, BOX 131-A, COYLE 73027 & N \\
\hline 2484 & $E$ & $\begin{array}{l}\text { RONNIE DILLON, } 3050 \text { GEORGIA STREET, EDMOND } \\
73034\end{array}$ & $\mathrm{~N}$ \\
\hline 2770 & $\mathrm{E}$ & $\begin{array}{l}\text { DENISE \& DWAYNE DOCKERY, HC } 69, \text { BOX 205, HUGO } \\
74743\end{array}$ & $\mathrm{~N}$ \\
\hline 2771 & E & PHALLS DOCKERY. RT. 2, BOX 278-B, ARDMORE 73401 & N17 \\
\hline 2550 & E & GORDON S. DODD, RT. 2, BOX 53, ADAIR 74330 & \\
\hline 1685 & $E$ & RONALD DODD, 1902 EAST 66TH STREET, TULSA 74126 & N28 \\
\hline 2422 & $E$ & JASON DODGE, RT. 1, BOX 379, CLINTON 73601 & $\mathrm{~N} 17 \ldots$ \\
\hline 2515 & $E$ & JOHNNY DODSON. P.O. BOX 177. STERLING 73567 & N19 \\
\hline 2083 & $E$ & $\begin{array}{l}\text { DOE WOLF GAME FARM, J. JONES, } 41 \text { TERRACE } \\
\text { RIDGE. BLANCHARD } 73101\end{array}$ & $\mathrm{~N}$ \\
\hline 2212 & $E$ & JERRY DOLLARHIDE, RT. 1, BOX 223, ROSE 74364 & N N \\
\hline 2462 & $E$ & $\begin{array}{l}\text { MIKE DOUGLAS, } 28232 \text { WEST } 47 T H \text { STREET SOUTH, } \\
\text { SAND SPRINGS } 74063\end{array}$ & N17 \\
\hline 2236 & $E$ & BRENT DOUGLASS, P.O. BOX 396, COLLINSVILLE 74021 & N \\
\hline 2078 & $E$ & $\begin{array}{l}\text { FRED DOVER, } 13 \text { WEST VENISON STREET, SAND } \\
\text { SPRINGS } 74063\end{array}$ & N..._. \\
\hline 2102 & $E$ & $\begin{array}{l}\text { REBECCA GLOVER \& LARRY DOVER, RT. 3, BOX } 162 \text {, } \\
\text { SALLISAW } 74955\end{array}$ & $\begin{array}{l}\text { N109, N110, N17, N28, N32, N34,. } \\
\text { N41, N43, N44 }\end{array}$ \\
\hline 1830 & $E$ & VICTOR DOWDY, RT. 3, BOX 370, BRISTOW 74010 & $\mathrm{~N}$ \\
\hline 2642 & $E$ & $\begin{array}{l}\text { JESUS DOZAL, } 1424 \text { SOUTH BLACKWELDER, } \\
\text { OKLAHOMA CITY } 73109\end{array}$ & N17 \\
\hline 2193 & $E$ & JERRY DRAIN, RT. 1, BOX 279-A, ARDMORE 73401 & $\mathrm{~N} 17$ \\
\hline
\end{tabular}


Table 1. HATCHERIES, DEALERS, AND INDEPENDENT FLOCKS PARTICIPATING IN

THE NATIONAL POULTRY IMPROVEMENT PLAN

WATERFOWL, EXHIBITION POULTRY AND GAME BIRDS

$\begin{array}{lll}\text { APP. } & \text { SUB- } \\ \text { NO. } & \text { PART }\end{array}$

$\begin{array}{cc}\text { HATCHING } & \text { PRODUCTS CLASSIFIED } \\ \text { EGG } & \text { U.S. PULLORUM-TYPHOID } \\ \text { CAPACITY } & \text { CLEAN }\end{array}$

ADDITIONAL

CLASSIFICATIONS FOR WHICHPRODUCT QUALIFIED

\begin{tabular}{|c|c|c|}
\hline 2423 & $E$ & KACEY DRESSER, RT. 2, BOX 431, SULPHUR 73086 \\
\hline 2139 & $E$ & CHARLES DRY, P.O. BOX 71, BRAGGS 74423 \\
\hline 2424 & $E$ & IVAN DUARTE, P.O. BOX 712, WEWOKA 74884 \\
\hline 2350 & $E$ & $\begin{array}{l}\text { THOMAS DUERR \& REX FINNELL, RT. 2, BOX 11-A. } \\
\text { FOSS } 73647\end{array}$ \\
\hline 2499 & $E$ & ARVIL DUGGER, RT. 3, BOX 2660, STILWELL 74960 \\
\hline 1454 & $E$ & RONNIE DUKE, RT. 3, BOX 447, BRISTOW 74010 \\
\hline 2313 & $E$ & BARRY DURHAM, 5806 EAST TYLER, TUTTLE 73089 \\
\hline 1386 & $E$ & $\begin{array}{l}\text { LARRY A. DYE, } 8551 \text { WEST HIGHLAND, PONCA CITY } \\
74601\end{array}$ \\
\hline 2108 & $E$ & DAVID EARLS, RT. 2, BOX 34-A, TECUMSHE 74873 \\
\hline 2666 & $E$ & MIKE EARP, P.O. BOX 312 , JOY 74346 \\
\hline 2561 & $E$ & $\begin{array}{l}\text { GREGG EASTERLING, } 4531 \text { 73RD STREET E, } \\
\text { MUSKOGEE } 74403\end{array}$ \\
\hline 2079 & $E$ & $\begin{array}{l}\text { KEITH EASTERLING, } 7511 \text { SOUTH } 45 \text { TH STREET EAST, } \\
\text { MUSKOGEE } 74403\end{array}$ \\
\hline 989 & $E$ & KEN EBERT, P.O. BOX 103, HULBERT 74441 \\
\hline 2698 & $E$ & $\begin{array}{l}\text { DOUG EBERT, } 411 \text { SOUTH 72RD WEST AVENUE, TULSA } \\
74127\end{array}$ \\
\hline 2672 & $E$ & IVAN EDEN, RT. 1, BOX 770 , ANTLERS 74523 \\
\hline 2562 & $E$ & JESSIE EDENDINE, RT. 2, BOX 290 \#36, SAPULPA 74066 \\
\hline & $E$ & SON, RT. 1, BOX 636, IDABEL 74745 \\
\hline
\end{tabular}

1326 E DOUGLAS EGBERT, 411 SOUTH 72ND WEST AVENUE, TULSA 74127

1453 E BUDDY EGGLESTON, 2801 NW. 16TH, NEWCASTLE 73065

179

$2516 \quad E$

$1221 \quad E$

2723

2385

2222
MIKE EGNOR, 31502 SOUTH 4330TH ROAD, VINITA 74301

E DALE EGNOR, 432855 EAST 230TH ROAD, VINITA 74301

IKE EISENHAUER POULTRY, 210 SOUTH OAK, HENNESSEY 73742

E TERRY \& TOMMY EITEL, RT. 1, BOX 113-2, TUTTLE 73089

E DENNY ELSTON, RT, 1, BOX 86, ROCKY 73661
E NICK EMERY, 18900 NORTH PEORIA, SKIATOOK 74070
N.

N17.

B15, C12

N.

N.

N127, N17, N41

N17.

R11, R12, R18, R19, R3, R44, R49, R51, W12, W38, W54. $\mathrm{X} 135, \mathrm{X2}, \mathrm{X} 3, \mathrm{Y} 14, \mathrm{Y} 3, \mathrm{Y} 8$

N

N24, N41, N65

N17

N17.

N.

N28

N17

W115, W123, W128, W141. W142, W149, W151, W187, W200, W25, W26, W262, W275, W276, W28, W29, W48, W50, W51, W52, W545, W568, W569, W579, W58, W69, W88, W89. W91, X3, X4, X6, Z202, Z204, Z205, Z230

N107, N28.

N17

N.

N17, N28, N32, N41, N90

R130, R227, W

N17

N 
Table 1. HATCHERIES, DEALERS, AND INDEPENDENT FLOCKS PARTICIPATING IN

THE NATIONAL POULTRY IMPROVEMENT PLAN

WATERFOWL, EXHIBITION POULTRY AND GAME BIRDS

\begin{tabular}{|c|c|c|c|c|c|}
\hline $\begin{array}{l}\text { APP. } \\
\text { NO. }\end{array}$ & $\begin{array}{l}\text { SUB- } \\
\text { PART }\end{array}$ & PARTICIPANTS NAME AND ADDRESS & $\begin{array}{l}\text { HATCHING } \\
\text { EGG } \\
\text { CAPACITY }\end{array}$ & $\begin{array}{l}\text { PRODUCTS CLASSIFIED } \\
\text { U.S. PULLORUM-TYPHOID } \\
\text { CLEAN }\end{array}$ & $\begin{array}{c}\text { ADDITIONAL } \\
\text { CLASSIFICATIONS FOR } \\
\text { WHICH PRODUCT QUALIFIED }\end{array}$ \\
\hline
\end{tabular}

\begin{tabular}{|c|c|c|}
\hline 2806 & $E$ & $\begin{array}{l}\text { ROBERT ENGLAND, } 13720 \text { WALKER ROAD, MCLOUD } \\
74851\end{array}$ \\
\hline 769 & $\mathrm{E}$ & $\begin{array}{l}\text { JACK ENGLISH, } 10912 \text { EAST 158TH STREET NORTH, } \\
\text { COLLINSVILLE } 74021\end{array}$ \\
\hline 1844 & E & JERRY ESTEP, RT. 2, BOX 1912, ATOKA 74525 \\
\hline 288 & $\mathrm{E}$ & R. J. ETCHIESON, P.O. BOX 164, ROFF 74865 \\
\hline 228 & E & $\begin{array}{l}\text { RICHARD E. ETCHIESON, } 300 \text { EAST MAIN, P.O. BOX } 65 \text {, } \\
\text { ROFF } 74865\end{array}$ \\
\hline 770 & E & EUGENE EUBANKS, P.O. BOX 560, STILWELL 74960 \\
\hline 2517 & $E$ & TIMOTHY J. EUBANKS, RT. 2, BOX 37, ARDMORE 73401 \\
\hline 2802 & $E$ & $\begin{array}{l}\text { R. A. EVERETT, } 1401 \text { MISSISSIPPI STREET, CHICKASHA } \\
73018\end{array}$ \\
\hline 2782 & E & $\begin{array}{l}\text { HEATHER \& ERIN FAHERTY, } 6705 \text { SOUTH REDBUD } \\
\text { AVENUE, BROKEN ARROW } 74011\end{array}$ \\
\hline 1487 & E & $\begin{array}{l}\text { ALBERT FAIR, } 17816 \text { EAST } 626 \text { ROAD, TAHLEQUAH } \\
74464\end{array}$ \\
\hline 704 & E & BOBBY FAIRCHILD, RT. 1, BOX 1050, STILWELL 74960 \\
\hline 2425 & $\mathrm{E}$ & JIM FAIRCHILD, P.O. BOX 3142, MCALESTER 74501 \\
\hline 2724 & E & TOMMY FANNIN, RT. 4, BOX 6690, ATOKA 74525 \\
\hline 454 & $E$ & $\begin{array}{l}\text { ROBERT M. FARABOUGH, } 500 \text { MEMORIAL DRIVE, } \\
\text { PERRY } 73077-9233\end{array}$ \\
\hline 2426 & E & BILL FARMER, 18987 WILSHIRE BLVD., JONES 73049 \\
\hline 2258 & E & SHANE FARRIS, RT. 1, BOX 75-A, OKEMAH 74859 \\
\hline 1857 & E & LARRY FASSINO, P.O. BOX 612, KREBS 74554 \\
\hline 1877 & E & NIKEY FASSINO, RT. 8, BOX 308, MCALESTER 74501 \\
\hline 2427 & $E$ & WAYNE FATHERREE, RT. 3, BOX 1527, STILWELL 74960 \\
\hline 2463 & $E$ & C. G. FATHERREE, RT. 4, BOX 1675, STILLWEL 74960 \\
\hline 1878 & $E$ & BILL FAUST, P.O. BOX 777, KREBS 74554 \\
\hline 1387 & E & $\begin{array}{l}\text { DANNY FEATHERS, } 3227 \text { WEST HIGHLAND, PONCA } \\
\text { CITY } 74601\end{array}$ \\
\hline 2500 & E & EVAN FELKER, HC 74, BOX 679-1, WRIGHT CITY 74766 \\
\hline 1548 & $\mathrm{E}$ & ROBBY FENNELL, 6661 SE 132, NOBLE 73068 \\
\hline 1993 & E & RAYMOND FENT, 54 OAK POND, LUTHER 73054 \\
\hline 2281 & $\mathrm{E}$ & WILLIS FERGUSON, P.O. BOX 711, SULPHUR 73086 \\
\hline
\end{tabular}

N17

N.

W115, W187

N122. N123, N51

N17

N17, N229, N236, N28, N41, N63_

N

N.

N17

$\mathrm{N} 113, \mathrm{~N} 172$

W119, W74, Z11, Z4

N17

N

N17

N28

N17

$\mathrm{N}$

N17

N

N17.

N17.

R22, W115, W123, W128, W174, W175, W200, W23, W293,

W294, W295, W48, W50, W542, W69, W88

N161, N32, N44

W23, W6, W7 
Table 1. HATCHERIES, DEALERS, AND INDEPENDENT FLOCKS PARTICIPATING IN THE NATIONAL POULTRY IMPROVEMENT PLAN

WATERFOWL, EXHIBITION POULTRY AND GAME BIRDS

\begin{tabular}{|c|c|c|c|c|c|}
\hline $\begin{array}{l}\text { APP. } \\
\text { NO. }\end{array}$ & $\begin{array}{l}\text { SUB- } \\
\text { PART }\end{array}$ & PARTICIPANTS NAME AND ADDRESS & $\begin{array}{l}\text { HATCHING } \\
\text { EGG } \\
\text { CAPACITY }\end{array}$ & $\begin{array}{l}\text { PRODUCTS CLASSIFIED } \\
\text { U.S. PULLORUM-TYPHOID } \\
\text { CLEAN }\end{array}$ & $\begin{array}{c}\text { ADDITIONAL } \\
\text { CLASSIFICATIONS FOR } \\
\text { WHICH PRODUCT QUALIFIED }\end{array}$ \\
\hline
\end{tabular}

$$
73 \text { - OKLAHOMA }
$$

\begin{tabular}{|c|c|c|c|}
\hline 2428 & $E$ & $\begin{array}{l}\text { JASON FINCANNON, } 22126 \text { WEST 48TH, SAND SPRINGS } \\
74063\end{array}$ & \\
\hline 2351 & $E$ & ASHLEY FISH, 502 WEST 47TH, SAND SPRINGS 74063 & N. \\
\hline 2243 & $E$ & $\begin{array}{l}\text { JOSHUA \& JOCEL YN FLETCHER, } 1408 \text { NORTH UNION } \\
\text { PLACE, TULAS } 74127\end{array}$ & \\
\hline 872 & $E$ & $\begin{array}{l}\text { CHARLES FLETCHER, } 12001 \text { SOUTH 13TH STREET } \\
\text { EAST, OKTAHA } 74401\end{array}$ & $N$ \\
\hline 1654 & $E$ & ANGEL FLORES, RT. 1, BOX 167-C, TERLTON 74081 & N104, N41, N43, N46 \\
\hline 2522 & $E$ & $\begin{array}{l}\text { STEVE FLORES, } 4209 \text { SOUTH TRIPLE X ROAD, } \\
\text { CHOCTAW } 73020\end{array}$ & N_________n \\
\hline 1973 & $E$ & $\begin{array}{l}\text { WILKIE FLOWERS, JR., RT. 1, BOX 88-A, WYNNEWOOD } \\
73098\end{array}$ & $\mathrm{~N} 134, \mathrm{~N} 44, \mathrm{~N} 65$ \\
\hline 2807 & $E$ & BOBBY FOSTER, P.O. BOX 229-F, RUSH SPRINGS 73082 & N28, N32, N41_. \\
\hline 2563 & $E$ & $\begin{array}{l}\text { FOUTCH FAMILY POULTRY, } 916 \text { SOUTH CANADIAN } \\
\text { AVENUE, PURCELL } 73080\end{array}$ & W25, W48_______________ \\
\hline 1832 & $E$ & $\begin{array}{l}\text { FOWL PLAY POULTRY, D \& J SHEPHERD, } 53501 \text { EAST } \\
120 \text { ROAD, MIAMI } 74354\end{array}$ & $\mathrm{X} 10, \times 135, \times 2, \times 23, \times 9,2202$ \\
\hline 2119 & $E$ & AL FOWLER, RT. 2, BOX 239, HOLLIS 73550 & R196. W200 \\
\hline 2163 & $E$ & $\begin{array}{l}\text { JOHN FOWLER, } 219 \text { NORTH CHEROKEE, MUSKOGEE } \\
74403\end{array}$ & N17_____________ \\
\hline 1863 & $\mathrm{E}$ & $\begin{array}{l}\text { ROLAND (BUTCH) FRAILICKS, RT. 2, BOX } 245 \text {, } \\
\text { BOKCHITA } 74726\end{array}$ & N17___ \\
\hline 1536 & $\mathrm{E}$ & GARY FRANCIS, RT. 2, BOX 65, POTEAU 74943 & N25, N41, N63 \\
\hline 2746 & $E$ & $\begin{array}{l}\text { RAY J. FRANKLIN, } 611 \text { WEST MT. CARMEL ROAD, } \\
\text { CADDO } 74727\end{array}$ & N28 \\
\hline 1446 & $E$ & JIM FRAZIER, RT, 3, BOX 289-A, SAPULPA 74066 & $\mathrm{~N}$ \\
\hline 1403 & $\mathrm{E}$ & DAVID L. FRYAR, RT. 1, BOX 1070, HARRAH 73045 & N128, N28, N33 \\
\hline 1925 & E & RAY FRYAR, P.O. BOX 33, COOKSON 74427 & $\mathrm{~N} 112, \mathrm{~N} 28, \mathrm{~N} 32$ \\
\hline 2699 & $\mathrm{E}$ & $\begin{array}{l}\text { G \& G POULTRY FARM, } 808 \text { DANA STREET, HINTON } \\
73047\end{array}$ & W114, W115, W119, W28, W48 \\
\hline 2747 & E & BRUCE GANT, RT. 3, BOX 615, KEOTA 74941 & N41 \\
\hline 1974 & $E$ & STEVE \& BRUCE GANT, RT. 3, BOX 615, KEOTA 74941 & $\mathrm{~N} 28, \mathrm{~N} 41 \ldots$ \\
\hline 2760 & E & LUIS A. GARCIA, HC 64, BOX 169-F, MARLOW 73055 & $\mathrm{~N}$ \\
\hline 2808 & $E$ & TONY GARCIA, P.O. BOX 15-5, VIAN 74962 & $\mathrm{~N}$ \\
\hline 2649 & $E$ & LEON GARDENHIRE, RT. 2, BOX 237-0, SALLISAW 74955 & N17 \\
\hline 705 & $E$ & $\begin{array}{l}\text { RANDY H. GARDENHIRE, RT. 1, BOX 234, ELMORE CITY } \\
73433\end{array}$ & N28, N33, N44 \\
\hline 2748 & $E$ & GARY GARNER, HC 60, BOX 33-A, FAIRVIEW 73737 & N17 \\
\hline 2712 & $E$ & $\begin{array}{l}\text { JAMES W. GARRETT, JR., RT. 1, BOX 12-5, GARVIN } \\
74736\end{array}$ & $\begin{array}{l}\text { R139, R52, R86, R87, W17, } \\
\text { W170, W223, W86 }\end{array}$ \\
\hline 827 & $\mathrm{E}$ & HARVEY GARRETT, RT. 1, BOX 284, COMANCHE 73529 & N28, N32 \\
\hline
\end{tabular}


Table 1. HATCHERIES, DEALERS, AND INDEPENDENT FLOCKS PARTICIPATING IN

THE NATIONAL POULTRY IMPROVEMENT PLAN

WATERFOWL, EXHIBITION POULTRY AND GAME BIRDS

\begin{tabular}{|c|c|c|c|c|c|}
\hline $\begin{array}{l}\text { APP. } \\
\text { NO. }\end{array}$ & $\begin{array}{l}\text { SUB- } \\
\text { PART }\end{array}$ & PARTICIPANTS NAME AND ADDRESS & $\begin{array}{l}\text { HATCHING } \\
\text { EGG } \\
\text { CAPACITY }\end{array}$ & $\begin{array}{l}\text { PRODUCTS CLASSIFIED } \\
\text { U.S. PULLORUM-TYPHOID } \\
\text { CLEAN }\end{array}$ & $\begin{array}{c}\text { ADDITIONAL } \\
\text { CLASSIFICATIONS FOR } \\
\text { WHICH PRODUCT QUALIFIED }\end{array}$ \\
\hline
\end{tabular}

\begin{abstract}
1659 E RICHARD GARZA, RT. 1, BOX 2096, THACKERVILLE 73459

2667 E SAM GASKINS, P.O. BOX 103, SLICK 74071

1975 E TIM GAULF, P.O. BOX 137-4, BRISTOW 74010

2223 E DAVID GEE, 12316 WEST 81 ST, SAPULPA 74066

1776 E MELVIN GIBBY \& AL BURROUGH, P.O. BOX 985 , COLBERT 74733

673 E FLOYD GIBSON, 19523 WEST 6 TH STREET SOUTH, SAND SPRINGS 74063

2084 E JIMMY GIBSON, 648 NORTH CLEVELAND, SHAWNEE 74801

1889 E THOMAS GIBSON, P.O. BOX 145, LINDSAY 73052

2272 E DON GIBSON, 1009 NORTH PESOTUM, SHAWNEE 74801

2643 E GARY GIBSON, HC 64, BOX 4095, HODGEN 74937

773 E GARY GILLIAM. 24392 S 450 ROAD, TAHLEQUAH 74464

1753 E JAMES A. GIRDNER, 4611 SOUTH 63RD WEST AVENUE, TULSA 74107

2464 E JERRY GIRDNER, RT. 3, BOX 638, TAHLEQUAH 74464

2636 E DOUG GIRDNER, 18021 HILLTOP CIRCLE, TAHLEQUAH 74464
\end{abstract}

2749 E M. J. GIRDNER, 14813 WEST 806 ROAD, TAHLEQUAH 74464

707 E CECIL GITININGS, 401 SOUTH MORGAN ROAD, TUTTLE 73089

1705 E LAWRENCE GLACER, 5751 NORTH UNION STREET, PONCA CITY 74601

2798 E EILEEN GLADDEN, RT.1, BOX 130, ASHER 74826

LAURA GLADDEN, RT. 1, BOX 130-2, ASHER 74826

$\begin{array}{lll}2713 & \text { E } & \text { TINA GLASCO, 1808 EAST 16TH STREET, TULSA } 74104 \\ 1557 & \text { E } & \text { DANNY GLASS, RT. 1, BOX 1270, CEMENT } 73017 \\ 2287 & \text { E } & \begin{array}{l}\text { FRED GLOVER, 13 WEST VENISON, SAND SPRINGS } \\ 74063\end{array} \\ 2810 & \text { E } & \begin{array}{l}\text { FELIMON GONZALES, } 824 \text { NEW YORK STREET, ALTUS } \\ 73521\end{array} \\ 2526 & \text { E } & \begin{array}{l}\text { KENNETH \& KANDY GORDON, RT. 2, BOX 548, } \\ \text { SULPHUR 73086 }\end{array} \\ 1927 & \text { E } & \text { RICHARD GORDON, RT. 1, BOX 102-C, CACHE 73527 } \\ 1229 & \text { E } & \text { ED GRAHAM, RT. 2, BOX 182, SALLISAW 74955 }\end{array}$

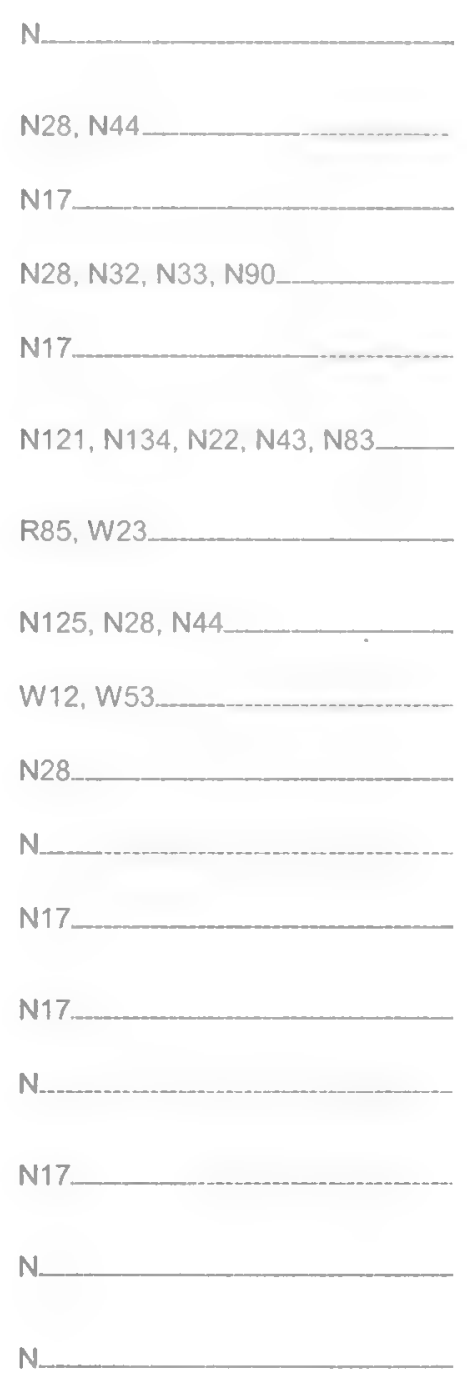

R1, R12, R7, R76, W12, W150, W270, W28, W3, W301, W50, W60, W69, W74, W88, W94

H5, R42, R44, R7, W100, W150, W276, W33, W4, W50, W51, W54, W561, W578, W60, W69, W88, W89

N69, W115, W17, W18, W29, _ W484, W56, W88

N1, N6, W35

N17

N28, N32, N44

N.

N127, N28, N32, N8

R91, W13, W136, W146, W149, W25, W26, W29, W32, W35, W38, W442, W48, W52, W6, 


\begin{tabular}{|c|c|c|c|c|c|}
\hline $\begin{array}{l}\text { APP. } \\
\text { NO. }\end{array}$ & $\begin{array}{l}\text { SUB- } \\
\text { PART }\end{array}$ & PARTICIPANTS NAME AND ADDRESS & $\begin{array}{l}\text { HATCHING } \\
\text { EGG } \\
\text { CAPACITY }\end{array}$ & $\begin{array}{l}\text { PRODUCTS CLASSIFIED } \\
\text { U.S. PULLORUM-TYPHOID } \\
\text { CLEAN }\end{array}$ & $\begin{array}{c}\text { ADDITIONAL } \\
\text { CLASSIFICATIONS FOR } \\
\text { WHICH PRODUCT QUALIFIED }\end{array}$ \\
\hline
\end{tabular}

\begin{tabular}{|c|c|c|c|}
\hline & & & W89, W94 \\
\hline 2386 & $E$ & BOBBY GRAHAM, P.O. BOX 182, BOKOSHE 74931 & N28, N41__ \\
\hline 2429 & $E$ & GREGORY GRAHAM, RT. 1, BOX 24, GARVIN 74736 & N. R \\
\hline 2324 & $\mathrm{E}$ & $\begin{array}{l}\text { STEVE GRANT, } 1321 \text { EAST LAFFYLETT, OKMULGEE } \\
74447\end{array}$ & N104, N17, N32 \\
\hline 650 & $E$ & LLOYD GRATZ, HC 71, BOX 99, KINGSTON 73439 & $\mathrm{~N} 44, \mathrm{~N} 63, \mathrm{~N} 90$ \\
\hline 2788 & $E$ & FLOYD GRAVES, RT. 3, BOX 502-AA, WAGONER 74467 & $\mathrm{~N}$ \\
\hline 2551 & $E$ & $\begin{array}{l}\text { JOHN GRAY, 14620-9 EAINDIAN HILLS ROAD. NEWALLA } \\
74857\end{array}$ & N17 \\
\hline 1044 & $\mathrm{E}$ & DON L. GRAY, RR 2, BOX 432-1, MULDROW 74948 & N28, N32, N41_ \\
\hline 2564 & $\mathrm{E}$ & $\begin{array}{l}\text { GARY GREEN, } 18198 \text { WEST } 874 \text { ROAD, PARK HILL } \\
74451\end{array}$ & N. \\
\hline 2545 & $\mathrm{E}$ & JIMMIE LEE GREENE, RT. 1, BOX 458-B, SAPULPA 74066 & N17 \\
\hline 2293 & $\mathrm{E}$ & $\begin{array}{l}\text { RODNEY GREGORY, RT. 3, BOX 247, BLANCHARD } \\
73010\end{array}$ & \\
\hline 828 & $\mathrm{E}$ & $\begin{array}{l}\text { MURLE E. GREGORY, RT. 1, BOX 918, THACKERVILLE } \\
73459\end{array}$ & N34 \\
\hline 2565 & E & TOBY GRIFFIN, P.O. BOX 176, FITTSTOWN 74842 & $\mathrm{~N}$ \\
\hline 2604 & $E$ & RICK GRIFFIN, RT. 1, BOX 226, KIOWA 74553 & N17 \\
\hline 2590 & E & $\begin{array}{l}\text { TONY GRANT GRIGSBY, HC 64, BOX 172, MARLOW } \\
73055\end{array}$ & $\mathrm{~N} 28, \mathrm{~N} 31, \mathrm{~N} 44$ \\
\hline 2725 & $E$ & $\begin{array}{l}\text { MIKE \& BILLY GRIGSLY, } 2937 \text { SW 19TH. OKLAHOMA } \\
\text { CITY } 73108\end{array}$ & N17 \\
\hline 1890 & $\mathrm{E}$ & $\begin{array}{l}\text { GENE \& TERRY GRINSTEAD, P.O. BOX 838, MULDROW } \\
74948\end{array}$ & $N 17$ \\
\hline 2080 & E & RICHARD GRUENWALD, P.O. BOX 123, AVANT 74001 & $\ldots$ \\
\hline 2523 & E & $\begin{array}{l}\text { CLINT GRUENWALD, } 501 \text { TIMBERLENE, SKIATOOK } \\
74070\end{array}$ & $\mathrm{~N} 127, \mathrm{~N} 43, \mathrm{~N} 44, \mathrm{~N} 63, \mathrm{~N} 90 \ldots$ \\
\hline 1812 & E & $\begin{array}{l}\text { H \& H FARMS, ACE HARRISON, RT. 2, BOX } 244 \text {, } \\
\text { STROUD } 74079\end{array}$ & $\mathrm{~N} 25, \mathrm{~N} 28, \mathrm{~N} 41, \mathrm{~N} 44, \mathrm{~N} 90$ \\
\hline 999 & E & $\begin{array}{l}\text { TOM HACKNEY, } 3427 \text { COUNTRY CLUB ROAD, } \\
\text { CHICKASHA } 73018\end{array}$ & $\mathrm{~N} 28, \mathrm{~N} 32 \ldots$ \\
\hline 876 & E & JAMES HAGAR, P.O. BOX 1025. JAY 74346 & N105, N28, N32, N90___ \\
\hline 1409 & E & ERNEST HALE, P.O. BOX 67, WHITEFIELD 74472 & $\mathrm{~N} 32, \mathrm{~N} 43, \mathrm{~N} 44$ \\
\hline 1948 & E & JOHN C. HALL, 18615 REDBUD DRIVE, CATOOSA 74015 & N104, N117. N63 \\
\hline 2176 & $\mathrm{E}$ & GLEN HALLMARK, P.O. BOX 393, ROFF 74865 & N123, N28, N32, N33, N63, N90__ \\
\hline 2691 & $\mathrm{E}$ & $\begin{array}{l}\text { HAMES FAMILY POULTRY, LEROY HAMES, RT. 1, BOX } \\
\text { 502, TUTTLE } 73089\end{array}$ & $\begin{array}{l}\text { R196, W123, W128, W175, } \\
\text { W187, W245, W293, W6, W88, } \\
\text { x3 }\end{array}$ \\
\hline 2259 & E & $\begin{array}{l}\text { ERIC HAMIL, } 18300 \text { NORTH PEORIA AVENUE, } \\
\text { SKIATOOK } 74070\end{array}$ & $\begin{array}{l}\text { N, W168, W17, W198, W38, } \\
\text { W484, W54 }\end{array}$ \\
\hline 709 & E & JACK HAMILTON. RT. 3, BOX 311-H, SEMINOLE 74868 & N131, N32, N41__ \\
\hline
\end{tabular}


Table 1. HATCHERIES, DEALERS, AND INDEPENDENT FLOCKS PARTICIPATING IN

THE NATIONAL POULTRY IMPROVEMENT PLAN

WATERFOWL, EXHIBITION POULTRY AND GAME BIRDS

APP. SUB- PARTICIPANTS NAME AND ADDRESS NO
HATCHING
EGG

CAPACITY
PRODUCTS CLASSIFIED

U.S. PULLORUM-TYPHOID CLEAN
ADDITIONAL

CLASSIFICATIONS FOR WHICH PRODUCT QUALIFIED

\section{3 - OKLAHOMA}

\begin{tabular}{|c|c|c|}
\hline 1864 & E & J.D. HAMLETT. RT. 1, BOX 98-1, DILL CITY 73641 \\
\hline 2049 & $E$ & EDDIE HAMLIN, 1400 SOUTH 12TH, COLLINSVILLE 74021 \\
\hline 2605 & $E$ & ANTHONY HAMMOCK, P.O. BOX 309, BOWLEGS 74830 \\
\hline 2430 & E & ROGER HAMMONDS, P.O. BOX 1952, IDABEL 74745 \\
\hline 1686 & E & $\begin{array}{l}\text { TILMAN L. HAMMONDS, HC } 63, \text { BOX 2490, FT. TOWSON } \\
74735\end{array}$ \\
\hline 2269 & $E$ & COLE HAMMONS, 801 SOUTH 29TH, CHICKASHA 73018 \\
\hline 1700 & $\mathrm{E}$ & $\begin{array}{l}\text { JOHNNY MAC HAMPTON, RT. 5, BOX 270, COALGATE } \\
74538\end{array}$ \\
\hline 2566 & $\mathrm{E}$ & DAN HANCOCK, 8154 EAST 122ND, WETUMKA 74883 \\
\hline 2352 & $\mathrm{E}$ & JAY HANSEN, 1411 MONTA AVENUE, MUSKOGEE 74403 \\
\hline 1046 & $E$ & AAGE HANSEN, JR., RT. 3, BOX 204-10, SALLISAW 74955 \\
\hline 2606 & E & $\begin{array}{l}\text { SCOTT HARBAUGH, } 20363 \text { EAST } 613 \text { ROAD, } \\
\text { TAHLEQUAH } 74464\end{array}$ \\
\hline 2325 & $E$ & MARVIN HARDISON, P.O. BOX 22, EUCHA 74342 \\
\hline 1433 & E & TOM HARGUS, 9 JAMES RAY DRIVE, SHAWNEE 74801 \\
\hline 629 & $\mathrm{E}$ & $\begin{array}{l}\text { HARMONY DOWNS, ED PYLE, P.O. BOX } 32070 \text {, } \\
\text { EDMOND } 73083\end{array}$ \\
\hline 2518 & E & GEORGE HARPER, P.O. BOX 536, BOKOSHE 74930 \\
\hline 2326 & E & $\begin{array}{l}\text { SHELBY HARRIS, RT. 2, BOX 83-A, THACKERVILLE } \\
73448\end{array}$ \\
\hline 2501 & $\mathrm{E}$ & DICKIE HARRIS, RT. 1, BOX 575, DEPEW 74028 \\
\hline 2177 & $\mathrm{E}$ & SONNY HARRISON, RT. 2, BOX 17, ROFF 74865 \\
\hline 2465 & E & THOMAS HARRISON, P.O. BOX 1114, ANTLERS 74523 \\
\hline 2714 & $E$ & RAY HARRISON, 3215 WOODRUFF LOOP, ATOKA 74525 \\
\hline 2111 & E & ROY HARRISON, RT. 2, BOX 408, CARNEGIE 73015 \\
\hline 382 & $\mathrm{E}$ & $\begin{array}{l}\text { ANSEL Z. HARSHBARGER, P.O. BOX 75, HALLETT } \\
74034\end{array}$ \\
\hline 2567 & $E$ & $\begin{array}{l}\text { GLENN \& JERRY HART, } 1717 \text { NORTH ALLEN LANE, } \\
\text { OKLAHOMA CITY } 73127\end{array}$ \\
\hline 2479 & $\mathrm{E}$ & HERB HASBEL, RT. 1, BOX 197, ASHER 74826 \\
\hline 983 & $\mathrm{E}$ & $\begin{array}{l}\text { LESTER HASBEL, } 8806 \text { NORTH HARRISON, SHAWNEE } \\
74804\end{array}$ \\
\hline 2208 & $\mathrm{E}$ & $\begin{array}{l}\text { GABE BOLENDER/SEAN \& DANIEL HAWKINS, } 1105 \\
\text { NORTH HICKORY PLACE, BROKEN ARROW } 74012\end{array}$ \\
\hline 566 & $E$ & ED HAWORTH, RT. 1, BOX 322, TAHLEQUAH 74464 \\
\hline 2726 & E & STEVE HAYDEN, RT. 3, BOX 184, COMANCHE 73529 \\
\hline 933 & $\mathrm{E}$ & $\begin{array}{l}\text { LARRY HAYES, } 19971 \text { EAST FLOURNOY ROAD, PARK } \\
\text { HILL } 74451\end{array}$ \\
\hline
\end{tabular}

N25, N32, N44, N90

N17

N134. N32. N63

N.

N17.

N32, N41

N

N17

N

N.

N28, N33, N41

N28, N46

N114, N131, N27, N28, N33, N41, N44, N81, N83

N125, N28, N32, N33, N41, N83 N.

N28

N123, N28, N32, N33, N63, N90 -

N

N17.

N.

N

N

N17

N198, N20, N25, N28, N41, N44, Z139, Z26, Z28, Z4

N

N 
Table 1. HATCHERIES, DEALERS, AND INDEPENDENT FLOCKS PARTICIPATING IN

THE NATIONAL POULTRY IMPROVEMENT PLAN

WATERFOWL, EXHIBITION POULTRY AND GAME BIRDS

\begin{tabular}{|c|c|c|c|c|c|}
\hline $\begin{array}{l}\text { APP. } \\
\text { NO. }\end{array}$ & $\begin{array}{l}\text { SUB- } \\
\text { PART }\end{array}$ & PARTICIPANTS NAME AND ADDRESS & $\begin{array}{l}\text { HATCHING } \\
\text { EGG } \\
\text { CAPACITY }\end{array}$ & $\begin{array}{l}\text { PRODUCTS CLASSIFIED } \\
\text { U.S. PULLORUM-TYPHOID } \\
\text { CLEAN }\end{array}$ & $\begin{array}{c}\text { ADDITIONAL } \\
\text { CLASSIFICATIONS FOR } \\
\text { WHICH PRODUCT QUALIFIED }\end{array}$ \\
\hline
\end{tabular}

\begin{tabular}{|c|c|c|}
\hline 2811 & E & JACK HAYS, RT. 2, BOX 119, PAULS VALLEY 73075 \\
\hline 1352 & E & $\begin{array}{l}\text { ODELL \& RODGER HEADRICK, P.O. BOX 694, DURANT } \\
74701\end{array}$ \\
\hline 2679 & E & $\begin{array}{l}\text { CARL D. HEARD, } 28415 \text { SOUTH } 4310 \text { ROAD, VINITA } \\
74301\end{array}$ \\
\hline 829 & $\mathrm{E}$ & ALBERT HEATH, RT. 3, BOX 328-B. NEWALLA 74857 \\
\hline 338 & E & $\begin{array}{l}\text { HEATH'S BANTAMS, } 13 \text { VERA LANE, SHAWNEE } 74801 \text { - } \\
3915\end{array}$ \\
\hline 2776 & $\mathrm{E}$ & $\begin{array}{l}\text { ODEL \& ROGER HEDRICK. } 1416 \text { HILLCREST STREET, } \\
\text { DURANT } 74702\end{array}$ \\
\hline 235 & E & $\begin{array}{l}\text { GLENN \& DEWAYNE HEFFINGTON. P.O. BOX } 123, \\
\text { BRADLEY } 73011\end{array}$ \\
\hline 1048 & $\mathrm{E}$ & JON HELLER, RT, 1, BOX 412, MULDROW 74948 \\
\hline 2696 & $\mathrm{E}$ & TERRY HELLER, P.O. BOX 264, GOTEBO 73041 \\
\hline 2303 & E & JOE HENLEY, 8015 NORTH 145 ROAD, BEGGS 74421 \\
\hline 2277 & $\mathrm{E}$ & $\begin{array}{l}\text { KYLE HENRY, } 2827 \text { EAST 176TH NORTH, SKIATOOK } \\
74070\end{array}$ \\
\hline 2431 & $\mathrm{E}$ & RICKY HENSLEY, RT. 1, BOX 196-25, CALERA 74730 \\
\hline 2591 & $E$ & JAMES HENSLEY, RT. 4, BOX 1240, STILWELL 74960 \\
\hline 2092 & $\mathrm{E}$ & CARMON HENSLEY, RT. 3, BOX 359, SALLISAW 74955 \\
\hline 1104 & $E$ & $\begin{array}{l}\text { DONNIE HENSON, } 14620 \text { EAST INDIAN HILL ROAD, } \\
\text { NEWALLA } 74857\end{array}$ \\
\hline 2387 & $E$ & DON HERELL, RT. 6, BOX 11. ARDMORE 73401 \\
\hline 1858 & E & LAVERNE HERRING, P.O. BOX 356, TIPTON 73570 \\
\hline 1196 & E & H. D. HESTER, 3201 SOUTH. HENNY, CHOCTAW 73020 \\
\hline 2750 & $E$ & HEY FAMILY, 1309 EAST FOX LANE, NEWCASTLE 73065 \\
\hline 2480 & E & $\begin{array}{l}\text { CAROLYN HICKS, } 621 \text { FLAMINGO AVENUE, OKLAHOMA } \\
\text { CITY } 73127\end{array}$ \\
\hline 1116 & $\mathrm{E}$ & $\begin{array}{l}\text { MARCY HICKS, } 15900 \text { SOUTH MERIDIAN AVENUE, } \\
\text { OKLAHOMA CITY } 73173\end{array}$ \\
\hline 712 & E & JIMMIE ROY HIEMER, P.O. BOX 193, MEEKER 74855 \\
\hline 2327 & $E$ & ROBERT HIGDON, RT. 1, BOX 360, TUTTLE 73089 \\
\hline 2373 & $\mathrm{E}$ & OLEN \& PAM HILL, P.O. BOX 402, SALLISAW 74955 \\
\hline 2568 & $\mathrm{E}$ & EDDIE HILL, RT. 3, BOX 1445, ATOKA 74525 \\
\hline 2620 & $\mathrm{E}$ & RICKY HILL, P.O. BOX 512, HUGO 74743 \\
\hline 1294 & E & STEVEN HILL, HC 83, BOX 472, ANTLERS 74523 \\
\hline 2182 & E & FRANK HILTON, HC 66, BOX 315, MOYERS 74557 \\
\hline 40 & $\mathrm{E}$ & BOBBY HINES, RT. 3, BOX 355-D 2. LINDSAY 73052 \\
\hline
\end{tabular}

N28, N44, N90

N17.

N121, N17, N28, N33, N41

N17

W232, W271, W81, W92

N17

N161, N174, N99

N17.

R222, R7, W27, W29, W88

N17

N44, N65, N8, N90

$\mathrm{N}$

N17.

N17.

N17

R42, W102, W17, W30, W370, W387

$\mathrm{N}$

W119. W28

N17, W25, W26, W588

N128, N28

N

N17.

$\mathrm{N} 148, \mathrm{~N} 17, \mathrm{~N} 43$

N17

N17

N.

N28, N32, N8. 
Table 1. HATCHERIES, DEALERS. AND INDEPENDENT FLOCKS PARTICIPATING IN

THE NATIONAL POULTRY IMPROVEMENT PLAN

WATERFOWL, EXHIBITION POULTRY AND GAME BIRDS

\begin{tabular}{|c|c|c|c|c|c|}
\hline $\begin{array}{l}\text { APP. } \\
\text { NO. }\end{array}$ & $\begin{array}{l}\text { SUB- } \\
\text { PART }\end{array}$ & PARTICIPANTS NAME AND ADDRESS & $\begin{array}{l}\text { HATCHING } \\
\text { EGG } \\
\text { CAPACITY }\end{array}$ & $\begin{array}{l}\text { PRODUCTS CLASSIFIED } \\
\text { U.S. PULLORUM-TYPHOID } \\
\text { CLEAN }\end{array}$ & $\begin{array}{c}\text { ADDITIONAL } \\
\text { CLASSIFICATIONS FOR } \\
\text { WHICH PRODUCT QUALIFIED }\end{array}$ \\
\hline
\end{tabular}
$\begin{array}{lll}2146 & E & \text { JERRY HINES, RT. 1, BOX 24, MINCO } 73059 \\ 2678 & \text { E } & \text { AMOS HINTON, } 513 \text { NORTH COYOTE STREET. } \\ & & \text { MARLAND } 74644\end{array}$

2374 E TERRAL HOGAN。417 ROUNDHOUSE ROAD, GUTHRIE 73044

2432 E VALDON L. HOLLAND, RT. 1, BOX 19-A, DAVIS 73030

2481 E MIKE HOLMES, P.O. BOX 400, MAYSVILLE 73057

1949 E WALLACE HOLMES, RT. 5, BOX 1338, MULDROW 74948

955 E LARRY HOLT, 4509 NE 48TH, OKLAHOMA CITY 73121

2214 E MIKE HOOKER, HC 64, BOX 1054, LOCUST GROVE 74352

1617 E JUSTIN \& MARY HOPPER, RT. 1, BOX 138, PRYOR 74361

2502 E LEANNE L. HORNBACK, RT. 1, BOX 101, PAWNEE 74058

2701 E BRYAN HOUGH, RT. 1, BOX 585, MUTUAL 73853

2178 E DAN HOUSER, RT. 2, BOX 45, WEWOKA 74884

2314 E BOBBY HOWARD, P.O. BOX 33, ELMORE CITY 73433

2328 E WILLIAML. HOWARD, 412 OAKRIDGE DRIVE, PAULS VALLEY 73075

2486 E BRIAN HOWARD, 318 NORTH DATE AVENUE, BROKEN

ARROW 74012

$956 \quad E$

$1184 \quad E$

$2686 \quad E$

$1647 \quad E$

1961

1295

1655

1715

2592

216

2569

2305

2304

986
SUNDOWN HOWARD, P.O. BOX 465, MANNFORD 74044

KENNETH HOWELL, RT. 5. BOX 1279, MULDROW 74948

WILLIAM B. HOWELL, RT. 2, BOX 118-A, MARLOW 73055

CHAD HUCKLEBERRY, RT. 2, BOX 217, KONAWA 74849

$E$

E

R. E. HUDSON, P.O. BOX 50, SMITHVILLE 74957

GEORGE W. HUFFMAN, 601 SOUTH ST. PAUL, MIDWEST CITY 73130

E STEVEN \& DIANA HUGHES, 105 WEST FRANKLIN STREET, MANGUM 73554

E BO HUGHES, 336 SOUTH ELM STREET, NOWATA 74048

ROGER HUGHES, RT. 2, BOX 245-5, NOWATA 74048

E ALVA HULL, RT. 2, BOX 96-A, WELLSTON 74881

E RANDY HULL, 7711 EAST HANCOCK, MUSKOGEE 74403

E LARRY D. HULL, RT. 2, BOX 45, MARIETTA 73448

E LARRY HULL, RT. 2, BOX 62, MARIETTA 73448

E
Z43

H5, H8, R15, R208, R222, R86, W107, W123, W130, W166, W20, W3, W306, W348, W57, $W 74, X 135, X 17, Y 3$

N28, N34

N19

$\mathrm{N} 17, \mathrm{Z}$

$\mathrm{N} 17$

N

N

W32, w86, w94

N141, N155, N194.

N24, N65, N94

R117, W484, W578

$\mathrm{N}$

N28, N44, N90

N17

$R, X, Y, Z$

N28, N32, N41

N28, N32, N34

N41

W20

N17

N127, N28, N32, N33, N41

N28, N32, N33

N109, N131, N183, N28, N41

N28

N

N

N228 
HATCHING
PRODUCTS CLASSIFIED

U.S. PULLORUM-TYPHOID CLEAN
ADDITIONAL CLASSIFICATIONS FOR WHICH PRODUCT QUALIFIED

\section{3 - OKLAHOMA}

\begin{tabular}{|c|c|c|}
\hline 830 & $E$ & DANNY HUNT, RT. $1,80 \times 84$, GORE 74435 \\
\hline 2194 & $E$ & MICKEY HUNT, RT. 2, BOX 38-E, SKIATOOK 74070 \\
\hline 2315 & E & RANDALL HUNTER, P.O. BOX 241, BOKOSHE 74930 \\
\hline 1082 & $E$ & REX HURD, HC 61, BOX 23, ALEX 73002 \\
\hline 808 & $\mathrm{E}$ & HENRY HUSSFELDT, P.O. BOX 36, BLANCHARD 73010 \\
\hline 2702 & $\mathrm{E}$ & $\begin{array}{l}\text { JAMES HUTCHINS, } 605 \text { SE COUNTY ROAD } 3140 \text {, } \\
\text { CORSICANA } 75109\end{array}$ \\
\hline 1280 & $\mathrm{E}$ & $\begin{array}{l}\text { INDIAN HILLS GAME FARM, CHARLES WILSON, RT. } 3 \text {, } \\
\text { BOX } 290 \text { W-W, TECUMSEH } 74873-9362\end{array}$ \\
\hline 2388 & $E$ & DENNIS INGERHAM, 2610 SE 156TH, NORMAN 73026 \\
\hline 2389 & $\mathrm{E}$ & $\begin{array}{l}\text { HAL INGERHAM, } 16201 \text { EAST BANNER ROAD, } \\
\text { LEXINGTON } 73051\end{array}$ \\
\hline 2282 & $E$ & BILL INGRAM, RT. 2, BOX 419, DUNCAN 73533 \\
\hline 1004 & $\mathrm{E}$ & $\begin{array}{l}\text { ROY \& TERESSA INGRAM, } 7275 \text { SOULIGNY ROAD, } \\
\text { PONCA CITY } 74601\end{array}$ \\
\hline 2237 & $\mathrm{E}$ & JOHN IVEY, P.O. BOX 2022, SAPULPA 74067 \\
\hline 2224 & $\mathrm{E}$ & J \& D GAME FARM, P.O. BOX 86, KEOTA 74941 \\
\hline 1625 & $\mathrm{E}$ & $\begin{array}{l}\text { J-J POULTRY, JACKIE COOK, RT. 3, BOX 121, CORDELL } \\
73632\end{array}$ \\
\hline 957 & $\mathrm{E}$ & ROBERT JACKSON, RT. 3, BOX 652, MULDROW 74948 \\
\hline 1891 & E & BOBBY JACKSON, RT. 2, BOX 582, CLEVELAND 74020 \\
\hline 2112 & $\mathrm{E}$ & KENNY JACKSON, HC 69, BOX 74, PARK HALL 74451 \\
\hline 2205 & $\mathrm{E}$ & JOHN JACKSON, P.O. BOX 1944, GROVE 74344 \\
\hline 2375 & $\mathrm{E}$ & JOE JACKSON, RT. 2, BOX 253-A, BOKCHITO 74726 \\
\hline 2570 & $\mathrm{E}$ & MIKE JACKSON, P.O. BOX 1273, MULDROW 74948 \\
\hline 2632 & $\mathrm{E}$ & $\begin{array}{l}\text { WILLIAM JACKSON, HC 73, BOX 232-X, BURNEYVILLE } \\
73430\end{array}$ \\
\hline 2487 & $\mathrm{E}$ & RONALD G. JACKSON, R1. 1, BOX 130, CANEY 74533 \\
\hline 2433 & $E$ & ROBERT JACKSON, RT, 2, BOX 26, WOODWARD 73801 \\
\hline 2618 & E & $\begin{array}{l}\text { JAMES FAMILY, } 2033 \text { S HIGHWAY 76, NEWCASTLE } \\
73065\end{array}$ \\
\hline 1284 & $\mathrm{E}$ & TONY JAMES, RT. 2, BOX 556-3, BROKEN BOW 74728 \\
\hline 2761 & $\mathrm{E}$ & FRANK JARED, P.O. BOX 549, FREDERICK 73542 \\
\hline 2531 & $\mathrm{E}$ & $\begin{array}{l}\text { ROGER JEFFCOATS, } 2405 \text { MCCLAIN ROAD, ARDMORE } \\
73401\end{array}$ \\
\hline 2353 & $\mathrm{E}$ & JON JENKINS, P.O. BOX 336, BOWLEGS 74830 \\
\hline 2673 & E & $\begin{array}{l}\text { JACK JENKINS, } 1004 \text { GRAND TERRACE, CHICKASHA } \\
73018\end{array}$ \\
\hline
\end{tabular}

N28, N41

N

N28, N33, N41, N44, N90

N28, N32, N44

N116, N28, N31, N44

N28, N32, N34, N44_

N121, N137, N24, N28, N32, N34. N41, N44, N90, N94

N17.

N17. N41

W12, W128, W175, W187, W28, W294, W295

N.

N17

N22, N28, N43, N44

$X, Y, Z$

N17

W12, W13, W198, W207, W30, W4, W54, W544, W560, W69

N.

N17

N17

N19

N17.

N.

W119, W123

N.

N32, N44, N90

N17.

N134, N32, N87

B1, W115, W18 
Table 1. HATCHERIES, DEALERS, AND INDEPENDENT FLOCKS PARTICIPATING IN

THE NATIONAL POULTRY IMPROVEMENT PLAN

WATERFOWL. EXHIBITION POULTRY AND GAME BIRDS

\begin{tabular}{|c|c|c|c|c|c|}
\hline $\begin{array}{l}\text { APP. } \\
\text { NO. }\end{array}$ & $\begin{array}{l}\text { SUB- } \\
\text { PART }\end{array}$ & PARTICIPANTS NAME AND ADDRESS & $\begin{array}{l}\text { HATCHING } \\
\text { EGG } \\
\text { CAPACITY }\end{array}$ & $\begin{array}{l}\text { PRODUCTS CLASSIFIED } \\
\text { U.S. PULLORUM-TYPHOID } \\
\text { CLEAN }\end{array}$ & $\begin{array}{c}\text { ADDITIONAL } \\
\text { CLASSIFICATIONS FOR } \\
\text { WHICH PRODUCT QUALIFIED }\end{array}$ \\
\hline
\end{tabular}

\begin{tabular}{|c|c|c|c|}
\hline 238 & $E$ & JOHN JENKINS, RT. 1, BOX 410, TERLTON 74084 & R19, W120, W19, W30, W35 \\
\hline 1414 & $\mathrm{E}$ & $\begin{array}{l}\text { CARLISLE \& KAY JENKS. RT. 3, BOX 334, TECUMSEH } \\
74873\end{array}$ & $\begin{array}{l}\text { R22, W11, W12, W54, W56, W6, } \\
\text { W88 }\end{array}$ \\
\hline 2783 & E & $\begin{array}{l}\text { JOHNATHAN LANE, } 9855 \text { EAST 96TH PLACE, TULSA } \\
74133\end{array}$ & -non \\
\hline 1339 & E & J. L. JOHNS, RT. 5, BOX 570, MCALESTER 74501 & N17 \\
\hline 1085 & $E$ & KENNY JOHNSON, RT. 1, BOX 430, WELLING 74471 & N109, N28, N33, N63 \\
\hline 1086 & E & TIM JOHNSON, RT. 1, BOX 430, WELLING 74471 & N107, N32, N43 \\
\hline 1105 & E & $\begin{array}{l}\text { ROBERT JOHNSON, } 7501 \text { SW 109TH, OKLAHOMA CITY } \\
73173\end{array}$ & N152, N236, N34___ \\
\hline 1929 & E & $\begin{array}{l}\text { KENNETH JOHNSON, } 1828 \text { EAST 54TH NORTH, TULSA } \\
74130\end{array}$ & $\mathrm{~N}$ \\
\hline 1994 & E & RAYMOND JOHNSON, RT. 1, BOX 470, CAMERON 74932 & N28, N41 \\
\hline 1544 & E & JOSEPH JOHNSON, P.O. BOX 44, PANAMA 74951 & N41, N63__. \\
\hline 2703 & E & TERRY JOHNSON, HC 67, BOX 3A, RATLIFF CITY 73481 & W89 \\
\hline 2164 & $E$ & JOE JOHNSON, 1005 WEST TISH, MADILL 73446 & $\mathrm{~N}$ \\
\hline 2330 & $E$ & DOUGLAS JOHNSON, P.O. BOX 12, KELLYVILLE 74039 & $\mathrm{~N} 32, \mathrm{~N} 41, \mathrm{~N} 90$ \\
\hline 2329 & $E$ & DANNY JOHNSON, RT. 3, BOX 160-B, ANADARKO 73005 & N110, N28, N32. \\
\hline 2225 & $\mathrm{E}$ & DARRYEL JONES, P.O. BOX 535, COLBERT 74733 & N24__ \\
\hline 2226 & E & JACKIE JONES, RT. 2, BOX 23-A, SKIATOOK 74070 & R151, R95 _ \\
\hline 2571 & $E$ & STEVEN JONES, RT. 1, BOX 1217, BUNCH 74960 & -_-n-1-n \\
\hline 2434 & $E$ & KENT JONES, 1900 PATRIOT LANE, HARRAH 73045 & N17__.................. \\
\hline 2151 & $E$ & $\begin{array}{l}\text { JACK JONES, } 8649 \text { SOUTH 161ST WEST AVENUE, } \\
\text { SAPULPA } 74066\end{array}$ & ב- \\
\hline 1164 & E & J. D. JORDAN, RT. 5, BOX 1635, STILWELL 74960 & N17 \\
\hline 2260 & $\mathrm{E}$ & $\begin{array}{l}\text { JAKE KANNADY, } 11119 \text { EAST 106TH STREET NORTH. } \\
\text { OWASSO } 74055\end{array}$ & 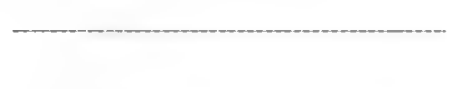 \\
\hline 1188 & $E$ & $\begin{array}{l}\text { CHARLES KEAR, } 10901 \text { SOUTH ANDERSON ROAD, } \\
\text { OKLAHOMA CITY } 73165\end{array}$ & N17 \\
\hline 1930 & $\mathrm{E}$ & KENNETH KEELER, RT. 2, BOX 251, BLANCHARD 73010 & N28, N32 \\
\hline 934 & $E$ & $\begin{array}{l}\text { ROBERT KEESE, } 2201 \text { SOUTH DOBBS ROAD. HARRAH } \\
73045\end{array}$ & N28, N32, N41_ \\
\hline 2196 & $E$ & EDDIE KEITH, 6451 NW 220TH. EDMOND 73003 & N_- \\
\hline 1497 & E & MATTHEW KELLEY, P.O. BOX 354, NINNEKAH 73067 & W6 \\
\hline 430 & $E$ & $\begin{array}{l}\text { DONALD E. KELSEY, } 4225 \text { WEST 82ND STREET, TULSA } \\
74132-3021\end{array}$ & $\ldots$ \\
\hline 1441 & $E$ & JIMMY KENNEDY, RT 2, BOX 160, LINDSAY 73052 & N17 \\
\hline 2104 & $E$ & JOE KENNEDY, HC 69, BOX 240, FINLEY 74543 & N17 \\
\hline
\end{tabular}


HATCHING

EGG

CAPACITY
PRODUCTS CLASSIFIED

U.S. PULLORUM-TYPHOID CLEAN
ADDITIONAL CLASSIFICATIONS FOR WHICH PRODUCT QUALIFIED

73 - OKLAHOMA

\begin{tabular}{|c|c|c|}
\hline 2633 & $\mathrm{E}$ & ALLEN KENT, RT. 1, BOX 26, COLCORD 74338 \\
\hline 2751 & $\mathrm{E}$ & R.C. KERNS, RT. 1, BOX 129-B, WEWOKA 74884 \\
\hline 2572 & $\mathrm{E}$ & GENE KETCHER, P.O. BOX 894, STILWELL 74960 \\
\hline 2601 & E & NEIL. KETCHUM, P.O. BOX 144, INDIANOLA 74442 \\
\hline 832 & E & $\begin{array}{l}\text { TONY KILLGORE, } 739 \text { SOUTH MARKET ROAD, } \\
\text { MCCLOUD } 74851\end{array}$ \\
\hline 2179 & E & KEITH KIMBLE, RT. 1, BOX 59, HYDRO 73048 \\
\hline 2021 & $\mathrm{E}$ & LARRY KINDER, RT. 2, BOX 858, CARNEGIE 73015 \\
\hline 1754 & $\mathrm{E}$ & KEN KING, 23750 COUNTY ROAD 220, MORRISON 73061 \\
\hline 2532 & E & CLIFF KING, RT. 1, BOX 85-AA, MEEKER 74855 \\
\hline 240 & $\mathrm{E}$ & $\begin{array}{l}\text { DEAN \& CHRISTINA KISER, } 14400 \text { SOUTH } \\
\text { WESTMINISTER, ARCADIA } 73007\end{array}$ \\
\hline 2634 & $\mathrm{E}$ & JERRY L. KLEIN, RT. 1, BOX 367, ELMORE CITY 73433 \\
\hline 923 & $\mathrm{E}$ & JAMES D. KNIGHT, 5101 LAKE DRIVE, HARRAH 73045 \\
\hline 1507 & 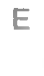 & $\begin{array}{l}\text { BOB KNIGHT, KNIGHT RIDER, RT. 6, BOX } 790 \text {, } \\
\text { BLANCHARD } 73010\end{array}$ \\
\hline 2306 & E & TIM KNOWLES, RT. 1, BOX 730, CEMENT 73017 \\
\hline 935 & E & BENNY KOGER, 8226 EAST 129TH, WETUMKA 74883 \\
\hline 2435 & E & $\begin{array}{l}\text { TOMMY KUEPKER, } 18430 \text { VALLEY DRIVE, NORMAN } \\
73026\end{array}$ \\
\hline 1052 & $E$ & JOHN KULL, RT. 1, TALIHINA 74571 \\
\hline 2436 & $E$ & LEON LABOR, P.O. BOX 89, KIOWA 74553 \\
\hline 2503 & $\mathrm{E}$ & LEON LABORS, P.O. BOX 89, KIOWA 74553 \\
\hline 2715 & $E$ & ROBERT LACKEY, RT, 3, BOX 408-D, WELLSTON 74881 \\
\hline 2473 & $\mathrm{E}$ & EMIL LACQUEMENT, RT. 1, BOX 137-A, MACOMB 74852 \\
\hline 2376 & E & $\begin{array}{l}\text { JAMES \& PHILLIP LACY, } 520 \text { COUNTRY CLUB ROAD, } \\
\text { DURANT } 74701\end{array}$ \\
\hline 1689 & $\mathrm{E}$ & THEO N. LAMB, RT. 1, BOX 235, MARLOW 73055 \\
\hline 2593 & E & KEVIN LANDIS, P.O. BOX 176, AVANT 74001 \\
\hline 2288 & $\mathrm{E}$ & $\begin{array}{l}\text { DONNA J. LANE, } 14593 \text { SOUTH HIGHWAY } 48 \text {, BRISTOW } \\
74010\end{array}$ \\
\hline 2619 & E & JOHN LANGLEY, 7700 W OAKLAND, PONCA CITY 74601 \\
\hline 1007 & $\mathrm{E}$ & $\begin{array}{l}\text { RONALD \& MIKE LANGLEY, RT. 2, BOX 617, SALINA } \\
74365\end{array}$ \\
\hline 242 & E & $\begin{array}{l}\text { RICHARD D. LANGSTON, } 2103 \text { WEST 121ST STREET, } \\
\text { SAPULPA } 74066\end{array}$ \\
\hline 2474 & $E$ & RUSSELL LANHAM, P.O. BOX 1011 , EUFAULA 74432 \\
\hline
\end{tabular}

N.

N17

N33, N43, N90

$A 1, B 1, N 28, N 32, N 83, W 12$, $W 132, W 150, X 22, Y 4$

N128, N28, N33

N19, R244, W316, W393, W511_.

N17.

Z243. Z9

N19. W139

W120, W387, w7

N157, N93

N17

N17

N.

N17.

N17

N28, N41

N28, N32

N32, N33, N41, N43

N17

N121, N17, N41, N43, N56, N57, N65

N17

N125, N28, N32, N41

N17, N28, N41

N109, N17, N33, N44

N17

R168, R3, W270, W271, W37, W54. W86

R183, R192, R247, R253, W30, W35, W64, W7

N17. 
Table 1. HATCHERIES, DEALERS, AND INDEPENDENT FLOCKS PARTICIPATING IN THE NATIONAL POULTRY IMPROVEMENT PLAN

WATERFOWL, EXHIBITION POULTRY AND GAME BIRDS

\begin{tabular}{|c|c|c|c|c|c|}
\hline $\begin{array}{l}\text { APP. } \\
\text { NO. }\end{array}$ & $\begin{array}{l}\text { SUB- } \\
\text { PART }\end{array}$ & PARTICIPANTS NAME AND ADDRESS & $\begin{array}{l}\text { HATCHING } \\
\text { EGG } \\
\text { CAPACITY }\end{array}$ & $\begin{array}{l}\text { PRODUCTS CLASSIFIED } \\
\text { U.S. PULLORUM-TYPHOID } \\
\text { CLEAN }\end{array}$ & $\begin{array}{c}\text { ADDITIONAL } \\
\text { CLASSIFICATIONS FOR } \\
\text { WHICHPRODUCT QUALIFIED }\end{array}$ \\
\hline
\end{tabular}

2727 E KEVIN LARSH, RT. 1, BOX 40-B, ROFF 74865

2331 E ROBIN LAWSON, 6606 SOUTH 80TH WEST AVENUE, TULAS 74107

2354 E JOHN LAWSON, RT. 1, BOX 1760, TALALA 74080

1817 E LAZY D ACRES, ROBERT LOPEZ, 1B LAZY D ACRES, BLANCHARD 73010

2294 E JAMES W. LEE, P.O. BOX 367, SAND SPRINGS 74063

2752 E WILLIAM LEE, HC 70, BOX 227M, ARDMORE 73401

1962 E RICKY LEMONS, P.O. BOX 424, CALERA 74730

1619 E WD "BILL" LEONARD, 628 SOUTH 18TH, CLINTON 73601

1656 E BUDDY LEWIS, P.O. BOX 443, SEMINOLE 74868

2674 E JERRY LEWIS, RT. 1, BOX 360, MANNFORD 74044

2728 E VIRGIL \& ARLENE LEWIS, HC 68, BOX 415, CHECOTHA 74426

2762 E ELMO LEWIS, RT. 1, BOX 300-A, MACOMB 74852

2215 E B.J.LEWIS, 8912 SOUTH 33RD WEST AVENUE, TULSA 74132

2627 E JOSH LINDAMOOD, P.O. BOX 1092, TUTTLE 73089

2152 E BRYAN LINDLEY, RT. 2, BOX 10-A, HINTON 73047

2390 E DENNIS LINDSEY, 5026 SOUTH HIGHWAY 77, PURCELL 73080

1950 E BILLY LINNE, P.O. BOX 328, HOWE 74940

2628

2332

2180

2183

2093

1951

1952

1607

2650
N107, N28, N32, N44

N17

N125, N28, N32, N91

N.

N17

N41, N65

R196, W119, W120, W23, W54

N22, N34, N36, N41

N28

B1, C1

N17

N131, N32, N65

W28

N17

N17

N104, N28, N32, N41

N28, N32, N41, N44

N.

$R, W$

N

N90

N.

N32, N44, N90

N17

A6, R19, R81, W60, W75, Y4, Z205, Z212, Z22, Z62

N28, N41

N17

N155, N164, N32, N58, N80 
Table 1. HATCHERIES, DEALERS, AND INDEPENDENT FLOCKS PARTICIPATING IN

THE NATIONAL POULTRY IMPROVEMENT PLAN

WATERFOWL, EXHIBITION POULTRY AND GAME BIRDS

\begin{tabular}{|c|c|c|c|c|c|}
\hline $\begin{array}{l}\text { APP. } \\
\text { NO. }\end{array}$ & $\begin{array}{l}\text { SUB- } \\
\text { PART }\end{array}$ & PARTICIPANTS NAME AND ADDRESS & $\begin{array}{l}\text { HATCHING } \\
\text { EGG } \\
\text { CAPACITY }\end{array}$ & $\begin{array}{l}\text { PRODUCTS CLASSIFIED } \\
\text { U.S. PULLORUM-TYPHOID } \\
\text { CLEAN }\end{array}$ & $\begin{array}{c}\text { ADDITIONAL } \\
\text { CLASSIFICATIONS FOR } \\
\text { WHICH PRODUCT QUALIFIED }\end{array}$ \\
\hline
\end{tabular}

\begin{tabular}{|c|c|c|}
\hline 1879 & E & $\begin{array}{l}\text { MACK MAHSETKY, } 630 \text { EAST OHIO STREET, WALTERS } \\
73572\end{array}$ \\
\hline 2603 & $E$ & CHARLES MAKEY, RT. 2, BOX 140, BLANCHARD 73010 \\
\hline 2391 & $E$ & CHIP MALCHOW, P.O. BOX 96, ADAIR 74330 \\
\hline 718 & E & LEROY MANTOOTH, 114 NORTH 6TH, YUKON 73099 \\
\hline 2437 & $E$ & MELVIN MANUEL, RT. 8, BOX 196, ADA 74820 \\
\hline 1679 & $E$ & BOB MARKHOLT, RT. 2, BOX 59-A, LINDSAY 73052 \\
\hline 2377 & E & DAVID MARR, 917 H STREET NE, ARDMORE 73401 \\
\hline 2438 & E & JERRY MARSHALL, RT. 1, BOX 714, MEEKER 74855 \\
\hline 2439 & $\mathrm{E}$ & $\begin{array}{l}\text { JERRY MARSHALL, } 1022 \text { HARBER STREET, SEMINOLE } \\
74868\end{array}$ \\
\hline 2334 & E & CHRIS MARTIN, RT. 2, BOX 237-B10, SALLISAW 74955 \\
\hline 1680 & $E$ & NOEL MARTIN, RT. 1, BOX 83, KENEFIC 74748 \\
\hline 1587 & $E$ & JESUS MARTINEZ. RT. 5, BOX 139, JAY 74346 \\
\hline 2335 & E & $\begin{array}{l}\text { VICTOR MARTINEZ, } 3215 \text { WEST } 81 S T \text { STREET, TULAS } \\
74132\end{array}$ \\
\hline 2573 & $E$ & $\begin{array}{l}\text { FRANCISCO MARTINEZ, P.O. BOX 1072, STILWELL } \\
74760\end{array}$ \\
\hline 2378 & E & RENE MARTINEZ, 421 LIVE OAK, ALTUS 73521 \\
\hline 2147 & $E$ & MICHAEL MASON, RT. 3, BOX 343, WALTERS 73572 \\
\hline
\end{tabular}

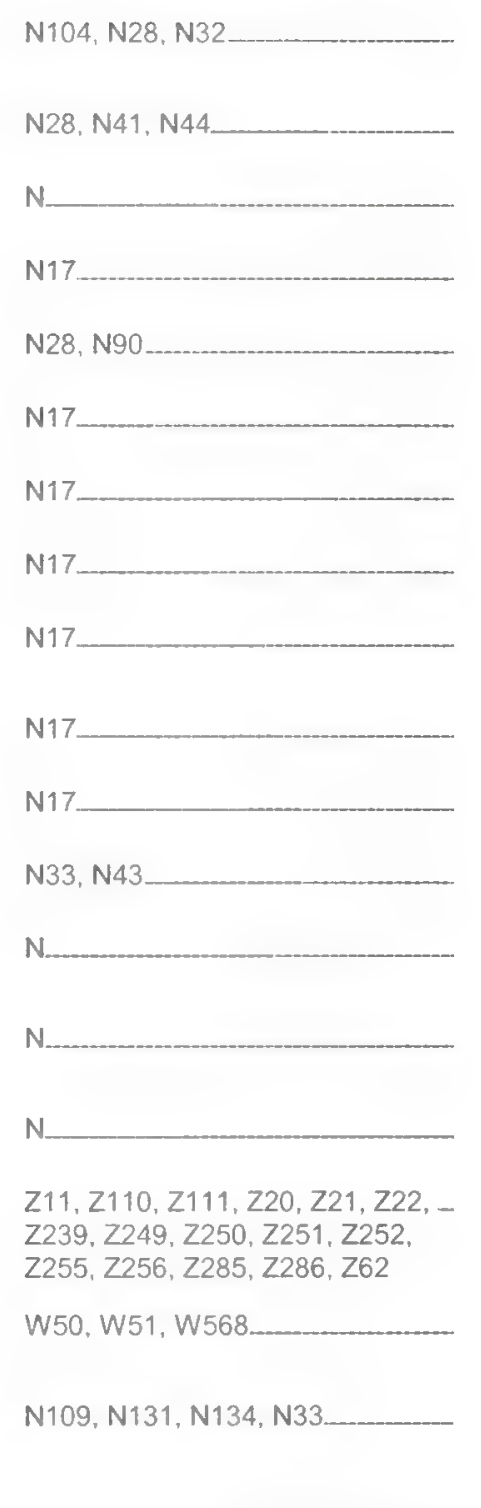

W102, W121, W140,W168 W169, W17, W18, W223, W60 N134, N17.

N17

$\mathrm{N}$

W17, W28, W45, W48, W50, $W 88, \times 10, \times 17$

N

$\mathrm{N} 28, \mathrm{~N} 32, \mathrm{~N} 44$

R22, R27, W20, W23, W38, W54, W7, X135, X96, Y21

W19. W20 
Table 1. HATCHERIES, DEALERS, AND INDEPENDENT FLOCKS PARTICIPATING IN

THE NATIONAL POULTRY IMPROVEMENT PLAN

WATERFOWL, EXHIBITION POULTRY AND GAME BIRDS

\begin{tabular}{lllll}
\hline & & HATCHING & PRODUCTS CLASSIFIED \\
APP. SUB- & PARTICIPANTS NAME AND ADDRESS & EGG & U.S. PULLORUM-TYPHOID & CLLASSIFICATIONAL \\
NO. PART & CAPACITY & CLEAN & WHICHPRODUCT QUALIFIED \\
\hline
\end{tabular}

73 - OKLAHOMA

\begin{tabular}{|c|c|c|}
\hline 780 & $E$ & DON MCCLURE, RT. 2, BOX 198, TAHLEQUAH 74464 \\
\hline 1977 & $E$ & $\begin{array}{l}\text { ROBERT MCCONNELL, } 2949 \text { INDIAN HILLS ROAD, } \\
\text { PONCA CITY } 74601\end{array}$ \\
\hline 2238 & E & DEWAYNE MCCOY, RT. 3, BOX 128, BLANCHARD 73010 \\
\hline 1913 & E & CHRIS MCCRAW, P.O. BOX 1762, COLBERT 74733 \\
\hline 2705 & E & $\begin{array}{l}\text { KYLER MCCUTCHEN, } 40491 \text { NORTH } 3950 \text { ROAD, } \\
\text { SKIATOOK } 74070\end{array}$ \\
\hline 2283 & $\mathrm{E}$ & $\begin{array}{l}\text { KYLER MCCUTHEN, } 414 \text { NORTH QUAPAW, SKIATOOK } \\
74070\end{array}$ \\
\hline 1088 & $E$ & CLARENCE MCDANIEL, RT. 2, BOX 736, HUGO 74743 \\
\hline 2318 & $\mathrm{E}$ & $\begin{array}{l}\text { HARROLD MCDERMOTT, RT. 2, BOX 59-A, LINDSAY } \\
73052\end{array}$ \\
\hline 2763 & $\mathrm{E}$ & JOHN MCDONALD, P.O. BOX 133, ROSE 74364 \\
\hline 2062 & $E$ & EDWARD MCDONALD, P.O. BOX 173 , KENEFIC 74748 \\
\hline 2629 & $\mathrm{E}$ & ERICK MCGAHAN, P.O. BOX 209, BURNEYVILLE 73430 \\
\hline 2441 & $E$ & JEFF MCGEE, RT. 1, BOX 220-B, DAVIS 73030 \\
\hline 1394 & $\mathrm{E}$ & MARVIN MCGEHEE, P.O. BOX 580175, TULSA 74158 \\
\hline 1498 & $E$ & FRED MCGEHEE, 7320 EAST 22ND PLACE, TULSA 74129 \\
\hline 2594 & $E$ & $\begin{array}{l}\text { BOBBY MCGHEE, } 535 \text { SOUTH 55TH WEST AVENUE, } \\
\text { TULSA } 74126\end{array}$ \\
\hline 2181 & $E$ & $\begin{array}{l}\text { LEW MCGINNIS FAMILY, } 2550 \text { OVERHOLSER, } \\
\text { OKLAHOMA CITY } 73127\end{array}$ \\
\hline 2355 & $\mathrm{E}$ & LYNDALL MCGLOCKLIN, RT, 1, BOX 123, MILBURN 73450 \\
\hline 888 & $\mathrm{E}$ & BILL MCGUIRE, P.O. BOX 841, VELMA 73491 \\
\hline 2249 & $\mathrm{E}$ & TIM MCKEE, 408 WEST D STREET, ELMORE CITY 73433 \\
\hline 2789 & $\mathrm{E}$ & $\begin{array}{l}\text { STEVE MCMILLEN, } 301 \text { EAST FRISCO STREET, ROFF } \\
74865\end{array}$ \\
\hline 1357 & E & BILL MCNATT, P.O. BOX 432, KEOTA 74941 \\
\hline 748 & $\mathrm{E}$ & C. DAVID MCPEAK, RT. 1, BOX 12, SHIDLER 74652 \\
\hline 2764 & $\mathrm{E}$ & LORENZO MEDINA, 408 F STREET NE, MIAMI 74354 \\
\hline 2753 & E & COY MEEKS, RT. 1, BOX 819, HARRAH 73045 \\
\hline 2052 & $\mathrm{E}$ & $\begin{array}{l}\text { LLOYD MEHR, } 5000 \text { ROYAL RIDGE ROAD, OKLAHOMA } \\
\text { CITY } 73135\end{array}$ \\
\hline 666 & $\mathrm{E}$ & BOB MELTON, RT, 4, BOX 410, MUSKOGEE 74401 \\
\hline 1914 & $E$ & MIKE MELTON, RT. 2, BOX 152, DAVIS 73030 \\
\hline 2804 & E & MENEFEE POULTRY, 922 SW 24, NEWCASTLE 73065 \\
\hline 2630 & E & GARY MERIDITH, RT. 1, BOX 414, PROCTOR 74457 \\
\hline
\end{tabular}

N

N.

N32, N41, N43

N17.

N17

$\mathrm{N}$

$\mathrm{N}$

N17

N32, N44, N9

W128, W50, W568, W69, W88

N28.

$\times 10, \times 17, \times 2$

N17

N28, N41, N44

N1, N125, N28

N

N

N104, N28, N32, N41

$\mathrm{N}$

N17

N.

N134, N137, N34, N46

N28, N34, N44

R96, W12, W7

N 
Table 1. HATCHERIES, DEALERS, AND INDEPENDENT FLOCKS PARTICIPATING IN

THE NATIONAL POULTRY IMPROVEMENT PLAN

WATERFOWL, EXHIBITION POULTRY AND GAME BIRDS

\begin{tabular}{|c|c|c|c|c|}
\hline $\begin{array}{l}\text { APP. } \\
\text { NO. }\end{array}$ & $\begin{array}{l}\text { SUB- } \\
\text { PART }\end{array}$ & PARTICIPANTS NAME AND ADDRESS & $\begin{array}{l}\text { HATCHING } \\
\text { EGG } \\
\text { CAPACITY }\end{array}$ & $\begin{array}{l}\text { PRODUCTS CLASSIFIED } \\
\text { U.S. PULLORUM-TYPHOID } \\
\text { CLEAN }\end{array}$ \\
\hline & & 73 & - OKLAHOMA & \\
\hline 1892 & $\mathbf{E}$ & SHELBY MERRILL, P.O. BOX 1231, SALLISAW 74955 & & N17 \\
\hline 2790 & $E$ & BILLY MERRITT, P.O. BOX 765, RUSH SPRINGS 73082 & & N24, N28, N41 \\
\hline 1931 & $E$ & $\begin{array}{l}\text { WALTER MERRYMAN, } 16015 \text { HEMLOCK DRIVE, } \\
\text { NEWALLA } 74857\end{array}$ & & N17______ \\
\hline 2261 & $\mathbf{E}$ & $\begin{array}{l}\text { SHANE METCALF, } 10720 \text { COKER ROAD, SHAWNEE } \\
74804\end{array}$ & & $N 17+2$ \\
\hline 373 & $E$ & $\begin{array}{l}\text { METHENY FAMILY POULTRY, } 39 \text { S.E. 23RD STREET, } \\
\text { EDMOND } 73013\end{array}$ & & $\begin{array}{l}\text { R12, R237, R81, W162, W23, - } \\
\text { W26, W302 }\end{array}$ \\
\hline 258 & $\mathbf{E}$ & LEON MEYER, RT. 1, BOX 6, FAIRMONT 73736 & & $\begin{array}{l}\text { H8, R227, R237, W102, W120, } \\
\text { W121, W17, W18, W198, W4, } \\
x 146, x 43, x 53, x 6, x 63\end{array}$ \\
\hline 1978 & $\mathbf{E}$ & BOBBY MILBURN, RT. 1, BOX 11, RATLIFF CITY 73481 & & N125, N128, N28, N32, N41, N44. \\
\hline 2199 & $\mathbf{E}$ & BILLY MILES, 25355 S. 4375 DRIVE, VINITA 74301 & & $\mathrm{~N} 28, \mathrm{~N} 44$ \\
\hline 2201 & $\mathrm{E}$ & JOHNNY MILLER, RT. 1, BOX 176, WELLSTON 74881 & & N17 ___ \\
\hline 977 & $E$ & MARK MILLER, RT. 3, BOX 219, DURNAT 74701 & & $\mathrm{~N} 28, \mathrm{~N} 32, \mathrm{~N} 44$ \\
\hline 1732 & $\mathbf{E}$ & BOB MILLER, RT. 1, BOX 890, BUNCH 74931 & & N17 _._._._. \\
\hline 1363 & $\mathbf{E}$ & DANNY MILLER, RT. 1, BOX 2750, ANTLERS 74523 & & $N 17 \ldots$ \\
\hline 2729 & $\mathbf{E}$ & $\begin{array}{l}\text { LARRY MILLIGAN, } 2801 \text { WEST COTTONWOOD ROAD, } \\
\text { TISHOMINGO } 73460\end{array}$ & & W115, W187, W28 \\
\hline 2754 & $E$ & TERRY MILLS, 110 JOHNSON, KENEFIC 74748 & & N24_____n_............. \\
\hline 2730 & $E$ & $\begin{array}{l}\text { RICK MINCHER, } 1012 \text { SOUTH CHEYENNE ROAD, } \\
\text { CADDO } 74729\end{array}$ & & N17 \\
\hline 722 & $\mathbf{E}$ & DON MINK, RT. 1, BOX 45, CEMENT 73017 & & N_- \\
\hline 2731 & $E$ & RICHARD MITCHELL, RT. 1, BOX 21, COLCORD 74337 & & $\mathrm{~N}$ \\
\hline 2661 & $E$ & TROY MITCHUM, RT. 2, BOX 220, BLANCHARD 73010 & & $N_{-}$ \\
\hline 1483 & $E$ & $\begin{array}{l}\text { JERRY MODENA, } 65 \text { INDUSTRIAL PARK, WOODWARD } \\
73801\end{array}$ & & $\begin{array}{l}\text { N5, R12, R52, W275, W28, } \\
\text { W394, W395, W6, X10, X17, V14 }\end{array}$ \\
\hline 2273 & $\mathbf{E}$ & $\begin{array}{l}\text { JAMES MOENING, } 9288 \text { HIGHWAY } 48 \text { SOUTH, } \\
\text { MANNFORD } 74044\end{array}$ & & $\begin{array}{l}\text { H5, H8, N17, R19, R196, R20, - } \\
\text { R30, R40, R56, R7 }\end{array}$ \\
\hline 2528 & $E$ & $\begin{array}{l}\text { GENE CODY/ABRAHAM MOLINA, P.O. BOX 202, RATTAN } \\
74562\end{array}$ & & \\
\hline 1703 & $\mathbf{E}$ & ISAAC MONDEN, 6050 120TH, NOBLE 73068 & & N17 \\
\hline 751 & $E$ & TOMMY MONKS, RT. 1, BOX 96-A, SEMINOLE 74868 & & N134, N161, N24, N32, N87 \\
\hline 2392 & $\mathbf{E}$ & JOHN MONTENRY, RT. 1, BOX 60, RED OAK 74567 & & $\mathrm{~N} 21, \mathrm{~N} 28$ \\
\hline 2595 & $E$ & FRANKLIN MONTGOMERY, RT. 1, BOX 241, SPIRO 74959 & & N129, N28, N44, N90_. \\
\hline 381 & $\mathbf{E}$ & $\begin{array}{l}\text { MOONLIGHT STABLES/JOE SIMPSON, } 24971 \text { SOUTH } 638 \\
\text { ROAD, GROVE } 74344\end{array}$ & & N28, N32 \\
\hline 2716 & $\mathbf{E}$ & $\begin{array}{l}\text { VIRGIL MOORE, } 5720 \text { EAST } 93 \text { SOUTH, MUSKOGEE } \\
74403\end{array}$ & & \\
\hline 2791 & $\mathbf{E}$ & ANTHONY MOORE, P.O. BOX 65, MANNFORD 74044 & & \\
\hline
\end{tabular}




\begin{tabular}{|c|c|c|c|c|c|}
\hline $\begin{array}{l}\text { APP. } \\
\text { NO. }\end{array}$ & $\begin{array}{l}\text { SUB- } \\
\text { PART }\end{array}$ & PARTICIPANTS NAME AND ADDRESS & $\begin{array}{l}\text { HATCHING } \\
\text { EGG } \\
\text { CAPACITY }\end{array}$ & $\begin{array}{c}\text { PRODUCTS CLASSIFIED } \\
\text { U.S. PULLORUM-TYPHOID } \\
\text { CLEAN }\end{array}$ & $\begin{array}{c}\text { ADDITIONAL } \\
\text { CLASSIFICATIONS FOR } \\
\text { WHICH PRODUCT QUALIFIED }\end{array}$ \\
\hline
\end{tabular}

2442 E BRUCE MOORE, P.O. BOX 65, MANNFORD 74044

1435 E LYNN D. MOORE, 10508 EAST 136TH STREET NORTH, COLLINSVILLE 74021

1979 E RICK MOORE, RT. 1, BOX 597, HULBERT 74441

2505 E VALQUEZ MORALES, RT. 2, BOX 567-3, BROKEN BOW 74722

2651 E RONALD MORGAN, 12117 WEST 775 ROAD, HULBERT 74441

2765 E WILLARD L. MORGAN, 13109 WEST 40TH STREET, SAND SPRINGS 74063

2679 E MICHAEL MORRIS, RT. 1, BOX 74, FOSTER 73434

1717 E RICKEY MORRIS, RT. 1, BOX 67, FOSTER 73434

2482 E BRIAN MORRISON, 609 EAST CHARLES, PAULS VALLEY 73075

726 E CHARLES MOXLEY, RT. 2, BOX 140, BLANCHARD 73010

2202 E CHARLES MOZLEY, RT. 2, BOX 140, MCLOUD 73010

2295 E ROBERT MUHLHAUSER, RT. 1, BOX 119-C1, CHOUTEAU 74337

2732 E ROBERT MULHOUSER, RT. 1, BOX 119-C1, CHOUTEAU 74337

202 E EUGENE MURNAN \& SONS, 700 REDWOOD DRIVE, NOBLE 73068

2688 E JAMES MURRAY, 5930 JONES ROAD, OKMULGEE 74447

2784 E TYMUSSYAL, 17401 NORTH TRIPLE XXX ROAD, LUTHER 73054

DENNIS NAIL, RT, 1, BOX 810, SHARON 73857

OKLAHOMA

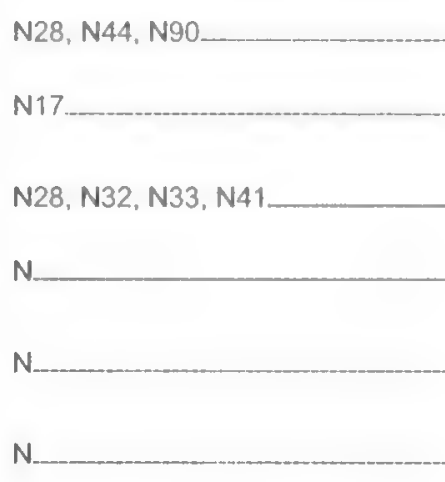

Z106, Z110, Z111, Z21, Z22

Z220, Z62, Z63

N116, N28, N31, N44

N17

N28, N41, N44

N28, N41, N44

N17.

R146, R247, R43, R74, W20, W7.

R87, W119, W121, W140, W17, W28, W484, Z204, Z22

243

N17.

R146, R169, R22, R228, R248, R6, R60, R89, W134, W15. W198, W20, W3, W30, W34, W478, W5, W54, X10, X14, X17, X96, Z202, Z205, Z4

N17.

N41

N28, N32, N41

N.

N17

$\mathrm{N} 28, \mathrm{~N} 41$

N17. 


\begin{tabular}{|c|c|c|c|c|c|}
\hline $\begin{array}{l}\text { APP. } \\
\text { NO. }\end{array}$ & $\begin{array}{l}\text { SUB- } \\
\text { PART }\end{array}$ & PARTICIPANTS NAME AND ADDRESS & $\begin{array}{l}\text { HATCHING } \\
\text { EGG } \\
\text { CAPACITY }\end{array}$ & $\begin{array}{c}\text { PRODUCTS CLASSIFIED } \\
\text { U.S. PULLORUM-TYPHOID } \\
\text { CLEAN }\end{array}$ & $\begin{array}{c}\text { ADDITIONAL } \\
\text { CLASSIFICATIONS FOR } \\
\text { WHICH PRODUCT QUALIFIED }\end{array}$ \\
\hline
\end{tabular}

\begin{tabular}{|c|c|c|}
\hline 2662 & $E$ & $\begin{array}{l}\text { JERRY NOE, } 716 \text { SOUTH } 51 \text { WEST AVENUE, TULSA } \\
74127\end{array}$ \\
\hline 2574 & $E$ & CLYDE NOEL, RT. 1, BOX 158-0, VIAN 74962 \\
\hline 2227 & $E$ & STACY NORTHCOTT, P.O. BOX 81, BOWLEGS 74830 \\
\hline 2575 & $E$ & DAVID NORTHCOTT, RT. 4, BOX 1040, SEMINOLE 74868 \\
\hline 2812 & $E$ & $\begin{array}{l}\text { EVERARDO NUNEZ, } 1001 \text { NORTH WILLARD STREET, } \\
\text { ALTUS } 73521\end{array}$ \\
\hline 2443 & $E$ & $\begin{array}{l}\text { JOE NUNICO, } 824 \text { NORTH 5TH STREET, FREDERICK } \\
73542\end{array}$ \\
\hline 2444 & $E$ & THOMAS NUNLEY, 612 EVANS. MARLOW 73055 \\
\hline 1058 & $E$ & DON L. NUTTER, RT. 2, BOX 115, WAYNOKA 73860 \\
\hline 1092 & $E$ & $\begin{array}{l}\text { JIM C. NUTTER, } 513 \text { HIGHWAY } 76 \text { SOUTH, NEWCASTLE } \\
73065\end{array}$ \\
\hline 1059 & $E$ & RAY NUTTER, RT. 1, BOX 10, WAYNOKA 73860 \\
\hline 2534 & $\mathrm{E}$ & BILLY O'REAR, RT, 1. BOX 703, IDABEL 74745 \\
\hline
\end{tabular}

E JOHNNY L. ODOM, 303 GAMECOCK LANE, FRANCIS 74844

2717 E ROBBIE ODOM, RT. 2., BOX 87-B, STONEWALL 74871

2631 E JERRY OGDEN, RT. 1, BOX 2542, QUINTON 74561

902 E OKLAHOMA BAD BOYS, JAMES EARL WELLS, 6830 WOOD ACRES, NOBLE 73068

883 E OKLAHOMA PRIDE GAME FARM,CARL LANCASTER, 2305 SE 143RD AVENUE, NORMAN 73071

1560 E KURT T. OLEKSUK. RT. 2, BOX 193-1, LINDSAY 73052

2663 E HENRY OLIVER, P.O. BOX 426, MOUNDS 74047

1513 E BETSY OLSEN, 3955 SOUTH 4200 ROAD, CHELSEA 74016

2393 E CIRILO OLVERO, 804 WEST TISHOMINGO, MADILL 73446

2203 E GARY ORR, 327 SOUTH EUCHER, DRUMRIFHT 74030

1060 E DENNIS ORR, RT. 1, BOX 1880, HOWE 74940

2216 E LARRY OTEY, P.O. BOX 153, ALEX 73002

2805 E JASON OT, P.O.BOX 242, DIBBLE 73031

1418 E ELMER OTT, RT. 2, BOX 115-D, PAULS VALLEY 73075

1998 E VERLON OWENS, P.O. BOX 1771, SALLISAW 74955
N109, N28, N44, N65

N17

N2, N24, N32, N33, N41, N43, N83

N109, N32, N33

N28, N32, N44.

N22, N34, N44.

N125, N28, N44

N

$\mathbf{N}$

N

R146, R20, R245, R247, R97 W140, W198. W208, W28, W286. W504, W519, W555, W81, W82, W89, Z15, Z179, Z19, Z20, Z204, Z205, Z211, Z22. Z230, Z27, Z62

N.

N.

N113, N22, N28, N44

N17

N17.

N9, N90

N

R225, W115, W25, W54, W560

N

N121, N32, N41, N43, N44, N63

N28.

N28, N32, N41.

N32, N44

N28, N32, N44

N17. 
Table 1. HATCHERIES, DEALERS, AND INDEPENDENT FLOCKS PARTICIPATING IN

THE NATIONAL POULTRY IMPROVEMENT PLAN

WATERFOWL, EXHIBITION POULTRY AND GAME BIRDS

\begin{tabular}{llccc}
\hline & & HATCHING & PRODUCTS CLASSIFIED \\
APP. SUB- & PARTICIPANTS NAME AND ADDRESS & EGG & U.S. PULLORUM-TYPHOID & CLASIFITIONAL \\
NO. PART & CAPACITY & & CLEAN & WHICH PRODUCT QUALIFIED \\
\hline
\end{tabular}

2476 E RIGOBERTO PADILLA, 810 NORTH 6TH, KINGFISHER 73750

2777 E BEN PAGE, 16046 SOUTH 655 ROAD, WYANDOTTE 74370

1459 E PAINTER POULTRY. 114 SOUTH CHEROKEE, HENNESSEY 73742

1061 E JIMM. PALMER, RT. 4, BOX 223-C, DUNCAN 73533

1199 E SAUL A. PANDO, RT. 1, BOX67, PAULS VALLEY 73075

1868 E RILEY PARIS, P.O. BOX 68, HOWE 74940

2694 E ROBIN PARKER, 341 WEST CHESTNUT STREET, NOBLE 73068

2535 E DILLON PARKER, 9650 WEST OAK STREET, SKIATOOK 74070

2148 E JIM PARNELL, P.O. BOX 322, TISHOMINGO 73460

667 E JOHN D. PARTON, RT. 1, BOX 285, CLEVELAND 74020

1311 E ANGELO B. PASQUALE, P.O. BOX 845, SHAWNEE 74801
E PEACH POULTRY, RT. 1, BOX 22, MARSHALL 73056

E JEFF PEARCE, P.O. BOX 1014, SALLISAW 74955 74872

BEN PEARSON, HC 64, BOX 169-A, MARLOW 73055

DONNA PEARSON, P.O. BOX 313, RUSH SPRINGS 73082

DOYLE PEBBLES, P.O. BOX 388, PORUM 74455

BOB PEDRIOLI, P.O. BOX 173, ST. LOUIS 74866

JACK PEEPLES, 3412 LAKE ROAD, PONCA CITY 74604

ALFRED PENNINGTON, RT. 1, BOX 198, STRATFORD

WAYNE PENNINGTON, RT. 1, BOX 202 C, STRATFORD 74872

RON PEPPER, P.O. BOX 515, KANSAS 74347

E MIGUEL ANGEL PEREZ, 119 NORTH WALNUT, COMMERCE 74339

E MITCHELL PERKEY, RT. 2, BOX 233, HOLLIS 73550

E MIKE PERKINS, P.O. BOX 223, KELLYVILLE 74039

E RANDY PERKINS, RT. 1, BOX 2375, HOWE 74940

E LEE PETERS, 4120 WEST RED EAGLE, SKIATOOK 74070
N

N17

$\mathrm{H} 8, \mathrm{~W} 50$

N17

N28, N32, N41

N113, N22, N25, N28, N44, N90

W3

N17

N-

N103, N108, N109, N129, N138, N156, N165, N23, N24, N27, N42. N61, N62, N65, N84, N87, N88, N94, N96, N97

R237, R30, R74

N17, N98

N125, N28, N32, N83, N90

N28, N41, N44

N28, N32, N44

N17

N17

N32, N41, N90

W119, W128, W204, W262 W28, W3, W301, W353, W454, W52, W54, W69, Z20, Z21, Z5

C1, W12, W2, W204, W24, W3, W4, W48, W50, W56, W568, W88, Y2, Z5

N

N

N41, N63

N17 
Table 1. HATCHERIES, DEALERS, AND INDEPENDENT FLOCKS PARTICIPATING IN

THE NATIONAL POULTRY IMPROVEMENT PLAN

WATERFOWL, EXHIBITION POULTRY AND GAME BIRDS

\begin{tabular}{|c|c|c|c|c|}
\hline $\begin{array}{l}\text { APP. } \\
\text { NO. }\end{array}$ & $\begin{array}{l}\text { SUB- } \\
\text { PART }\end{array}$ & PARTICIPANTS NAME AND ADDRESS & $\begin{array}{l}\text { HATCHING } \\
\text { EGG } \\
\text { CAPACITY }\end{array}$ & $\begin{array}{l}\text { PRODUCTS CLASSIFIED } \\
\text { U.S. PULLORUM-TYPHOID } \\
\text { CLEAN }\end{array}$ \\
\hline & & 73 & - OKLAHOMA & \\
\hline 2576 & $E$ & $\begin{array}{l}\text { EVAN \& SUSAN PETERSON, } 1020 \text { SOUTH CANADIAN, } \\
\text { PURCELL } 73080\end{array}$ & & $\begin{array}{l}\text { R56, W119, W262, W28, W3, } \\
\text { W568 }\end{array}$ \\
\hline 1627 & $E$ & DUKE PETITT, HC 64, BOX 4150, HEAVNER 74937 & & $N 28, N 32$ \\
\hline 2734 & $E$ & DUKE PETTIT, HC 64, BOX 4150, HEAVENER 74937 & & N105, N32 \\
\hline 78 & $E$ & $\begin{array}{l}\text { EDGAR L. PETTY, JR., } 10008 \text { NORTH EASTERN } \\
\text { AVENUE, OKLAHOMA CITY } 73131\end{array}$ & & $\begin{array}{l}\text { W117, W338, W364, W38, } \\
\text { W466, W79, W89, W94 }\end{array}$ \\
\hline 2394 & $E$ & JOE PFLASTERER, P.O. BOX 1941, MULDROW 74948 & & $\mathrm{~N} 28, \mathrm{~N} 32, \mathrm{~N} 44 \ldots$ \\
\hline 2263 & $E$ & TROY PHILLIPS, RT. 1, BOX 3640, ADAIR 74330 & & $\mathrm{~N}$ \\
\hline 2477 & $E$ & $\begin{array}{l}\text { BEVERLY PHILLIPS, } 705 \text { WEST MILL STREET, } \\
\text { MARRIETTA } 73448\end{array}$ & & $\mathrm{~N}$ \\
\hline 1012 & $E$ & REAFORD A. PICKENS, RT. 2, BOX 74, GERONIMO 73543 & & $\mathrm{~N}$ \\
\hline 2251 & $E$ & NATHAN PIERCE, 2417 CHICAGO, MUSKOGEE 74401 & & N105 \\
\hline 2445 & $E$ & MICHAEL PIERCE, 1004 SW H AVENUE, LAWTON 73501 & & N17 \\
\hline 2446 & $E$ & STEVE PLANK, RT. 4, BOX 9-2, MULDROW 74948 & & N17 \\
\hline 2395 & E & JASON PLUM, RT. 1, BOX 307-C. BRISTOW 74010 & & N_ \\
\hline 2596 & $\mathrm{E}$ & JASON PLUM, RT. 1, BOX 307-6, BRISTOW 74010 & & \\
\hline 1838 & E & DAVID PLUM. RT. 4, BOX 532, BRISTOW 74010 & & N._. \\
\hline 2447 & $E$ & $\begin{array}{l}\text { MARK N. POLVADORE, HC 71, BOX 130-A, KINGSTON } \\
73439\end{array}$ & & N17__ \\
\hline 2793 & $E$ & CLIFTON POOL, RT. 2, BOX 154, RUSH SPRINGS 73082 & & N28, N41, N44 \\
\hline 2616 & $E$ & $\begin{array}{l}\text { LARRY PORTER, } 445 \text { S. 84TH. STREET WEST, } \\
\text { MUSKOGEE } 74401\end{array}$ & & N \\
\hline 2307 & $E$ & DANNY PORTWOOD, P.O. BOX 548, CRESCENT 73028 & & $\mathrm{~N}$ \\
\hline 2735 & $E$ & SAMMY POWELL, P.O. BOX 184, SPRINGER 73458 & & N17 \\
\hline 1312 & $\mathrm{E}$ & $\begin{array}{l}\text { G. W. POYNOR \& SARAH HUMPHRIES, HC } 69, \text { BOX } 222 \text {, } \\
\text { HUGO } 74743\end{array}$ & & $N_{-}$ \\
\hline 2032 & $\mathrm{E}$ & $\begin{array}{l}\text { MARY \& GEORGE PRATER, P.O. BOX 457, ELMORE } \\
\text { CITY } 73433\end{array}$ & & N24, N28, N32, N41 \\
\hline 2772 & E & JIMMY F. PRICE, JR., P.O. BOX 2432, ADA 74820 & & $243 \ldots$ \\
\hline 2794 & $E$ & $\begin{array}{l}\text { PRICE FAMILY POULTRY, } 418 \text { NE 16TH, NEWCASTLE } \\
73065\end{array}$ & & \\
\hline 2577 & $E$ & TODD PRIEE, 1100 S 690 ROAD, QUAPAW 74363 & & N41, N65 \\
\hline 2669 & $E$ & JUAN PRIETO, RT. 4, BOX 114, SEMINOLE 74868 & & $\mathrm{~N}$ \\
\hline 2308 & $\mathrm{E}$ & RICKY PRINCE, RT. 1, BOX 2800, STONEWALL 74871 & & N32, N41, N90__ \\
\hline 2768 & $E$ & $\begin{array}{l}\text { DONALD \& JEREMY PRYOR, P.O. BOX 326, SAVANNA } \\
74565\end{array}$ & & $\mathrm{~B} 1, \mathrm{~N} 24, \mathrm{~N} 28, \mathrm{~N} 32, \mathrm{~N} 83$ \\
\hline 2336 & E & $\begin{array}{l}\text { DESERT OAK GAME FARM, HOWARD PUGH, HC } 71 \text {, } \\
\text { BOX } 145 \text {. KINGSTON } 73439\end{array}$ & & N83 \\
\hline 2478 & $\mathrm{E}$ & AUBREY PUGH, RT. 1, BOX 119 , MARRIETTA 73448 & & N... \\
\hline
\end{tabular}


Table 1. HATCHERIES. DEALERS, AND INDEPENDENT FLOCKS PARTICIPATING IN

THE NATIONAL POULTRY IMPROVEMENT PLAN

WATERFOWL, EXHIBITION POULTRY AND GAME BIROS

APP. SUB- PARTICIPANTS NAME AND ADDRESS

NO. PART

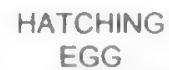

EGG

CAPACITY

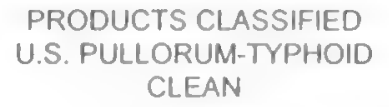

PRODUCTS CLASSIFIED

U.S. PULLORUM-TYPHOID CLEAN

ADDITIONAL

CLASSIFICATIONS FOR WHICH PRODUCT QUALIFIED

\section{3 - OKLAHOMA}

2396 E MAX QUAID, P.O. BOX 553, QUINTON 74561

2552 E PAUL QUAINTANCE, P.O. BOX 133. WELLING 74471

959 E MIKE QUINN. P.O. BOX 594, MANNFORD 74044

2357 E NORMAN RACKLIFF, RT. 3, BOX 2640, BARTLESVILLE 74003

1405 E CHRIS L. RADER, 16800 183RD STREET, LEXINGTON 73051

2264 E STEVE RAGAN, 40641 NORTH 3950TH ROAD, SKIATOOK 74070

837 E RAYMOND RANGE, JR., STAR ROUTE, BOX 24-A, ARNETT 73832

2397 E COZRATLIFF. P.O. BOX 304, MILBURN 73450 DANNY RAWLS, 209 NORTH B STREET, DAVIS 73030 OAKHURST 74050 KEVIN REECE, 35615 SOUTH 4198 ROAD, INOLA 74036 JAMES REECE, 1601 HARRISON, MUSKOGEE 74401 RONNY REED, 24810 WEST 49TH STREET S, SAND SPRINGS 74063

E JIMMIE DON REICH, RT. 4, BOX 123-C, HOLDENVILLE 74848

E ROBERT REID, RT. 1, BOX 106-6, SALLISAW 74955

ARTEMIO REYES, 11909 EAST ROBIN ROAD, MIDWEST CITY 73130 RICHARD RIALS, HC 62, BOX 243, ALTUS 74701 JIM RICHARDSON, RT. 1, BOX 148-E, SEMINOLE 74868 KENNY RICHARDSON, 28315 SOUTH 590 ROAD, WELLING 74471

E JOHN RICHBOURG, RT. 1, BOX 728, BROKEN BOW 74728

E FINIS RIDDLE, P.O. BOX 1126, ROLAND 74954

E SANDRA RUDICK, RT. 3, BOX 414, MULDROW 74948
N25, N28, N41, N44, N98

N17

N

N17

N.

N17

N105, N28, N34, N41

N17

N

N17

N.

N

N136, N174, N240, N243, N33, ... N44, N83

N17

N17

N134.

N17

N17

R168, W56, W576, W77

N113, N116, N63

N

N19

N17

R119, R139, R140, R20, R30. R527, R53, R60, R86, R87, W26, W435, W504, W74, W75, Z101. 
Table 1. HATCHERIES, DEALERS, AND INDEPENDENT FLOCKS PARTICIPATING IN

THE NATIONAL POULTRY IMPROVEMENT PLAN

WATERFOWL, EXHIBITION POULTRY AND GAME BIRDS

\begin{tabular}{lllll}
\hline & & HATCHING & PRODUCTS CLASSIFIED \\
APP. & SUB- & PARTICIPANTS NAME AND ADDRESS & ADDITIONAL \\
NO. & PART & CAPACITY & $\begin{array}{c}\text { C.S. PULLORUM-TYPHOID } \\
\text { CLEAN }\end{array}$ \\
\hline
\end{tabular}

73 - OKLAHOMA

\begin{tabular}{|c|c|c|c|}
\hline 1650 & E & CAREY RILEY, RT. 5, BOX 1710, COALGATE 74538 & N28_______ \\
\hline 1298 & $E$ & STEVEN RITCHIE, RT. 2, BOX 209-2, TAHLEQUAH 74464 & $\mathrm{~N}$ \\
\hline 217 & $\mathrm{E}$ & BENNY RITTER, RT. 4, BOX 11, MARLOW 73055 & Z43 \\
\hline 1442 & $\mathrm{E}$ & $\begin{array}{l}\text { PAUL RITTER, } 3103 \text { SPECK WRIGHT ROAD, MANNFORD } \\
74044\end{array}$ & $\ldots$ \\
\hline 674 & $\mathrm{E}$ & BILL RITTER, 2004 EAST WILLOW, DUNCAN 73533 & N____ \\
\hline 2337 & $E$ & KENNETH RITTER, HC 61, BOX 213, SALLISAW 74955 & $\mathrm{~N} 28, \mathrm{~N} 41, \mathrm{~N} 90$ \\
\hline 2398 & $E$ & PAUL ROARK, RT. 1, BOX 91, GORE 74435 & $\mathrm{~N} 33, \mathrm{~N} 43, \mathrm{~N} 44 \ldots$ \\
\hline 2095 & $E$ & STONEY ROBBINS, RT. 3, BOX 446-C, LINDSAY 73052 & N105, N24, N32, N44_. \\
\hline 2536 & $\mathrm{E}$ & ROBERTS FAMILY, RT.1, BOX 464, ADA 74820 & W54 _..._. \\
\hline 2488 & $\mathrm{E}$ & WALTER ROBINSON, 209 SOUTH 5TH, CYRIL 73029 & $N_{-}$ \\
\hline 2578 & $E$ & $\begin{array}{l}\text { GENE ROBINSON, } 6440 \text { SOUTH 161ST STREET. BIXBY } \\
74008\end{array}$ & \\
\hline 549 & $E$ & $\begin{array}{l}\text { DENNIS J. ROBINSON, P.O. BOX 1764, TAHLEQUAH } \\
74465\end{array}$ & $\mathrm{~N}$ \\
\hline 1935 & $E$ & BILLY ROBISON. P.O. BOX 752. MULDROW 74948 & N_n- \\
\hline 1010 & $E$ & $\begin{array}{l}\text { ROCK HILL POULTRY, RON HALL, P.O. BOX } 566 \text {, } \\
\text { STONEWALL } 74871\end{array}$ & $\mathrm{R} 35, \mathrm{~W} 3, \mathrm{~W} 4, \mathrm{X10}, \mathrm{X17}$ \\
\hline 2134 & $E$ & JOHN \& KELLI RODELA, RT. 6, BOX 344-A, ADA 74820 & $\mathrm{~N} 28, \mathrm{~N} 32, \mathrm{~N} 44, \mathrm{~N} 83 \ldots$ \\
\hline 1916 & $E$ & PAUL RODEN, P.O. BOX 176, COMANCHE 73529 & N125, N28, N32, N41_.. \\
\hline 2579 & $E$ & JOSE RODRIQUES, RT. 2, BOX 1312, STILWELL 74960 & $\ldots$ \\
\hline 1399 & $\mathrm{E}$ & $\begin{array}{l}\text { EDWARD C. ROEBUCK GAME FARM, RT. 5, BOX } 1505 \text {, } \\
\text { COALGATE } 74538\end{array}$ & $\mathrm{~N} 32, \mathrm{~N} 41, \mathrm{~N} 44 \ldots$ \\
\hline 2289 & $E$ & $\begin{array}{l}\text { RICK ROGERS, RT. 2, BOX 1092-J, LOCUST GROVE } \\
74352\end{array}$ & $\mathrm{~N}$ \\
\hline 2580 & $\mathrm{E}$ & $\begin{array}{l}\text { CHARLES \& RUTH ROSE, } 620 \text { NORTH MAIN, SOPER } \\
74759\end{array}$ & N17 \\
\hline 2165 & $\mathrm{E}$ & GENE ROSS, RT. 1, BOX 1980, ADAIR 74330 & $\mathrm{~N}_{-}$ \\
\hline 2448 & $\mathrm{E}$ & EARL \& ROSIE ROSS, HC 67, BOX 35, DAVIS 73030 & N19 \\
\hline 1821 & $\mathrm{E}$ & CLAUDE ROSS, 1749 MIMMOSA, NEWCASTLE 73065 & $\mathrm{~N}$ \\
\hline 2297 & $\mathrm{E}$ & TROY ROSSON, RT. 1, BOX 117, CHOUTEAU 74337 & N17. \\
\hline 731 & $\mathrm{E}$ & ROGER ROYSTON, RT 2, BOX 1120, POTEAU 74953 & $\mathrm{~N} 28, \mathrm{~N} 32, \mathrm{~N} 41_{-}$ \\
\hline 2449 & $\mathrm{E}$ & $\begin{array}{l}\text { MARSHALL RUSHE, } 16717 \text { TRIPLE X CIRCLE, CHOCTAW } \\
73020\end{array}$ & $\mathrm{~N} 17 \ldots$ \\
\hline 1682 & $E$ & $\begin{array}{l}\text { THOMAS C. RUSHING. RT. 2, BOX 59-C2, RANDLETT } \\
73562\end{array}$ & \\
\hline 2399 & $\mathrm{E}$ & DON RUSSELL, 211 NORTH ELLISON, EL RENO 73036 & N19. \\
\hline
\end{tabular}


TabIe 1. HATCHERIES, DEALERS, AND INDEPENDENT FLOCKS PARTICIPATING IN

THE NATIONAL POULTRY IMPROVEMENT PLAN

WATERFOWL, EXHIBITION POULTRY AND GAME BIRDS

APP. SUB- PARTICIPANTS NAME AND ADDRESS

NO. PART
HATCHING

EGG

CAPACITY
PRODUCTS CLASSIFIED

U.S. PULLORUM-TYPHOID CLEAN
ADDITIONAL

CLASSIFICATIONS FOR WHICH PRODUCT QUALIFIED

73 - OKLAHOMA

1683 E RUSSELL RUTHERFORD, RT. 4, BOX 564, GROVE 74344

2316 E SAMRYALS, RT. 1, BOX 29-A, MILBURN 73450

2779 E STEWART RYAN, RT. 1, BOX 158, ADA 74820

1255 E GENE RYBURN, P.O. BOX 902, WISTER 74966

2059 E LUTHER FRANK SAGER, P.O. BOX 236, INDIANOLA 74442

2755 E JUAN SALINAS, P.O. BOX 493, SALLISAW 74955

2737 E ROY WALLACE SALSMAN, P.O. BOX 122, STILWELL 74960

2400 E SALTY DOG, RICHARD PALMER, RT. 1, BOX 901, STIGLER 74063

2359 E JESUS SANCHEZ, RT. 2, BOX 57-R, MARIETTA 73445

2507 E ISIDORA SANCHEZ, P.O. BOX 643, BROKEN BOW 74728

2773 E PABLO SANCHEZ, RT, 1, BOX 57-R, MARIETTA 73445

2796 E LAZARO SANCHEZ, RT. 2, BOX 148, MADILL 73446

2338 E WILBUR SANDERS, RT. 1, BOX 220, GRANITE 73547

2346 E ROBERT SANDERS, P.O. BOX 910, WISTER 74966

2401 E DUSTIN SANDLIN, 13919 MCGUIRE ROAD, NOBLE 73068

2217 E RON SANFORD, RT. 4, BOX 454, SEMINOLE 74868

1572 E DEWAYNE SARTEN, RT. 3, BOX 128, COLCORD 74338

1243 E BUTCH SAVAGE, P.O BOX 173, GRANITE 73547

2228 E DEE SCARBERRY, HC 68, BOX 68, NASHOBA 74558

2458 E SCORPION HILL GAME FARM, MICHAEL CASPERS, RT. 1, BOX 267, MACOMB 74852

2166 E DAVID SCOTT, P.O. BOX 1676, KINGSTON 73439

2244 E JEREMY SCOTT,P.O. BOX 134, SALINA 74365

2653 E RONNIE SCOTT. 8300 SUNDOWN LANE, HARRAM 73045

1460 E DINK SCOTT, P.O. BOX 104, VIAN 74962

1734 E ROY SCOTT, P.O.BOX 51, MERIDIAN 73058

2265 E JOSHUA SEALS, 40643 NORTH 3954TH ROAD, SKIATOOK 74070

2617 E RON SELF, RT. 4, BOX 107-1, CHICKASHA 73018

1846 E PHILLIP SETTLEMYRE, RT. 5, BOX 490, TAHLEQUAH 74464

2680 E JOSE M. SEVIELLA, P.O. BOX 176, GORE 74435
N17.

N17.

N32, N41, N43

N28, N41

N44, N83, N84, W30, Y2, Z118_-

N17.

N17

N25, N28, N41, N44, N90

$\mathrm{N}$

N

N.

N.

N.

N41.

N.

N17

N

N.

N

N100, N105, N128, N144, N151, N167, N172, N182, N190, N20, N221, N224, N24, N32, N65

N...

N.

N171, N230, N28, N41

N121, N32, N43, N84

N121, N32

N28, N32

N.

N17 
Table 1. HATCHERIES, DEALERS, AND INDEPENDENT FLOCKS PARTICIPATING IN

THE NATIONAL POULTRY IMPROVEMENT PLAN

WATERFOWL, EXHIBITION POULTRY AND GAME BIRDS

\begin{tabular}{|c|c|c|c|c|}
\hline $\begin{array}{l}\text { APP. } \\
\text { NO. }\end{array}$ & $\begin{array}{l}\text { SUB- } \\
\text { PART }\end{array}$ & PARTICIPANTS NAME AND ADDRESS & $\begin{array}{l}\text { HATCHING } \\
\text { EGG } \\
\text { CAPACITY }\end{array}$ & $\begin{array}{l}\text { PRODUCTS CLASSIFIED } \\
\text { U.S. PULLORUM-TYPHOID } \\
\text { CLEAN }\end{array}$ \\
\hline & & 73 & - OKLAHOMA & \\
\hline 2718 & $E$ & $\begin{array}{l}\text { SHADY TREE BANTAMS, RT. 2, BOX 59-BB, LAWTON } \\
73501\end{array}$ & & $\begin{array}{l}\text { N12, N126, N14, N15, W13, } \\
\text { W198 }\end{array}$ \\
\hline 1065 & $E$ & $\begin{array}{l}\text { CRAIG \& CHARLENE SHEA, BLACK \& BLUE GF, } 2600 \\
108 T H \text { NE., NORMAN } 73026\end{array}$ & & N24, N94, W136 \\
\hline 1611 & $E$ & $\begin{array}{l}\text { SHELL CREEK GAME FARM. J. BROWN, P.O. BOX } 434 \text {, } \\
\text { DEWAR } 74431\end{array}$ & & $N 17$ \\
\hline 2339 & $\mathbf{E}$ & DOUG SHEPARD, 1006 NW 7TH, LINDSAY 73052 & & 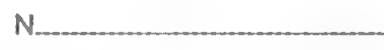 \\
\hline 2622 & $\mathbf{E}$ & $\begin{array}{l}\text { HAROLD SHERE, } 2753 \text { SOUTH } 4390 \text { ROAD, WELCH } \\
74369\end{array}$ & & N104, N17, N28 \\
\hline 2184 & $E$ & $\begin{array}{l}\text { JOSEPH SHERRILL, RT, 3, BOX 168, PAULS VALLEY } \\
73075\end{array}$ & & $\mathrm{~N} 28, \mathrm{~N} 44, \mathrm{~N} 90$ \\
\hline 2470 & $\mathbf{E}$ & $\begin{array}{l}\text { ED SHERWOOD, } 22 \text { GREENFIELD DRIVE, SHAWNEE } \\
74801\end{array}$ & & N17, N43, N44, N90, N99 \\
\hline 2239 & $E$ & WILLIAM SHIELDS, 5131 WEST 6TH, TULSA 74127 & & $\mathrm{~N} 28, \mathrm{~N} 41, \mathrm{~N} 44$ \\
\hline 2240 & $E$ & STEVE SHONKWILER, P.O. BOX 1093 , MULDROW 74948 & & N17 \\
\hline 1003 & $E$ & JIM SHONTS, P.O. BOX 144, NICOMA PARK 73066 & & $\mathrm{~N}$ \\
\hline 1638 & E & SAM SHRUM, RT. 5, BOX 146, JAY 74346 & & N17 \\
\hline 2519 & $E$ & $\begin{array}{l}\text { BOBBY SHUMWAY, } 507 \text { WEST COAL STREET, } \\
\text { MCALESTER } 74501\end{array}$ & & N32 \\
\hline 2537 & $E$ & $\begin{array}{l}\text { JAVIER SILVA, } 205 \text { NORTH COSTILOW, BROKEN BOW } \\
74728\end{array}$ & & N___- \\
\hline 2086 & $E$ & ANTONIO SILVA, 104 CONSER AVENUE, POTEAU 74953 & & $\mathrm{~N} 28, \mathrm{~N} 41 \ldots$ \\
\hline 2402 & E & $\begin{array}{l}\text { ALVIN SIMMONS, } 3921 \text { EAST AVENUE, OKLAHOMA CITY } \\
73121\end{array}$ & & $\mathrm{~N}$ \\
\hline 2623 & $\mathbf{E}$ & $\begin{array}{l}\text { WALTER K. SIMMONS, } 15254 \text { MEDLIN ROAD. HULBERT } \\
74441\end{array}$ & & N__ \\
\hline 757 & $E$ & MIKE SIMON, RT. 3, BOX 148, CHANDLER 74834 & & \\
\hline 2252 & $E$ & WALTER SIMPSON, 527 WEST 68TH, TULSA 74132 & & \\
\hline 2266 & $E$ & $\begin{array}{l}\text { LAURA SIMPSON, } 1021 \text { EAST 181ST NORTH, SKIATOOK } \\
74070\end{array}$ & & \\
\hline 2245 & $E$ & HARLEY SITSLER, RT. 1, BOX 1245, ROSE 74364 & & N \\
\hline 2624 & $\mathrm{E}$ & $\begin{array}{l}\text { HARVEY SITSLER, P.O. BOX 912, LOCUST GROVE } \\
74352\end{array}$ & & N17 \\
\hline 2508 & $E$ & MIKE SKAGGS, P.O. BOX 579, PORUM 74455 & & N17 \\
\hline 1896 & $E$ & JIM SKAGGS, HC 67, BOX 920, RATTAN 74562 & & N17 \\
\hline 1987 & $E$ & $\begin{array}{l}\text { JACKIE SKIPWORTH, RT. 2, BOX 96, MOUNTAIN VIEW } \\
73062\end{array}$ & & N17_ \\
\hline 683 & $\mathrm{E}$ & GARY SMITH, 318 EAST ATLANTA, OKEMAH 74859 & & \\
\hline 1242 & $E$ & $\begin{array}{l}\text { CLAYTON SMITH, } 7540 \text { COTTONWOOD ROAD. } \\
\text { SAPULPA } 74066\end{array}$ & & $\mathrm{~N}$ \\
\hline 1318 & $E$ & DAVID SMITH, RT. 2, BOX 306, WYANDOTTE 74370 & & N17 \\
\hline 1472 & $E$ & JIMMY SMITH, P.O. BOX 304, KREBS 74554 & & N17 \\
\hline
\end{tabular}


HATCHING EGG

NO. PART
PRODUCTS CLASSIFIED

U.S. PULLORUM-TYPHOID CLEAN
ADDITIONAL

CLASSIFICATIONS FOR WHICH PRODUCT QUALIFIED

73 - OKLAHOMA

\begin{tabular}{|c|c|c|c|}
\hline 1247 & E & $\begin{array}{l}\text { SEAMON SMITH, } 34664 \text { WEST } 301 \text { STREET SOUTH, } \\
\text { BRISTOW } 74010\end{array}$ & - \\
\hline 1917 & $E$ & $\begin{array}{l}\text { CHARLES SMITH, } 5404 \text { SOUTH } 385 T H \text { WEST AVENUE, } \\
\text { MANNFORD } 74044\end{array}$ & N12, N32, N41, N44, N91___ \\
\hline 1955 & $E$ & JERRY SMITH, RT, 3, BOX 94-A, OKEMAH 74859 & N17 _ _ _ \\
\hline 1989 & E & LESLIE SMITH, 21315 NE 146 TH STREET, LUTHER 73054 & N17_ \\
\hline 1936 & $E$ & $\begin{array}{l}\text { DEVIN SMITH, } 16317 \text { SOUTH MIDWEST BLVD., } \\
\text { OKLAHOMA CITY } 73165\end{array}$ & $\mathrm{~N}$ \\
\hline 1988 & $\mathrm{E}$ & $\begin{array}{l}\text { CHARLES SMITH, } 21277 \text { ROBIN WAY, CLAREMORE } \\
74017\end{array}$ & $\mathrm{~N} 28, \mathrm{~N} 32, \mathrm{~N} 41 \ldots$ \\
\hline 2310 & $E$ & CLIFF SMITH, RT. 1, BOX 305, WISTER 74966 & N28, N41 \\
\hline 2340 & $E$ & $\begin{array}{l}\text { FLOYD SMTH, } 5741 \text { SOUTH 113TH WEST AVENUE, } \\
\text { SANDSPRINGS } 74063\end{array}$ & $\mathrm{~N} 24, \mathrm{~N} 28, \mathrm{~N} 32, \mathrm{~N} 41$ \\
\hline 2379 & $E$ & JESSE SMITH, 2880 GLENDA LANE, SKIATOOK 74070 & $\mathrm{~N}$ \\
\hline 2403 & $E$ & PAUL SMITH. 14105 MILES DRIVE, NEWALLA 74857 & $\mathrm{~N}$ \\
\hline 2471 & $\mathrm{E}$ & THERNON SMITH, JR., RT. 1, BOX 839, HARRAH 73045 & $\mathrm{~N}$ \\
\hline 2489 & $E$ & BUDDY SMITH, HC 70, BOX 193, ARDMORE 73401 & N17 \\
\hline 2664 & $E$ & BUDDY SMITH, HC 75, BOX 234, WILSON 73463 & N \\
\hline 2542 & $E$ & $\begin{array}{l}\text { RODNEY SMITH/DENNIS WARE, P.O. BOX 422, NOBLE } \\
73068\end{array}$ & N17 ___ \\
\hline 2538 & $E$ & MICHAEL T. SMITH, RT. 1, BOX 803, IDABEL 74745 & $\begin{array}{l}\text { W128, W142, W151, W200, } \\
\text { W202, W276, W28, W48, W50, } \\
\text { W509, W51, W52, W596, W69, } \\
\text { W81, W88, W89, Z4 }\end{array}$ \\
\hline 2450 & $E$ & BENNY SMITH, 119 EAST TEXAS, DURANT 74701 & $\mathrm{~N} 41, \mathrm{~N} 44$ \\
\hline 2341 & $E$ & WILLIAM SMITH, 5934 NORTH ROCKFORD, TULSA 74126 & N17 \\
\hline 2719 & $\mathbf{E}$ & TADD SMITH, RT. 1, BOX 176, MARTHA 73566 & $\mathrm{~N}$ \\
\hline 2785 & E & $\begin{array}{l}\text { BLAKE SMITH, } 32227 \text { EAST 141ST SOUTH. COWETA } \\
74429\end{array}$ & \\
\hline 2024 & $\mathrm{E}$ & TODD SNEED, RT. 2, BOX 42, ANADARKO 73005 & $\mathrm{~N}_{-}$ \\
\hline 1490 & $\mathbf{E}$ & CHRIS SNOW, P.O. BOX 363, GRANITE 73547 & \\
\hline 1783 & $\mathbf{E}$ & JOHN SPARKMAN, RT. 4; BOX 556, GROVE 74344 & \\
\hline 813 & E & $\begin{array}{l}\text { DALE \& JOE SPARKS, RT. 2, BOX 185-B, SALLISAW } \\
74955\end{array}$ & N17 \\
\hline 785 & $\mathbf{E}$ & $\begin{array}{l}\text { PAUL SPARKS, } 10801 \text { WEST } 805 \text { ROAD, FT. GIBSON } \\
74434\end{array}$ & $\mathrm{~N}$ \\
\hline 2284 & E & MIKE SPENCER, RT. 2, BOX 1282, MANNFORD 74044 & N17 _ \\
\hline 2096 & $E$ & $\begin{array}{l}\text { MICHAEL SPILLER, } 310 \text { SOUTH WASHINGTON, } \\
\text { DELAWARE } 74027\end{array}$ & N28, N32, N44, N91 \\
\hline 1853 & $E$ & EDWARD SROCK, RT. 2, BOX 36-AA, CORDELL 73632 & $\begin{array}{l}\text { N38, W102, W106, W117, W12, } \\
\text { W121, W129, W132, W140, } \\
\text { W164, W17, W18, W216, W24, }\end{array}$ \\
\hline
\end{tabular}


Table 1. HATCHERIES, DEALERS, AND INDEPENDENT FLOCKS PARTICIPATING IN

THE NATIONAL POULTRY IMPROVEMENT PLAN

WATERFOWL, EXHIBITION POULTRY AND GAME BIRDS

APP. SUB- PARTICIPANTS NAME AND ADDRESS

NO. PART

\section{HATCHING}

EGG

CAPACITY
PRODUCTS CLASSIFIED

U.S. PULLORUM-TYPHOID

CLEAN
ADDITIONAL

CLASSIFICATIONS FOR WHICH PRODUCT QUALIFIED

73 - OKLAHOMA

\begin{tabular}{|c|c|c|}
\hline 2472 & $E$ & LARRY SROCK, 237 BARTLEY DRIVE, NEWALLA 74852 \\
\hline 2520 & $E$ & $\begin{array}{l}\text { VERNON ST. CLAIR, } 2915 \text { MCCLAINE ROAD, ARDMORE } \\
73401\end{array}$ \\
\hline 1882 & $E$ & KENNETH ST. CLAIR, 601 LEE STREET, DURANT 74701 \\
\hline 2135 & $E$ & HANSEL ST. CLAIR, P.O. BOX 70, KENEFIC 74748 \\
\hline 2597 & $\mathrm{E}$ & $\begin{array}{l}\text { STEVEN FLOYD STAFFORD, RT. 1, BOX 180. STUART } \\
74570\end{array}$ \\
\hline 2229 & $E$ & EARL STANLEY, RT. 3, BOX 265, STROUD 74079 \\
\hline 2380 & $E$ & JOHN STANLEY, RT. 2, BOX 266, SPIRO 74959 \\
\hline 2015 & $E$ & ELVIN E. STANLEY, RT. 3, BOX 265, STROUD 74079 \\
\hline 1096 & $E$ & FRANK STANSBERRY, RT. 1, BOX 595, BOSWELL 74727 \\
\hline 661 & $E$ & GARY STARNS, RT. 7, BOX 112, ADA 74820 \\
\hline 2124 & $E$ & BILL STEEL, RT. 1. BOX 750, POTEAU 74953 \\
\hline 1661 & E & $\begin{array}{l}\text { DANNY STEPHENSON, HC } 64, \text { BOX } 2300, \text { HEALDTON } \\
73438\end{array}$ \\
\hline 2218 & $E$ & RICHARD STEWARD, RT. 2, BOX 283, PRYOR 74361 \\
\hline 2360 & $E$ & MIKE STEWART, RT. 2, BOX 430. WASHINGTON 73093 \\
\hline 2521 & $E$ & DOUG STIDLEY, 2002 NORTH 15TH, MCALESTER 74501 \\
\hline 2361 & $E$ & JAMES STILLWELL, RT. 2, BOX 9420, PORUM 74455 \\
\hline 2581 & $E$ & JAMES STILWELL, P.O. BPX 468, WARNER 74469 \\
\hline 2774 & $E$ & BOB STOCKTON, HC 70, BOX 221, ARDMORE 73402 \\
\hline 759 & $E$ & $\begin{array}{l}\text { CLARENCE STOCKTON, } 1304 \text { SOUTH DICKERSON } \\
\text { AVENUE, ATOKA } 74525\end{array}$ \\
\hline 1956 & $E$ & HIAWATHA STOKES, P.O. BOX 273, GRANITE 73547 \\
\hline 2246 & $E$ & $\begin{array}{l}\text { STONE GAP GAME FARM, RT. } 3, \text { BOX 162, SALLISAW } \\
74955\end{array}$ \\
\hline 2451 & $E$ & $\begin{array}{l}\text { STEVEN STRONG, } 6216 \text { SADDLEBACK WAY, SPENCER } \\
73084\end{array}$ \\
\hline 1629 & $E$ & DONNIE STROUD, RT. 1, BOX 1490, HOWE 74940 \\
\hline 1735 & $E$ & $\begin{array}{l}\text { LARRY STUDIE, } 16845 \text { NORTH } 609 \text { ROAD, STILWELL } \\
74960\end{array}$ \\
\hline 2033 & $E$ & $\begin{array}{l}\text { STEVE STURM, } 27470 \text { NORTH } 3940 \text { ROAD, OCHELATA } \\
74051\end{array}$ \\
\hline 2654 & $E$ & CHARLES M. STURM. RT. 1, BOX 219, PORTER 74454 \\
\hline 2689 & $E$ & TOMMY C. SULLIVAN, RT. 1, BOX 271-A, WILSON 73463 \\
\hline
\end{tabular}

W27, W277, W3, W33, W48 W53, W55, W74, W94, X10, $\mathrm{X} 143, \mathrm{X6}, \mathrm{Z146}$

N1, N28, N47

N17

N17.

N17.

N28, N32, N44, N90

N.

N17.

N.

$\mathrm{N} 17$.

N28, N32

W231, W301, W357, W359, W360, W361, W363, W364

N28, N44

N17

B1, C1, N18, N32, W17, W368, X24

N17

N17

N17

N113, N172

N145, N87.

N17

N127, N131, N43, N44

N28, N41, N44

N17.

N125, N28, N32, N41

R18, R237, R34, R36, R7, R71, _ R85, W13, Z22

W20, W3, W306, W57, w97 
Table 1. HATCHERIES, DEALERS. AND INDEPENDENT FLOCKS PARTICIPATING IN

THE NATIONAL POULTRY IMPROVEMENT PLAN

WATERFOWL, EXHIBITION POULTRY AND GAME BIRDS

$\begin{array}{lll}\text { APP. } & \text { SUB- } \\ \text { NO. PARTICIPANTS NAME AND ADDRESS } & \text { PART }\end{array}$

$\begin{array}{cc}\text { HATCHING } & \text { PRODUCTS CLASSIFIED } \\ \text { EGG } & \text { U.S.PULLORUM-TYPHOID } \\ \text { CAPACITY } & \text { CLEAN }\end{array}$

ADDITIONAL

CLASSIFICATIONS FOR WHICH PRODUCT QUALIFIED

73 - OKLAHOMA

\begin{tabular}{|c|c|c|}
\hline 2167 & $E$ & WILLIAM C. SUMMERS, P.O. BOX 1452, PURCELL 73080 \\
\hline 2274 & $E$ & $\begin{array}{l}\text { ERIC SUTHERLAND, } 14868 \text { WEST BROADWAY, YUKON } \\
73099\end{array}$ \\
\hline 1828 & E & EDDIE SUTPHIN, RT. 1, BOX 1635, CAMERON 74932 \\
\hline 2195 & E & $\begin{array}{l}\text { RONNIE \& DAN SWAFFORD, } 1006 \text { WEST WILLOW, } \\
\text { DURANT } 74701\end{array}$ \\
\hline 2675 & $E$ & SONDRA SWANSON, RT. 2, BOX 431, SULPHUR 73086 \\
\hline 2404 & $\mathrm{E}$ & MIKE SWEETEN, RT. 1, BOX 2314, CAMERON 74532 \\
\hline 2756 & E & $\begin{array}{l}\text { THEARON TALLEY, } 11009 \text { NE 50TH, OKLAHOMA CITY } \\
73084\end{array}$ \\
\hline 2362 & $E$ & JERRY TALLON, RT. 2, BOX 5200, PORUM 74455 \\
\hline 1690 & E & JAMES M. TALLY, 36 5TH STREET, CARTWRIGHT 74731 \\
\hline 1510 & $\mathrm{E}$ & STEVE TANKERSLEY, RT. 1, BOX 265, ARDMORE 73401 \\
\hline 2582 & $E$ & STEVE TANKERSLY, RT, 4, BOX 1330, CHECOTAH 74426 \\
\hline 1691 & $E$ & JERALD TANNER, 63972 EAST 333RD, JAY 74346 \\
\hline 1918 & $E$ & GENE TAYLOR, RT. 1, BOX 810, ANTLERS 74523 \\
\hline 2230 & $\mathrm{E}$ & JEFF TAYLOR, 1514 NORTH LEE STREET, ALTUS 73521 \\
\hline 2290 & $E$ & MARK TAYLOR, RT. 3, BOX 317, PAWNEE 74058 \\
\hline 2231 & $E$ & JEFF TAYLOR, P.O. BOX 158, MOODYS 74444 \\
\hline 2813 & $E$ & ROBERT TAYLOR, RT. 1, BOX 1144, ANTLERS 74523 \\
\hline 2232 & E & $\begin{array}{l}\text { DANNY TEAGUE (TEAGUE FAMILY), } 15702 \text { EAST 101ST, } \\
\text { BROKEN BOW } 74011\end{array}$ \\
\hline 2625 & $E$ & $\begin{array}{l}\text { GARY LEE TEAGUE, } 34738 \text { W 51ST. STREET SOUTH, } \\
\text { MANNFORD } 74044\end{array}$ \\
\hline 2660 & $\mathrm{E}$ & MIKE G. TEAGUE, RT. 2, BOX 806, MANNFORD 74404 \\
\hline 1390 & $E$ & GARY TEAGUE, RT. 2, BOX 1710, MANNFORD 74044 \\
\hline 1992 & $\mathrm{E}$ & JOHN TEDFORD, P.O. BOX 285, CAMERON 74932 \\
\hline 1785 & $\mathrm{E}$ & DAVID THOMAS, P.O. BOX 664, KINGSTON 73439 \\
\hline 1639 & $E$ & $\begin{array}{l}\text { CRAWFORD THOMASON, RT. 2, BOX 52, MANGUM } \\
73554\end{array}$ \\
\hline 2490 & $\mathrm{E}$ & CARL THOMPSON, RT. 1, BOX 2415, HEAVENER 74937 \\
\hline 2491 & $E$ & MAX THOMPSON, RT. 1, BOX 2255, ROSE 74364 \\
\hline 2539 & $E$ & TYLER THOMPSON, P.O. BOX 524. STONEWALL 74871 \\
\hline 1854 & $\mathrm{E}$ & ELMER THURBER, RT. 1, BOX 1012, STILWELL 74960 \\
\hline 1594 & $E$ & $\begin{array}{l}\text { HERCHEL TIMMONS, JR., } 20306 \text { SOUTH 105TH WEST } \\
\text { AVENUE, MOUNDS } 74047\end{array}$ \\
\hline
\end{tabular}

Ni7.

R85, W138, W142, W176, W198, W28, W81

N.

N121, W195

N28, N41

$\mathrm{N}$

N17

N104, N43, N56, N83

N17

N17

N17

N121, N28, N32

N

N.

N17

N28

N17, N32, N33, N43, N44, N65, N76, N90

N28.

W20, W23, W57, W88

$\mathrm{H} 5$, W30

N17

N17, R169, R196, W231, W301, W351, W357, W358, W360, W363, W364, W53, W54, W90

N.

R196, W28, W3, W4, W54

N17

N28 
Table 1. HATCHERIES, DEALERS, AND INDEPENDENT FLOCKS PARTICIPATING IN

THE NATIONAL POULTRY IMPROVEMENT PLAN

WATERFOWL, EXHIBITION POULTRY AND GAME BIRDS

\begin{tabular}{|c|c|c|c|c|}
\hline $\begin{array}{l}\text { APP. } \\
\text { NO. }\end{array}$ & $\begin{array}{l}\text { SUB- } \\
\text { PART }\end{array}$ & PARTICIPANTS NAME AND ADDRESS & $\begin{array}{l}\text { HATCHING } \\
\text { EGG } \\
\text { CAPACITY }\end{array}$ & $\begin{array}{l}\text { PRODUCTS CLASSIFIED } \\
\text { U.S. PULLORUM-TYPHOID } \\
\text { CLEAN }\end{array}$ \\
\hline & & 73 & - OKLAHOMA & \\
\hline 2150 & $E$ & $\begin{array}{l}\text { THERON TOLLETT, } 24814 \text { WEST 49TH AVENUE, SAND } \\
\text { SPRINGS } 74063\end{array}$ & & 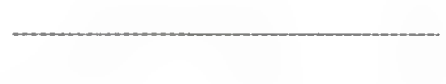 \\
\hline 795 & $E$ & JACK TRAVIS, RT. 1, BOX 135, WEBBERS FALLS 74470 & & $\mathrm{~N}$ \\
\hline 1451 & $E$ & HAROLD TREAT, P.O. BOX 718, NOBLE 73068 & & W69. \\
\hline 2504 & $E$ & $\begin{array}{l}\text { THOMAS \& MENDY TREJO-MARTINEZ, } 321 \text { EAST } \\
\text { CEDAR, STILWELL } 74960\end{array}$ & & N \\
\hline 2540 & $E$ & $\begin{array}{l}\text { AMANDA TROXELL, } 10928 \text { NORTH 145TH EAST } \\
\text { AVENUE, OWASSO } 74055\end{array}$ & & \\
\hline 2291 & $E$ & $\begin{array}{l}\text { GORGONIO TRUJILLO, RT. 2, BOX 1225, LOCUST } \\
\text { GROVE } 74352\end{array}$ & & N17 \\
\hline 2738 & $E$ & KENNETH TUCKER, P.O. BOX 291, LONE GROVE 73443 & & \\
\hline 2452 & $\mathrm{E}$ & CURT TULLY, RT. 2, SULPHUR 73086 & & N19. \\
\hline 2285 & $E$ & $\begin{array}{l}\text { TUMBLING DICE FARMS, KEVIN BIRCH, P.O. BOX } 382 \text {, } \\
\text { DAVIS } 73030\end{array}$ & & N \\
\hline 2405 & $\mathrm{E}$ & MARVIN TURMAN, 22625 NE 50TH, HARRAH 73045 & & N17___ \\
\hline 2406 & $E$ & $\begin{array}{l}\text { ROBERT TURMAN \& JOHN GRAY, P.O. BOX } 192 \text {, } \\
\text { HARRAH } 73045\end{array}$ & & N17 \\
\hline 1589 & E & TOM TURNER, RT. 2, BOX 1851, JENNINGS 74038 & & $\mathrm{~N} 131, \mathrm{~N} 17$ \\
\hline 1981 & $E$ & $\begin{array}{l}\text { GEORGE TURNEY, } 18033 \text { EAST } 690 \text { ROAD, TAHLEQUAH } \\
74464\end{array}$ & & N17 \\
\hline 2599 & E & BILLY C. TYER, P.O. BOX 92 OAS, LEACH 74359 & & $N_{-}$ \\
\hline 1336 & $E$ & JAMES TYLER, P.O. BOX 1060, BLANCHARD 73010 & & - \\
\hline 2144 & $E$ & ED TYNER, 35891 SOUTH 4430 ROAD, VINITA 74301 & & N_L \\
\hline 842 & E & $\begin{array}{l}\text { DONALD UNDERWOOD, P.O. BOX 349, ELMORE CITY } \\
73433\end{array}$ & & N._._._._._._. \\
\hline 2342 & $E$ & DALE UPTON, RT. 2, BOX 71-A, APACHE 73006 & & \\
\hline 2466 & $E$ & WALTER UPTON, RT. 1, BOX 129-B. WEWOKA 74884 & & N17_____._. \\
\hline 1847 & E & MEGAN UTTERBACK, 1031 4TH STREET, ALVA 73717 & & W20 \\
\hline 1321 & $E$ & $\begin{array}{l}\text { FLOYD M. VANDERBURG, HC } 70, \text { BOX 164, ARDMORE } \\
73401\end{array}$ & & N- \\
\hline 2219 & E & MATT VANFLEET, 3101 ERIC LANE, EDMOND 73034 & & W119, W20, W28, W48, W56 \\
\hline 1479 & $E$ & $\begin{array}{l}\text { TOM VARNER, } 20282440 \text { ROAD, UNIT D, CLAREMORE } \\
74017\end{array}$ & & $\mathrm{Z1}, \mathrm{Z27}, \mathrm{Z} 43$ \\
\hline 1737 & $E$ & ALLAN VASSAR, RT. 1, BOX 99, AGRA 74824 & & N___- \\
\hline 2626 & $E$ & MIKE VAUGHAN, P.O. BOX 654, LOCUST GROVE 74352 & & N17_ \\
\hline 1416 & $E$ & MIKE VAUGHN, RT. 2, BOX 281, LINDSAY 73052 & & $\mathrm{~N} 28, \mathrm{~N} 32, \mathrm{~N} 44$ \\
\hline 2583 & $E$ & $\begin{array}{l}\text { MICHAEL VEACH, } 15310 \text { SOUTH HARVARD AVENUE, } \\
\text { BIXBY } 74008\end{array}$ & & $\mathrm{~N}$ \\
\hline 1999 & $E$ & TONY VILLALOBOS, RT. 4, BOX 5535, STIGLER 74462 & & N. \\
\hline
\end{tabular}


Table 1. HATCHERIES, DEALERS, AND INDEPENDENT FLOCKS PARTICIPATING IN

THE NATIONAL POULTRY IMPROVEMENT PLAN

WATERFOWL, EXHIBITION POULTRY AND GAME BIRDS

\begin{tabular}{|c|c|c|c|c|c|}
\hline $\begin{array}{l}\text { APP. } \\
\text { NO. }\end{array}$ & $\begin{array}{l}\text { SUB- } \\
\text { PART }\end{array}$ & PARTICIPANTS NAME AND ADDRESS & $\begin{array}{l}\text { HATCHING } \\
\text { EGG } \\
\text { CAPACITY }\end{array}$ & $\begin{array}{c}\text { PRODUCTS CLASSIFIED } \\
\text { U.S. PULLORUM-TYPHOID } \\
\text { CLEAN }\end{array}$ & $\begin{array}{c}\text { ADDITIONAL } \\
\text { CLASSIFICATIONS FOR } \\
\text { WHICH PRODUCT QUALIFIED }\end{array}$ \\
\hline
\end{tabular}

73 - OKLAHOMA

\begin{tabular}{|c|c|c|c|}
\hline 2363 & $E$ & $\begin{array}{l}\text { THOMAS VOYLES, RT. 1, BOX 2096, THACKERVILLE } \\
73459\end{array}$ & N17_ \\
\hline 2492 & $E$ & $\begin{array}{l}\text { KENNETH WAAG, } 9912 \text { EAST NORTHPARK, } \\
\text { CLAREMORE } 74017\end{array}$ & $\mathrm{~N} 28, \mathrm{~N} 33, \mathrm{~N} 41$ \\
\hline 2453 & $E$ & BRAXTON WADE, RT. 1, BOX 140, RINGLING 73456 & \\
\hline 901 & $E$ & $\begin{array}{l}\text { CHARLES WAGNER, RT. 3, BOX 572, BROKEN BOW } \\
74728\end{array}$ & $\mathrm{~N}$ \\
\hline 1670 & $E$ & GRANVILL WALKER, RT. 1, BOX 63, KENEFIC 74748 & Ni7. \\
\hline 1919 & $E$ & $\begin{array}{l}\text { DONNIE WALKER \& STEVE BOURGUE, RT. 2, BOX 198- } \\
\text { C, DURANT } 74701\end{array}$ & N17. N65 \\
\hline 2233 & $E$ & TRAVIS C. WALKER, RT. 2, BOX 38-M, SKIATOOK 74070 & W25 \\
\hline 2343 & $E$ & CARL WALKER, P.O. BOX 922, ATOKA 74525 & N65, N70 \\
\hline 2682 & $E$ & TERRY WALKER, RT. 2, BOX 38-M, SKIATOOK 74070 & 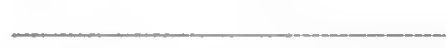 \\
\hline 2781 & $E$ & MATTHEW WALKER, RT. 5, BOX 110-B, ADA 74820 & R53, R56, R76 \\
\hline 1713 & $\mathrm{E}$ & WAYNE WALLACE, 1402 SE 3RD, DURANT 74701 & N107, N28, N32 \\
\hline 920 & $E$ & JERRY WALLIS, RT. 1, BOX 52, PADEN 74860 & N17_______n \\
\hline 2739 & $E$ & $\begin{array}{l}\text { CHARLES WALLIS, } 711 \text { TTH AVENUE, P.O. BOX } 87 . \\
\text { WARNER } 74469\end{array}$ & N17 _ \\
\hline 2637 & $E$ & MIKE WALTERS, RT. 3, BOX 1409, STILWELL 74960 & $\begin{array}{l}\text { Z182, Z201, Z202, Z204, Z205, } \\
\text { Z206, Z209, Z230 }\end{array}$ \\
\hline 2656 & $E$ & $\begin{array}{l}\text { DONALD \& MELISA WALTERS, P.O. BOX } 178, \\
\text { THACKERVILLE } 73459\end{array}$ & $\mathrm{~N}$ \\
\hline 2541 & $E$ & $\begin{array}{l}\text { HOUSTON H. WARD, JR., } 1513 \text { SOUTH } 74 \text { WEST } \\
\text { AVENUE, TULSA } 74127\end{array}$ & N28 \\
\hline 2720 & $E$ & JUSTIN WARE, 8500 SE 154TH STREET, NOBLE 73068 & 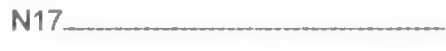 \\
\hline 2071 & $E$ & $\begin{array}{l}\text { OKIE WARLORD (ROBERT FANNON), HC } 66, \text { BOX } 79 \text {, } \\
\text { FOSS } 73647\end{array}$ & $\begin{array}{l}\text { N106, N121, N41, N46, N47, N63, } \\
\text { N8 }\end{array}$ \\
\hline 1303 & $E$ & RALPH WARNER, P.O. BOX 161, DURANT 74701 & N17 \\
\hline 733 & $E$ & BYRON WATKINS, 1620 SOUTH CEDAR, PADEN 74860 & N17 \\
\hline 2706 & $E$ & JUNIOR WEAVER, HC 71, BOX 3223, KINGSTON 73439 & $\begin{array}{l}\text { N28, N32, N41, N83, R1, Z1, Z35, } \\
\text { Z41 }\end{array}$ \\
\hline 2344 & $E$ & BILL WEAVER, RT. 2, BOX 225, BEGGS 74421 & N104___........... \\
\hline 2345 & $E$ & BOB WEBB, 17744 GRANDVIL ROAD, BRISTOW 74010 & $\mathrm{~N}$ \\
\hline 1268 & E & RALPH WEBB, P.O. BOX 245, MONROE 74949 & N1 16, N34, N43 \\
\hline 1595 & E & BRAD WEGER, P.O. BOX 1477, COLBERT 74733 & N17_ \\
\hline 2044 & $E$ & $\begin{array}{l}\text { GARY WELCH, } 3666 \text { SOUTH MCCORD ROAD, PONCA } \\
\text { CITY } 74604\end{array}$ & W30 \\
\hline 2657 & $E$ & $\begin{array}{l}\text { CLARENCE D. WELDON, } 19221 \text { SOUTH HIGHWAY } 49 \text {, } \\
\text { BRISTOW } 74010\end{array}$ & N17 \\
\hline 2797 & $E$ & BOB WENNINGER, RT. 1, BOX 1485, CEMENT 73017 & N28, N74 \\
\hline
\end{tabular}


Table 1. HATCHERIES, DEALERS, AND INDEPENDENT FLOCKS PARTICIPATING IN

THE NATIONAL POULTRY IMPROVEMENT PLAN

WATERFOWL, EXHIBITION POULTRY AND GAME BIRDS

\begin{tabular}{|c|c|c|c|c|c|}
\hline $\begin{array}{l}\text { APP. } \\
\text { NO. }\end{array}$ & $\begin{array}{l}\text { SUB- } \\
\text { PART }\end{array}$ & PARTICIPANTS NAME AND ADDRESS & $\begin{array}{l}\text { HATCHING } \\
\text { EGG } \\
\text { CAPACITY }\end{array}$ & $\begin{array}{c}\text { PRODUCTS CLASSIFIED } \\
\text { U.S. PULLORUM-TYPHOID } \\
\text { CLEAN }\end{array}$ & $\begin{array}{c}\text { ADDITIONAL } \\
\text { CLASSIFICATIONS FOR } \\
\text { WHICH PRODUCT QUALIFIED }\end{array}$ \\
\hline
\end{tabular}

73 - OKLAHOMA

1672 E RANDY WENNINGER, RT. 1, BOX 47-A, CORDELL 73632

2407 E CHARLES WEST, 21851 PERRY-LUCAS ROAD, HARRAH 73045

2298 E TIM WHEELER, RT. 1, BOX 308-B, CHOUTEAU 74337

2454 E HAROLD WHEELER, P.O. BOX 31, THACKERVILLE 73459

2190 E ALBERT WHITE, RT. 2, BOX 500, HOWE 74940

2204 E JACK WHITE, P.O. BOX 64, GENE AUTRY 73436

2234 E L.J.WHITE, P.O. BOX 144, KENEFIC 74748

2740 E JOHNNIE R. WHITE, 3589 NORTH 379 ROAD, HOLDENVILLE 74848

2097 E LARRY WHITE, RT. 2, BOX 208, BENNINGTON 74423

2155 E JERRY WHITE, 901 SOUTH MYERS ROAD, APACHE 73006

2117 E HUBERT WHITEHOUSE, 28125 SOUTH 321ST. WEST AVENUE, BRISTOW 74010

2621 E RANDY WHITEHOUSE, 42968 W. 311 TH STREET. DEPEW 74028

1869 E CLAYTON WHITLOCK, RT. 1, BOX 336, WEWOKA 74884

2364 E GARY WIGGS, RT.2, BOX 2570, CUSHING 74023

2769 E JACKY WILKETT, RT. 3, BOX 1480, WILBURTON 74578

2800 E JARED WILKINS, P.O. BOX 579, SUMMERFIELD 74966

2721 E JOE WILLIAMS, 18700 DEE STREET, NEWALLA 74857

2786

2455

2543

1562

1640

1746

$$
\text { KELLIE JO WILLIAMS, } 9702 \text { SOUTH } 4210 \text { ROAD, }
$$
CHELSEA 74016

E JUNIOR WILLIAMS, RT. 1, BOX 155, ASHER 74826

E JOHN WILLIAMS, RT. 4. BOX 42, DUNCAN 73533

E DAVID C. WILLIAMS, RT. 2, BOX 62-A, MAYSVILLE 73057

E BOB WILLIAMS, RT. 2, BOX 173-9, PORTER 74454

E ODIS WILLIAMSON, RT. 2, BOX 287-B, HENRYETTA 74437

E JONATHAN E. WILLIS, 1721 TREECE ROAD N.E., PIEDMONT 73078

E KELLY WILLIS, RT, 1, BOX 98, WAYNE 73095

E SCOTT WILSON, 2125 WILSON DRIVE, NEWCASTLE 73065

E RANDALL WIMMER, 10492 WEST SHELL CREEK ROAD, SAND SPRINGS 74063

E KIRK WINFORD, 2021 SOUTH BLACKBERRY DRIVE, MCCLOUD 74851

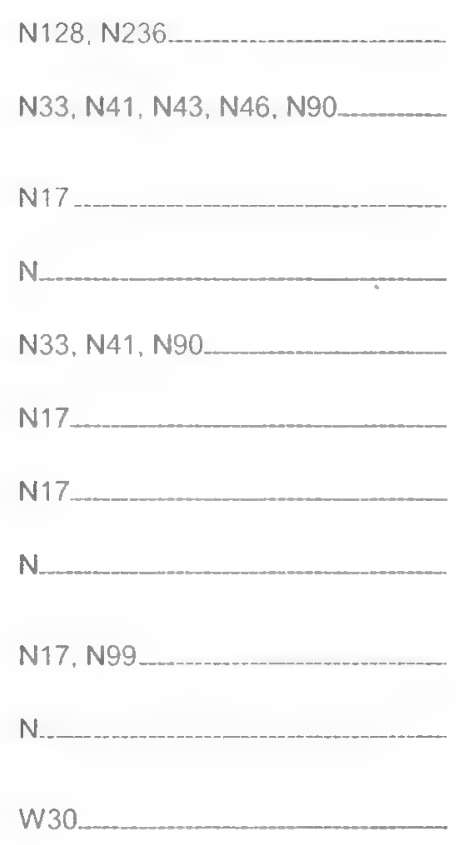

W30

N28, N41.

N.

N41, N43, N65

B1, C1, H9, W114, W198, W20, $\mathrm{X} 107, \mathrm{X} 2, \mathrm{Y} 1, \mathrm{Y} 4, \mathrm{Z} 132, \mathrm{Z} 139$

$\mathrm{N} 17$

N.

N17

N.

N17.

N25, N44, N56.

N17

W12, W142, W263, W50

W301, W357

N17 
Table 1. HATCHERIES, DEALERS, AND INDEPENDENT FLOCKS PARTICIPATING IN THE NATIONAL POULTRY IMPROVEMENT PLAN

WATERFOWL, EXHIBITION POULTRY AND GAME BIRDS

\begin{tabular}{|c|c|c|c|c|c|}
\hline $\begin{array}{l}\text { APP. } \\
\text { NO. }\end{array}$ & $\begin{array}{l}\text { SUB- } \\
\text { PART }\end{array}$ & PARTICIPANTS NAME AND ADDRESS & $\begin{array}{l}\text { HATCHING } \\
\text { EGG } \\
\text { CAPACITY }\end{array}$ & $\begin{array}{l}\text { PRODUCTS CLASSIFIED } \\
\text { U.S. PULLORUM-TYPHOID } \\
\text { CLEAN }\end{array}$ & $\begin{array}{c}\text { ADDITIONAL } \\
\text { CLASSIFICATIONS FOR } \\
\text { WHICH PRODUCT QUALIFIED }\end{array}$ \\
\hline
\end{tabular}

73 - OKLAHOMA

\begin{tabular}{|c|c|c|}
\hline 2509 & $E$ & MIKE WISDOM, RT, 2, BOX 370, ADAIR 74330 \\
\hline 2267 & $E$ & $\begin{array}{l}\text { JOHNNY WISELY, } 13334 \text { NORTH } 77 \text { TH EAST AVENUE, } \\
\text { COLLINSVILLE } 74021\end{array}$ \\
\hline 736 & E & JIM WIXOM, RT. 4, BOX 130, WHITEFIELD 74472 \\
\hline 265 & E & $\begin{array}{l}\text { MICHAEL P. WOLF, } 7301 \text { EAST PRAIRIE GROVE ROAD, } \\
\text { GUTHRIE } 73044\end{array}$ \\
\hline 1172 & E & $\begin{array}{l}\text { PAUL \& RUTH WOLF, } 7301 \text { EAST PRAIRIE GROVE } \\
\text { ROAD, GUTHRIE } 73044\end{array}$ \\
\hline 2602 & E & $\begin{array}{l}\text { WONDER GAME FARM, JESSE LAWSON, HC } 68, \text { BOX } \\
740 \text {, CHECOTAH } 74426\end{array}$ \\
\hline 2493 & E & $\begin{array}{l}\text { ORVILLE WOOD, } 7419 \text { SOUTH } 161 \text { ST WEST AVENUE, } \\
\text { SAPULPA } 74066\end{array}$ \\
\hline 1440 & E & $\begin{array}{l}\text { ERIC E. WOOD, } 39178 \text { WEST 54TH PLACE SOUTH, } \\
\text { MANNFORD } 74044\end{array}$ \\
\hline 254 & $E$ & $\begin{array}{l}\text { DARWIN \& DONITA WOODALL, } 12664 \text { SOUTH 33RD } \\
\text { WEST AVENUE. SAPULPA } 74066-7400\end{array}$ \\
\hline 2467 & $E$ & SID WOODALL, RT. 2, BOX 415, BRISTOW 74010 \\
\hline 2468 & E & TRAVIS WOODSON, 5400 EXPLORER, ORLANDO 73073 \\
\hline 2585 & $E$ & JERELL WOOLWINE, RT. 1, BOX 185-H, DUNCAN 73533 \\
\hline 669 & E & GARY WOOLWINE, RT. 4, BOX 221-F, DUNCAN 73533 \\
\hline 1898 & $\mathrm{E}$ & $\begin{array}{l}\text { DORTHY WORCHESTER, RT. 2, BOX 181, ARDMORE } \\
73401\end{array}$ \\
\hline 2655 & $E$ & JOE WORKMAN, RT. 3, BOX 69-W, OKEMAH 74859 \\
\hline 1424 & E & $\begin{array}{l}\text { JACK \& VIC WORLEY, HC } 64, \text { BOX 2620, FT. TOWSON } \\
74735\end{array}$ \\
\hline 2213 & E & GREG WORTHAM, RT. 2, BOX 81-F, MARLOW 73055 \\
\hline 2280 & E & RICK WRIGHT, RT. 2, BOX 130-B, BRISTOW 74010 \\
\hline 2469 & E & $\begin{array}{l}\text { ROBERT LEE WRIGHT, JR., RT. 1, BOX 1144. ANTLERS } \\
74523\end{array}$ \\
\hline 2598 & E & $\begin{array}{l}\text { MAURICE WRIGHT, } 3940 \text { NORTH STELLA, SPENCER } \\
73084\end{array}$ \\
\hline 1444 & $E$ & JODIE WRIGHT, RT. 2, BOX 58-B, RUSH SPRINGS 73082 \\
\hline 2510 & $E$ & TERRY WYATT, RT. 1, BOX 259, PAULS VALLEY 73075 \\
\hline 1100 & $E$ & J. C. WYRICK, P.O. BOX 242, HUGO 74743 \\
\hline 2365 & E & JUAN YANEZ, HC 60, BOX 78, SPRINGER 73458 \\
\hline 497 & E & TERRY YELL, 618 EAST BENTON, SAYRE 73662 \\
\hline 2241 & $E$ & ROY YOCHAM, P.O. BOX 7, HITCHITA 74438 \\
\hline 2156 & E & $\begin{array}{l}\text { BLACK LOBRA FARMS, DAMON YORKMAN, RT. 1, BOX } \\
\text { 301, CHELSEA } 74016\end{array}$ \\
\hline 2511 & E & $\begin{array}{l}\text { RAMUNDO YOUNG, } 605 \text { NW FAIRVIEW STREET, IDABEL } \\
74745\end{array}$ \\
\hline
\end{tabular}

N.

N17.

R168, W119, W160, W4, W56, W576, W6

$W 12, \times 10, \times 6, Z 205$

N17

N17, W2

W30, W7

W11, W12, W231, W301, W357, W361

N17

N17.

$\mathrm{N} 28, \mathrm{~N} 32, \mathrm{~N} 44$

$\mathrm{N}$

N17

N17

N28, N32, N41

N17

N17.

N28, N32, N44

N28, N32, N8

N17

N17

N38

N17

$N$ 
Table 1. HATCHERIES, DEALERS, AND INDEPENDENT FLOCKS PARTICIPATING IN

THE NATIONAL POULTRY IMPROVEMENT PLAN

WATERFOWL. EXHIBITION POULTRY AND GAME BIRDS

\begin{tabular}{lllcl}
\hline & & HATCHING & PRODUCTS CLASSIFIED \\
APP. & SUB- & PARTICIPANTS NAME AND ADDRESS \\
NO. & PART & EGG & U.S. PULLORUM-TYPHOID & CLEAN \\
\hline
\end{tabular}

73 - OKLAHOMA

$\begin{array}{lll}2137 & \text { E } & \begin{array}{l}\text { ISAAC YOUNGBIRD, GENERAL DELIVERY, TWIN OAKS } \\ 74368\end{array} \\ 2317 & \text { E } & \text { GARY ZACHARY, RT. 1, BOX 198-D, DURANT } 74701 \\ 1197 & \text { E } & \begin{array}{l}\text { W.P. (WILLIAM) ZIMMERMAN, 35565 SOUTH 4470 ROAD, } \\ \text { WELCH 74369 }\end{array}\end{array}$

N

N17.

N28, N32

92 - OREGON

DR. ANDREW A. CLARK

STATE VETERINARIAN

OREGON DEPARTMENT OF AGRICULTURE

635 CAPITOL STREET, NE

SUITE 100

SALEM, OR $97310-2532$

Phone: (503)986-4680

Fax: (503) $986-4734$

\section{Hatcheries}

226 E CANYON CREEK GAME FARM, ROBERT ALEXANDER, 63439 DUFUR GAP ROAD, DUFUR 97021

140 E HOLDERREAD'S WATERFOWL FARM, 2679 WEST HILLS ROAD, PHILOMATH 97370

55

SHANK'S HATCHERY, P.O. BOX 38, HUBBARD 97032

\section{Dealers}

242 E EARLY BIRD GAME FARM \& HATCHERY, 4300 FOOTHILLS BLVD., GRANTS PASS 97526 Phone: (541)955-8527

232 E UNCLE TOM'S FARM, TOM JOHNSON, P.O. BOX 42 , GARDINER 97441

Independent Flocks

$\begin{array}{ccl}227 & E & \begin{array}{l}\text { A.L. ADAMS, } 41728 \text { KINGSTON JORDAN ROAD, } \\ \text { STAYTON } 97383\end{array} \\ 244 \quad \text { E } & \begin{array}{l}\text { LARRY ANDERSON, 1800 ROXY ANN PLACE, MEDFORD } \\ \text { 97504 }\end{array} \\ 240 \quad \text { E } \quad \begin{array}{l}\text { BIRCH CREEK BIRD FARM, RONALD BENJAMIN, } 85594 \\ \text { WINESAP ROAD, MILTON-FREEWATER } 97862\end{array}\end{array}$

DR. BRUCE. MUELLER

OREGON DEPARTMENT OF AGRICULTURE 635 CAPITOL STREET, NE

SALEM, OR 97310-0110

Phone: (503)986-4680

Fax: $\quad(503) 986-4734$

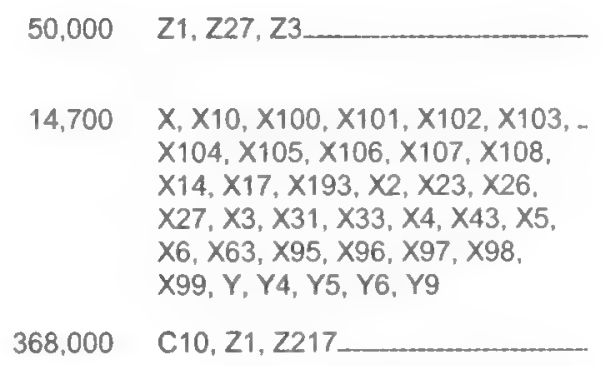

$14,700 \quad X, X 10, X 100, X 101, \times 102, \times 103, \ldots$ $X 104, X 105, X 106, X 107, X 108$. $\times 14, \times 17, \times 193, \times 2, \times 23, \times 26$. $x 27, x 3, x 31, x 33, x 4, x 43, x 5$, $X 6, X 63, X 95, X 96, X 97, X 98$, $X 99, Y, Y 4, Y 5, Y 6, Y 9$

$368,000 \quad \mathrm{C} 10,21,2217$

E\&C N9, R10, R146, R196, R20, R227, R237, R239, R240, R247, R34, R6, R7, W25, W326, Z1, Z118, Z129, Z141, Z142, Z15, Z188, Z21, Z235, Z24, Z257, Z26, Z260, Z27, Z34, Z4, Z41, Z43, Z51, Z52. $\mathrm{Z} 53, \mathrm{Z} 5 \mathrm{7}, \mathrm{Z68}, \mathrm{Z72}, \mathrm{Z93}$

E\&C R18, R259, R57, W248, W28, W33, W518, X187, X85, Z118, Z142, Z169, Z188, Z24, Z257, Z26, Z27, Z283, Z290, Z34, Z49, Z57, 29

$Z 1, Z 27, Z 9$

Z107.

$\mathrm{Z1}, \mathrm{Z2}, \mathrm{Z} 43$ 
Table 1. HATCHERIES, DEALERS, AND INDEPENDENT FLOCKS PARTICIPATING IN

THE NATIONAL POULTRY IMPROVEMENT PLAN

WATERFOWL, EXHIBITION POULTRY AND GAME BIRDS

$\begin{array}{lll}\text { APP. } & \text { SUB- } \\ \text { NO. } & \text { PART }\end{array}$

HATCHING
EGG
CAPACITY

PRODUCTS CLASSIFIED

U.S. PULLORUM-TYPHOID CLEAN
ADDITIONAL

CLASSIFICATIONS FOR WHICH PRODUCT QUALIFIED

92 - OREGON

\begin{abstract}
226
E

CANYON CREEK GAME FARM, ROBERT ALEXANDER, 63439 DUFUR GAP ROAD, DUFUR 97021

248

E KARL CASCO, 14000362 ND AVENUE, BORING 97009

CRAFTYS QUAIL FARM, 21050 NW SPRUCE AVENUE, REDMOND 97756

E ROBERT \& LAURA FLOWERS, 1121 MARQUERITE WAY. NEWBERG 97132

E FOWL. PLAY, EVELYN BAKER \& SHANNON MORGAN 28598 STAFFORD HANSELL ROAD, HERMISTON 97838 Phone: (541)567-6670

E PAMELA HOGAN, 650 SARDINE CREEK ROAD, GOLD HILL 97525

Phone: (541)855-7674

HOLDERREAD'S WATERFOWL FARM, 2679 WEST HILLS ROAD, PHILOMATH 97370
\end{abstract}

ROCK CREEK BIRD FARM, H \& J. JORDAN, 15034 MIDDLE ROCK CREEK LANE, ARLINGTON 97812

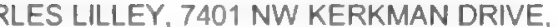
CORNELIUS 97113 ABBEY ROAD, CARLTON 97111

E PAUL LONG, 29380 NE OWLS LANE, NEWBERG 97132 Phone: (503)685-8752

E DON NICHOLS, 5915 S.E. 174TH, PORTLAND 97236

E RESTON GAMEBIRD FARM, D. DOUTHITT, 3621 RESTON ROAD, ROSEBURG 97470
BILL SOVAY, P.O. BOX 12411,2197 DOAKS FERRY ROAD, SALEM 97309

E GLENNL. THOMA, 11388 SOUTH HIGHWAY 211,P.O. BOX 617 MOLALLA 97038

Phone: (503)651-3408

E MATTHEW TUTTLE, 30608 SE PIPELINE, GRESHAM 97080

E GUY WHEATLEY, 11118 SOUTHMT. HOPE ROAD. MOLLALA 97308
Z1, Z2, Z27, Z3, Z30, Z31, Z43, _ Z44, Z48, Z74, Z88, Z93

N17.

$\mathrm{X} 3, \mathrm{Z1}, \mathrm{Z142}, \mathrm{Z24}, \mathrm{Z259}, \mathrm{Z27}$, Z293, Z31, Z33, Z43, Z45, Z60.

293

Z142, Z43.

R232, R248, R71, R86, W132, W207, W209, W415, W573, X2

X5, Z129, Z219, Z220, Z27, Z28, $\mathrm{Z4}$

$X, X 10, \times 100, \times 101, \times 102, \times 103$, $\mathrm{X} 104, \times 105, \times 106, \times 107, \times 108$. $\times 14, \times 17, \times 193, \times 2, \times 23, \times 26$. $\times 27, \times 3, \times 31, \times 33, \times 4, x 43, \times 5$, $X 6, X 63, X 95, X 96, X 97, X 98$ $X 99, Y, Y 4, Y 5, Y 6, Y 9$

Z2.

Z27

$\mathrm{Z} 112, \mathrm{Z43}$

N17.

Z1, Z118, Z12, Z126, Z141, Z142, Z149, Z24, Z26, Z260, Z268, Z269, Z27, Z34, Z43, Z45, Z48, Z52, Z53, Z55, Z57, Z69, Z72

267

Z2, Z48

N17

R, R57, Z118, Z126, Z57

Z202, Z203, Z227 
Table 1. HATCHERIES, DEALERS, AND INDEPENDENT FLOCKS PARTICIPATING IN

THE NATIONAL POULTRY IMPROVEMENT PLAN

WATERFOWL, EXHIBITION POULTRY AND GAME BIRDS

\begin{tabular}{|c|c|c|c|c|c|}
\hline $\begin{array}{l}\text { APP. } \\
\text { NO. }\end{array}$ & $\begin{array}{l}\text { SUB- } \\
\text { PART }\end{array}$ & PARTICIPANTS NAME AND ADDRESS & $\begin{array}{l}\text { HATCHING } \\
\text { EGG } \\
\text { CAPACITY }\end{array}$ & $\begin{array}{l}\text { PRODUCTS CLASSIFIED } \\
\text { U.S. PULLORUM-TYPHOID } \\
\text { CLEAN }\end{array}$ & $\begin{array}{c}\text { ADDITIONAL } \\
\text { CLASSIFICATIONS FOR } \\
\text { WHICH PRODUCT QUALIFIED }\end{array}$ \\
\hline
\end{tabular}

23 - PENNSYLVANIA

DR. JOHNI. ENCK, JR

and

DIRECTOR, ANIMAL. INDUSTRY

PENNSYLVANIA DEPARTMENT OF AGRICULTURE

2301 NORTH CAMERON STREET

HARRISBURG, PA 17110

Phone: (717)783-6677

Fax: $\quad(717) 787-1868$

\section{Hatcheries}

183

$E$

E

ERVIN BURKHOLDER, 1000 LONG LANE ROAD, DENVER 17517

294

$E$

CLEARVIEW STOCK FARM HATCHERY, BOX 399 , GRATZ 17030

\begin{tabular}{|c|c|c|}
\hline 357 & $E$ & $\begin{array}{l}\text { ERDMAN GAME FARM, D. ERDMAN, RD 1, BOX } 1386 \text {, } \\
\text { HERNDON } 17830\end{array}$ \\
\hline 246 & $E$ & $\begin{array}{l}\text { DICK GREUBEL, } 441 \text { FIRST AVENUE, DUNCANSVILLE } \\
16635\end{array}$ \\
\hline 68 & $E$ & $\begin{array}{l}\text { GRIMAUD FARMS, } 886 \text { EAST MOUNTAIN ROAD, HEGINS } \\
17938\end{array}$ \\
\hline 72 & $E$ & $\begin{array}{l}\text { KENNETH GRIMM, } 126 \text { EAST END ROAD, } \\
\text { CONNELLSVILLE } 15425\end{array}$ \\
\hline 81 & $E$ & $\begin{array}{l}\text { HIDDEN CREEK HATCHERY, M. LAITY, RR 2, BOX 2068- } \\
\text { B. SHICKSHINNY } 18655\end{array}$ \\
\hline 39 & $E$ & HOFFMAN HATCHERY, PINIC ROAD, GRATZ 17030 \\
\hline 44 & $E$ & $\begin{array}{l}\text { HORAN'S TURKEY FARM, RT. 4, BOX } 417 \text {, TAMAQUA } \\
18252\end{array}$ \\
\hline 69 & $E$ & $\begin{array}{l}\text { NEVIN KEGERREIS, } 5101 \text { GUITNER ROAD, } \\
\text { CHAMBERSBURG } 17201\end{array}$ \\
\hline 88 & $E$ & DONALD L. KRAHE, 2108 WAGER ROAD. ERIE 16509 \\
\hline 69 & $E$ & $\begin{array}{l}\text { MAHANTONGO GAME FARMS, RR 1, BOX 70, DALMATIA } \\
17017\end{array}$ \\
\hline 95 & $E$ & MARTZ GAME FARM, RR 1, BOX 85. DALMATIA 17017 \\
\hline 27 & $E$ & $\begin{array}{l}\text { MEADOWBROOK GAME BIRDS, BOX } 127, \text { RICHFIELD } \\
17086\end{array}$ \\
\hline 4 & $E$ & $\begin{array}{l}\text { MOYER'S CHICKS, INC., } 266 \text { EAST PALETOWN ROAD, } \\
\text { QUAKERTOWN } 18951\end{array}$ \\
\hline & $E$ & $\begin{array}{l}\text { WILLIAM L. MUSSER, } 12136 \text { PEN MAR ROAD, } \\
\text { WAYNESBORO } 17268\end{array}$ \\
\hline
\end{tabular}

DR. PHILLIP C. DEBOK.

ANIMAL \& POULTRY HEALTH DIVISION

PENNSYLVANIA DEPARTMENT OF AGRICULTURE

2301 NORTH CAMERON STREET

HARRISBURG, PA 17110

Phone: (717)783-8555

1,200 A, D, R11, R169, R19, R22, R23, R34, R51, R86, R93, W11, W12, $\mathrm{X} 21, \mathrm{Y} 4, \mathrm{Y} 6$

$120,000 \quad$ A, B, C, D, E, H, X10, X11, X17, $\mathrm{Z1}$

$209,000 \quad$ A, B, C, D, H, W, X10, X14, X17, $X 2, X 25, X 27, X 3, X 37, X 4, X 48$ $X 6, X 64, X 9, Y 1, Y 13, Y 2, Y 4, Z 1$, Z110, Z20, Z202, Z212, Z22, Z62, Z88

160 Z108, Z15, Z179, Z19

$1.400 \quad \mathrm{R} 181, \mathrm{~W} 110, \mathrm{~W} 129, \mathrm{~W} 131$, W155, W304, W386, W39, W424

$67,200 \times 21$

$11,000 \quad Z 1, Z 12, Z 2, Z 28$

MG; MS; Al
Fax: (717)787-1868

$128,000 \times 11, \times 14, \times 17, \times 2, \times 21, \times 25$, $\times 27, X 3, X 37, X 4, X 45, X 5, X 6$, $X 9, Y 1, Y 2, Y 3, Y 4, Z 20, Z 22, Z 62$

$10,000 \quad Z 1, Z 110, Z 12, Z 2, Z 20, Z 212, \ldots$ Z22, Z3, Z30, Z48, Z62, Z9

200,000 R19, R93, W74, W75, W76, X1, $\mathrm{X} 11, \mathrm{X} 14, \mathrm{X} 15, \mathrm{X} 17, \mathrm{X} 2, \mathrm{X} 25$, $X_{28}, X 3, X 4, X 6, X 9, Y 1, Y 10, Y 2$, $Y 3, Y 4, Y 5, Y 7, Z 24, Z 57$

900 R111, W166, W25, W26, X10, $\times 17$

135,135 Z1, Z2
WHICH PRODUCT QUALIFIED 
Table 1. HATCHERIES, DEALERS, AND INDEPENDENT FLOCKS PARTICIPATING IN

THE NATIONAL POULTRY IMPROVEMENT PLAN

WATERFOWL, EXHIBITION POULTRY AND GAME BIRDS

\begin{tabular}{|c|c|c|c|c|c|}
\hline $\begin{array}{l}\text { APP. } \\
\text { NO. }\end{array}$ & $\begin{array}{l}\text { SUB- } \\
\text { PART }\end{array}$ & PARTICIPANTS NAME AND ADDRESS & $\begin{array}{l}\text { HATCHING } \\
\text { EGG } \\
\text { CAPACITY }\end{array}$ & $\begin{array}{c}\text { PRODUCTS CLASSIFIED } \\
\text { U.S. PULLORUM-TYPHOID } \\
\text { CLEAN }\end{array}$ & $\begin{array}{c}\text { ADDITIONAL } \\
\text { CLASSIFICATIONS FOR } \\
\text { WHICH PRODUCT QUALIFIED }\end{array}$ \\
\hline & & 23 & - PENNSYLVANIA & & \\
\hline 130 & E & NOLL'S POULTRY FARM, , KLEINFELTERSVILLE 17039 & 217,000 & $\mathrm{~B}, \mathrm{~B} 7, \mathrm{C}, \mathrm{C} 6, \mathrm{D} \ldots$ & \\
\hline 364 & $E$ & DAVID H. REBER, JR., 251 POND ROAD, FELTON 17322 & 300 & W26 & \\
\hline 162 & $E$ & REICH POULTRY FARM, INC., RR 1, MARIETTA 17547 & 113,000 & W26 & \\
\hline 336 & $E$ & $\begin{array}{l}\text { LORETTA K. SCHMIDGALL, RD 2, BOX } 785 \text {, } \\
\text { THOMASVILLE } 17364\end{array}$ & 1,000 & $\begin{array}{l}\text { A, W132, W138, W415, W45, } \\
\text { W81, Y14, Y25, Y3, Y4, Y6 }\end{array}$ & \\
\hline 359 & $E$ & $\begin{array}{l}\text { ARNOLD R. SENSENIG, } 1365 \text { INDIANTOWN ROAD, } \\
\text { STEVENS } 17578\end{array}$ & 74,000 & CUSTOM, HATCHING & \\
\hline 346 & $E$ & $\begin{array}{l}\text { TRACE PHEASANTRY, } 288 \text { LEVENGOOD ROAD, } \\
\text { DOUGLASVILLE } 19518\end{array}$ & 55,600 & Z1, Z12, Z27, Z30, Z43, Z48, Z93_ & \\
\hline 360 & $E$ & $\begin{array}{l}\text { ALVIN B. ZIMMERMAN, RD 2, BOX 188, WILLIAMSBURG } \\
16693\end{array}$ & 44,000 & $\mathrm{Z} 1$ & \\
\hline \multirow[t]{2}{*}{362} & $E$ & $\begin{array}{l}\text { DAVID H. ZIMMERMAN, } 995 \text { WEST FAIRVIEW ROAD, } \\
\text { LITITZ } 17543\end{array}$ & 19,000 & $\mathrm{Z1}$ & \\
\hline & & Dealers & & & \\
\hline 366 & $E$ & $\begin{array}{l}\text { JUNIATA RIVER GAME FARM, D. BOREMAN, } 52 \text { GOOSE } \\
\text { LANE, MCCLURE } 17841\end{array}$ & $\mathrm{E \& C}$ & $X 28, X 4, X 8, Y 9, Z 1, Z 2, Z 43$ & \\
\hline \multirow[t]{2}{*}{371} & $E$ & $\begin{array}{l}\text { WOLFE'S GAMEBIRD HATCHERY, RD. \#2, BOX } 359 \text {, } \\
\text { BROOKVILLE } 15825\end{array}$ & $\mathrm{E \& C}$ & $\mathrm{Z1}, \mathrm{Z88}$ & \\
\hline & & Independent Flocks & & & \\
\hline 373 & $E$ & BRIAN H. PIFER, RR 2, BOX 55-E, LERAYSVILLE 18829 & & $\mathrm{R} 20, \mathrm{R} 42, \mathrm{~W} 12, \mathrm{~W} 387, \mathrm{~W} 55$ & MG; MS \\
\hline
\end{tabular}

DR. CHRISTOFER HANNAFIN

STATE VETERINARIAN

RHODE ISLAND DIVISION OF AGRICULTURE

235 PROMENADE STREET

PROVIDENCE, RI 02908-5767

Phone: (401)222-2781, EXT. 4503

Fax: $\quad(401) 222-6047$

Independent Flocks

57 E ADDIEVILLE EAST FARM, GEOFF GAEBE, P.O. BOX 157 , MAPLEVILLE 02835

64 E HARRY "BUZZ" BARBOUR, 190 FRY POND ROAD, WEST GREENWICH 02817

87 E DAVE BETHEL, 1 SHERMAN AVENUE, LINCOLN 02865

W89, W91

W151, W28, W568, W88.

79 E SUSAN BROWN, P.O. BOX 237. EXETER 02822-2907

54 E WARREN CARLOW, JR., 955 PLAINFIELD PIKE, NORTH SCITUATE 02857

227

R11, R227, W142, W556, W568, KINGSTOWN 02852

R183, W544, W6, W8

W120, W28, W3, W48, W67 
Table 1. HATCHERIES, DEALERS, AND INDEPENDENT FLOCKS PARTICIPATING IN

THE NATIONAL POULTRY IMPROVEMENT PLAN

WATERFOWL, EXHIBITION POULTRY AND GAME BIRDS

APP. SUB- PARTICIPANTS NAME AND ADDRESS

NO. PART
PARTICIPANTS NAME AND ADDRESS

HATCHING
EGG
CAPACITY

PRODUCTS CLASSIFIED

U.S. PULLORUM-TYPHOID

CLEAN
ADDITIONAL

CLASSIFICATIONS FOR WHICH PRODUCT QUALIFIED

\section{5 - RHODE ISLAND}

RICHARD GEE, P.O. BOX 157, FISKVILLE 02823

$86 \quad E$

TREVOR GREENE, 12 RICHMOND TOWN HOUSE ROAD, WYOMING 02898

29 E ROBERT HARMON, P.O. BOX 94, GREEN 02827

70 E DAVID HARTLEY, 36 QUEENS FORT LANE, EXETER 02822

74 E ROBERT HARTLEY, 1711 FLAT RIVER ROAD, COVENTRY 02816

96 E THOMAS HARTSHORN, 17 TAYLOR ROAD, JOHNSTON 02919

80 E ASHLEY HAY, 21 RED OAK DRIVE, WYOMING 02898

89 E RICHARD IADEVAIA, 575 SPRING LAKE ROAD, GLENDALE 02826

71 E RICHARD KENYON, P.O. BOX 268, ASHAWAY 02804

82 E DAVE LOFGREN, 462 ROBIN HOLLOW ROAD, WEST GREENWICH 02817

75 E ROBERT MINER, P.O. BOX 231, ASHAWAY 02804

28 E NATALINO MIZZONI, 229 WESTCOTT ROAD, NORTH SCITUATE 02857

90 E MARCO MIZZONI, 26 EAST KILLINGLY ROAD, FOSTER 02825

76 E JOSEPHMONIZ, 363 FAIRVIEW AVENUE, COVENTRY 02816

91 E JAMES MONK, 30 WENDY LANE, CAROLINA 02812

51 E DONALD NELSON, P.O. BOX 345, WYOMING 02898

67 E ROBERT PADULA, 129 LIBERTY ROAD, EXETER 02822 Phone: (401)295-8466

92 E PAUL PAIVA, P.O. BOX 648, SLATERSVILLE 02876-0648

84 E RONALD PIERCE, 60 SHORE DRIVE, NORTH KINGSTOWN 02852

62 E WILLIAM RICHARDSON, 1945 MIDDLE ROAD, EAST GREENWICH 02818

83 E NORMAN RICHMOND, 874 FRENCHTOWN ROAD, EAST GREENWICH 02818

66 E GEORGE \& DONNA SCHOPAC.60 CAMP AVENUE, NORTH KINGSTON 02852

97 E JOHN SUNDELIN, 280 FRY POND ROAD, WEST GREENWICH 02817

93 E CARL SWANSON, 47 GOODWIN STREET, EAST GREENWICH 02818

94 E JAYTEKLENSKI, P.O. BOX 43, NORTH KINGSTOWN 02852

17 E UNIVERSITY OF RHODE ISLAND, 213 WOODWARD HALL, NORTH KINGSTON 02852
}

R114, R9, W11, W119, W28, W34. W69, W7, W8

W102, W121

Z1, Z43

W69, W88

W200, W28, W50, W53, W56, W88

R227, R233, R237

W18, W568

R146, R6

R37, R52, R71, R86, W25, W26 W89

W119, W26

W211, W57, W61, W74, W75, W88

W125, W28

W69.

R204, W3

R183, W125, W568, W76, W8 W120, W2, W492, W52, W69, W89

R183, W121, W29, W570, W76

W190, W281, W38, W54, W576, . W67, W79

N4, W142, W2, W200, W28, W48, W5, W51, W568

W23, W52, W89

W128, W28, W29, W48

W220, W69

R93, W134, W578

W11. 293

$\mathrm{R} 183, \mathrm{Z27}$ 
Table 1. HATCHERIES, DEALERS, AND INDEPENDENT FLOCKS PARTICIPATING IN

THE NATIONAL POULTRY IMPROVEMENT PLAN

WATERFOWL, EXHIBITION POULTRY AND GAME BIRDS

\begin{tabular}{|c|c|c|c|c|c|}
\hline $\begin{array}{l}\text { APP. } \\
\text { NO. }\end{array}$ & $\begin{array}{l}\text { SUB- } \\
\text { PART }\end{array}$ & PARTICIPANTS NAME AND ADDRESS & $\begin{array}{l}\text { HATCHING } \\
\text { EGG } \\
\text { CAPACITY }\end{array}$ & $\begin{array}{l}\text { PRODUCTS CLASSIFIED } \\
\text { U.S. PULLORUM-TYPHOID } \\
\text { CLEAN }\end{array}$ & $\begin{array}{c}\text { ADDITIONAL } \\
\text { CLASSIFICATIONS FOR } \\
\text { WHICH PRODUCT QUALIFIED }\end{array}$ \\
\hline & & 15 & - RHODE ISLAND & & \\
\hline 85 & E & $\begin{array}{l}\text { STEVE VAN HORN, } 73 \text { MAXON HILL ROAD, ASHAWAY } \\
02804\end{array}$ & & R227, W153__________________ & \\
\hline 95 & $E$ & $\begin{array}{l}\text { JANE WILBUR, } 397 \text { CHESTNUT HILL ROAD, } \\
\text { CHEPACHET } 02814\end{array}$ & & $\begin{array}{l}\text { R104, R122, R179, R20, R222, } \\
\text { R30, R45, R51, R53, R66, R88, } \\
\text { W13 }\end{array}$ & \\
\hline 22 & $E$ & $\begin{array}{l}\text { GERRY YEAW, } 105 \text { WILLIAM HENRY ROAD, NORTH } \\
\text { SCITUATE } 02857\end{array}$ & & $\begin{array}{l}\text { R114, W138, W174, W188, } \\
\text { W274, W35, W76, W88 }\end{array}$ & \\
\hline
\end{tabular}

DR. JULIE D. HELM

CLEMSON LABORATORY

LIVESTOCK-POULTRY HEALTH DEPARTMENT

P.O. BOX 102406

COLUMBIA, SC 29224

Phone: (803)788-2260

Fax: $\quad(803) 736-0885$

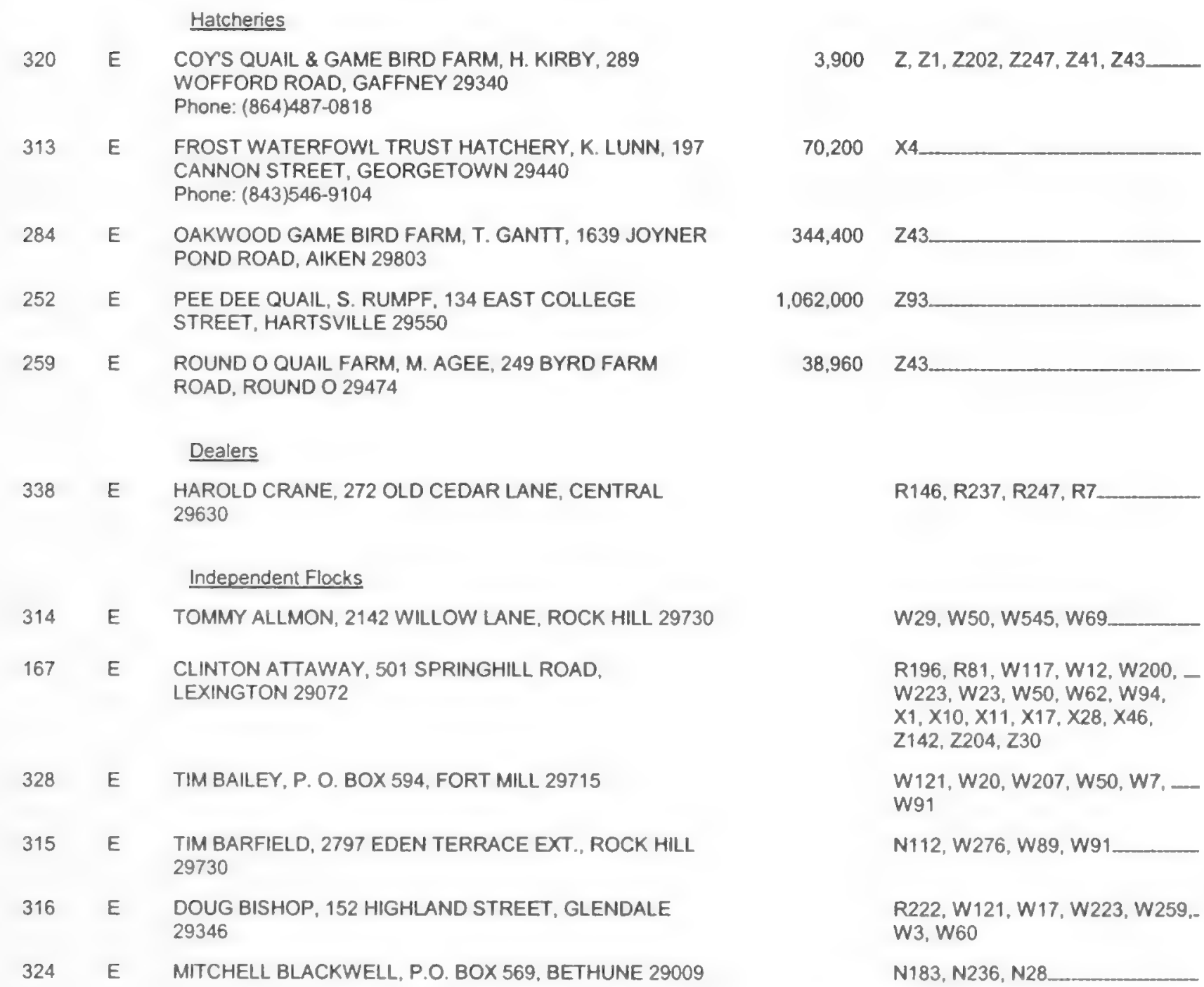


Table 1. HATCHERIES, DEALERS, AND INDEPENDENT FLOCKS PARTICIPATING IN

THE NATIONAL POULTRY IMPROVEMENT PLAN

WATERFOWL, EXHIBITION POULTRY AND GAME BIRDS

\begin{tabular}{|c|c|c|c|c|c|}
\hline $\begin{array}{l}\text { APP. } \\
\text { NO. }\end{array}$ & $\begin{array}{l}\text { SUB- } \\
\text { PART }\end{array}$ & PARTICIPANTS NAME AND ADDRESS & $\begin{array}{l}\text { HATCHING } \\
\text { EGG } \\
\text { CAPACITY }\end{array}$ & $\begin{array}{l}\text { PRODUCTS CLASSIFIED } \\
\text { U.S. PULLORUM-TYPHOID } \\
\text { CLEAN }\end{array}$ & $\begin{array}{c}\text { ADDITIONAL } \\
\text { CLASSIFICATIONS FOR } \\
\text { WHICH PRODUCT QUALIFIED }\end{array}$ \\
\hline
\end{tabular}

$\begin{array}{lll}305 & \text { E } & \begin{array}{l}\text { NICK BOLIN, } 237 \text { ROCK SPRINGS ROAD, BLACKSBURG } \\ 29702\end{array} \\ 341 & \text { E } & \text { GENE BRANCHE, 2821 DALE HURSL, ROCK HILL } 29731 \\ 329 & \text { E } & \begin{array}{l}\text { PETE BRANHAM, 1578 FIRE TIRE ROAD, ROCK HILL } \\ 29730\end{array} \\ 330 & \text { E } & \begin{array}{l}\text { HERMAN EARL CAIN, BOX 370, CAIN PATCH LANE, MT. } \\ \text { CROGAN 29727 }\end{array} \\ 331 & \text { E } & \text { GREG CARVER, 385 KIMBERLY LANE, TAMASSEE 29686 }\end{array}$

MIKE LOUDERMILK, 250 WINDY HOLLOW ROAD, WALHALLA 29691

E

BILLY CORDER, 336 MARION STREET, ROCK HILL 29730

E GARY DEBRUHL, 571 FLETCHER DRIVE, KERSHAW 29067

E JANICE GEIGER, 251 CROSBY ROAD, GILBERT 29054

E DAVID N. HILL, 255 ANDERSON HILL ROAD, BISHOPVILLE 29010

E DENNIS HOOVER, 717 EDDIE WATKINS ROAD, CASSATT 29032

E ROCKY HUGHEY, 163 COYL ROAD, GAFFNEY 29340

E INGLESIDE PLANTATION, LLC., JIM HILLS, 107 CANNON STREET, GEORGETOWN 29440

E DAVID JETT, 855 BETHESDA ROAD, SPARTANBURG 29302

E W. D. JONES, 110 KING STREET, LEXINGTON 29072

E THOMAS LEWIS, 1844 HIGHWAY 57 SOUTH, DILLON 29536

E JASON LEWIS, 922 STERRA ROAD, YORK 29745
E LYMAN MATHIS, 192 MATHIS RANCH DRIVE, GRAY COURT 29645

E PETE MCCONNELL, 712 BISHOP BRANCH ROAD, CENTRAL 29630

E MiCHELLE NUOVO, 232 WOODCREST LANE, GASTON 29053
W132

W151, W28, W50, W88

W151, W28, W69, W88

N17.

N106, N109, N116, N131, N139, .. N15, N24, N240, N28, N32, N41, N43, N63, N90, N97, R20, R62,

R86, W121, W149, W17, W18, W28, W3, W50, W570, W573, W88

R246, W17, W415, W81, W83, ... W88

N17.

W18, W4, W559, Z4_

N104, N137, N139, N163, N165, . N181, N182, N25, N43, N63

N114, N17, N183, N236, N28, N44

W28, W69, W91

$\mathrm{X} 48$

R21, W36

W115, W128, W187, W88

W121, W142, W145, W164 W19, W27, W308, W69, W83, Z204, Z41, Z43, Z44, Z48

W121, W28, W303, W88, X143, $\mathrm{X} 65, \mathrm{Z} 118, \mathrm{Z2}, \mathrm{Z211}$

N63, R1, R123, R146, R178, R19.. R222, R227, R229, R245, R70,

R88, R89, W1, W12, W121, W13. W140, W142, W149, W164, W169, W17, W179, W18, W23, W247, W263, W28, W29, W3, W301, W33, W38, W387, W48, W50, W518, W52, W568, W573, W579, W596, W60, W615, W7. W74, W75, W86, W89, W91. W97, X1, X131, X25, X27, Y4, Z22, Z93, Z94

W142, W28, W566, W88

W120, W128, W187, W4, W54, .. W7

W17, W19, W20, W57. 
Table 1. HATCHERIES, DEALERS, AND INDEPENDENT FLOCKS PARTICIPATING IN

THE NATIONAL POULTRY IMPROVEMENT PLAN

WATERFOWL, EXHIBITION POULTRY AND GAME BIRDS

\begin{tabular}{|c|c|c|c|c|c|}
\hline $\begin{array}{l}\text { APP. } \\
\text { NO. }\end{array}$ & $\begin{array}{l}\text { SUB- } \\
\text { PART }\end{array}$ & PARTICIPANTS NAME AND ADDRESS & $\begin{array}{l}\text { HATCHING } \\
\text { EGG } \\
\text { CAPACITY }\end{array}$ & $\begin{array}{l}\text { PRODUCTS CLASSIFIED } \\
\text { U.S. PULLORUM-TYPHOID } \\
\text { CLEAN }\end{array}$ & $\begin{array}{c}\text { ADDITIONAL } \\
\text { CLASSIFICATIONS FOR } \\
\text { WHICH PRODUCT QUALIFIED }\end{array}$ \\
\hline
\end{tabular}
59, 119 SOUTH STREET, PAGELAND 29728

242 E OTES RAY, 315 PEARSON STREET, BLACKSBURG 29702

321 E DAVID, RANDY, \& BILLY RISNER, 302 BALL ROAD, HONEA PATH 29654

165 E RALPH SHERIFF, 3717 RIVER ROAD, PIEDMONT 29673

333 E DONALD SMALL, 2284B HIGHWAY 1 NORTH, CASSATT 29032

336 E LERAY STEPHENS, 6038 OLD GEORGETOWN ROAD, BISHOPVILLE 29010

$312 E$ MARK \& MARSHALL STILL, 115 HENRY DRIVE, BARNWELL 29812

282 E TIM SULLENS, 111 LORRAINE DRIVE, SUMMERVILLE 29485

339 E KATHERINE VALENTINE, 852 1/2 FOLLY ROAD, JAMES

ISLAND 29412
E JESSICA WARD, 305 CROSBY ROAD, GILBERT 29054

303

340

323

334

307

E BILL WATKINS, 929 MCKENZIE ROAD, MCBEE 29101
335 E QUALL VALLEY FARMS, INC. STAN REDFERN, P.O. BOX
E KATE WATSON, 2725 OLD GREENVILLE HIGHWAY, CENTRAL 29630

E RODGER D. WINN, 2528 OLD DUTCH ROAD, LITTLE MOUNTAIN 29075

E BRIAN PAUL WITT, 2056 OLD STAGECOACH ROAD, CASSATT 29032

Phone: (803)432-1067

\author{
Z43. \\ R30, W121, W128, W17, W60. \\ W169, W175, W187, W200, \\ W30, W308, W50, W568, W596, \\ W69, W7, W88 \\ W25, W28, W88 \\ N17 \\ N134, N175, N183, N224, N32, . \\ N34, N43 \\ W28, W48, W568. \\ W142, W151, W28, W88, W91 \\ N105, N106, N110, N121, N181, . \\ N27, N97, R229 \\ R89, W559, W75 \\ N183, N25, N28, N33 \\ W128, W142, W4 \\ $R 213, R 225$ \\ N90, R34, W20, W4, X1, X12, \\ $X 15, X 18, X 180, X 194, X 195, X 2$, \\ $X 21, X 32, X 46, X 67, Y 63, Y 64$, \\ $\mathrm{Y} 65, \mathrm{Y} 73, \mathrm{Y} 74$
}

46 - SOUTH DAKOTA
DR. SAM HOLLAND -DR. THOMAS F. CLINE AMIMAL INDUSTRY BOARD 411 SOUTH FORT STREET

PIERRE, SD 57501

Phone: (605)773-3321

Fax: (605)773-5459 and DR. DARWIN BRITZMAN

SOUTH DAKOTA ANIMAL INDUSTRY BOARD 2208 ST. CHARLES CIRCLE

SIOUX FALLS, SD 57103

Phone: (605)332-1116

Fax: $\quad(605) 688-6170$

\section{Hatcheries}
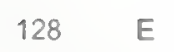
ABSOLUTELY WILD RANCH GAMEBIRDS \& HTY., RR 2, BOX 141-A, GREGORY 57533

\section{5} 122
E CONNIE FARMS, HCR 57, BOX 38, IDEAL 57541

$E$ COUNTRY CORNER EXOTICS, RT, 1, BOX 76, WORTHING 57077

E

$\begin{array}{rl}28,000 & Z 27 \\ 5,000 & Z 27 \\ 400 \quad X, Y, Z 27, Z 48, Z 94 \\ 70,000 \quad Z 27, Z 30, Z 43, Z 48, Z 9\end{array}$


Table 1. HATCHERIES, DEALERS, AND INDEPENDENT FLOCKS PARTICIPATING IN

THE NATIONAL POULTRY IMPROVEMENT PLAN

WATERFOWL, EXHIBITION POULTRY AND GAME BIRDS

\begin{tabular}{lllll}
\hline & & HATCHING & PRODUCTS CLASSIFIED \\
APP. & SUB- & PARTICIPANTS NAME AND ADDRESS & U.S. PULLORUM-TYPHOID \\
NO. & PART & CLEAN & CLASSIFICATIONS FOR & WHICH PRODUCT QUALIFIED
\end{tabular}

46 - SOUTH DAKOTA

\begin{tabular}{|c|c|c|c|c|}
\hline 127 & E & $\begin{array}{l}\text { DAKOTA HUNTING FARM, BILL MITCHELL, } 10095 \\
\text { MITCHELL ROAD, HECLA } 57446\end{array}$ & 16,000 & 227 \\
\hline 132 & $E$ & $\begin{array}{l}\text { DAKOTA RIDGE, INC., DAKOTA DUCKS, } 47618 \text { 173RD } \\
\text { STREET, CLEAR LAKE 57226-5106 }\end{array}$ & 3,000 & X4_______ \\
\hline 129 & E & $\begin{array}{l}\text { FARMING-N-MORE, TIM \& BILL ERK, RR 1, BOX } 21 \text {, } \\
\text { NEWELL } 57760\end{array}$ & 4,300 & $\mathrm{Z1}, \mathrm{Z27}, \mathrm{Z} 48$ \\
\hline 100 & E & $\begin{array}{l}\text { G \& C CENTURY PHEASANTS, RT. 1, BOX } 52 \text {, } \\
\text { CHAMBERLAIN } 57325\end{array}$ & 15,000 & $\mathrm{Z27}$ \\
\hline 120 & $E$ & $\begin{array}{l}\text { GISI PHEASANT FARM, } 13720363 \text { AVENUE, IPSWICH } \\
57451 \\
\text { Phone: }(605) 426-6066\end{array}$ & 84,000 & Y9, Z129, Z176, Z27, Z28, Z79 \\
\hline 89 & E & $\begin{array}{l}\text { HIDDEN VALLEY HUNT CLUB, HC } 56, \text { BOX } 51 \text {, ORAL } \\
57766\end{array}$ & 10,000 & Z27. Z43. Z48 \\
\hline 99 & $\mathrm{E}$ & $\begin{array}{l}\text { HIGH PLAINS GAME RANCH, HC 76, BOX 192, NISLAND } \\
57762\end{array}$ & 35.000 & $\mathrm{Z27}$ \\
\hline 131 & $E$ & $\begin{array}{l}\text { HILLSIDE COLONY, } 18248 \text { HILLSIDE LANE, DOLAND } \\
57436\end{array}$ & 72,000 & $227 \ldots$ \\
\hline 22 & $E$ & $\begin{array}{l}\text { INMAN HATCHERY, } 3000 \text { THIRD AVENUE SE., } \\
\text { ABERDEEN } 57401\end{array}$ & 368,000 & $\begin{array}{l}\mathrm{A} 16, \mathrm{~B} 10, \mathrm{C}, \mathrm{D} 9, \mathrm{X} 131, \mathrm{X} 14, \mathrm{X} 2, \\
\mathrm{X} 3, \mathrm{X} 4, \mathrm{X} 5, \mathrm{X} 6, \mathrm{Y} 1, \mathrm{Y} 2, \mathrm{Y} 3, \mathrm{Y} 4 \\
\mathrm{Z27}\end{array}$ \\
\hline 94 & E & HAROLD KLIMISCH, RR 1, BOX 39, UTICA 57067 & 15,000 & 227 \\
\hline 130 & $E$ & $\begin{array}{l}\text { MOUNTAIN VIEW GAME BIRDS, CURT NULLE, RR } 2 \text {, } \\
\text { BOX } 2577, \text { BELLE FOURCHE } 57717\end{array}$ & 400 & 227,243 \\
\hline 124 & E & $\begin{array}{l}\text { MILTON SCHOLTEN, RT. 1. BOX 1310, VALLEY SPRINGS } \\
57068\end{array}$ & 500 & Y9___ \\
\hline 82 & E & SHILTZ FOODS, INC.. . SISSETON 57262 & 68,000 & $Y 17, Y 18, Y 19$ \\
\hline 104 & $E$ & $\begin{array}{l}\text { STONE HILL, RR 1, BOX 60, GARY } 57237 \\
\text { Dealers }\end{array}$ & 48,000 & Z27 \\
\hline 41 & $E$ & DAKOTA BEST, INC.. , REDFIELD 57469 & C & A16, B, C, R209 \\
\hline 11 & E & $\begin{array}{l}\text { MORROW PRODUCE, , ARMOUR } 57313 \\
\text { Independent Flocks }\end{array}$ & C & A16, D, HD, R209 \\
\hline 84 & $E$ & ARLYN FRERICHS, RR 1, BROOKINGS 57006 & & W_ \\
\hline 75 & $E$ & REYNOLD LOECKER, RR 4, BOX 131, YANKTON 57078 & & w \\
\hline
\end{tabular}

63 - TENNESSEE

DR. RONALD B. WILSON

STATE VETERINARIAN

TENNESSEE DEPARTMENT OF AGRICULTURE

BOX 40627. MELROSE STATION

NASHVILLE, TN 37204

Phone: (615)837-5120

Fax: $\quad(615) 837-5250$ and TINA ROGERS

TENNESSEE DEPARTMENT OF AGRICULTURE ELLINGTON AGRICULTURAL CENTER BOX 40627. MELROSE STATION NASHVILLE, TN 37204 Phone: (615)837-5120

Fax: (615)837-5250 
Table 1. HATCHERIES, DEALERS, AND INDEPENDENT FLOCKS PARTICIPATING IN

THE NATIONAL POULTRY IMPROVEMENT PLAN

WATERFOWL, EXHIBITION POULTRY AND GAME BIRDS

\begin{tabular}{|c|c|c|c|c|c|}
\hline $\begin{array}{l}\text { APP. } \\
\text { NO. }\end{array}$ & $\begin{array}{l}\text { SUB- } \\
\text { PART }\end{array}$ & PARTICIPANTS NAME AND ADDRESS & $\begin{array}{l}\text { HATCHING } \\
\text { EGG } \\
\text { CAPACITY }\end{array}$ & $\begin{array}{l}\text { PRODUCTS CLASSIFIED } \\
\text { U.S. PULLORUM-TYPHOID } \\
\text { CLEAN }\end{array}$ & $\begin{array}{c}\text { ADDITIONAL } \\
\text { CLASSIFICATIONS FOR } \\
\text { WHICH PRODUCT QUALIFIED }\end{array}$ \\
\hline
\end{tabular}

\begin{tabular}{|c|c|c|c|c|}
\hline \multicolumn{5}{|c|}{ Hatcheries } \\
\hline 163 & $E$ & $\begin{array}{l}\text { KEITH DANIEL, } 499 \text { COFFEE MCGEE ROAD, } \\
\text { LAWRENCEBURG } 38464\end{array}$ & 1.200 & Z43. Z93 \\
\hline 155 & E & $\begin{array}{l}\text { FRED GREEN, } 1351 \text { NEW HEARMAN ROAD, } \\
\text { SHELBYILLE } 37160\end{array}$ & 20,000 & $\begin{array}{l}\text { R104, R12, R179, R18, R183, } \\
\text { R20, R21, R237, R239, R240, } \\
\text { R42, R44, R69, R72, R77, R81, } \\
\text { R82, R83, R84, R94, R95, W102, } \\
\text { W138, W140, W141, W145, } \\
\text { W169, W17, W176, W248, W25, } \\
\text { W26, W30, W302, W313, W427, } \\
\text { W45, W48, W518, W60, W69, } \\
\text { W70, W74, W75, W81, W91, } \\
\text { W96, X1, X11, X131, X134, X14, } \\
\text { X146, X161, X2, X23, X25, X3, } \\
\text { X37, X4, X6, X64, X9, X96, X98, } \\
\text { Z20, Z202, Z205, Z22, Z227, } \\
\text { Z285, Z62, Z78 }\end{array}$ \\
\hline 170 & $E$ & DEWEY HEWITT, 8201 TERRY LANE, NASHVILLE 37076 & 300 & $\times 4$ \\
\hline 121 & $\mathrm{E}$ & $\begin{array}{l}\text { J \& J GAME COCK FARM, P.O. BOX 8674, FLINTVILLE } \\
37335\end{array}$ & 540 & N28, N32, N41, N44 \\
\hline 159 & $E$ & $\begin{array}{l}\text { KENNETH E. WALL, } 1469 \text { FREEMAN LOOP, } \\
\text { CUMBERLAND FURNACE } 37051\end{array}$ & 360 & N183, N28, N41, N44, N63 \\
\hline 118 & $E$ & $\begin{array}{l}\text { THOMAS R. WILSON, } 269 \text { COUNTY ROAD } 611 \text {, ATHENS } \\
37303\end{array}$ & 1,200 & N28, N32, N44, N63 _ _ \\
\hline \multicolumn{5}{|c|}{ Independent Flocks } \\
\hline 51 & $E$ & $\begin{array}{l}\text { OSCAR C. AKINS, } 4309 \text { MARTIN CHAPEL ROAD, } \\
\text { SPRINGFIELD } 37172\end{array}$ & & 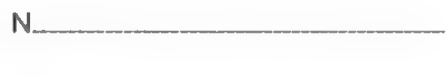 \\
\hline 168 & $E$ & $\begin{array}{l}\text { C. W. BROWN, } 1602 \text { LIBERTY AVENUE, } \\
\text { LAWRENCEBURG } 38464\end{array}$ & & Z43 _ \\
\hline 110 & E & $\begin{array}{l}\text { GARY F. CUNNINGHAM, } 2334 \text { BURDETT ROAD, } \\
\text { GRANDVIEW } 37337\end{array}$ & & $\begin{array}{l}\text { R22, W119, W128, W207, W542, } \\
\text { W568, W88 }\end{array}$ \\
\hline 169 & $E$ & ROBERT KITCHELL, P.O. BOX 54, STEWART 37175 & & $\begin{array}{l}\mathrm{R} 222, \mathrm{R} 233, \mathrm{~W} 131, \mathrm{~W} 3, \mathrm{Z11}, \\
\mathrm{Z231}, \mathrm{Z4}\end{array}$ \\
\hline 164 & $E$ & $\begin{array}{l}\text { ANTON MARCIUC, } 8501 \text { CUB CREEK ROAD, NASHVILLE } \\
37209\end{array}$ & & $\begin{array}{l}\text { R222, R233, R7, R96, W13, Z11, . } \\
\text { Z3, Z4 }\end{array}$ \\
\hline 166 & $E$ & BENNY NEASE, 167 PINE RIDGE WAY, NEWPORT 37821 & & $\mathrm{~N} 28, \mathrm{~N} 33, \mathrm{~N} 41 \ldots$ \\
\hline 171 & E & $\begin{array}{l}\text { ALBERT RIDLEY, } 2233 \text { STANDING ROCK ROAD, SIGNAL } \\
\text { MOUNTAIN } 37377\end{array}$ & & W142, W25, W26 \\
\hline 162 & E & $\begin{array}{l}\text { DORIS ROBINSON, } 810 \text { SWEETWATER ROAD, } \\
\text { PHILADELPHIA } 37846\end{array}$ & & R160, R19, R51, R93, R94 \\
\hline 161 & $E$ & $\begin{array}{l}\text { DARRELL SEIBER, } 115 \text { WEST MAGNOLIA LANE, OAK } \\
\text { RIDGE } 37830\end{array}$ & & W30 \\
\hline 167 & E & $\begin{array}{l}\text { BRIAN SHAMBLIN, } 271 \text { OLD CHATTANOOGA PIKE. } \\
\text { CLEVELAND } 37311\end{array}$ & & $\mathrm{R} 145, \mathrm{R} 94, \mathrm{Z1}, \mathrm{Z231}$ \\
\hline 165 & E & $\begin{array}{l}\text { BRYAN SIMMONS, } 80 \text { PHIPPS ROAD, CROSSVILLE } \\
38571\end{array}$ & & w30, w39 \\
\hline
\end{tabular}

Hatcheries

163 E KEITH DANIEL, 499 COFFEE MCGEE ROAD,

155 E FRED GREEN, 1351 NEW HEARMAN ROAD, SHELBYILLE 37160 LAWRENCEBURG 38464

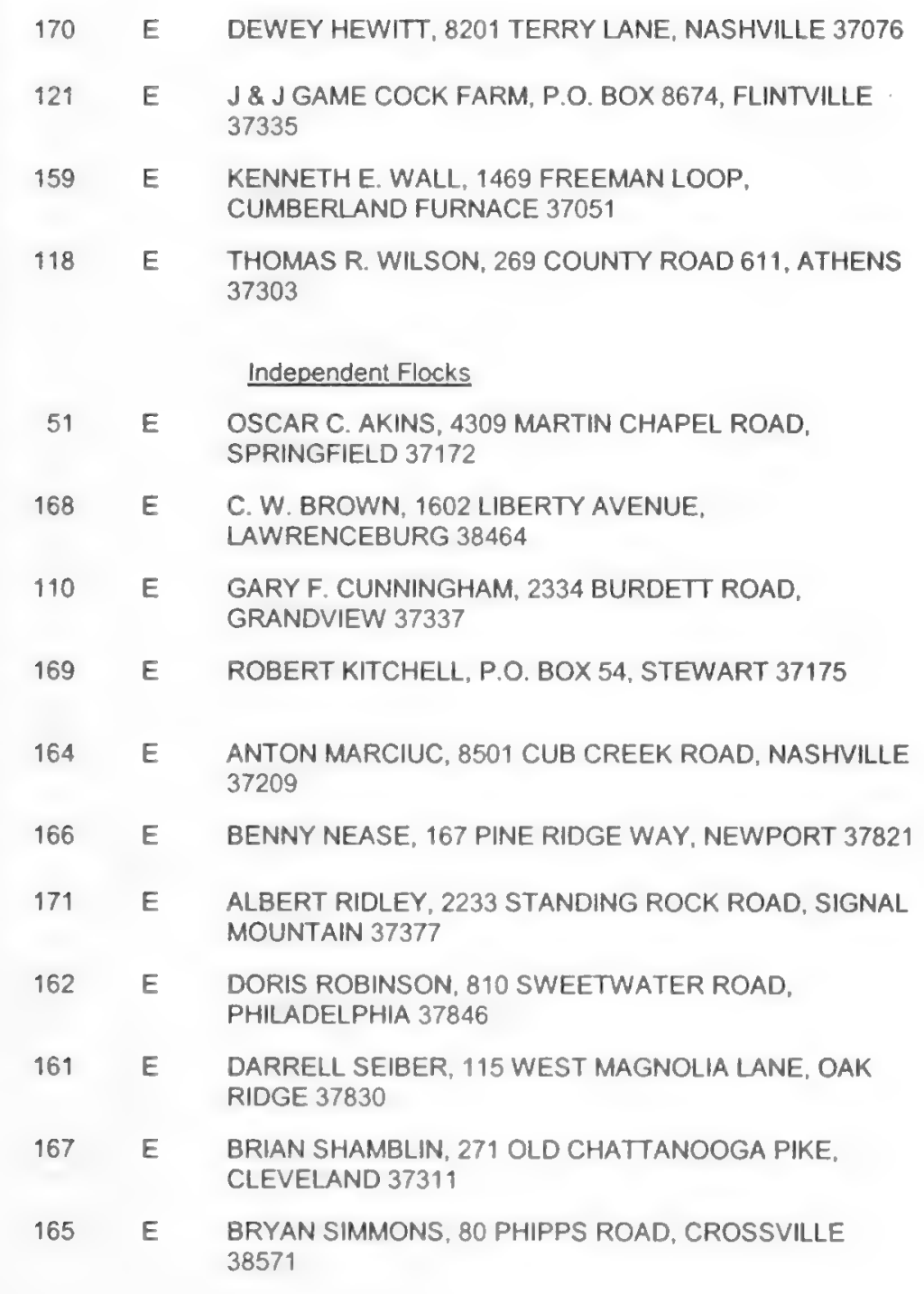

ADDITIONAL WHICH PRODUCT QUALIFIED 
Table 1. HATCHERIES, DEALERS, AND INDEPENDENT FLOCKS PARTICIPATING IN

THE NATIONAL POULTRY IMPROVEMENT PLAN

WATERFOWL, EXHIBITION POULTRY AND GAME BIRDS

\begin{tabular}{llcccc}
\hline & & HATCHING & PRODUCTS CLASSIFIED \\
APP. & SUB- & PARTICIPANTS NAME AND ADDRESS & EGG & U.S. PULLORUM-TYPHOID \\
NO. PART & CLEAN & CAPACITY & CLASSIFICATIONS FOR \\
\hline
\end{tabular}

74 - TEXAS

\author{
DR. FLOYD A. GOLAN \\ TEXAS VETERINARY MEDICAL DIAGNOSTIC \\ LABORATORY \\ P.O. DRAWER 3040 \\ COLLEGE STATION, TX 77841-3040 \\ Phone: (979)845-4186 \\ Fax: $\quad(979) 845-1794$
}

\begin{tabular}{|c|c|c|c|c|}
\hline & & Hatcheries & & \\
\hline 3707 & $E$ & $\begin{array}{l}\text { A\&P EXOTICS, AL NOVOSAD, } 4201 \text { WONDAR HILL } \\
\text { ROAD, CHAPPELL HILL } 77426\end{array}$ & 500 & $W 25, W 26, X, Y, Z 2$ \\
\hline 3663 & $E$ & $\begin{array}{l}\text { BARRETT'S QUAIL FARM, O.G. BARRETT, } 12102 \\
\text { WESTGARD BLVD, HOUSTON } 77044-2734\end{array}$ & 10,000 & $\mathrm{Z} 27, \mathrm{Z} 43$ \\
\hline 3661 & $\mathrm{E}$ & $\begin{array}{l}\text { BEAR BAYOU QUAIL FARM, A.K. RHODES, JR., P.O. BOX } \\
\text { 514, CHANNELVIEW } 77530\end{array}$ & 150 & $Z 1, Z 43, Z 93$ \\
\hline 2386 & $\mathrm{E}$ & BENOIST HATCHERY, RR 1, BOX 74, LAMPASAS 76550 & 1,000 & $\mathrm{~N}$ \\
\hline 3632 & $\mathrm{E}$ & BLACKSMITH FARM, RR 1, BOX 102D4, WEIMAR 78962 & 2,500 & $\mathrm{~N}$ \\
\hline 3700 & $E$ & $\begin{array}{l}\text { DIAMOND H RANCH, T.E. HERRINGTON, } 5322 \text { HIGHWAY } \\
16 \text { NORTH. BANDERA } 78003\end{array}$ & 90,000 & 243,293 \\
\hline 3650 & $E$ & $\begin{array}{l}\text { MARTIN C. DIVER, } 545 \text { MCDOUGAL, SAN ANTONIO } \\
78223\end{array}$ & 1,500 & \\
\hline 3703 & $E$ & $\begin{array}{l}\text { DOUBLE-D-GAMEBIRDS, DAVID KENNEDY, } 1313 \text { BECKY } \\
\text { DRIVE, ALEDO } 76008\end{array}$ & 5,000 & $Z 247, Z 43, Z 93, Z 94$ \\
\hline 3701 & $E$ & $\begin{array}{l}\text { 3-D GAME BIRDS, GLYNN DURRETT, P.O. BOX } 8 \text {, } \\
\text { ABERNATHY } 79311\end{array}$ & 6.800 & $\mathrm{Z} 180, \mathrm{Z2}, \mathrm{Z} 43$ \\
\hline 3694 & $E$ & $\begin{array}{l}\text { FOWL SHOE FARM, SUSAN LEWIS, } 644 \text { FLYING T } \\
\text { RANCH ROAD, BANDERA } 78003\end{array}$ & 1,000 & $\begin{array}{l}\text { R31, W102, W104, W17, W193, } \\
\text { W247, W265, W270, W302, } \\
\text { W304, W330, W332, W415, } \\
\text { W518, W527, W573, W574, } \\
\text { W58, W615, W74, W75, W86, } \\
\text { W90 }\end{array}$ \\
\hline 3708 & $E$ & $\begin{array}{l}\text { AUNT MARTHA'S HATCHERY, MARTHA GUZMAN, P.O. } \\
\text { BOX } 1251 \text {, FRANKSTON } 75763\end{array}$ & 500 & $\begin{array}{l}\text { R7, W17, W208, W26, W427, } \\
\text { W484, W96, X6, Z22, Z28, Z57 }\end{array}$ \\
\hline 3639 & $E$ & $\begin{array}{l}\text { HARPER'S GAME BIRD FARM, RR 2, BOX 484, BOOKER } \\
79005\end{array}$ & 8.400 & $Z 27, Z 43, Z 444$ \\
\hline 3631 & $\mathrm{E}$ & HIGH PLAINS GAME BIRDS, P.O. BOX 467, VEGA 79092 & 2,100 & $Z 142, Z 43,272$ \\
\hline 3688 & $E$ & $\begin{array}{l}\text { HOFFART GAME BIRDS, } 15547 \text { O'CONNOR, } \\
\text { SPLONDORA } 77372\end{array}$ & 2,000 & R146, R19, R196, R247, Z43 \\
\hline 6 & $\mathrm{E}$ & $\begin{array}{l}\text { IDEAL POULTRY BREEDING FARM, INC., BOX } 591 . \\
\text { CAMERON } 76520\end{array}$ & 50,000 & $\begin{array}{l}\text { R146, R169, R20, R270, R271, } \\
\text { R272, R5, R6, R7, R95, W1, } \\
\text { W102, W120, W121, W131, } \\
\text { W19, W20, W213, W256, W257, } \\
\text { W259, W26, W424, W86 }\end{array}$ \\
\hline 3662 & $E$ & $\begin{array}{l}\text { JONES HATCHERY, ROUTE 5, BOX 567-B, GATESVILLE } \\
76528\end{array}$ & 1,000 & N181, N182, N28, N32 \\
\hline 3704 & $\mathrm{E}$ & $\begin{array}{l}\text { KEENE FARMS, DAVID KEENE, } 12019 \text { NORTH COUNTY } \\
\text { ROAD } 1740 \text {, LUBBOCK } 79415\end{array}$ & 1,000 & R196, R222, R247, X4, Z204 \\
\hline 3690 & $\mathrm{E}$ & LOWE'S HATCHERY, ROSA LOWE, 20213 MEADOW & 500 & R42, W570, W72 \\
\hline
\end{tabular}


Table 1. HATCHERIES, DEALERS, AND INDEPENDENT FLOCKS PARTICIPATING IN

THE NATIONAL POULTRY IMPROVEMENT PLAN

WATERFOWL, EXHIBITION POULTRY AND GAME BIRDS

\begin{tabular}{llccc}
\hline & & HATCHING & PRODUCTS CLASSIFIED \\
APP. SUB- & PARTICIPANTS NAME AND ADORESS & EGG & U.S. PULLORUM-TYPHOID \\
NO. PART & CAPACITY & CLEAN & CLASSIFICATIONS FOR \\
\hline
\end{tabular}

74 - TEXAS 158 E JEFFE. MAXWELL, 916 COUNTY ROAD \#676, DAYTON
77535

129 E CECIL MOORE, 1406 ETAIN ROAD, IRVING 75060

3677 E C\&F GAME BIRD FARM, D \& S PENETT, 17087 HIGHWAY 64 EAST, TYLER 75707

3695 E PREMIER GAMEBIRD RANCH, C. MITCHELL, 1608 KIRKPATRICK ROAD, FLOWER MOUND 75028

3686 E ROBINSON HATCHERY, MARC ROBINSON, 6720 AN.COUNTY ROAD 448, PALESTINE 75803

3684 E ROCKING R. GAMEBIRDS, BUD R. ROMINE, 2505 FISHER, BIG SPRING 79720

3680 E ROCKING TRANCH \& POULTRY FARM, P. THEER, ROUTE 2, BOX 76, KEMPER 76539

200 E ROSEBUD CHUKAR FARM, RR 2, BOX 215, ROSEBUD 76570

3692 E SANDY ROOST ACRES, GERRY THOMPSON, 25839 BANK HEAD DRIVE, BEDIAS 77831

3693 E SOUTHARD HATCHERY, BRENT A. SOUTHARD, 1212 FM 991, TEXARKANA 75501

190 E STEVENSON GAME BIRD FARM, P.O. BOX 426, FM 405, RIVERSIDE 77367

3678 E STUEARTS GAME BIRD RANCH \& HATCHERY, RR 3, BOX 40, LITTLEFIELD 79339

3702

E

DAVID H. STURN, RR 4, BOX 800, MT. PLEASANT 75455

3668

E TEXAS HILL COUNTRY QUAIL FARM, J.VALLONE, 1380 SERENITY DRIVE, FISCHER 78623

3706 E TNT RARE POULTRY, TOMMY POWELL, RT, 9, BOX 5800, LUFKIN 75901

3656 E TOP FLIGHT GAMEBIRDS, ROUTE 3, BOX 201-A, ALICE 78332

3659 E TURTLE CREEK GAME FARM, C. MENDEZ, JR., ROUTE 1, BOX 57-C, THRALL 76578

371 E C. D. WHEELER, 29519 WEST HAWTHORNE, SPRING 77386

3698 E X-TREME GAMEBIRDS \& POULTRY, HAYES, 280 LACY DRIVE, ELGIN 78621
$500 \mathrm{H} 10, \mathrm{H} 8, \mathrm{~W} 106, \mathrm{~W} 115, \mathrm{~W} 119$, W120, W123, W128, W176. W187, W188, W192, W198, W20, W209, W22, W27, W3, W32, W36, W57, W69, W7, W81. W91, X10, X17

600 R17, R44, R49, W23, W3

$16,896 \quad Z 43$

$6.000 \quad Z 180, Z 43$

$200 N$

$2,400 \quad Z 235, Z 24, Z 247, Z 28, Z 44, Z 60, \ldots$ Z72, Z90, Z93

400 W24, W25, W26, W3, W30, W35.

$5,000 \quad \mathrm{Z} 1$

1,500 R1, R16, R19, R20, R222, R237, $R 247, R 51, R 71, R 77, R 85, R 89$, R94, W13

25

H10

$80,000 \quad Z 1, Z 11, Z 27, Z 43, Z 48$

$8,340 \quad \mathrm{Z} 180, \mathrm{Z} 247$

500 R, R247, R249, R71, W1, W111, . W13, W131, W132, W24, W387, W554, W595, W94

$10,000 \quad Z 43$

350 R213, R249, R7, R89, W. W142, W554, W74

$10,000 \quad Z 41, Z 43$

$2,500 \quad \mathrm{~N} 28, \mathrm{~N} 32, \mathrm{~N} 44$

$99,999 \quad$ Z1, Z27, Z43.

1,200 A, C2, D, H, R1, R146, R15, R19, R196, R20, R227, R237, R247, R256, R40, R7, R81, R9, T9, X3, $X 4, Y 1, Y 2, Y 3, Z 118, Z 218, Z 22$. Z26, Z260, Z27, Z43, Z57, Z93

R, R146, R15, R168, R20, R227, $R 237, R 7, R 83, W, W 13, X, X 1$, $X 102, X 13, X 131, X 14, X 2, X 25$, $X 3, X 4, X 6, X 98, Y, Y 13, Y 21$, Y35, Z102, Z108, Z11, Z110, Z129, Z137, Z179, Z19, Z2, Z21. 


\begin{tabular}{lllll}
\hline & & HATCHING & PRODUCTS CLASSIFIED \\
APP. SUB- & PARTICIPANTS NAME AND ADDRESS & EGG & U.S. PULLORUM-TYPHOID & CLLASSIONAL \\
NO. PART & CAPACITY & CLATIONS FOR & WHICH PRODUCT QUALIFIED \\
\hline
\end{tabular}

$$
74 \text { - TEXAS }
$$

3636 E VANCE HATCHERY, 16485 FM 1957. SAN ANTONIO
$78253-6883$

Z22, Z239, Z24, Z249, Z251, Z254, Z26, Z27, Z41, Z43, Z49, Z62, Z68

$\begin{array}{lll} & & \text { Z22, Z239, Z24, Z249, Z251, } \\ 3636 \text { Z } \quad \begin{array}{l}\text { VANCE4, Z26, Z27, Z41, Z43, Z49, } \\ \text { 78253-6883 }\end{array} & \text { Z62, Z68 }\end{array}$

DR. MICHAEL R. MARSHALL

STATE VETERINARIAN

UTAH DEPARTMENT OF AGRICULTURE

BOX 146500

SALT LAKE CITY, UT 84114-6500

Phone: (801)538-7160

Fax: $\quad(801) 538-7169$

\section{Hatcheries}

157

E

JACK FOY, 2730 SOUTH DESERT ROAD, MOAB 84532 Phone: (435)259-5570

143 E ROYD HATT, P.O. BOX 134, GREEN RIVER 84525 Phone: 801/564-3224

148 E ALAN LARSEN, TR GAME BIRDS \& HATCHERY, 10194 NORTH 9500 EAST, TRIDELL 84076

Phone: (801)247-2311

160 E DANNY MERRILL, 85 NORTH 200 WEST, RICHMOND 84333

Dealers

$159 \quad$ E

PLEASANT VALLEY PHEASANTS, KEITH HICKEN, RT. 3 , BOX 3736, MYTON 84052

Phone: (435)646-3020

\section{Independent Flocks}

151 E C R CHUKARS, DIANE JENSEN, P.O. BOX 372, GREEN RIVER 84525

143 E ROYD HATT, P.O. BOX 134, GREEN RIVER 84525

Phone: 801/564-3224

148 E ALEN LARSEN, TR GAME BIRDS \& HATCHERY, 10194 NORTH 9500 EAST, TRIDELL 84076

Phone: (801)247-2311 and DR. EARL ROGERS

ASSISTANT STATE VETERINARIAN

UTAH DEPARTMENT OF AGRICULTURE

BOX 146500

SALT LAKE CITY, UT 84114-6500

Phone: (801)538-7162

Fax: $\quad(801) 538-7169$

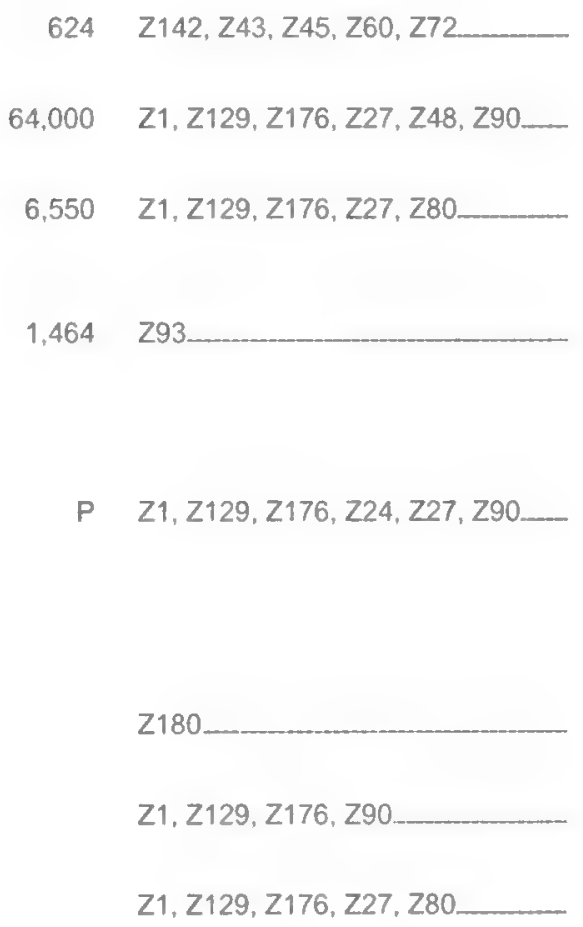


Table 1. HATCHERIES, DEALERS, AND INDEPENDENT FLOCKS PARTICIPATING IN

THE NATIONAL POULTRY IMPROVEMENT PLAN

WATERFOWL. EXHIBITION POULTRY AND GAME BIRDS

\begin{tabular}{|c|c|c|c|c|c|}
\hline $\begin{array}{l}\text { APP. } \\
\text { NO. }\end{array}$ & $\begin{array}{l}\text { SUB- } \\
\text { PART }\end{array}$ & PARTICIPANTS NAME AND ADDRESS & $\begin{array}{l}\text { HATCHING } \\
\text { EGG } \\
\text { CAPACITY }\end{array}$ & $\begin{array}{l}\text { PRODUCTS CLASSIFIED } \\
\text { U.S. PULLORUM-TYPHOID } \\
\text { CLEAN }\end{array}$ & $\begin{array}{c}\text { ADDITIONAL } \\
\text { CLASSIFICATIONS FOR } \\
\text { WHICH PRODUCT QUALIFIED }\end{array}$ \\
\hline
\end{tabular}

DR. TODD JOHNSON

STATE VETERINARIAN

DEPARTMENT OF AGRICULTURE, FOOD \& MARKETS

116 STATE STREET, DRAWER 20

MONTPELIER, VT 05620-2901

Phone: (802)828-2421

Fax: $\quad(802) 828-5983$

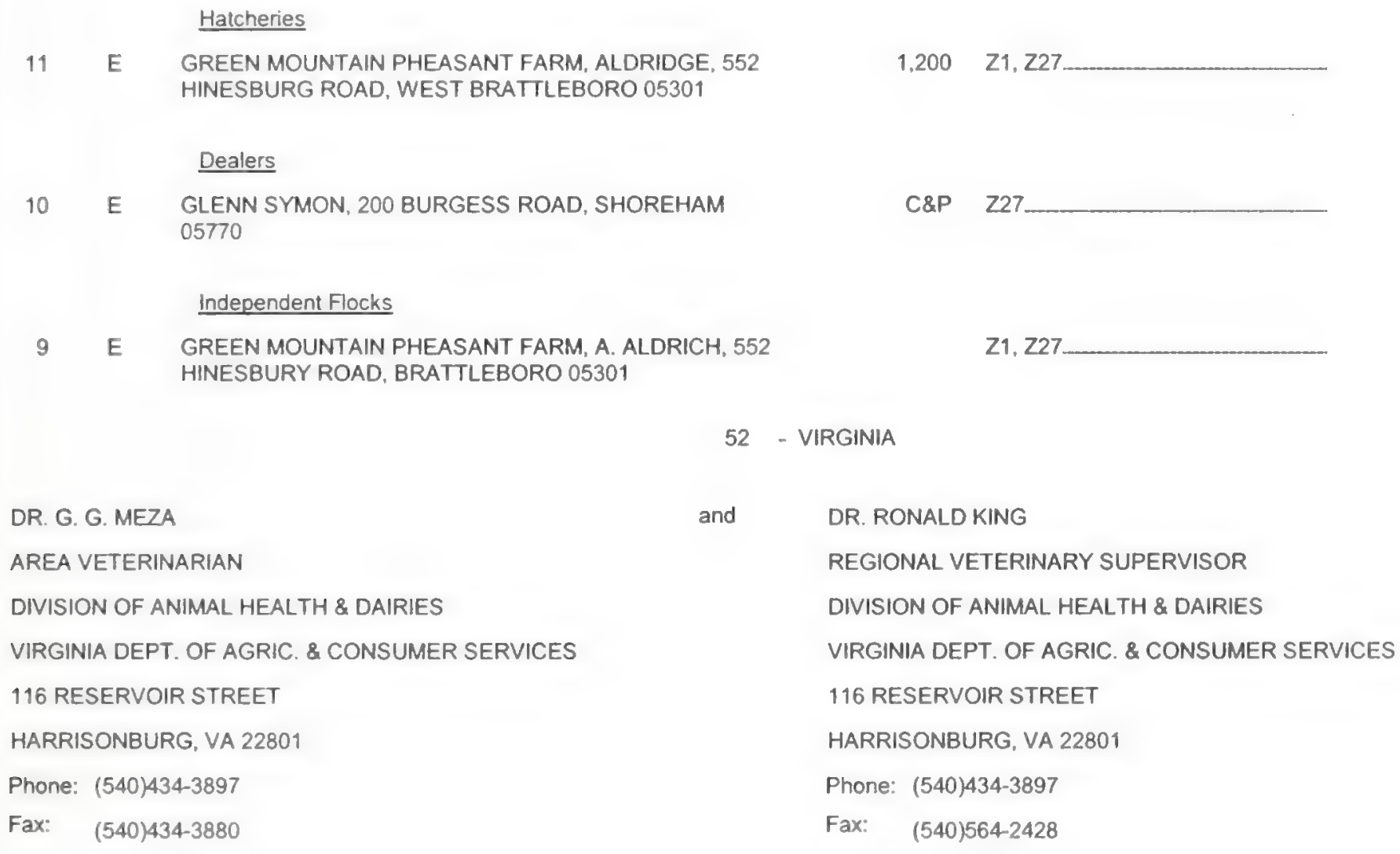

Independent Flocks

153 E LUTHERL. ALSTON, 6044 KNOTTS CREEK LANE, SUFFOLK 23435

$X 10, X 17, X 28, X 3, X 4, X 40, X 52,-$ $X 8, Y 15, Y 9, Z 107, Z 43$

176 E CHAD BLAIR, 461 RICKEY ROAD, MAX MEADOWS 24360

W30

166 E CECILL. BOGGS, JR., 764 DOOMS CROSSING ROAD, WAYNESBORO 22980

155 E DANIEL A. DYSART, 946 MILL ROAD, WOODSTOCK 22664

175 E ROGER W. JONES, 1413 PRIDEMOORE ROAD, GALAX 24333

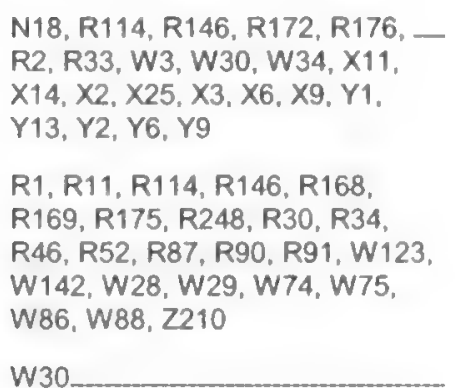




\begin{tabular}{|c|c|c|c|c|c|}
\hline $\begin{array}{l}\text { APP. } \\
\text { NO. }\end{array}$ & $\begin{array}{l}\text { SUB- } \\
\text { PART }\end{array}$ & PARTICIPANTS NAME AND ADDRESS & $\begin{array}{l}\text { HATCHING } \\
\text { EGG } \\
\text { CAPACITY }\end{array}$ & $\begin{array}{l}\text { PRODUCTS CLASSIFIED } \\
\text { U.S. PULLORUM-TYPHOID } \\
\text { CLEAN }\end{array}$ & $\begin{array}{c}\text { ADDITIONAL } \\
\text { CLASSIFICATIONS FOR } \\
\text { WHICH PRODUCT QUALIFIED }\end{array}$ \\
\hline
\end{tabular}

\begin{tabular}{|c|c|c|}
\hline 180 & $E$ & $\begin{array}{l}\text { JOHN ROBERT LEWIS, JR., } 3606 \text { PLANK ROAD, } \\
\text { NATURAL BRIDGE } 24578\end{array}$ \\
\hline 57 & $E$ & CINDY LONGERBEAM, RR 1, BOX 533, PHENIX 23959 \\
\hline 72 & E & $\begin{array}{l}\text { G. L. LOUDERBACK, } 561 \text { EGYPT BEND ROAD, LURAY } \\
22835\end{array}$ \\
\hline 79 & $E$ & $\begin{array}{l}\text { ANDY MARSINKO, } 191 \text { MAJOR WADE ROAD, BLUE } \\
\text { RIDGE 24064-1924 }\end{array}$ \\
\hline 58 & $E$ & $\begin{array}{l}\text { ORMSBY'S BANTAMS, } 252 \text { NORTH LIBERTY SPRINGS } \\
\text { ROAD. SUFFOLK } 23435\end{array}$ \\
\hline 174 & $E$ & $\begin{array}{l}\text { BRENDA PRICE, } 2195 \text { OLINGER ROAD, BLACKSBURG } \\
24060\end{array}$ \\
\hline 178 & E & MERRILL T. RIFE, P.O. BOX 91, BASTIAN 24314 \\
\hline 177 & $E$ & $\begin{array}{l}\text { JAMES SALYER, SR, \& JAMES SALYER, JR., RT, 2, BOX } \\
535 \text {, NICKELSVILLE } 24271\end{array}$ \\
\hline J & $\mathrm{E}$ & $\begin{array}{l}\text { CARLTON \& JUDY SANDERLIN, } 4136 \text { CHARLES CITY } \\
\text { PLACE, RICHMOND } 23231\end{array}$ \\
\hline
\end{tabular}

SPIRITHAVEN FARM, SCOTT EUTSLER, 88 DABYNS CHURCH ROAD, STUART 24171
TOMMY \& SANDY STANLEY, 12009 YOWELL ROAD, ASHLAND 23005

$\begin{array}{lll}165 \text { E } & \begin{array}{l}\text { LINWOOD A. STONE, JR., 643 RICHARDSON ROAD, } \\ \text { COLLINSVILLE 24078 }\end{array} \\ 151 \text { E } & \text { BETSY THORNHILL, RR 3, BOX 214, CULPEPER 22701 } \\ 124 \text { E } & \begin{array}{l}\text { WILLOW GAME FARM, 884 COTTONTAIL TRAIL, MT. } \\ \text { CRAWFORD 22841 }\end{array}\end{array}$

N1, R15, R31, R65, R72, R8, R9, Z219

R20, W1, W123, W130, W14, W166, W18, W207, W293, W50, W573, W96, X, Y, Z11, Z210, Z26. Z28, Z4

W30

W30

W115, W123, W145, W188, W81, X28

W30

W30

W30

R123, R30, R53, W115, W121, W125, W138, W145, W174,

W18, W187, W198, W211 W224, W245, W262, W270, W276, W29, W293, W308 W320, W33, W401, W50, W552. W606, W607, W74, W81, W86, W91, X27, X28, X8, Y10, Z202

W30

R1, R10, R11, R114, R117, R12, R146, R15, R168, R169, R17, R183, R196, R2, R22, R227, R23, R248, R3, R30, R34, R37, R38, R39, R44, R49, R52, R6, R7. R71, R78, R81, R85, R87, R9, R90, R91, W104, W106, W11, W12, W120, W126, W127 W134, W159, W16, W23, W3. W36, W38, W4, W54, W7

W136, W16, W231, W301, W357, W364, W560, W600, 278

R, W, Z3, Z5

$X 10, X 143, X 16, X 17, X 2, X 28$ $X 38, X 4, Y 64, Y 8, Y 9, Z 108, Z 11$, Z139, Z142, Z175, Z24, Z26, Z28, Z3, Z44, Z48, Z49, Z51, Z53, Z56. Z57, Z6, Z60, Z72, Z75, Z88 
Table 1. HATCHERIES DEALERS AND INDEPENDENT FLOCKS PARTICIPATING IN

THE NATIONAL POULTRY IMPROVEMENT PLAN

WATERFOWL. EXHIBITION POULTRY AND GAME BIRDS

\begin{tabular}{lllll}
\hline & & HATCHING & PRODUCTS CLASSIFIED \\
APP. & SUB- & PARTICIPANTS NAME AND ADDRESS & EGG & U.S. PULLORUM-TYPHOID \\
NO. PART & CAPACITY & CLEAN & CLASSIFICATIONS FOR \\
\end{tabular}

91 - WASHINGTON

DR. KATHLEEN M. CONNELL

ASSISTANT STATE VETERINARIAN

FOOD SAFETY \& ANIMAL HEALTH DIVISION

DEPARTMENT OF AGRICULTURE

P.O. BOX 42577

OLYMPIA, WA 98504-2577

Phone: (360)902-1878

Fax: (360)902-2087

\section{Hatcheries}

56 E HARDER HATCHERY, 624 NORTH COW CREEK ROAD, RITZVILLE 99169

Phone: (509) 659-1423

81 E NORTHWEST GAME BIRDS, 228812 EAST GAME FARM ROAD, KENNEWICK $99337-7487$

Phone: (509) 586-0150
17 E PHINNEY HATCHERY, 1331 DELL AVENUE, WALLA WALLA 99362
Phone: (509) 525-2602

80 E PITTS GAME FARM, HCR 2, BOX 357-A, COULEE CITY 99115

Phone: (509) 632-5212

Independent Flocks

85 E DRY CREEK WATERFOWL, ARNOLD SCHOUTEN, 367 WALKABOUT WAY, PORT ANGELES 98363

Phone: (360) 452-3464

94 E ELKHORN RIDGE FARM, JOHN DAILY, 1642 PHEASANT
LANE, ELLENSBURG 98926 Phone: (509)962-4960

89 E MATSON FARMS, MIKE \& JENNIFER MATSON, 24414 NORTH ELK-CHATTAROY ROAD, CHATTAROY 99003 Phone: (509)238-3146 87 E MOUNTAIN BROOK GAME FARM, B. \& P. CLARK, P.O.

Phone: (509)365-5182

88 E NESTLER GAME FARM, 861 COOK CANYON ROAD, ELLENSBURG 98926 Phone: (509)968-4844

86 E NORTHWEST WILDFOWL, PAUL DYE, 1011454 TH PLACE NE, EVERETT 98205

Phone: (360)334-8223

\author{
$5,000 \quad E_{1}, \times 22$ \\ $4,500 \quad 293$ \\ 4,500 A, B, H1, R146, R20, R227, R57. \\ R7, R96, W, Z1, Z202, Z227, Z27 \\ $2,083 \quad \mathrm{Z} 264, \mathrm{Z} 27$
}

$\times, \times 133, \times 159, \times 172, \times 173, \times 174$. $X 175, X 176, X 177, X 58, X 81$, Y39, Y 61

Z44, Z45, Z60, Z88, Z89

W13, W132, W566, X14, X146, $X 15, X 2, X 20, X 5, X 99, Y 10, Y 14$, $Y 2, Y 54, Y 55, Y 6, Y 70, Y 71$

Z1, Z27

$\mathrm{Z1}, \mathrm{Z27}, \mathrm{Z3}, \mathrm{Z9}$

$\mathrm{X}, \mathrm{X} 109, \mathrm{X} 111, \mathrm{X} 113, \mathrm{X} 116, \mathrm{X} 124$, $\times 133, X 159, X 171, X 172, X 174$.

$\times 177, \times 182, \times 183, \times 184, X 185$,

$\times 186, \times 187, \times 188, \times 189, \times 28, \times 4$, $\times 40, \times 49, \times 52, \times 53, \times 57, \times 58$.

$\times 59, X 60, X 62, X 7, X 73, X 74$

$X 78, X 79, X 88, X 89, Y 20, Y 28$.

Y32, Y39, Y41, Y55, Y66, Y67

Z122, Z123, Z161, Z187, Z247.

Z262, Z65, Z74, Z79

227 
Table 1. HATCHERIES, DEALERS, AND INDEPENDENT FLOCKS PARTICIPATING IN

THE NATIONAL POULTRY IMPROVEMENT PLAN

WATERFOWL, EXHIBITION POULTRY AND GAME BIRDS

\begin{tabular}{llccc}
\hline & & HATCHING & PRODUCTS CLASSIFIED \\
APP. SUB- & PARTICIPANTS NAME AND ADDRESS & EGG & U.S. PULLORUM-TYPHOID & CLEAN \\
NO. PART & CAPACITY & CLASSIFICATIONS FOR & WHICH PRODUCT QUALIFIED
\end{tabular}

\section{1 - WASHINGTON}

91 E PIONEER PARK AVIARY, JOANNA LANNING, P.O. BOX 478, WALLA WALLA 99362

Phone: (509)527-4403
$X \times 109, \times 113, \times 114 \times 118, \times 122$ $\times 130, X 141, X 142, \times 149, \times 150$ $\mathrm{X} 159, \times 163, \times 166, \times 184, \times 185$ $\mathrm{X} 187, \mathrm{X} 189, \mathrm{X} 197, \mathrm{X} 198, \mathrm{X} 20$, $\mathrm{X} 28, \mathrm{X} 4, \mathrm{X} 40, \mathrm{X} 49, \mathrm{X} 51, \mathrm{X} 52$

$\times 53, \times 54, x 57, \times 58, x 60, x 61$ $X 62, X 7, X 73, X 78, X 88, X 89$, $X 93, Y, Y 22, Y 26, Y 28, Y 32, Y 39$ Y41, Y51, Y75, Y9, Z, Z11, Z118, Z129, Z149, Z153, Z161, Z19, Z24, Z26, Z260, Z269, Z34, Z46, Z49, Z52, Z57, Z99

N183, N28, N32, N90, W30

W.

C

54 - WEST VIRGINIA

DR. JEWELL PLUMLEY

ASSISTANT DIRECTOR ANIMAL HEALTH DIVISION

WEST VIRGINIA DEPARTMENT OF AGRICULTURE

HC 85, BOX 302

MOOREFIELD, WV 26836

Phone: (304)538-2397

Fax: $\quad(304) 538-7088$

\section{Hatcheries}

33 E GREGG \& KIM FALLS, HC 64, BOX 136, PARSONS 26287 Phone: (304)478-3224

116

B, R243, W11, W16, W283, W442, W578

$35-$ WISCONSIN

DR. DONALD P. O'CONNOR

and

DR. CLARENCE J. SIROKY

NPIP COORDINATOR

STATE VETERINARIAN

DEPARTMENT OF AGRICULTURE

DEPARTMENT OF AGRICULTURE

DIVISION OF ANIMAL HEALTH

DIVISION OF ANIMAL HEALTH

P.O. BOX 8911

P.O. BOX 8911

MADISON, WI 53708-8911

MADISON, WI 53708-8911

Phone: (608)224-4882

Phone: (608)224-4872

Fax: $\quad(608) 224-4871$

Fax:

(608)224-4871 
Table 1. HATCHERIES. DEALERS. AND INOEPENDENT FLOCKS PARTICIPATING IN

THE NATIONAL POULTRY IMPROVEMENT PLAN

WATERFOWL, EXHIBITION POULTRY AND GAME BIRDS

APP. SUB- PARTICIPANTS NAME AND ADDRESS NO. PART

HATCHING
EGG
CAPACITY

CAPACITY
PRODUCTS CLASSIFIED

U.S. PULLORUM-TYPHOID

CLEAN
ADDITIONAL

CLASSIFICATIONS FOR WHICH PRODUCT QUALIFIED

$35-$ WISCONSIN

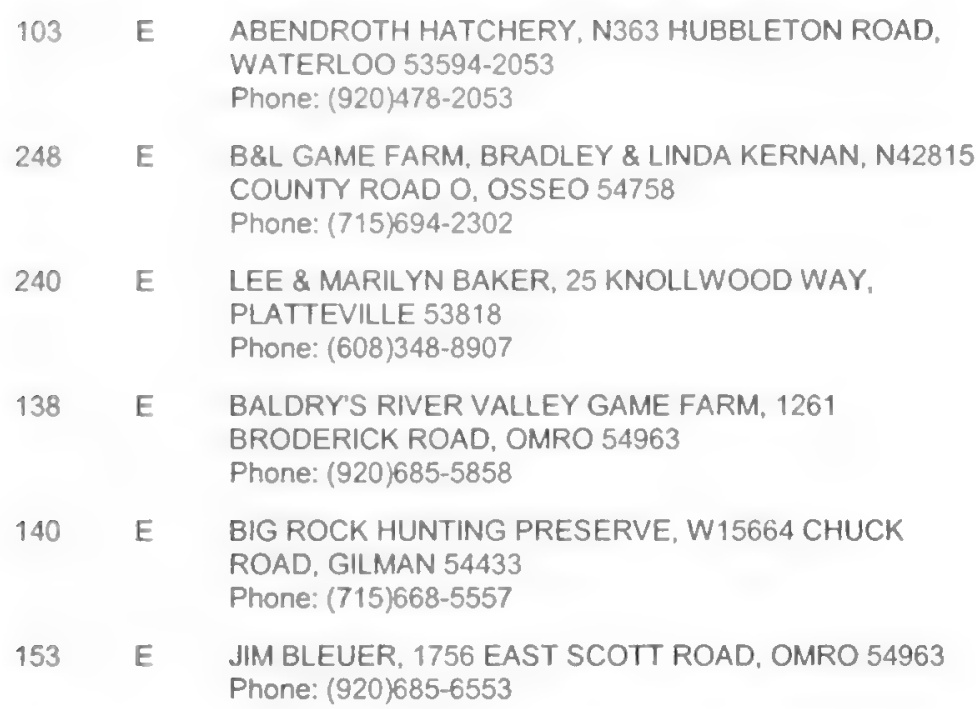

E HICKORY RIDGE FARM, N330 14TH ROAD.

PARDEEVILLE 53954

Phone: 9608)429-2657

131 E CLIFFORD JOHNSON, W226 HIGHWAY 98, SPENCER 54479

Phone: (715)659-3892

245 E BYRON KERSHAW, N5514 DUNNING ROAD,

PARDEEVILLE 53954

Phone: (608)742-3267

148 E KRUEGERS KLUCKERS, 7025 CENTER ROAD,

MANITOWOC 54220

Phone: (920)758-2832

142 E JERRY \& SUSAN KRUG, W11216 RAHMEL LANE, MEDFORD 54451

Phone: (715)785-7836

134 E PATRICIA A. LACEY, W5055 HIGHWAY 10, NEILLSVILLE 54456

Phone: (715)743-4799

E LAKE ELAINE GAME BIRDS, BOX 92, NELSONVILLE 54458

Phone: (715)824-2661

E MACFARLANE PHEASANT FARM, 2821 HIGHWAY 51 SOUTH, JANESVILLE 53545

Phone: (608)757-7881

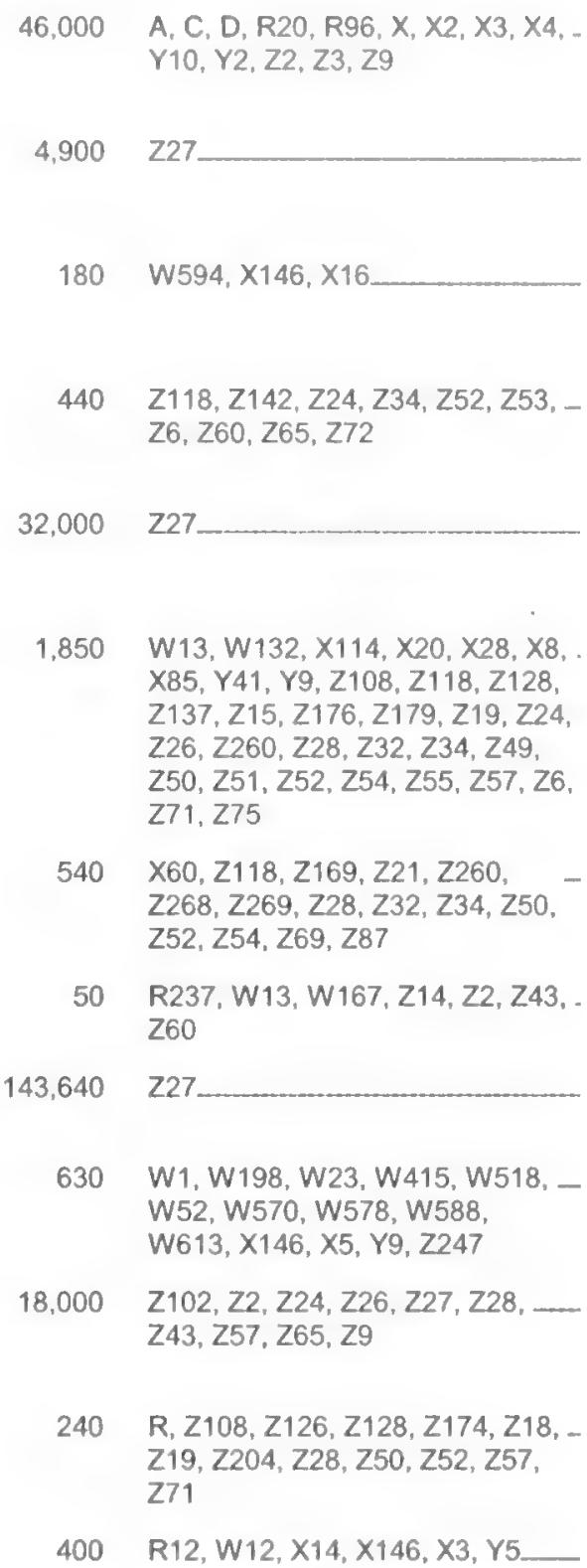

Z118, Z142, Z24, Z34, Z52, Z53, Z6, Z60, Z65, Z72

$32,000 \quad Z 27$

$1,850 \quad \mathrm{~W} 13, \mathrm{~W} 132, \mathrm{X} 114, \mathrm{X} 20, \mathrm{X} 28, \mathrm{X} 8$, X85, Y41, Y9, Z108, Z118, Z128, Z137, Z15, Z176, Z179, Z19, Z24. Z26, Z260, Z28, Z32, Z34, Z49, Z50, Z51, Z52, Z54, Z55, Z57, Z6 Z71, Z75

$540 \times 60, Z 118, Z 169, Z 21, Z 260$, Z268, Z269, Z28, Z32, Z34, Z50, Z52, Z54, Z69, Z87

50 Z60

$143,640 \quad 227$ W1, W198, W23, W415, W518, W52, W570, W578, W588, W613, X146, X5, Y9, Z247

$18,000 \quad Z 102, Z 2, Z 24, Z 26, Z 27, Z 28, \ldots$ Z43, Z57, Z65, Z9

240 R, Z108, Z126, Z128, Z174, Z18, Z19, Z204, Z28, Z50, Z52, Z57, 271 $R 12, W 12, X 14, X 146, X 3, Y 5$

R10, R146, R248, R34,W102 W104, W206, W24, W3, W36, W4, W5, W54

$99,000 \quad Z 11, Z 257, Z 27, Z 28, Z 9$ $\mathrm{Z27}$ 


\begin{tabular}{|c|c|c|c|c|}
\hline $\begin{array}{l}\text { APP. } \\
\text { NO. }\end{array}$ & $\begin{array}{l}\text { SUB- } \\
\text { PART }\end{array}$ & PARTICIPANTS NAME AND ADDRESS & $\begin{array}{l}\text { HATCHING } \\
\text { EGG } \\
\text { CAPACITY }\end{array}$ & $\begin{array}{l}\text { PRODUCTS CLASSIFIED } \\
\text { U.S. PULLORUM-TYPHOID } \\
\text { CLEAN }\end{array}$ \\
\hline & & 35 & - WISCONSIN & \\
\hline 244 & $E$ & $\begin{array}{l}\text { MAPLE LEAF FARMS, INC., DOWNY HATCHERY, } 24830 \\
\text { WASHINGTON AVENUE, KANSASVILLE } 52139 \\
\text { Phone: }(262) 534-2275\end{array}$ & 733,800 & $x 3$ \\
\hline 143 & $E$ & $\begin{array}{l}\text { EDWARD NIENHAUS, } 31810-45 \text { TH STREET, } \\
\text { BURLINGTON } 53105 \\
\text { Phone: }(262) 537-4434\end{array}$ & 350 & R247, W166, W593, W94____ \\
\hline 254 & E & $\begin{array}{l}\text { BRUCE NOVAK, } 1575 \text { NOVAK ROAD, HIGHLAND } 53543 \\
\text { Phone: }(608) 623-2091\end{array}$ & 1,080 & $\mathrm{Z} 27, \mathrm{Z43}$ \\
\hline 130 & $E$ & $\begin{array}{l}\text { OAK RIDGE PHEASANT FARM, T. \& K. ZINDL, W2241 } \\
\text { GOPHER HILL ROAD, WATERTOWN } 53094 \\
\text { Phone: }(920) 262-8334\end{array}$ & 28,176 & $\mathrm{Z} 121, \mathrm{Z31} \ldots$ \\
\hline 226 & $E$ & $\begin{array}{l}\text { POPPLE LAKE GAME FARM, } 14630181 \text { TH STREET, } \\
\text { CHIPPEWA FALLS } 54729 \\
\text { Phone: }(715) 382-4145\end{array}$ & 1,100 & $\begin{array}{l}\text { W11, W119, W121, W131, W17, } \\
\text { W182, W3, W370, W435, W57, } \\
\text { W570, X114, X115, X117, X120, } \\
\text { X124, X125, X126, X127, X130, } \\
X 144, X 146, X 150, X 171, X 184, \\
X 187, X 28, X 4, X 40, X 42, X 48, \\
X 49, X 5, X 51, X 52, X 53, X 54 \\
X 57, X 60, X 61, X 62, X 7, X 70 \\
X 73, X 74, X 75, X 76, X 77, X 78, \\
X 89, Y 15, Y 26, Y 28, Y 32, Y 39, \\
Y 41, Y 51, Z 107, Z 109, Z 126, \\
Z 142, Z 161, Z 27, Z 284, Z 30, Z 43, \\
Z 48, Z 60, Z 65, Z 72, Z 78, Z 8, Z 86, \\
Z 93, Z 94\end{array}$ \\
\hline 253 & $E$ & $\begin{array}{l}\text { TRENT RASMUSSEN, RENEGADE GAMEBIRDS, } 5561 \\
\text { REDDIN ROAD, WISCONSIN RAPIDS } 54495 \\
\text { Phone: }(715) 424-5561\end{array}$ & 13,250 & 243,248 \\
\hline 264 & $E$ & JEFF RICE, 29800 BUSHNELL ROAD, BURLINGTON & 8,000 & $\mathrm{Z1}, \mathrm{Z} 27, \mathrm{Z} 3$ \\
\hline
\end{tabular}
246 E GARY W. ROSSMAN, 19120 WASHINGTON AVENUE, UNION GROVE 53182 Phone: (262)878-2862
231 E GREG SERRES, 28312 MILL STREET, MERTON 53056 Phone: (262)538-1808
136 E TRESA STILLMAN, E6325 RAILROAD GRADE ROAD, WEYAUWEGA 54983 Phone: (920)867-4544

106 E TOUBL GAME BIRD FARMS, 8716 WEST ST LAWRENCE ROAD, BELOIT 53511 Phone: (608)635-9273

229 E WILD WINGS ESTATE, B. COCHRANE, 10801 WEST HIGHWAY 81, BELOIT 53511

Phone: (608)365-3256

\begin{tabular}{|c|c|c|}
\hline 232 & $E$ & $\begin{array}{l}\text { WINDRIDGE POULTRY, H } 2946 \text { COUNTY ROAD NORTH, } \\
\text { COLBY } 54421\end{array}$ \\
\hline 152 & $E$ & $\begin{array}{l}\text { WOODS \& MEADOWS GAME FARM, SCOTT GOETZKA, } \\
\text { RT. } 1 \text {, BOX } 40 \text {, N4335 POTTERS ROAD, WARRENS } 54666 \\
\text { Phone: }(608) 378-4223\end{array}$ \\
\hline 86 & $E$ & $\begin{array}{l}\text { LEON ZIMDARS, } 767 \text { PALMYRA STREET, SULLIVAN } \\
53178 \\
\text { Phone: (262)593-8873 }\end{array}$ \\
\hline
\end{tabular}

ADDITIONAL

CLASSIFICATIONS FOR WHICH PRODUCT QUALIFIED 
Table 1. HATCHERIES, DEALERS. AND INDEPENDENT FLOCKS PARTICIPATING IN

THE NATIONAL POULTRY IMPROVEMENT PLAN

WATERFOWL, EXHIBITION POULTRY AND GAME BIROS

\begin{tabular}{|c|c|c|c|c|c|}
\hline $\begin{array}{l}\text { APP. } \\
\text { NO. }\end{array}$ & $\begin{array}{l}\text { SUB- } \\
\text { PART }\end{array}$ & PARTICIPANTS NAME AND ADDRESS & $\begin{array}{l}\text { HATCHING } \\
\text { EGG } \\
\text { CAPACITY }\end{array}$ & $\begin{array}{l}\text { PRODUCTS CLASSIFIED } \\
\text { U.S. PULLORUM-TYPHOID } \\
\text { CLEAN }\end{array}$ & $\begin{array}{c}\text { ADDITIONAL } \\
\text { CLASSIFICATIONS FOR } \\
\text { WHICH PRODUCT QUALIFIED }\end{array}$ \\
\hline
\end{tabular}

\section{$\underline{\text { Dealers }}$}

235 E ROBERT BLAHA, W2186 POPPLE RIVER ROAD, UNITY 54488

Phone: (715)223-2753

242 E CHET'S FEED \& SEED, 373 WEST 6TH STREET,

$E \& C$

231 E MERTON FEED COMPANY, 28312 MILL STREET, P.O. BOX 465, MERTON 53056-0465

Independent Flocks
125 E BLONHAVEN GAME FARM, 8006 NORTH JOHN PAUL ROAD, MILTON 53563 Phone: (608)868-3176
265 E DARWIN DEAN DAEBLER, W6796 STATE ROAD 156. SHIOCTON 54170
Phone: (715)758-8106
262 E HAPPY FARM, LLC, UE THAO, 2518 CITY ROAD DD, GLENWOOD CITY 54013
150 E ELISA NOEL, 2288 HILL ROAD, GREENLEAF 54126 Phone: (920)532-4367
230 E PHEASANTS ON THE LEDGE, S. MESSNER, N2560 CENTER LINE ROAD, OAKFIELD 53065 Phone: (920)582-3662
263 E DAN SPECHT, 9861 CITY ROAD EAST, AUBURNDALE 54412
Phone: (715)652-6407
259 E CHESTER SUMWALT, 18313 HIGHWAY 56, RICHLAND CENTER 53581
Phone: (608)647-8833
236 E SUNNYSIDE OF BEAVER DAM, INC., M. WILKE, P.O. BOX 452, 104 BELTLINE DRIVE, DEAVER DAM 53916 Phone: (920)887-2122

$\mathrm{Z} 27$

227

$\mathrm{Z1}, \mathrm{Z} 27, \mathrm{Z43}$

W. W16, W20, W578, W609

R, W, Z231

227

C. R147, R19, R20, R233, R239. R247, R6, R7, R81, X161, X2, X3, $\mathrm{X} 4, \mathrm{Y} 1, \mathrm{Y} 2, \mathrm{Y} 3, \mathrm{Y} 4$

$\mathrm{Z} 27$.

$X_{2}, X_{3}, X_{4}, Y 1, Y 31$

MR. DOUGLAS J. LEINART

WYOMING LIVESTOCK BOARD

ANIMAL HEALTH DIVISION

2020 CAREY AVENUE, 4TH FLOOR

CHEYENNE, WY 82002

Phone: (307)777-6437

Fax: (307)777-6561

Hatcheries

2 E KARL BEAR, 1175 LANE 10, POWELL 82414 
HATCHING

EGG

CAPACITY
PRODUCTS CLASSIFIED U.S. PULLORUM-TYPHOID CLEAN
ADDITIONAL

CLASSIFICATIONS FOR WHICH PRODUCT QUALIFIED
RAY HILBURN

POULTRY PATHOLOGIST

DEPARTMENT OF AGRICULTURE \& INDUSTRIES

376 BRYAN AVENUE

LUVERNE, AL 36049

Phone: (334)240-7255

Fax: (334)223-7352

No Participants
64 - ALABAMA

and

DR.TONY FRAZIER

STATE VETERINARIAN

DEPARTMENT OF AGRICULTURE \& INDUSTRIES

P.O. BOX 3336

MONTGOMERY, AL 36109-0336

Phone: (334)240-7253

Fax: (334)240-7198

86 - ARIZONA

DR. E. J. BICKNELL

EXTENSION VETERINARY SPECIALIST

MARICOPA AGRICULTURAL CENTER

37860 WEST SMITH ENKE ROAD

MARICOPA, AZ 85239

Phone: (520)568-2273

Fax: $\quad(520) 568-2556$

No Participants

DR. H. M. GHORI

ARKANSAS LIVESTOCK \& POULTRY COMMISSION

1 NATURAL RESOURCES DRIVE

LITTLE ROCK, AR 72205

Phone: (501)907-2434

Fax: (501)907-2259

No Participants
71 - ARKANSAS

and MELODY PARSLEY

ARKANSAS LIVESTOCK \& POULTRY COMMISSION

1 NATURAL RESOURCES DRIVE

LITTLE ROCK, AR 72205

Phone: (501)907-2447

Fax: (501)907-2259

93 - CALIFORNIA

BILL MATTOS

CALIFORNIA POULTRY HEALTH BOARD

3117-A MCHENRY AVENUE

MODESTO. CA 95350

Phone: $(209) 576-6355$

Fax: $\quad(209) 576-6119$

\section{Independent Flocks}

" Does not report Hatching egg capacity 
Table 2. HATCHERIES, DEALERS, AND INDEPENDENT FLOCKS PARTICIPATING IN

THE NATIONAL POULTRY IMPROVEMENT PLAN

Ratites

\begin{tabular}{|c|c|c|c|c|c|}
\hline $\begin{array}{l}\text { APP. } \\
\text { NO. }\end{array}$ & $\begin{array}{l}\text { SUB- } \\
\text { PART }\end{array}$ & PARTICIPANTS NAME AND ADDRESS & $\begin{array}{l}\text { HATCHING } \\
\text { EGG } \\
\text { CAPACITY }\end{array}$ & $\begin{array}{l}\text { PRODUCTS CLASSIFIED } \\
\text { U.S. PULLORUM-TYPHOID } \\
\text { CLEAN }\end{array}$ & $\begin{array}{c}\text { ADDITIONAL } \\
\text { CLASSIFICATIONS FOR } \\
\text { WHICHPRODUCT QUALIFIED }\end{array}$ \\
\hline & & 93 & - CALIFORNIA & & \\
\hline 269 & $\mathrm{~F}$ & $\begin{array}{l}\text { NEWBERRY FARMS, PHILIP MIZRAHIE, } 4105 \text { WEST } \\
\text { JEFFERSON BLVD., LOS ANGELES } 90016 \\
\text { Phone: (323)734-1445 }\end{array}$ & & O & \\
\hline
\end{tabular}

WILLIAM C. LOWER

COLORADO POULTRY IMPROVEMENT BOARD, INC.

4816 EAST COUNTY ROAD \#30

FORT COLLINS, CO 80528-9324

Phone: $(970) 226-3680$

No Participants

DR. BRUCE SHERMAN

STATE VETERINARIAN

DEPARTMENT OF AGRICULTURE

765 ASYLUM AVENUE

HARTFORD, CT 06105

Phone: (860)713-2504

Fax: $\quad(860) 713-2515$

No Participants

DR. H. WESLEY TOWERS, JR.

STATE VETERINARIAN

STATE DEPARTMENT OF AGRICULTURE

2320 SOUTH DUPONT HIGHWAY

DOVER, DE 19901

Phone: (302)739-4811

Fax: (302)697-6287
16 - CONNECTICUT

and

\author{
DR. LOUIS VAN DER HEIDE \\ DEPARTMENT OF PATHOLOGY \\ UNIVERSITY OF CONNECTICUT \\ STORRS, CT 06268 \\ Phone: $(860) 486-4000$ \\ Fax: (860)486-2794
}

50 - DELAWARE

No Participants 
Table 2. HATCHERIES, DEALERS, AND INDEPENDENT FLOCKS PARTICIPATING IN

THE NATIONAL POULTRY IMPROVEMENT PLAN

Ratites

\begin{tabular}{lllll}
\hline & & HATCHING & PRODUCTS CLASSIFIED \\
APP. SUB- & PARTICIPANTS NAME AND ADDRESS & A.S. PULLORUM-TYPHOID & CLEAN \\
NO. PART & CAPACITY & CLASSIFICATIONS FOR & WHICH PRODUCT QUALIFIED \\
\hline
\end{tabular}

58 - FLORIDA

JENNIFER JENNINGS-GLOVER

and DR. CAROLYN PARKINS

FLORIDA DEPARTMENT OF AGRICULTURE AND

FLORIDA DEPARTMENT OF AGRICULTURE AND

CONSUMER SERVICES

CONSUMER SERVICES

2145 LAMBERT LANE

P.O. DRAWER "O"

TALLAHASSEE, FL 32317

LIVE OAK, FL 32064

Phone: (850)251-1226

Phone: (386)362-1216

Fax: $\quad(850) 921-3647$

Fax: (386)362-4213

Independent Flocks $\begin{aligned} & 540 \text { F } \text { LITTLE CREEK OSTRICH, LLC., P.O. BOX 2375. FT. } \\ & \text { MYERS BEACH } 33932\end{aligned}$

O.

57 - GEORGIA

JAMES SCROGGS

GEORGIA POULTRY IMPROVEMENT ASSOCIATION, INC.

P.O. BOX 20

OAKWOOD, GA 30566

Phone: (770)535-5996

Fax: $\quad(770) 535-5941$

No Participants
DR. DOUG WALTMAN

GEORGIA POULTRY LABORATORY

P.O. BOX 20

OAKWOOD, GA 30566

Phone: (770)535-5996

Fax: (770)535-5941

82 - IDAHO

DR. KENDAL G. EYRE

BUREAU CHIEF

BUREAU OF ANIMAL HEALTH LABORATORIES

2230 OLD PENITENTIARY ROAD

BOISE. ID 83712

Phone: (208)332-8570

Fax: $\quad(208) 334-4619$

No Participants 
Table 2. HATCHERIES, DEALERS, AND INDEPENDENT FLOCKS PARTICIPATING IN

THE NATIONAL POULTRY IMPROVEMENT PLAN

Ratites

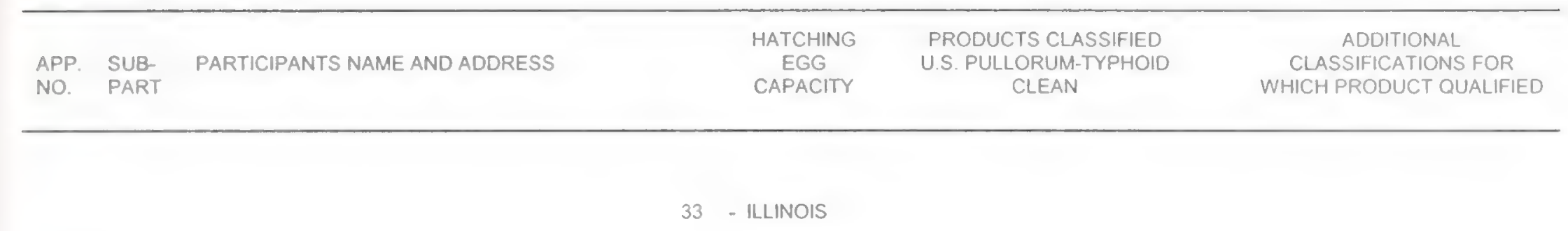

\begin{abstract}
JAMES E. KUNKLE
P.O. BOX 19281

STATE FAIRGROUNDS

SPRINGFIELD, IL 62794

Phone: (217)782-4944

Fax: (217)524-7702
\end{abstract}

ILLINOIS DEPARTMENT OF AGRICULTURE

DIVISION OF ANIMAL INDUSTRIES

Independent Flocks

856 F ELDE RATITE FAMILY RANCH, R.D. HALL, 346 OSWEGO STREET, PARK FOREST 60466-2104

O.

32 - INDIANA

\title{
PAUL BRENNAN
}

INDIANA STATE POULTRY ASSOCIATION, INC.

PURDUE UNIVERSITY

1151 LILLY HALL

WEST LAFAYETTE, IN 47907-1151

Phone: (765)494-8517

Fax: $\quad(765) 496-1600$

No Participants

42 - IOWA

KEVIN S. VINCHATTLE

IOWA POULTRY ASSOCIATION

8515 DOUGLAS AVENUE, SUITE 9

URBANDALE, IA 50322-2924

Phone: (515)727-4701

Fax: (515)727-4707

No Participants

48 - KANSAS

\begin{abstract}
DR. SCOTT BEYER
\end{abstract}
EXTENSION SPECIALIST, POULTRY SCIENCES

CALL HALL, KANSAS STATE UNIVERSITY

MANHATTAN, KS 66506

Phone: (785) 532-1201

Fax: (785) 532-5681 
Table 2. HATCHERIES, DEALERS, AND INDEPENDENT FLOCKS PARTICIPATING IN

THE NATIONAL POULTRY IMPROVEMENT PLAN

Ratites

\begin{tabular}{|c|c|c|c|c|c|}
\hline $\begin{array}{l}\text { APP. } \\
\text { NO. }\end{array}$ & $\begin{array}{l}\text { SUB- } \\
\text { PART }\end{array}$ & PARTICIPANTS NAME AND ADDRESS & $\begin{array}{l}\text { HATCHING } \\
\text { EGG } \\
\text { CAPACITY }\end{array}$ & $\begin{array}{l}\text { PRODUCTS CLASSIFIED } \\
\text { U.S. PULLORUM-TYPHOID } \\
\text { CLEAN }\end{array}$ & $\begin{array}{c}\text { ADDITIONAL } \\
\text { CLASSIFICATIONS FOR } \\
\text { WHICH PRODUCT QUALIFIED }\end{array}$ \\
\hline
\end{tabular}

48 - KANSAS

No Participants

61 - KENTUCKY

DR. ANTHONY PESCATORE

UNIVERSITY OF KENTUCKY

DEPARTMENT OF ANIMAL SCIENCES

604 GARRIGUS BUILDING

LEXINGTON, KY 40546

Phone: (859)257-7529

Fax: (859)323-1027

\section{Independent Flocks}

51 F THREE FORKS CREEK OSTRICH RANCH, 1010 CROSS ROAD PIKE, CORINTH 41010

0

72 - LOUISIANA

DR. MARTHA LITTLEFIELD

ASSISTANT STATE VETERINARIAN

OFFICE OF ANIMAL HEALTH SERVICES

P.O. BOX 1951

BATON ROUGE. LA 70821

Phone: (225)925-3980

Fax: $\quad(225) 925-4103$

No Participants

11 - MAINE

DR. DONALD E. HOENIG

STATE VETERINARIAN

MAINE DEPARTMENT OF AGRICULTURE

28 STATE HOUSE STATION

AUGUSTA. ME 04333-0028

Phone: (207)287-3701

Fax: $\quad(207) 624-5044$ 
Table 2. HATCHERIES, DEALERS, AND INDEPENDENT FLOCKS PARTICIPATING IN THE NATIONAL, POULTRY IMPROVEMENT PLAN

Ratites

\begin{tabular}{|c|c|c|c|c|c|}
\hline $\begin{array}{l}\text { APP. } \\
\text { NO. }\end{array}$ & $\begin{array}{l}\text { SUB- } \\
\text { PART }\end{array}$ & PARTICIPANTS NAME AND ADDRESS & $\begin{array}{l}\text { HATCHING } \\
\text { EGG } \\
\text { CAPACITY }\end{array}$ & $\begin{array}{l}\text { PRODUCTS CLASSIFIED } \\
\text { U.S. PULLORUM-TYPHOID } \\
\text { CLEAN }\end{array}$ & $\begin{array}{c}\text { ADDITIONAL } \\
\text { CLASSIFICATIONS FOR } \\
\text { WHICH PRODUCT QUALIFIED }\end{array}$ \\
\hline
\end{tabular}

51 - MARYLAND

DR. FIDELIS HEGNGI

MARYLAND DEPARTMENT OF AGRICULTURE

ANIMAL HEALTH LABORATORY

P.O. BOX 2599

SALISBURY, MD 21802

Phone: (410)543-6610

Fax: $\quad(410) 543-6676$

No Participants and

KIM JONES

MARYLAND DEPARTMENT OF AGRICULTURE

ANIMAL HEALTH LABORATORY

P.O. BOX 2599

SALISBURY, MD 21802

Phone: $(410) 543-6610$

Fax: $\quad(410) 543-6676$

14 - MASSACHUSETTS

\begin{abstract}
EDWARD HAGEMAN
\end{abstract}
MASSACHUSETTS DEPARTMENT OF AGRICULTURE

DIVISION OF POULTRY \& POULTRY PRODUCTS

251 COSWAY STREET, SUITE 500

BOSTON, MA 02114-2151

Phone: (617)626-1796

Fax: $\quad(617) 626-1850$

No Participants

34 - MiCHIGAN

GEORGE HOUSE

EXECUTIVE DIRECTOR/NPIP REPRESENTATIVE 5635 FOREST GLEN DRIVE SE

P.O. BOX 242

ADA, MI 49301

Phone: (616)676-5593

Fax: (616)676-1494 and -

DR. R. M. (MICK FULTON

\section{AVIAN PATHOBIOLIST}

ANIMAL HEALTH DIAGNOSTIC LABORATORY

G-1304 VETERINARY MEDICAL CENTER

MICHIGAN STATE UNIVERSITY

EAST LANSING, MI 48824-1314

Phone: (517)353-3701

Fax: (517)355-2152

Independent Flocks

436 F NELL WARD, 30078 ALLEN ROAD, DOWAGIAC 49047 
Table 2. HATCHERIES, DEALERS, AND INDEPENDENT FLOCKS PARTICIPATING IN

THE NATIONAL POULTRY IMPROVEMENT PLAN

Ratites

\begin{tabular}{llccc}
\hline & & HATCHING & PRODUCTS CLASSIFIED \\
APP. SUB- & PARTICIPANTS NAME AND ADDRESS & EGG & U.S. PULLORUM-TYPHOID \\
NO. PART & CLEAN & CLAPACITY & WHICH PROACATIONS FOR \\
\hline
\end{tabular}

41 - MINNESOTA

DR. KEITH FRIENDSHUH

BOARD OF ANIMAL HEALTH

90 WEST PLATO BLVD., SUITE 119

ST. PAUL, MN 55107

Phone: (651)296-2942

Fax: (651)296-7417

\section{Dealers}

860 F THE FEED STORE, 18961 STREET HIGHWAY 23, P.O. BOX 18961, RICHMOND 56368

C

Independent Flocks

341 F MIKET. LOSS, 5869 BARBEAU ROAD, BRAINERD 56401

P

65 - MISSISSIPPI

DANNY THORNTON

POULTRY EPIDEMIOLOGIST

4239 EBENEZER ROAD

CARTHAGE, MS 39051

Phone: (601)267-3844

Fax: (601)359-1177

No Participants

43 - MISSOURI
DR. JIM WATSON

STATE VETERINARIAN

MISSISSIPPI BOARD OF ANIMAL HEALTH

P.O. BOX 3889

JACKSON, MS 39207

Phone: (601)359-1170

Fax: (601)359-1177

\section{ROSE FOSTER}

MISSOURI DEPARTMENT OF AGRICULTURE

P.O. BOX 630

JEFFERSON CITY, MO 65102

Phone: (573)522-3377

Fax: $\quad(573) 751-5279$

No Participants 
Table 2. HATCHERIES, DEALERS, AND INDEPENDENT FLOCKS PARTICIPATING IN

THE NATIONAL POULTRY IMPROVEMENT PLAN

Ratites

\begin{tabular}{llccc}
\hline & & & PRODUCTS CLASSIFIED \\
APP. & SUB. & PARTICIPANTS NAME AND ADDRESS & HATCHING & ADDITIONAL \\
NO. & PART & EGG & U.S. PULLORUM-TYPHOID \\
\end{tabular}

81 - MONTANA

DR. THOMAS F.T. LINFIELD

ASSISTANT STATE VETERINARIAN

MONTANA DEPARTMENT OF LIVESTOCK

ANIMAL HEALTH DIVISION

P.O. BOX 202001

HELENA, MT 59620-2001

Phone: (406)444-2043

Fax: $\quad(406) 444-1929$

No Participants

47 - NEBRASKA

\begin{abstract}
DR. DELWIN WILMOT
P.O. BOX 94787

LINCOLN, NE 68509-4787

Phone: (402)471-2351

Fax: (402)471-3252
\end{abstract}

DEPUTY STATE VETERINARIAN

DEPARTMENT OF AGRICULTURE

BUREAU OF ANIMAL INDUSTRY

No Participants

94 - NEVADA

DR. RONALD D. ANDERSON

NEVADA ANIMAL DISEASE LABORATORY

350 CAPITOL HILL AVENUE

RENO, NV 89502

Phone: (702)688-1182 EXT.233

Fax: $\quad(702) 688-1198$

No Participants

12 - NEW HAMPSHIRE

DR. CLIFFORD W. MCGINNIS

STATE VETERINARIAN

NH DEPT. OF AGRICULTURE \& DIV. OF ANIMAL INDUSTRY

P.O. BOX 2042

CONCORD, NH 03302-2042

Phone: (603)271-2404

Fax: $\quad(603) 271-1109$ 
Table 2. HATCHERIES, DEALERS, AND INDEPENDENT FLOCKS PARTICIPATING IN

THE NATIONAL POULTRY IMPROVEMENT PLAN

Ratites

\begin{tabular}{lllll} 
& & & ADDITIONAL \\
APP. & SUB- & PARTICIPANTS NAME AND ADDRESS & HATCHNG & PRODUCTS CLASSIFIED \\
NO. & PART & EGG & U.S. PULLORUM-TYPHOID & CLEAN \\
\hline
\end{tabular}

12 - NEW HAMPSHIRE

Independent Flocks $\begin{array}{ll}186 \text { F } & \text { SHERRI TOWLE-BRUN, } 379 \text { TOLAND ROAD, DOVER } \\ 03820 & \end{array}$

22 - NEW JERSEY

DR. KAREN M. FROBERG

and

DR. ERNEST ZIRKLE

DIVISION OF ANIMAL HEALTH

DIVISION OF ANIMAL HEALTH

DEPARTMENT OF AGRICULTURE

DEPARTMENT OF AGRICULTURE

P.O. BOX CN-330

P.O. BOX CN-330

TRENTON, NJ 08625

TRENTON, NJ 08625

Phone: (609)292-3965

Phone: (609)292-3965

Fax: (609)633-2550

Fax: $\quad(609) 633-2550$

No Participants

85 - NEW MEXICO

DR. RON PARKER

DEPARTMENT HEAD

AGICULTURAL EXTENSION SERVICE

BOX $3 A E$

LAS CRUCES, NM 88003

Phone: (505)646-1709

Fax: (505)646-3164

Independent Flocks

41 F DAVID WEST, P.O. BOX 657, FORT SUMNER 88119

$01,02,03,04$

21 - NEW YORK

DR. JOHN HUNTLEY

STATE VETERINARIAN

DIVISION OF ANIMAL INDUSTRY

DEPARTMENT OF AGRICULTURE \& MARKETS

1 WINNERS CIRCLE

ALBANY, NY 12235

Phone: (518)457-3502

Fax: (518)485-7773

No Participants 


\begin{tabular}{|c|c|c|c|c|c|}
\hline $\begin{array}{l}\text { APP. } \\
\text { NO. }\end{array}$ & $\begin{array}{l}\text { SUB- } \\
\text { PART }\end{array}$ & PARTICIPANTS NAME AND ADDRESS & $\begin{array}{l}\text { HATCHING } \\
\text { EGG } \\
\text { CAPACITY }\end{array}$ & $\begin{array}{c}\text { PRODUCTS CLASSIFIED } \\
\text { U.S. PULLORUM-TYPHOID } \\
\text { CLEAN }\end{array}$ & $\begin{array}{c}\text { ADDITIONAL } \\
\text { CLASSIFICATIONS FOR } \\
\text { WHICH PRODUCT QUALIFIED }\end{array}$ \\
\hline
\end{tabular}

73 - OKLAHOMA

\section{RALPH DUNCAN}

ANIMAL INDUSTRY DIVISION

OKLAHOMA STATE DEPARTMENT OF AGRICULTURE

2800 NORTH LINCOLN BLVD.

OKLAHOMA CITY, OK 73105

Phone: (405)521-3864

Fax: (405)522-0756

\section{No Participants}

92 - OREGON

DR. ANDREW A. CLARK

STATE VETERINARIAN

OREGON DEPARTMENT OF AGRICULTURE

635 CAPITOL STREET, NE

SUITE 100

SALEM, OR $97310-2532$

Phone: (503)986-4680

Fax: $\quad(503) 986-4734$

Independent Flocks

253 F A \& N LIVESTOCK. ANN \& NELS OLSSON. 18315 BRIDGEPORT ROAD, DALLAS 97338

23 - PENNSYLVANIA

DR. JOHN I. ENCK, JR.

and

DIRECTOR, ANIMAL INDUSTRY

PENNSYLVANIA DEPARTMENT OF AGRICULTURE

2301 NORTH CAMERON STREET

HARRISBURG, PA 17110

Phone: (717)783-6677

Fax: $\quad(717) 787-1868$ and DR. BRUCE. MUELLER

OREGON DEPARTMENT OF AGRICULTURE

635 CAPITOL STREET, NE

SALEM, OR 97310-0110

Phone: (503)986-4680

Fax: (503)986-4734
P

DR. PHILLIP C. DEBOK.

ANIMAL \& POULTRY HEALTH DIVISION

PENNSYLVANIA DEPARTMENT OF AGRICULTURE

2301 NORTH CAMERON STREET

HARRISBURG. PA 17110

Phone: (717)783-8555

Fax: $\quad(717) 787-1868$

No Participants 
Table 2. HATCHERIES. DEALERS. AND INDEPENDENT FLOCKS PARTICIPATING IN

THE NATIONAL POULTRY IMPROVEMENT PLAN

$$
\text { Ralites }
$$

\begin{tabular}{|c|c|c|c|c|c|}
\hline $\begin{array}{l}\text { APP. } \\
\text { NO. }\end{array}$ & $\begin{array}{l}\text { SUB- } \\
\text { PART }\end{array}$ & PARTICIPANTS NAME AND ADDRESS & $\begin{array}{l}\text { HATCHING } \\
\text { EGG } \\
\text { CAPACITY }\end{array}$ & $\begin{array}{l}\text { PRODUCTS CLASSIFIED } \\
\text { U.S. PULLORUM-TYPHOID } \\
\text { CLEAN }\end{array}$ & $\begin{array}{c}\text { ADDITIONAL } \\
\text { CLASSIFICATIONS FOR } \\
\text { WHICHPRODUCT QUALIFIED }\end{array}$ \\
\hline
\end{tabular}

15 - RHODE ISLAND

DR. CHRISTOFER HANNAFIN

STATE VETERINARIAN

RHODE ISLAND DIVISION OF AGRICULTURE

235 PROMENADE STREET

PROVIDENCE, RI 02908-5767

Phone: (401)222-2781, EXT. 4503

Fax: (401)222-6047

No Participants

56 - SOUTH CAROLINA

DR. JULIE D. HELM

CLEMSON LABORATORY

LIVESTOCK-POULTRY HEALTH DEPARTMENT

P.O. BOX 102406

COLUMBIA, SC 29224

Phone: (803)788-2260

Fax: $\quad(803) 736-0885$

Independent Flocks

337 F SAM CUNNINGHAM, P.O. BOX 1137, WOOORUFF 29388

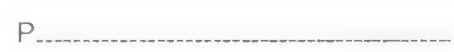

46 - SOUTH DAKOTA

DR. SAM HOLLAND -DR. THOMAS F. CLINE AMIMAL INDUSTRY BOARD

411 SOUTH FORT STREET

PIERRE, SD 57501

Phone: (605)773-3321

Fax: (605)773-5459 and DR. DARWIN BRITZMAN

SOUTH DAKOTA ANIMAL INDUSTRY BOARD

2208 ST. CHARLES CIRCLE

SIOUX FALLS, SD 57103

Phone: (605)332-1116

Fax: $\quad(605) 688-6170$

No Participants

63 - TENNESSEE

DR. RONALD B. WILSON

STATE VETERINARIAN

TENNESSEE DEPARTMENT OF AGRICULTURE

BOX 40627, MELROSE STATION

NASHVILLE, TN 37204

Phone: (615)837-5120

Fax: (615)837-5250 and TINA ROGERS

TENNESSEE DEPARTMENT OF AGRICULTURE

ELLINGTON AGRICULTURAL CENTER

BOX 40627, MELROSE STATION

NASHVILLE, TN 37204

Phone: (615)837-5120

Fax: $\quad(615) 837-5250$ 
Ratites

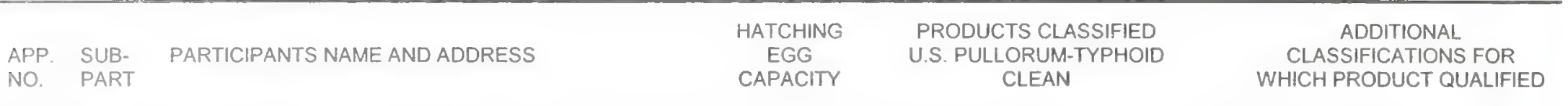

63 - TENNESSEE

No Participants

74 - TEXAS

DR. FLOYD A. GOLAN

TEXAS VETERINARY MEDICAL DIAGNOSTIC

LABORATORY

P.O. DRAWER 3040

COLLEGE STATION, TX 77841-3040

Phone: (979)845-4186

Fax: (979)845-1794

Hatcheries

3689 F SRL FARM, EDWIN A. CANNON, 30134 BINFORD ROAD, WALLER 77484

$252 P$

87 - UTAH

DR. MICHAEL R. MARSHALL

STATE VETERINARIAN

UTAH DEPARTMENT OF AGRICULTURE

BOX 146500

SALT LAKE CITY, UT 84114-6500

Phone: (801)538-7160

Fax: (801)538-7169

No Participants and DR. EARL ROGERS

ASSISTANT STATE VETERINARIAN

UTAH DEPARTMENT OF AGRICULTURE

BOX 146500

SALT LAKE CITY, UT 84114-6500

Phone: (801)538-7162

Fax: $\quad(801) 538-7169$

13 - VERMONT

DR. TODD JOHNSON

STATE VETERINARIAN

DEPARTMENT OF AGRICULTURE, FOOD \& MARKETS

116 STATE STREET. DRAWER 20

MONTPELIER, VT 05620-2901

Phone: (802)828-2421

Fax: $\quad(802) 828-5983$

No Participants 
Table 2. HATCHERIES, DEALERS, AND INDEPENDENT FLOCKS PARTICIPATING IN

THE NATIONAL POULTRY IMPROVEMENT PLAN

Ratites

\begin{tabular}{llccc}
\hline & & HATCHING & PRODUCTS CLASSIFIED \\
APP. SUB- & PARTICIPANTS NAME AND ADDRESS & ERG & U.S. PULLORUM-TYPHOID & CLEATIONAL \\
NO. PART & CAPACITV & CLASSIFICATIONS FOR & WHICH PRODUCT QUALIFIED
\end{tabular}

52 - VIRGINIA

DR. G. G. MEZA

AREA VETERINARIAN

DIVISION OF ANIMAL HEALTH \& DAIRIES

VIRGINIA DEPT. OF AGRIC. \& CONSUMER SERVICES

116 RESERVOIR STREET

HARRISONBURG, VA 22801

Phone: (540)434-3897

Fax: $\quad(540) 434-3880$

\section{No Participants}

and DR. RONALD KING

REGIONAL VETERINARY SUPERVISOR

DIVISION OF ANIMAL HEALTH \& DAIRIES

VIRGINIA DEPT. OF AGRIC. \& CONSUMER SERVICES

116 RESERVOIR STREET

HARRISONBURG, VA 22801

Phone: (540)434-3897

Fax: (540)564-2428

91 - WASHINGTON

DR. KATHLEEN M. CONNELL

\section{ASSISTANT STATE VETERINARIAN}

FOOD SAFETY \& ANIMAL HEALTH DIVISION

DEPARTMENT OF AGRICULTURE

P.O. BOX 42577

OLYMPIA, WA 98504-2577

Phone: (360)902-1878

Fax: (360)902-2087

No Participants

54 - WEST VIRGINIA

DR. JEWELL PLUMLEY

ASSISTANT DIRECTOR ANIMAL HEALTH DIVISION WEST VIRGINIA DEPARTMENT OF AGRICULTURE HC 85, BOX 302

MOOREFIELD, WV 26836

Phone: (304)538-2397

Fax: (304)538-7088

No Participants 


\begin{tabular}{lllcc}
\hline & & HATCHING & PRODUCTS CLASSIFIED \\
APP. & SUB- & PARTICIPANTS NAME AND ADDRESS & ADEITIONAL \\
NO. PART & P.S. PULLORUM-TYPHOID & CLEAN & CLASSIFICATIONS FOR \\
\end{tabular}

$35-$ WISCONSIN

DR. DONALD P. O'CONNOR

NPIP COORDINATOR

DEPARTMENT OF AGRICULTURE

DIVISION OF ANIMAL HEALTH

P.O. BOX 8911

MADISON, WI 53708-8911

Phone: (608)224-4882

Fax: (608)224-4871

\section{No Participants}

and DR. CLARENCE J. SIROKY

STATE VETERINARIAN

DEPARTMENT OF AGRICULTURE

DIVISION OF ANIMAL HEALTH

P.O. BOX 8911

MADISON, WI 53708-8911

Phone: (608)224-4872

Fax: (608)224-4871

83 - WYOMING

MR. DOUGLAS J. LEINART

WYOMING LIVESTOCK BOARD

ANIMAL HEALTH DIVISION

2020 CAREY AVENUE, 4TH FLOOR

CHEYENNE, WY 82002

Phone: (307)777-6437

Fax: (307)777-6561 
Table 3. POULTRY BREEDS, VARIETIES, STRAINS, AND TRADE NAMES, WITH THEIR CODE NUMBERS LISTED NUMERICALLY BY KINDS OF STOCK

A - WHITE LEGHORN (NON-EXHIBITION)

\begin{tabular}{|c|c|c|c|c|c|}
\hline A1 & ARBOR ACRES & $A 2$ & BABCOCK & $A 6$ & EURIBRID \\
\hline A8 & H AND N & A 16 & WELP & A19 & HYLINE \\
\hline
\end{tabular}

B - RHODE ISLAND RED (NON-EXHIBITION)

\begin{tabular}{lllll}
\hline B1 & ARBOR ACRES & B3 & CORBETT & B7 \\
B10 WARDY
\end{tabular}

C - BARRED PLYMOUTH ROCK (NON-EXHIBITION)

\begin{tabular}{llllll}
\hline C1 & ARBOR ACRES & C2 & COLONIAL & C4 & DAVIS \\
C5 & HARCO & C6 & HARDY & C10 & WELP \\
C12 & IDEAL & & & &
\end{tabular}

D - WHITE PLYMOUTH ROCK (NON-EXHIBITION)

\begin{tabular}{|c|c|c|c|c|c|}
\hline D1 & ARBOR ACRES & D4 & COLONIAL & D5 & HARDY \\
\hline D6 & HUBBARD & D9 & WELP & D11 & CEBE, RECESSIVE \\
\hline $\mathrm{D} 12$ & IDEAL & & & & \\
\hline
\end{tabular}

E - NEW HAMPSHIRE (NON-EXHIBITION)

\begin{tabular}{|c|c|c|c|c|c|}
\hline E१ & COLONIAL & E2 & HUBBARD & E3 & ROWLEY \\
\hline & & & $\mathrm{H}$ - CORNISH (NON-EXHIBITION) & & \\
\hline $\mathrm{H} 1$ & ARBOR ACRES & $\mathrm{H} 2$ & COBB & $\mathrm{H} 3$ & HARMAN \\
\hline H5 & DARK & $\mathrm{H} 6$ & RED & $\mathrm{H} 8$ & WHITE LACED RED \\
\hline H9 & BUFF & $\mathrm{H} 10$ & WHITE & $\mathrm{H} 11$ & CEBE RED \\
\hline $\mathrm{H} 12$ & CEBE GOLD & $\mathrm{H} 14$ & CEBE BLACK & & \\
\hline
\end{tabular}

N - GAME CHICKEN

\begin{tabular}{|c|c|c|c|c|c|}
\hline N1 & MODERN, BLACK BREASTED RED & N2 & MODERN, BROWN RED & N4 & MODERN. SILVER DUCKWING \\
\hline N5 & MODERN, BIRCHEN & N6 & MODERN, RED PYLE & N7 & MODERN, WHITE \\
\hline N8 & MODERN, BLACK & N9 & $\begin{array}{l}\text { OLD ENGLISH, BLACK BREASTED } \\
\text { RED }\end{array}$ & N10 & OLD ENGLISH, BROWN RED \\
\hline N11 & OLD ENGLISH, GOLD DUCKWING & $\mathrm{N} 12$ & OLD ENGLISH. SILVER DUCKWING & N14 & OLD ENGLISH, WHITE \\
\hline N15 & OLD ENGLISH, BLACK & N16 & OLD ENGLISH, SPANGLED & N17 & PIT \\
\hline N18 & OLD ENGLISH & N19 & MODERN & N20 & PIT, WHITE HACKLE \\
\hline N21 & SAM BIGHAM & N22 & MCCLANHANS & N23 & CLIPPERS \\
\hline N24 & MINER BLUES & N25 & LAW GRAYS & N26 & OLD ENGLISH. BLACK BREASTED \\
\hline
\end{tabular}


N GAME CHICKEN (Continued)

\begin{tabular}{|c|c|c|c|c|c|}
\hline N27 & SPANISHPIT & N28 & HATCH & N30 & WILERSON RED \\
\hline N31 & WILKERSON GRAY & N32 & GRAY & N33 & CLARET \\
\hline N34 & HATCH, GRAY & N36 & BIGHAM RED & N37 & CAPITOL HILL RED \\
\hline N38 & CAPITOL HILL GRAY & N41 & ROUNDHEAD & $\mathrm{N} 42$ & HATCH, MURPHY \\
\hline N43 & HATCH, MCLAIN & N44 & KELSO & N46 & GRAY, MADIGIN \\
\hline N47 & WALLACE FOWL & N50 & OLD ENGLISH, BLUE & N51 & ROBIN HOOD BROWN RED \\
\hline N52 & DARK CORNISH & N55 & OLD ENGLISH, BLUE RED & N56 & JUMPER RED \\
\hline N57 & JUMPER GRAY & N58 & MCRAE, RED & N61 & LOWHMANN WHITE HACKLE \\
\hline N62 & MARSH BUTCHER & N63 & BUTCHER & N65 & HATCH, BLUE FACE \\
\hline N69 & NIPNUC BLUE & N70 & NIPNUC RED & N74 & MODERN, BLUE RED \\
\hline$N / 7$ & GLEASON WHITE HACKOE & N78 & OLD ENGLISH, BRASSY BLACK & N80 & ASEEL, DEVARAJA \\
\hline N81 & GOWDY JAP & N82 & NARRAGANSETT & N83 & ALBANY \\
\hline N84 & DEMOCRAT & N85 & GRIFFIN & N87 & BROWN RED, WINGATE \\
\hline N88 & ASEEL. KAHN & N89 & ASEEL, SONATOL & N90 & BROWN RED \\
\hline N91 & HENNIES, BLACK & N93 & THOMPSON WHITE & N94 & RED QUILL \\
\hline N96 & PEARCE SHUFFLER & N97 & DOMINIQUE & N98 & HATCH, PERRY \\
\hline N99 & GRAY, BUMBLEFOOT & N100 & BROWN RED, FREDRICK'S & N101 & GREY TOPPIES, JACK WILSON \\
\hline N102 & GREY, MISSISSIPP & N103 & PINNON & N104 & ASEEL \\
\hline N105 & MUG. RACEY & N106 & TAYLOR & N107 & HUSLEY \\
\hline N108 & TRAVLERS & N109 & TOPPY & N110 & CUBAN \\
\hline N111 & HENNY, SPANGLED & $\mathrm{N} 112$ & BLUE SPLASH & N113 & BROWN RED, SHUFFLER \\
\hline N114 & ORIENTAL & N116 & PIT, WHITE HACKLE, MORGAN & N117 & RENE VALLE \\
\hline N119 & OLD ENGLISH, BRASSY BLUE & N121 & HATCH, YELLOW LEG & N122 & ROBIN HOOD REDS \\
\hline N123 & ROBIN HOOD BLACK GREY & N125 & WHITE HACKLE, NORTH BRITTON & N126 & BAMA GREY \\
\hline N127 & MURPHYS & N128 & WHITES & N129 & MUFFS \\
\hline N130 & COPPERHEADS & N131 & HATCH, LEPER & N132 & LAWSHE BLUE \\
\hline N133 & WHITE DOME & N134 & ROUNDHEAD, LACY & N135 & WARHORSE \\
\hline N136 & CLARET, GRIFFIN & N137 & HATCH, RUBLE & N138 & HATCH, HAROLD BROWN \\
\hline N139 & GRAY, SWEATER & N140 & KELSO, RADIO & N141 & KELSO, BLUE LEG \\
\hline N142 & HATCH. SPECKLED & N143 & MUG. BLACK & N144 & BENOIST. SLIM BLUE \\
\hline N145 & BENOIST, SLIM RED & N146 & HATCH, SPANGLED BRASS BACK & N147 & MUG, BLACK MANGEL \\
\hline N148 & MUG & N149 & SPANISH, JEREZANO & N150 & GRAY, FINDLEY \\
\hline N151 & MCCRAE, BLACK & N152 & HATCH, SWEATER & N155 & HATCH, KELSO \\
\hline N156 & GRAY. IRISH & N157 & HATCH, BLACK & N159 & BUCKALOO \\
\hline N160 & ROUNDHEAD, BRUNNER & N161 & HATCH, GREEN LEG & N162 & HATCH, ROUND HEAD \\
\hline \multirow[t]{2}{*}{ N163 } & ROUNDHEAD, BOSTON & N164 & ROUNDHEAD, ALLEN & N165 & WARHORSE, BACON \\
\hline & & & 214 & & \\
\hline
\end{tabular}


$N$ GAME CHICKEN (Continued)

\begin{tabular}{|c|c|c|c|c|c|}
\hline N167 & GILMORE & N168 & GAVLIN & N169 & WHITE HACKLE, KEARNEY \\
\hline N171 & GRAY, JOE GOOD & N172 & HATCH, DOC ROBINSON & N174 & MURPHY WHITE HACKLE \\
\hline N175 & GREY, BULLOCK & $N 180$ & MODERN, LEMON BLUE & N181 & KELSO, YELLOW LEG \\
\hline N182 & KELSO, WHITE LEG & $N 183$ & WHITE HACKLE & N187 & HATCH, BLUE LEGGED \\
\hline N188 & MAHONEY GULL & N189 & HATCH, BILOXI & N190 & GARRARD \\
\hline N193 & GREY. WAYNE WOOD & N194 & BROWN RED, BOSTON & N195 & HATCH, OAK GROVE \\
\hline N197 & GREY, FROST & N198 & CLARET, WHITE & N199 & KELSO, HORSESHOE \\
\hline N206 & SHUFFLER & N207 & ORIENTAL, BLACK SHAMO & N211 & ORIENTAL, MALAYSION \\
\hline N216 & BUTCHER, RED & N219 & BUTCHER, BLACK & N221 & ROUNDHEAD, REBEL \\
\hline N222 & BUTCHER, SPANGLED & N224 & CLARET, MADIGIN & N225 & HATCH, DEMOCRAT \\
\hline N226 & HATCH, SANDY & N227 & KELSO, OUT \& OUT & N228 & ROUNDHEAD, JARRETT \\
\hline N229 & SHUFFLER, BLACK & N230 & WHITE HACKLE, MORGAN & N231 & WHITE HACKLE, RADIO \\
\hline N236 & GREY & N239 & GREY, MUG & N240 & BLUE FACE \\
\hline N241 & GRAY, GOLDEN & N243 & HATCH, GILMOORE & N247 & GREY, RICHARDSON \\
\hline N248 & WILDCAT BLUE & N249 & GREY, SLAVIN & N250 & $\begin{array}{l}\text { ROUNDHEAD, SLAVIN CRIPPLE } \\
\text { TON }\end{array}$ \\
\hline N251 & ROUNDHEAD, SLAVIN & N252 & HATCH, SLAVIN BLACK & N253 & BLACK, DENT COUNTY \\
\hline N254 & GREY, DENT COUNTY & N255 & RED, DENT COUNTY & N263 & HATCH CLARET, NACHA \\
\hline N264 & HATCH, WALTON & N268 & SIMMACK & N269 & BURN'S WHITE \\
\hline N270 & ALBANY, RADIO & N271 & RADIO & & \\
\hline
\end{tabular}

$R$ - OTHER LARGE BREEDS \& VARIETIES

\begin{tabular}{|c|c|c|c|c|c|}
\hline R1 & LEGHORN, SINGLE COMB BROWN & R2 & LEGHORN, SINGLE COMB BUFF & R3 & MINORCA, SINGLE COMB BLACK \\
\hline R10 & PLYMOUTH ROCK, COLUMBIAN & $\mathrm{R} 11$ & WYANDOTTE, WHITE & R12 & WYANDOTTE, SILVER LACED \\
\hline R13 & WYANDOTTE, COLUMBIAN & R14 & $\begin{array}{l}\text { RHODE ISLAND WHITE, SINGLE } \\
\text { COMB }\end{array}$ & R15 & JERSEY GIANT, BLACK \\
\hline R16 & JERSEY GIANT, WHITE & $\mathrm{R} 17$ & DELAWARE & R18 & BRAHMA \\
\hline R19 & BRAHMA, LIGHT & R20 & ARAUCANA & R21 & HAMBURG, SILVER SPANGLED \\
\hline R22 & LANGSHAN, BLACK & $\mathrm{R} 23$ & LANGSHAN, WHITE & R24 & EXPERIMENTAL \\
\hline R25 & LEGHORN, SINGLE COMB BLACK & R26 & AUSTRALORP, WHITE & R27 & EXHIBITION \\
\hline R36 & BARNEVELDER & R37 & WYANDOTTE, BLACK & R38 & WYANDOTTE, BUFF \\
\hline R39 & WYANDOTTE, PARTRIDGE & R40 & WYANDOTTE, SILVER PENCILED & R41 & CAMPINE, SILVER \\
\hline R42 & DOMINIQUE & R43 & PLYMOUTH ROCK, SILVER & R44 & ORPINGTON, BLACK \\
\hline
\end{tabular}


R OTHER LARGE BREEDS \& VARIETIES (Continued)

\begin{tabular}{|c|c|c|c|c|c|}
\hline R45 & POLISH, WHITE CRESTED BLACK & R46 & POLISH, NON-BEARDED WHITE & R47 & POLISH, NON-BEARDED BUFF \\
\hline$R+8$ & MALAY & R49 & ORPINGTON, WHITE & R50 & POLISH. NON-BEARDED BLACK \\
\hline R51 & BRAHMA, DARK & R52 & COCHIN. BLACK & R53 & COCHIN, PARTRIDGE \\
\hline R54 & HOUDAN. MOTTLED & R55 & JUNGLEFOWL & R56 & ANDALUSIAN \\
\hline R57 & JUNGLEFOWL, RED & $\mathrm{R} 60$ & SUMATRAS & R62 & YOKAHAMA \\
\hline R64 & CAMPINE, GOLDEN & R65 & IOWA BLUES & R66 & POLISH, WHITE CRESTED BLUE \\
\hline R67 & POLISH. WHITE SPLASH & R68 & HAMBURG. SILVER & R69 & FAYOUMI, EGYPTIAN \\
\hline R70 & SULTAN. WHITE & R71 & FAVEROLLE, SALMON & R72 & LAKENVELDER \\
\hline$R 73$ & HAMBURG & R74 & LEGHORN, SILVER & R75 & POLISH. NON-BEARDED GOLDEN \\
\hline R76 & HAMBURG. GOLDEN PENCILED & R77 & BUTTERCUP & R78 & JAVA \\
\hline R81 & WYANDOTTE, GOLDEN LACED & R82 & POLISH. BUFF LACED & R83 & POLISH. SILVER LACED \\
\hline R84 & $\begin{array}{l}\text { POLISH, NON-BEARDED GOLDEN } \\
\text { LACED }\end{array}$ & R85 & SUSSEX. SPECKLED & R86 & SUMATRAS, BLACK \\
\hline R87 & COCHIN, WHITE & R88 & CREVECOEURS & R89 & PHOENIX, SILVER \\
\hline 290 & $\begin{array}{l}\text { LEGHORN, ROSE COMB LIGHT } \\
\text { BROWN }\end{array}$ & R91 & $\begin{array}{l}\text { PLYMOUTH ROCK. SIVER } \\
\text { PENCILED }\end{array}$ & R92 & HAMBURG, SILVER PENCILED \\
\hline R93 & BRAHMA, BUFF & R94 & COCHIN & R95 & WYANDOTTE \\
\hline R96 & AUSTRALORP & R97 & SPANISH, WHITE FACED BLACK & R98 & LEGHORN, SINGLE COMB RED \\
\hline R99 & POLISH, GOLDEN & R100 & POLISH, BEARDED SILVER & R103 & ORPINGTON \\
\hline R104 & POLISH & R105 & FRIZZLE & R107 & ROSE COMB, BLACK \\
\hline R109 & HAMBURG. GOLDEN SPANGLED & R110 & HAMBURG, WHITE & R111 & HAMBURG, BLACK \\
\hline R112 & $\begin{array}{l}\text { BARTHAEHNER, SILVER } \\
\text { THOERINGER }\end{array}$ & R113 & BARTHAEHNER & R114 & LEGHORN, ROSE COMB WHITE \\
\hline R115 & LA FLECHE & R116 & ASEEL & R117 & ORPINGTON, BLUE \\
\hline R118 & PHOENIX. GOLDEN & R119 & COCHIN, GOLDEN LACED & R120 & COCHIN, SILVER LACED \\
\hline R121 & COCHIN. RED LACED & R122 & $\begin{array}{l}\text { POLISH, NON-BEARDED BUFF } \\
\text { LACED }\end{array}$ & R123 & POLISH, BEARDED BUFF LACED \\
\hline R124 & POLISH, NON-BEARDED SILVER & R125 & REDCAP & R126 & JAPANESE, WHITE TAIL BLACK \\
\hline R130 & NEW HAMPSHIRE, WHITE & R134 & MOZISECK SHOW BROILER & $\mathrm{R} 136$ & JAPANESE. BLACK TAIL WHITE \\
\hline R139 & COCHIN, BLUE & R140 & COCHIN, BARRED & R145 & PHOENIX, LONG TAIL \\
\hline R146 & PLYMOUTH ROCK, BARRED & R147 & LEGHORN, DANISH BROWN & R148 & STEELE SHOW BIRDS \\
\hline R151 & COLUMBIA & R153 & RUMPLESS & R158 & FRIZZLE, BUFF \\
\hline R160 & COCHIN. FRIZZLE BUFF & R165 & IDEAL SHOW BIRD & R166 & SPITZ HAUBEN \\
\hline R168 & $\begin{array}{l}\text { LEGHORN, SINGLE COMB LIGHT } \\
\text { BROWN }\end{array}$ & R169 & $\begin{array}{l}\text { LEGHORN, SINGLE COMB DARK } \\
\text { BROWN }\end{array}$ & R170 & SAMATRAS WHITE \\
\hline R171 & SPITZ HAUBEN, GERMAN SILVER & R172 & $\begin{array}{l}\text { ARAUCANA, TUFFED RUMPLESS } \\
\text { PEA COMB }\end{array}$ & R173 & NEW HAMPSHIRE, BLACK \\
\hline R175 & $\begin{array}{l}\text { LEGHORN, ROSE COMB DARK } \\
\text { BROWN }\end{array}$ & R176 & ANCONA, ROSE COMB & R177 & POLISH, BEARDED BUFF \\
\hline R178 & POLISH, BEARDED GOL.DEN & R179 & $\begin{array}{l}\text { POLISH, BEARDED WHITE CREST } \\
\text { BLACK }\end{array}$ & R180 & POLISH, BEARDED WHITE \\
\hline R181 & HOUDAN, WHITE & R182 & $\begin{array}{l}\text { POLISH. BEARDED WHITE } \\
\text { CRESTED BLUE } \\
216\end{array}$ & R183 & RHODE ISLAND RED, ROSE COMB \\
\hline
\end{tabular}




\section{R OTHER LARGE BREEDS \& VARIETIES (Continued)}

\begin{tabular}{|c|c|c|c|c|c|}
\hline R185 & CHANTECLERS, PARTRIDGE & R186 & CHANTECLERS, WHITE & R187 & LEGHORN, ROSE COMB BLACK \\
\hline R188 & MINORCA. ROSE COMB BLACK & R192 & $\begin{array}{l}\text { CUBALAYAS, BLACK BREASTED } \\
\text { RED }\end{array}$ & R195 & BUCKEYE \\
\hline R196 & AUSTRALORP, BLACK & R199 & ORLOFF, RUSSIAN & R200 & LEGHORN, ROSE COMB BUFF \\
\hline R201 & COCHIN, SPLASH & $\mathrm{R} 202$ & SUMATRAS, WHITE & R203 & PHOENIX, WHITE \\
\hline R204 & BRAHMA, BLACK & R206 & PLYMOUTH ROCK, BLUE & R208 & JERSEY GIANT, BLUE \\
\hline R209 & CALIFORNIA WHITE & $R 210$ & CALIFORNIA GRAY & R211 & FAVEROLLE, WHITE \\
\hline $\mathrm{R} 213$ & MARANS & $\mathrm{R} 214$ & PHOENIX, BLACK BREASTED RED & R219 & MALAY, BLACK BREASTED RED \\
\hline R220 & SAIPANS & $\mathrm{R} 222$ & AMERAUCANA & R223 & LANGSHAN, BLUE \\
\hline R224 & VORWERK & $\mathrm{R} 225$ & WELSUMMERS & R227 & NEW HAMPSHIRE, RED \\
\hline R228 & TRIPLE PRODUCTION RED & R229 & MIXED & $\mathrm{R} 230$ & LEGHORN, SINGLE COMB BARRED \\
\hline R231 & LEGHORN, DAVID'S AUTOMATIC & R232 & LANGSHAN & R233 & SEX LINK \\
\hline R234 & LEGHORN, SILVERUDD & R236 & LEGBAR & R237 & LEGHORN, SINGLE COMB WHITE \\
\hline R238 & PRODUCTION BLACK & R239 & SEX LINK, BLACK & R240 & SEX LINK, GOLD \\
\hline R241 & BROILER CROSS, BLACK & R242 & BROILER CROSS, RED & R243 & GOLDEN COMET EX. \\
\hline R244 & SHAMO, BLACK & R245 & $\begin{array}{l}\text { POLISH, NON-BEARDED WHITE } \\
\text { CRESTED BLACK }\end{array}$ & R246 & $\begin{array}{l}\text { POLISH, NON-BEARDED WHITE } \\
\text { CRESTED BLUE }\end{array}$ \\
\hline R247 & $\begin{array}{l}\text { RHODE ISLAND RED, SINGLE } \\
\text { COMB }\end{array}$ & $\mathrm{R} 248$ & PLYMOUTH ROCK, WHITE & $\mathrm{R} 249$ & $\begin{array}{l}\text { OLD ENGLISH GAME، BLACK } \\
\text { BREASTED RED }\end{array}$ \\
\hline R250 & OLD ENGLISH GAME, BROWN RED & R251 & $\begin{array}{l}\text { OLD ENGLISH GAME, GOLDEN } \\
\text { DUCKWING }\end{array}$ & R253 & $\begin{array}{l}\text { OLD ENGLISH GAME, SILVER } \\
\text { DUCKWING }\end{array}$ \\
\hline R254 & OLD ENGLISH GAME. SPANGLED & R255 & OLD ENGLISH GAME, WHEATEN & R256 & PRODUCTION RED \\
\hline R257 & JAVA, MOTTLED & $\mathrm{R} 258$ & CATALANA, BUFF & R259 & JUNGLEFOWL, GREY \\
\hline$R 260$ & AMERAUCANA, BLACK & R261 & AMERAUCANA, BLUE & R262 & AMERAUCANA, WHITE \\
\hline R263 & $\begin{array}{l}\text { NECKED NECK, BLACK TAILED } \\
\text { RED }\end{array}$ & R264 & SULTAN, BLACK & R265 & SULTAN, BLUE \\
\hline R268 & LA FLECHE, BLACK & R269 & LA FLECHE, BLUE & R270 & KRAIENKOPPE \\
\hline R271 & NORWEGIAN JAERS & $\mathrm{R} 272$ & BARNEVELDERS & & \\
\hline
\end{tabular}

W - BANTAMS

$\begin{array}{llllll}\text { W1 } & \text { JAPANESE } & \text { W2 } & \text { MIXED } & \text { W3 } & \text { PLYMOUTH ROCK, BARRED } \\ \text { W4 } & \text { PLYMOUTH ROCK, WHITE } & \text { W5 } & \text { PLYMOUTH ROCK, PARTRIDGE } & \text { W6 } & \text { LEGHORN, SINGLE COMB WHITE } \\ \text { W7 } & \begin{array}{l}\text { RHODE ISLAND RED, SINGLE } \\ \text { COMB }\end{array} & \text { W8 } & \text { RHODE ISLAND RED, ROSE COMB } & \text { W9 } & \text { BRAHMA, DARK } \\ \text { W10 } & \text { BRAHMA, RED } & \text { W11 } & \text { BRAHMA, LIGHT } & \text { W12 } & \text { BRAHMA, BUFF } \\ \text { W13 } & \text { SILKIE } & \text { W14 } & \text { LANGSHAN, WHITE } & \text { W15 } & \text { LANGSHAN, BLACK } \\ \text { W16 } & \text { ORPINGTON, BUFF } & \text { W17 } & \text { COCHIN, BLACK } & \text { W18 } & \text { COCHIN, WHITE } \\ \text { W19 } & \text { CORNISH, WHITE LACED RED } & \text { W20 } & \text { CORNISH, DARK } & \text { W21 } & \text { ROSECOMB, BIRCHEN } \\ \text { W22 } & \text { ROSECOMB, WHITE } & \text { W23 } & \text { WYANDOTTE, PARTRIDGE } & \text { W24 } & \text { PLYMOUTH ROCK, COLUMBIAN } \\ \text { W25 } & \text { SEBRIGHT SILVER } & \text { W26 } & \text { SEBRIGHT, GOLDEN } & \text { W27 } & \text { WYANDOTTE, BUFF }\end{array}$


W BANTAMS (Continued)

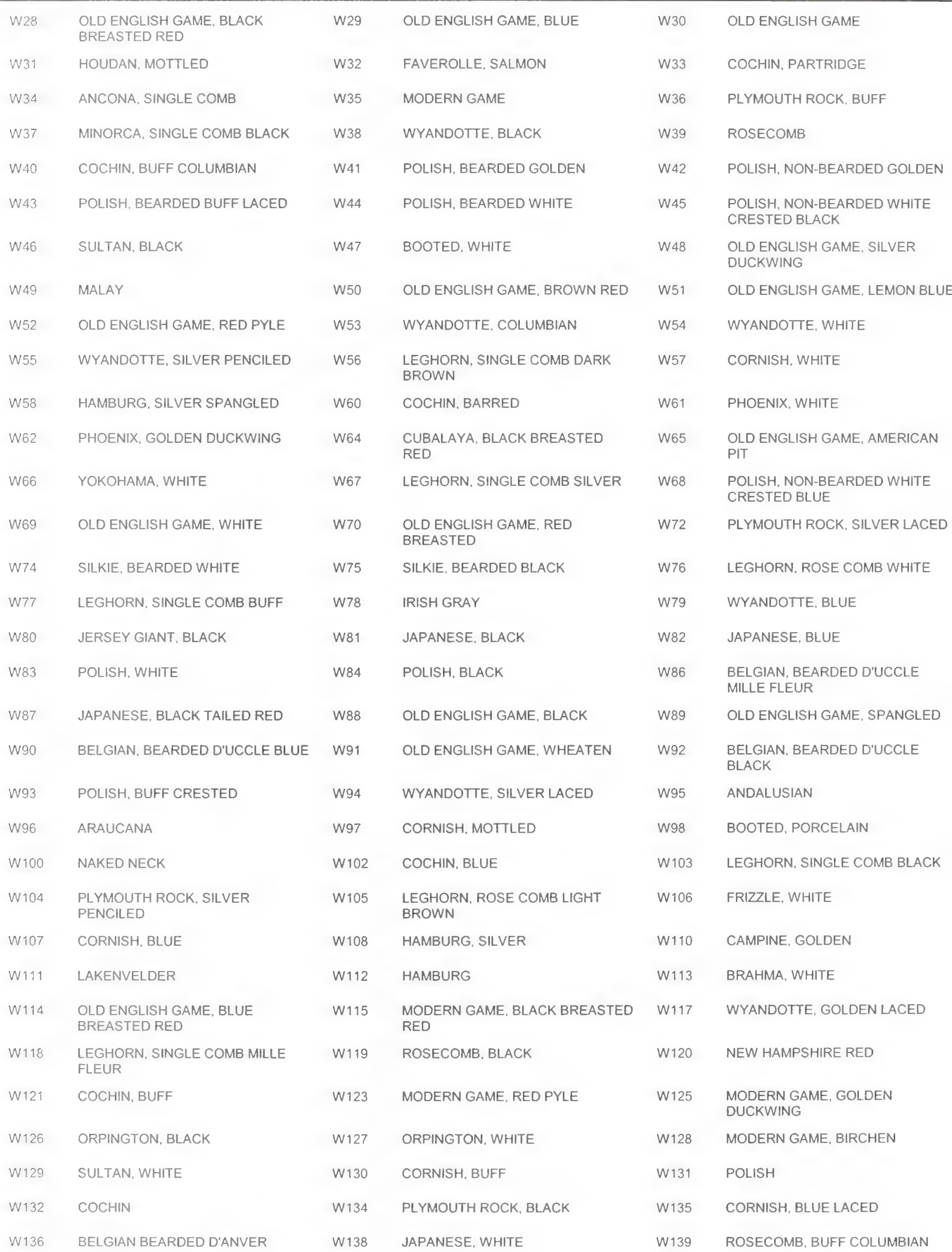


W BANTAMS (Continued)

\begin{tabular}{|c|c|c|c|c|c|}
\hline W140 & COCHIN, RED & W141 & OLD ENGLISH GAME, BARRED & W142 & OLD ENGLISH GAME, CRELE \\
\hline W144 & MALAY, BLACK BREASTED RED & W145 & JAPANESE, MOTTLED & W146 & FRIZZLE, BLACK \\
\hline W149 & OLD ENGLISH GAME. SELF BLUE & W150 & $\begin{array}{l}\text { OLD ENGLISH GAME, GOLDEN } \\
\text { OUCKWING }\end{array}$ & W151 & OLD ENGLISH GAME, BLUE RED \\
\hline W152 & POLISH, BEARDED SILVER & W153 & HAMBURG, GOLDEN PENCILED & W155 & LA FLECHE \\
\hline W159 & ORPINGTON, BLUE & W160 & ROSECOMB, BLUE & W162 & POLISH, NON-BEARDED SILVER \\
\hline W164 & COCHIN, MOTTLED & W165 & CORNISH, BLUE LACED RED & W166 & SUMATRA BLACK \\
\hline W167 & COCHIN, BROWN & W168 & COCHIN, SILVER LACED & W169 & COCHIN, GOLDEN LACED \\
\hline W170 & COCHIN, BROWN RED & W173 & $\begin{array}{l}\text { FRIZZLE, WHITE FEATHER } \\
\text { LEGGED }\end{array}$ & W174 & MODERN GAME, WHITE \\
\hline W175 & MODERN GAME, LEMON BLUE & W176 & JAPANESE, GRAY & W177 & JAPANESE, BLUE TAILED WHITE \\
\hline W179 & FRIZZLE, RED FEATHER LEGGED & W180 & FRIZZLE, BLUE FEATHER LEGGED & W182 & CORNISH, RED LACED \\
\hline W184 & SILKIE, NON-BEARDED PARTRIDGE & W187 & MODERN GAME, BROWN RED & W188 & MODERN GAME, WHEATEN \\
\hline W189 & JUNGLEFOWL & W190 & DELAWARE & W192 & MAX CROSS \\
\hline W193 & $\begin{array}{l}\text { BELGIAN, BEARDED D'UCCLE } \\
\text { MOTTLED }\end{array}$ & W198 & JAPANESE, BLACK TAILED WHITE & W200 & OLD ENGLISH GAME, BIRCHEN \\
\hline W206 & COCHIN, COLUMBIAN & W207 & CORNISH & W208 & SILKIE, NON-BEARDED WHITE \\
\hline W209 & $\begin{array}{l}\text { FRIZZLE, BLACK FEATHER } \\
\text { LEGGED }\end{array}$ & W211 & JAPANESE, BLACK TAILED BUFF & W213 & COCHIN, DARK \\
\hline W215 & SILKIE, NON-8EARDED BUFF & W216 & WYANDOTTE, BARRED & W219 & CORNISH, ROCK \\
\hline W220 & ROSECOMB, SPLASH & W221 & MODERN GAME, BARRED & W223 & COCHIN, BIRCHEN \\
\hline W224 & JAPANESE, BROWN RED & W230 & BOOTED, MOTTLED & W231 & $\begin{array}{l}\text { BELGIAN, BEARDED D'ANVER } \\
\text { WHITE }\end{array}$ \\
\hline W232 & $\begin{array}{l}\text { BELGIAN, BEARDED D'UCCLE } \\
\text { WHITE }\end{array}$ & W241 & JAPANESE, BARRED & W242 & FRIZZLE, WHITE COCHIN \\
\hline W243 & FRIZZLE, BUFF COCHIN & W244 & FRIZZLE, BLACK COCHIN & W245 & $\begin{array}{l}\text { MODERN GAME, SILVER } \\
\text { DUCKWING }\end{array}$ \\
\hline W247 & SILKIE, BEARDED PARTRIDGE & W248 & SILKIE, BEARDED BUFF & W249 & FRIZZLE, RED \\
\hline W250 & SILKIE, JAPANESE & W251 & $\begin{array}{l}\text { FRIZZLE, PARTRIDGE FEATHER } \\
\text { LEGGED }\end{array}$ & W252 & RUSSIAN RED \\
\hline W253 & FRIZZLE, BUFF FEATHER LEGGED & W255 & LEGHORN, SINGLE COMB RED & W256 & $\begin{array}{l}\text { SILKIE, NON-BEARDED WHITE } \\
\text { JAPANESE }\end{array}$ \\
\hline W257 & $\begin{array}{l}\text { SILKIE, NON-BEARDED BLACK } \\
\text { JAPANESE }\end{array}$ & W259 & COCHIN, SILVER PENCILED & W261 & FRIZZLE, WYANDOTTE \\
\hline W262 & OLD ENGLISH GAME, GINGER RED & W263 & OLD ENGLISH GAME, CUCKOO & W265 & COCHIN, SPLASH \\
\hline W270 & $\begin{array}{l}\text { BELGIAN, BEARDED D'UCCLE } \\
\text { PORCELAIN }\end{array}$ & W271 & $\begin{array}{l}\text { BELGIAN, BEARDED D'UCCLE } \\
\text { GOLD NECK }\end{array}$ & W274 & $\begin{array}{l}\text { OLD ENGLISH GAME, BLACK } \\
\text { TAILED BUFF }\end{array}$ \\
\hline W275 & OLD ENGLISH GAME, SILVER BLUE & W276 & OLD ENGLISH GAME, SPLASH & W278 & $\begin{array}{l}\text { BELGIAN, BEARDED D'ANVER } \\
\text { BLUE }\end{array}$ \\
\hline W281 & $\begin{array}{l}\text { LEGHORN, ROSE COMB DARK } \\
\text { BROWN }\end{array}$ & W282 & $\begin{array}{l}\text { POLISH, NON-BEARDED BUFF } \\
\text { LACED }\end{array}$ & W283 & REDCAP \\
\hline W286 & JAPANESE, SELF BLUE & W289 & LEGHORN, BARRED SILVER GOLD & W290 & SILKIE, NON-BEARDED BLACK \\
\hline W291 & SILKIE, SILVER & W293 & MODERN GAME, BLACK & W294 & MODERN GAME, SILVER BLUE \\
\hline W295 & MODERN GAME, SPLASH & W297 & $\begin{array}{l}\text { BELGIAN, BEARDED D'UCCLE SELF } \\
\text { BLUE }\end{array}$ & W299 & $\begin{array}{l}\text { LEGHORN, SINGLE COMB } \\
\text { COLUMBIAN }\end{array}$ \\
\hline W300 & LEGHORN, BUFF COLUMBIAN & W301 & $\begin{array}{l}\text { BELGIAN, BEARDED D'ANVER } \\
\text { QUAIL }\end{array}$ & W302 & SPANISH, WHITE FACED BLACK \\
\hline
\end{tabular}




\section{W BANTAMS (Continued)}

\begin{tabular}{|c|c|c|c|c|c|}
\hline W303 & POLISH, NON-BEARDED WHITE & W304 & BUTTERCUP & W305 & $\begin{array}{l}\text { DORKING. SINGLE COMB SILVER } \\
\text { GRAY }\end{array}$ \\
\hline W306 & CORNISH. BLACK & W307 & ROSECOMB, BROWN RED & W308 & $\begin{array}{l}\text { OLD ENGLISH GAME, BLACK } \\
\text { TAILED RED }\end{array}$ \\
\hline W309 & SILKIE. NON-BEARDED GRAY & W313 & SILKIE, BEARDED GRAY & W314 & FRIZZLE, MOTTLED \\
\hline W316 & AMERICAN GAME & W319 & $\begin{array}{l}\text { AMERICAN GAME, BLACK } \\
\text { BREASTED RED }\end{array}$ & W320 & AMERICAN GAME, BLUE \\
\hline W322 & AMERICAN GAME, BROWN RED & W323 & $\begin{array}{l}\text { AMERICAN GAME, GOLDEN } \\
\text { DUCKWING }\end{array}$ & W326 & $\begin{array}{l}\text { AMERICAN GAME, SILVER } \\
\text { DUCKWING }\end{array}$ \\
\hline W327 & AMERICAN GAME, WHEATEN & W328 & AMERICAN GAME, WHITE & W330 & $\begin{array}{l}\text { ARAUCANA, BLACK PLAIN } \\
\text { RUMPLESS }\end{array}$ \\
\hline W331 & $\begin{array}{l}\text { ARAUCANA, BLACK TUFTED } \\
\text { RUMPLESS }\end{array}$ & W332 & ARAUCANA, BLACK PLAIN TUFTED & W333 & $\begin{array}{l}\text { ARAUCANA, BLACK TUFTED } \\
\text { TAILED }\end{array}$ \\
\hline W334 & $\begin{array}{l}\text { ARAUCANA, SILVER PLAIN } \\
\text { RUMPLESS }\end{array}$ & W335 & $\begin{array}{l}\text { ARAUCANA, SILVER TUFTED } \\
\text { RUMPLESS }\end{array}$ & W337 & ARAUCANA, SILVER TUFTED TAIL \\
\hline W338 & $\begin{array}{l}\text { ARAUCANA. WHITE TUFTED } \\
\text { RUMPLESS }\end{array}$ & W346 & $\begin{array}{l}\text { ARAUCANA, DARK BROWN PLAIN } \\
\text { RUMPLESS }\end{array}$ & W348 & ASEEL, BLACK BREASTED RED \\
\hline W351 & ASEEL, WHITE & W352 & AUSTRALORP & W353 & AUSTRALORP, BLACK \\
\hline W357 & $\begin{array}{l}\text { BELGIAN, BEARDED D'ANVER } \\
\text { BLACK }\end{array}$ & W358 & $\begin{array}{l}\text { BELGIAN, BEARDED D' ANVER } \\
\text { BLACK BREASTD }\end{array}$ & W359 & $\begin{array}{l}\text { BELGIAN, BEARDED D'ANVER } \\
\text { BUFF }\end{array}$ \\
\hline W360 & $\begin{array}{l}\text { BELGIAN, BEARDED D'ANVER } \\
\text { CUCKOO }\end{array}$ & W361 & $\begin{array}{l}\text { BELGIAN, BEARDED D'ANVER } \\
\text { MILLE FLEUR }\end{array}$ & W363 & $\begin{array}{l}\text { BELGIAN, BEARDED D'ANVER } \\
\text { PORCELAIN }\end{array}$ \\
\hline W364 & $\begin{array}{l}\text { BELGIAN, BEARDED D'ANVER SELF } \\
\text { BLUE }\end{array}$ & W365 & BELGIAN, BEARDED D'UCCLE & W367 & BOOTED, BUFF \\
\hline W368 & BOOTED, MILLE FLEUR & W370 & BRAHMA & W372 & BUCKEYE \\
\hline W373 & BUCKEYE, RED & W380 & CORNISH, SPANGLED & W386 & CREVECOEUR, BLACK \\
\hline W387 & DOMINIQUE & W390 & DORKING, ROSECOMB WHITE & W393 & DUTCH \\
\hline W394 & DUTCH, BLACK & W395 & DUTCH, BLACK BREASTED RED & W396 & DUTCH, BLUE \\
\hline W397 & DUTCH, BLUE RED & W401 & DUTCH, SILVER & W415 & FRIZZLE \\
\hline W416 & FRIZZLE, BLUE & W422 & HAMBURG, BLACK & W424 & HOUDAN \\
\hline W427 & JAPANESE, BUFF & W435 & JUNGLEFOWL, RED & W439 & LA FLECHE, BLACK \\
\hline W440 & LANGSHAN & W442 & LEGHORN & W453 & MODERN GAME, BLUE \\
\hline W454 & MODERN GAME, BLUE RED & W455 & MODERN GAME, CRELE & W456 & MODERN GAME, GINGER RED \\
\hline W457 & MODERN GAME, SELF BLUE & W466 & NAKED NECK, SINGLE COMB BUFF & W468 & NAKED NECK, SINGLE COMB RED \\
\hline W469 & $\begin{array}{l}\text { NAKED NECK, SINGLE COMB } \\
\text { WHITE }\end{array}$ & W470 & NANKIN ROSE COMB & W473 & ORLOFF, SPANGLED \\
\hline W475 & PHOENIX, GOLDEN & W477 & PHOENIX, SILVER & W478 & PLYMOUTH ROCK, BLUE \\
\hline W479 & POLISH. BEARDED BLUE & W480 & POLISH. NON-BEARDED BLUE & W481 & PYCHEON, MILLE FLEUR \\
\hline W484 & $\begin{array}{l}\text { ROSECOMB, BLACK BREASTED } \\
\text { RED }\end{array}$ & W486 & ROSECOMB, BLUE RED & W492 & ROSECOMB, MOTTLED \\
\hline W494 & ROSECOMB, RED & W501 & $\begin{array}{l}\text { RUMPLESS, BLACK BREASTED } \\
\text { RED }\end{array}$ & W504 & SILKIE, RED-BEARDED \\
\hline W507 & RUMPLESS, GOLDEN DUCKWING & W508 & RUMPLESS, RED & W509 & RUMPLESS, SILVER DUCKWING \\
\hline W510 & RUMPLESS, WHITE & W511 & SHAMO, BLACK BREASTED RED & W516 & SHAMO, WHEATEN \\
\hline W518 & SILKIE, BEARDED BLUE & W519 & SILKIE, NON-BEARDED BLUE & W526 & SUSSEX, RED \\
\hline W527 & SUSSEX, SPECKLED & W531 & WYANDOTTE, BIRCHEN & W535 & WYANDOTTE, BUFF COLUMBIAN \\
\hline
\end{tabular}


W BANTAMS (Continued)

\begin{tabular}{|c|c|c|c|c|c|}
\hline W537 & WYANDOTTE, SPLASH & W538 & WYANDOTTE, WHITE LACED RED & W542 & OLD ENGLISH GAME, COLUMBIAN \\
\hline W554 & COCHIN, FRIZZLE & W555 & SILKIE, POLISH & W556 & $\begin{array}{l}\text { OLD ENGLISH GAME, WHEATON } \\
\text { BLUE }\end{array}$ \\
\hline W566 & OLD ENGLISH GAME. 713 & W567 & WELSUMMER, BROWN & W568 & $\begin{array}{l}\text { OLD ENGLISH GAME, BRASSY } \\
\text { BACK }\end{array}$ \\
\hline W569 & OLD ENGLISH GAME, QUAIL & W570 & MILLE FLEUR & W573 & AMERAUCANA \\
\hline W574 & SPITSHAUBEN & W576 & $\begin{array}{l}\text { LEGHORN, SINGLE COMB LIGHT } \\
\text { BROWN }\end{array}$ & W577 & DUTCH, QUILL \\
\hline W590 & $\begin{array}{l}\text { PORCELAIN, MILLE FLEUR, NON } \\
\text { BEARDED }\end{array}$ & W593 & PHOENIX & W594 & WYANDOTTE \\
\hline W595 & PORCELAN & W596 & $\begin{array}{l}\text { OLD ENGLISH GAME, BRASSY } \\
\text { BACK, BLUE }\end{array}$ & W599 & $\begin{array}{l}\text { OLD ENGLISH GAME, BLUE } \\
\text { GOLDEN DUCKWING }\end{array}$ \\
\hline W600 & BELGIAN ANTWERP, QUAIL & W603 & JAPANESE, BARRED FRIZZLE & W604 & SILKIE, RED PYLE \\
\hline W606 & DUTCH, CREAM LIGHT BROWN & W607 & DUTCH, BLUE LIGHT BROWN & W609 & ORPINGTON \\
\hline W610 & PLYMOUTH ROCK & W613 & $\begin{array}{l}\text { OLD ENGLISH GAME, FAWN } \\
\text { DUCKWING }\end{array}$ & W615 & SILKIE, BEARDED SPLASH \\
\hline W616 & $\begin{array}{l}\text { OLD ENGLISH GAME, WHITE- } \\
\text { TAILED BUFF }\end{array}$ & W617 & OLD ENGLISH, BUFF & W619 & WYANDOTTE, DUN LACED \\
\hline W638 & LA FLECHE, BLUE MOTTLED & W639 & LA FLECHE, MOTTLED & W640 & LA FLECHE, SPLASH \\
\hline W641 & LA FLECHE, WHITE & W642 & OLD ENGLISH GAME, MILLE FLEUR & W643 & OLD ENGLISH GAME, PEARL \\
\hline W644 & OLD ENGLISH GAME, PORCELAIN & W645 & OLD ENGLISH GAME, SILVER DUN & W646 & OLD ENGLISH GAME MOTTLED \\
\hline
\end{tabular}

W647 POLISH, BLACK WHITE CRESTED

x - DUCKS

\begin{tabular}{|c|c|c|c|c|c|}
\hline $\mathrm{x}_{1}$ & BUFF & $\times 2$ & ROUEN & $\times 3$ & PEKIN, WHITE \\
\hline$\times 4$ & MALLARD & $\times 5$ & INDIAN RUNNER & $x_{6}$ & CAMPBELL, KHAKI \\
\hline$x 7$ & REDHEAD & $x 8$ & WOOD, CAROLINA & X9 & EAST INDIA, BLACK \\
\hline$\times 10$ & CALL, GRAY & $\mathrm{X} 11$ & CRESTED, WHITE & $x_{12}$ & MUSCOVY, BLUE \\
\hline$\times 13$ & SHEL, DUCK EUROPEAN & $\mathrm{X} 14$ & CAYUGA & $\times 15$ & AYLESBURY \\
\hline $\mathrm{x} 16$ & EAST INDIA & $\mathrm{X} 17$ & CALL, WHITE & $\times 18$ & MUSCOVY, BLACK \\
\hline$x_{20}$ & MUSCOVY & $\times 21$ & MUSCOVY, WHITE & $\times 22$ & INDIAN RUNNER, FAWN \\
\hline
\end{tabular}


DUCKS (Continued)

\begin{tabular}{|c|c|c|c|c|c|}
\hline$\times 23$ & INDIAN RUNNER, WHITE & $\times 24$ & MUSCOVY, COLORED & $\times 25$ & SWEDISH, BLUE \\
\hline$\times 26$ & INDIAN RUNNER, PENCILED & $\times 27$ & $\begin{array}{l}\text { INDIAN RUNNER, FAWN AND } \\
\text { WHITE }\end{array}$ & $\times 28$ & MANDARIN \\
\hline$\times 29$ & MALLARD, WHITE ALBINO & $\times 30$ & $\begin{array}{l}\text { NORTH AMERICAN MIGRATORY } \\
\text { WATERFOWL }\end{array}$ & $\times 31$ & MAGPIE \\
\hline$\times 32$ & MUSCOVY, CHOCOLATE & $\times 33$ & $\begin{array}{l}\text { INDIAN RUNNER, CUMBERLAND } \\
\text { BLUE }\end{array}$ & $\times 34$ & INDIAN RUNNER, BUFF \\
\hline$\times 35$ & INDIAN RUNNER, CHOCOLATE & $\times 37$ & CRESTED, BLACK & $\times 38$ & CALL, BLUE \\
\hline$\times 40$ & TEAL, RING-NECKED & $x 41$ & CALL, SILVER & $\times 42$ & TREE, WHITE FACE \\
\hline$x 43$ & CAMPBELL, WHITE & $\mathrm{X} 44$ & CAMPBELL, ENGLISH WHITE & $\times 45$ & CRESTED, BUFF \\
\hline$x 46$ & MALLARD, GRAY & $\mathrm{X} 47$ & CALL, MUTATION & $x 48$ & BLACK \\
\hline$\times 49$ & WIGEON, AMERICAN & $x 50$ & PINTAIL, AMERICAN & $\times 51$ & POCHARD, RED CRESTED \\
\hline$\times 52$ & TEAL, CINNAMON & $\times 53$ & TEAL, BLUE-WING & $\times 54$ & TEAL, EUROPEAN GREEN-WING \\
\hline$\times 55$ & TEAL, BRAZILIAN & $\times 57$ & MERGANSER HOODED & $\times 58$ & RUDDY \\
\hline$\times 59$ & TREE, FULVUS & $x 60$ & CANVASBACK & $x 61$ & SHOVELER, COMMON \\
\hline$x 62$ & GADWALL & $x 63$ & CAMPBELL, DARK & $x 64$ & CRESTED, BLUE \\
\hline$\times 65$ & CALL, BLACK & $\times 67$ & MUSCOVY, PASTEL & $x 68$ & MUSCOVY. BUFF \\
\hline$x 70$ & WIGEON, CHILOE & $x 71$ & PINTAIL, RED-BILLED & $X 72$ & RINGBILLS \\
\hline$x 73$ & SCAUP, LESSER & $X 74$ & SCAUP, GREATER & $\times 75$ & TUFTED \\
\hline$x 76$ & WIGEON, EUROPEAN & $x 77$ & TEAL. FALCATED & $x 78$ & GOLDENEYE \\
\hline$x 79$ & GOLDENEYE, BARROWS & $\times 81$ & SCOTER, WHITE WINGED & $\times 85$ & PINTAIL \\
\hline$\times 88$ & EIDER & $\times 89$ & RINGNECK & $x 93$ & MERGANSER \\
\hline X94 & TREE & $\times 95$ & CAMPBELL, PIED & X96 & INDIAN RUNNER, BLACK \\
\hline$\times 97$ & INDIAN RUNNER, GRAY & $x 98$ & HARLEQUIN, WELSH & $x 99$ & SILVER APPLEYARD \\
\hline$\times 100$ & AMERICAN SAXONY & $\times 101$ & ANCONA & $\times 102$ & GOLDEN CASCADE \\
\hline$\times 103$ & CAYUGA, BLUE & $\times 104$ & INDIAN RUNNER, SAXONY & $\times 105$ & INDIAN RUNNER, GOLDEN \\
\hline$\times 106$ & INDIAN RUNNER, EMERY & $\times 107$ & INDIAN RUNNER, CINNAMON & $x 108$ & MALLARD, GOLDEN \\
\hline$\times 109$ & TEAL, BAIKAL & $\times 110$ & TEAL, CHESTNUT-BREASTED & $\mathrm{X} 111$ & TEAL, HOTTENTOT \\
\hline$\times 112$ & TEAL, NORTHERN SILVER & $\times 113$ & TEAL, CAPE & $\mathrm{X} 114$ & TEAL, MARBLED \\
\hline X115 & GARGANEY & $\mathrm{x} 116$ & TEAL, VERSICOLOR & $\mathrm{X} 117$ & TEAL, LAYSAN \\
\hline X118 & TEAL, CHESTNUT & $\mathrm{X} 120$ & PINTAIL, CHILEAN & $X 121$ & PINTAIL, BAHAMA \\
\hline $\mathrm{x} 122$ & PINTAIL, SILVER BAHAMA & $\mathrm{X} 123$ & PINTAIL, LESSER BAHAMA & $\times 124$ & TREE, BLACK-BELLIED \\
\hline$\times 125$ & FERRUGINOUS WHITE EYES & $\times 126$ & SCAUP, NEW ZEALAND & $\times 127$ & POCHARD, ROSYBILL \\
\hline$\times 130$ & FULVOUS & $\mathrm{X} 131$ & SWEDISH, BLACK & $\times 133$ & BUFFLEHEAD \\
\hline$\times 134$ & SNOWY & $\times 135$ & CAYUGA, BLACK & $\times 136$ & MAGPIE, BLACK \& WHITE \\
\hline$\times 137$ & MAGPIE, BLUE \& WHITE & $\times 140$ & MALLARD, BLACK & $\times 141$ & SHEL, DUCK PARADISE \\
\hline$\times 142$ & SHEL, DUCK COMMON & $x+143$ & CALL, SNOWY & $\times 144$ & TEAL, CHILEAN \\
\hline$\times 145$ & CALL. BUFF & $\mathrm{X} 146$ & CALL & $\times 147$ & CALL, PASTEL \\
\hline
\end{tabular}


Z - OTHERBIRDS (Continued)

\begin{tabular}{|c|c|c|c|c|c|}
\hline $\mathrm{zi}$ & CHUKAR & $\mathrm{Z2}$ & PHEASANT & $\mathrm{Z3}$ & QUAIL \\
\hline Z4 & GUINEA & Z6 & FRANCOLIN & $\mathrm{Z7}$ & SWAN \\
\hline 28 & PRAIRIE CHICKEN & 29 & TURKEY, WILD & $\mathrm{Z} 11$ & PEAFOWL \\
\hline Z12 & PARTRIDGE, RED LEGGED & $\mathrm{Z14}$ & PEACOCK & Z15 & PEAFOWL, BLUE INDIA \\
\hline $\mathrm{Z} 18$ & PEAFOWL, BLUE & Z19 & PEAFOWL, WHITE & $\mathrm{Z} 20$ & GUINEA, LAVENDER \\
\hline $\mathrm{Z21}$ & GUINEA, BUFF DUN DOTTE & $\mathrm{Z22}$ & GUINEA, PEARL & $Z 23$ & TINAMOU \\
\hline 224 & PHEASANT, REEVES' & $\mathrm{Z} 25$ & PHEASANT, MUTANT & $\mathrm{Z} 26$ & PHEASANT, SILVER \\
\hline$Z 27$ & $\begin{array}{l}\text { PHEASANT, CHINESE RING- } \\
\text { NECKED }\end{array}$ & $\mathrm{Z} 28$ & PHEASANT, GOLDEN & $\mathrm{Z} 29$ & PARTRIDGE, INDIAN CHUKAR \\
\hline $\mathrm{Z30}$ & PARTRIDGE, BARBARY CHUKAR & 231 & $\begin{array}{l}\text { PHEASANT, MONGOLIAN RING- } \\
\text { NECKED }\end{array}$ & $\mathrm{Z} 32$ & PHEASANT, AMHERST WHITE \\
\hline Z33 & PHEASANT, CHINESE GREEN & $\mathrm{Z} 34$ & PHEASANT, SWINEHOE'S & Z38 & QUAIL, BRITISH RANGE \\
\hline 239 & QUAIL, ENGLISH WHITE & $\mathrm{Z} 40$ & QUAIL. MANCHURIAN GOLDEN & Z41 & QUAIL, PHARAOH \\
\hline Z42 & QUAIL, TUXEDO & $\mathrm{Z} 43$ & QUALL, BOBWHITE & $\mathrm{Z} 44$ & QUAIL, CALIFORNIA VALLEY \\
\hline Z45 & QUAIL. TENNESSEE RED & $\mathrm{Z} 48$ & PARTRIDGE, HUNGARIAN & $Z 49$ & PHEASANT, BLUE-EARRED \\
\hline 250 & PHEASANT, BROWN-EARRED & Z51 & $\begin{array}{l}\text { PHEASANT, DARK THROATED } \\
\text { GOLDEN }\end{array}$ & 252 & PHEASANT, ELLIOT'S \\
\hline 253 & $\begin{array}{l}\text { PHEASANT, GIGI'S YELLOW } \\
\text { GOLDEN }\end{array}$ & $\mathbf{Z 5 4}$ & PHEASANT, HUME'S BAR-TAILED & $z 55$ & PHEASANT, IMPERIAL \\
\hline 256 & $\begin{array}{l}\text { PHEASANT, KALIJ WHITE- } \\
\text { CRESTED }\end{array}$ & $\mathrm{Z} 57$ & PHEASANT, LADY AMHERST'S & 259 & QUAIL, CHESTNUT BELLY \\
\hline 260 & QUAIL, GAMBEL & $\mathrm{Z} 61$ & TURKIN & $\mathrm{Z} 62$ & GUINEA, WHITE \\
\hline $\mathrm{Z} 63$ & GUINEA, SIVER POLISHED & Z65 & GROUSE, RUFFED & Z66 & GROUSE, SHARPTAIL \\
\hline 267 & PHEASANT, MANCHURIAN & $\mathrm{Z} 68$ & PHEASANT, EDWARDS' & Z69 & PHEASANT, IJIMA \\
\hline 270 & PHEASANT, HUME & $\mathrm{Z71}$ & PHEASANT, MIKADO & $\mathrm{Z72}$ & QUAIL, BLUE SCALE \\
\hline $\mathrm{Z73}$ & QUAIL, TEXAS BLUE & 274 & QUAIL, MOUNTAIN & 275 & FRANCOLIN, ERCKEL'S \\
\hline 277 & PHEASANT, CHEER & 278 & QUAIL, BELGIUM & 279 & PHEASANT, BLACK-NECKED \\
\hline$Z 80$ & PHEASANT, WHITE-WINGED & $\mathbf{Z} 86$ & QUAIL, BOBWHITE TEXAS BLONDE & $Z 87$ & PHEASANT, GOLDEN SALMON \\
\hline 288 & QUAIL, NORTHERN BOBWHITE & Z89 & QUAIL, JUMBO WISCONSIN & Z90 & PHEASANT, CALIFORNIA BUFF \\
\hline 293 & QUAIL, COTURNIX & Z94 & PARTRIDGE & Z99 & PHEASANT, RED-LEGGED \\
\hline $\mathrm{Z100}$ & PHEASANT, BLACK & Z101 & $\begin{array}{l}\text { PEAFOWL, BLACK SHOULDER } \\
\text { PURPLE }\end{array}$ & Z102 & PHEASANT, YELLOW \\
\hline $\mathrm{Z104}$ & PHEASANT, MELARCTIC BLACK & $\mathrm{Z} 105$ & PHEASANT. MELARCTIC BRONZE & Z106 & GUINEA, SPECKLED \\
\hline Z107 & QUAIL, BUTTON & Z108 & PEAFOWL, BLACK SHOULDERED & $\mathrm{Z} 109$ & SWAN, MUTE \\
\hline 2110 & GUINEA, BLUE & $\mathrm{Z} 111$ & GUINEA, ROYAL PURPLE & $\mathrm{Z} 112$ & PHE,ASANT, MONGOLIAN \\
\hline 2118 & PHEASANT, GOLDEN RED & $\mathrm{Z} 119$ & SWAN, WHISTLING & $\mathrm{Z120}$ & QUAIL, AUSTRIAN FAWN \\
\hline Z121 & $\begin{array}{l}\text { PHEASANT, CHINESE RING- } \\
\text { NECKED, BLUE BACK }\end{array}$ & $\mathrm{Z} 122$ & SWAN. TRUMPETER & $\mathrm{Z123}$ & SWAN, BLACK \\
\hline Z124 & SWAN, WHOOPER & $\mathrm{Z} 126$ & PHEASANT, TRAGOPAN & $Z 127$ & QUAIL, JAPANESE \\
\hline Z128 & PEAFOWL, JAVANESE GREEN & $Z 129$ & PHEASANT, MELANISTIC MUTANT & $\mathrm{Z} 130$ & PEAFOWL, CAMEO PIED \\
\hline Z131 & PEAFOWL, OATEN & Z134 & GROUSE, SPRUCE & Z136 & PHEASANT, PIED \\
\hline
\end{tabular}




\section{$Z$ OTHER BIROS (Continued)}

2137 PEAFOWL, CAMEO

Z140 PEAFOWL, SPALOING

Z147 HELMET

Z159

Z169

Z173

2176

$\mathrm{Z} 179$

Z182

Z188

Z203

Z206

Z210

2213

Z216

Z219

$Z 227$

$\mathbf{Z 2 3 1}$

2235

2239

2244

2249

$\mathrm{Z} 252$

$\mathrm{Z} 255$

$\mathrm{Z} 258$

$\mathrm{Z} 262$

2266

$Z 270$

$\mathrm{Z} 283$

$\mathrm{Z} 286$

2289

Z295
PARTRIDGE, ANDREWS' CHUKAR

PHEASANT, SILVER, GOLDEN

QUAIL, BOBWHITE BUTLER GIANT

PHEASANT, WHITE JUMBO

PEAFOWL, BLACK SHOULDER PIED

TURKEY, EASTERN

PHEASANT, ALASKA SNOW

TURKEY, BELTSVILLE EXHIBITION

TURKEY, BLACK

TURKEY, EXHIBITION

TURKEY, NEBRASKA ROYAL BLUE

TURKEY, ARKANSAS RED

TURKEY, JERSEY BUFF

TURKEY, WHITE

TURKEY

QUAIL, BOBWHITE, CUNNINGHAM GIANT

GUINEA, FRENCH

PHEASANT, AFGHAN WHITE WING

GUINEA, BUFF

GUINEA, BROWN

GUINEA, PEWTER

TURKEY, WILD EASTON

SWAM, TUNDRA

PHEASANT, BLACK SHOULDER

PEAFOWL, PURPLE

PEAFOWL, INDIA BLUE

GUINEA, PIED

PEAFOWL, WHITE SHOULDERED

TURKEY, BARRED
PEAFOWL, BLUE PIED

QUAIL, MEXICAN SPECKLED

QUAIL, BOBWHITE, MASKED

SWAN, COSCOROBA

$\mathrm{Z} 170$

$\mathrm{Z174}$

2177

2180

Z183

Z201

Z204

Z207

Z211

Z214

Z217

$\mathrm{Z} 220$

Z229

Z233

Z236

Z242

Z247

Z250

Z253

Z256

2259

Z264

2268

$Z 274$

$Z 284$

Z287

$Z 290$
QUAIL, BOBWHITE, SOLID WHITE

PEAFOWL, PALAWAN

PHEASANT, CINNAMON

QUAIL, CHUCKAR

TURKEY, RIO GRANDE

TURKEY, WHITE HOLLAND

TURKEY, ROYAL PALM

TURKEY, SLATE

TURKEY, BLACK SPANISH

TURKEY, NARAGANSETT GOLDEN

TURKEY, WHITE BRONZE

TURKEY, LILAC

TURKEY, DARK AUBURN

PHEASANT, WHITE GOLD

PHEASANT, DILUTE

PHEASANT, BLUE MUTANT

PHEASANT, RINGNECK JUMBO

GUINEA, PORCELAIN

GUINEA, VIOLET

GUINEA, OPALINE

PHEASANT, WHITE RINGNECK

PHEASANT, BUFF

PHEASANT, SATYR

PEAFOWL, PURPLE PIED

SWAN, AUSTRALIAN BLACK

GUINEA, ROYAL BLUE

TURKEY, WILD WHITE
Z139

Z142

Z149

Z165

Z172

Z175

Z178

Z181

$Z 187$

$\mathrm{Z} 202$

$Z 205$

Z209

$\mathrm{Z} 212$

Z215

Z218

Z221

$Z 230$

Z234

$Z 237$

Z243

$\mathrm{Z} 248$

$\mathbf{Z} 251$

Z254

$\mathrm{Z} 257$

$\mathrm{Z} 260$

2265

Z269

$\mathrm{Z} 281$

Z285

Z288

$\mathbf{Z 2 9 3}$
PEACOCK, INDIA BLUE

QUAIL, VALLEY

PHEASANT, GREY PEACOCK

PARTRIDGE, BUFF CHUKAR

QUAIL, BOBWHITE INDIANA GIANT

PARTRIDGE, CHINESE BAMBOO

PHEASANT, FORMOSA

PHEASANT, GREEN

GROUSE, BLUE

TURKEY, BRONZE

TURKEY, BOURBON RED

TURKEY, NARAGANSETT

TURKEY, BROADWHITE LARGE EXHIBITION

TURKEY, KENT

TURKEY, SILVER

TURKEY, DOMESTIC MERRIAM

TURKEY, BLUE SLATE

PEAFOWL, BURMESE GREEN

PHEASANT, COPPER

PHEASANT, ORNAMENTAL

PHEASANT, HIMALAYAN MONAL GUINEA, SLATE

GUINEA, BRONZE

PHEASANT, BLACK MUTANT

PHEASANT, IMPEYAN

PHEASANT, ISABEL BUFF

PHEASANT, TIMNEK

PHEASANT, BLUE IDAHO

GUINEA, CORAL

PEAFOWL, BLUE SHOULDERED

CHUKAR, WHITE 
A - WHITE LEGHORN (NON-EXHIBITION)

\begin{tabular}{|c|c|c|c|c|c|}
\hline A1 & ARBOR ACRES & $A 2$ & BABCOCK & A6 & EURIBRID \\
\hline \multirow[t]{2}{*}{ A8 } & HAND N & A19 & HYLINE & A16 & WELP \\
\hline & & \multicolumn{2}{|l|}{ - RHODE ISLAND RED (NON-EXHIBITION) } & \\
\hline B1 & ARBOR ACRES & B3 & CORBETT & B7 & HARDY \\
\hline B15 & IDEAL & B10 & WELP & & \\
\hline & & & - BARRED PLYMOUTH ROCK (NON-EXHIBI & TION) & \\
\hline C1 & ARBOR ACRES & $\mathrm{C} 2$ & COLONIAL & C4 & DAVIS \\
\hline $\mathrm{C} 5$ & HARCO & C6 & HARDY & $\mathrm{C}_{12}$ & IDEAL \\
\hline $\mathrm{C} 10$ & WELP & & & & \\
\hline
\end{tabular}

D - WHITE PLYMOUTH ROCK (NON-EXHIBITION)

\begin{tabular}{|c|c|c|c|c|c|}
\hline D1 & ARBOR ACRES & D11 & CEBE, RECESSIVE & D4 & COLONIAL \\
\hline D5 & HARDY & D6 & HUBBARD & D12 & IDEAL \\
\hline D9 & WELP & & & & \\
\hline
\end{tabular}

E - NEW HAMPSHIRE (NON-EXHIBITION)

\begin{tabular}{|c|c|c|c|c|c|}
\hline E1 & COLONIAL & E2 & HUBBARD & E3 & ROWLEY \\
\hline & & $\mathrm{H}$ & - CORNISH (NON-EXHIBITION) & & \\
\hline $\mathrm{H} 1$ & ARBOR ACRES & $\mathrm{H} 9$ & BUFF & $\mathrm{H} 14$ & CEBE BLACK \\
\hline $\mathrm{H} 12$ & CEBE GOLD & $\mathrm{H} 11$ & CEBE RED & $\mathrm{H} 2$ & COBB \\
\hline H5 & DARK & $\mathrm{H} 3$ & HARMAN & $\mathrm{H} 6$ & RED \\
\hline \multirow[t]{2}{*}{$\mathrm{H} 10$} & WHITE & $\mathrm{H} 8$ & WHITE LACED RED & & \\
\hline & & N & - GAME CHICKEN & & \\
\hline N83 & ALBANY & N270 & ALBANY, RADIO & N104 & ASEEL \\
\hline N80 & ASEEL, DEVARAJA & N88 & ASEEL, KAHN & N89 & ASEEL, SONATOL \\
\hline N126 & BAMA GREY & N144 & BENOIST, SLIM BLUE & N145 & BENOIST, SLIM RED \\
\hline N36 & BIGHAM RED & N253 & BLACK, DENT COUNTY & N240 & BLUE FACE \\
\hline N112 & BLUE SPLASH & N90 & BROWN RED & N194 & BROWN RED, BOSTON \\
\hline N100 & BROWN RED, FREDRICK'S & $\mathrm{N} 113$ & BROWN RED, SHUFFLER & N87 & BROWN RED, WINGATE \\
\hline N159 & BUCKALOO & N269 & BURN'S WHITE & N63 & BUTCHER \\
\hline N219 & BUTCHER, BLACK & N216 & BUTCHER, RED & N222 & BUTCHER, SPANGLED \\
\hline N38 & CAPITOL HILL GRAY & N37 & CAPITOL HILL RED & N33 & CLARET \\
\hline N136 & CLARET, GRIFFIN & N224 & CLARET, MADIGIN & N198 & CLARET, WHITE \\
\hline N23 & CLIPPERS & N130 & COPPERHEADS & N110 & CUBAN \\
\hline N52 & DARK CORNISH & N84 & DEMOCRAT & N97 & DOMINIQUE \\
\hline
\end{tabular}




\begin{tabular}{|c|c|c|c|c|c|}
\hline N190 & GARRARD & N168 & GAVLIN & N167 & GILMORE \\
\hline N77 & GLEASON WHITE HACKOE & N81 & GOWDY JAP & N32 & GRAY \\
\hline N99 & GRAY, BUMBLEFOOT & N150 & GRAY, FINDLEY & N241 & GRAY, GOLDEN \\
\hline N156 & GRAY, IRISH & N171 & GRAY, JOE GOOD & N46 & GRAY, MADIGIN \\
\hline N139 & GRAY, SWEATER & N236 & GREY & N101 & GREY TOPPIES, JACK WILSON \\
\hline N175 & GREY, BULLOCK & N254 & GREY, DENT COUNTY & N197 & GREY, FROST \\
\hline N102 & GREY, MISSISSIPP & N239 & GREY, MUG & N247 & GREY, RICHARDSON \\
\hline N249 & GREY, SLAVIN & N193 & GREY, WAYNE WOOD & N85 & GRIFFIN \\
\hline N28 & HATCH & N263 & HATCH CLARET, NACHA & N189 & HATCH, BILOXI \\
\hline N157 & HATCH. BLACK & N65 & HATCH, BLUE FACE & N187 & HATCH, BLUE LEGGED \\
\hline N225 & HATCH, DEMOCRAT & N172 & HATCH, DOC ROBINSON & N243 & HATCH, GILMOORE \\
\hline N34 & HATCH, GRAY & N161 & HATCH, GREEN LEG & N138 & HATCH, HAROLD BROWN \\
\hline N155 & HATCH, KELSO & N131 & HATCH, LEPER & N43 & HATCH, MCLAIN \\
\hline N42 & HATCH, MURPHY & N195 & HATCH, OAK GROVE & N98 & HATCH, PERRY \\
\hline N162 & HATCH, ROUND HEAD & N137 & HATCH, RUBLE & N226 & HATCH, SANDY \\
\hline N252 & HATCH, SLAVIN BLACK & N146 & HATCH, SPANGLED BRASS BACK & N142 & HATCH, SPECKLED \\
\hline N152 & HATCH, SWEATER & N264 & HATCH. WALTON & N121 & HATCH. YELLOW LEG \\
\hline N91 & HENNIES, BLACK & N111 & HENNY, SPANGLED & N107 & HUSLEY \\
\hline N57 & JUMPER GRAY & N56 & JUMPER RED & N44 & KELSO \\
\hline N141 & KELSO, BLUE LEG & N199 & KELSO, HORSESHOE & N227 & KELSO, OUT \& OUT \\
\hline N140 & KELSO, RADIO & N182 & KELSO, WHITE LEG & N181 & KELSO, YELLOW LEG \\
\hline N25 & LAW GRAYS & N132 & LAWSHE BLUE & N61 & LOWHMANN WHITE HACKLE \\
\hline N188 & MAHONEY GULL & N62 & MARSH BUTCHER & N22 & MCCLANHANS \\
\hline N151 & MCCRAE, BLACK & N58 & MCRAE, RED & N24 & MINER BLUES \\
\hline N19 & MODERN & N5 & MODERN, BIRCHEN & N8 & MODERN, BLACK \\
\hline N1 & MODERN, BLACK BREASTED RED & N74 & MODERN, BLUE RED & N2 & MODERN, BROWN RED \\
\hline N180 & MODERN, LEMON BLUE & N6 & MODERN, RED PYLE & N4 & MODERN, SILVER DUCKWING \\
\hline N7 & MODERN, WHITE & N129 & MUFFS & N148 & MUG \\
\hline N143 & MUG, BLACK & N147 & MUG, BLACK MANGEL & N105 & MUG. RACEY \\
\hline N174 & MURPHY WHITE HACKLE & N127 & MURPHYS & N82 & NARRAGANSETT \\
\hline N69 & NIPNUC BLUE & N70 & NIPNUC RED & N18 & OLD ENGLISH \\
\hline N15 & OLD ENGLISH, BLACK & N26 & OLD ENGLISH, BLACK BREASTED & N9 & $\begin{array}{l}\text { OLD ENGLISH, BLACK BREASTED } \\
\text { RED }\end{array}$ \\
\hline N50 & OLD ENGLISH, BLUE & N55 & OLD ENGLISH, BLUE RED & N78 & OLD ENGLISH, BRASSY BLACK \\
\hline N119 & OLD ENGLISH, BRASSY BLUE & N10 & OLD ENGLISH, BROWN RED & N11 & OLD ENGLISH, GOLD DUCKWING \\
\hline N12 & OLD ENGLISH, SILVER DUCKWING & N16 & OLD ENGLISH, SPANGLED & N14 & OLD ENGLISH, WHITE \\
\hline N114 & ORIENTAL & N207 & ORIENTAL, BLACK SHAMO & N211 & ORIENTAL, MALAYSION \\
\hline N96 & PEARCE SHUFFLER & N103 & PINNON & N17 & PIT \\
\hline N20 & PIT, WHITE HACKLE & N116 & PIT, WHITE HACKLE, MORGAN & N271 & RADIO \\
\hline N94 & RED QUILL & N255 & RED, DENT COUNTY & N117 & RENE VALLE \\
\hline N123 & ROBIN HOOD BLACK GREY & N51 & ROBIN HOOD BROWN RED & N122 & ROBIN HOOD REDS \\
\hline N41 & ROUNDHEAD & N164 & ROUNDHEAD, ALLEN & N163 & ROUNDHEAD, BOSTON \\
\hline
\end{tabular}


GAME CHICKEN (Continued)

\begin{tabular}{|c|c|c|c|c|c|}
\hline N160 & ROUNDHEAD, BRUNNER & N228 & ROUNDHEAD, JARRETT & N134 & ROUNDHEAD, LACY \\
\hline N221 & ROUNDHEAD, REBEL & N251 & ROUNDHEAD, SLAVIN & N250 & $\begin{array}{l}\text { ROUNDHEAD, SLAVIN CRIPPLE } \\
\text { TON }\end{array}$ \\
\hline N21 & SAM BIGHAM & N206 & SHUFFLER & N229 & SHUFFLER, BLACK \\
\hline N268 & SIMMACK & N27 & SPANISH PIT & N149 & SPANISH, JEREZANO \\
\hline N106 & TAYLOR & N93 & THOMPSON WHITE & N109 & TOPPY \\
\hline N108 & TRAVLERS & N47 & WALLACE FOWL & N135 & WARHORSE \\
\hline N165 & WARHORSE, BACON & N133 & WHITE DOME & N183 & WHITE HACKLE \\
\hline N169 & WHITE HACKLE, KEARNEY & N230 & WHITE HACKLE, MORGAN & N125 & WHITE HACKLE, NORTH BRITTON \\
\hline N231 & WHITE HACKLE, RADIO & N128 & WHITES & N248 & WILDCAT BLUE \\
\hline N30 & WILERSON RED & N31 & WILKERSON GRAY & & \\
\hline
\end{tabular}

R - OTHER LARGE BREEDS \& VARIETIES

\begin{tabular}{|c|c|c|c|c|c|}
\hline R222 & AMERAUCANA & $\mathrm{R} 260$ & AMERAUCANA, BLACK & $\mathrm{R} 261$ & AMERAUCANA, BLUE \\
\hline R262 & AMERAUCANA, WHITE & R176 & ANCONA, ROSE COMB & R6 & ANCONA, SINGLE COMB \\
\hline R56 & ANDALUSIAN & $\mathrm{R} 20$ & ARAUCANA & R172 & $\begin{array}{l}\text { ARAUCANA, TUFFED RUMPLESS } \\
\text { PEA COMB }\end{array}$ \\
\hline $\mathrm{R} 116$ & ASEEL & R96 & AUSTRALORP & R196 & AUSTRALORP, BLACK \\
\hline R26 & AUSTRALORP, WHITE & R36 & BARNEVELDER & $\mathrm{R} 272$ & BARNEVELDERS \\
\hline $\mathrm{R} 113$ & BARTHAEHNER & $\mathrm{R} 112$ & $\begin{array}{l}\text { BARTHAEHNER, SILVER } \\
\text { THOERINGER }\end{array}$ & R18 & BRAHMA \\
\hline R204 & BRAHMA, BLACK & R93 & BRAHMA, BUFF & R51 & BRAHMA, DARK \\
\hline R19 & BRAHMA, LIGHT & $\mathrm{R} 241$ & BROILER CROSS, BLACK & $\mathrm{R} 242$ & BROILER CROSS, RED \\
\hline R195 & BUCKEYE & $\mathrm{R} 77$ & BUTTERCUP & $\mathrm{R} 210$ & CALIFORNIA GRAY \\
\hline R209 & CALIFORNIA WHITE & R64 & CAMPINE, GOLDEN & R41 & CAMPINE, SILVER \\
\hline R258 & CATALANA, BUFF & $\mathrm{R} 185$ & CHANTECLERS, PARTRIDGE & $\mathrm{R} 186$ & CHANTECLERS, WHITE \\
\hline R94 & $\mathrm{COCHIN}$ & R140 & COCHIN, BARRED & R52 & COCHIN, BLACK \\
\hline R139 & COCHIN, BLUE & $\mathrm{R} 30$ & COCHIN, BUFF & $\mathrm{R} 160$ & COCHIN, FRIZZLE BUFF \\
\hline R119 & COCHIN, GOLDEN LACED & R53 & COCHIN, PARTRIDGE & R121 & COCHIN, RED LACED \\
\hline$R: 20$ & COCHIN. SILVER LACED & R201 & COCHIN, SPLASH & R87 & COCHIN, WHITE \\
\hline R151 & COLUMBIA & R88 & CREVECOEURS & R192 & $\begin{array}{l}\text { CUBALAYAS, BLACK BREASTED } \\
\text { RED }\end{array}$ \\
\hline $\mathrm{R} 17$ & DELAWARE & R42 & DOMINIQUE & R35 & DORKING, SILVER GRAY \\
\hline R27 & EXHIBITION & $\mathrm{R} 24$ & EXPERIMENTAL & $\mathrm{R} 28$ & FANCY BREEDS \\
\hline R71 & FAVEROLLE, SALMON & $\mathrm{R} 211$ & FAVEROLLE, WHITE & R69 & FAYOUMI, EGYPTIAN \\
\hline R105 & FRIZZLE & R158 & FRIZZLE, BUFF & $\mathrm{R} 243$ & GOLDEN COMET EX. \\
\hline R73 & HAMBURG & R111 & HAMBURG, BLACK & R76 & HAMBURG, GOLDEN PENCILED \\
\hline R109 & HAMBURG, GOLDEN SPANGLED & R68 & HAMBURG, SILVER & R92 & HAMBURG, SHLVER PENCILED \\
\hline R21 & HAMBURG, SILVER SPANGLED & R110 & HAMBURG, WHITE & R31 & HOLLAND, BARRED \\
\hline R54 & HOUDAN, MOTTLED & $\mathrm{R} 181$ & HOUDAN, WHITE & R165 & IDEAL SHOW BIRD \\
\hline R65 & IOWA BLUES & R136 & JAPANESE, BLACK TAIL WHITE & $\mathrm{R} 126$ & JAPANESE, WHITE TAIL BLACK \\
\hline R78 & JAVA & R257 & JAVA, MOTTLED & R15 & JERSEY GIANT, BLACK \\
\hline R208 & JERSEY GIANT, BLUE & R16 & JERSEY GIANT, WHITE & R55 & JUNGLEFOWL \\
\hline R259 & JUNGLEFOWL, GREY & R57 & JUNGLEFOWL, RED & R270 & KRAIENKOPPE \\
\hline
\end{tabular}


R

OTHER LARGE BREEDS \& VARIETIES

(Continued)

\begin{tabular}{|c|c|c|c|c|c|}
\hline R115 & LA FLECHE & $\mathrm{R} 268$ & LA FLECHE, BLACK & R269 & LA FLECHE, BLUE \\
\hline R72 & LAKENVELDER & $\mathrm{R} 232$ & LANGSHAN & R22 & LANGSHAN. BLACK \\
\hline R223 & LANGSHAN. BLUE & R23 & LANGSHAN, WHITE & R236 & LEGBAR \\
\hline R147 & LEGHORN, DANISH BROWN & $\mathrm{R} 231$ & LEGHORN, DAVID'S AUTOMATIC & $\mathrm{R} 187$ & LEGHORN, ROSE COMB BLACK \\
\hline R200 & LEGHORN, ROSE COMB BUFF & $\mathrm{R} 175$ & $\begin{array}{l}\text { LEGHORN, ROSE COMB DARK } \\
\text { BROWN }\end{array}$ & $\mathrm{R} 90$ & $\begin{array}{l}\text { LEGHORN, ROSE COMB LIGHT } \\
\text { BROWN }\end{array}$ \\
\hline R114 & LEGHORN, ROSE COMB WHITE & R74 & LEGHORN, SILVER & $\mathrm{R} 234$ & LEGHORN. SILVERUDD \\
\hline R230 & LEGHORN, SINGLE COMB BARRED & R25 & LEGHORN, SINGLE COMB BLACK & R1 & LEGHORN. SINGLE COMB BROWN \\
\hline R2 & LEGHORN, SINGLE COMB BUFF & R169 & $\begin{array}{l}\text { LEGHORN, SINGLE COMB DARK } \\
\text { BROWN }\end{array}$ & $\mathrm{R} 168$ & $\begin{array}{l}\text { LEGHORN, SINGLE COMB LIGHT } \\
\text { BROWN }\end{array}$ \\
\hline R98 & LEGHORN, SINGLE COMB RED & $\mathrm{R} 237$ & LEGHORN, SINGLE COMB WHITE & R48 & MALAY \\
\hline R219 & MALAY, BLACK BREASTED RED & R213 & MARANS & $\mathrm{R} 188$ & MINORCA, ROSE COMB BLACK \\
\hline R3 & MINORCA, SINGLE COMB BLACK & R5 & MINORCA, SINGLE COMB BUFF & $\mathrm{R} 4$ & MINORCA, SINGLE COMB WHITE \\
\hline R229 & MIXED & $\mathrm{R} 134$ & MOZISECK SHOW BROILER & R33 & NAKED NECK \\
\hline R263 & $\begin{array}{l}\text { NECKED NECK, BLACK TAILED } \\
\text { RED }\end{array}$ & R173 & NEW HAMPSHIRE, BLACK & $\mathrm{R} 227$ & NEW HAMPSHIRE, RED \\
\hline R130 & NEW HAMPSHIRE, WHITE & $\mathrm{R} 271$ & NORWEGIAN JAERS & $\mathrm{R} 249$ & $\begin{array}{l}\text { OLD ENGLISH GAME, BLACK } \\
\text { BREASTED RED }\end{array}$ \\
\hline R250 & OLD ENGLISH GAME, BROWN RED & R251 & $\begin{array}{l}\text { OLD ENGLISH GAME, GOLDEN } \\
\text { DUCKWING }\end{array}$ & $\mathrm{R} 253$ & $\begin{array}{l}\text { OLD ENGLISH GAME, SILVER } \\
\text { DUCKWING }\end{array}$ \\
\hline R254 & OLD ENGLISH GAME, SPANGLED & R255 & OLD ENGLISH GAME, WHEATEN & R199 & ORLOFF, RUSSIAN \\
\hline R103 & ORPINGTON & R44 & ORPINGTON, BLACK & $\mathrm{R} 117$ & ORPINGTON, BLUE \\
\hline R7 & ORPINGTON, BUFF & R49 & ORPINGTON, WHITE & $\mathrm{R} 214$ & PHOENIX, BLACK BREASTED RED \\
\hline R118 & PHOENIX, GOLDEN & R145 & PHOENIX, LONG TAIL & $\mathrm{R} 89$ & PHOENIX, SILVER \\
\hline R203 & PHOENIX, WHITE & $\mathrm{R} 146$ & PLYMOUTH ROCK, BARRED & R206 & PLYMOUTH ROCK, BLUE \\
\hline R9 & PLYMOUTH ROCK, BUFF & R10 & PLYMOUTH ROCK, COLUMBIAN & R34 & PLYMOUTH ROCK, PARTRIDGE \\
\hline R43 & PLYMOUTH ROCK, SILVER & R91 & $\begin{array}{l}\text { PLYMOUTH ROCK, SIVER } \\
\text { PENCILED }\end{array}$ & $\mathrm{R} 248$ & PLYMOUTH ROCK, WHITE \\
\hline R104 & POLISH & R177 & POLISH, BEARDED BUFF & $\mathrm{R} 123$ & POLISH, BEARDED BUFF LACED \\
\hline R178 & POLISH, BEARDED GOLDEN & R100 & POLISH, BEARDED SILVER & $\mathrm{R} 180$ & POLISH, BEARDED WHITE \\
\hline R179 & $\begin{array}{l}\text { POLISH, BEARDED WHITE CREST } \\
\text { BLACK }\end{array}$ & R182 & $\begin{array}{l}\text { POLISH, BEARDED WHITE } \\
\text { CRESTED BLUE }\end{array}$ & $\mathrm{R} 82$ & POLISH, BUFF LACED \\
\hline R99 & POLISH, GOLDEN & R50 & POLISH, NON-BEARDED BLACK & R47 & POLISH, NON-BEARDED BUFF \\
\hline R122 & $\begin{array}{l}\text { POLISH, NON-BEARDED BUFF } \\
\text { LACED }\end{array}$ & $\mathrm{R} 75$ & POLISH, NON-BEARDED GOLDEN & $\mathrm{R} 84$ & $\begin{array}{l}\text { POLISH, NON-BEARDED GOLDEN } \\
\text { LACED }\end{array}$ \\
\hline R124 & POLISH, NON-BEARDED SILVER & R46 & POLISH, NON-BEARDED WHITE & $\mathrm{R} 245$ & $\begin{array}{l}\text { POLISH, NON-BEARDED WHITE } \\
\text { CRESTED BLACK }\end{array}$ \\
\hline R246 & $\begin{array}{l}\text { POLISH, NON-BEARDED WHITE } \\
\text { CRESTED BLUE }\end{array}$ & R83 & POLISH, SILVER LACED & R45 & POLISH, WHITE CRESTED BLACK \\
\hline R66 & POLISH, WHITE CRESTED BLUE & R67 & POLISH, WHITE SPLASH & R238 & PRODUCTION BLACK \\
\hline R256 & PRODUCTION RED & R125 & REDCAP & R183 & RHODE ISLAND RED, ROSE COMB \\
\hline R247 & $\begin{array}{l}\text { RHODE ISLAND RED, SINGLE } \\
\text { COMB }\end{array}$ & R14 & $\begin{array}{l}\text { RHODE ISLAND WHITE, SINGLE } \\
\text { COMB }\end{array}$ & $\mathrm{R} 107$ & ROSE COMB, BLACK \\
\hline R153 & RUMPLESS & $\mathrm{R} 220$ & SAIPANS & $\mathrm{R} 170$ & SAMATRAS WHITE \\
\hline R233 & SEX LINK & $\mathrm{R} 239$ & SEX LINK, BLACK & $\mathrm{R} 240$ & SEX LINK, GOLD \\
\hline R244 & SHAMO, BLACK & R97 & SPANISH, WHITE FACED BLACK & R166 & SPITZ HAUBEN \\
\hline R171 & SPITZ HAUBEN, GERMAN SILVER & R148 & STEELE SHOW BIRDS & $\mathrm{R} 264$ & SULTAN, BLACK \\
\hline R265 & SULTAN, BLUE & R70 & SULTAN, WHITE & R60 & SUMATRAS \\
\hline R86 & SUMATRAS, BLACK & $\mathrm{R} 202$ & SUMATRAS, WHITE & $\mathrm{R} 8$ & SUSSEX \\
\hline R85 & SUSSEX, SPECKLED & R228 & TRIPLE PRODUCTION RED & R224 & VORWERK \\
\hline
\end{tabular}


$R$ OTHER LARGE BREEDS \& VARIETIES (Continued)

$\begin{array}{llllll}\text { R225 } & \text { WELSUMMERS } & \text { R95 } & \text { WYANDOTTE } & \text { R37 } & \text { WYANDOTTE, BLACK } \\ \text { R38 } & \text { WYANDOTTE, BUFF } & \text { R13 } & \text { WYANDOTTE, COLUMBIAN } & \text { R81 } & \text { WYANDOTTE, GOLDEN LACED } \\ \text { R39 } & \text { WYANDOTTE, PARTRIDGE } & \text { R12 } & \text { WYANDOTTE, SILVER LACED } & \text { R40 } & \text { WYANDOTTE, SILVER PENCILED } \\ \text { R11 } & \text { WYANDOTTE, WHITE } & \text { R62 } & \text { YOKAHAMA } & \end{array}$

W - BANTAMS

\begin{tabular}{|c|c|c|c|c|c|}
\hline W573 & AMERAUCANA & W316 & AMERICAN GAME & W319 & $\begin{array}{l}\text { AMERICAN GAME, BLACK } \\
\text { BREASTED RED }\end{array}$ \\
\hline W320 & AMERICAN GAME, BLUE & W322 & AMERICAN GAME, BROWN RED & W323 & $\begin{array}{l}\text { AMERICAN GAME, GOLDEN } \\
\text { DUCKWING }\end{array}$ \\
\hline W326 & $\begin{array}{l}\text { AMERICAN GAME, SILVER } \\
\text { DUCKWING }\end{array}$ & W327 & AMERICAN GAME, WHEATEN & W328 & AMERICAN GAME, WHITE \\
\hline W34 & ANCONA, SINGLE COMB & w95 & ANDALUSIAN & w96 & ARAUCANA \\
\hline W330 & $\begin{array}{l}\text { ARAUCANA, BLACK PLAIN } \\
\text { RUMPLESS }\end{array}$ & W332 & ARAUCANA, BLACK PLAIN TUFTED & W331 & $\begin{array}{l}\text { ARAUCANA, BLACK TUFTED } \\
\text { RUMPLESS }\end{array}$ \\
\hline W333 & $\begin{array}{l}\text { ARAUCANA, BLACK TUFTED } \\
\text { TAILED }\end{array}$ & W346 & $\begin{array}{l}\text { ARAUCANA, DARK BROWN PLAIN } \\
\text { RUMPLESS }\end{array}$ & W334 & $\begin{array}{l}\text { ARAUCANA, SILVER PLAIN } \\
\text { RUMPLESS }\end{array}$ \\
\hline W335 & $\begin{array}{l}\text { ARAUCANA, SILVER TUFTED } \\
\text { RUMPLESS }\end{array}$ & W337 & ARAUCANA, SILVER TUFTED TAIL & W338 & $\begin{array}{l}\text { ARAUCANA, WHITE TUFTED } \\
\text { RUMPLESS }\end{array}$ \\
\hline W348 & ASEEL, BLACK BREASTED RED & W351 & ASEEL, WHITE & W352 & AUSTRALORP \\
\hline W353 & AUSTRALORP, BLACK & W621 & BARNEVELDER & W560 & BELGIAN ANTWERP \\
\hline W600 & BELGIAN ANTWERP, QUAIL & W136 & BELGIAN BEARDED D'ANVER & W358 & $\begin{array}{l}\text { BELGIAN, BEARDED D' ANVER } \\
\text { BLACK BREASTD }\end{array}$ \\
\hline W357 & $\begin{array}{l}\text { BELGIAN, BEARDED D'ANVER } \\
\text { BLACK }\end{array}$ & W278 & $\begin{array}{l}\text { BELGIAN, BEARDED D'ANVER } \\
\text { BLUE }\end{array}$ & W359 & $\begin{array}{l}\text { BELGIAN, BEARDED D'ANVER } \\
\text { BUFF }\end{array}$ \\
\hline W360 & $\begin{array}{l}\text { BELGIAN, BEARDED D'ANVER } \\
\text { CUCKOO }\end{array}$ & W361 & $\begin{array}{l}\text { BELGIAN, BEARDED D'ANVER } \\
\text { MILLE FLEUR }\end{array}$ & W363 & $\begin{array}{l}\text { BELGIAN, BEARDED D'ANVER } \\
\text { PORCELAIN }\end{array}$ \\
\hline W301 & $\begin{array}{l}\text { BELGIAN, BEARDED D'ANVER } \\
\text { QUAIL }\end{array}$ & W364 & $\begin{array}{l}\text { BELGIAN, BEARDED D'ANVER SELF } \\
\text { BLUE }\end{array}$ & W231 & $\begin{array}{l}\text { BELGIAN, BEARDED D'ANVER } \\
\text { WHITE }\end{array}$ \\
\hline W365 & BELGIAN, BEARDED D'UCCLE & W92 & $\begin{array}{l}\text { BELGIAN, BEARDED D'UCCLE } \\
\text { BLACK }\end{array}$ & W90 & BELGIAN, BEARDED D'UCCLE BLUE \\
\hline W549 & $\begin{array}{l}\text { BELGIAN, BEARDED D'UCCLE } \\
\text { COLUMBIAN }\end{array}$ & W271 & $\begin{array}{l}\text { BELGIAN, BEARDED D'UCCLE } \\
\text { GOLD NECK }\end{array}$ & W86 & $\begin{array}{l}\text { BELGIAN, BEARDED D'UCCLE } \\
\text { MILLE FLEUR }\end{array}$ \\
\hline W193 & $\begin{array}{l}\text { BELGIAN, BEARDED D'UCCLE } \\
\text { MOTTLED }\end{array}$ & W270 & $\begin{array}{l}\text { BELGIAN, BEARDED D'UCCLE } \\
\text { PORCELAIN }\end{array}$ & W544 & $\begin{array}{l}\text { BELGIAN, BEARDED D'UCCLE } \\
\text { QUAIL }\end{array}$ \\
\hline W297 & $\begin{array}{l}\text { BELGIAN, BEARDED D'UCCLE SELF } \\
\text { BLUE }\end{array}$ & W232 & $\begin{array}{l}\text { BELGIAN, BEARDED D'UCCLE } \\
\text { WHITE }\end{array}$ & W367 & BOOTED, BUFF \\
\hline W368 & BOOTED, MILLE FLEUR & W230 & BOOTED, MOTTLED & W98 & BOOTED, PORCELAIN \\
\hline W47 & BOOTED, WHITE & W370 & BRAHMA & $W 12$ & BRAHMA, BUFF \\
\hline W9 & BRAHMA, DARK & W11 & BRAHMA, LIGHT & W634 & BRAHMA, PARTRIDGE \\
\hline W10 & BRAHMA, RED & W113 & BRAHMA, WHITE & W372 & BUCKEYE \\
\hline W373 & BUCKEYE, RED & W304 & BUTTERCUP & W110 & CAMPINE, GOLDEN \\
\hline W132 & COCHIN & w60 & COCHIN, BARRED & W223 & COCHIN, BIRCHEN \\
\hline W17 & COCHIN, BLACK & W546 & COCHIN, BLACK RED & W102 & COCHIN, BLUE \\
\hline W167 & COCHIN, BROWN & W170 & COCHIN, BROWN RED & W121 & COCHIN, BUFF \\
\hline W40 & COCHIN, BUFF COLUMBIAN & W206 & COCHIN, COLUMBIAN & W213 & COCHIN, DARK \\
\hline W554 & COCHIN, FRIZZLE & W169 & COCHIN, GOLDEN LACED & W164 & COCHIN, MOTTLED \\
\hline W33 & COCHIN, PARTRIDGE & $W 140$ & COCHIN, RED & W168 & COCHIN, SILVER LACED \\
\hline W259 & COCHIN, SILVER PENCILED & W265 & COCHIN, SPLASH & W18 & COCHIN, WHITE \\
\hline W207 & CORNISH & W306 & CORNISH, BLACK & W107 & CORNISH, BLUE \\
\hline W135 & CORNISH, BLUE LACED & W165 & CORNISH, BLUE LACED RED & W130 & CORNISH, BUFF \\
\hline
\end{tabular}


W BANTAMS (Continued)

\begin{tabular}{|c|c|c|c|c|c|}
\hline W20 & CORNISH, DARK & W97 & CORNISH, MOTTLED & W182 & CORNISH, RED LACED \\
\hline W219 & CORNISH, ROCK & W380 & CORNISH, SPANGLED & W57 & CORNISH, WHITE \\
\hline W19 & CORNISH, WHITE LACED RED & W386 & CREVECOEUR, BLACK & W64 & $\begin{array}{l}\text { CUBALAYA, BLACK BREASTED } \\
\text { RED }\end{array}$ \\
\hline$W+90$ & DELAWARE & W387 & DOMINIQUE & W390 & DORKING, ROSECOMB WHITE \\
\hline W305 & $\begin{array}{l}\text { DORKING, SINGLE COMB SILVER } \\
\text { GRAY }\end{array}$ & W393 & DUTCH & W394 & DUTCH. BLACK \\
\hline W395 & DUTCH, BLACK BREASTED RED & W396 & DUTCH, BLUE & W607 & DUTCH, BLUE LIGHT BROWN \\
\hline W397 & DUTCH, BLUE RED & W606 & DUTCH, CREAM LIGHT BROWN & W552 & DUTCH, LIGHT BROWN \\
\hline W577 & DUTCH, QUILL & W401 & DUTCH, SILVER & W548 & FAVEROLLE, BIRCHEN \\
\hline W32 & FAVEROLLE, SALMON & W415 & FRIZZLE & W146 & FRIZZLE, BLACK \\
\hline W244 & FRIZZLE, BLACK COCHIN & W209 & $\begin{array}{l}\text { FRIZZLE, BLACK FEATHER } \\
\text { LEGGED }\end{array}$ & W416 & FRIZZLE, BLUE \\
\hline W180 & FRIZZLE, BLUE FEATHER LEGGED & W243 & FRIZZLE, BUFF COCHIN & W253 & FRIZZLE, BUFF FEATHER LEGGED \\
\hline W314 & FRIZZLE, MOTTLED & W251 & $\begin{array}{l}\text { FRIZZLE, PARTRIDGE FEATHER } \\
\text { LEGGED }\end{array}$ & W249 & FRIZZLE, RED \\
\hline W179 & FRIZZLE, RED FEATHER LEGGED & W106 & FRIZZLE, WHITE & W242 & FRIZZLE, WHITE COCHIN \\
\hline W173 & $\begin{array}{l}\text { FRIZZLE, WHITE FEATHER } \\
\text { LEGGED }\end{array}$ & W261 & FRIZZLE, WYANDOTTE & W112 & HAMBURG \\
\hline W422 & HAMBURG, BLACK & W153 & HAMBURG, GOLDEN PENCILED & W108 & HAMBURG, SILVER \\
\hline W58 & HAMBURG, SILVER SPANGLED & W424 & HOUDAN & W31 & HOUDAN, MOTTLED \\
\hline W78 & IRISH GRAY & w1 & JAPANESE & W241 & JAPANESE, BARRED \\
\hline W603 & JAPANESE, BARRED FRIZZLE & W81 & JAPANESE, BLACK & W211 & JAPANESE, BLACK TAILED BUFF \\
\hline W87 & JAPANESE, BLACK TAILED RED & W198 & JAPANESE, BLACK TAILED WHITE & W82 & JAPANESE, BLUE \\
\hline W177 & JAPANESE, BLUE TAILED WHITE & W224 & JAPANESE, BROWN RED & W427 & JAPANESE, BUFF \\
\hline W176 & JAPANESE, GRAY & W145 & JAPANESE, MOTTLED & W286 & JAPANESE, SELF BLUE \\
\hline W138 & JAPANESE, WHITE & W636 & JERSEY GIANT & W80 & JERSEY GIANT, BLACK \\
\hline W635 & JERSEY GIANT, BLUE & W189 & JUNGLEFOWL & W435 & JUNGLEFOWL, RED \\
\hline W155 & LA FLECHE & W439 & LA FLECHE, BLACK & W637 & LA FLECHE, BLUE \\
\hline W638 & LA FLECHE, BLUE MOTTLED & W639 & LA FLECHE, MOTTLED & W640 & LA FLECHE, SPLASH \\
\hline W641 & LA FLECHE, WHITE & W111 & LAKENVELDER & W440 & LANGSHAN \\
\hline W15 & LANGSHAN, BLACK & W14 & LANGSHAN, WHITE & W442 & LEGHORN \\
\hline W289 & LEGHORN, BARRED SILVER GOLD & W300 & LEGHORN, BUFF COLUMBIAN & W564 & LEGHORN, GOLDEN DUCKWING \\
\hline W281 & $\begin{array}{l}\text { LEGHORN, ROSE COMB DARK } \\
\text { BROWN }\end{array}$ & W105 & $\begin{array}{l}\text { LEGHORN, ROSE COMB LIGHT } \\
\text { BROWN }\end{array}$ & W76 & LEGHORN, ROSE COMB WHITE \\
\hline W103 & LEGHORN, SINGLE COMB BLACK & W77 & LEGHORN, SINGLE COMB BUFF & W299 & $\begin{array}{l}\text { LEGHORN, SINGLE COMB } \\
\text { COLUMBIAN }\end{array}$ \\
\hline W56 & $\begin{array}{l}\text { LEGHORN, SINGLE COMB DARK } \\
\text { BROWN }\end{array}$ & W576 & $\begin{array}{l}\text { LEGHORN, SINGLE COMB LIGHT } \\
\text { BROWN }\end{array}$ & W118 & $\begin{array}{l}\text { LEGHORN, SINGLE COMB MILLE } \\
\text { FLEUR }\end{array}$ \\
\hline W255 & LEGHORN, SINGLE COMB RED & W67 & LEGHORN, SINGLE COMB SILVER & W6 & LEGHORN, SINGLE COMB WHITE \\
\hline W49 & MALAY & W144 & MALAY, BLACK BREASTED RED & W192 & MAX CROSS \\
\hline W570 & MILLE FLEUR & w37 & MINORCA, SINGLE COMB BLACK & w2 & MIXED \\
\hline W35 & MODERN GAME & W221 & MODERN GAME, BARRED & W128 & MODERN GAME, BIRCHEN \\
\hline W293 & MODERN GAME, BLACK & W115 & $\begin{array}{l}\text { MODERN GAME, BLACK BREASTED } \\
\text { RED }\end{array}$ & W453 & MODERN GAME, BLUE \\
\hline W454 & MODERN GAME, BLUE RED & W187 & MODERN GAME, BROWN RED & W455 & MODERN GAME, CRELE \\
\hline W456 & MODERN GAME, GINGER RED & W125 & $\begin{array}{l}\text { MODERN GAME, GOLDEN } \\
\text { DUCKWING }\end{array}$ & W175 & MODERN GAME. LEMON BLUE \\
\hline W629 & MODERN GAME, MOTTLED & W123 & MODERN GAME, RED PYLE & W457 & MODERN GAME, SELF BLUE \\
\hline
\end{tabular}


W BANTAMS (Continued)

\begin{tabular}{|c|c|c|c|c|c|}
\hline W294 & MODERN GAME, SILVER BLUE & W245 & $\begin{array}{l}\text { MODERN GAME. SILVER } \\
\text { DUCKWING }\end{array}$ & W295 & MODERN GAME, SPLASH \\
\hline W188 & MODERN GAME, WHEATEN & W174 & MODERN GAME, WHITE & W100 & NAKED NECK \\
\hline W466 & NAKED NECK. SINGLE COMB BUFF & W468 & NAKED NECK, SINGLE COMB RED & W469 & $\begin{array}{l}\text { NAKED NECK. SINGLE COMB } \\
\text { WHITE }\end{array}$ \\
\hline W470 & NANKIN ROSE COMB & W120 & NEW HAMPSHIRE RED & W30 & OLD ENGLISH GAME \\
\hline W646 & OLD ENGLISH GAME MOTTLED & W566 & OLD ENGLISH GAME, 713 & W65 & $\begin{array}{l}\text { OLD ENGLISH GAME. AMERICAN } \\
\text { PIT }\end{array}$ \\
\hline W141 & OLD ENGLISH GAME, BARRED & W200 & OLD ENGLISH GAME, BIRCHEN & W88 & OLD ENGLISH GAME, BLACK \\
\hline W28 & $\begin{array}{l}\text { OLD ENGLISH GAME, BLACK } \\
\text { BREASTED RED }\end{array}$ & W274 & $\begin{array}{l}\text { OLD ENGLISH GAME, BLACK } \\
\text { TAILED BUFF }\end{array}$ & W308 & $\begin{array}{l}\text { OLD ENGLISH GAME, BLACK } \\
\text { TAILED RED }\end{array}$ \\
\hline W29 & OLD ENGLISH GAME. BLUE & W114 & $\begin{array}{l}\text { OLD ENGLISH GAME, BLUE } \\
\text { BREASTED RED }\end{array}$ & W545 & $\begin{array}{l}\text { OLD ENGLISH GAME, BLUE } \\
\text { DUCKWING }\end{array}$ \\
\hline W599 & $\begin{array}{l}\text { OLD ENGLISH GAME, BLUE } \\
\text { GOLDEN DUCKWING }\end{array}$ & W579 & OLD ENGLISH GAME, BLUE QUAIL & W151 & OLD ENGLISH GAME, BLUE RED \\
\hline W568 & $\begin{array}{l}\text { OLD ENGLISH GAME, BRASSY } \\
\text { BACK }\end{array}$ & W596 & $\begin{array}{l}\text { OLD ENGLISH GAME, BRASSY } \\
\text { BACK. BLUE }\end{array}$ & W50 & OLD ENGLISH GAME, BROWN RED \\
\hline W542 & OLD ENGLISH GAME, COLUMBIAN & W142 & OLD ENGLISH GAME, CRELE & W263 & OLD ENGLISH GAME, CUCKOO \\
\hline W613 & $\begin{array}{l}\text { OLD ENGLISH GAME, FAWN } \\
\text { DUCKWING }\end{array}$ & W262 & OLD ENGLISH GAME, GINGER RED & W150 & $\begin{array}{l}\text { OLD ENGLISH GAME, GOLDEN } \\
\text { DUCKWING }\end{array}$ \\
\hline W51 & OLD ENGLISH GAME, LEMON BLUE & W642 & OLD ENGLISH GAME, MILLE FLEUR & W643 & OLD ENGLISH GAME, PEARL \\
\hline W644 & OLD ENGLISH GAME, PORCELAIN & W569 & OLD ENGLISH GAME, QUAIL & W70 & $\begin{array}{l}\text { OLD ENGLISH GAME, RED } \\
\text { BREASTED }\end{array}$ \\
\hline W52 & OLD ENGLISH GAME, RED PYLE & W149 & OLD ENGLISH GAME. SELF BLUE & W275 & OLD ENGLISH GAME, SILVER BLUE \\
\hline W48 & $\begin{array}{l}\text { OLD ENGLISH GAME, SILVER } \\
\text { DUCKWING }\end{array}$ & W645 & OLD ENGLISH GAME. SILVER DUN & W89 & OLD ENGLISH GAME, SPANGLED \\
\hline W276 & OLD ENGLISH GAME. SPLASH & W91 & OLD ENGLISH GAME、 WHEATEN & W556 & $\begin{array}{l}\text { OLD ENGLISH GAME, WHEATON } \\
\text { BLUE }\end{array}$ \\
\hline W69 & OLD ENGLISH GAME, WHITE & W616 & $\begin{array}{l}\text { OLD ENGLISH GAME, WHITE- } \\
\text { TAILED BUFF }\end{array}$ & W617 & OLD ENGLISH, BUFF \\
\hline W473 & ORLOFF. SPANGLED & W609 & ORPINGTON & W126 & ORPINGTON, BLACK \\
\hline W159 & ORPINGTON, BLUE & W16 & ORPINGTON, BUFF & W127 & ORPINGTON, WHITE \\
\hline W593 & PHOENIX & W559 & PHOENIX. BLACK BREASTED RED & W475 & PHOENIX. GOLDEN \\
\hline W62 & PHOENIX, GOLDEN DUCKWING & W477 & PHOENIX, SILVER & W61 & PHOENIX, WHITE \\
\hline W610 & PLYMOUTH ROCK & W3 & PLYMOUTH ROCK, BARRED & W134 & PLYMOUTH ROCK, BLACK \\
\hline W478 & PLYMOUTH ROCK, BLUE & W36 & PLYMOUTH ROCK, BUFF & W24 & PLYMOUTH ROCK, COLUMBIAN \\
\hline W5 & PLYMOUTH ROCK. PARTRIDGE & W72 & PLYMOUTH ROCK. SILVER LACED & W104 & $\begin{array}{l}\text { PLYMOUTH ROCK, SILVER } \\
\text { PENCILED }\end{array}$ \\
\hline W4 & PLYMOUTH ROCK, WHITE & W131 & POLISH & W628 & $\begin{array}{l}\text { POLISH, NON-BEARDED BLACK } \\
\text { CRESTE WHITE }\end{array}$ \\
\hline W479 & POLISH, BEARDED BLUE & W43 & POLISH, BEARDED BUFF LACED & W41 & POLISH, BEARDED GOLDEN \\
\hline W152 & POLISH, BEARDED SILVER & W44 & POLISH, BEARDED WHITE & W84 & POLISH, BLACK \\
\hline W647 & POLISH, BLACK WHITE CRESTED & W93 & POLISH, BUFF CRESTED & W585 & POLISH, GOLD LACED \\
\hline W480 & POLISH, NON-BEARDED BLUE & W282 & $\begin{array}{l}\text { POLISH, NON-BEARDED BUFF } \\
\text { LACED }\end{array}$ & W42 & POLISH, NON-BEARDED GOLDEN \\
\hline W162 & POLISH, NON-BEARDED SILVER & W303 & POLISH, NON-BEARDED WHITE & W45 & $\begin{array}{l}\text { POLISH, NON-BEARDED WHITE } \\
\text { CRESTED BLACK }\end{array}$ \\
\hline W68 & $\begin{array}{l}\text { POLISH. NON-BEARDED WHITE } \\
\text { CRESTED BLUE }\end{array}$ & W83 & POLISH, WHITE & W590 & $\begin{array}{l}\text { PORCELAIN, MILLE FLEUR, NON } \\
\text { BEARDED }\end{array}$ \\
\hline W595 & PORCELAN & W481 & PYCHEON, MILLE FLEUR & W283 & REDCAP \\
\hline W578 & RHODE ISLAND RED & W8 & RHODE ISLAND RED, ROSE COMB & W7 & $\begin{array}{l}\text { RHODE ISLAND RED, SINGLE } \\
\text { COMB }\end{array}$ \\
\hline W39 & ROSECOMB & W21 & ROSECOMB, BIRCHEN & W119 & ROSECOMB, BLACK \\
\hline W484 & $\begin{array}{l}\text { ROSECOMB, BLACK BREASTED } \\
\text { RED }\end{array}$ & W160 & ROSECOMB, BLUE & W486 & ROSECOMB, BLUE RED \\
\hline
\end{tabular}


BANTAMS (Continued)

\begin{tabular}{|c|c|c|c|c|c|}
\hline W307 & ROSECOMB, BROWN RED & W139 & ROSECOMB, BUFF COLUMBIAN & W492 & ROSECOMB, MOTTLED \\
\hline W494 & ROSECOMB, RED & W220 & ROSECOMB, SPLASH & W22 & ROSECOMB, WHITE \\
\hline W501 & $\begin{array}{l}\text { RUMPLESS, BLACK BREASTED } \\
\text { RED }\end{array}$ & W507 & RUMPLESS, GOLDEN DUCKWING & W508 & RUMPLESS, RED \\
\hline W509 & RUMPLESS, SILVER DUCKWING & W510 & RUMPLESS, WHITE & W252 & RUSSIAN RED \\
\hline W588 & SEBRIGHT & W25 & SEBRIGHT SILVER & W26 & SEBRIGHT, GOLDEN \\
\hline W511 & SHAMO, BLACK BREASTED RED & W516 & SHAMO, WHEATEN & W13 & SILKIE \\
\hline W75 & SILKIE, BEARDED BLACK & W518 & SILKIE, BEARDED BLUE & W248 & SILKIE, BEARDED BUFF \\
\hline W313 & SILKIE, BEARDED GRAY & W247 & SILKIE, BEARDED PARTRIDGE & W615 & SILKIE, BEARDED SPLASH \\
\hline W74 & SILKIE, BEARDED WHITE & W250 & SILKIE, JAPANESE & W290 & SILKIE, NON-BEARDED BLACK \\
\hline W257 & $\begin{array}{l}\text { SILKIE, NON-BEARDED BLACK } \\
\text { JAPANESE }\end{array}$ & W519 & SILKIE, NON-BEARDED BLUE & W215 & SILKIE, NON-BEARDED BUFF \\
\hline W309 & SILKIE, NON-BEARDED GRAY & W184 & SILKIE, NON-BEARDED PARTRIDGE & W208 & SILKIE, NON-BEARDED WHITE \\
\hline W256 & $\begin{array}{l}\text { SILKIE, NON-BEARDED WHITE } \\
\text { JAPANESE }\end{array}$ & W555 & SILKIE, POLISH & W604 & SILKIE, RED PYLE \\
\hline W504 & SILKIE, RED-BEARDED & W291 & SILKIE, SILVER & W302 & SPANISH, WHITE FACED BLACK \\
\hline W574 & SPITSHAUBEN & W46 & SULTAN, BLACK & W624 & SULTAN, GOLDEN NECK \\
\hline W625 & SULTAN, MILLE FLEUR & W626 & SULTAN, RED & W627. & SULTAN, WHEATEN \\
\hline W129 & SULTAN, WHITE & W166 & SUMATRA BLACK & W526 & SUSSEX, RED \\
\hline W527 & SUSSEX, SPECKLED & W567 & WELSUMMER, BROWN & W594 & WYANDOTTE \\
\hline W216 & WYANDOTTE, BARRED & W531 & WYANDOTTE, BIRCHEN & W38 & WYANDOTTE, BLACK \\
\hline W79 & WYANDOTTE, BLUE & W620 & WYANDOTTE, BLUE LACED GOLD & W27 & WYANDOTTE, BUFF \\
\hline W535 & WYANDOTTE, BUFF COLUMBIAN & W53 & WYANDOTTE, COLUMBIAN & W619 & WYANDOTTE, DUN LACED \\
\hline W117 & WYANDOTTE, GOLDEN LACED & W23 & WYANDOTTE, PARTRIDGE & W586 & WYANDOTTE, SILKIE LACED \\
\hline W587 & WYANDOTTE, SILVER & W94 & WYANDOTTE, SILVER LACED & W55 & WYANDOTTE, SILVER PENCILED \\
\hline W537 & WYANDOTTE, SPLASH & W54 & WYANDOTTE, WHITE & W538 & WYANDOTTE, WHITE LACED RED \\
\hline
\end{tabular}

W66 YOKOHAMA, WHITE

$x$ - DUCKS

\begin{tabular}{|c|c|c|c|c|c|}
\hline$\times 100$ & AMERICAN SAXONY & $\times 185$ & AMERICAN, BLACK & $\times 101$ & ANCONA \\
\hline$\times 15$ & AYLESBURY & $\times 48$ & BLACK & $x 1$ & BUFF \\
\hline $\mathrm{X} 133$ & BUFFLEHEAD & $\times 146$ & CALL & $\times 65$ & CALL, BLACK \\
\hline$\times 38$ & CALL, BLUE & $\times 145$ & CALL, BUFF & $\times 168$ & CALL, BUTTERSCOTCH \\
\hline$\times 10$ & CALL, GRAY & $\times 47$ & CALL, MUTATION & $\times 147$ & CALL, PASTEL \\
\hline $\mathrm{x} 41$ & CALL, SILVER & $\times 143$ & CALL, SNOWY & $\mathrm{x} 17$ & CALL, WHITE \\
\hline$x 63$ & CAMPBELL, DARK & $\times 44$ & CAMPBELL, ENGLISH WHITE & $x_{6}$ & CAMPBELL, KHAKI \\
\hline X95 & CAMPBELL, PIED & $x 43$ & CAMPBELL, WHITE & $\times 60$ & CANVASBACK \\
\hline $\mathrm{x} 14$ & CAYUGA & $\times 135$ & CAYUGA, BLACK & $\times 103$ & CAYUGA, BLUE \\
\hline $\mathrm{X} 161$ & CRESTED & $\times 37$ & CRESTED, BLACK & $\times 64$ & CRESTED, BLUE \\
\hline$\times 45$ & CRESTED, BUFF & $\times 11$ & CRESTED, WHITE & $\mathrm{X} 193$ & DUTCH HOOKBILLED \\
\hline $\mathrm{X} 16$ & EAST INDIA & $\times 9$ & EAST INDIA, BLACK & $x 88$ & EIDER \\
\hline $\mathrm{X} 175$ & EIDER, ATLANTIC & $\times 177$ & EIDER, KING & $\times 183$ & EIDER, NORTHERN \\
\hline $\mathrm{X} 176$ & EIDER, PACIFIC & $\times 182$ & EIDER, SPECTACLED & $\times 125$ & FERRUGINOUS WHITE EYES \\
\hline
\end{tabular}




\begin{tabular}{|c|c|c|c|c|c|}
\hline $\mathrm{x} 130$ & FULVOUS & x62 & GADWALL & $\times 115$ & GARGANEY \\
\hline$\times 102$ & GOLDEN CASCADE & $x 78$ & GOLDENEYE & $x 79$ & GOLDENEYE, BARROWS \\
\hline$\times 174$ & HARLEQUIN & $\times 98$ & HARLEQUIN, WELSH & $\times 5$ & INDIAN RUNNER \\
\hline$\times 96$ & INDIAN RUNNER, BLACK & $\times 34$ & INDIAN RUNNER, BUFF & $\times 35$ & INDIAN RUNNER, CHOCOLATE \\
\hline $\mathrm{x} 107$ & INDIAN RUNNER, CINNAMON & $\times 33$ & $\begin{array}{l}\text { INDIAN RUNNER, CUMBERLAND } \\
\text { BLUE }\end{array}$ & $\times 106$ & INDIAN RUNNER, EMERY \\
\hline$\times 22$ & INDIAN RUNNER, FAWN & $\times 27$ & $\begin{array}{l}\text { INDIAN RUNNER, FAWN AND } \\
\text { WHITE }\end{array}$ & $\times 105$ & INDIAN RUNNER, GOLDEN \\
\hline X97 & INDIAN RUNNER, GRAY & $\times 26$ & INDIAN RUNNER, PENCILED & $\times 104$ & INDIAN RUNNER, SAXONY \\
\hline$\times 23$ & INDIAN RUNNER, WHITE & $\times 160$ & KAIYA & $\times 31$ & MAGPIE \\
\hline$\times 136$ & MAGPIE. BLACK \& WHITE & $\times 137$ & MAGPIE, BLUE \& WHITE & $\times 4$ & MALLARD \\
\hline$\times 140$ & MALLARD, BLACK & $\times 108$ & MALLARD, GOLDEN & $\times 46$ & MALLARD, GRAY \\
\hline X199 & MALLARD, RUNNER & $\times 158$ & MALLARD, SILVER & $\times 29$ & MALLARD, WHITE ALBINO \\
\hline$\times 28$ & MANDARIN & $\times 166$ & MANDARIN, WHITE & $\times 93$ & MERGANSER \\
\hline$\times 57$ & MERGANSER HOODED & $\mathrm{X} 172$ & MERGANSER, AMERICAN & $\times 173$ & MERGANSER, REDBREASTED \\
\hline $\mathrm{X} 162$ & METZER, GOLDEN 300 & $\times 192$ & METZER, WHITE GOLDEN LAYER & $\times 20$ & MUSCOVY \\
\hline$\times 18$ & MUSCOVY, BLACK & $\times 12$ & MUSCOVY, BLUE & $x 68$ & MUSCOVY, BUFF \\
\hline$\times 32$ & MUSCOVY, CHOCOLATE & $\times 24$ & MUSCOVY, COLORED & $\mathrm{X} 194$ & MUSCOVY, DARK RIPPLE \\
\hline$\times 195$ & MUSCOVY, LILAC & $x 67$ & MUSCOVY, PASTEL & $\mathrm{X} 180$ & MUSCOVY, SILVER \\
\hline$\times 21$ & MUSCOVY, WHITE & $\times 186$ & MUSCOVY, WILD & $\times 30$ & $\begin{array}{l}\text { NORTH AMERICAN MIGRATORY } \\
\text { WATERFOWL }\end{array}$ \\
\hline$\times 3$ & PEKIN, WHITE & $x 85$ & PINTAIL & $\times 50$ & PINTAIL, AMERICAN \\
\hline$\times 121$ & PINTAIL, BAHAMA & $\times 120$ & PINTAIL, CHILEAN & $x 123$ & PINTAIL, LESSER BAHAMA \\
\hline$\times 184$ & PINTAIL, NORTHERN & $x 71$ & PINTAIL, RED-BILLED & $\mathrm{X} 122$ & PINTAIL, SILVER BAHAMA \\
\hline$\times 51$ & POCHARD, RED CRESTED & $\times 127$ & POCHARD, ROSYBILL & $x 7$ & REDHEAD \\
\hline$x 72$ & RINGBILLS & $\times 89$ & RINGNECK & $\times 2$ & ROUEN \\
\hline$x 58$ & RUDDY & X74 & SCAUP, GREATER & $x 73$ & SCAUP, LESSER \\
\hline$\times 126$ & SCAUP, NEW ZEALAND & $x 81$ & SCOTER, WHITE WINGED & $\mathrm{X} 142$ & SHEL, DUCK COMMON \\
\hline$\times 13$ & SHEL, DUCK EUROPEAN & $\mathrm{X} 141$ & SHEL, DUCK PARADISE & $\mathrm{X} 198$ & SHOVELER, ARGENTINA RED \\
\hline$\times 197$ & SHOVELER. AUSTRALIAN & X61 & SHOVELER, COMMON & $\mathrm{X} 163$ & SHOVELER, NEW ZEALAND RED \\
\hline x99 & SILVER APPLEYARD & $\times 159$ & SMEW & $\times 134$ & SNOWY \\
\hline$\times 154$ & SWEDISH & $\times 131$ & SWEDISH, BLACK & $\times 25$ & SWEDISH, BLUE \\
\hline$\times 150$ & TEAL & $x 188$ & TEAL, AMERICAN GREENWING & $\times 109$ & TEAL, BAIKAL \\
\hline$\times 53$ & TEAL, BLUE-WING & $\times 55$ & TEAL, BRAZILIAN & $x 113$ & TEAL, CAPE \\
\hline$\times 118$ & TEAL, CHESTNUT & $\times 110$ & TEAL, CHESTNUT-BREASTED & $\times 144$ & TEAL, CHILEAN \\
\hline$\times 52$ & TEAL, CINNAMON & $\times 54$ & TEAL, EUROPEAN GREEN-WING & $x 77$ & TEAL, FALCATED \\
\hline X189 & TEAL, GARANEY & $\mathrm{x} 111$ & TEAL, HOTTENTOT & $\mathrm{X} 117$ & TEAL, LAYSAN \\
\hline X114 & TEAL, MARBLED & $\mathrm{X} 112$ & TEAL, NORTHERN SILVER & $\times 40$ & TEAL, RING-NECKED \\
\hline$\times 149$ & TEAL, SHARP-WINGED & X116 & TEAL, VERSICOLOR & $x 94$ & TREE \\
\hline$x 124$ & TREE, BLACK-BELLIED & $\times 165$ & TREE, CUBAN & $x 171$ & TREE, EYTON \\
\hline$\times 59$ & TREE. FULVUS & $\times 42$ & TREE, WHITE FACE & $x 75$ & TUFTED \\
\hline$\times 49$ & WIGEON, AMERICAN & $x 70$ & WIGEON, CHILOE & $x 76$ & WIGEON, EUROPEAN \\
\hline$\times 187$ & WOOD & $x_{8}$ & WOOD, CAROLINA & $\mathrm{X} 167$ & WOOD, WHITE \\
\hline
\end{tabular}


$Y$ - GEESE (Continued)

\begin{tabular}{|c|c|c|c|c|c|}
\hline Y50 & ABYSSINIAN BLUE-WINGED & Y4 & AFRICAN & $Y 72$ & AFRICAN, BUFF \\
\hline V71 & AFRICAN, GIANT DEWLAP & Y53 & AFRICAN, GRAY & Y61 & AFRICAN, PYGMY \\
\hline Y68 & AFRICAN, SUPER & Y35 & AFRICAN, WHITE & Y56 & ANDEAN \\
\hline Y36 & ASHY HEADED & Y75 & BARHEADED & Y26 & BARNACLE \\
\hline$Y 16$ & BLUE & Y 66 & BRANT, ATLANTIC & Y67 & BRANT, PACIFIC BLACK \\
\hline$Y 7$ & BUFF & Y21 & BUFF, AMERICAN & Y69 & BUFF, TUFTED \\
\hline Y9 & CANADA & Y40 & CANADA, DUSKY & Y57 & CANADA, MINIATURE \\
\hline Y6 & CHINESE, BROWN & $Y_{2}$ & CHINESE, WHITE & Y15 & COCKLER \\
\hline Y25 & CRESTED, ROMAN & Y8 & EGYPTIAN & Y60 & EGYPTIAN, WHITE \\
\hline Y13 & EMBDEN (EMDEN) & Y12 & EMBDEN, ENGLISH & Y70 & EMBDEN, GIANT \\
\hline Y3 & EMBDEN, WHITE & Y28 & EMPEROR & Y49 & GREATER MAGELLAN \\
\hline Y27 & INDIAN BAR HEADED & $\mathrm{Y} 37$ & MANED & Y51 & NE-NE \\
\hline Y45 & ORINOCO & Y23 & PIED BUFF & Y5 & PILGRIM \\
\hline Y29 & PINK-FOOTED & Y65 & POMERANIAN, BUFF & Y64 & POMERANIAN, GRAY \\
\hline$Y_{14}$ & POMERANIAN, SADDLEBACK & Y74 & POMERANIAN, SADDLEBACK BUFF & Y73 & POMERANIAN, SADDLEBACK GRAY \\
\hline Y63 & POMERANIAN, WHITE & Y39 & RED-BREASTED & Y59 & RICHARDSON \\
\hline Y54 & ROMAN, TUFTED WHITE & $\mathrm{Y}_{32}$ & ROSS'S & $Y_{44}$ & RUDDY-HEADED \\
\hline Y24 & SADDLE PINCER & Y19 & SCHILTZ, HEAVY GRAY & Y18 & SCHILTZ, HEAVY WHITE \\
\hline Y17 & SCHILTZ, LINE 306 & $Y 22$ & SEBASTOPOL & Y55 & SIBERIAN \\
\hline Y1 & TOULOUSE & Y10 & TOULOUSE, DEWLAP & Y52 & TOULOUSE, DEWLAP BUFF \\
\hline Y41 & WHITE FRONTED, LESSER & Y20 & WHITE SNOW & Y31 & WHITE-FRONTED \\
\hline
\end{tabular}

Z - OTHER BIRDS

\begin{tabular}{|c|c|c|c|c|c|}
\hline Z1 & CHUKAR & $\mathrm{Z} 293$ & CHUKAR, WHITE & Z6 & FRANCOLIN \\
\hline 275 & FRANCOLIN, ERCKEL'S & Z187 & GROUSE, BLUE & Z65 & GROUSE, RUFFED \\
\hline$Z 66$ & GROUSE, SHARPTAIL & Z134 & GROUSE, SPRUCE & $\mathrm{Z} 4$ & GUINEA \\
\hline $\mathbf{Z 1 1 0}$ & GUINEA, BLUE & $\mathrm{Z254}$ & GUINEA, BRONZE & $\mathrm{Z} 252$ & GUINEA, BROWN \\
\hline $\mathrm{Z249}$ & GUINEA, BUFF & $\mathrm{Z} 21$ & GUINEA, BUFF DUN DOTTE & 2285 & GUINEA, CORAL \\
\hline 2239 & GUINEA, FRENCH & $\mathrm{Z20}$ & GUINEA, LAVENDER & 2256 & GUINEA, OPALINE \\
\hline 222 & GUINEA, PEARL & $\mathrm{Z} 255$ & GUINEA, PEWTER & $\mathrm{Z} 286$ & GUINEA, PIED \\
\hline $\mathrm{Z} 250$ & GUINEA, PORCELAIN & 2287 & GUINEA, ROYAL BLUE & $\mathbf{Z 1 1 1}$ & GUINEA, ROYAL PURPLE \\
\hline 263 & GUINEA, SIVER POLISHED & $\mathrm{Z} 251$ & GUINEA, SLATE & Z106 & GUINEA, SPECKLED \\
\hline 2253 & GUINEA, VIOLET & $\mathrm{Z} 62$ & GUINEA, WHITE & $\mathrm{Z} 147$ & HELMET \\
\hline Z94 & PARTRIDGE & Z159 & PARTRIDGE, ANDREWS' CHUKAR & $\mathbf{Z} 30$ & PARTRIDGE, BARBARY CHUKAR \\
\hline Z165 & PARTRIDGE, BUFF CHUKAR & $\mathbf{Z 1 7 5}$ & PARTRIDGE, CHINESE BAMBOO & $\mathrm{Z} 48$ & PARTRIDGE, HUNGARIAN \\
\hline 229 & PARTRIDGE, INDIAN CHUKAR & $\mathrm{Z} 12$ & PARTRIDGE, RED LEGGED & $\mathrm{Z14}$ & PEACOCK \\
\hline Z139 & PEACOCK, INDIA BLUE & $\mathrm{Z11}$ & PEAFOWL & $\mathrm{Z179}$ & PEAFOWL, BLACK SHOULDER PIED \\
\hline Z101 & $\begin{array}{l}\text { PEAFOWL, BLACK SHOULDER } \\
\text { PURPLE }\end{array}$ & Z108 & PEAFOWL, BLACK SHOULDERED & $\mathrm{Z} 18$ & PEAFOWL, BLUE \\
\hline Z15 & PEAFOWL, BLUE INDIA & 2138 & PEAFOWL, BLUE PIED & $Z 288$ & PEAFOWL, BLUE SHOULDERED \\
\hline $\mathbf{Z 2 3 4}$ & PEAFOWL, BURMESE GREEN & 2137 & PEAFOWL, CAMEO & $\mathrm{Z} 130$ & PEAFOWL, CAMEO PIED \\
\hline$Z 283$ & PEAFOWL, INDIA BLUE & $\mathrm{Z} 128$ & PEAFOWL, JAVANESE GREEN & Z131 & PEAFOWL, OATEN \\
\hline
\end{tabular}


2174 PEAFOWL. PALAWAN

Z140 PEAFOWL, SPALDING

22 PHEASANT

232 PHEASANT, AMHERST WHITE

2266 PHEASANT, BLACK SHOULDER

2242 PHEASANT, BLUE MUTANT

Z264 PHEASANT, BUFF

Z33 PHEASANT, CHINESE GREEN

2177 PHEASANT. CINNAMON

Z236 PHEASANT, DILUTE

Z178 PHEASANT, FORMOSA

Z118 PHEASANT, GOLDEN RED

Z149 PHEASANT, GREY PEACOCK

254 PHEASANT, HUME'S BAR-TAILED

Z260 PHEASANT, IMPEYAN

257 PHEASANT, LADY AMHERST'S

Z104 PHEASANT, MELARCTIC BLACK

Z112 PHEASANT, MONGOLIAN

2243 PHEASANT, ORNAMENTAL

Z24 PHEASANT, REEVES'

226 PHEASANT, SILVER

2269 PHEASANT, TIMNEK

Z176 PHEASANT, WHITE JUMBO

Z102 PHEASANT, YELLOW

Z120 QUAIL, AUSTRIAN FAWN

243

286

$Z 170$

244

293

7.127

QUAIL, BOBWHITE

QUAIL, BOBWHITE TEXAS BLONDE

QUAIL, BOBWHITE, SOLID WHITE

QUAIL, CALIFORNIA VALLEY

QUAIL, COTURNIX

QUAIL, JAPANESE

QUAIL, MEXICAN SPECKLED

QUAIL. PHARAOH

QUAIL, TUXEDO

SWAN

SWAN, COSCOROBA

SWAN, WHISTLING

TURKEY

TURKEY, BELTSVILLE EXHIBITION

TURKEY. BLUE SLATE
$\mathrm{Z} 270$

Z19

Z244

$\mathrm{Z} 100$

$\mathrm{Z79}$

Z49

Z90

$\mathrm{Z} 27$

Z237

Z68

Z53

Z87

$\mathrm{Z248}$

Z69

Z265

Z67

Z105

Z31

$Z 136$

Z247

$Z 169$

Z126

Z259

Z8

278

Z173

Z235

$\mathrm{Z} 38$

259

Z39

Z89

Z74

Z45

$\mathrm{Z} 142$

$\mathrm{Z} 284$

2109

$\mathrm{Z} 124$

Z216

Z206

Z205
PEAFOWL, PURPLE

PEAFOWL, WHITE

PHEASANT, AFGHAN WHITE WING

PHEASANT, BLACK

PHEASANT, BLACK-NECKED

PHEASANT, BLUE-EARRED

PHEASANT, CALIFORNIA BUFF

PHEASANT, CHINESE RING-

NECKED

PHEASANT, COPPER

PHEASANT, EDWARDS'

PHEASANT, GIGI'S YELLOW

GOLDEN

PHEASANT, GOLDEN SALMON

PHEASANT, HIMALAYAN MONAL

PHEASANT, IJIMA

PHEASANT, ISABEL BUFF

PHEASANT, MANCHURIAN

PHEASANT, MELARCTIC BRONZE

PHEASANT, MONGOLIAN RING-

NECKED

PHEASANT. PIED

PHEASANT, RINGNECK JUMBO

PHEASANT, SILVER, GOLDEN

PHEASANT, TRAGOPAN

PHEASANT, WHITE RINGNECK

PRAIRIE CHICKEN

QUAIL, BELGIUM

QUAIL, BOBWHITE BUTLER GIANT

QUAIL, BOBWHITE, CUNNINGHAM GIANT

QUAIL, BRITISH RANGE

QUAIL, CHESTNUT BELLY

QUAIL, ENGLISH WHITE

QUAIL, JUMBO WISCONSIN

QUAIL, MOUNTAIN

QUAIL, TENNESSEE RED

QUAIL, VALLEY

SWAN, AUSTRALIAN BLACK

SWAN, MUTE

SWAN, WHOOPER

TURKEY, ARKANSAS RED

TURKEY, BLACK

TURKEY, BOURBON RED
Z274

$Z 289$

Z188

$Z 257$

Z281

250

$\mathrm{Z} 77$

Z121

Z51

Z52

$\mathrm{Z} 28$

$\mathrm{Z181}$

270

Z55

Z56

$\mathbf{Z 1 2 9}$

271

Z25

$Z 99$

Z268

$\mathrm{Z} 34$

Z233

$\mathrm{Z} 80$

$\mathrm{Z3}$

$\mathrm{Z} 72$

$\mathrm{Z172}$

Z148

Z107

Z180

$\mathrm{Z} 60$

240

288

273

2262

$Z 123$

$\mathrm{Z122}$

223

Z295

Z211

$\mathrm{Z} 212$
PEAFOWL, PURPLE PIED

PEAFOWL, WHITE SHOULDERED

PHEASANT, ALASKA SNOW

PHEASANT, BLACK MUTANT

PHEASANT, BLUE IDAHO

PHEASANT, BROWN-EARRED

PHEASANT, CHEER

PHEASANT, CHINESE RINGNECKED, BLUE BACK

PHEASANT, DARK THROATED GOLDEN

PHEASANT, ELLIOT'S

PHEASANT, GOLDEN

PHEASANT, GREEN

PHEASANT, HUME

PHEASANT, IMPERIAL

PHEASANT, KALIJ WHITE.

CRESTED

PHEASANT, MELANISTIC MUTANT

PHEASANT, MIKADO

PHEASANT, MUTANT

PHEASANT, RED-LEGGED

PHEASANT, SATYR

PHEASANT, SWINEHOE'S

PHEASANT, WHITE GOLD

PHEASANT, WHITE-WINGED

QUAIL

QUAIL, BLUE SCALE

QUAIL, BOBWHITE INDIANA GIANT

QUAIL, BOBWHITE, MASKED

QUAIL, BUTTON

QUAIL, CHUCKAR

QUAIL, GAMBEL

QUAIL, MANCHURIAN GOLDEN

QUAIL, NORTHERN BOBWHITE

QUAIL, TEXAS BLUE

SWAM, TUNDRA

SWAN, BLACK

SWAN, TRUMPETER

TINAMOU

TURKEY, BARRED

TURKEY, BLACK SPANISH

TURKEY, BROADWHITE LARGE

EXHIBITION 
OTHER BIRDS (Continued)

\begin{tabular}{|c|c|c|c|c|c|}
\hline 2202 & TURKEY, BRONZE & 2229 & TURKEY, DARK AUBURN & $\mathrm{Z} 221$ & TURKEY, DOMESTIC MERRIAM \\
\hline $\mathrm{Z} 182$ & TURKEY, EASTERN & $\mathrm{Z} 210$ & TURKEY, EXHIBITION & Z219 & TURKEY, JERSEY BUFF \\
\hline $\mathrm{Z} 215$ & TURKEY, KENT & 2220 & TURKEY, LILAC & Z209 & TURKEY, NARAGANSETT \\
\hline $\mathrm{Z} 214$ & TURKEY, NARAGANSETT GOLDEN & $z 213$ & TURKEY, NEBRASKA ROYAL BLUE & Z183 & TURKEY, RIO GRANDE \\
\hline 2204 & TURKEY, ROYAL PALM & $Z 218$ & TURKEY, SILVER & $\mathrm{Z} 207$ & TURKEY, SLATE \\
\hline 2227 & TURKEY, WHITE & Z217 & TURKEY, WHITE BRONZE & Z201 & TURKEY, WHITE HOLLAND \\
\hline $\mathrm{Z9}$ & TURKEY, WILD & $\mathrm{Z} 258$ & TURKEY, WILD EASTON & Z290 & TURKEY, WILD WHITE \\
\hline
\end{tabular}

Z61 TURKIN 
TABLE 5. RATITE BREEDS, VARIETIES, STRAINS, AND TRADE NAMES WITH THEIR CODE NUMBERS LISTED ALPHABETICALLY BY KINDS OF STOCK

O - OSTRICH

01 OSTRICH, BLUE NECK

$\mathrm{O} 2$

OSTRICH, BLUE-BLACK CROSS

$\mathrm{O} 3$

OSTRICH, RED NECK

O4 OSTRICH, SOUTH AFRICAN BLACK

P - EMU

Q - REA

U - CASSOWARY 

3

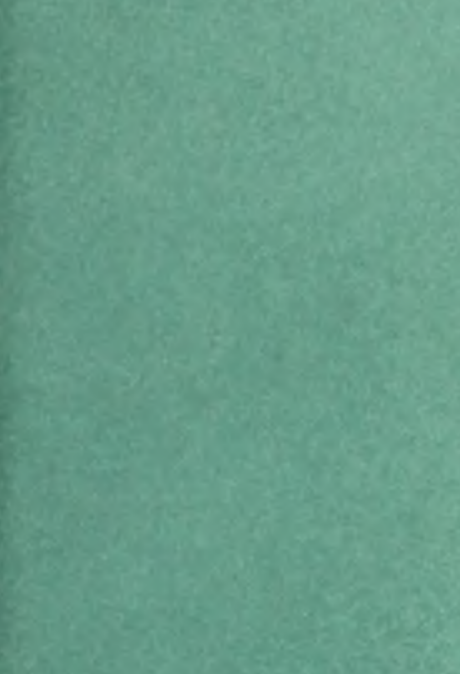

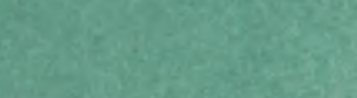

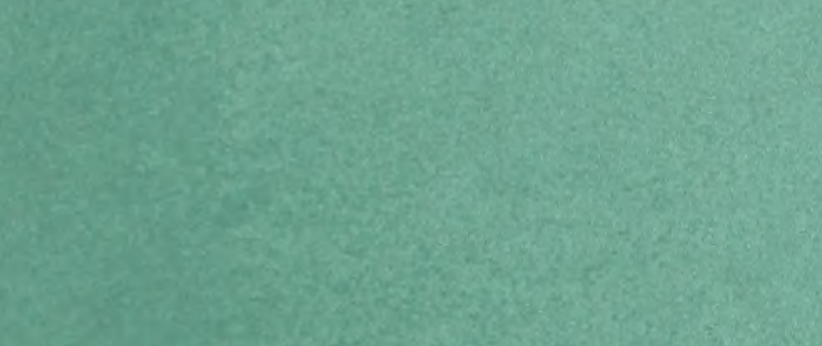

(5)

(3)

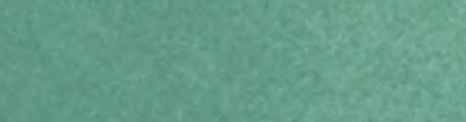

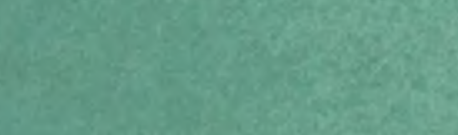

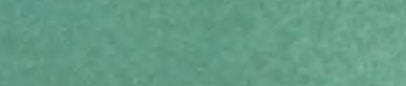

$c^{2}+a^{2} x^{2}$

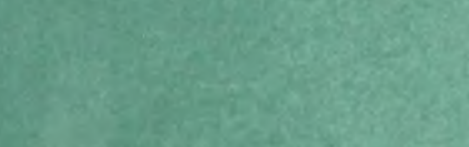

(x)

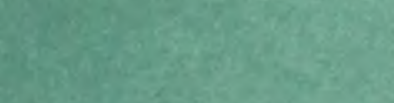

3.tons

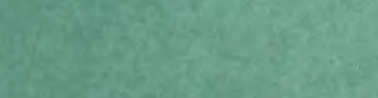

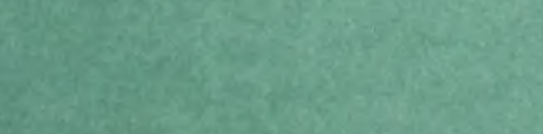

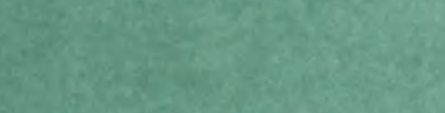




\section{United States}

Department of Agriculture

APHIS-VS

National Poultry Improvement Plan

Suite 200

1498 Klondike Road

Conyers, GA 30094

OFFICIAL BUSINESS

Penalty for Private Use, $\$ 300$ 